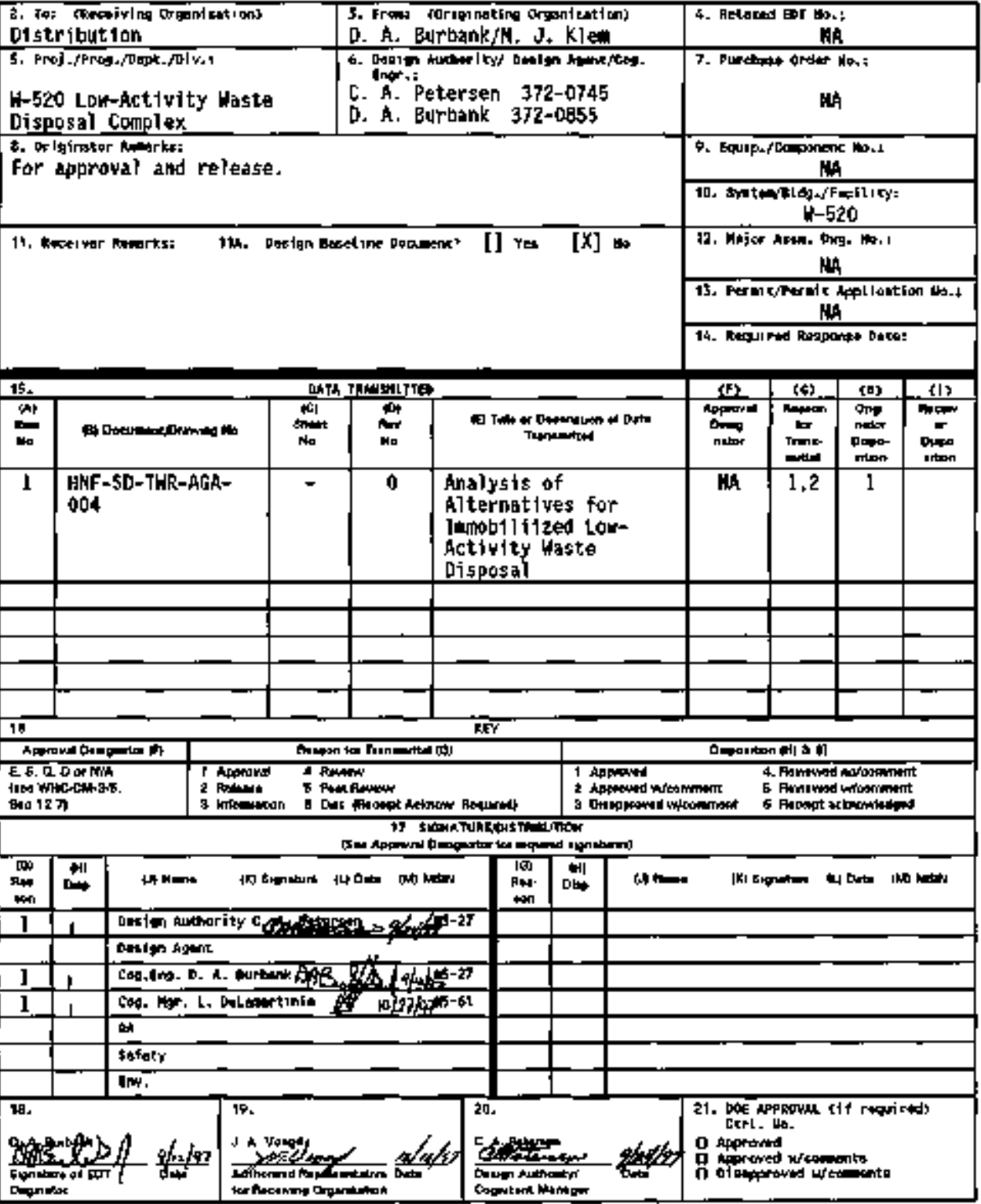




\title{
Analysis of Alternatives for Immobilized Low- Activity Waste Disposal
}

\author{
D. A. Eurbank ând H. J. Klon
}

5GN Eurisys Services Corporation, Richland, 99352

U.S. Dopartetent of Energy Contract of-ACO6-96RL13200

\author{
EDT/ECN: 622667 UC: 721 \\ Org Code: 51400 Charge Code: 04toxE \\ Bak Code: Ex\}130010 Total Pages: 109
}

Key Mords: Low-actirity wasto, ILAn, TuRS

Abstract: Th1s raport presents a study of altemative disposal system arcbitectures and limplewentation strateglos to provide onsite nearsurface disposal capacity to receive the imobilized low-actirity waste produced by the pritute vendors. The amalysis shors that a flexible unit strategy that provides a suite of design solutions tailored to the characteristich of the immobilized low-activity waste will provide a disposal systain that best neets the program goajs of reducing the enrirononta1, health, and safaty ingacts; meeting the schedule mijestenes; and mininizing the life-cycle cost of the program.

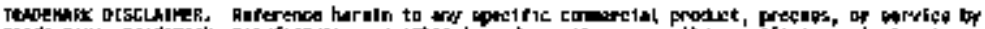

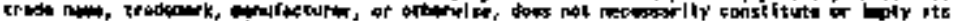

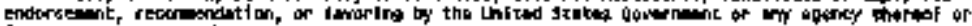

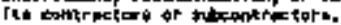

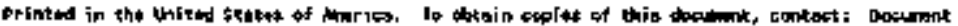

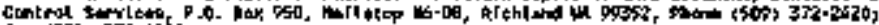

far $\{50 \%$ \} $376-40$.
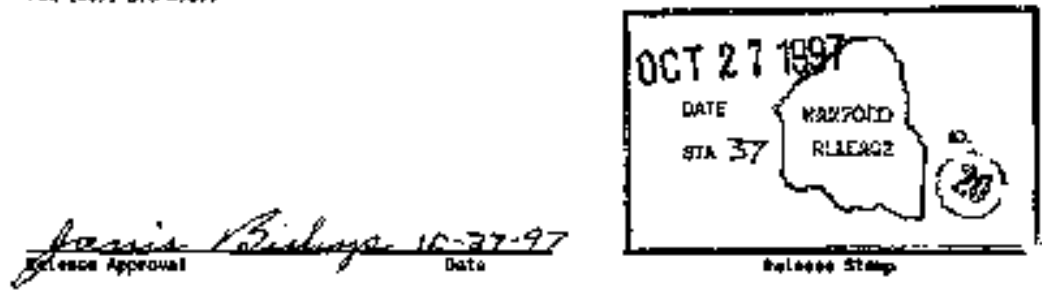


\title{
ANALYSIS OF ALTERNATIVES FOR IMMOBLIZED LOW-ACTIVITY WASTE DISPOSAL
}

September Is

\author{
D. A. Burbank \\ M. J. Klem \\ \$GN Eurisyb \$ervipta Corporation \\ Ricbland, Washington
}

\section{Prepared for}

U.S. Department of Eneryy

Richtand, Washington 
HNF-SD.TWR-AGA-O0A

Revision 0

Thls page inkentionally left blank. 


\section{HNF-\$D-TKR-AGA-D04}

Revision 0

\section{DECIYION ANALYSTS GUMMARY}

On Segrember 25, 1996, the U.S. Departuren of Energy andrded two contracts for the

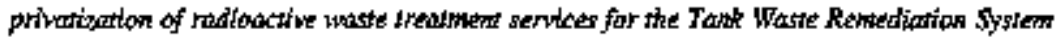
(TWRS at the Houford Site near Richiond, Washimgron. The priwatization schedide requiles the vendor to begin treannent of tow-activity waste in 2002. The THRS Eavironmental impact

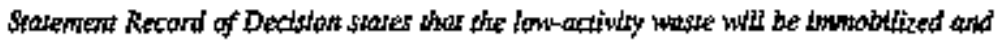
dispoted of onsite in nearsurface disposal facitities.

This roport presents a study of altemative disposal system anchitections ard inplementation strategies to provide onsite near-swface dispanal cappacity to receive the

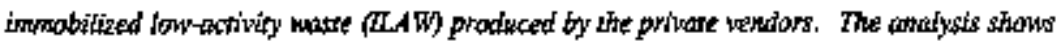
that a flextbite whit strategy that provides a sulte of design solutions taitored to the characteristics of the ILAW widt provide a disposal gystem that best weets the program gads of

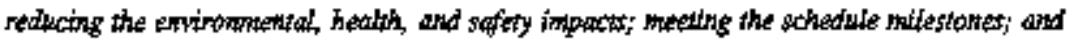
mintmizing the thereyte cost of the progrom.

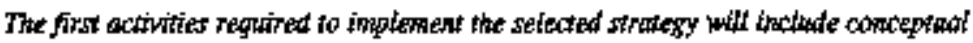

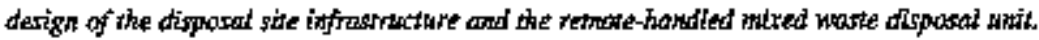
The completion of these conceptus designs witl make a conplate suite of design solations awallable for WAW disposal architectare selections to be made at a dater dott. 
HDF-SD-TWR-AGA-004

Revision 0

This page intentionally left blank. 


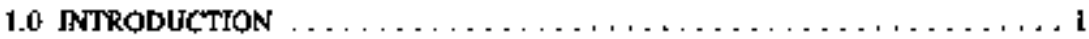

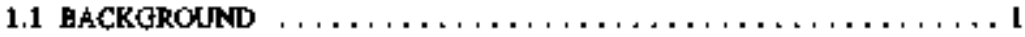

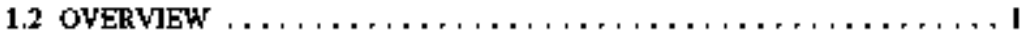

1.3 ARCHTTECTURE ALTERNATTVES $\ldots \ldots \ldots \ldots \ldots \ldots \ldots \ldots \ldots$

1.3.1 Site Locstion for Immobilized Low-Activity Waste Disposad Facility . . 3

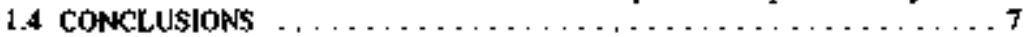

is RBCOMMENDATIONS $\ldots \ldots \ldots \ldots \ldots \ldots \ldots \ldots \ldots \ldots$

1.6 DMAOBILIZED LOW-ACTTVITY DISPOSAL ARCHITECTURE DECISION . 7

2.0 PROBLEM STATEMAENT $\ldots \ldots \ldots \ldots \ldots \ldots \ldots \ldots \ldots \ldots \ldots$

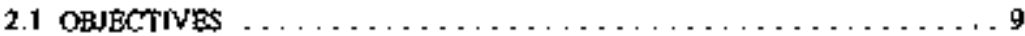

2.2 KEY I\$SUES . . . . . . . . . . . . . . . . . . . . . .

2.2.1 Waste Package Dose Rato . . . . . . . . . . . . . . . . 9

2.2 .2 Hazardous Waste Regolatory Stahla $\ldots \ldots \ldots \ldots \ldots \ldots \ldots \ldots$

2.2 .3 Disposal Autworization Schedne . . . . . . . . . . . . . . . . . 10

2.3 SCOPE AND BASTS . . . . . . . . . . . . . . . . . 10

2.3.1 Disposal Faceility Data For The Loterme Performance Atsestmenl . . . . J0

2.3.2 Tank Waste Remediation System Privatization Request for Proposals , I 1

2.3.3 Tank Waste Remediation System Fiscal Year 1997 Mnlti-Yoar Work Plan $\ldots \ldots \ldots \ldots, \ldots \ldots \ldots \ldots \ldots \ldots \ldots, \ldots \ldots$

2.3.4 Tank Waste Remediakion System Privationation Process Technical Easeling . . . . . . . . . . . . . . . . . . . . 11

2.3.5 Bopford Low-Level Tank Waste Interim Performance Assessment , . , 12

2.4 IMMOBILIZED LOW-ACTTVTTY WASTE DISPOSAL SYSTEM $\ldots \ldots \ldots, 12$

25 PROGRAMMATTC RISTK MINDMIZATTON $\ldots \ldots \ldots \ldots \ldots \ldots \ldots, \ldots$

3.0 CONSTRAJNTS AND ASSUMTTIONS $\ldots \ldots \ldots \ldots \ldots \ldots \ldots \ldots, \ldots, 1$ ?

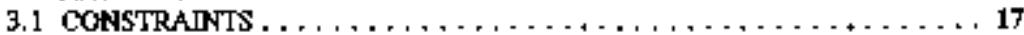

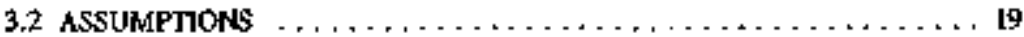

3.2.1 Immotilized Low-Aclivify Waste Prochuct Spexificalions $\ldots \ldots \ldots$ I9

3.2.2 Amound of Immobilized Low-Activity Waste $\ldots \ldots \ldots \ldots \ldots \ldots .20$

3.2.3 Radioactine Waste Regulabry Stalus $\ldots \ldots \ldots \ldots \ldots \ldots \ldots \ldots 20$

3.3 UNCERTAJNTES $\ldots \ldots \ldots \ldots \ldots \ldots \ldots \ldots \ldots \ldots \ldots \ldots \ldots \ldots, \ldots \ldots \ldots$

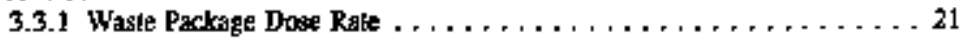

3.3.2 Hazardous Waste Regulatocy Stams $\ldots \ldots \ldots \ldots \ldots \ldots \ldots \ldots, \ldots \ldots$

3.3.3 Dispossal Authotimation $\ldots \ldots \ldots \ldots \ldots \ldots \ldots \ldots \ldots \ldots \ldots$

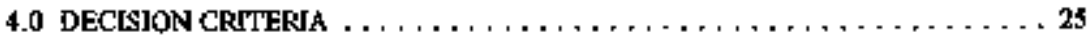

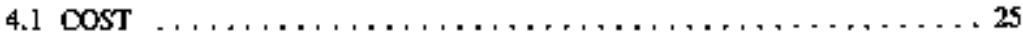

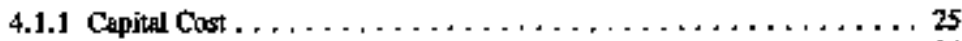

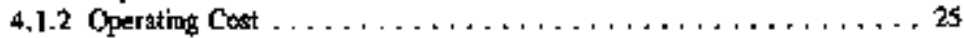




\section{HINF-SD-TWR-AGA-004 \\ Revision D}

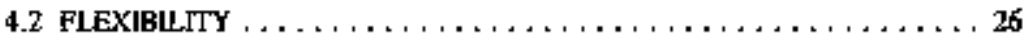

4.2.1 Multiple Waste Types $\ldots \ldots \ldots \ldots \ldots \ldots \ldots \ldots \ldots \ldots \ldots, 26$

4.2 .2 Waste Retrieval . . . . . . . . . . . . . . . . . . . 26

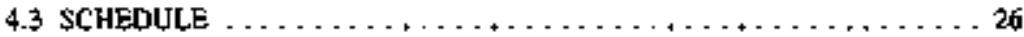

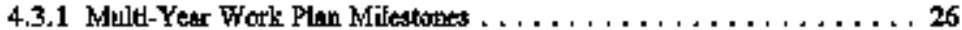

4.3.2 Tri Party Agreement Milestobe $\ldots \ldots \ldots \ldots \ldots \ldots \ldots \ldots \ldots$ 27

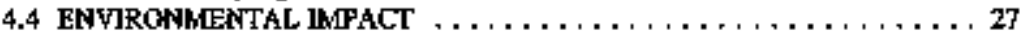

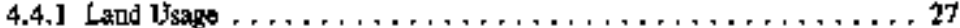

4.4 .2 Secondary Waste Generation $\ldots \ldots \ldots \ldots \ldots \ldots, \ldots, 27$

4.9 OPERATION, MALNTENANKE, AND SAFETY $\ldots \ldots \ldots \ldots \ldots \ldots, 27$

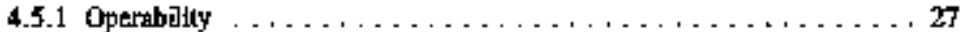

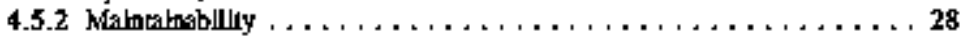

4.5 .3 Safety $\ldots \ldots \ldots \ldots \ldots \ldots \ldots \ldots \ldots \ldots \ldots \ldots \ldots \ldots, \ldots \ldots \ldots$

4.5.4 Radiation Expesure $\ldots \ldots \ldots \ldots \ldots \ldots \ldots \ldots \ldots \ldots \ldots \ldots$

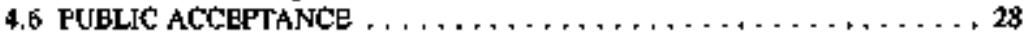

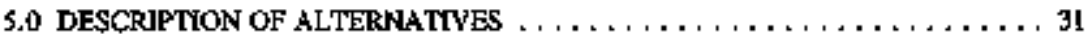

5.1 BASELINE WASTE DISPOSAL CONCEPT $\ldots \ldots \ldots \ldots \ldots \ldots \ldots \ldots$

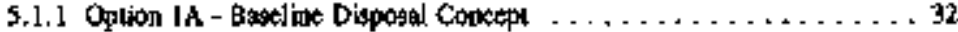

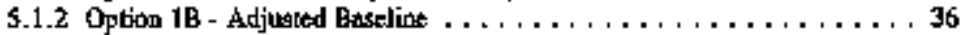

5.2 FLEXIBLE UNTT APPROACH $\ldots \ldots \ldots \ldots \ldots \ldots \ldots \ldots$

5.2.1 Option 2A - Remote-Handled Mined Waste Disposal Vaults , . . . . 39

5.2.2 Option 2B - Remote-Handled Nop-Mred Wate Dikpossl Vaults . . . 44

5.2.3 Option 2C - Contact-Haddled Mixed Waste Dlsposal Trenches . . . . 47

\$.2.4 Option 2D - Coutact-Hendled Non-Mixed Waste Disposal Trenches . . 51

5.3 OPTION 3 - SINGLE SHELL TANKS DISPOSAL $\ldots \ldots \ldots \ldots \ldots \ldots$ S5

5.4 OPTION 4 - EXISTING CANYON BULLDINGS $\ldots \ldots \ldots \ldots \ldots$. . . . .

5.4.1 Option $4 A$ - Canyon Euildings Entombment with Intenal Waste

Disposed . . . . . . . . . . . . . . . . . . . . . 60

5.4.2 Option 4B - Canjosn Buildings Entorabment soith Interanl and External Waste ........................65

\$.\$ OPTION $\$$ - EXPANDED INTERIM \$TORAGE . . . . . . . . . . . . . 69

$\$ .6$ OFTION 6 - SHELDED DISPOSABLE OVRRPACKS $\ldots \ldots \ldots \ldots \ldots$, . .

S. 7 REJECTED IMMOBLLZED LOW-ACTIVTTY WASTE DISPOSAL

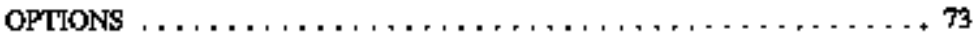

\$.7.1 Options Rejected by the Tank Waste Remediation System Einviroumestea Jmpact Statement $\ldots \ldots \ldots \ldots \ldots \ldots \ldots \ldots \ldots$

5.7 .2 Additional Rejected Disposal Options $\ldots \ldots \ldots \ldots \ldots \ldots \ldots$

6.0 ALTERNATIVES EYALUATION $\ldots \ldots \ldots \ldots \ldots \ldots \ldots \ldots \ldots \ldots$

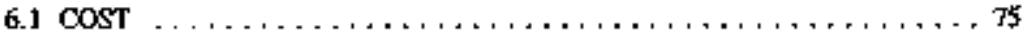

6.1 .1 Capital Cost $\ldots \ldots \ldots \ldots \ldots \ldots \ldots \ldots \ldots \ldots \ldots \ldots$

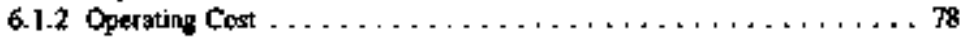

6.2 flexIBILITY $\ldots \ldots \ldots \ldots \ldots \ldots \ldots \ldots \ldots \ldots \ldots \ldots$ 
6.2 .1 Muttipte Waste Types $\ldots \ldots \ldots \ldots \ldots \ldots \ldots \ldots \ldots \ldots \ldots$

6.2 .2 Warte Ketrieval $\ldots \ldots \ldots \ldots \ldots \ldots \ldots \ldots \ldots \ldots \ldots \ldots \ldots$

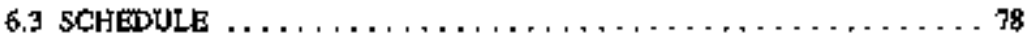

$6.3,1$ Multi-Year Wot Plan Wilestones . . . . . . . . . . . . . . 79

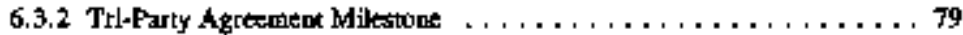

6.3 .3 Privatization Schedule $\ldots \ldots \ldots \ldots \ldots \ldots \ldots \ldots \ldots \ldots \ldots$

6.4 ENVIRONHENTAL IMPACT $\ldots \ldots \ldots \ldots \ldots \ldots \ldots \ldots \ldots \ldots$

$6.4,1$ Land Usage $\ldots \ldots \ldots \ldots \ldots, \ldots \ldots \ldots$

6.4,2 Secondary Wadte Generation . . . . . . . . . . . . . B1

6.5 OPERATION, MAINTENANCE, AND \$AFETY . . . . . . . . 81

6.5 .1 Operability . . . . . . . . . . . .

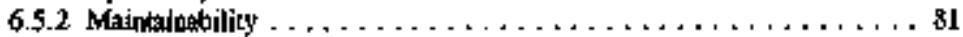

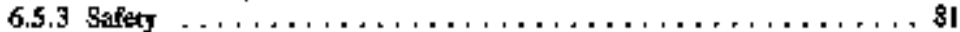

6.5.4 Radiatten Exposure $\ldots \ldots \ldots \ldots \ldots \ldots \ldots \ldots \ldots \ldots \ldots \ldots$

6.6 SUMMARY AND CONCLUSIONS . . . . . . . . . . . . . 84

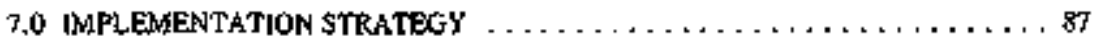

8.0 OPEN ISSUES AND ACTIONS $\ldots \ldots \ldots \ldots \ldots \ldots \ldots \ldots \ldots \ldots, \ldots, \ldots 1$

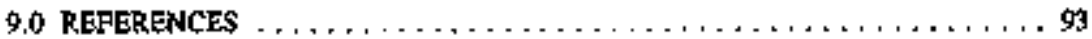

\section{APPENDTKES}

A - COST ESTIMATING DATA $\ldots \ldots \ldots \ldots \ldots \ldots \ldots \ldots \ldots \ldots$ A-I

B - CONSTRAINTS ON DESIGN OF THE IMROEILIZED LOW-ACTIVITY

WASTE DISPOSAL FACILITY $\ldots \ldots \ldots \ldots \ldots \ldots \ldots \ldots \ldots$.1 
HNNE-SD-TWR-AGA-D04

Revision 0

This page intentionally left blank. 


\section{HNTF-SD-TWR-AGA- 004}

Revisision 0

\section{LSTT OF FIGURRS}

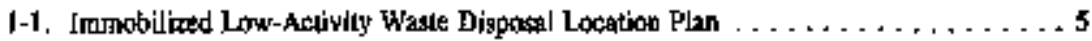

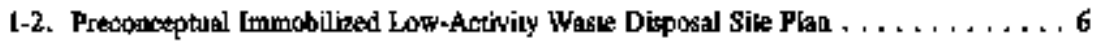

2.1. Inmobilized Low-Activity Waste \$lorage and Dieposal Logic $\ldots \ldots \ldots \ldots \ldots$ is

5-1. Dispospl Facility Concept Basejine - Option $14 \ldots \ldots \ldots \ldots \ldots \ldots \ldots$ 33

5-2. Cross Section of Baselize Disposal Facility Concept $\ldots \ldots \ldots \ldots \ldots \ldots \ldots \ldots$

5-3. Ressore-Handled Mired Wrate Disposal Facility Concept . . . . . . . . . . 40

54. Cross Section of Closed Immobilized Lon-Aclivlly Waste Disposal Unil for

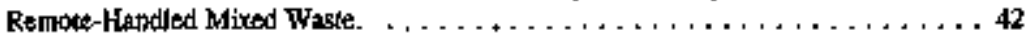

5-5. Remote-Handled Non-Mixed Waste Dispesal Facility Concept $\ldots \ldots \ldots \ldots, \ldots, 46$

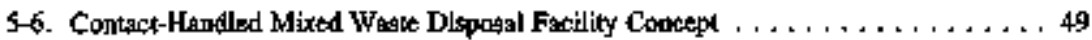

5.7. Contach-Handled Non-Mixed Waste Disposal Facility Concept . . . . . . 52

5-8. Arrangemenr of Immohilized Low-Activity Waste Packages in an Empty Tank . . . 56

\$-9. Canyon Building Entomboment with Internal Waste Dlsposal . . . . . . . . 6 61

5-10. Canyon Buildings Entombment with Internal and External Waste Disposal . . . . 66

\$11. Dispossble Oveppact Concepts . . . . . . . . . . . . . . . . 72

6-1. Totral Lift-Cycle Cost Versus Renote-Handled Fraction. , . . . . . . . . . Tt

7.1. Immabilized Lon-Activity Waste Disposal Decision Trac . . . . . . . . 88

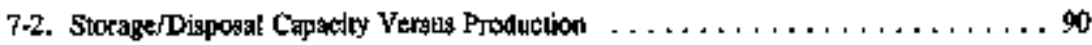


HNF-\$D-TWR-AG/A-004

Revizion 0

This page inemtionally left blank. 
HNF-5D-TWR-AGA-004

Revision: 0

\section{LET OF TABLES}

1-1. Summary Misstion Requirements. $\ldots \ldots \ldots \ldots \ldots \ldots \ldots \ldots \ldots \ldots$

1-2. Summary of Immobilized Low-Activity Waste Dieposal Alternatives $\ldots \ldots \ldots \ldots$. 3

2-1. Dezign Requirements by Immobilized Low-Activity waste Type $\ldots \ldots \ldots \ldots \ldots$ Is

3-1. Key Consuraints For Jpmobilized Low-Activily wabe Disposal Pacility , . . . . . . IT

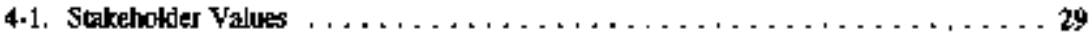

5-1. Summary of Estimated Costs For Desigh, Construction, and Closure of the Bascline Immobilized Low-Actiyity Wase Disposal Facility (Option 1A) , . . . . . . . . 35

5-2. Sundiary of Estmated Custs For Conaroction atod Closure of the Adjosted Baseling Immobilized Lon-Activity Waste Dispogal Facility (Option 1B) . . . . . . . . . . 36

5-3. Eslimaled Operatiog Cosss for Imurobilized Lom-Activity Waske Storage and Dłsposa[ Based on 1997 Mulij-Year Work Pan Baseline (Option 1A) . . . . . . . 37

54. Estimated Operating Costs for Immobilized Low-Activity Waste \$torage and Disposal Based on 1997 Multi-Year Work Plan as Adjusted for Throughput (Option IB) . . . 38

5-5. Summary of Estimated Cepital Cods For Diposal of Remote-Handled Mired Waste (Opton 2A)

5-6. Estimated Dperating Costs fox Disposal Of Remole-Handled Mixed Waste (Option 2A) . . . . . . . . . . . . . . . . . . . . . 4

5-7. Summary of Estimatad Capital Costs For Disposal of Rernote-Findled Non-Mixed Wagk (Option 2B) $\ldots \ldots \ldots \ldots \ldots \ldots \ldots \ldots \ldots \ldots \ldots \ldots$

5.8. Estmated Operating Cosus for Dlsposal Of Remole-Handled Non-klked Waste (Option 2B) $\ldots \ldots \ldots \ldots \ldots \ldots \ldots \ldots \ldots \ldots \ldots \ldots \ldots \ldots \ldots$

5-9. Summary of Estimated Capital Conts For Desposal of Combact-Handled Mixed Waste

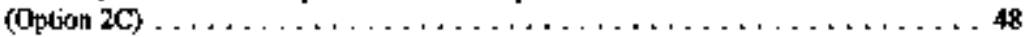

5-10. Estimated Optrating Coses for Disposal of Contact-Fandled Mixed Waste (Option 2C) $\ldots \ldots \ldots \ldots \ldots \ldots \ldots \ldots \ldots \ldots \ldots \ldots \ldots \ldots$ so 
HINF-SD-TTR-AGA- 004

Revigion o

LLST OP TABLES (CONTINUKO)

5-11. Summary of Estimared Caplel Costs Fơr Dispogal of Conkpct-Handled Non-Mixed

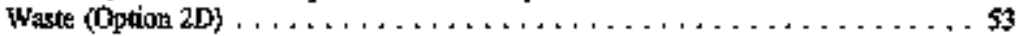

5.12. Estimaled Operating Cosk for Disposal of Contact-Hatrdled Non-Mixed Waste

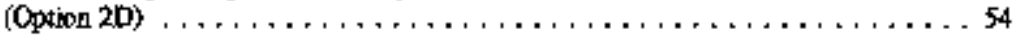

5-13. Capacity of l00 Series Simgle-Shell Tanks for Immobilized Lonv-Aclivity Wast

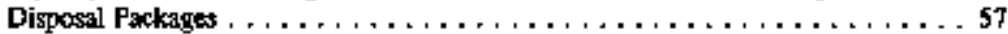

5-14. Availability of 100 Series Stagle-Stell Tanks for Potental Ditgonsal of Imrobilimad Low-Activity Waste $\ldots \ldots \ldots \ldots \ldots \ldots \ldots \ldots \ldots \ldots \ldots, \ldots \ldots$

5-15. Surbmary of Rough Order Magnitude Capital Cost fror Addition of a Large Opening to Dome of 100 \$eries Single-Shall Tarks (Option 3) . . . . . . . . s s9

5-16. Sunmary of Potential Remode-Handled Immobilized Low-Axcivity Waste Disposal Inside Canyon Buildings

5-17. Summary of Estiraated Cosss Poe Construction and Closure of Four Canyon Buildings for Disposal of Imnobilized lout-Activity Waste and Other Haste

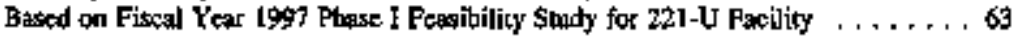

5-18. Summary of Estlmated Operatlog Costs For Disposition of Fourt Canyon Butldiugs Eased on Internal waste Disposal (Option 4A) $\ldots \ldots \ldots \ldots \ldots \ldots \ldots$, f

5-19. Summary of Potential Immobilized Low-Actisity Wasle Disposal Inside and Oulside Camyon Bunldings $\ldots \ldots \ldots \ldots \ldots, \ldots \ldots \ldots, \ldots \ldots$,

5-20. Summary of Estimated Capital Costs for Carryon Buildings With Iattrinal and External Dispesal (Option 4B) . . . . . . . . . . . . . . . . . . . . 6 B

5-21. Surnorary of Estimared Operaling Cosas Fur Disposition of Four Canyon Buikdings Based on Internal and External Waste Dispoxal (Option 4B) $\ldots \ldots \ldots \ldots \ldots 68$

5.22. Summary of Estinated Costs For Contruction and Closure of Unita for Interim Storage of Contact-Handled Mrxed Inmabilized Low-Acrivity Waste Containers Babed an Project W-112

5.23. Estimated Operating Costs for Immabilized Low-Activity Waste Storage Based on Preliminary Operatung Sequence for Contajoer Transpoxtation $\ldots \ldots \ldots \ldots \ldots$ T0

5-24. Summary of Capitai Cost Estirnate for Oisposable Overpacks . . . . . . . . . 7I 
HNF-SD-TWR-AGA-004

Revision 0

\section{LIST OF TADLES (CONTINUED)}

6-1. Capital, Operating, and Life-Cycle Cout Summary $\ldots \ldots \ldots \ldots \ldots \ldots \ldots \ldots$.

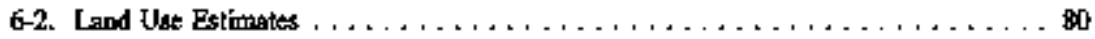

6-3. Radiation Exposure Estimale tor Trabeporting Remote-Handked Immobilized Low. Activity Waste Contsitiers to Disposal Bayed an Transportation Requitements . . . . 82

64. Radítlon Expouste Estimate for Transporting Condact-Hatedled Inmobllized LotwActvicy Waste Coutainers to Storage or Disposal Based ou Transportatton Requirements . . . . . . . . . . . . . . . . . . . . . . 83

6.5. Summary Evalvation Matrix $\ldots \ldots \ldots \ldots \ldots \ldots \ldots \ldots \ldots \ldots, \ldots \ldots$ 
HNF-SD-TWR-AGA-004

Reviagon 0

Thit page intentlonally left blank. 
HNF-\$̣D-TWR-ACA-004

Revision 0

\section{ACROWYMS AND ABRREVLATONS}

AGA

ALARA

CERCENA

CFR

$\mathrm{CH}$

DOE

DST

Ecology

EIS

ETP

EPA

ERDF

FR

PSAR,

HWF

HPT

JLAW

IPA

LAW

LLW

MW

MYWP

NMW

NRC

PA

PFTB

PUREX
Alternatives Genteration Analysis

As Low As Reasombly Achievable

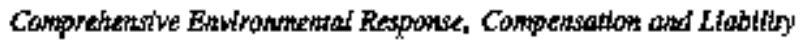
Act of 1980

Code of Federal Regulationts

Conlact Handled

U.S. Depsisment of Energy

Druble-shell ank

Wakington State Department of Ecalogy

Ewronmencal Impact Statement

Extutent Treatment Facilizy

U.S. Environmenulal Protection Ageacy

Enrironmental Restoration Disposal Facility

Federal Register

Final Safety Analysis Report

Harardous Waste Identification Rule

Health Physics Technician

Immobilized low-activity waste

Interim Performance Asoessmetrir

Low-activily waste

Low-level waste

Mixed Waste

Multi-Year Work Plan

Non-Mixed Wagte

U.S. Nuclear Regulatory Commission

Performance Assossment

Privatization Procegs Technical Baseline

Plntoniuntr-Utandum Extraction Plant 
HNE-SD-TWR-AGA-004

Revision o

\section{ACRONYMS AND ABZREVIATIONS (COATTNUEO)}

RCRA Reroxuce Conservation and Recovery Act of 1976

RFP Privatization Request for Prepposals

RH Reurote Handled

ROD Record of Decision

ROM Rough Onder of Magnitude

SST Simgle-shell tank

TEDF Trealed Effluent Discharge Factifity

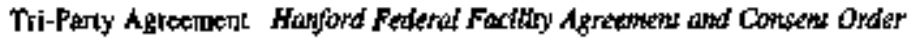

TWRS Tank Waste Remediation System

WAC Washington dethinistrative Cade

WHC Westinghouse Hanford Company 
HONF-SD-TWR-AGA-004

Revisiogn 0

\section{ANALYSLS OF ALTERNATTVES FOR MOMOBDUZED LOW-ACTIVITX \\ WASTE DISPOSAL}

\subsection{INTRODUCTION}

This report presents descriptions and analysis of altornative syotem archilectures for

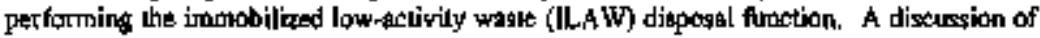
programmatic uncertalntices and their impacts on the disposal system requirements is also presented. An moplementation strategy for cost-effectinely responding to changining requirements and programmatic uncertainties is proposed.

\section{J.1 BACKGROUND}

It September 1996, the U.S. Department of Enengy (DOE) awarded two contracts to privalize the treatment and immobilization of Hanford Sice tank waste. The Tank Wagte Remediation Systers (TWRS) privatization stralegy is described in the privatization Request for

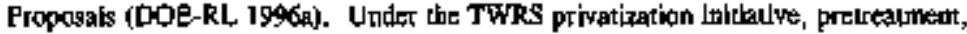
cadionuclide separationg, end immobilizallon will be perfonmed in two pheses by private contractors for the U.S. Departmenl of Energy. In Phase LA of privatimation, vendors wero selected to establish the tectinical, operational, regulalory, and finaccial elements of the privatized services program. In Ptsque [B, the selected vendor(s) will perforer demonstraltont scale troatment of 6 percent to I3 percent of Hanford \$ite tank waste in facilities that are

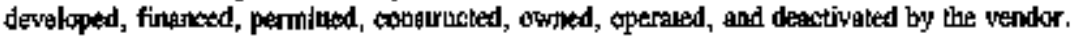
Phase Il would coulimue privtaltzed trealment of the remainder of the tank waste at higher throughput rales in full-scale facilitis.

The TWRS Emvironmental Impart Statemend (TWRS EIS) (DCE 1996) stares wat all

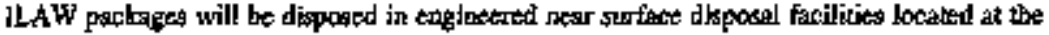
Hanford Site. The disposal facilities will be contered will a surface protective barrier durm clostre $\omega$ inhibit reoisture lofilutuation, minimize transport of contaminats to the ground water, and mitigate intrusion from plants, apimals and hemans. Monitoring will contiate for at least 10 yearg during the post closure care period.

\section{OVDRVIEW}

The mlsslon of the TWRS LAW disposal project is to receive, transport, store, and dispose of treated low sctivity tank waste in a safe and environmentylly sound mamer. It 
iscludes anoitoring, closere, and post closure mronizoring af the disposel facility. Disposal ghall be in a near-surfact facility on the Hanford gile.

The ILAW disposal program sehectule shows disposal capacity w be required beginning

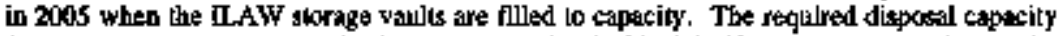
for the maximum order quanlity in Phase I (ending in 2011 ) is 13,200 packages. The total reacnired disposal sybtem cepacily is projected to be 98,200 packages, with ILAW package restipt to be completed by 2025. Table 1-1 summarbed the disposal program mlssion. requirements.

Table 1-1. Summary Mission Reyuriements.

\begin{tabular}{|c|c|c|c|}
\hline ltem & Phase I & Phase ll & Tatal \\
\hline Hot Operations Slart Date & $12 / 31 / 02$ & $1 / 3 / 12$ & $12 / 31,002$ \\
\hline Hot Operations End Date & $12 / 30 / 11$ & $7 / 31 / 25$ & $7 / 31 / 25$ \\
\hline Post-closure Monitoring Start & & & $B / 1 / 2 S$ \\
\hline Post-closure Monitoring End & & & $4 \sqrt{28 \sqrt{34}}$ \\
\hline Nominal Waste Package Coant & 13,200 & 85,000 & 98,200 \\
\hline Nominal Package Receint Rate & 4 per day & 17 per day & \\
\hline Pzalk Package Receipl Rate & 9 pet day & 27 per day & \\
\hline Nominal Waste Package Size & \multicolumn{3}{|c|}{$1.2 \mathrm{~m} \times 1.2 \mathrm{~m} \times 1.8 \mathrm{~m}=2.59 \mathrm{~m}^{3}$} \\
\hline
\end{tabular}

The primtaty gords of the lLAW digaseal progetam (Armacost at al. 1994) are as folkows:

- Protect public and workes health and safety

- Minimize capital costs

- Minimiza operating cossts

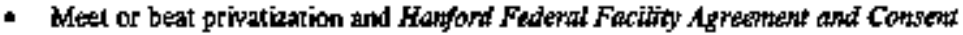
Order (Tri-Party Agreement) (Ecology et al. 1996) schedule milestones

- Mindmiza environmenatal intopats

- Enable fatore abe options. 
The objective of this atudy is to select a dighost architechre and implementation strategy that minimbes the rlsk of not achleving these prostammalic goals. This atudy wra performed prior to the reles of the Iittel Performance Assessment (PA) or Final Safty Aratygig Report (FSAR) for ILAW dispesal. The conchusions of this study could be affected by the results of the PA or FSAR.

\subsection{ARCHITECTURE ALTERNATTYES}

Architectore alternatives for disposal of ILAW were developed by revlewing the exising soutce documents described in Section 2.3. Surveys of existing radtwaste disposal woils were also consulted to determine the common feames of [LAW disposal facilitics (Han et al, 1997, Gerton et al. 1995. Templekn et al. 1994). Those stodies confintmed the decíaion of the TWRS EIS that the disposal sile should be a near-wurace faciliry on the Hanford Site.

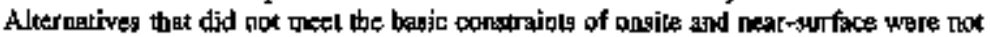
considered in this atralyais. Table 1-2 summarizes the ILAW disposil architectures that wrese developed and analyzed in wis sturdy.

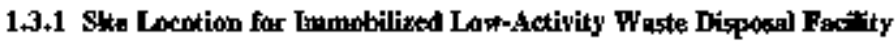

The location of the LAA disposal facilify will be on 90 acres of unconaminated, vacans land in 200 East Area and soutbwest of PUREX (\$hord 1995, Reidel et al. 1995). This afgpogal sile kocation was part of the TWRS complex lagout that ranked the highest and had the most desicable features. DOE has poncurred with the recomnended site location (Rutherford 1997). Figures I-I and 1-2 depict the ILAW depoosal sive location plan and a preconceptupal site plan (based on the option that would use the largest land area) of the propored dispospal location.

Table 1-2. Summary of Immobllized Low-Actirlty Wase Dlsposal Aliernatlyes. (2 \$̦bets)

\begin{tabular}{|c|c|}
\hline Alternative & Descriolion \\
\hline $\begin{array}{l}\text { Buscelln: } \\
\text { Optron IA }\end{array}$ & 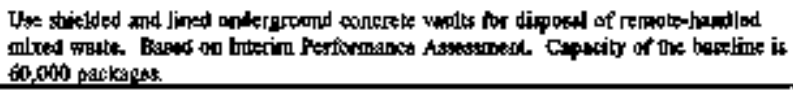 \\
\hline 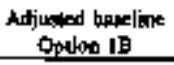 & 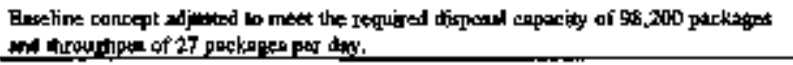 \\
\hline 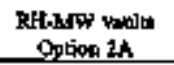 & 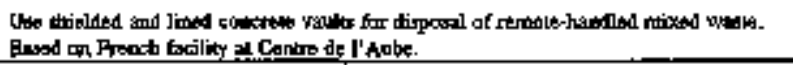 \\
\hline $\begin{array}{l}\text { RH-ILW Yeuk } \\
\text { Option } 2 \mathrm{~B}\end{array}$ & 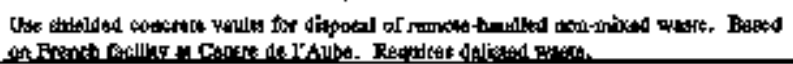 \\
\hline
\end{tabular}




\section{Tabie 1-2. Summary of Immobjlized Low-Actrvity Waste Disposal Alterinatues} (2 Shats)

\begin{tabular}{|c|c|}
\hline Alternalrve & Descriptepn \\
\hline 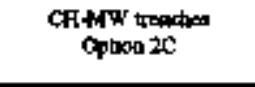 & 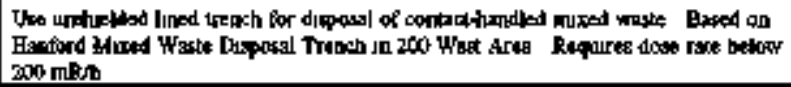 \\
\hline 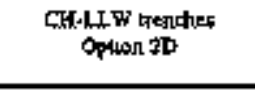 & 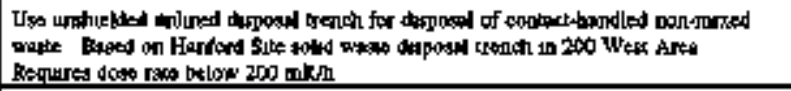 \\
\hline 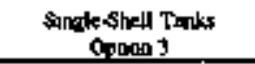 & 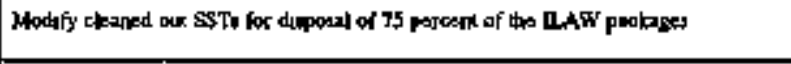 \\
\hline 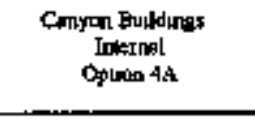 & 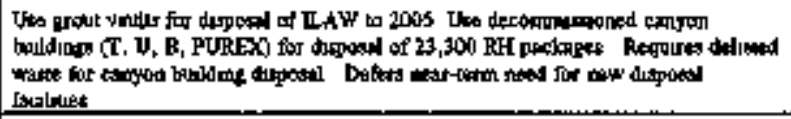 \\
\hline 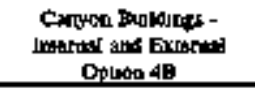 & 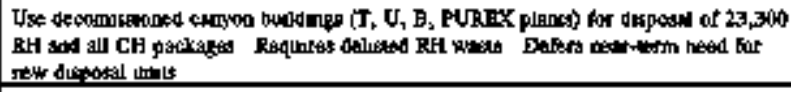 \\
\hline 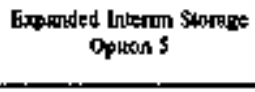 & 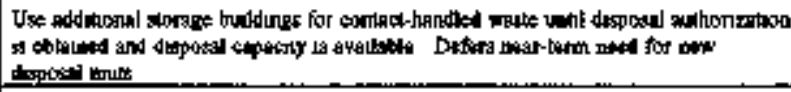 \\
\hline 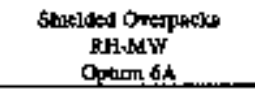 & 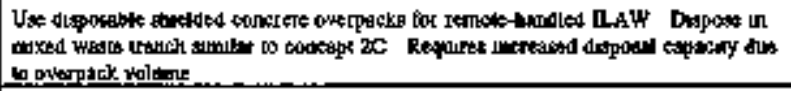 \\
\hline $\begin{array}{l}\text { Stotided Orespocks } \\
\text { RB-LLW } \\
\text { Optop } 6 \mathrm{~B}\end{array}$ & 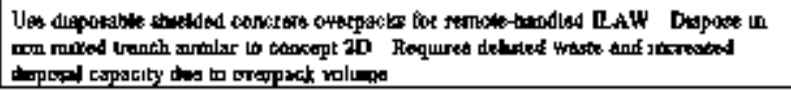 \\
\hline
\end{tabular}

cH - coeara Hindela

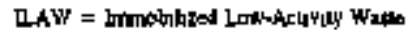

LLW = Lom-leved Woute

MW - Broed Whits

RH $=$ Reaure Hamlad

SST $=$ Singleastenl lonks 
Pigure l-1. Immobilized Low-Activity Waste Disposal Location Plan.

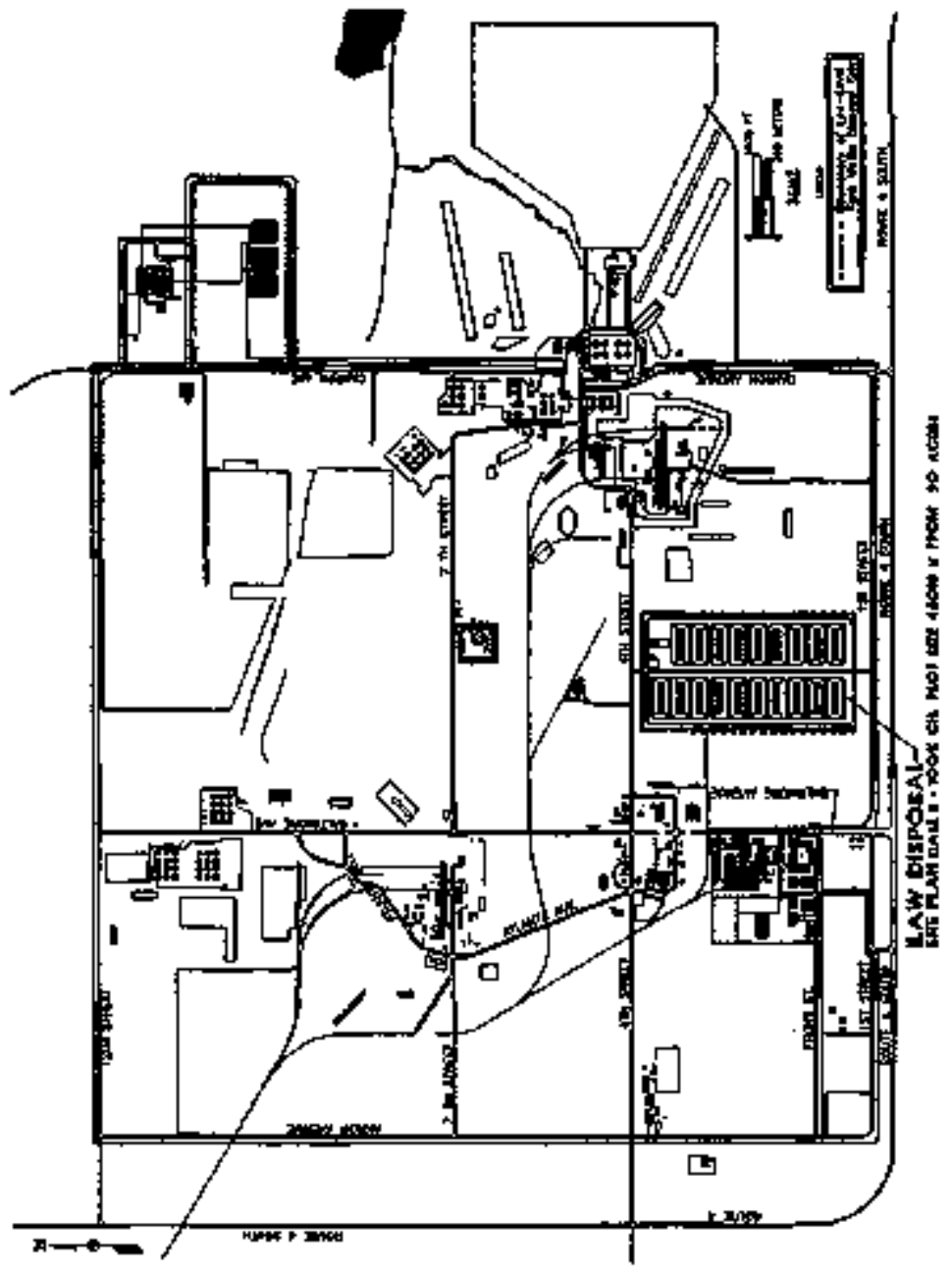


HNF-SD-TWR-AGA-004

Revigión 0

Figure 1-2. Preconoeptuel Itrmobilized Low-Activity Waste Disposal Sile Plan.

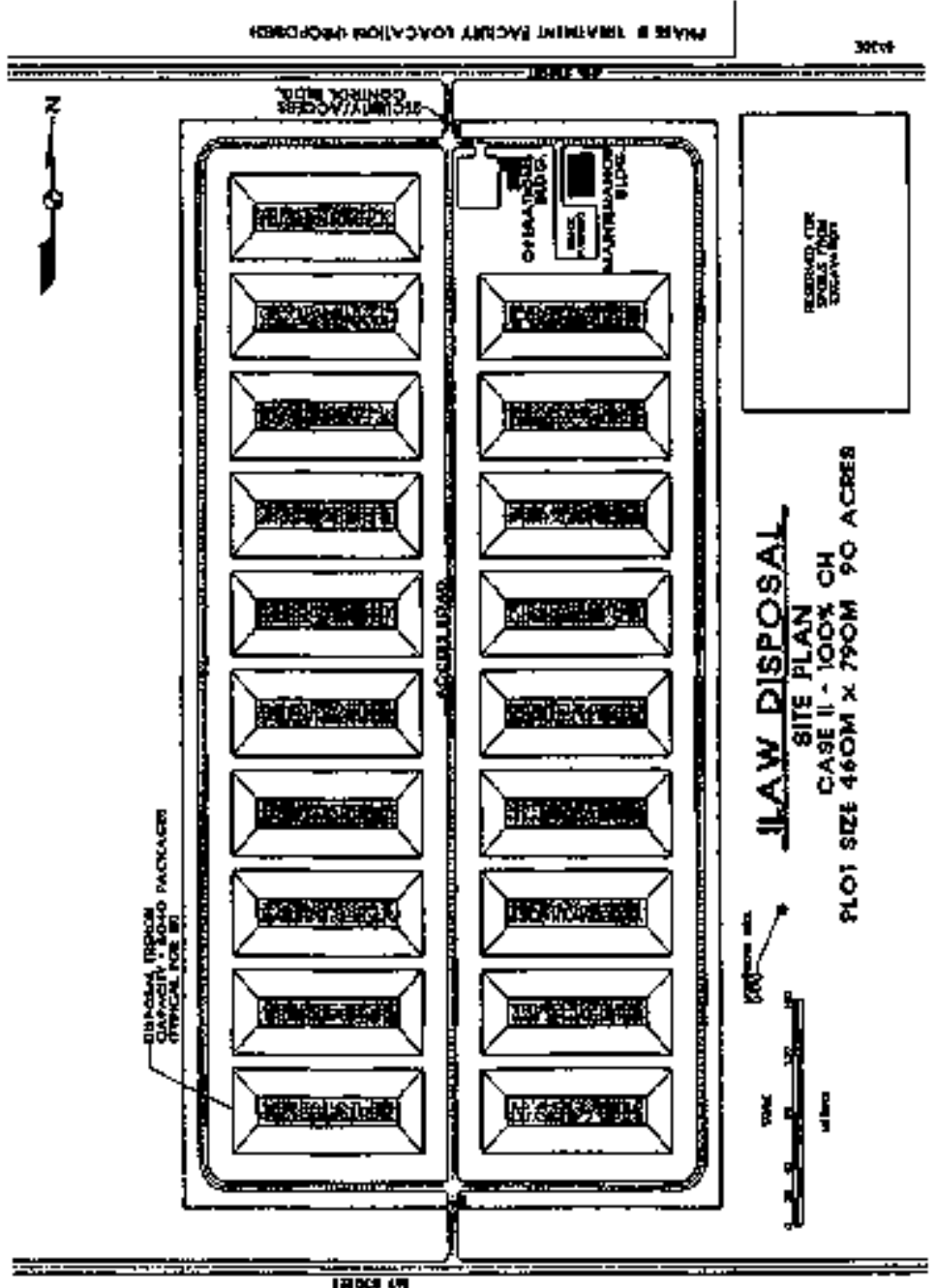




\section{Revision 0}

\subsection{CONCLUSHONS}

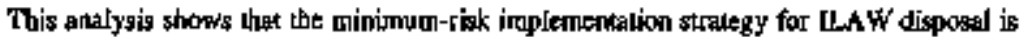
the flexible unit alternaliye. The flexible unit altaroative provides a suite of disposal unit destgn solutlons that meet the requiremems dictaved by the chiracteristics of the ditfetent ILAW waste types that may be recejed from the tank waste tetituent vendors. The stratedy provides a series of manageable subprojects that: (I) prowide the disposel system. infraglructure, (2) deploy multiple modolar disposal units taikred to specific [LAW uraste lypes, and (3) construct surface banrier closure systems o prevent intrusion by water, homans, plants and antmals. The staingy providio ILAW disposal capacity on a just-in-time schedule, with the flexibility to aljnst the mumber and types of modular disposal units based of operating experience andior changes in the regisinements for ILAW dipposal.

\subsection{RECOMAENDATJONS}

Due to the uncertainties sarrounding the characteristics of the ILAW packages, the flexible anit girategy should be selected for implementation. The rexi activity required to

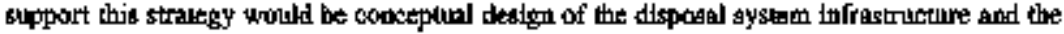
remote-handled miked wasce disposal unit. Conceptual destgen of this unit woukd provlde information needed to validate both Options 2A and 28. Degigo information for existing facilities is sufficient on servo a condeptual design basis for the other toro types of disposal unit (Options 2C, and 2D) that may be deployed under this otategy. Conceptual design of a

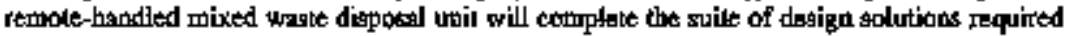
to implement the strategy.

\subsection{MAMOBILIZED LOW-ACTIYTYY DISFOSAL ARCFIECTURE DECISION}

This document doss not cotalan a decision on the ILAW disposal system archilecture. A decision board wag courvened in early hay 1997. The decigion plan for this activity (Petersen. 1997) stakes was DCE concturence on the decisjon will be requested by September 30, 1997. 
HNF-SD-TWR-AGA-004

Rayision 0

This page imtentionatly keft blank. 


\section{Rerision 0}

\subsection{PROBLEM STATEMENT}

Thls section describes the problem statebtet fot this analys the analysis, key issues identified during the antlyals, the source documents uped for the anslysis, and the approach used to develop the analysis.

\subsection{OBJECTTVES}

The problets statement for this shody is: What lype of engineted near-surface dlsposal faciliry and implementation surategy would mininize the programmotle rlsks of the JLAW disposal program?

The abjeclives of the analyzis are twafold. Firsti, select an implementation stratcoy that

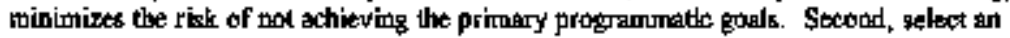
archnecure for the disposs system that flus wilth the implementation strategy.

\subsection{KEY ISSUES}

This section describes the key issues that have been identified during the course of thes analysis. The course of action that will be taken during deploynent of the ILAW djsposal facilities dopends on the regolution of these isues.

\subsubsection{Waste Package Doee Rovt}

Previons studies addressing ILAW disposal bave all been predicated on the assumption that the IL,AW woukd be remote handled and disposed in sbielded below grownd vapits

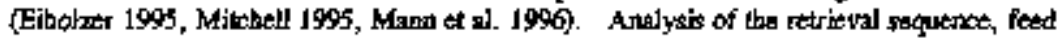
batch composirions, waste package speciffachons, and stelelding calculatons ited deate that the may not be pexessary for all of the RLAW. The likelibood that remote handing and shielded fricilities may nol be required for a sippinicanl fraction of the ILAW provides an opportusity for substantial cost gavings ower the life of the dipposal program.

\subsubsection{Earaxdous Wapte Refolatory Status}

Under currenl errironmental regulations, Hanford tank wagte and the residus from its treatroent (e.g, the ILAW product) fall uader the purview of the Resource Congernation and Aecovery Act of 1976 (RCRA). The privatization contracts provide incentiva for production of ILAW that is mot regulated ats a RCRA waste. Addillotrally, the U.S. Envitonimental Protectlon Agency (EPA) has proposed tew moles (EPA 1995) that would allow listed wastes 


\section{HNF-SD-TWR-AGA-OQ4 \\ Revision 0}

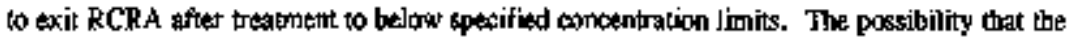
ILAW product may not be subject os RCRA requirements provides an opportunity for additional cost savings over the Iife of the disposal program.

\section{2,2,3 Dtwposs Anthorizstipn Schedule}

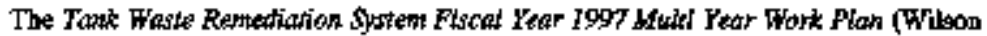
1996) shows additional disposal capacity becorving ayailable in 2005 or about 1 year before

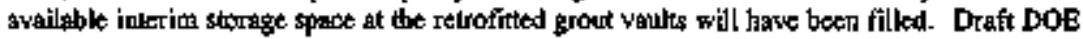
Order 435.1, Rasloocilve Waste Btonggennent, which will replate DOE Onder 5820.2A, regulres that the program receive a disposal authorization slatement based on an approwed PA and composite analysis for each disposal facility privor to starl of consirnction. The chatiging requirements for disposad suthorization and required approvals coukd adversely binpact the implementation scheatule for ILAWW disposal, and conold result in the need for additional interim skorape facilitites.

\subsection{SCOPE AND BASIS}

The scope of this aralysis is the ILAW Disposal System, atchfiecure elemeat L.S.2 of the TWRS systems engineering database. This architechure performs fubction 4.2.4.2.4, Dispose of Imtrobilized Lon-Activity Wasto.

The techtical basks for this acurty is derived from the TWRS ElS and the opper-kervel performance requirements described in the followigg related documents: Disporel Focllity Data For The Interim Peformapte Assessnuent (Eibolzer 1995), TWRS Privatization Request for Proposats (RFP) (DOE-RL 1996a). TWRS Privatization Contracts (DOE RL 1996b), Tark Waste Remediation System Fiscat Year 1 S97 Multi-Year Work Plon (Wilson 1990) and Tonk Waste Remediation S)ertem Privatization Procest Techalical Baseline (Orme 1996). These source documents are described it more detail in the following subsections.

\subsubsection{IHsposal Focility Daxa For The Interf Performance Assesmeax}

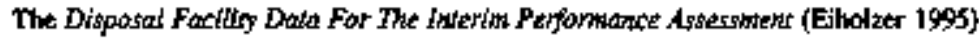
contlilutes the techtrical basis for the ILAW dieposal faciliry cost estimates in the fiscal year (FY) 1997 muli-year program plan. This document provides initial estimates and eraobling assumption on the ILAW package size axd two disprosal utuit concepts for the interim.

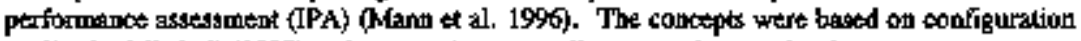
stixies by Micchell (J995) and assumptions regarding waste form and volume.

The technical basis presented in the document is significandy differen from the privatization basis. For the IPA concept, the basis was 6,804 sanisters, esch $2 \mathrm{~m} \times 2 \mathrm{~m} \times 8 \mathrm{~m}$ 
and maile of carton steel. The contsiners pere to be placed in undergound concrete vaults with rempte hardling e्pripment, radiation shielding, asd surface protective barrior. RCRA

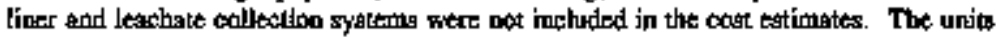
described in this concepl meet the reguiremets for disposal of remote-handled non-mired waste.

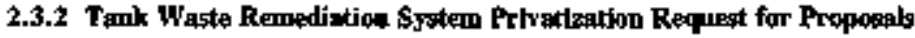

The TWRS Privatization Reguest for Proposols (RFP) (DOE-RL 19964) idenlifies the services that DOB will provide to the low-activity waste (LAW) treatrment contractors and key ILAW product requirements for Phase I privatimalion. These requiremeuts wers oubsequently written irto the Phase IA contracts fioc the private veadors (DOE-RL, 1996). Throughout this report, all references to requiremeats in the RFP also refer to reguirements is the priwatization contracts. The specifications in the RFP and the contracts proyide the primary requirements for this disposal system analysis. A fondanental gugannption of this shoty is that the ILAW produci will meet or exceed the recpirements of the product specificatisols.

The ILAW proctuct specification in the RFP reguires sealing the ILAW in steel packages. The aonalnal dimensions of the package are $1.8 \mathrm{~m}$ loth $\times 1.2 \mathrm{~m}$ high $\times 1.2 \mathrm{~m}$ wide, and it can

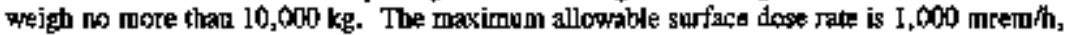
and the average cesium concentration can be no more than $3 \mathrm{Cli}^{\prime} \mathrm{m}^{3}$. The allowable surface contamination on the pachage is limited to levels that arc considered clean athd fits of coutaminallob. The waste packenges muss meet requlirtments for ghalkw land disposal in the state of Washinglod. The package must be able to tupport $50,000 \mathrm{~kg}$, resulting in a slacking limit of six packages high unless additional administralive corkols are in plece. Information on the amoont of lLAW, number of D.AW packiges and the disposal unit requirements were not identified in the Pbase I specifications.

\subsubsection{Tank Waste Remedlation System Flsed Year 1997 Mplti-Year Work Plan}

The Tent Waste Remedinton Systen Fiscal Year 1907 Mall-Year Work Plan (Wilsun 1996) ls bayed on constrbetion of three utederground modules between 2004 and 2020 for disposal of ILAW as remote-handled mixed waste. The modules were to be concrete vaules (18 vaults each module) and based on the consept of Eiboizer (1955). The modnles woukd be backfilled, sealed, and covered wlth a provective barrier at the time of closore.

\subsubsection{Tank Waste Remediatinn System Privatizhtion Process Technisol Baseilme}

The TWRS Privarization Process Technicat Daselime (PPTB) (Orme 1996) ptowdes the process flow diaprams and mass balance for the TWRS treatment concept under the privatizalion iniliative. It esilmates 13,200 pactages of JLAW for Pbase I and aboot \$5,000 
HNF-\$D-TWR-AGA-1004

Rerision 0

parkatges of ILAW for Pbase li. These projections are lased on the product specifications for Phoge I and a standerd sel of assumpticone regarding waste togding, waste form density. package fill fraction, and and imventory (Brown 1996). The PPTB provides the bagis for the disposal system sapacity (98,200 packages) and the peak throughput requirements of uine packages per day for Ptabe I and 27 packages per day for Phase If.

\subsubsection{Bonford Lav-Level Tants Waste Interim Petfarnowe Astent}

The RA (Mann et al. 1996) provides an anslysls of the potential impacts of ILAW disposal on the general public, inadvertent introders, groundwater, surface water, and air Iesources. The IPA concludes that wastes meeting the privalization product specifications, if disposed beneath a surface barrier that performb simikarly to the Hanford Barrier (BSyers and Durascegu 1994), can गneet all performance objectives found in DOE Order $5820.2 \mathrm{~A}$ and 10 CFk 61 for protection of human health and the environment. Additional sensitivity studies

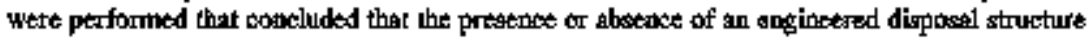
has a negligible effect on the long tern dlsposal system performance. Ttiege findings show that the engineered disposal structure need not perform ang kng-term containinent functions, or bong as the waste form muets the specificationg and the closure tarsier performs as desiggned.

\subsection{DMMOBDLIZED LOW-ACTIVTTY WASTE DLSPOSAL SYSTEM}

The TWRS Final Euritonmental Impact Statement (DOE 1996) and TWRS Record of Decision (DOE 1997) establish that the ILAW "Will be prepared for future onsite disposal in existing grout vauls and similary designed near surface dispessal facilities." The grout vanlts will be witially nsed for interim storage to allow for a proper performance agsessment of the ILAW dispesal system and better detercainalion of the disposal facility performance requirements.

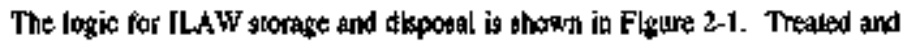
immobilized LAW from Phase I production is received from the vendor in 2002 and expplaced In the retrofitted grout valus for interim storage until 2005 when the additional storage and deposal vauls are ayailable. The hnterim storage alternatives generacion and analysis (Butbank 1996), design requirements (Burbark 1997) and DOE guidapoe provide technical and

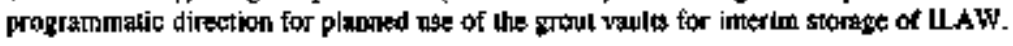

This AGA and subsequent work cower the treated and immobilized LAW during Phase IB and Pbage II. During this time period (2005 to 2025) ILAW is received from the vendor(b) and emplaced be authorized dispessal units. Additional interion storage nuits may be constructed for some LAW packsges until authorized dispogal units are constructed. All interim-gtored waste will be retriesed for trangport to the authorized fisposal utrits. 


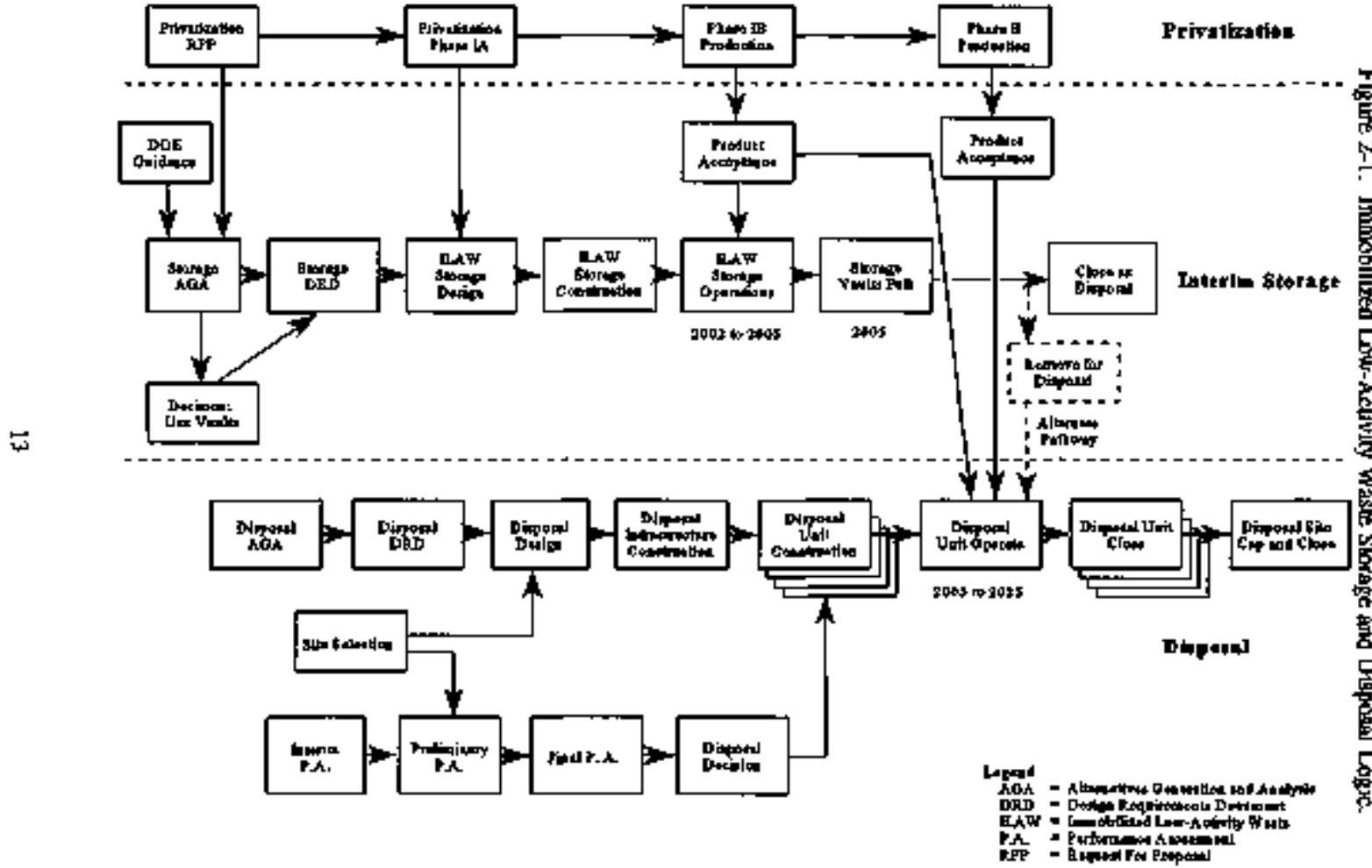




\section{Revision 0}

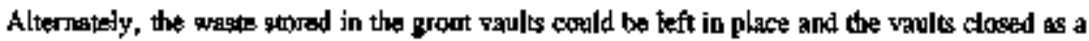
dlsposal unis, saving the cost of retrleval and transportalion to the train disposal oito. A performance assessmen and composile analysis will be prepated to verify the appropriateness of the site selection and disposal unit design.

After fisposal authorization, the filled disposal units will be backfilled with soil, sand or grasel wo elimirate woid space and a concrete roof added to enclese each shielded vanil that cortains remote-handled wagte. At closure, a protective barrier will be constructed over the

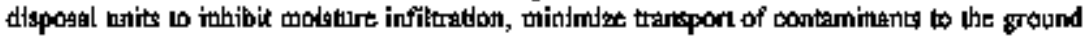
Watter, ated mitigate the effects of intrustion from plants, animals, and humans.

\subsection{PROGRAMAMTTC RISTK MINIMTEATION}

Programmatic risk is the probability that one or more progran gosls will not be met by the irgplementation strangy. The primary goals of the IlAW disposal program are as follows:

- Protect pablice and worker besllb and safiety

- Minimize capkal costo

- Mlnimize operating costs

- Meet or beat privatization and Tri-Party Agreemeat schedole milestones

- Miniojize errironanonal imapacts

- Enable future uate options.

The measures used to evaluave these goals are as folkwy:

- Publictorker exposore (man-rems)

- Total capital cost (conslant-year dollers)

- Total operating cost (constanl-year dallars)

- Time-phased implenentation schedule (years)

- Total land area permatetenly alloctated to waste dtsposal (bectares)

- Total waste volume/source tem (cubic neters/curies).

For all alurnatives, the total wate wolume and source verm it assumed to be the same; i.e., the waste o be disposed is the same as that described in the Privatization Process Technical Baseline (PPTB) (Orme 1996) for all cases. Similarly, the DOE and stakebolders have agreed, through the Hanford Future Site Uges Working Group (DOE 1992), that the furute use of the 200 Ared ceutral plateaul will be for waste management, thus all on-site alernatives are consistent with thls use of the latad.

The higher-risk atpecus of the existog programmatic strakegy for lmplementation of the ILAW disposal tystem are as follows: 
- Comrent plars are to construct three large capacity disposal modules betwten 2004 and 2020 and dispose of all LAW as remote-findled mixed waste. This entails a large early commitrment of resources and little flexibility to adjust to changing requirements.

- The current concept has bo provigions for lest expensiwe dlaposal units for low dost rate DAW packages that can be concact hadiled.

- The currtont concept has no protyisions for less expensive disposal units for delisted ILAW pactages thal tan be classified ay nop-dpixpd waste.

The risks posed by the curent strategy cocld be mitigated by implementing a strategy that provides a suike of malker expacity degign sohutions, each tailored to the characteristics and requirements of a particular ILAW waske type so shown in Table 2-1. This would provide a flexible dlsposal architecture with the ability to quickly adjust to changes in the mix of waste types being proxtuced or changing requirements tor disposal of ILAW. The smaller size of 2 disposal unil under this stratergy would ayojd cormiting a large amoun of resources for a oingle disposal architechure, thus allowing lower cost architsetures to be deployed as noeded to meel the fequirements applicalble to the ILAW being produced. This strategy would enable the disposal program to meet its mission while minimiting the lifec-cycle cost of the dispospl system. Section 7.0 of this report provides additional detals on the proposed implementiotion stratiegy for the LAW dispesal system.

Tabie 2-1. Desien Requitements by Impabbilized Lop-Activity Wapte Type,

\begin{tabular}{|c|c|c|c|c|}
\hline Desinn Requirement & RHA-MW & RH-LLWH & $\mathrm{CH}-\mathrm{MWW}$ & CH.LLWW \\
\hline Remole Handling Equipment (Crans) & $\mathrm{x}$ & $\underline{x}$ & & \\
\hline Concrete Radistion Shielding & $\mathbf{x}$ & $\underline{x}$ & & \\
\hline Remote Video Mondototes and Inspeccion & $\mathrm{x}$ & $\underline{x}$ & & \\
\hline RCRA Double Linter System & $\mathrm{x}$ & & $\mathbf{x}$ & \\
\hline RCRA Leacluate Collectlon System & $\mathrm{x}$ & & $\mathrm{x}$ & \\
\hline Conlart Handl bng Equiqument, (Forkلlic) & & & $\mathbf{X}$ & $\mathbf{x}$ \\
\hline Encineered Surface Closure Barrier & $\mathrm{x}$ & $\mathbf{x}$ & $\mathrm{x}$ & $\mathrm{x}$ \\
\hline
\end{tabular}

CH $=$ Contact handled

LLW = Low-lewel waste

MW $=$ MLked waste

RCRA = Resource Consenution and Recovery Act of I976

RH = Remote hadiled. 
HNF-SD-TWR-AGA-004

\section{Reyisinan 0}

This page latentionally left blank. 


\section{HINF-SD-TWR-AGA-004}

Ravision 0

\subsection{CONSTRADTS AND ASSIMIPTIONS}

This section describes the constraints and assumptions laal defite the framework of this analysis.

\subsection{CONSTRANTS}

Constraints are requirements that are imposed on the system by outside agencites and are beyond controd of design, constroction, and operating organizations. The key constraints that arive the ILAW disposal gygken pome frem the Code of Federal Regulations (CPR), the Washington Adruindstrative Code (WAC), and DOE Orders. In addition there are gujdelines

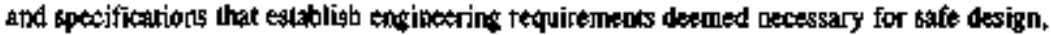
construction and operation of the system. The techaical basis of the desijn concepts that were developed atd analyzed in this study are derived directly from the requirements and guidelines established in liese orders, regulations, codes, and agreements.

Table 3-1 list the primaty cortstraints for the ILAW disposal system. It is brsed on the Hanford Site 19\%6 RDD-100 data base, TWRS Functions and Reguiremears (WHC 1996) and subsequent review of the regubalons. A comprehtensive review of the regulatious applicable to ILAW dispasal is summarized in Appendix B. Results of the tequlrements revlew were uted as a sereening loot to develop raw concepts and eliminate some cousepu from consideration.

Table 3.1. Key Conatraints For Immobilized Low-Actirity Waste Disposal Facility. (3 Shets)

\begin{tabular}{|c|c|}
\hline Doxoment dentifike & Tick \\
\hline 10 CFR 20 & Standards for Protection Arainst Ratiation \\
\hline $10 \mathrm{CFR} 61$ & $\begin{array}{l}\text { Licensing Requirements for Land Disposal of Radioactive } \\
\text { Waste }\end{array}$ \\
\hline 10 CFR 830 & Nuckear Safety Management \\
\hline 10 CFR 834 (draft) & Radiation Protection of Prblic and the Braviropment \\
\hline 10 CPR 835 & Ocapational Radiation Frotection \\
\hline 40 CFR 50 & $\begin{array}{l}\text { Narional PrImary and Secondary Amblent Air Quality } \\
\text { Standards }\end{array}$ \\
\hline 40 CFR 61 & Nationgl Emission Standards for Hazandougs Pollhuants \\
\hline 40 CER 260 & 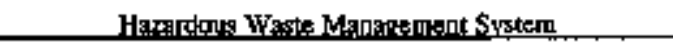 \\
\hline
\end{tabular}


Table 3. L. Key Constraints For Irumobilized Low-Acriyly Waste Disporal Facility. (3 Sheetk)

\begin{tabular}{|c|c|}
\hline Document Identifier & Tille \\
\hline 40 CFR 264 & $\begin{array}{c}\text { Standanits for Owhers and Operators of Harardous Waste } \\
\text { Trealment, Starage, and Disposal Facilitices }\end{array}$ \\
\hline 40 CFR 193 (draft) & $\begin{array}{l}\text { Etrvironmential Rediation Frotection Standards for the } \\
\text { Managemenl, Streage and Dispossal of Low-Level } \\
\text { Radicaclive Waste. }\end{array}$ \\
\hline DOE Order 435. I (drafl) & Radioactine Waste Manghement \\
\hline DOE Order 5400.1 & Generral Ervironmenkal Prowoction Proporarl \\
\hline DOE Order 3400.5 & 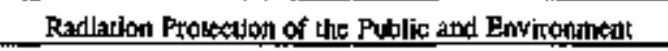 \\
\hline DOE Order $54 \$ 0.11^{\circ}$ & $\begin{array}{c}\text { Worker Radiological Sofety: Hanford Site } \\
\text { Rarthologleal Contro! Matural } \\
\end{array}$ \\
\hline DOE 5480.19 & Condact of Operalion Requiremente for DOE Factlides \\
\hline DOE Order 5480.204 & $\begin{array}{c}\text { Personiel Sekecrion, Qualificalion, and Training for DOE } \\
\text { Nocker Pacilities }\end{array}$ \\
\hline DoE Order 5480.21 & Unreviewed Sofety Question \\
\hline DOE Order 5480.22 & Teshetical Safery Renuinements \\
\hline DOE Order $\$ 480.23$ & Nuckear Safisty Analyoju Reports \\
\hline DOE OTder 5480.28 & Natural Phenomena Harards Miligalion \\
\hline DOE Order 5480.4 & Enviromental Protection, Safely, and Health Protection \\
\hline $\begin{array}{c}\text { DOE Drder } \\
\text { 5480.7A2/RLID5480.7 }\end{array}$ & Fire Protection \\
\hline DOE Order 5483.1.' & $\begin{array}{c}\text { Cocupational Safety att Health Program for DOE } \\
\text { Confrackors at Government Owned Contractor Operated } \\
\text { Facilities }\end{array}$ \\
\hline DOE Onder $\$ 880.2 \lambda$ & Radioactive Waste Manarement \\
\hline DOE Onder 6430.1A & Generel Design Criteria \\
\hline $\begin{array}{c}\text { Secremry of Energy Notice } \\
35-91\end{array}$ & Nuckespr Safety Policy \\
\hline NFPA $70(1996)$ & National Elecurical Code \\
\hline NFPA 101 (1997) & Life Soifty Code \\
\hline $\mathrm{UBC}(1997)$ & Uniform Buildinr code \\
\hline
\end{tabular}


Table 3-L. Key Conatraims For Immobilized Low-Activity Waste Disposal Facility. (3 Sherets)

\begin{tabular}{|c|c|}
\hline Document Identifier. & Tidte \\
\hline Tri.Parky Agrezment (1996) & 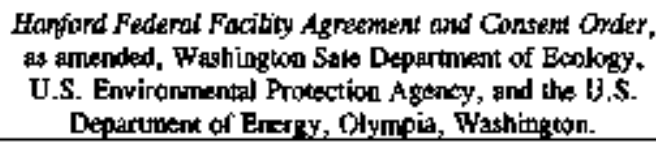 \\
\hline WAC 173.303. & Dangercus Waste Mamagement \\
\hline WAC $173-460$ & Controls for New Sonnces of Toris Air Poilutants \\
\hline WAC 173-475 & $\begin{array}{c}\text { Aubient Air Quality Standards for Carton Monoxide, } \\
\text { Ozgne and Nitrogen }\end{array}$ \\
\hline WAC $173-490$ & $\begin{array}{l}\text { Emisgipn Standerds and Controls for Soturcers } \\
\text { Emilling Volaulle Organic Compounds }\end{array}$ \\
\hline WAC 246-220 & Radialion Pratection - General Provistions \\
\hline WAC 246-247 & Radialion Prottection Air Emission \\
\hline WAC 296-24 & Geperal Safety and Health Slandards \\
\hline WAC 296-62 & Ocsonoational Health Standards \\
\hline ESRCM-1 & Hanford Sito Radioloxical Confrol Manual \\
\hline WHC-SD-ETF-WAC-001. & Efflutert Tyealmetr Facilly Waste Aocentance Criteria \\
\hline $\begin{array}{l}\text { WHC-SD-W049-JCI-001, } \\
\text { Rey } 2\end{array}$ & $\begin{array}{l}200 \text { Area Treaved ElDhent Disposal Facility } \\
\text { [nverface Conirol Doxument }\end{array}$ \\
\hline WHC-SD-GN-DGS-300t1 & Șhlelduto Crikeria \\
\hline
\end{tabular}

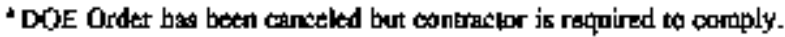

\subsection{ASSUMPTIONS}

Assumptions were made fox areas where limited iuformation is available and the required atralysis has not been completed. The mein assumptions used in this analysis and the rationale behind them are described below.

\subsubsection{Immobllbed Low-hcalvity Weste Produm Specillcations}

The first atsumpton is that the Phate I conlractors will mett or exceed all technical specificatlous for the LLAW product and package as dsfined in the RFP (DOE-RL I996is) and the Phase IA contracts (DOe-RL 1996 ). There are no specifications available for Phase II al 
this ime, so the Ptase I specificalions ate asoumed to also apply to all Phate II prothet. Changes in the Phase II specificalions woukd impect the correnl ILAW dikposal desten tasis (e. g. , minioutn waste losaling, package size, maximum dose rate, delisting of waste, etc.).

This assumption is tey to the anglysis of altercative digposal systen architectures, primarily because it is the cocnersiouse of the lnterim Parfitrmatuce hasessment finding that the only significanl engimeered fearures of the disposal system are the wagte form and the chosante cover. Sjuce a waste form that deets the priqatization profoct specification will also meet the dispossal facility performances bujectives when emplaced beneath a closure cover giminar in construction to the Hanford Barrier, the disposal facility is rot recpained to perform a long-kerm contaiment function. For this atalyais, all aluercotives have the same waste form specifications and closure cover design. Thus, they would all be expected w meet the disposplat system performance objectives required by DOE Onder 5820.2A and 10 CFR 61.

\subsubsection{Amoun or hmmobllzed Lor-ketivity Wast:}

The second agsumption is that the curreant D.AW product waste lowding assumptions will produce aboull 13,200 packenges form Phase I and shoos 85,000 packatges for Phage II (Brown 1996, Orme 1996). This provides the basis for the diepposal system capakiry requirement of 98,200 peckages. The rationale for the assumed waste leading was to keep the alteritattyes analysis basis consistent pith the Priyatization Process Technical Baseline (Orme 1996).

Under the worts case essunpotions for waste loading and package sim, the contractually allowed package count could be up to $16 \$, 000$. The design approench should therefore be flexible enough to handle potentially large changes in the expected number of ILAW pactages over the life of the disposal program.

\subsubsection{Rarioartive Waste Regulatory Status}

The last major assumpton is that the recent U.S. Nuclear Regulatory Cormmisation (NRC) determination that ILAW is "incidendal waste" (NRC 1997) will remain valid. This classification remowes the waste from the bigh lewel waste disposal licensing authority of the NRC (currendly baged on doubte-shell tank [DST] waste classification) and" permits disposal of ILAW from both single-shell lanks (SST) and DSTs under DOE requirements in shallow land disposal facilities. DOE Ordes 5820.2A. Chapter 3 contains DOE policy and requirements for management of low-activity waste.

Petersen (1996) provided the tectinical basts to suppont the NRC determinalion to clastify treated and immobilized waste as incidental waste. Key radionnclides considered for removal

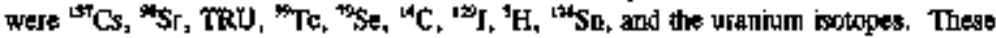

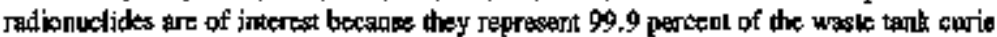

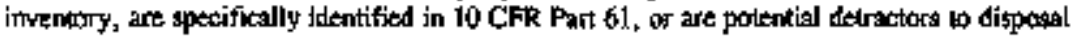


MIN-\$D-TWR-AGA-004

Revision 0

system pertormance bectause they (of thair dayghter products) may exceed dose limits for a short term tortuder scenario (100 mrem/yr) or a long kem drinkiol water scensaro (4 mrem/yr).

The ${ }^{195} \mathrm{Cs}$ content of mIprocessed LAW represents the largesi opportunify to meet the "techuically and sconomitally" practical" criteria of incidental waste. For most of the

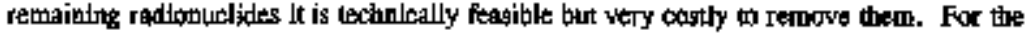

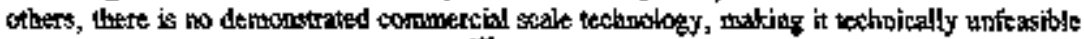
to renowe then. If efficient removal of ${ }^{15} \mathrm{Cs}$ frome alkaline squeous thastes is acoomplished, the incidental waste criteria cas be mer without need to remove additional soluble radjomuclides.

The NRC incidental walke determiation may be re-evaluated if any of the following factors art foud to differ siguilleably from the antalysis in Peversea (1996):

- The radionuclide inventory in the tanks is found to be significantly higher than or difterent from that described in the technical basis report.

- The LAW fraction is not vitrified, or the final waste form volume is sterifictintly different from the projections of the technical basis seport, or

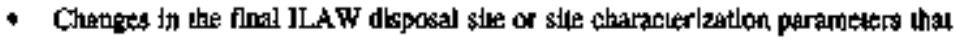
adverstely affect the conchusions of the final performance astesstment.

\subsection{UNCERTADNTES}

Unoertadnties art idedified where insufficient ioformation exigts to performa a required atalysis. The matn uncertuinties identified in this analysis are described in the following sectious.

\subsubsection{Waste Package Dose Rute}

The first key umcertainty that drives the design requirements ts the radiation dose rate dietribution of the ILAW packages. The RFP prodnct specification allows a maximum surface

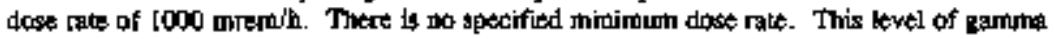
radiation requires remole bandling, shielding, and administrattye controls to meed As Low As Reasonably Achievable (ALARA) exposure lewels and to protect the health axd safety of workers. Designing the radiation protection of the entire disposal system based on the assumption that all ILAW packenges will have the maximum allowable dose rate is ovarly conservaltve and expensive. 
Predimicary analygis of the Phase I retrieval seqpence, feed bach composiliong, and discuggions with the treatnent vetndors thow thal a significant. fraction (corrently estimated to be from 25 percent to 40 percent product packages are expected to be kower dose rate and condact-handled weste ( $<200$ inrem/h). The unmber of contact-handlestble packages will depend primarily on the retrieved waste type and eflleiency of the separation process for ${ }^{137} \mathrm{Cs}$ and other sadionuclides. For some feed batches, cesiom removal efticietacies of ondy S5 percent masy be suffecient to produce coutaci-handled ILAW, while other batches condd require remoral efticiepeive 4 tr 99.9 percent to meet the minimum produst spexifieations. A high separation efficitacy will mimimize the mumber of ILAW packaces that require resoote haniling and gamma shielding al the disposal unit.

\subsubsection{Haxardons Waste Refulatory Sistw}

A second key uncertainty that drives the design requitements is the regulatory stans of the ILAW produre Under the current U.S. Environment Protection Agency (EPA) "mixtmrer and "derived-from" rules, the DAW would contme be managod ag a Retoturce Contervation and Recovery fot of 1976 (RCRA) regulated mixtd (haxartious and radioactive) waste, becouss it is derived from bnk wasto that contairs listed hazandoens constiments. A]though the vitrification procesp woold result in a product that is compliant with the land dispossal reglrictions, the ILAW tisposal facility woold stitl hase to meet the requirements of a RCRA Clas I bazardous wase landfill, inchudlang double liners, leashate collection sybtems, and closure/post-slosure monitoring sybutms.

Two possibilities conld provide relief front the requirements of RCRA for the disposal facility. First, the privatization vendors have been astod to propose methods o provide nonRCCRA Jezulated final waste prothucts. Under the cerstant rogolations this could be achieved by petdidinin the EPA to deligh the ILAW product The degign reguirements for digpesal of a deligted wagte form would be considerably less stlongtat than for miked waste.

The second avenue for exiting RCRA lies in the proposed Hezardous Waste Identification Rule (HWIR) (EPA [995). If pronnalgated, the HWIR would reyise the "mixtare" and "derived-from" noles and develop rigk-baged exje kevels for listod constituents in hazardous waste treatmett tesifites. Baged on the inilial proposed rolte, ILAW product ahould be able to exit the RCRA rules under HWLR, and could therefore be mankged as ordinary lowlevel waste doring storage, dispospl, and closere cptrations.

If ont of these apenus for exiting RCRA is successfully completed, the design requirements would be less strimgent and less costly than those found in a facility that incorporates the RCRA diposal unit design features. 


\section{HNF-SD-TWR-AGA-004 \\ Revision 0}

\subsubsection{Disposal Authorinuthon}

The last key uncertanty is based on draft DOB Order 435.1, Rufloactive Waste Maptigement. When imptemented, this order will roplace DOE Order 5820.2A. The main impact on ILAW disposal will be in the following areas:

- Stronger emphasis on overall waste management (e.g., planolug document for wagte from generation through dispossl)

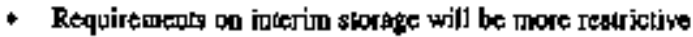

- Requires approval of performance assessment and composite analysis before obtaining a digposal anthorizabion statement (needed to start construction).

The draft DOE Order 435.1 is not final yet and is still subject to change. The prinary impact of the changing requirements for disposal authorization woold be potential schedple delays while auchatzation tocumente are preptared, keadiog to a nees for addilipal interim storage facitities to meet the schedole and capaclly requirements to suppont the privaldzation conrackors. 


\section{HNF-SD-TWR-AGA-(X)d}

Revisision 0

This page intentionslly left blank. 
HANP-\$D-TWR-ACA-OOA

Revision 0

\subsection{DRCISION CRITERIA}

This section describes the decision criteria that were used w differentiate betwest the types of IAW dispostal uuits and implementation strategy. They can be protped into the major cakegories of cost, flexibility, schedule, risk, environnental impact, operability, maintainability, safety, radiation exposure, and public moceptance.

These crilleris represebt a mixture of quanliative and posillative faclors. Some of the criteria, such as cost and schetule, represem directly measurable yariables. Other PETfortragoce measures, such as operability and maintainability, are moro dependent on the

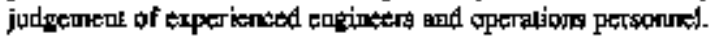

\section{$4.1 \cos T$}

Toral life-cycle costs were deyeloped for each almative. For the purposes of this anabysis, all costs were indexed bo constant 2002 dollars. Cost sumanaries tor each alterisative are presented througgout Section 5.0, and cost estimate detrils are presented in Appendix A.

\subsubsection{Capital Cost}

Capital costs for newly developed concepts were estimated by using unit rate parametric methods applied to excavation, concrete, and steel guantilies developed from preconceptoal facility layouls, along with wendor quotations for major equipment items. All consanction cest estrmates incinde a mizimum 30 percent contingency. Coste for existing fecilitiss and

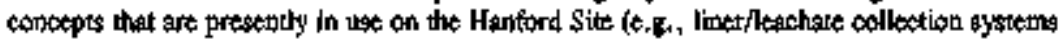
and closuce batriers) were hased ont historitzal cost data.

Coppital costs uere evahualed objectively baged an ponstroction cost estimates developed by ICF Kaber Englbeering Hatford and Fluor Datilel NorthoresL. These esulmates were at a rough onder of magnitude level of accuracy due wo the limited amount of detailed degign data. Capital cost estimales prestented in this analysis are for contruction only. There is no

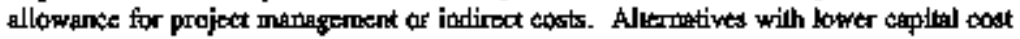
reseived higher scores.

\section{1+2 Operating Cost}

Life-cycle operating costs were developed tased on pretiminary tiane and motion shedies and operatlons scenarios based on the required throughput for both Phase I and Phase I] proxfuction rates. Opprating crews size was basped on peak throughpurt requirtments. Labor 
HNF-SD-TWR-AOA-OOA

Revision 0

tales artre baged on Hanford acganizational iabor rates indered to year 2002. Strategies with lower operaing cost received blgher rankings.

\subsection{FLEXIBILITY}

The flexibtidty of a dikposal vuit to perform Inultiple wagk disposal functions was eviluaked under this calegary. The stratepies and options with the ability to be meel minimum requirements for disposal of multipite waste types and on the eage of retriteval of waste packages were scored higher.

\subsubsection{Malifie Waste Types}

Flexibillty of the facility to handle mulupie waste types such as remote-handled mlxed waste, remole-handled non-nized waste, contact-handled mized wagle, and contact-handled

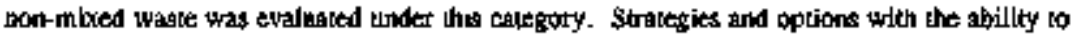
neet multiple requismens were scored higher.

\subsubsection{Worte Refripital}

Retrlevabllity was eraltaled subjeclively based on the estimaned ease of teentering the facility for package retrieval. Strategies that provided easy retrievabijity were scored higher.

\subsection{SCHFDULE}

The ability of an option to met critical schedule uilestones was considered under this caregory. The optiong were objectively evalusted on their ability o meel the I 997 Multi-Year Work Plan (MYWP) schedile (WWllson 1996) and Tri-Party Agreement (Eoclogy et al. 1996) mikestone M-90-10.

\subsubsection{Mukl-Yeer Work Plan Milestones}

The FY 199] MYWP achedule ideulfies a 3.3 year operaing fife of the U-AW Lnterim Starage Facllly (retrofited groot valls berween 2002 and 2005) and showg that the firtat ILAW digposal module shall be constructed by Septamber 2004, This criterion was uged th compliment the go'po-go best to meet Tri-Party Agroetinent millestune M-90-10. 


\subsubsection{Tht Party Agreenend Mlipstone}

The Tri-Farty Agreement prowides the legally enforceable milestones for TWRS. It is the primary schedole driver for new ILAW disposal facility. A disposal facility is needed by December 2D05 because the LAW inkerim storage capacity of the grout yaults will have been nsepd. This criteriod wag a go/po-go test bo determine if it wass possible to implement an option thal jnitiales hot diesposal operations by Docember 2006.

\section{4,4 ENVIRONMENTAL IMPACT}

The environmental impacus of the ILAW dispossal system can be assessed by amount of land usage and secondary qaste gemeralion.

\subsubsection{Land Usage}

The amount of land noed for consuruction of Iow ILAW disposal facilities was evaluated under this catesory. Options with smaller land requirements scared hipher. Only land we withiv the TWRS dicposal program was considerext. Use of land that is already committed to other cleanup programs (t. 8 -, istide and around canyou buildings, or in emply tanks) was not considered to be used by the TWRS dlsposal program.

\section{4,4.2 Secondary Wagle Generatlos}

Contaminated waste from consiruidion and cperation of the dlsposal tacilieies Fere evaluated subjectively under thls catcercisy. Options with the potenulal to generate lesg secondery waste scored bightr.

\subsection{OPERATHON, MATNTENANCE, AND SAFETY}

The cptions were sobjectively evahuated from a daiby operations sedodpoint under this category. The category was further subdivided into subcategories of operability, maintiatability, safety, and radialion exposure.

\subsubsection{Operabliaty}

Operability pas evaluated aubjectively based on the estimated complexity of procedures that wotld be nsed during the daily cperations. Options with fewer pieces of equipinenl and simpler operating procedures spored hivger. 


\subsubsection{Matptaingbility}

Maintainability was evaluated aubjectively based on the mimated complexity of the equipment teded to support the disposal facility. Equppment acossstbility, zone entry, and

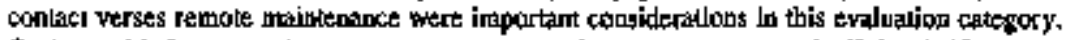
Options with fener moving parts, easy contact maintenance access, and off-lhe-shelf equiptrent scersed higher.

\subsubsection{Safty}

Worker liwes/lost time accidenss during constnucton, disposal and potential retrieval

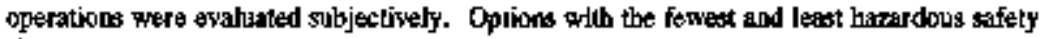
tisks were tared bighest.

\subsubsection{Radhation Exposure}

Worker radiakion exposure was evaluated objectively based on estbmates of time etrd proximilly to radlation soumces during dispostal and potential retrieval operations. Potential public exposone to radionoclides vin the air, surlace, and groundwater pathwags is evaluated by the Interim Peformance Ascessment, which shows that the public dose commitment is insensitive to the disposal architectore. Oplions that had lowtr estimaterd exposares for personnal were scored hightest.

\subsection{PUBLIC ACCEPTANCQE}

This criterion considesed the acceptabillty of at option relative to expressed stakeholder vahes and concerns. The kevel of public acceptance wo an option is messured against a subjeclive set of volues as described in various position proters prepared by the Hanford Advisory' Bostd, otter stakebolder stoups and guidere decision groups (Armacost et al, 1994, Keeney and von Wibterfeld 1996). The performane mesgeves were selected to bound and consolidare the various satkeholder vahtes. Table 4-I summarizes the ksy stakebolder values and assessment that afe consibered in this amysis. 
HNF-SD-TWR-AGA-004

Revision o

Table 4-J. Stakeholder Vahues.

\begin{tabular}{|c|c|}
\hline End objective & Study performance measure \\
\hline $\begin{array}{l}\text { Protect Public/Worker Health } \\
\text { and Safety }\end{array}$ & Astessed in safely and enyironmental performance nesasores \\
\hline Protect environment & Assested in safty and enviranmental performante messures \\
\hline $\begin{array}{l}\text { Enable future use options to } \\
\text { oscur }\end{array}$ & Assetsed in safety and enticonmental per formance measures \\
\hline Ptobed the Columblin Rlver & Assessed by environmental performance measures \\
\hline Ensure compliance & $\begin{array}{l}\text { Assessed by environmenral and regulatory performance } \\
\text { measares }\end{array}$ \\
\hline Reduce costs & Directly assessed by cost data for the alternatives \\
\hline $\begin{array}{l}\text { Enhance technokogy } \\
\text { developinent }\end{array}$ & $\begin{array}{l}\text { Assessed by operability, maintainability and risk performance } \\
\text { шсев:тея }\end{array}$ \\
\hline $\begin{array}{l}\text { Capture economical } \\
\text { developinear locallyy }\end{array}$ & Not tossessed \\
\hline $\begin{array}{l}\text { Proted righly of Native } \\
\text { Amerieat } \\
\text { lndians }\end{array}$ & Assetsed by tentronemenul performance meagures \\
\hline
\end{tabular}


HNF-SD-TWR-AGA-DOA

Revision 0

This page toten Lonally left blank. 
HWF-SD-TWR-AGA-004

Resvision 0

\section{DESCRIPITON OF ALTERNATTYS}

The droposal syslem architectures analyzed in thts sndy are the baseline, flesible muit apptoach, SST usage, existing facilities, and shieked disposable overpacks. An analysis of extended interion storage facilities is also incladed. Previously rejected disposal alternatives and those that do bot mete the haslc consuralus of trear-surface and onslte were thot recrongidered.

The first alternative considered is the tase]ine approach, which reflects the concept that provides the basis for the cost estimates in the PY 1997 MYWP. The tecthoical trasis for this concept is not the anme as the privalizalion basis; it is carried torpugh the athalygis ag a point of

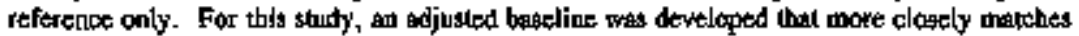
the privatization basis. This adjusted baselite wat used bo the evaluation of alternalive architectures.

The flekible unit appromb altermalive resulted from a delailed revisw of lLAW digposal requirements (See Appendlx B). This epproach establishes design rectuiremonts based on the characteristies of the diffentht wastes types that moy be producted turiug immobilizattou of the

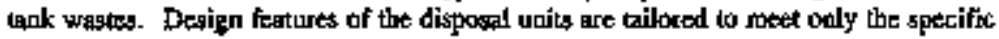
requirements for a perticular waste type.

The next alternative proposes bo use the eraptied SSTE 29 disposal units for ILAW wate packages. It resulted from input to FY 1997 Hanford Site Tecknotogy Coordination group. This alternative does not meet the near-term schedule requirements but may be suitable for nutyear implezontation.

The fouth alternalive is to uge existion factlilleg anch as the canyon buildings for final dikposal of the ILAW packages. This altertiative does not meet the near-tenn schedule requirements but may be suitable for outyear implementation.

The fifth alterrative is to extend interim storage of contacl-tand alled ILAW packages in regulated facilitics until dispogal euthotization is chtaimed (draft DOE Order 435.1) and ditposal unith are consinucted. This alternative would be used to provide the required nearterm capacity uril outyear alternatives could be implemented.

The final alkeraative is to use dilsposabie ghielded orerpaciss for remote-bandled waste pactrages and dispose of the overpacked waste in a contact-handled dispasal facility similat to those discribed in the flexible unit approach.

Options that were Tejected by the TWR's EIS (DOE 1996) or those that did not meet key' requirements of the RFP (DOE-RL 19969) gare summarlzed in Sectlon 5.7. They were not considered firterter in this atralysis. 
HNJF-SD-TWR-AGGA-DOT

Revision $\theta$

\subsection{BASELIRE WASTE DISPOSAL CONCEPT}

The baseline waste disposal concept and the boeline concept as adjusted to atcouth for capacity and throughput discrepancies are described in the following sections.

\subsubsection{Optlon 14 - Basellpe Dtsposel Comcept}

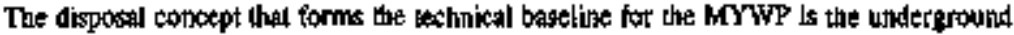

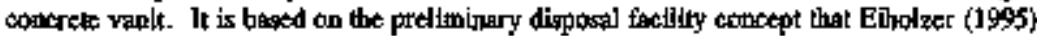
developed tor the interim petrormance asgessment (Mann ea al. 1990).

The MYWP planaing is based on conteructhon of three large underground disposal modules ( 18 concrete vavits each Inodule, 54 vants total) between 2004 and 2020 for dispossi

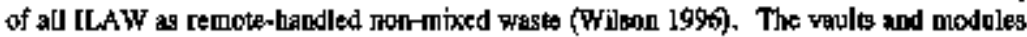

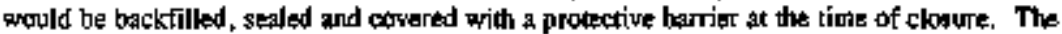
folkwing descriplion is from Eiholzer (19s).

Two disposal facility concepts were developed for the interim performance assestment They wete based on the configuration studies by Mlichell (I99). The initial concept contained 42 vault bays $\left(31,000 \mathrm{~m}^{2}\right.$ vault floor area axd $36,000 \mathrm{~m}^{2}$ total floor area) for disposal of the proliminary $2 \mathrm{~m}$ $2 \mathrm{~m} \times \mathrm{m}$ m (nargo) ILAW coptainers. The facility would cortain remote handling equipment, skiekding walls, and a surface barrier with a inoisture diverter and water conditioning layer.

The second cooctept th a variation of the iritiol concept. It was selected the baseline conkept for ILAW disposal based on die interlm performance assessment due to its larger size and additional features. Figure 5-1 shous the dispogal facility concept. Figure 5-2 ghows a cross section view.

The utit conlained 54 yault bays $\left(40,000 \mathrm{mi}^{2}\right.$ vault floor area and $86,500 \mathrm{~m}^{2}$ total floor area), additional backfill between vaplt groups, and a muiti-slopped bottom pad. The additlonal backfill would help to keep moishure away from the waste conbinets and provide a stronger gtil column to prevent sultoidence. The multi-glope bottom ped improves drainge.

The technical and cost baseline for the FY 1997 MYWP is to design ard construct this type of acillity as three modules of 18 vaults each for disposal of all ILAW as remoti-handled waste. The yaults wopald be made from reinforcend cortrate.

Far this concepl, the botton pad of the disposal facility would be $246 \mathrm{~m} \times 351.5 \mathrm{~m} x$ $0.76 \mathrm{~m}\left(86,500 \mathrm{~m}^{\mathrm{t}}\right)$. The iodividalal vallt bry pads would be $38 \mathrm{~m} \mathrm{~K} 19.5 \mathrm{~m} \times 0.76 \mathrm{~m}$ ( $740 \mathrm{~m}^{2}$ ). The otater bay walls woukd be $2 \mathrm{~m}$ thick abd $9 \mathrm{~m}$ all. The itterior bay walls would be $1 \mathrm{~m}$ thick and $9 \mathrm{n}$ latl. There would be openings into each bay from the center aisle, 


\section{HNF-SD-TWR-AGA-O0)4 \\ Reurswo 0}

Figure \$-] Disposal Facilnty Concept Baselure Optuor 1A

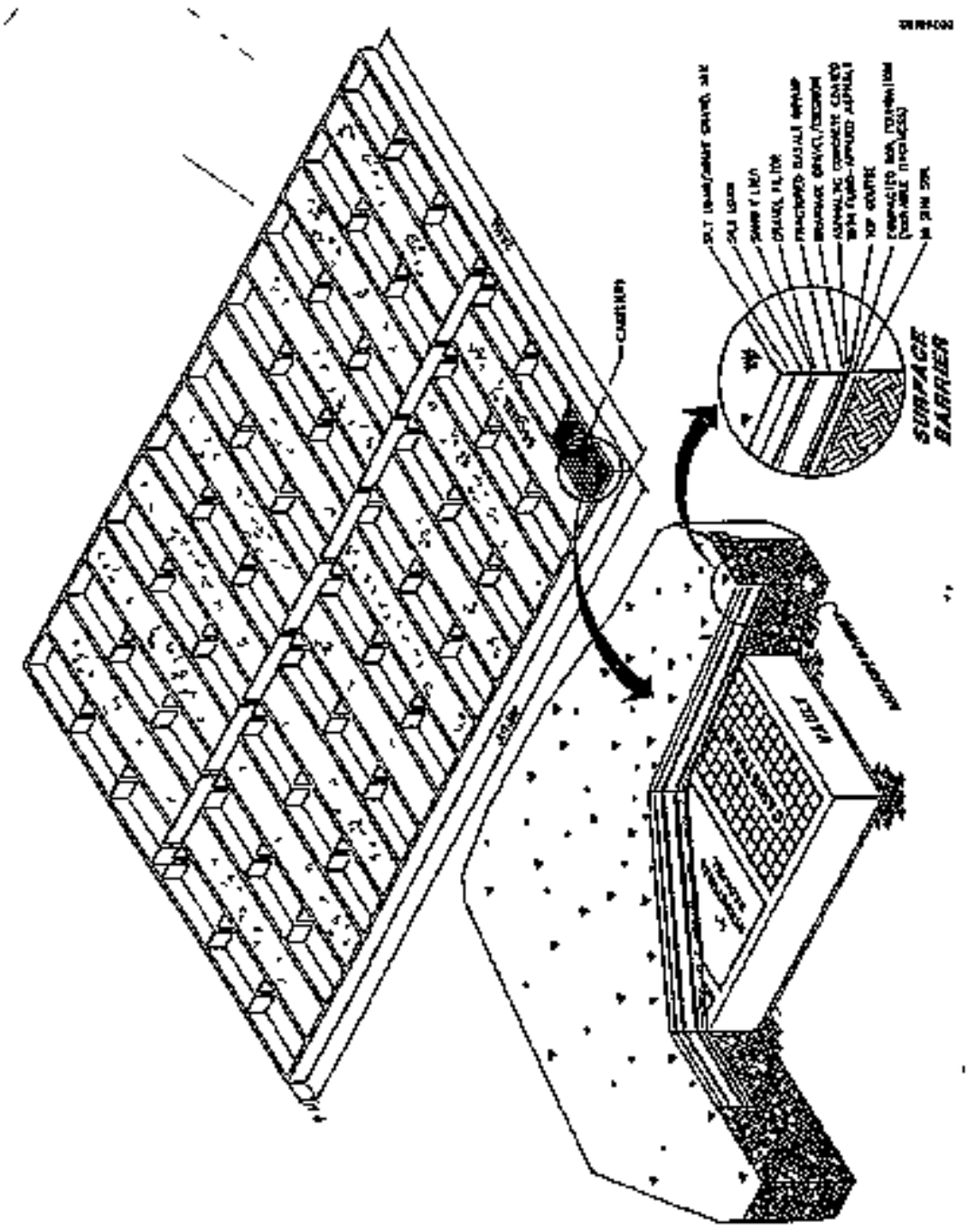




\section{HNF-SD-TWR-AGA-004}

Revision 0

Fieare 5-2. Cross Section of Baseline Disoosal Farility Concept.

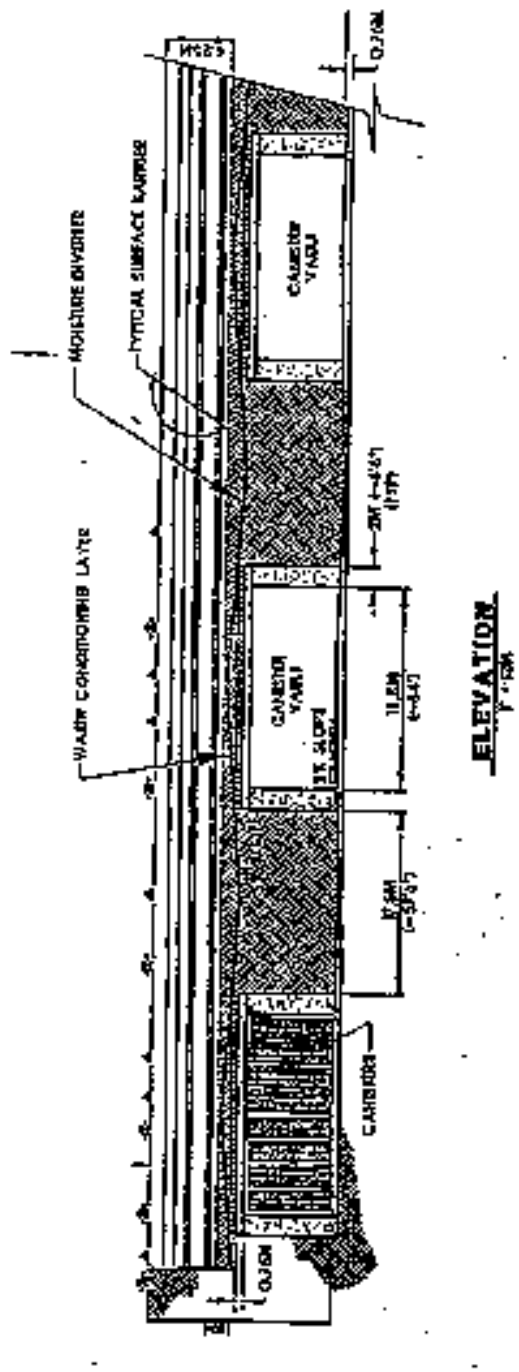


For Phape I, the analysis atsumes the ILAW packages ate transported by thuts to the

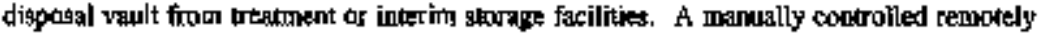
opersted crane would move the packages from the transporter to their disposal position in the vault bayg. The parkage marker iufouthation would be chected aqginst be wasto manifest

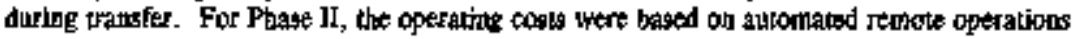
and tranqport via rail from a close-colipled trealment facility dete to the bigher throughput requirements.

The operations and control building world inchude systems for remote yiewing. conkrolling access to the active portion, alarm disp]xys, communications, a dedicated computer for scorage of records, persounel training area, and standard office butldine equipmetis. Maintenance of the crane and other failed equipment would be performed in a fow radiation zone of the disposal facility or ar a degignated maintenance facility in the 200 East Area, assumed to be thared with the vitrifleation plant.

The closure cover or surtace protective barrier corstste of muli-layer cower consisting of soil, sand and rock thowt $4.6 \mathrm{~m}$ thick. [I will be p]aced over the ILAW dieposal usits at clorure. A maisture conditioning layer has been proposed to potentially reduce glass

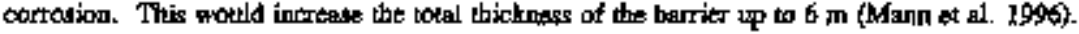
Delails on the surface protective barrier are given in the des Jen besis document for the ffortond Site Bavier Program (Myers and Durancean 1994) and the Futerint Perfomence Assesment ( $M$ Antst et al, 1996).

Table 5-1 summarines the estimated conglutetion costs for this facility as found it the

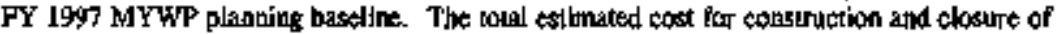
the facility is $\$ 393$ millton (includes $\$ 66$ million for infrastruture inems such as sle work, roads. buildings. fences, utilities, power water, sewer, transport equipment). The consirvction cost of one nochule is $\$ 96$ million. All costs ane escalated to 2002 . See Appendix A, Table A-3 for details.

Table 5-1. Summary of Estimsted Cogst For Detiph, Constructipr, and Closure of the Baseline Immobilimed Low-Activity Wagte Dispposal Facjlity (Option 1A).

\begin{tabular}{|c|c|c|c|c|}
\hline $\begin{array}{c}\text { Disposal } \\
\text { unit }\end{array}$ & $\begin{array}{l}\text { Construction } \\
\$ \text { million }\end{array}$ & $\begin{array}{l}\text { Infirastruchure } \\
\text { \& milliog }\end{array}$ & $\begin{array}{l}\text { Corsure } \\
\text { \$ million. }\end{array}$ & Total \$ million \\
\hline Moduk 109804 & 96 & 66 & 13 & 175 \\
\hline Mochule 2 o9:15 & 96 & 0 & 13 & 109 \\
\hline Hodhule 3 09/20 & 으. & 0 & 13 & 100 \\
\hline Total & 288 & 66 & 39 & 393 \\
\hline
\end{tabular}

tThe cupactey basls for these costs is 60,000 packiages. 


\section{Revision 0}

\subsubsection{Option 1B - Adjusted Buselline}

The cost estimate for the Option 1A Baseline was baped to incomplete infernation regarding the privatization initiacive and was built using factored cost estimating methods. Several design features (e.g. RCRA Jiner and leachate coliection system) nere sketchy or omitted completely. Snhsequent apalysis of this concept (Bibolzer 1996) agairst the privalization specifications shows that the copacky of the oplion is nominally 60,000 XLAW packages. The required tapracily is 98,200 packeges.

For comparative punposes, the construction costs for the disposel mis and closure for Option IA have been adjusted to the langer required capecits. Adjustunents were based on the anparity ratio for the troo optionos. Intriastructure casts prese not adjusted since they sbould bs largely indepetadent of the facility caparity. Table 5-2 reflects the adjusted onstruction costs for a ficility comparable to Option 1A, but with the required capacity of 98,200 [LAW packages.

Table 5-2. Sumamary of Estimaued Coss For Condtruction and Clouse of the Adjusted Baseline Immobilized Low-Aclivity Waste Digposal Facility (Option 1B).'

\begin{tabular}{|c|c|c|c|c|}
\hline $\begin{array}{c}\text { Dteposel } \\
\text { unit }\end{array}$ & $\begin{array}{l}\text { Cousenutction } \\
\$ \text { million }\end{array}$ & $\begin{array}{l}\text { Infrastrucemer } \\
\text { \$ million }\end{array}$ & $\begin{array}{l}\text { Cloture } \\
\$ \text { mblion }\end{array}$ & $\begin{array}{c}\text { Total } \\
\$ \text { million }\end{array}$ \\
\hline Motule 10904 & 159 & 66 & 22 & 247 \\
\hline Module $209 / 15$ & 159 & 0 & 22 & 181 \\
\hline Motule 3 09/20 & 159 & D & 22 & 181 \\
\hline Tokel & 477 & 66 & 66 & 609 \\
\hline
\end{tabular}

The capacity bagin for these cossts is 98,200 pactopes.

Operating cost estimates for the Baseline concept were chtained from the FY97 MYWP, and are shown in Table 5-3. The basiz for the Bxseline operating cost escinale is presented in Appendix A, Tahles A.1 and A.2. The operating costs for the Adjugted Baseline ware derived from the Baseline casts by multiplying by the throughput ratlo. The Adjusted Baselloe operaling costs are shown in Tabke 5.4. 


\section{HDF-SD-TWR-AOA-OOH}

Revision D

Table 5-3. Estimated Operating Costs for Immabilized Low-Activity Waste Storage and Disposal Based on 1997 Multi-Year York Flan Baseline (Option LA).

\begin{tabular}{|c|c|c|c|c|c|}
\hline Mode & $\begin{array}{l}\text { Operational } \\
\text { Feparation } \\
\text { \& million }\end{array}$ & $\begin{array}{c}\text { Hox } \\
\text { operation } \\
\text { \$ m million } \\
\end{array}$ & $\begin{array}{l}\text { Facility } \\
\text { desctivalion } \\
\$ \text { million }\end{array}$ & $\begin{array}{l}\text { Long term } \\
\text { monitoring } \\
\$ \text { midlion }\end{array}$ & $\begin{array}{l}\text { Total east } \\
\$ \text { mition }\end{array}$ \\
\hline $\begin{array}{l}\text { Phase I Remple- } \\
\text { handled JLAW } \\
\text { Dirposal" } \\
\text { (12,520 conpiners) }\end{array}$ & 4,0 & 38.7 & 2.0 & - & 44.8 \\
\hline $\begin{array}{l}\text { Phase II Rempte- } \\
\text { haudlod ILAW } \\
\text { Disposel* } \\
\text { (83,200 contamers) }\end{array}$ & 5.3 & 76.3 & . & 5.9 & 87,4 \\
\hline Total & 9.3 & 115.0 & 2.0 & 5.9 & 132.2 \\
\hline
\end{tabular}

'Production rate is:

5.4 patkenes per day (27 packapes per 5 day weeck)

12 year dunation (2 yr pre-operation, 9 yr operation, 1 yr descthation)

3 shifi, I grout valt storageidisposal operaljon at a time, 5 days/areek

(I.8 package/shift $\times 3$ shifts/day $\times 5$ days/week -27 packages/week), manual conkrol of remole operations.

'Production tate is:

16.8 packages per day (118 packages per 7 day weck)

16 year duralion (2 yr pre-operation, 14 ys operation, wo deactivation), plus 9 year long term mosiltoring

S shifts (4 + troining shift), 1 module disposal operation at a time

(3 total), 7 days tweek (5.6 package/shift $x 3$ hifis/day $x 7$ days/ $/$ geak $=118$ packages/week\}, automatic control of semote operations. 
Table 5-4. Eqtimated Operating Coest for Imonobillzed Low-Activity Waste Storage and Ddsposal Based on 199 ? Multi-Year Wotk Phan as Adjugled for Throngheul (Option IB).

\begin{tabular}{|c|c|c|c|c|c|}
\hline Mode & $\begin{array}{l}\text { Optrational } \\
\text { preparation } \\
\text { \& million }\end{array}$ & $\begin{array}{c}\text { Hot } \\
\text { operation } \\
5 \text { million } \\
\end{array}$ & $\begin{array}{c}\text { Pacility } \\
\text { deaclivalion } \\
\text { Imilion }\end{array}$ & $\begin{array}{c}\text { Long trm } \\
\text { monitoring } \\
\$ \text { million }\end{array}$ & $\begin{array}{l}\text { Total cost } \\
\$ \text { million }\end{array}$ \\
\hline 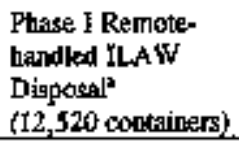 & 9.4 & 90.4 & 4.8 & - & 104.6 \\
\hline 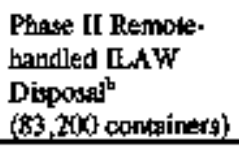 & 8.4 & 122.0 & - & 9.5 & 139.9 \\
\hline Total & 17.8 & 212,4 & 4.8 & 9.5 & 244.5 \\
\hline
\end{tabular}

IL.AW = Immobilized low-activity waste

Based on Table 5-3 and adjusted for throughnol ration of 2.33 for Phase 1. Producidots rate is 9 packages per day (63 packages per 7 day wesk), mantally controlled remove operations.

'Based on Table 5-3 and adjusted for throughput ratio of 1.60 for. Phase If. Production rate is 27 packages per day (189 packages por 7 day woek), autocratically coptrolled remote operations.

\subsection{FLEXIBLE UNTT APPROACH}

The flexibde woil approach alternative resuilted from a debiled retiew of ILAW dispesal requirements (WHC 1996). Il is based on the expectation that pretreatment processes for SST and DST waskes and regulatoxy delisting will result in waste forms that are bubjoct to fewer constraints, which allows for construction of simplex disposal unitg. See Appendix B for the detailed astestment of the disposed sysuet design requirements.

For the lexible unit approach, a disposal unit size of 5,040 packages in an array 10 packages wids $x$ 84 long $\mathbf{x} 6$ bigh was chosen on the basis of proyiding capacity to hold approximstely four years of Phase I production in a cost effective manner. The anit size was sejected to provide a flextb]e systimn withoul the freed for commiting large capilat expenditures for facilities that may not be neoded or used for several years. For Phase II, the approach provides for mulliple units apereaing concurrently to more easily handle the expected throughpot of op bo 27 packages per day. See Appendix A, Section A2. 1 , for descriptions of the components included in each of the flexible unit comoepts. 
HNF-SD-TWR-AGA-OOA

Revijerion 0

\subsubsection{Optian 2A - Renote-Handled Mlued Waste Dippost Vanls}

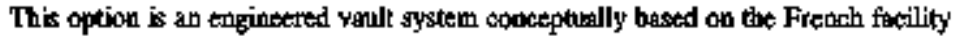
managed by ANDRA at Centre de l'Aube for LAW disposal and the premise that all of the projicted 98,200 packages will requite disposel as remole-kandled mlked waste. It astitures that the existing grout vaults will be retroftted and used for interim storage of ILAW. The current schedule shows permanent disposal capacicy becoming ayailable in 2005 or about one year before storage spaca at existing pornt vald is tilled.

The unmber of remote-handled mixed waste dlsposal unils can not be flrmly tstablished at this time the to incomplete ILAW prodact periormance specifications and regulatory jsates on land disposal restrictions for treated dangerons waste. For the purpose of this analysis, it was assumed that each disposal uqit wonld hase a copaciky for about 5,040 packanges of ILAW, for a maximutn 19 disposal unils. Further sludy of fued olaging plans and privare contractor process flow diagrams is requitried to determlae the actual number of remote-bathded mixed waste packages that will be produced.

The engineserd system featurts include remote handling, ahielding for gamma radation provecton of operating and maintenance personnel, double lines and leachate collection system, moisture drainape or accumulation monitor, and surface closure barrier. The concept is sbown in Figme \$. 3 .

The disposal unit wovid be a conerete van]t divided into six cello. Each cell would have concrete walls and flows. A matbile toof with bridge crante would be used for addition and retrieval of packages and would provide protection from the environment. After a cell is filled, a temporary roof wrould be placed over the cell, allowing retrieval of the waste packages etrit authorhation for disposeal is recelsed.

The facility would have a double limer and leachate collection spstem for compliance with RCRA regulations. A surface protective barrler over the units would be constructed at ckosure. The design, operation, and cloome wonld conpply with regulatory requiresneats for near surface dispossal of mixed watste, DOE Ordert, and NRC tequlathons for destgnated incidentad antute are excoseding NRC Class C.

During operations, the ILAW packages (1.8 $\mathrm{m} \times 1.2 \mathrm{~m} \times 1.2 \mathrm{~m})$ world be removed from the shiolded tranport conkimer, moved to the digposal vault, and stacked up to slx high ( $7.2 \mathrm{~m}$ ) by the bridge crane. During this operatlon the package makker information would be checkred against the waste manifest.

Sufficient spacing between packages $(10 \mathrm{tm})$ would be provided to allow for subsequent packinge identification checks, retrieval, and addlion of filler materia1. The crane opopld be remotely operated and connrolled fiom an operations boilding at the disposal bitr. A kocal backup contool panel would be avalable at each valult for manusi crate operation. 


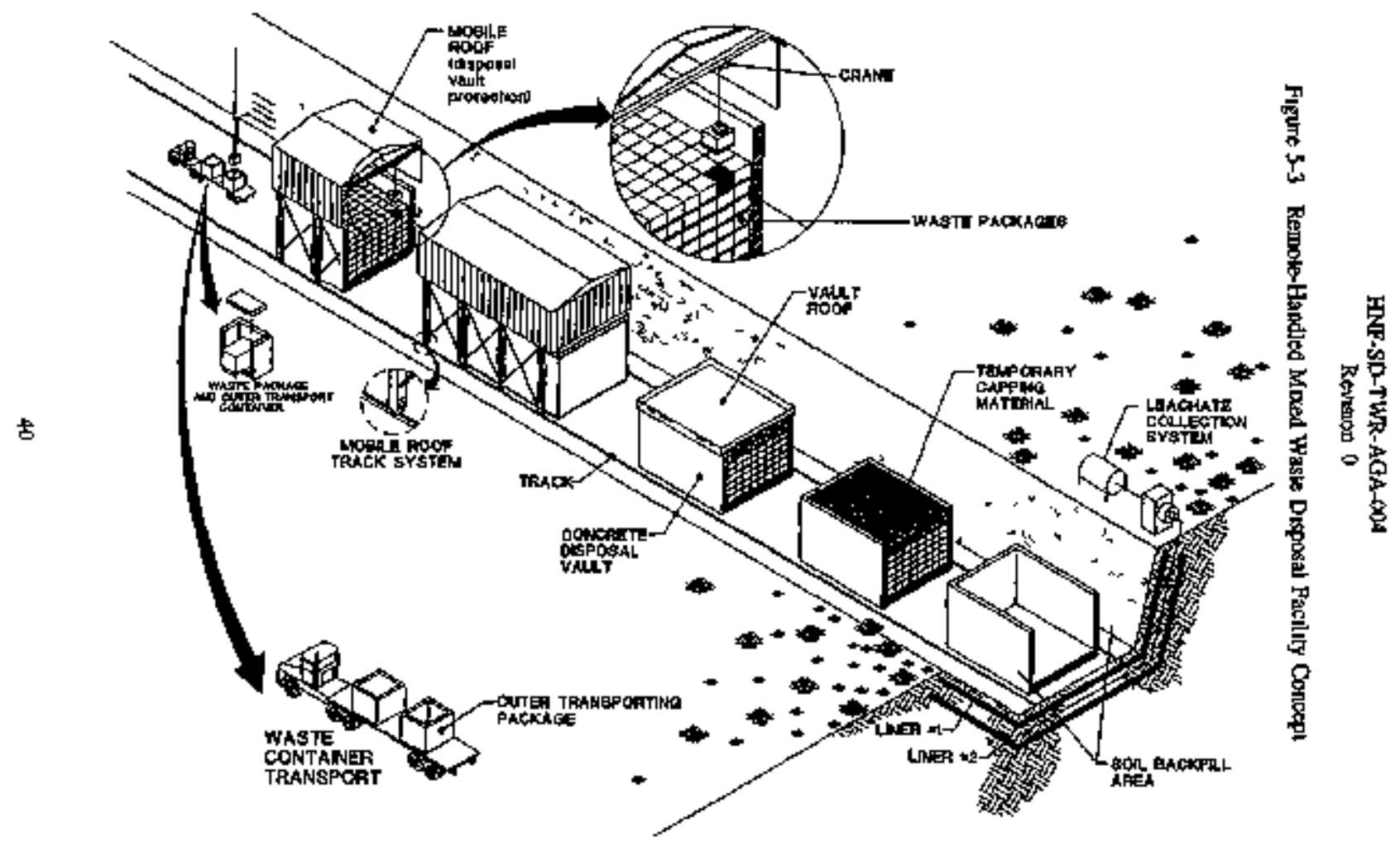


After a vanlt cell is tilled with waste packages, a new cell would be aclivaled. A temporary metal roof would be placed over the filled cell ko probect the waste packages from weather and animalk. A moisture monitor, double linar, and keachare colleclion system will be

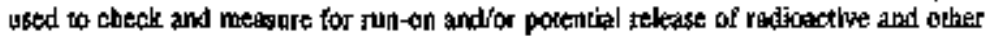

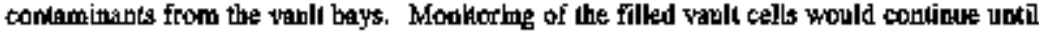
closure.

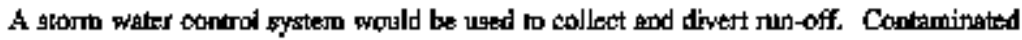
or potebtially contamisgted stotn water and leachale would requite disposal at the Effluent Treatontenl Facility (ETF). Uncomtaminater storm water atd leachate would be disposed at the Treated Effluent Disposal Facllity.

At chostre, the emporary metal roof system noold be renowed and the cells would be

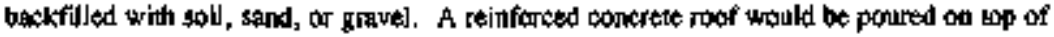
the becksfill. The dispogal unil would then be backfilled o grade and a surface peotective barrier system placed over the vaul's to prevent intrusion and minimize water influtation into the disposal facility. Pigure 5-4 shouts a cross section of a closed digposal unit.

The operations and control bulld big wowd liclude systems such as a control room, monitors for remote yiewing, access controls to the active portion, alarm displass, communications, a dedicated computer for storage of records, a personntl kaining area, and standard oftice building equipment.

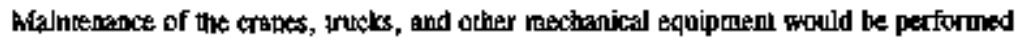
in a low radiation zonce ar the disposal unit or at the dispossil site equipment mainterarice facility. 
HNR-STD-THR-AGA-004

Rerrision 0

Figure 5-4, Cross Sectron of Cloded Immobilized Lop-Activity Waste Disposal Unit for

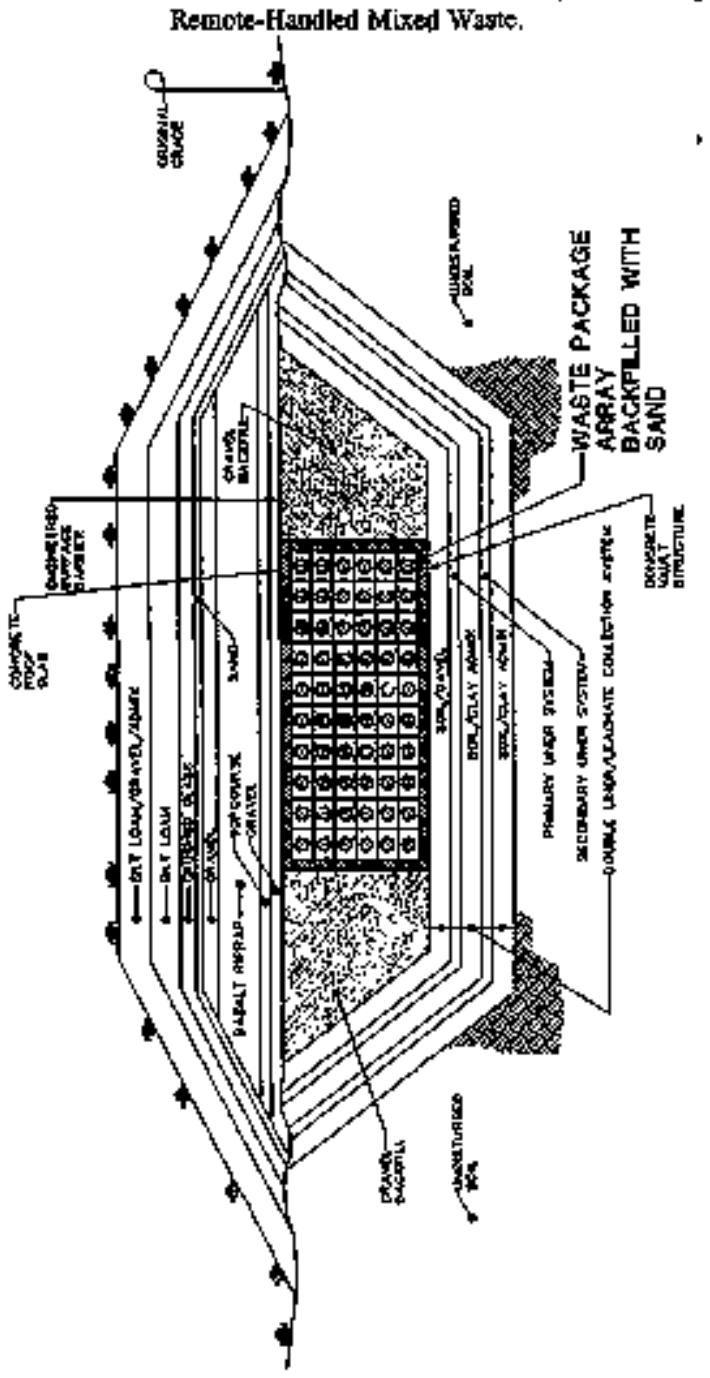


Tab]e 5-5 summarizes the capital cost estimales for this type of disposal facility. The total estimated coen for constroction and closure of 18 of these type of digpoenl units is \$554.5 miltion. including \$24.4 milion lor infrestructure ltems and \$47.5 mollon for clasure. The cansuruction cost for otte disposal unit is \$25.4 million. See Appendix A, Tables A-4, A.5, and A-6 for details.

Tabje 5-5. Sumanary of Egrimated Capital Costs For Digpostal of Remote-Handked Muxed Wade (Opton 2A).

\begin{tabular}{|c|c|c|c|c|}
\hline DHsposal unit & $\begin{array}{l}\text { Construction } \\
\text { \$ millikn }\end{array}$ & $\begin{array}{c}\text { Isfragtrukture" } \\
\text { \$ million }\end{array}$ & $\begin{array}{l}\text { Site tlosure } \\
\text { \$ million }\end{array}$ & $\begin{array}{c}\text { Total } \\
\$ \text { million } \\
\end{array}$ \\
\hline Module 1 & $\$ 25.4$ & 24.4 & 2.5 & 52.3 \\
\hline 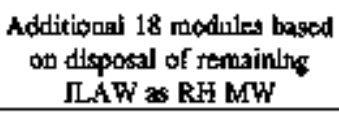 & 457.2 & 0 & 45.0 & 502.2 \\
\hline Total & 482,6 & 24,4 & 47.5 & 554.5 \\
\hline
\end{tabular}

CH MW $=$ Conchach-handled mixed worgto

ILAW = Immobilized low-ectivity waste

RH $M W=$ Remoto-handled mixed waste

- The infrastructure is a one time cost for construction of any of the disposal onits for the flexibde alternative options.

${ }^{2}$ Unit and site ckosure coste are 2 one time cost. They are the same for any of the units for the flexible altornative options.

Table 5-6 summarize the estimaled operaling cossts for this facility. The total cont for operational preparation, bot operations, deactivation and kong-term monitoring is $\$ 40$. I million. See Appeadix A, Table A-7 and Table A-8 for details. 
HINE-\$D-TWR-AGA-DOA

Reviaion o

Table 5-6. Estimated Operating Cogls for Disposal Of Remoto-Handled Mixed Waste (Option 2A).

\begin{tabular}{|c|c|c|c|c|c|}
\hline Mode & $\begin{array}{l}\text { Oparaliontal } \\
\text { preparation } \\
\text { \$ million }\end{array}$ & $\begin{array}{c}\text { Hort } \\
\text { opertition } \\
\mathbf{5} \text { million } \\
\end{array}$ & $\begin{array}{c}\text { Facility } \\
\text { desaclivation } \\
5 \text { million }\end{array}$ & $\begin{array}{l}\text { Long term } \\
\text { monitoring } \\
\$ \text { milliogl }\end{array}$ & $\begin{array}{l}\text { Total cost } \\
5 \text { nillion }\end{array}$ \\
\hline $\begin{array}{l}\text { Phase [' } \\
\text { (13,2@0 } \\
\text { contain:erg) }\end{array}$ & 6.4 & 63.4 & 3.2 & - & 73.0 \\
\hline $\begin{array}{l}\text { Plage II } \\
(85,000(0) \\
\text { containes })\end{array}$ & 18.5 & 266.6 & 9.3 & 40.7 & 335.1 \\
\hline Total & 24.9 & 330.0 & 12.5 & 40.7 & 408.1 \\
\hline
\end{tabular}

Phase [ Disposal Bases:

12 year duration (2 yr pre-pperation, 9 yr operation, 1 yr desctivation) pesk production 9 continders pert day (63 coralliners per 7 day wsek) peak production of 13 conteiners per thy based on $\$$ day wrek

1 container per trailer and 2 trailess per shift based on 3.25 heftrailer turmarouthd 3 shift, 2 vault disposal operation handles 12 contelners per day and

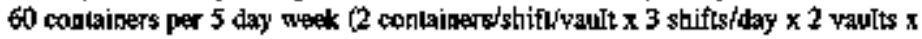
5 day rwetk $=60$ containets wetk).

"Phage II Disposal Baseg:

17 year duraton (2 yr pre-operation, 14 yr operation, 1 yr deactivation) plus 9 yt long term monitoring

peak production 27 contriners per day (189 containers per 7 day weak)

I container per trailer and 2 trailers per shift based on 3.25 heftriler tarnaround 5 shifis (4 + taining shift), 4 vanlt dispossal operation handles 24 containers per day and 168 coutaikers per 7 day week (2 containersishift vault $x 3$ shifulday $x 4$ vauls $x 7$ day/week $=168$ conlainers/tek).

\subsubsection{Optlow $2 B$ - Reance-Hondled Not-Mixed Waste Disposal Vantes}

This alternalive is based on the premise that aot all of the ILAW parkagots reguire digosal as remote handle mixed wagte due to delisting of vikrified LAW. A shielded unit or yault facility prould be required for disponal of ILA'W packages that meet per formance specitications for remote-bandled non mixed waste. The engineered system feahses Include remote handlinge, stiveldmg fir gamma radlaton protection of operaulng and mainegance personnel, moistart accutnulation monitos or drainage in filled soctions, and surface protective bartier. 
HNF-SD-TWR-AGA-004

Revision 0

This cotrcept is dientital to the mobile crane and vaul syster described in Sextion 5.2.1 except there is no requlrement for a double liner and leschale colkection system (see Ficiorte 5-5).

Table \$.7 sumamarizes the capilal cust esumales for a remote-handled non-mized waste disposal facility. The total estimated capital cost for construction and closure of 19 disposal units is \$4\$.6 million. The construction cost for one disposel unit is $\$ 22.3 \mathrm{million}$. See Appendix A, Tables A-4, A-5, and A-6 for delails.

Table 5-7. Summary of Estirrated Capital Costs For Disposal of Remote-Handed Non-Miked Waste (Option 2B).

\begin{tabular}{|c|c|c|c|c|}
\hline $\begin{array}{c}\text { Dispossil } \\
\text { unit }\end{array}$ & $\begin{array}{c}\text { Construction } \\
\$ \text { million }\end{array}$ & $\begin{array}{c}\text { Infrastructure" } \\
\text { \$ million }\end{array}$ & $\begin{array}{l}\text { Sile closure } \\
\$ \text { million }\end{array}$ & $\begin{array}{c}\text { Total } \\
\text { \$ million }\end{array}$ \\
\hline Module 1 & 22.3 & 24.4 & 2.5 & 49.2 \\
\hline $\begin{array}{l}\text { Additional } 18 \text { units for } \\
\text { disposal of retinditning } \\
\text { n.WW as RH MWW }\end{array}$ & 401.4 & 0 & 45,0 & 446.4 \\
\hline Tobal & 423.7 & 24,4 & 47,5 & 495.6 \\
\hline
\end{tabular}

ILAW = Immobilized low-activity waste

RH MW - Renote-handled mixed waste

"The infrastructure it one-ime cost for construction of any of the dispossal units for the flexkle alternative optlons.

"Unit and site closure costs are $\mathbf{2}$ one-time cost. They are the same for any' of the units for flexible alternative options.

Tabje 5-8 summarjes the estlmated operaling cosis for this factlly. The rotal cost for operational preparation, bot operations, deactivation and long-term monitoring is $\$ 408$. I million. See Appendix A, Table A-7 and Table A-8 for debils. 


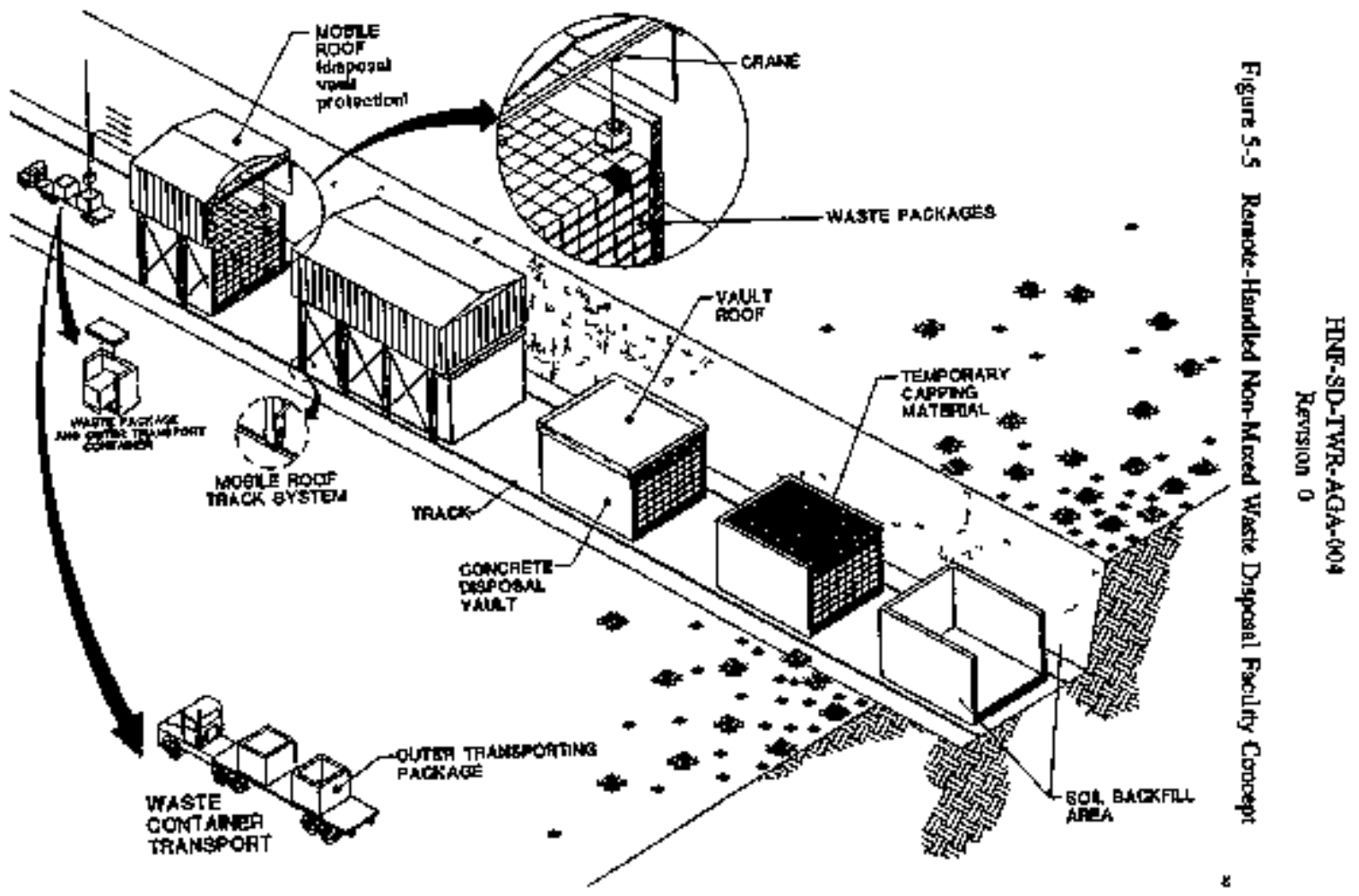


Table 5-8. Estimated Operaling Cowts for Digposal Of Remote-Handled Fion-Mixed Waste (Option 2B).

\begin{tabular}{|c|c|c|c|c|c|}
\hline Mopde & $\begin{array}{l}\text { Opetationat } \\
\text { prefparalion } \\
\text { \$ million }\end{array}$ & $\begin{array}{c}\text { Hot } \\
\text { operation } \\
\text { S million }\end{array}$ & $\begin{array}{l}\text { Facility } \\
\text { deactivation } \\
\text { \$ million }\end{array}$ & $\begin{array}{l}\text { Long term } \\
\text { monitoring } \\
\$ \text { milljion }\end{array}$ & $\begin{array}{l}\text { Total cost } \\
\$ \text { m railisinn }\end{array}$ \\
\hline $\begin{array}{l}\text { Phasse It } \\
(13,200 \\
\text { containers) }\end{array}$ & 6.4 & 63.4 & 3.2 & - & 73.0 \\
\hline 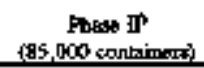 & 18.5 & 266.6 & 9.3 & 40.7 & 335.1 \\
\hline Total & 24.9 & 330.0 & 12.5 & 40.7 & 408.1 \\
\hline
\end{tabular}

APlager I Disprosat Bases:

12 year deralíon (2 yr pre-dperation, 9 yг operation, ] yr desctivation)

Peak production 9 containers per day (69 contamers per 7 day woek)

Peak production of 13 containers per day baed on 5 day week

1 container per trailer and 2 traikers per shift based on $3.25 \mathrm{hr} /$ trailer tornaround

3 shift, 2 verolt disposal operation handles 12 containets per dsy anded

60 coulainers pet 5 day woek (2 coulaibersighifi'vault $x 3$ gbifis/day $\times 2$

vaults $x 5$ day/week = 60 containers/week)

'Phase Hi Disposal Bases:

IT year duration (2 yr pre-operation, I4 yr operation, I yr deactivation) plos 9 year long term montitoring

Peak production 27 containers per day (I89 conlaimers per 7 day wetek)

I container per urailer and 2 urailers per shift based on $3.25 \mathrm{hr} /$ trailer earnaround 5 shifls (4 + trabning shif), 4 vaull disposal operstion handlos 24 containers per day and 168 containers per 7 dgy week $Q 2$ contaisers/shift'vault $x 3$ shiftsiday $x$ 4 vaults $\times 7$ dag/week $=168$ containers'areek)

\subsubsection{Option 2C - Contact-Handled Mtied Waste Diposol Treaches}

This alternative is based on the premise that not all of the $\amalg A W$ requires disposal as remote-handled waste. Comtact-handied waste covld result from efficient removal of radjornalides during trealment or low concentrations of radipmectides in the feed. A double lined trenth with leachate collochlon would be required for digposal of ILAW that falls under the RCRA mixed waste rulet. The destgn, operation, and closture shall comply with regulatory requirements for near surface disposal of mixed waste, DOE Otders, and NRC designatiod incidenlal waste not exceeding NRC Class C. 
The concept is show in Figure 5-6. It is gimilar to the existing mixed waste digpasal trenches in the 200 West ares of Henford. Cost estimates were derived from project W-025, the Solid Wagte propram's Radiosctive Mixed Waste burial trenches. The concept includes double liners and a leachate collection system for compliance with RCRA mixed wask dispostal requirements. Addilional desiench feallurets such as a concrete pad under the disposal zone ceruld be required, depending on the results of the performance assessment.

The ILAW peckeses would be remorved frome the tratsport vathicle, moved to the disposal position, and uscked up to gir high by fork lift. Duriug this opesation the package jdentification would be checked against the watte manifesc

A storग water control system sould be used to collect and divert run-off. Contaminated or potentially contaminaled storm water and keachate would require disposal at the Etfluent Trealment Facility (ETF). Uncondaminared storm water and keachate Wriuld be diepoed at the Treated Effluent Disposal Facility.

Sufficient spacing ( $10 \mathrm{~cm}$ ) between packeges will be prowided to allow for subsequent parclage identification checks, retrieval, and/or addition of bachefill makerial. A sanface protective barrier sinilar to the thiter options will he added at clogure.

Table 5-9 summarizes the tatinated construction eests for this type of disposal unit. The total estimaned cost for conseruction and closure of 19 of these type dispasal units is $\$ 159.3$ million. The consiruction cost for one dispesal unit is $\$ 4.6$ million. Ses Appeadix A, Tables A-4, A-5, and A-6 for desails.

Table 5-9. Summary of Estimated Capilal Costs For Dlsposal of Contact-Handled Mixed Wasuc (Option 2C).

\begin{tabular}{|c|c|c|c|c|}
\hline $\begin{array}{c}\text { Dipposal } \\
\text { unit }\end{array}$ & $\begin{array}{l}\text { Consteuction } \\
\$ \text { million }\end{array}$ & $\begin{array}{c}\text { Infrestructure } \\
5 \text { million }\end{array}$ & $\begin{array}{l}\text { Sile closuret } \\
\text { \$ million }\end{array}$ & $\begin{array}{c}\text { Totaj } \\
\text { million }\end{array}$ \\
\hline Modole 1 & 4.6 & 24.4 & 2.5 & 31.5 \\
\hline 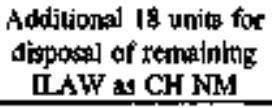 & 82.8 & 0 & 45.0 & 127.8 \\
\hline Tolal & 87.4 & 24.4 & 47.5 & 159.3 \\
\hline
\end{tabular}

CH NM = Contact-bandled pron-mixed waste

JLAW a Immobilized low-actirify waste

The infrastructure is one-dtene cast fax conseructlon of any of the disposal uniss for the flexible alsernative options.

'UTitit and site closure cooss are a one-tine cost. They are the darte for diny of the units for the flexlble alternalive optiots. 


\section{HRE-SD-TWR-AOH-OCOA \\ Reviston D}

Fugure 56 Contact-Handled Mloxed Waste Daqposal Facilly Cancept

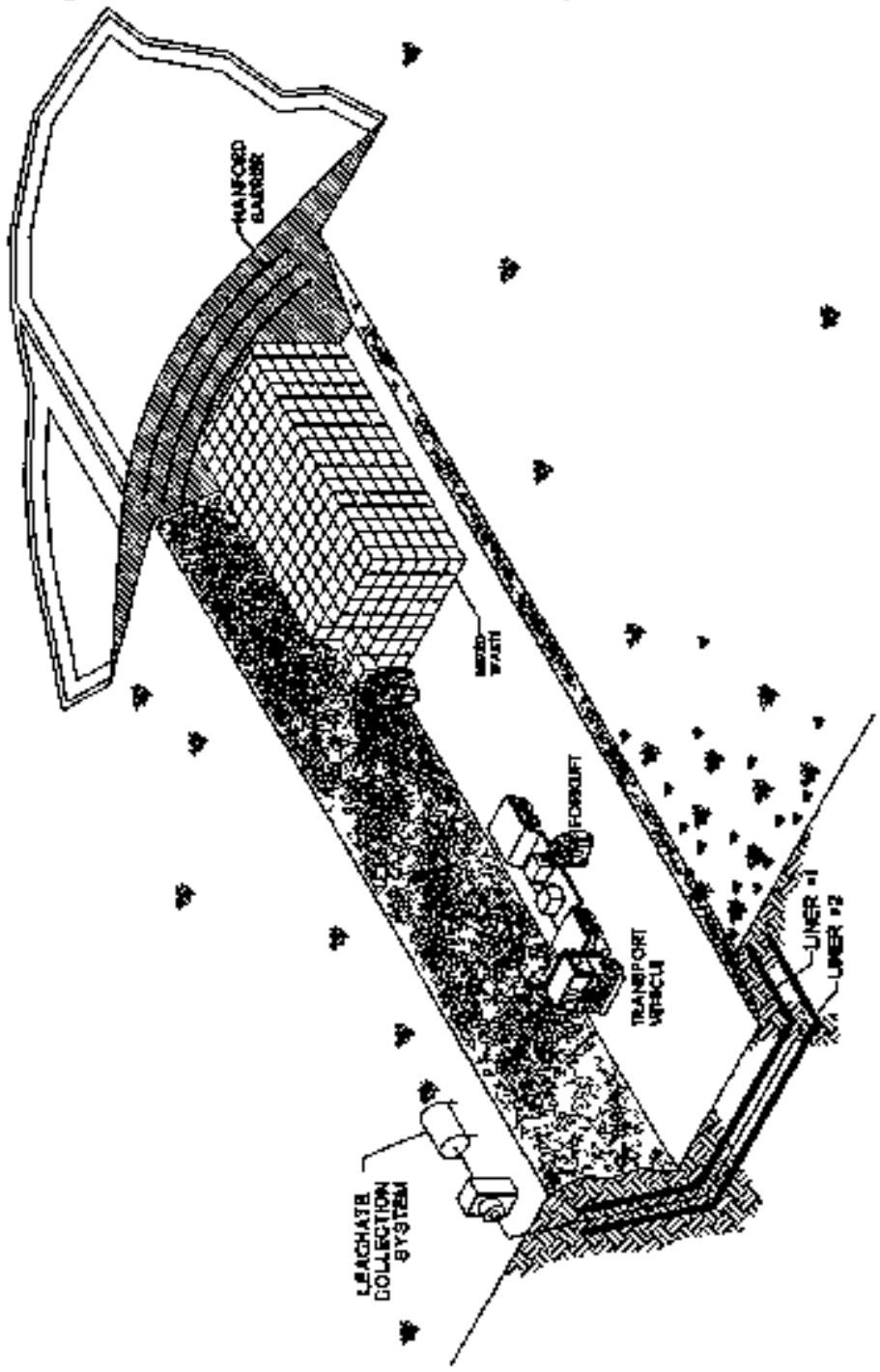


Table 5-10 summarizes the estimated operating costs for this facility. The total cost for operational preparation, bot operations, deactivation and long-term monitoring is $\$ 217.3$ million. See Appendix A, Table A-s and Table A-10 for details.

Table 5-10. Estimated Oporating Costs for Dipposal of Contacl-Handled Mirod Waste (Optom 2C).

\begin{tabular}{|c|c|c|c|c|c|}
\hline Bode & $\begin{array}{c}\text { Operational } \\
\text { prepparalion } \\
\$ \text { million }\end{array}$ & $\begin{array}{l}\text { Hot operation } \\
\text { \$ millipop }\end{array}$ & $\begin{array}{c}\text { Facility } \\
\text { deactivation } \\
\text { \$million }\end{array}$ & $\begin{array}{l}\text { Long term } \\
\text { monibring } \\
\text { \$ million }\end{array}$ & $\begin{array}{c}\text { Tol } \\
\text { cost } \\
\$ \text { million } \\
\end{array}$ \\
\hline $\begin{array}{c}\text { Phase } \mathbf{I}^{\mathbf{2}}(13.200 \\
\text { containers }\end{array}$ & 5.1 & 51.9 & 2.6 & . & 59.6 \\
\hline $\begin{array}{c}\text { Phase } \square^{b}(85,(000) \\
\text { containers }\end{array}$ & 8.1 & 127.6 & 4.1 & 17.9 & 157,7 \\
\hline Total & 13.2 & 179.5 & 6.7 & 17.9 & 257,3 \\
\hline
\end{tabular}

'Phage I Disposal Bres:

12 year duration (2 year pre-cperation, 9 year operation, 1 year deactivation) Petak production 9 conlainers pet day (69 contaltiert per 7 day wtek)

Peak produclion of 13 containers per day bated on 5 day week

2 package per trailer and 2 trailers per shift based on 3.5 he/trailer turnsound 3 shiffs, 1 digposal umil handles 12 containers per day and 60 conkainers per 5 day week ( 4 containers/shiftivalt $\times 3$ shifts $/ d a y$ x I pail $\times 5$ day/weck $=60$ containers/weet)

'Phase II Disposal Based;

17 year dufation (2 year propererathon, 14 year cperation, 1 yeer deactivation) plus 9 year loag kerm monitoring

Peak productign 27 containers per day (189 containers per 7 day week) Peak production of 37 condainers per day based on 5 day week 2 conrainers per uallec and 2 uailers per shift based on 3.5 heftrailer turnaround 3 shifts, 3 unit disposal operation provides for disposal of 36 containers per day

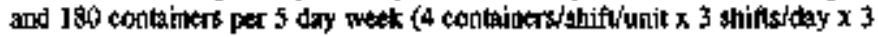
units $x$ \$ daytwerelk - 180 contaimentweelk) 


\subsubsection{Option 2D - Contact-Hagdied Non-Mised Wode Dtsposal Trenches}

This alternative is based on the premise that not all of the ILAW product requires

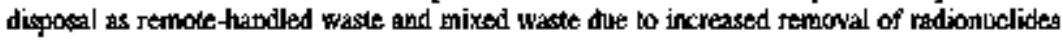
and dellsting of virifled LAW. The solid waste reach facility would permil disposal of ILAW that meet performance specifications for contact-handled non mixed waste. The design, operation, and clasure sball comply with near sur face disposal regulatory recuirements for nom mixed waste, DOE Orders, and NRC designaled incidental waste not texteditng NRC Cless C.

The concept is ghow'n in Figure 5-7, It is similat to the axigting golid waste disposal trenches in the 200 West area of Hanford. There is in requirement for liners and letachate collection system. Pesformance assessment results may require a concrete pad uncer the paste diaposal zone.

\$ufticient gpacing between packages will be allowed for packsage identification checks, retcieval, andior addition of backfill material. A surface protectve barrier will be added al closure.

Tab]e 5-11 summarizes the prelinimary tolinsated costs for this dispoesal facility. The total estimated case for construction and closure of 18 wits is 596.6 million, inchading $\$ 24.4$ million for infrastructore items. The construetion cost for one disposal unit is $\$ L, 3$ mition. See Appendix A, Tables A-4, A-5, and A-6 for details.

rabte 5-12 summantines the totionated operating costs for this facility. The wolal cool for operational prepafation, hot operations, deaclivation, and long-kerth monitoring is $\$ 217.3$ million. See Appendix A, Table A-9 and Table A-10 for details. 


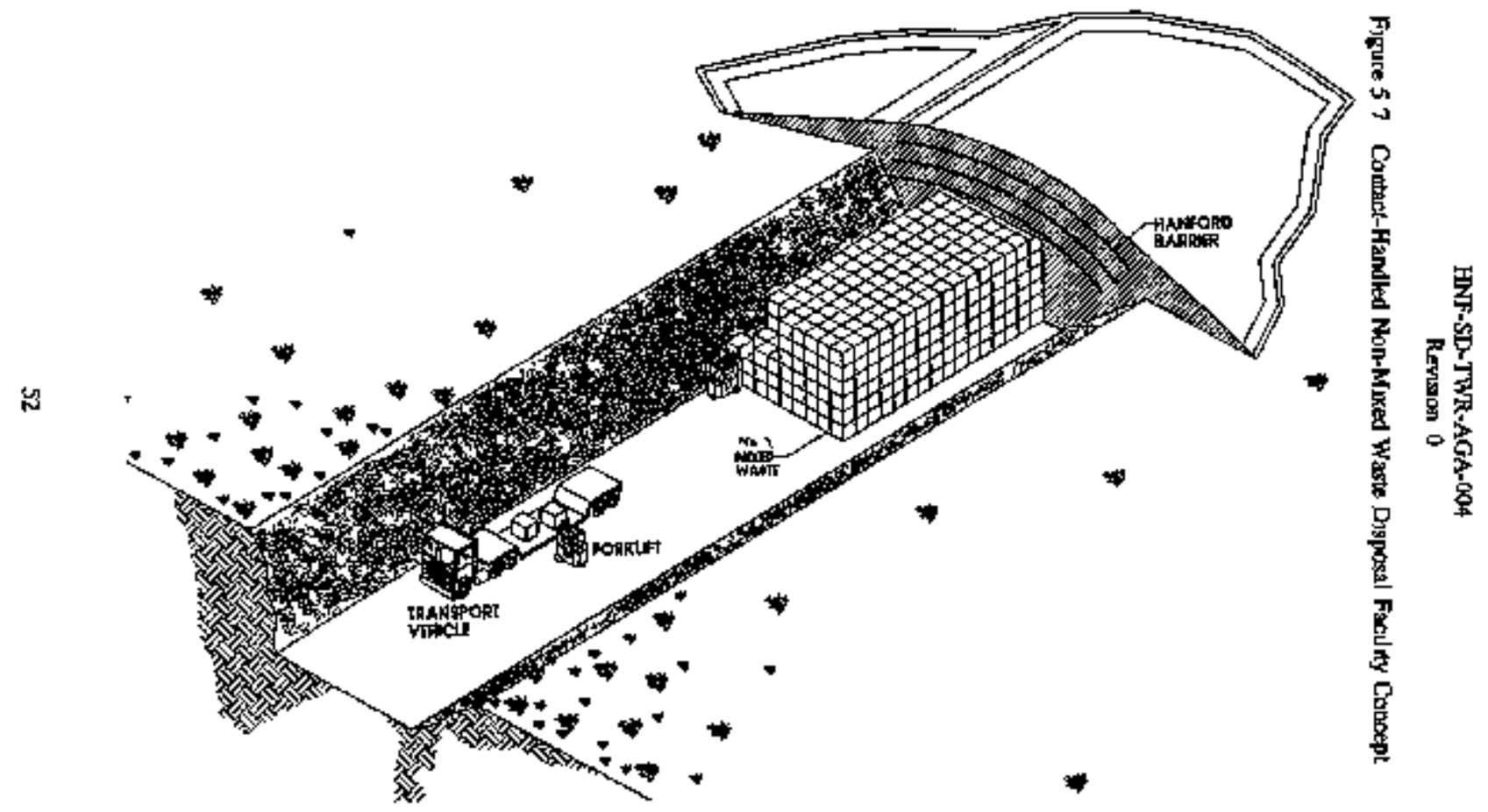


Table 5-11. Summary of Estimesed Capilal Costs For Disposal of Conotict-Handled NenMixed Waste (Option 2D).

\begin{tabular}{|c|c|c|c|c|}
\hline $\begin{array}{c}\text { Dispkssal } \\
\text { unj! }\end{array}$ & $\begin{array}{c}\text { Construction } \\
\text { \& quillion }\end{array}$ & $\begin{array}{l}\text { Infrastructure' } \\
\text { \& millivol }\end{array}$ & $\begin{array}{c}\text { Site elocure" } \\
\$ \text { million }\end{array}$ & $\begin{array}{c}\text { Total } \\
\$ \text { million }\end{array}$ \\
\hline Module 1 & 1.3 & 24.4 & 2.5 & 28.2 \\
\hline $\begin{array}{l}\text { Additional Is units for } \\
\text { digpesel of remaining } \\
\text { LLAW as CH NMM }\end{array}$ & 23,4 & 0 & 45.0 & 68.4 \\
\hline Tolal & 24.7 & 24.4 & 47.5 & 96.6 \\
\hline
\end{tabular}

ILAW = Immobilized low-2ctivity waste

CH NDA = Contact-handled noln-mixed wastt

'Infrastructure is one lime cost for construction of ang of the units for the flexible alternative options.

"Upit and site clesure costs are a one time cobl. They ate the same for any of the fiexdile allernative options. 
Table \$-12. Estimated Operating Costs for Diqposal of Contact-Handled Non-Mixed Waste (Opion 2D).

\begin{tabular}{|c|c|c|c|c|c|}
\hline Msodel & $\begin{array}{l}\text { Operationtal } \\
\text { preparathou } \\
\text { \$ million }\end{array}$ & $\begin{array}{l}\text { Hot operation } \\
\$ \text { mililion }\end{array}$ & $\begin{array}{c}\text { Paxility } \\
\text { teativalion } \\
5 \text { million }\end{array}$ & $\begin{array}{l}\text { Long term } \\
\text { mantivoring } \\
\$ \text { million }\end{array}$ & $\begin{array}{l}\text { Totil cost } \\
\text { \$ m milison }\end{array}$ \\
\hline $\begin{array}{c}\text { Phase ]' } \\
(13,200 \\
\text { conlainets) }\end{array}$ & 5.1 & 51.9 & 2.6 & . & 59.6 \\
\hline $\begin{array}{l}\text { Phase [I" } \\
(85,000 \\
\text { Dontalners) }\end{array}$ & 8.1 & 127.6 & 4.1 & 17.9 & 157.7 \\
\hline Tolal & 13.2 & 179.5 & 6.7 & 17.9 & 217,3 \\
\hline
\end{tabular}

A Phase I Disposal Bases:

12 year duration (2 yг pre-pperation, 9 yr operation, 1 yr denclivation) peak production 9 consaibert per day (63 contaliners per 7 day week) peak productuon of 13 containers per day based on 5 day week 2 package per trailer and 2 trailers per shift baged on 3.5 heforailex turnaround 3 shifts, 1 dispowal urit handes 12 containers per day and 60 containers per 5 day sweck (4 contạinergishifuvault $\times 3$ stilis/da $\times 1$ unil $\times 5$ day/wek $=60$ containers/week)

b Phrage II Disposal Eases:

13 year duration (2 yr pre-operation, 14 yt operatloh, 1 yr deaclivation) plus 9 yr term skoniloring

Peak production 27 conkainers per day (189 convainers per 7 day peek) Peak production of 37 conteiners per day based on 5 day week 2 containers per trailer and 2 trailess per shift babed on 3.5 he/traiker turnaround 3 shifts, 3 unil disposal operation provides for disposel of 36 cosntainers per day

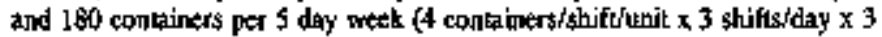

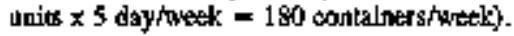


HNF-SD-TWR-AGA-AOS

Revision 0

\subsection{OPTION 3 - SINGLE SHRLL TANKS DISPOSAL}

This alternative is based on the concept of using cleaned out \$STs as digposal units tor the ILAW packapes. The scterdule for this option requires that a substantial emount of the ILAW be plared in regulated interim storage faciljtes to allow sime for waste retrievat, thenk inspection, tank modilloations, and disposal atuhoudzation. Tank inepection and secondary waste teuteval may require enlarging the dowe opendag of several tanks to remove in-rank equipnent such as thermocouples, air lift circolators, salt well screens, and other items. The additional clean out work is estimaled bo require at loset one year per tank bo compleke.

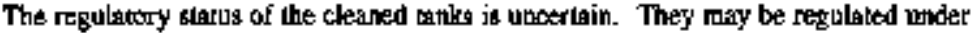

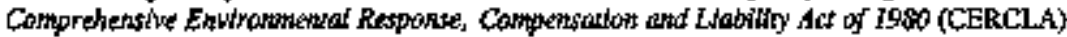

or RCRA. If uaed for disposal of LAW, the SSTs would not meet the requirements for mixed waste since there is no double finer or leachate collection systern in placte.

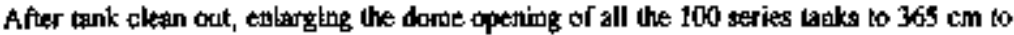
$1,460 \mathrm{~cm}$ (12 ft to $48 \mathrm{ft}$ ) or larger wouk be needed for addkion of remote handling equipment to insert and position ILAW packages inside the tanks. At this tirie, wask retrieval planning does not inclode enlarging the tank dome cpenings, of dispossal of contaminated in-tank. equipment and other debriv.

Figure \$-8 shows the arrangement of ILAW packages in empty tanks of varions sizes. Table 5-13 summarizes the estimated ILAW disposal copacity of the 100 peries SSTS. Results show there is space available for disposal of about 75 percent of the projectod 98,200 LAWN packarges. Additional units moold be recqujred for disposal of the temaining 23,000 packares. The 28 DSTs coold provide most of the ndditional disposal capacity (28 tanks $\times 792$

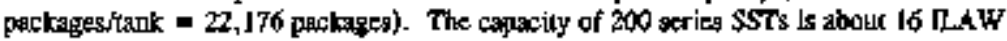
packages per tank and too low for further confideration.

Table S.14 summarizes the SST retrieval sebedule. The schedule shows AX Tank Farm (first tank completed in February 2004 and bast tank completed in February 20008). A Tink Farm (first tank porpleterd in April 2004, last tonk coupleted In Ocwber 2016), and C Tank Farm (first tank completed in May 2012, last tank couppleted in July 2018) are the first thes tank farms to be ayailable for [LAW disposal (Penaell 1996). An additional year or longer must be added to these projected comptetion dates to allow for tant ingpection, secondery clean out, and addition of a large openting in the domes for ILAW disposal. This schedule does not mett the near-termi requlrements for ILAW dlsposal. Therefore, addltional interim storage wowld be requlred (tee Option 5) to provide the near-term capacity abeded to support the privale vetador production schedule. 
HNF-SD-TWR-AGA-004

Ravizion 0

Figure 5-8. Arrategement of Immobilized Lord-Activity Waste Packages in an Enpty Tank.

DISPOSAL OF ILAW PACKACES IN EMPTY BST'B

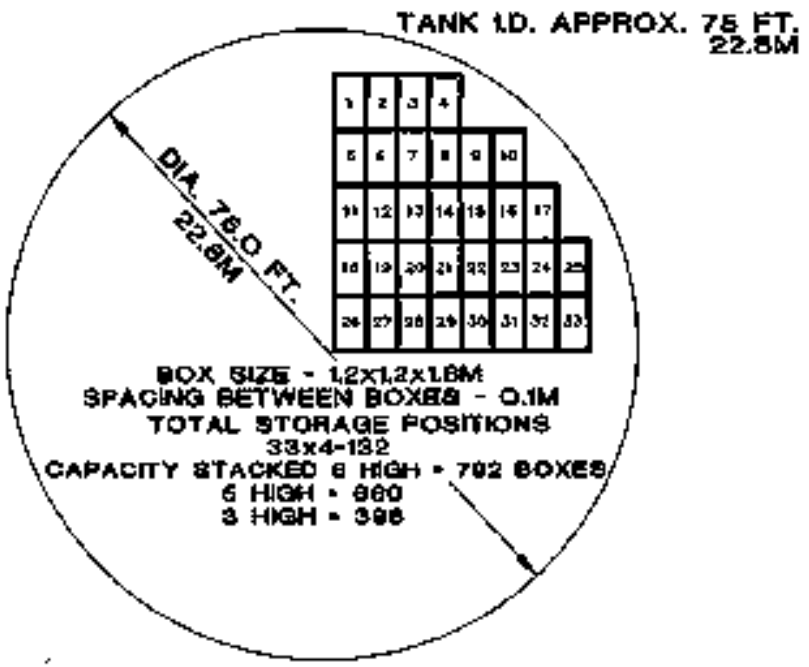

TANK HIEIGHT (DEPTH) LIMITS STACKING HEIGHT $180^{\prime+}$ - EOOK GA. - 16FT $\rightarrow$ ONLY CAN STACK 3 HCH $270^{\prime \prime}=750 K$ GA. - 22,5FT. $\rightarrow$ ONL Y CAN STACK 6 HKA

3E0" = 1ODOK GA. - SOFT $\rightarrow$ ONLY GAN BTACK 5 HCH
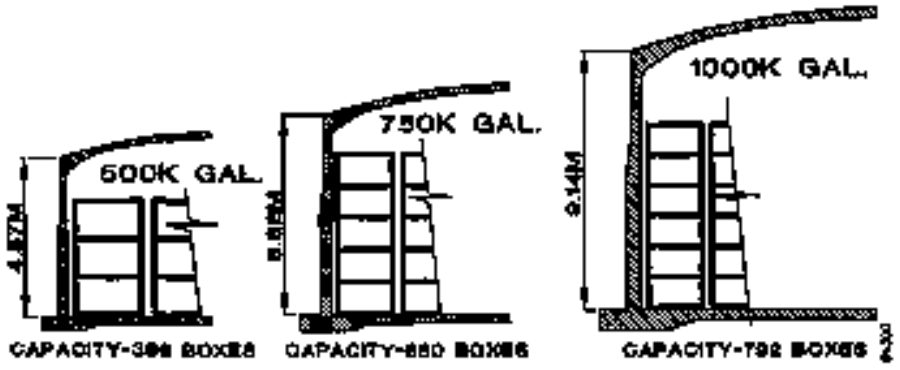
HNF-SD-THR-AGA-004

Rerition 0

Table 5-13. Capacity of 100 Series Single-Shell Tanks for lnmobilized Low-Activlty Waste Disposal Packans.

\begin{tabular}{|c|c|c|c|c|}
\hline \multirow{2}{*}{ Tank Farm } & \multicolumn{3}{|c|}{ Tank Count by Canacily } & \multirow{2}{*}{ Fackrges ILAW } \\
\hline & $1,000 \mathrm{~kg}$ al & 750 bxal & F0, kgal & \\
\hline A & 6 & $\cdot$ & & 4,752 \\
\hline $\mathrm{AX}$ & 4 & & & 3,168 \\
\hline 息 & & & 12 & $4,7 \$ 2$ \\
\hline $\mathrm{BX}$ & & & 12 & 4.752 \\
\hline BY. & & 12 & & 7,920 \\
\hline$c$ & & & 12 & 4,752 \\
\hline $\mathrm{s}$ & & 12 & & 7,920 \\
\hline$\$ \mathbf{x}$ & 15 & & & 11,880 \\
\hline$T$ & & & 12 & $4,7 \$ 2$ \\
\hline TX & & 18 & & $11,8,80$ \\
\hline TY & & 6 & & 3,960 \\
\hline $\mathbf{u}$ & & & 12 & 4,752 \\
\hline Total tanks & 25 & 48 & 60 & - \\
\hline Packanges/lank & $792^{\circ}$ & $660^{\circ}$ & $390^{\circ}$ & - \\
\hline $\begin{array}{c}\text { Total } \\
\text { packses }\end{array}$ & 19,800 & 31,680 & 23,760 & 75,240 \\
\hline
\end{tabular}

ILAW $=$ Imunobitimed low-3ctivity paste

'Based on stacking beight of $6 \mathrm{ILAW}$ packages ( $7.2 \mathrm{~m})$

"Based on stacking beight of 5 ILAW packages $(6.0 \mathrm{~m})$

"Based on Macking helokil of 3 D.AW packases $(3.6 \mathrm{~m})$. 
Table 5-Id, Availability of 100 Series Single-Shell Tanks for Potential Disposal of Inmobjilized Low-Activity Waste.

\begin{tabular}{|c|c|c|c|c|}
\hline \multirow[t]{2}{*}{ Tank farm } & \multicolumn{2}{|c|}{$\begin{array}{l}\text { Projected retrieval } \\
\text { connpiction date* }\end{array}$} & \multirow[t]{2}{*}{$\begin{array}{l}\text { Tanks available } \\
\text { for ILd W wsposal }\end{array}$} & \multirow[t]{2}{*}{$\begin{array}{c}\text { Total } \\
\text { pachlatges }\end{array}$} \\
\hline & First tank & Lsidu tank & & \\
\hline $\mathrm{A}$ & 여/(04 & $10 / 16$ & 6 & 4.752 \\
\hline $\mathrm{AX}$ & $02 \times 04$ & $06+08$ & 4 & 3,158 \\
\hline $\mathrm{B}$ & 00114 & $02 / 1 \mathrm{~g}$ & 12 & 4,752 \\
\hline EX & $10 / 14$ & $12 / 18$ & 12 & 4,752 \\
\hline BY & $07 / 16$ & $12 / 19$ & 12 & 7,920 \\
\hline$c$ & $03 / 04$ & $06 / 16$ & 12 & $4,7 \$ 2$ \\
\hline $\mathrm{s}$ & $05 / 12$ & $07 / 18$ & 12 & 7,920 \\
\hline $\mathbf{s x}$ & 12,04 & $05 / 20$ & 15 & 11,890 \\
\hline $\mathrm{T}$ & $11 / 1]$ & 0019 & 12 & 4,752 \\
\hline TX & $08 / 12$ & $12 / 18$ & 18 & 11,890 \\
\hline $\mathrm{TY}$ & $01 / 17$ & $01 / 18$ & 6 & 3,960 \\
\hline $\mathbf{U}$ & $09 / 07$ & $09 / 19$ & 12 & 4,752 \\
\hline Total & - & - & 133 & 75,240 \\
\hline
\end{tabular}

ILAW = Immobilized low-actipity waste

"Baged on Pentrel] 1996. 


\section{Revisina 0}

Table 5-15 summarizes the estimaled capital costs for addition of a 365 cm (12 f) th $1,460 \mathrm{~cm}$ ( $48 \mathrm{ft}$ ) opening to domes of the 100 series SSTs. The estimates are based on rough order of magtitude cost extimates from 1990 for one that and escalated to 2002. The estimated eapital cust for addition of a large opening to 133 \$STs wodild ranga from about $\$ 1,330$ million w $\$ 6,110$ million. See Appendix $A$, Tables A-11 and A-12 foc details. Additional funds would be needed for procurement and installation of a portable crane'manipulator systern(s) to lower, position and stack the ILAWW packeges inside the tanks and other remote systems for inspection and monitoring purposes.

Since the capital costs for this option are prohibitively bigh, an operaring cost estimate was not prepared. Por porpeses of the evaluation, operating cosis were assumed io be at lease as high as for Option $2 \mathrm{~A}$, remote-handled mixed watkt.

Table 5-15. Summary of Rough Order Magnitude Capital Cost for Addition of a Large Opening to Dorre of 1 cos Serios Siggle-Shell Tanks (Option 3).

\begin{tabular}{|c|c|c|c|c|}
\hline \multirow{2}{*}{$\begin{array}{l}\text { Tank } \\
\text { farm }\end{array}$} & \multirow{2}{*}{$\begin{array}{c}\text { Tanks } \\
\text { available for } \\
\text { ILAW disnosal }\end{array}$} & \multicolumn{2}{|c|}{ ROM capital cost $\$$ million } & \multirow{2}{*}{$\begin{array}{l}\text { Proliminary dispossal } \\
\text { cost } \$ 1,000 / \text { pacikage }\end{array}$} \\
\hline & & I2 fl cpening & 48 ft opening' & \\
\hline$A$ & 6 & 60 & 2 影 & $12 \cdot 60$ \\
\hline $\mathrm{AX}$ & 4 & 40 & 180 & $12-60$ \\
\hline$B$ & 12 & 120 & 550 & $25 \cdot 115$ \\
\hline$B X$ & 12 & 130 & 550 & $25 \cdot 115$ \\
\hline $\mathrm{BX}$ & 12 & $13 t$ & 550 & $15 \cdot 65$ \\
\hline $\mathrm{c}$ & 12 & 120 & 550 & 25.115 \\
\hline $\mathrm{s}$ & 12 & 120 & 550 & $15 \cdot 70$ \\
\hline SX & 15 & 150 & 690 & $12 \cdot 60$ \\
\hline $\mathbf{T}$ & $\underline{12}$ & 120 & 550 & $25 \cdot 115$ \\
\hline TX & 18 & 180 & 830 & $15-70$ \\
\hline TY & 6 & 60 & 280 & 35.70 \\
\hline $\mathbf{U}$ & 12 & 120 & 550 & $25 \cdot 115$ \\
\hline Total & 133 & 1,330 & 5,110 & $20 \cdot 80$ \\
\hline
\end{tabular}

ILAW = lmmobilized low-activity waste

"Based on ROM from 1990 eatimate for one tank and copalaved to 2002 using a factor of 1.46 for composite materials and labor. 


\subsection{OPTJON 4 - EXISTTNG CANYON BULLDEGS}

This alternative is based on the premise that surplug matn propessing faxilities in the 200 Area (B Plant, T Plant. U Plant and Phocoium Uranium Exaraction Facilizy) of the Fatrford Site may be usable for digposal of remoes and contact-handed ILAW.

A Canyon Task Team from the U.S. Department of Energ, Richland Operations Office, W.S. Enrironmental Protoclion Ageacy and State of Washington Department of Ecology conducted a series of workshops to identify an opproach for long-term disposition of the main processing facillties in the 200 Area (DOE-RL I997). Two of the propesed six dispossal alternatiyes are potentially applicsble to ILAW disporal based on the fespibility study (FS) for

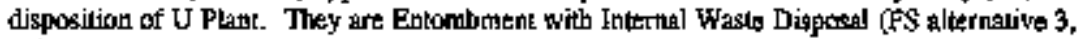
AGA Option 4A) and Entombmenr with Intertal and External Watst Dispcosal (FS alternative $4, A G A$ Option 4B). Both alternatives are discossed below for potential ILAW disposal at the B, T, U, and PUREX Plams.

The Canyon Task Team concluded that the CERCLA regulatory process ts the appropriate decision making process for disposition of the main processing faciliries. RCRA requjrements for disposal of mixed wate inzide the canyon buildings world be revisited once the canyou dispostlton decision las been made. The dedsuing of ILAW wonld simplify the regulations for disposal of CERCLA waste and RCRA tank waste at these facilities. Entombment with disposal of wagte wilh require expedited regulatory decision taking to neet the schedules for remediation of both past practict and tank wasles.

\section{5,4,1 Option 4A - Cangen Bolldigg Butombitent whh Internol Waste Disposed}

The Entombment with Internal Waste Dispesal altermative calls for disposal of Hanford Site remediation waste iuside the canjon facilities followed by in stu entonbment of the waste filled facilities, as shown in Figure 5-9. The ervisioned end state is an entombed waste disposal facility containing selected tacility legacy waste, plus approwed oraste front other Hanfard Site cleanup projects only on the inside of the entambed tacility.

The earlier AOA on inkerim skonge of remole-handled LAW packages (Buttank 1996) showed that a total of 18,600 pachages coold be piaced on the canyon dechs of $B, T, U$, and PUREX Plants and 4,670 packageg could be planed in the protess cells and storage gallery of the PUREX Plabt The oyallable clearance utder the existing cranes limited the stacking height w 4 or 5 hlgh on the eanjon decks. Table 5-16 suramarizes the potential disposal capacity isside each of the carryon beildinga and based on inserim slorage of ILAW by Burtonk (1996). 


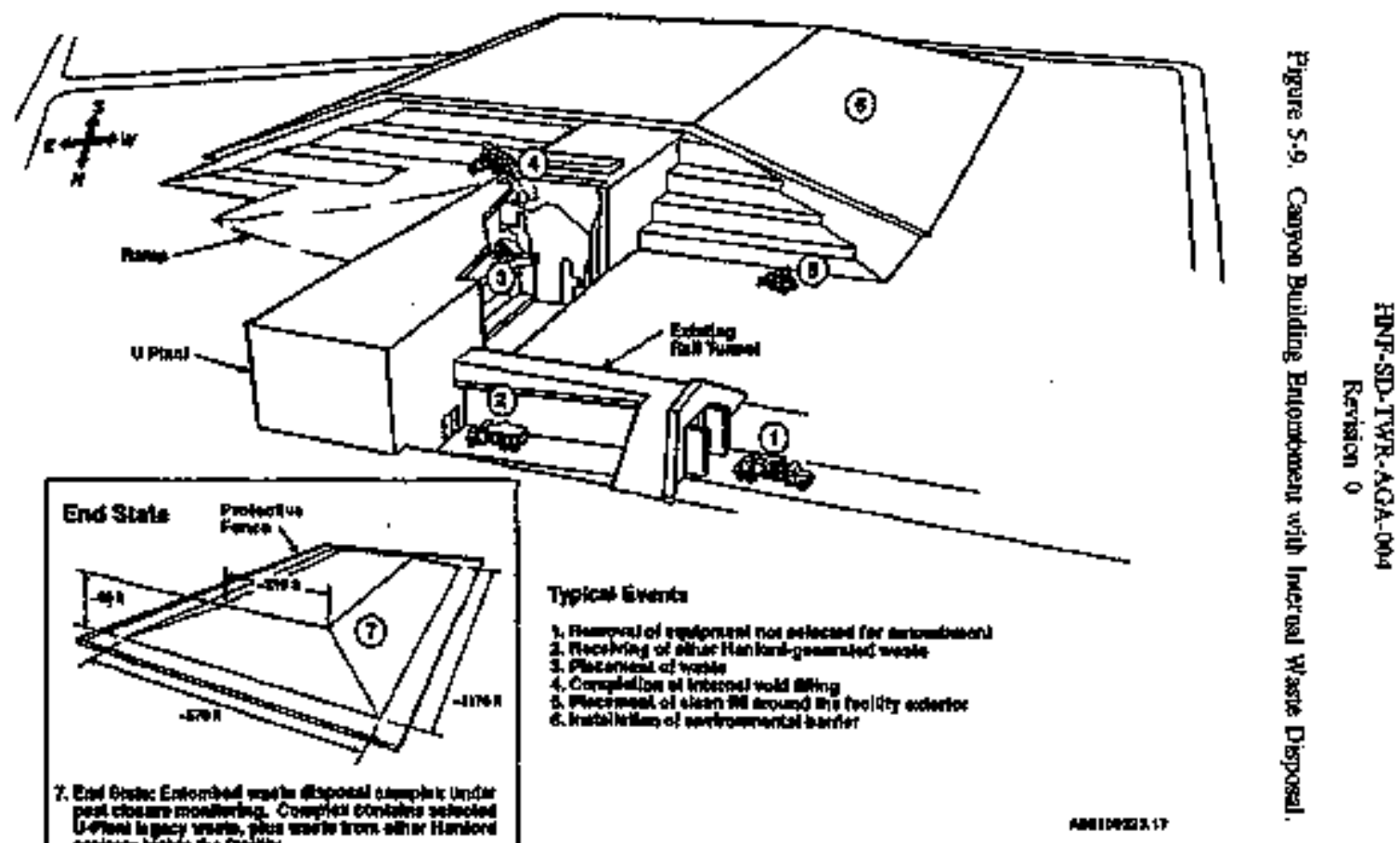


The canyon deck disposal concept uses a modular steel floor system about $0.9 \mathrm{~m}$ thith to raise the ILAW packages showe the cover block lifting bails and spread the laed evenly across the canyen deck. The ILAW containes trogsport wehicle woold anter the buildings directly

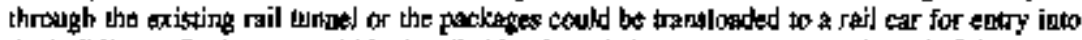
the buildings. Packages would be handled by the existing canyon craras. At PUREX, removing the main bridge crane from service and using the aluxiliary trate for package handling would increase the available capacity. Using the auxdlary crane would allow stacking of packages ajx bigh and rould iacrease the canyon deck disposal capactly by $50 \mathrm{~T}$ (equivalete to 2,130 additional ILAW pactages).

Table 5-16. Sumenay of Polential Remote-Handled Jrimobilized Low-Activiry Waste Disposal Inside Canyon Buildings.

\begin{tabular}{|c|c|c|c|}
\hline Descrjptlon & $\begin{array}{c}\text { Capacity } \\
\text { RH-JLAW packapet } \\
\text { count }\end{array}$ & $\begin{array}{c}\text { Package } \\
\text { gtsckiog heighy }\end{array}$ & $\begin{array}{l}\text { Pacility } \\
\text { section } \\
\text { size }\end{array}$ \\
\hline PUREX Camyon Deck & 4,260 & 4. & $8.4 \mathrm{~m} \times 248 \mathrm{~m}$ \\
\hline PUREX Stounge Gallesy & 2,700 & 5 & $19.5 \mathrm{~m} \times 232 \mathrm{~m}$ \\
\hline PUREX PTocess Cells" & 1,970 & 6 & $4.3 \mathrm{~m} \times 264 \mathrm{~m}$ \\
\hline U Plant Campon Deck: & 4,800 & $\underline{5}$ & $11.3 \mathrm{~m} \times 244 \mathrm{~m}$ \\
\hline T Plant Canyon Deck & 4.800 & 5 & $11.3 \mathrm{~m} \times 244 \mathrm{~m}$ \\
\hline$B$ Plank Canyon Decks & $4,8 \mathrm{POC}$ & 5 & $11.3 \mathrm{~m} \times 244 \mathrm{mil}$ \\
\hline Total & 23,330 & - & $16,000 \mathrm{~m}^{2}$ \\
\hline
\end{tabular}

ILAW = Immobllied low-activity waske

PUREX = Plutonimo-uranlum exiraction

RH $\leftarrow$ Remole hancled

- Based on adtition of moduiar steel floor system ahout 0.9 m high to raise the packapos above the cover block lifting bails and apread the load evenly serpos the canyon deck.

"Requires removial of cover blocks and digposel of contanithated proces equpment.

" Based on interim storage information in Burbank (1996). Assumes space availsbility of designated building cations for ILAW pactages.

The PUREX storage gallery has capacily for disposal of an estimated 2.700 packiges of ILAW. It it a clean area loxated on the kwer level of PUREX. Access would be from the existing annex buildigg loading dock, through the agpopous make up aros to the elevator and down to the lower level. ILAW package handing would be via semotely aperatod aulomated guided vehictes similar in concept to a forklift. The anmex losding dock and aqueous make up 


\section{Revition 0}

area would be modified to allow remole banding of the ILAW packates by the altomatc guided vethicles. Pachatides monld be teansported to the loading dock by trock trailes.

The 12 PUREX process cells have capaclly for disposal of about 1,970 ILAW packighe. The celis are contaminated and contein process equipmen. The cover blocks and all process equiproent must be remeved before the exlls copld be used for dispogal of ILAW. The IIAW parkages would be packaged ink new carton stetel cell liners (82 wew Jiners each combaining 24 ILAW packages) for placement in the cells using the existing canyon crame. The packages would be transported by truck trailer transport and would enter the buildings directly through the existing rail tonoel or the packnges coold be transiloaded $\mathrm{k}$ a rail car for entry into the buikdingy. There is not enough ropm in the process cells or operating galleries of $T$, U, or B Plant to make those ateas suitab]e for ILAW packesse disposal.

Table 5-17 summarizes the estimated capital costs for consuruction, infrastorcture, and closure for this alternative. The total construction cost is $\$ 65.1$ willion. See Appedix A, Tsble A-14 for details.

Table 5-17. Summary of Estimated Costa For Constrution and Clostre of Four Canyon Buildings for Disposil of Jmmobilized Low-Activity Waste and Other Waste Based on Fiscal Year 1997 Phase I Feagibility Study for 22L-U Facility:

\begin{tabular}{|c|c|c|c|c|}
\hline Option & Construction \$M & Infrastructire \$ M & Closure \$M & Told \$M \\
\hline $\begin{array}{c}4 A-\text { Internal } \\
\text { Waste Dispasal }\end{array}$ & 65.1 & 0 & 0 & $6 \$ .1$ \\
\hline
\end{tabular}

- Seurce of information is DOE-RL 1997 for 22l-U. Costs escalated to 2002 assuming similar costs for disposilion of other canyon buldings.

Table 5-18 summarizes the estimated operating costs for the 4 canyon boildings with incerpal wapte disposal includiag 23,330 ILAW contairess. The ropl cost for operational prepsagtion, hot operations, deactivalipn and long-term monitoring ig \$147 million. See Appendlx A, Table A-19 for detalls. 
FINF-SD-TWR-AGA-004

Revition o

Table 5-18. Summary of Escimated Operaling Costs For Disposition of Four Canyon Buildings Based on Intermat Waste Disposal (Option 4A).

\begin{tabular}{|c|c|c|c|c|c|}
\hline Option & $\begin{array}{c}\text { Operational } \\
\text { prepsration } \\
\$ M\end{array}$ & $\begin{array}{c}\text { Hot dperation } \\
\text { S M }\end{array}$ & $\begin{array}{c}\text { Deactivation } \\
\$ M\end{array}$ & $\begin{array}{c}\text { Sublotal } \\
\$ \mathbf{M}\end{array}$ & $\begin{array}{c}\text { Grend total } \\
\mathbf{S M}\end{array}$ \\
\hline $4 A(23,330$ & \multicolumn{2}{|c|}{117.8} & 0 & $117.8^{4}$ & \multirow[t]{2}{*}{145.8} \\
\hline Containers) & $1.9^{\circ}$ & $26.3^{\circ}$ & $0.6^{\circ}$ & $29.0^{b}$ & \\
\hline
\end{tabular}

ILAW = Immobilized kny-ativity waste

'Soure of iuforation is DOE-RL 1997 for 22J-U. Costs extrapolated to 20,02 asguming similar costs for disposition of otfier canyon buildengs.

'Source of information is Table A-19. Costs reduced to 24\% of full capacity operating crepr cost based on disposal of 23,330 renote-handled ILAW ontainers inside of canyon buildings. No cost incloded for use of available managentert and operating support pergonacl agsigned th cantyon building disposal. 


\section{HRNF-SD-TWR-AGA-1004 \\ Revision 0}

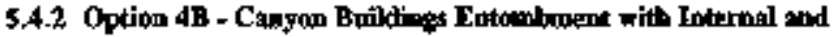 Externsol Waste Dispasad}

The Entombment with Internal and External Waste Disposal alterngive calls for disposal of Hanford \$ite remediation orabtes inside and around the optside of the canjon boildings followed by in situ thlopmbment (Figute 5-10). Remote-hatedled lLAW pachastes would be ptaced irside the buildieg and conract-handled ILAW pactages would placed along the culside wall of the bujidings.

Plans for external waste placement woold be to achiove regulatory compliance by using a double-liner leschate onllection tystem and provision for ground opater motuttoring. The external wasse materials and clasm fill finst the base for the protectlye barrier that mould cover the walte filled facilities. The envisioned end-3ate is an entorthed waste disposel complex contaituing selected factlity legacy waste, plus approved waste fron other Hanford Site cleapup located both on the inside and oursidts of the brildings.

Option 4A described the disposal of remete-handifed ILAW pactages inside the B, T, U, and PUREX Plants and showed thal 23,300 rempote-handled ILAW packages could be dispased inside the canyou buildings. Internal disposal of temote-handled praste nould precede of proceted in parallel pith contact-bandled waste disposal outside the buildings.

Contact-handled ILAW packarses would be transported by trucke urailer th the canyen buildings for off loading and placement by fortlifts. The packages would be placed aloug the one or both extertor walls and atacked 6 high to a dlstance of about $40 \mathrm{~m}$ from both exterior walls. This stacking antangement leaves a 5 mo thickness of protectise barrier cover oret the Jast ILAW/ package based on the proposed 1:3 slope of the proted tive berrier over the caryon building. This exterior arrangesment provides for placement of about 96,000 pectages of contast-handled ILAW based on a $\$ 0$ percebt availabiliny of the space aldug the exterior walls of the canyon buildings. Table S-19 suntmarizes the total package count information for the canyon buildiag dispossal altemative.

Table $\$ 20$ summarizes the estimated caphtal costs for construction, infrastructure, and closure for this alternative. The total constructions coat is $\$ 189$ million See Appendix $A$, Table A-14 for detajls.

Table 5-21 dermmarizes the estimated operating cous for the 4 canyon bulldings with

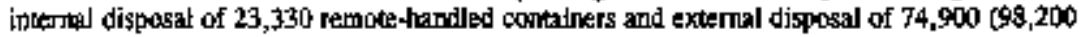
$-23,300=74,900$ ) conlact-handled containert. The kotal cost for operational preparation, hot operations, deactivation and long-term monitoring is $\$ 293$ million. Sex Table A-19 for details. 


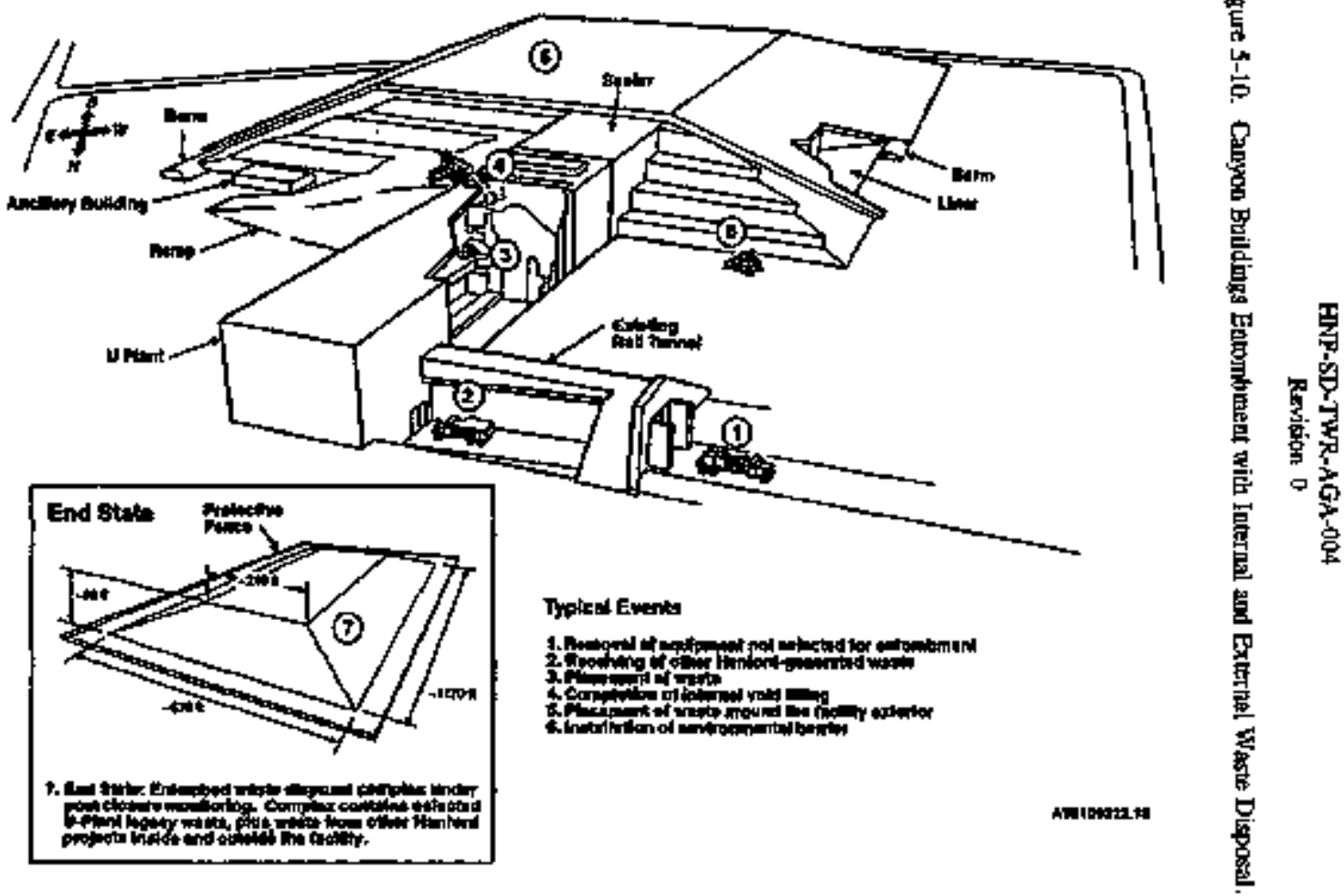


HNP-SD-TWR-AGA-(0)4

Reviकion D

Table 5-19. Summary of Potential Immobillzed Low-Activity Wagte Disposal Inside and Ousagide Canyon Buildings.

\begin{tabular}{|c|c|c|c|c|}
\hline \multirow[t]{2}{*}{ Bujlding } & \multicolumn{2}{|c|}{ Imtectior dispos; I } & \multicolumn{2}{|c|}{ Extariox dispossal } \\
\hline & Section sizet & $\begin{array}{c}\text { Capacity } \\
\text { RH-[LAW } \\
\text { pactage } \\
\text { countr }\end{array}$ & $\begin{array}{l}\text { Section size (both } \\
\text { sides of canyon) }\end{array}$ & $\begin{array}{l}\text { Copacity } \\
\text { CH-ILAW } \\
\text { packong } \\
\text { couni }\end{array}$ \\
\hline \multirow[t]{3}{*}{ PUREX Plant } & $8.4 \mathrm{~m} \times 248 \mathrm{~m}$ & 4,260 & \multirow[t]{3}{*}{$306 \mathrm{~m} \times 80 \mathrm{~m}$} & \multirow[t]{3}{*}{57,600} \\
\hline & $19.5 \mathrm{~m}=232 \mathrm{~m}$ & 2,700 & & \\
\hline & $4,3 \mathrm{~m} \times 264 \mathrm{~m}$ & $1,9 \% 0$ & & \\
\hline B Plant & $11.3 \mathrm{~m} \times 244 \mathrm{~m}$ & 4,800 & 247 m $x$ 影田 & 44,800 \\
\hline T Plant & 11.3 mा $\times 244 \mathrm{~m}$ & 4,800 & $247 \mathrm{~m} x 80 \mathrm{~m}$ & 44,800 \\
\hline U Plant & $11.3 \mathrm{~m} \times 244 \mathrm{~m}$ & 4,800 & $247 \mathrm{~m} \times 90 \mathrm{~m}$ & 44,800 \\
\hline Total & $16,000 \mathrm{~m}^{3}$ & 23,330 & $84,000 \mathrm{~m}^{3}$ & 192,000 \\
\hline
\end{tabular}

CH-[-AW = Contact-bandled immobilized low-activity waste PUREX = Plutroniom-Uranium extraxtion

RH-ILAW = Remote-bandled isnmobilized low-aclivity waste

' Ses Table S-16 for additional delaits. Atsumes apece avaibility of designared internal building sections for ILAW packages.

- Based on remoral of all canyou building prodrosious and unrestricted spece ayaliablllty on both long sides of the exterior canyon walls. 


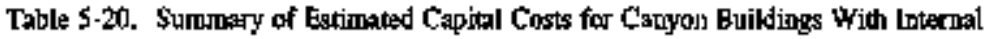
and External Disposal (Option 46)."

\begin{tabular}{|c|c|c|c|c|}
\hline Option & Consuruction $\$ \mathbf{M}$ & Inirastructure $\$ M$ & Clopure \$M & Total \& M \\
\hline $\begin{array}{c}\text { AB - } \\
\text { Injernal } \\
\text { and } \\
\text { External } \\
\text { Wagte } \\
\text { Disposal }\end{array}$ & 105.4 & 0 & 83.2 & 188.6 \\
\hline
\end{tabular}

-Source of jufixmialion is DOE-RL 1997 for $221-13$, Costs eacalated to 20012 assuming sidrilar cogts for disposition of other canyou buildings.

Table \$-21, \$unmary of Eatimated Operating Costs For Dieposition of Four Canyon Buildings Based on Internal and Exterted Waste Disposal (Option AB).

\begin{tabular}{|c|c|c|c|c|c|}
\hline Option & $\begin{array}{c}\text { Operaicional } \\
\text { preparation } \\
\$ M\end{array}$ & $\begin{array}{c}\text { Hot operatlots } \\
\$ M\end{array}$ & $\begin{array}{c}\text { Deactlvation } \\
\$ M\end{array}$ & $\begin{array}{c}\text { Subtotal } \\
\$ M\end{array}$ & $\begin{array}{c}\text { Grand toral } \\
\$ M\end{array}$ \\
\hline \multirow{2}{*}{$\begin{array}{l}4 \mathrm{~B}(98,200 \\
\text { ILAW } \\
\text { Containers) }\end{array}$} & \multicolumn{2}{|c|}{155.7} & $14.4^{*}$ & יו. & \multirow{2}{*}{292.5} \\
\hline & $7.8^{4,4}$ & $111.0^{b s}$ & $3.6^{* 4}$ & $123.4^{b, 0}$ & \\
\hline
\end{tabular}

' Sonrice of information is DOE-RL 1997 for 22L-U and Table A-19. Costs extrapolared to 2002 assuming simliar costs for disposition of other canyon buildings.

${ }^{2}$ Source of information is Tahle A-19. Costs reduced to 24 percent of operating arew cost based on disposal of 23,330 remove-handled ILAW coubiaks inskde of tranyen buildings. No cost imcluded for uge of avaibable manoigement and operating support personnel assigned to canjon building disposal.

- Soutce of informalion is Table A-19. Cost is sum of 76 percent of cperating crew cost for external disposal of conlacl-handled $\mathrm{ILAW}$ and 24 percent of crep cost for internal disposal of remote-handled ILAW. No cost included for use of available management and operating support pesionnei assigued to canyon buikting disposal. 
HNF-\$D-TWR-AGA-004

Revision 0

\section{S.5 OPTTON 5 - EXPANDED INTERIM STORAGE}

This altemative is based on extended storage of the ILAW packases due to potential delay of chtaining a DOE disposal authorization stateinent beyond 2002 and/or ab additional disposil units available by 2005. It assumes thal a significant amownt of ILAW product is contaci-hasdled due to bor dose rates from low radjomuclide concentration in the retrieved tank waste or eficient removal of radionuclides during pretreatment.

The contact-bandled ILAW packages would be placed into extepribd interim storage

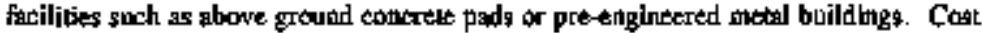
estibates were bated on a unil storage stee of 5,040 packiges. The unis would comply with tequirements for interim stotage of contact-handled inixed wasle. There is issuffictent infornation at this time to determine the amoun of contact-handled naW that may be receiwed.

The ILAW packages would be rempyed from the urangport webicle and moved th the storage position by focklift The opecation is similar to disposal of contact-handled ILAW except aisles art provided belween rows of packages 6 meet the inspection requirenents for mixed waste storage.

The storage concepl would position packares in $\$$ rows of 2 packages wide, 84 packages long, and 6 packages high by fork lif or mobile crant. Throe meter wide aishes would be provided between nows, resulting in floor dimensions of about $40 \mathrm{~m} \mathrm{x} 100 \mathrm{~m}$ for interim scorige of 5,040 packages. The floor area would be covered by pre-enginerred melal butidings.

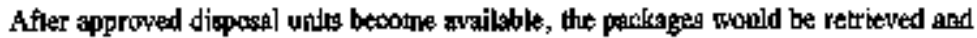
transported to disposal using the garte resources that were used for transporting them to the intering storage uniL

Two interian storage onits for contzet-handled ILAW phus the groul vaults would be

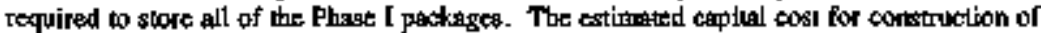
twa above ground thetal storage buildings atd ancillary equipment is about $\$ 9.6$ million based or bigkrical tosts for Project W-1 12, Enharced Radiosetive and Mixed Wagto Storago, excalated to year 2002. 
Table 5-22. Summary of Estimated Cests For Consirucioun and Closure of Units for Intexim Storage of Contact-Handled Mixed Iminobllized Low-Activity Waste Containers Based on Project W/L12.

\begin{tabular}{|c|c|c|c|c|}
\hline Optlon & $\begin{array}{c}\text { Capital } \\
5 \mathrm{M}\end{array}$ & $\begin{array}{c}\text { Infrastrucuure } \\
\text { \$ M }\end{array}$ & $\begin{array}{c}\text { Closure } \\
\$ \mathbf{M}\end{array}$ & $\begin{array}{l}\text { Toxal } \\
\$ M\end{array}$ \\
\hline $\begin{array}{l}\text { 1st unit } 5,040 \\
\text { containers }\end{array}$ & 3.2 & 3.2 & 0 & 6.4 \\
\hline $\begin{array}{l}\text { 2nd unit 5,040 } \\
\text { containess }\end{array}$ & 3.2 & 0 & 0 & 3.2 \\
\hline Tomal & 6.4 & 3.2 & 0 & 9.6 \\
\hline
\end{tabular}

'Source of information is Table A-21

"Source of informalion is Table A.22.

Table 5.23. Estimated Operating Costs for Immbilized Low-Aclivlty Waste Stunde Batad on Prelimimary Operatimg Sequenot for Container Transportation."

\begin{tabular}{|c|c|c|c|c|c|}
\hline Unit & $\begin{array}{l}\text { Operational } \\
\text { petparation } \\
5\end{array}$ & $\begin{array}{c}\text { Hot pperation } \\
\text { \$ M }\end{array}$ & $\begin{array}{c}\text { Short term } \\
\text { moniloring } \\
5 \mathrm{M}\end{array}$ & $\begin{array}{c}\text { Facility } \\
\text { desclivation } \\
\text { SM } \\
\end{array}$ & $\begin{array}{l}\text { Total } \\
\text { cost } \\
\$ \mathrm{MM} \\
\end{array}$ \\
\hline $\begin{array}{c}1 \\
5,040 \text { Contaiders }\end{array}$ & 5.1 & $\begin{array}{l}51.9 \text { (in) } \\
5 L .9 \text { (out) }\end{array}$ & \multirow{2}{*}{11.2} & 2.6 & 117.1 \\
\hline$\frac{2}{5,040 \text { Contriners }}$ & 0 & $\begin{array}{l}51.9 \text { (ia) } \\
51.9 \text { (ont) }\end{array}$ & & 2.6 & 112.0 \\
\hline $\begin{array}{c}\text { Total } \\
10,000 \text { Containers }\end{array}$ & 5.1 & 207.6 & 11.2 & $\$ .2$ & $229 . \mathrm{I}$ \\
\hline
\end{tabular}

"Source of information is Tables A-9 and A-10 for one voit. 
HNF-SD-TWR-ASA-0N4

Revision 0

\subsection{OPTION 6 - SEIIELDD DUSPOSABLE OVERPACKS}

This allermative is besed on construction of a shielded tisposal overpack for packages of ILAW that have g dose rate greater than 200 mremb. The shielding wond rectuot the ourface dose rave to less than 260 nurem/h and permll ditposal of the ILAW as conctaci-handled waste. The overpack woold be made from steel or concrete.

Prelimioary radiation dose rate calculations thowed that 46 en (18 in.) of concrete or

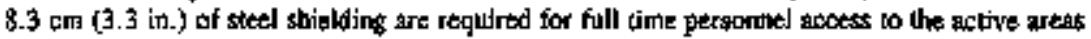

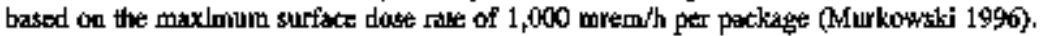
Figure 5-1 I shows the dispestal oxerpack concepts. Table 5-24 summarizes the estimated costs for the shielded disposable overpacks. The estimated cost of 93,000 overpacks is 3302 million for concrete and \$834 million for stect. The iddividual overpack bost is \$4662 for concrete and $\$ 8218$ for stoel. Costs are based on fabricator quotations for a lot size of 5040 ovierpacks.

The larger package sizze for concrete owerpacks (12 $\mathrm{m}^{3}$ versus $\left.2.6 \mathrm{~m}^{3}\right)$ would require the disposal units to be aboul 4.6 times larger (and 4.6 times more expensive) for disposal of the same mumber of contact-handled packages. Even bo, the total cappilal togl for concrete bueparacs would be less that for atted.

Table 5-24. Sunmary of Capital Cos Estimate for Disposable Ovecoachs.

\begin{tabular}{|c|c|c|c|c|}
\hline \multirow{2}{*}{$\begin{array}{l}\text { Matrerial of } \\
\text { constroctions }\end{array}$} & \multirow{2}{*}{$\begin{array}{c}\text { Mass of one } \\
\text { oreerpack, } \\
\mathrm{k}_{\mathrm{g}}\end{array}$} & \multicolumn{3}{|c|}{$\operatorname{Cos}^{*} *$ SK } \\
\hline & & I overpack & $\begin{array}{c}5,040 \\
\text { overpacks }\end{array}$ & $\begin{array}{c}93,000 \\
\text { overpicts }\end{array}$ \\
\hline Concreale & 12,000 & 4.7 & 23,3 & 302,000 \\
\hline Stetel & 19,650 & 8.2 & 41.3 & $\mathrm{~B} 34,000$ \\
\hline
\end{tabular}

'Requires larger contact-handled dispostal facility than Options 2C and 2D due to larger: vahume of disposal comainer

${ }^{b}$ Assumes Immobilized Low-Activity Wagte container is $\mathbf{i} 6 \mathrm{~cm}$ (5/8 in.) thick steel.

- See Appendin A, Table A-23 fọr estimagke details. 
HNF-SD-TWR-ACA-004

Reviston of

Fkgure 5-11. Disposable Overpack Concepts.
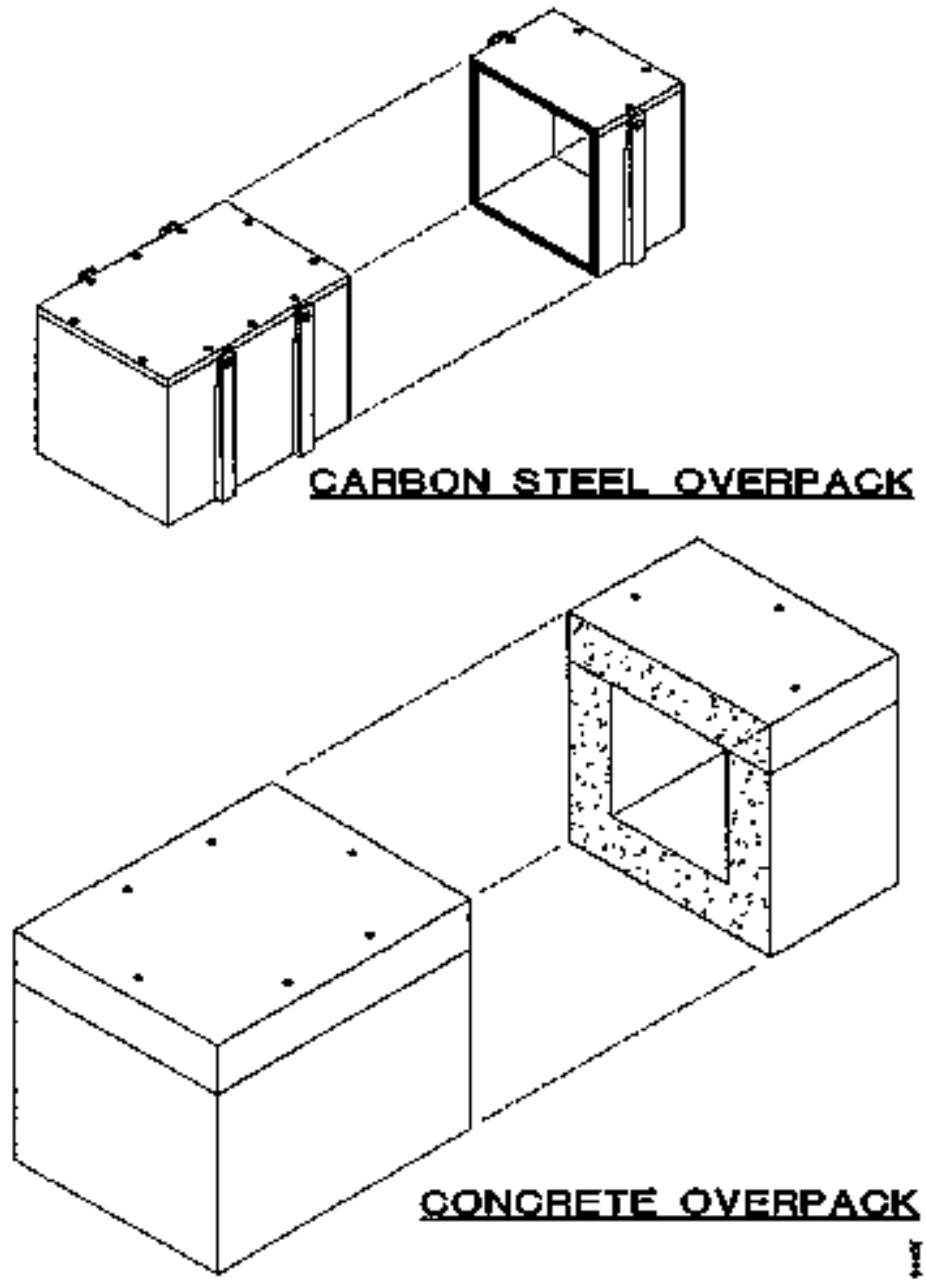


\subsection{REJECTED IMMOBIIZED LOW-ACTIVTY WASTE DIBPOSAL OPTIONS}

Several disposal options inciuding those proposed by the public were eqaluated by DOE and Washington Siate Departonent of Ecology (Ecology) or Westingbouse Hanford Company (WHC). The following sections describe these rejocted options. They have not been contidered further in ubis analysis.

\section{S.7.1 Optlous Rejected by tive Tamik Waste Remedibaton Syatem Enviromonental Impanct Statement}

The following optoas were considered but rejexked as insppropriate for waste disposal by DOE and Ecolory in the TWRS E[S. The criteria of technical viability, maturity of

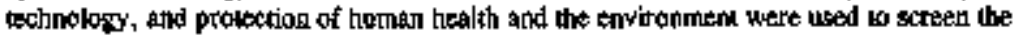
following al territatives purt of congideration.

- Seabed Disposal, space disposal, deep hole disposal, ice sheel disposal, ard island disposal

- Geplogic tisposel of tank contents, lanks, equipment, and contamintited sodl

- Rock melthis or infecting the waste into a deep mined carity:

- Transmutation

- Groputing the retired canyon facilities with hot grout

- Disposing of ghase loge in growk vauls and allowing golids ir lanks to decay.

Additional details on the evaluation of these technotogies can be found in Appendin $C$ of the TWRS EIS, and in the supporting data package (WHC 1995).

\subsubsection{Additional Kejected Disposal Opdions}

Two additional dipersal alternatives were proposed following the completion of TWRS ELS and Record of Dexislou (ROD). These alternatives recommend using the Environtrenual Restoration Disposal Facility (ERDF) and the bulk dispossl of gkas cullet in single-ghell tanks (SST).

The ERDF is a RCRA compliant land fill authorized under CERCLA. Al wasle placed in ERDF must meet dic ERDF Waste Acceptance Crleria (BHD 1995). The raste roceptance crileria for ERDF states that it may not be used for disposal of non-CERCLLA waste such at ILAW. 
Revision 0

The dispossl alternatre of adding ILAW collet to retieved SSTs as engineered fill malerial does aot comply with the product speciffeations in the TWRS privatization RPP (DOR-RL 1996). 
HIF-\$SD-TWR-AGA-004

Revision 0

\subsection{ALTERNATIVES EVALUATKON}

This section presents an evaluation of each of the alteruatives described in Section 5.0 in telation to the evaluation crileria tostablished in Sectlon 4.0.

The alterratives analysin was performed by engineering slaff familiar with the projecl. The evaluation method wap based on well establighed decigion aralysis techniques (Kkepner and Tregoe 1981) on apply the desigion criteria to the allernalivas. Esch coption was ranked from 1 to 10 for technical triterda. Individual rankingts were averaged to arrive at a compusite conoensus ranking for the options. The maximum posshle score was I40 points. Table 6-5 in Section 6.6 presents the composite seores.

\section{1 cost}

Total life-cycle costs for tach alternative pere deteloped. For the purposes of this analysis, all costs were irdexed to constant 2002 dollars. Table $6 \cdot 1$ summarizes the estimated life-cycle cost for constuktion and operation of the baseline and alternative disposal unity. Appendix A provides additional details for cepieal and operatimg cost eatimaties.

The flexlble unit option, consteling of a combination of options $2 \mathrm{~A}, 2 \mathrm{~B}, 2 \mathrm{C}$, and $2 \mathrm{D}$, sill have a variable total cost depeuding on the number of contact-handled, remote-handled, mired, and non-mixed waste disposal facilities that are constnucted. Figure 6.1 shows the estinated range of costs for the flexible unit option compared with the MYWP bageline costs for constroction and operation of the ILAW disposal facilitiea.

\section{$6+1+1$ Cagital Cost}

Capital costs were exaluated ohjectiwely bayed on capitol cost estimales developed by ICP Kaiber Engineers Hanford and Fhor Daniel Northwest. These estimates were at a tough

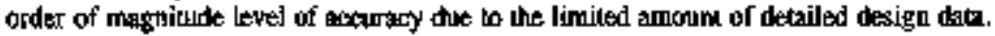
Capilal cost estinates of the alternatives are for construction obly. Athernatives with kower copital cost received better rankings.

The architectures with the lonest capilal cost are the conead-handled alternatives. The next lowest capital coats are for the canyon building altematives. The new remlote-haralled vevits are next, followed by the adjusted troseline concept and ghielded overpack. Disposel in the SST, has the hightest capital coot of any option. 
HNF-SD-TWR-AOA-004

Rovision 0

Table 6-1. Ceppital, Operatting, atod Lifte-Cyele Cosi Mummary.

\begin{tabular}{|c|c|c|c|c|}
\hline Optiont & $\begin{array}{l}\text { Construction cost, } \\
\text { I million }\end{array}$ & $\begin{array}{l}\text { Operating cost, } \\
\$ \text { million }\end{array}$ & $\begin{array}{l}\text { Total life- } \\
\text { cycle cost. } \\
\text { \$ million }\end{array}$ & $\begin{array}{l}\text { Lifo-pycle } \\
\text { cost } \$ \mathrm{~m}^{3}\end{array}$ \\
\hline $\begin{array}{c}\text { Option 1A } \\
\text { MYPP Baselines }\end{array}$ & 399 & 132 & 525 & 3,366 \\
\hline $\begin{array}{c}\text { Option 1B } \\
\text { Adjusted MYYWP } \\
\text { basseline }\end{array}$ & 609 & 245 & 854 & 3,344 \\
\hline $\begin{array}{c}\text { Opdlon } 2 \mathrm{~A}^{\mathrm{C}} \\
\text { RH MW disposal }\end{array}$ & 555 & 408 & 963 & 3,772 \\
\hline $\begin{array}{c}\text { Option 2B } \\
\text { RH NMW diqposal }\end{array}$ & 496 & 408 & .904 & 3,541 \\
\hline $\begin{array}{c}\text { Option 2C } \\
\text { cH MW Aispotal }\end{array}$ & 159 & 217 & 376 & 1,473 \\
\hline $\begin{array}{c}\text { Option } 20 \\
\text { CH NMW disposal }\end{array}$ & 97 & 217 & 314 & 1,230 \\
\hline $\begin{array}{c}\text { Option } 3 \text { th } \\
\text { \$STs disprosal }\end{array}$ & $>>1,330$ & 3408 & $31,73,8$ & 36,807 \\
\hline $\begin{array}{c}\text { Option } 4 \Lambda^{\text {xb }} \\
\text { Onyous }- \text { Jaternal }\end{array}$ & 65 & 147 & 212 & 3,513 \\
\hline $\begin{array}{l}\text { Option } \mathbf{A B} \mathbf{B}^{\mathrm{b} g} \\
\text { Canyons - Internal } \\
\text { and Extermal }\end{array}$ & 189 & 293 & 482 & 1,895 \\
\hline $\begin{array}{l}\text { Dption } \$ \\
\text { Exterudad Interim } \\
\text { Storage }\end{array}$ & 10 & 229 & 239 & 9,119 \\
\hline $\begin{array}{c}\text { Option 6A' } \\
\text { Overpacked RH } \\
\text { MW }\end{array}$ & 914 & 217 & 1,131 & 4,430 \\
\hline $\begin{array}{c}\text { Option 6B } \\
\text { Overpacked RH } \\
\text { NMW }\end{array}$ & 641 & 217 & 858 & 3,360 \\
\hline
\end{tabular}

CH $=$ Contact-handled

MW = Miked waste

NMW = Non-mixed waste

RH = Remote-jandled

SST = Single-shel] tank

-Does not provide required eapacity

boes not meet near-term gchedule requireerments

Meets desipn requincments for all waste lypes. 
Figure 6-1. Tolat Lib-Cycle Cost versus Remolo-Handled Fraction.
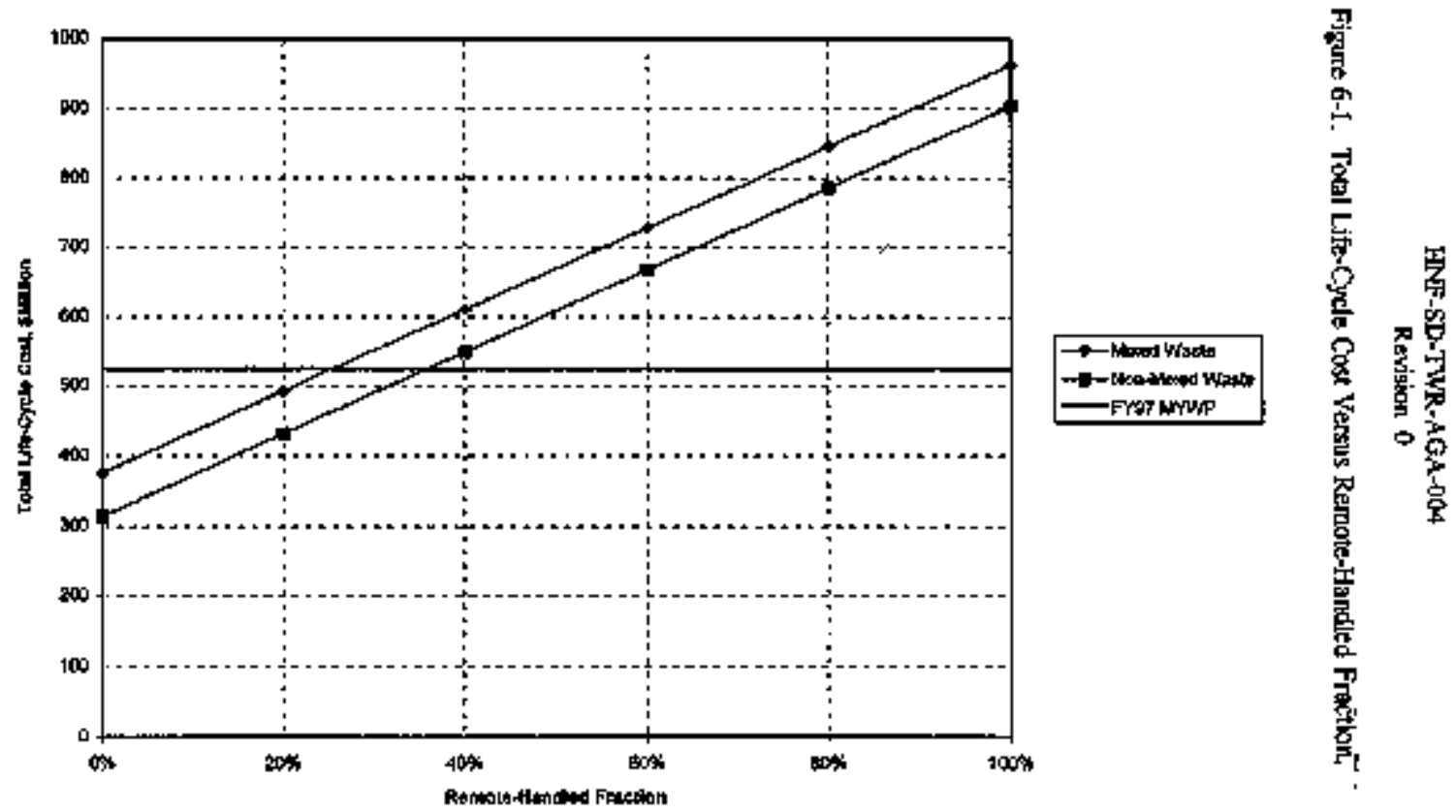


\section{6.t.2 Operofing Cost}

Life-cycle aperating costs were deweloped baned on preliminary time and motion atndies and operations tocenariog based on the required throughput fir both Phast I atad Phase II proptuction rates. Srattepies with kower cperating cost rectived highter rankings.

Operaling costs are lowest for contact-handled alterutives. Disposal inside the canyou byildings is next, followed by the adjugted bogeline cooceph. The rematc-bardled al terratives are next, followed by use of the external and internal portionte of the canyons. Operatiug costs for the \$ST option were not developed due to the extremely hígh caplal costs, but they are expected to be at least as bigh as the remote-habdled options.

\subsection{FLEXIBILITY}

The fiexibility of a disposal unit to perform multiple watote dispoqal funclions was evaloated under this category. The strategies and options with the ability to thet minimimn requirements for disposal of moltiple weste types and on the ease of reirieyal of waste packages were scored higher.

\subsubsection{Muiklple Wagte Types}

Flexibility of the facility to bandle multiple praste types such as nemote-handled mixed wagte, Jemote-handled non mixed sragte, contact-handled mixed wasto and contact-bandled noet mixed wate was evalutated onder thit categery. Strattgits and oplions wh the ability to meet multiple requirements were scored higher. Options $2 \mathrm{~A}, 4 \mathrm{~B}$, and $5 \mathrm{~A}$ scored highest in this calegory.

\subsubsection{Weste Retrivial}

Retrievability was evaluated subjectively boged on the eslimated tate of retertering the tacility for package retringal. Stratengies that provided easy retrievability were scored bigher. Options 3 and $4 \mathrm{~A}$ scored poorly for this criterion.

\subsection{SCHFDULE}

The ability of a option to met critical scheduk milestones was considered under this caregory. The options were objectively evaluated to meer the 19g7 MYWP scthedule (Wilson 1996) and Tri-Party Agreement (Ecology et al. 1996) milestone M(-90-10. Alteriadves 3, 4A, and $4 \mathrm{~B}$ could not meet one or more gehednle milesenes. 


\subsubsection{Mubti-Year Work Plan Milectones}

The FY 1997 MYWP schedale identifies a 3.5 year operating life of the ILAW Interim

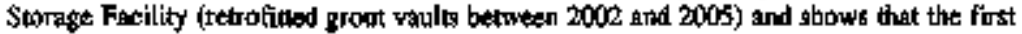
ILAW disposal module stall be constructed by Seplember 2004. This erterion was used to compliment the goino-go test to meat Tri-Party Agreement milestone M-90-10. Near-term schedule recroiroments are not met by alkernatiyes $3,4 \mathrm{~A}$, and $4 \mathrm{~B}$.

\subsubsection{Tri-Party Agreencht Whilestone}

The Tri-Paty Agreemenl provides the legath enforceable milestonca for TWRS. It it the primary schectile driver for new ILAW dispasal facility. A djaposal facility ts meded by December 2005 becanse the ILAW interim storage capacily of the grout vaults will bave been used. This criterion was a goinongo test to deternime if is was possible w implement a option that initiates bot disposal operations by Decenuber 2005. Near-term schedule requicements are not met by alternalives $3,4 \mathrm{~A}$, and $4 \mathrm{~B}$.

\subsubsection{Pripatizaloon Schedult}

The ability to ureet the reguirements of the privatization schedvle were eveluated unger this criterion. Oplions 3, $4 \lambda$, and AB canerot meet the near-trrm privatization schedulte. Concerns about the ability of the batelibe alteruatives to meet the privatization schectule also resulled in a lower seore for Oplons $1 \mathrm{~A}$ and $1 B$.

\subsection{ENVIRONDIENTAL MMPACT}

The envinonenenal impacts of the ILAW disposel system can be assessed by amount of lasd usage and secondary waste geberation.

\subsubsection{Land Usage}

The amount of land uged for disposal of ILAw wat evaluated under this cakgory.

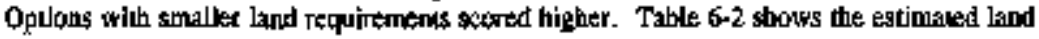
usage for each option. 
HNF-SD-TWR-AGA-064

Revision 0

Table 6-2, Land Uge Estimateg,

\begin{tabular}{|c|c|}
\hline Option & Land use, hectares \\
\hline $\begin{array}{l}\text { Option 1A } \\
\text { MYTP Baseline" }\end{array}$ & 13 \\
\hline $\begin{array}{c}\text { Option 1B } \\
\text { Adjusted MYWP baseline }\end{array}$ & 21 \\
\hline $\begin{array}{l}\text { Option 2A } \\
\text { RH MW dispasal }\end{array}$ & 24 \\
\hline $\begin{array}{c}\text { Option 2E } \\
\text { RH NatW disposal }\end{array}$ & 24 \\
\hline $\begin{array}{c}\text { Opton 2C } \\
\text { CH MW disposal }\end{array}$ & 36 \\
\hline $\begin{array}{c}\text { Option 2D } \\
\text { CH NMW disposal }\end{array}$ & 36 \\
\hline $\begin{array}{l}\text { Optipm } 3^{\circ} \\
\text { SSTi disposal }\end{array}$ & 12 \\
\hline $\begin{array}{c}\text { Option } 4 A^{\circ} \\
\text { Coutyotas - Internalal }\end{array}$ & 28 \\
\hline $\begin{array}{c}\text { Option 4B } \\
\text { Canyons - Inketrial and Excerinal }\end{array}$ & 0 \\
\hline $\begin{array}{l}\text { Option } 5^{\mathrm{d}} \\
\text { Extented Interim Storage }\end{array}$ & 4 \\
\hline $\begin{array}{c}\text { Option 6A } \\
\text { Ovetpacked RH MW }\end{array}$ & $B 7$ \\
\hline $\begin{array}{c}\text { Option 6B } \\
\text { Owtrpacked RH NMW }\end{array}$ & $\mathrm{BT}$ \\
\hline
\end{tabular}

CH NMW = Coblact-harddled noot-mlxed waste

MYWP • Multi-year work plan

KH NIMW = Remote-hoodted non-mixed waste

SST = Slingle-shell tank

'Capacity is only 60,000 packages

"Crpacity is only 23,300 packsges

"Capecly is otly' 75,240 parkages

deapacity is obly $1,0 B 0$ packages. 
HNF-SD-TWR-AGA-DOA

Revision 0

\subsubsection{Secondary Waste Generadion}

Contaminated waste from construction and opecalion of the disposal facifities were evaluated subjectively uader this category. Options with lower secondary waste score highter. Option 3 gored the worst for this eakegry.

\subsection{OPERATION, MAINTENANCE, AND SAFETY}

The alternatives rere evaloated with respect to daily operations under this category. The

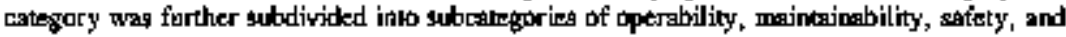
radjation exposure.

\subsubsection{Operabiltty}

Operablliry was evaluated subjecthely based on the estimated complexity of procedures that would be used during the daily operations. Options with fequer pieces of equipment and simpler operating procedores scored higher. Options 2C, 2D, SA and 5B scored well due bo the sienplicity of forkljif operations. Options 3, $4 A$, and $4 B$ scored poorly duc to the difficulty of remole operations it a coutaminated enviroumebl

\subsubsection{Maincimabilits}

Maintainability gas evaluated subjecthely bated on the estimated complexlly of the equipment neded to support the disposal facility. Equipment scossibility, zone entry, and contact verses remote maintenane were important considerations in this evaluation category. Options with feres moving parts, easy contact Iraintenance access, and off-the-shelf eqpipment scored higher. Options 3, 4A, and 4B goored poorty.

\subsubsection{Safety}

Warker livegilogt tirne atcidents darriag construction, dispasal and potential retrieval operations were evaluated subjectively. Dptions judged to have the fewest and least haxardous safexy risks were scored bighest. Options 3, 4A, and 43 scored pootly.

\section{6,5,4 Radlation Exporrere}

Prelindnary radiation exposure estimates pere developed for trangporting renove and contact-handled ILAW containess from the Vituification Building to positions in sticrage of dlsposal utilts. The time estumates were based on informalion from the Project W-465 
HNF-SD-TWR-AGA-004

Revision 0

preliminary operatung sequence block djagram for transporting R-AW conuainors and other extimates for an operating tacility. The radiacion dose rates were based on preliminary

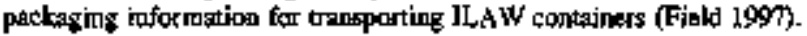

The results are summarized in Table 6-3 for remole-hrabdled waste and Table 6-4 for contact-handled waste. The operatiog sequence, container handling usethod, teme wo perform the steps, amound of shielding, and mumber of personnel have got been optimized at this thoe. They will be the subject of a filture study.

The radiation exposufs resuits for remote-handled waste fesulls show that the truth driver and health physics technician (HFT) afe the capscity limiting positions based on preliminary staffing levely, manual remote operation and a l,0 (Table 6-3). The 1,000 muediyr exposure limit does not iupact the Phasge I and Fherse II disposal based on the average produculon rate of containers. The tadlation exposure at the HPT position will litgit dispogal operations to alxout $\$ 0$ percent of the Phase ll peak disposal rate $(8,400$ conlainters jer ytar). Thelr short work interval during containes transport permits sharing of HPTs among the 4 disposal unlts doring Phase II. See Table A-24 for delails.

The estimated expoxure barden is 10 man-mrem per remote-handled container.

Toble 6-3. Radiatipg Exposure Estimate for Trangponting Remote-Handked Inmabilized LowActhily Waste Containers to Disposal Based on Transportation Requirethentia.

\begin{tabular}{|c|c|c|c|c|c|}
\hline Description & $\begin{array}{l}\text { Thuck } \\
\text { driver }\end{array}$ & $\begin{array}{l}\text { Prodess } \\
\text { Qpetanor }\end{array}$ & $\begin{array}{l}\text { Hesllh physics } \\
\text { exchniclan }\end{array}$ & Millwight & $\begin{array}{l}\text { Craile } \\
\text { operatox }\end{array}$ \\
\hline $\begin{array}{l}\text { Exposure per } 1 \\
\text { container traiker, InTesn }\end{array}$ & 2.6 & 2.3 & 1.4 & 0.8 & 0.8 \\
\hline $\begin{array}{l}\text { Exposure per person at } \\
\text { capaciny, mrem/ys }\end{array}$ & 1,280 & 570 & 720 & 420 & 420 \\
\hline $\begin{array}{l}\text { Exposure per captaiber } \\
\text { men mrem }\end{array}$ & \multicolumn{5}{|c|}{10} \\
\hline $\begin{array}{l}\text { No. containers disposed } \\
\text { at } 1,000 \text { mrem/yr lirajt } \\
\text { based on: }\end{array}$ & - & - & $\cdot$ & v & - \\
\hline $\begin{array}{l}\text { - } 3 \text { shifts and } 2 \text { disposel } \\
\text { unitg }\end{array}$ & 2,340 & 5,260 & 2,090 & 3,540 & 7,080 \\
\hline $\begin{array}{l}\text { - S Shifts and } 4 \text { disposal } \\
\text { unils }\end{array}$ & $9, \pi 70$ & 17,500 & 6,970 & 23,600 & 29,500 \\
\hline $\begin{array}{l}\text { Phase I production rete, } \\
\text { pontainerstyr }\end{array}$ & \multicolumn{5}{|c|}{1,470 werige and 3,800 peale } \\
\hline $\begin{array}{l}\text { Phase II production tate, } \\
\text { containers'yr }\end{array}$ & \multicolumn{5}{|c|}{6,400 average and 8,400 peak } \\
\hline
\end{tabular}




\section{HNF-SD-TWR-AGA-OOA}

Revision 0

The contact-handled waste dispospl results are summaxized in Table 6.4. The process operator and HPT are the capacity limiling positiens baged on $], 000$ mrem/yt exposure. The proceas operrater postition limits diaposal to about 50 percert of the Phase I peak rate 63,000 containers per year), about 50 percent of the Phase II ayerage race $(6,400$ contalseert per year) and atoout 30 percent of the Phase II peak rate $(9,000$ conlainers per year). The HPT position liraits disposal to about 80 percent of the Phase I peak rate, 80 percenl of the Phase II average rate and abourt 60 percent of the Fhase I peak rate. See Table A.25 for details.

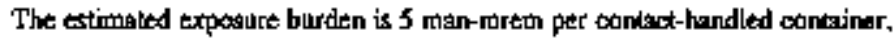

Tabie 6-4, Radiation Exposwe Estimate for Transporting Contact-Handled Lramobilized Lons-Activily Waske Contriners to Storage or Disposal Based on Transportation

Requirements.

\begin{tabular}{|c|c|c|c|c|c|}
\hline Description & $\begin{array}{l}\text { Truck } \\
\text { driwer }\end{array}$ & $\begin{array}{l}\text { Process } \\
\text { cperator }\end{array}$ & $\begin{array}{l}\text { Hetaluh physict } \\
\text { teclinician }\end{array}$ & Milworight & $\begin{array}{l}\text { Porklifi } \\
\text { opefakor }\end{array}$ \\
\hline $\begin{array}{l}\text { Exposure per } 2 \\
\text { container trailer. } \\
\text { mrem } \\
\end{array}$ & 2.3 & 3.7 & 2.4 & 0.0 & 2.2 \\
\hline $\begin{array}{l}\text { Exposure per person } \\
\text { at capacity, mirem/yr }\end{array}$ & 1,170 & 1,860 & 1,200 & 0 & 1,080 \\
\hline $\begin{array}{l}\text { Exposure per } \\
\text { container man mrem }\end{array}$ & & & 5 & & \\
\hline $\begin{array}{l}\text { No. containers } \\
\text { disposed at } 1000 \\
\text { mrem/yr limit Baged } \\
\text { on: }\end{array}$ & - & - & - & $\cdot$ & - \\
\hline $\begin{array}{l}\text { - } 3 \text { Shifis and } 2 \\
\text { dikposal units }\end{array}$ & 3,420 & 1,620 & 2,500 & - & 3,710 \\
\hline $\begin{array}{l}\text { - } 5 \text { shifts and } 4 \\
\text { disposal mil }\end{array}$ & 10,260 & 3,230 & 5,000 & - & 11,120 \\
\hline $\begin{array}{l}\text { Phase l productuons } \\
\text { rate, containerstyr }\end{array}$ & \multicolumn{5}{|c|}{ 1,470 averope and 3,000 peak } \\
\hline $\begin{array}{l}\text { Phase II production } \\
\text { rate, containers/yr }\end{array}$ & \multicolumn{5}{|c|}{6,400 averaye and 9,000 pestix } \\
\hline
\end{tabular}


The initial radiallon exposure fibdiugs thfer that trantsportation shielding atrd mathual type operations could impact the abllity of disposal operation to meet the required throughput the to radjation expostare. A continintion of additional shiejding, stortier task times, and remole operating methods may be neodod for the disposal operation wachicese and maintain peak throughputs. Shielding, operationsal requirements, and perominel will be part of a future study at optimization of the disposal unil cperation.

\subsection{SUMMARY AND COACLUSIONS}

The evaluation results matrix surnmarizing the rankings of al] alteriatives apaingt all ceriteria is shown in Table 6-\$. The results show that alternatives $2 C$ (contacl-hapdled rijed

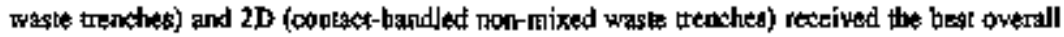

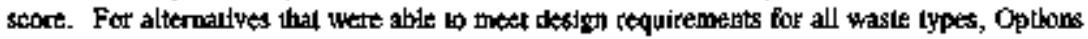
2A (remote-bandled mixed waste vauls) and 6A (ovepacked remote-bandled miked waste in trencles) scored the best. 
Table 6-5. Sumbrary Evaluation Matron.

Evaruswant Crinere

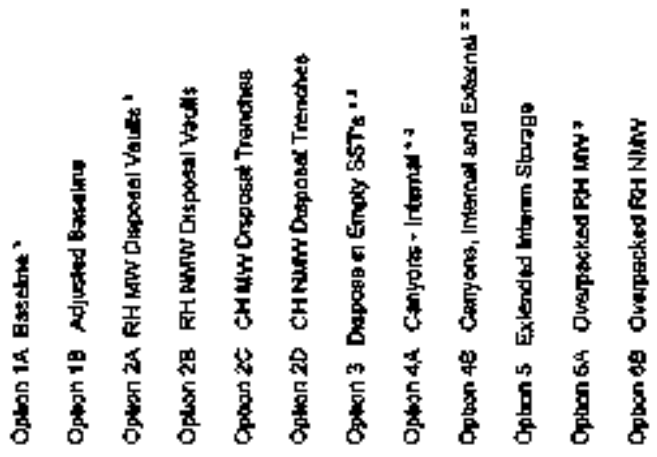

A COST

A I Cpprtal Cont

A I Operaing Cool

A. we Dicte Cost Frolle

B FLEXIEILITY

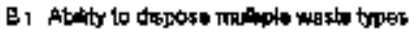

B. Refreistbilty

C BCEEDULE

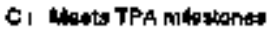

Co n Mand MYPP whostonts

C a Supports Pnutatizalion schedest

D ENVIRGAEUENTAL DOPACTS

C I Land Unepa

Oa Secondary What Gentertum

E DPERATONSNANNERANCERAFETY

EI Operantiby

$E$ u Mogramabuily

Ew Solow

E ru Radution expogire

TOTAL SCCise

$$
\begin{array}{rrrrrrrrrrrr}
4 & 4 & 4 & 5 & 9 & 10 & 1 & 5 & 5 & 3 & 3 & 5 \\
8 & 7 & 4 & 4 & 6 & 10 & 1 & 4 & 2 & 3 & 10 & 10 \\
7 & 5 & 4 & 5 & 9 & 10 & 1 & 5 & 4 & 4 & 2 & 5
\end{array}
$$

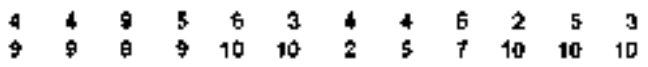

$\begin{array}{rrrrrrrrrrrr}10 & 10 & 10 & 10 & 10 & 10 & 0 & 3 & \$ & 7 & 10 & 10 \\ 10 & 10 & 10 & 10 & 10 & 10 & 0 & 3 & 3 & 7 & 10 & 10 \\ 8 & 8 & 10 & 10 & 10 & 10 & 0 & 2 & 2 & 7 & 10 & 10\end{array}$

$\begin{array}{rrrrrrrrrrrr}8 & 7 & 7 & 7 & 6 & 6 & 8 & 9 & 9 & 4 & 2 & 2 \\ 60 & 10 & 9 & 10 & 10 & 10 & 1 & 5 & 5 & 7 & 5 & 8\end{array}$

$\begin{array}{rrrrrrrrrrrr}7 & 7 & 7 & 7 & 9 & 10 & 7 & 5 & 5 & 9 & 9 & 10 \\ 8 & 9 & 7 & 7 & 9 & 10 & 3 & 4 & 5 & 9 & 9 & 10 \\ 7 & 7 & 8 & 8 & 9 & 9 & 3 & 4 & 5 & 9 & 9 & 9 \\ 9 & 9 & 7 & 8 & 8 & 9 & 4 & 7 & 6 & 7 & 10 & 10\end{array}$

109 104 106 $106 \quad 123 \quad 126$ 30 62 G

Nowe

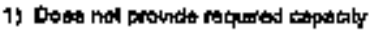

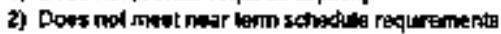

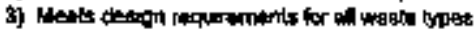


HNP-SD-TWR-AGA-004

Ravigion D

This pase intentionally loft blark. 


\section{IMPLEMENTATHON STRATRGY}

There ane lafge uncertainties surrounding the final waste form characteriscics and the assceiated desigh requirements for the disposal system. These unoertainties mate the seleclion of a singk preferred disyposal system architectmre difficalt To provide the lowest sost and mast flexible disposal system, a sulle of tesizen solutions shosuld be ayallable for deploymenal on a "just-in-time" basts.

The number of design golutions required to meet all epmtimpencies depends on the number of uncertainties. As the igsues and uncertainties are resolved, cerlain solutions wrold no longer be needed. Al th time, three key uncertainuies lead to a suite of six different design solutiong. Figure 7.1 shows the destgh salutions and the decision tree for selecting the proper solution based on tie answer to tach of the uncertsimties.

The first and most inporteut question from a cosi gtandpoitl is the radiation dose rate of the wagte package. If It $\mathbf{s}$ over $\mathbf{2 0 0}$ wrem/h, remote handling and steleled facllibes ate requined. Belon $200 \mathrm{mrenth}$, the packages can be coutact bandled. Initial analysis of the Phate I feed staging plans indicate that a signiticant fiaction (Iovghly 35 pervert) of the Phase I product could be contact handled. Analysis of the cegimon pontent of the tanka stows that the SST, (to be treared in Phase II) have less desinum that the DSTs (b be treaved in Plase l) indicallag that an even larger fraction of the Phase II product could be contect handled. Furcher analysis of feed batch compcsition and the privale contractor flowsheets is required to determine how moch of the Phase I and Phage I product would be expecked to be contact handled. Providing gome contact-handled facilities to complemethr the remote operated shicelded vanlts would decrtass the cotsteruction and opertalting costs for the overall digposal system.

The second important question is whether or not the prodoct has texited RCRA through

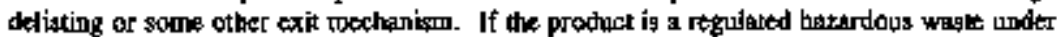

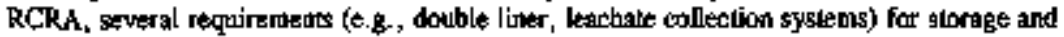
dispospl facilities are Imposed. Exitng RCRA wonld allow cost savings duo to the removel of these design requirements.

Fually, the process of obtaining a disposal authorizalion dectsion from DOE-HQ htenged on completion and approval of the disposal performance astessment and composke andysis reports (draft DOE Order 435.1). If auborization w perform a disposal action bas not been obtained before the atart of waste treatoment, extended interim skorage facilities sould be coeded. In the case of remote-handled waste, the distinction betwent storage and dispol is immaterial since a shieided vault wornld be uged in eidher case. Far conuact-handled waste, the requiretments (anonittoring, inspections, aisle space) for a storage facility are sulficiently different irom a disposal facility that they are depicted separately in Figure 7-1. 


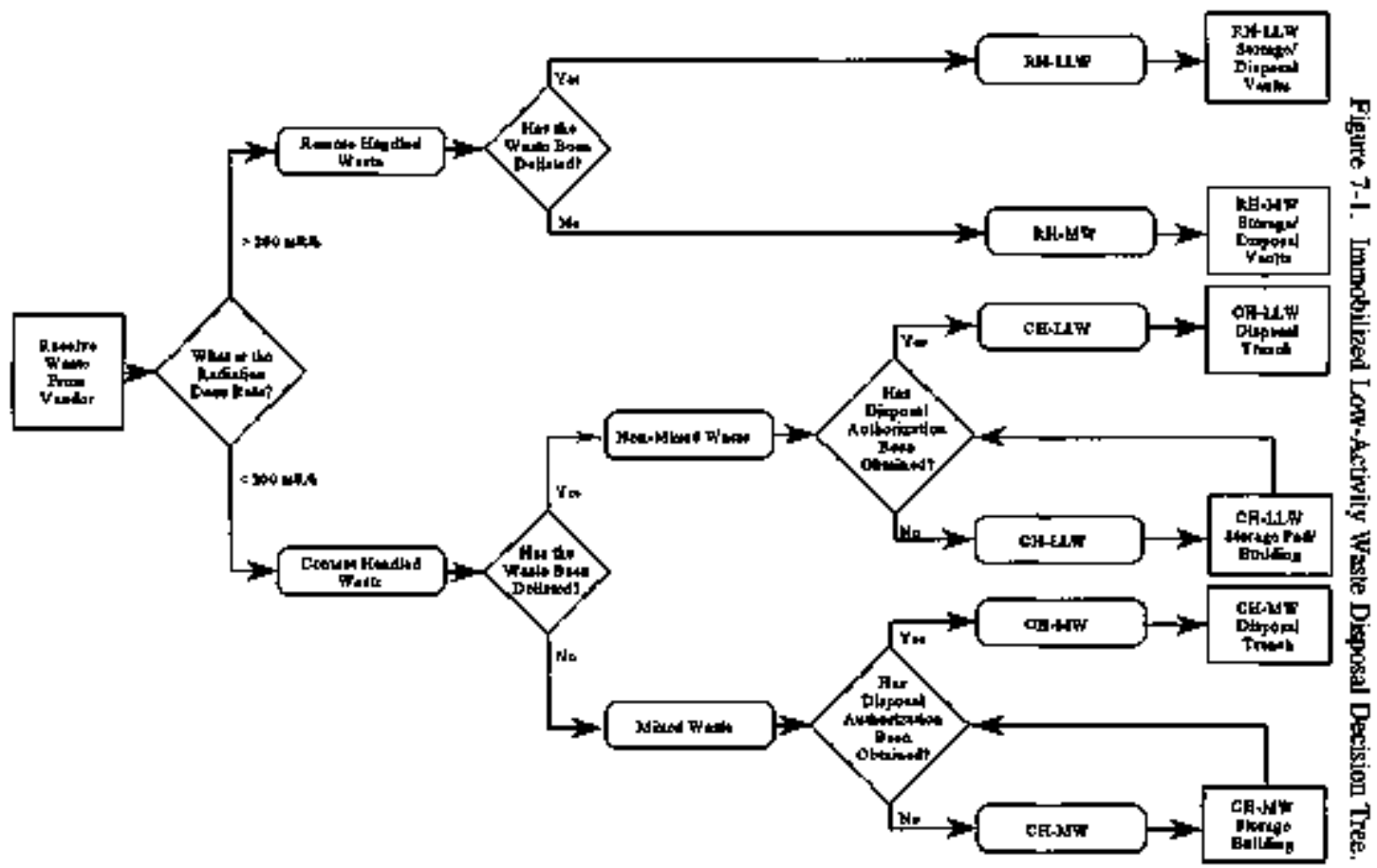

Lapip 811 Cerion Contra of W Wto
Inat 42 RCRH
Dotistint Ration
Japu IS Dispocai Sial

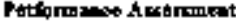




\section{HNF-SD.TWR-AOA-OMOA}

Revision 0

The available desigh sohultons would be deployed on a "just-in-lime" basis through 2 series of construction projects schednied al rooghly thee year intervals. This schednle provides enough lead time to obtain fundiag for the projects while awoiding the corrmitment of too many resonrces toward a particular desiza soletion. The initial project would provide the diaposal site infrasinscmere and the first additional diopogat unith \$ubsequente disposal units would be constructed as noeded to keep up with production raves. The last project would decommission the operatlons buttlings and consuruet the flasal closure barrler over the dlsposal site. Flgure 7-2 depicts one example of the available disposal capacily atrd production rates as a function of time using this approach.

This approach also allows the adjition of additional design schutions lo the stratiog as they hecoive ayailable in the coutyeart, such as Option 3. SST and Option 4, Canyou Buildings. When these alternatives become available, they can be reassessed to deternine their suitability for deployment wilhin the TWRS disposal strategy.

To implement this atrategy, the ILAW disposal program will wed to develop conceptual destgus of each elenent of the strategy. Design media for CH-MW and CH-NMW already exist at Hanfurd. While a design is also availathe for RH-WW (the grout vaulta), it is not optirnized for packaged waste, and iunprovernents could be made to this concept. All alternatives thal provinte new disposal fatilitles witl noed infrastructure support, and disposal authorizalon can nol be obtained whovt a closore plan for the facility. Therefore, the hight priority actions w implement this strategy should be:

- Devekp conceptual designs for disposal sile inftagluructure, RH vaults, and surface barrier clogare systrems.

- Examize the near-term processing schemes to determine how much CH waste should be expected during Phase l.

- Review and evaluate private contractor flowsheets to determine whecher the ILAW will be mixed watte ar bot. 


\section{HNF-SD-TWR-AGA-004 \\ Revition 0}

Figufe 7-2. Storadelbisposai Cappaclly Versus Production.

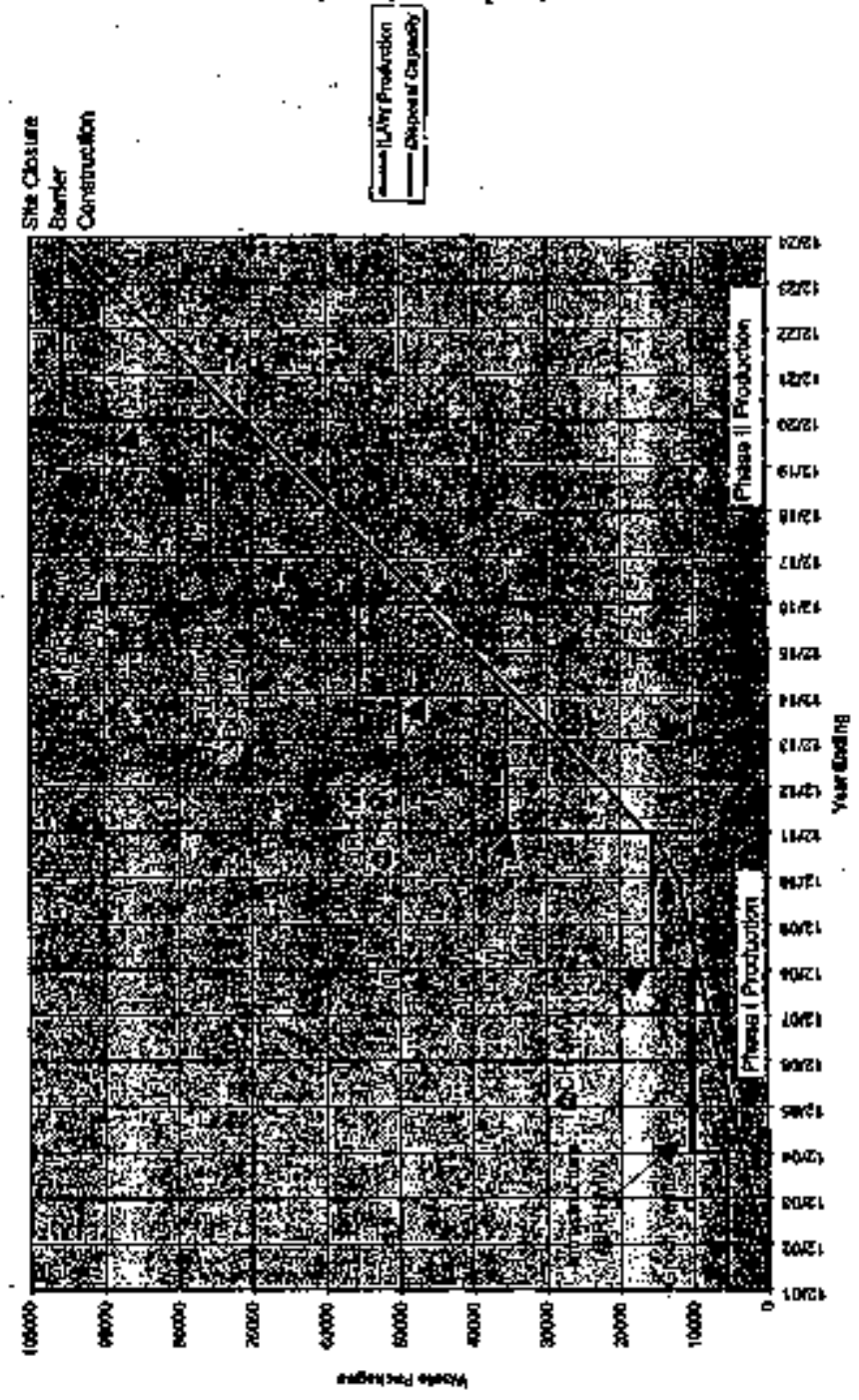




\section{HNF-SD-TWR-AGA-0OA \\ Revision 0}

\subsection{OPEN ISSUES AND ACTIONS}

Three open issles were ideulified th this study. This secthon summarizes the issuted and identifies the requined axtion.

1. Cesinm Content of Treated Waste (Packege Dose Rate)! The maximum surface dose eate requiremenl is less than 1,000 inrem/h for each LLAW packaste based on the Phase I RFP. A garmma radiation doge rate of $\mathrm{b}, 000$ mrent'h requires rempte handling, shiekding (maximum $46 \mathrm{~cm}$ concreve) and adminigarstive con irols to meet ALARA exposare levels for workers and the pablic. The curremt dispasel und dealen objectives for personned redjation protection are based on handling all of the projecled 99,200 packages at the maximum credible source term. This position is owethy conservative and expensive.

Requlared Action: Dewelopg a roliable rediation source terni that can be used to understand the doas rate distribution for the projected ILAW pactinges by performing a

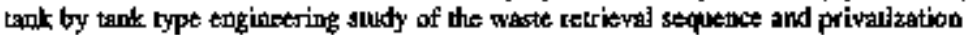
oesium separaion efficiency. Use the results to establish the types, mumbers, and need dilles of ILAW disposal mits.

2. Harsardaus Waste Regulatory Status (Dellsting of Theated Waste); Uoder the current EPA "mixture" and 'derived-from" rules, the ILAW would continue to be mataged as a RCRA regulated mixed hazardonofradioactive waste, because it is derined from tapk waste that contains liated hazardoes congtituentg. Although the vitrification proctss would resplt in a prochuct thot is compliant with the land disposal restrictions, the ILAW

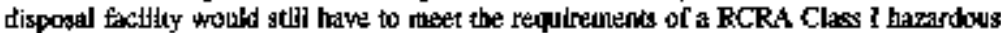
twaste landfill, including donble liness, leachate collection systems, and closure/pastclosure thonitoring.

Regined Action: Two possible strategies could be uged to obtain relief from the requirements of RCRA fir the disposal vnits. Firsh, be privalizallon vendord have been asked to propose methods to prowide non-RCRA regulated fltall waste products. Under the current regulations, this acold be achieved by petitioning the EPA to delist the ILAW product. Although the delisted ILAW woukd continue to be subject to certain RCRA regulations, the design requirements for the dispossal faxility would be consideribly less stringent-

The second aveme for exiting RCRA lies in the proposed HW/R. If promulgatud, the HWIR would reyige the "mintors" and "derived-from" rules athd develop rist-based exlt lovels for listed conththents in hazardous wate treatment residges. Bassed on the initial proposal, the ILAW product should be able to exic the RCRA rukes under HWIR, and

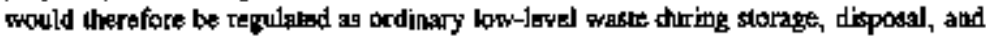
closure operational 
3. Disposal Authurtation Statement: When implemented DOE Order 435.1 (draft), Radioactive Waste Maragement will replece DOE Order 5820.2A. This aEF provision will impact ILAW alispopal in the following areas:

- Stronger eniphass on overall waste manggemen such as planning documentation frora waste generation to disposal

- Requirements on interim storage will be more restrietive

- Requires oblaining a disposal authorizstion statement based on approved performance assessment and contossite analyzis for a specific dispospl facility before starting ponstruction.

Requined Actlom: Two groups of actions are needed. The first group action is to select the specific LLAW disposal Bitte, develop ILAW disposal strateg', determine type of JLAW disposal anits (based on the enginetering abudy identified in logut \#1), and develop prelimimary conceplual design of the lanlus for the performance distessmeat. The second is to complete composilte athalysis of all sources of all radicaclive material which may interact with the disposel facility. Approval of these reports is neded to obtain a disposat auhorization statement to start construction of the disposal units.

If authorization for disposal has not boen oblained, exiended imterim storabe facilites would be needed. 


\section{HINF-5D-TWR-AGA-COS \\ Revision 0}

\subsection{BEDERENCES}

Armacost, L. L., D. won Wirterfeldt, J. Creighton, M. Roberthotte, 1994, Public Vatues Related to Dectivions is the Tank Waste Remediation Syotem Progran, PNL-1010?, UC-630, Pacific Northwesl Laboratery, Richland, Waskinglon.

BHI, 1995, Enwiransental Restonation Dappasal Facility Waste Acreptance Criteria, BHI-0139, Rev. B, Bechtel Hanford Incorporated, Rithlathd, Washington.

Brown, N. R. 1996, DS] to R. M. Orme, Westinghouse Hanford Company, $X L W$ Product Waste Lonting Assumptions for the TWRS Process Flowsheet, dated April 23.

Burbank, D. A., 1996, Attematives Generation asd Analysts Report for Inwobilized LowLevel Waste Intertin Storage Arthtecture, WHC-SD-W465-AGA-BO1, Rev, 0, Westimghoves Hanford Company, Richland, Washington.

Burbank, D. A., 1997, Design Requirements for Immabillzed Lou-Adolvity Waste Interint Storase, HNF-SD-W465-DRD-00], Rev, 0, SGN Eurisys Serviees Corperalon, Richland, Wastingtrn.

DOE Order 435.1, Rudlowstve Waste Managenten, U.S. Deparument of Energy, Washington, D.C.

DOE Order 5820.2A, Radianctive Waste Horagestestr, U.S. Depariment of Enargy, Washington, D.C.

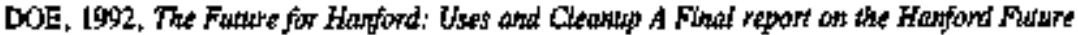
Site Uses Workthg Groxp, U.S. Department of Eneryy, Richlaud, Washington, December, 1992.

DOE, 1996, Final Enwhonnental Impact Statement for the Tank Waste Remediation Syster, Hanfard Site Richiand Washingrom, DOE/ELS-0189, U.S. Dephiment of Energy, Washinglon, D. C.

DOE, 1997, 'Record of Dexision for the Tank Waste Remedjation System, Hanford Sitt, Richland, Washing U.S. Department of Energg. Washington, D.C.

DOE-RL, 1996a, TWWS Privatzation Requetse for Propasals, Solicitation Nopbor DE-RP06-96RL13308 (Febsuary 1999) U.S. Department of Entrgy - Richlartd Operalions Office, Richland, Hishingtop. 
DOE-RL, 1996b, TWhs Privatization Contract Awards, Conlracts DE-RP06-96RL13308 and DE-RP06-96RL13309, dited September 25, 1996.

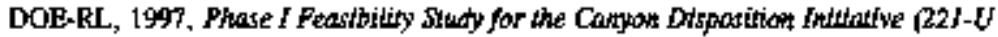
Facility), DOE/RL-97-11, Rov. 0, U.S. Depariment of Energy - Kichland Operations Office, Richland, Washingkon.

Ecolozy, EPA, DOE, 1996, Hantord Fedenal Facitity Agrement and Consent Onder, Sixth Afoendment, Washingent State Dequartment of Exology, U.S. Environmental Protection Agency, and U.S. Departmed of Energy, Olympia, Washingtop.

Eiholzer, C. R., 1995. Disposel Facilty Data for the Trerim Perfomance, WHC-SD-WhM-RPT-159, Rev 0, Westinghouse Hanford Company, Richland, Washington,

Eiholzer, C. R., 1996, Jetter to F, M. Mann, Westinghouse Hantord Compary, Changes to Facility Concepts Due to Privatization Droft Specifications for Lowsictiviy Waste

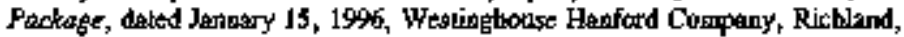
Wastington.

EPA, 1995, "Hadzardons Waste: Identificalion atd Listing; Proposed Rule," 60 FR 66343,

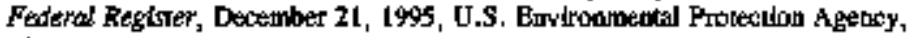
Wasbingon, D.C.

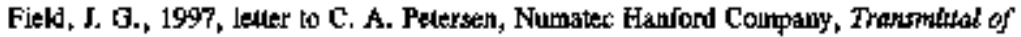

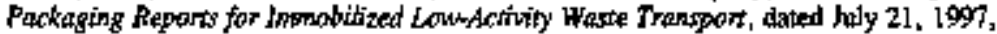
Waste Management Federal Seryices Inc., Richland, Washington.

Gerton, R. E., S. T. Burmum, D. J. Squires, 1995, "StoracedDisposal Stratepiss in U.N. Mermber Siates," Proceedings of the Syrmaxriwing on Waste Maragement, Tucsor. Arizonth.

Han, K. W., J. Heinesals, A. Bome, 1997, 'Radibaxtive waste disposal: Global experience

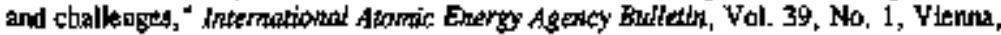
Austrin.

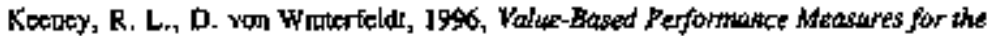
Honjand Tank Wart Remediction (TTRS) Program, PNNL.10946, UC-630, Pacific Northwest National Laboratory, Richland, Washington.

Kepner, C. H., and B. B. Tregon, 1981, The New Rational Bomager, Princeton Research Ptess, Princeton, New Jersey. 
Mann, F, M., C. R. Eihober, A. H. L, P. D. Rittman, G. F. Wulliangon, of Westinghouse Hanford Company, N. K. Kline of Boteing Computer Services, Y. Chen, B. P. MeGrail of Pacific Nortbwest Lstopatory, N. R. Brown of U. \$. Departinenl of Energy, 1996,

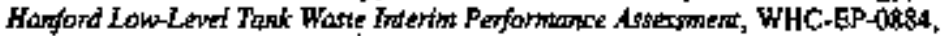
Rev 0, Westubabouse Hanford Company, Richland, Washingowo.

Myers, D. R., and D. A. Duranceau, 1994, Protofype Hanfort Surfoce Burrier: Destgn Basts Bocument, BHJ-00007, Rev. 0, Bechtel Hanford Jocorporated, Richland, Washington.

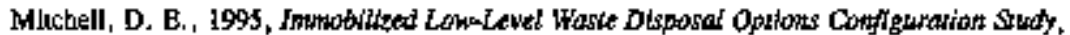
WHC-5D-WM-T7-686, Rev 0, Westinghruse Fanford Company, kichland, Washinglou.

Murkonviki, R. J., 1996, Jnmabilized Low-Level Waste Interin Storage Aftematives, Letter Number 9650431, Leter to P. E. Lallont, DOE (January 30), Weatingbouse Hatford Company, Richland, Washtugepn.

NRC, 1997, Letter from C. J. Papriello to J. Kinzer, DOE, Classinication of Hatrord LowActivity Tank Waste Fraction, Dated Inme \$, I\$97, United States Nuclest Regulatory Commission, Washington, D. C.

Ordne, R. M, 1996, TWhs Privetization Pracess Techwical Baseline, WHC-SD-WM-TI-774, Ret. 0, Westinghouse Hanford Company, Richland, Washington.

Penwell, D. L., 1996, Iwitial Retrieval Sequence and Blendint Stroftey, WHC-SD-WMI-RPT-220, Rev. 0, Westinghouse Hanford Company, Rlchland, Washington.

Peterken, C. A, 1996, Technical Bessis for Classification of Lowdaivity Watte Fraction from Hanford Site Takks, WHC-SD-WM-TI-699, Rev. 1, Wethinghouse Hanford Coumpany, Richland, Washutegton.

Petersen, C. A., 1997, Decision Ptan - Inthobilized Lowhevel Waste Disposal, Letter

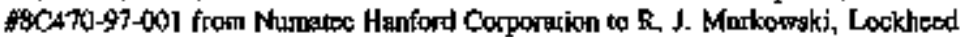
Martin Hanterd Conp., dated April 30, 1997.

Reidel, S. P. A. M. Tallmali, V. G. Johnson, C. J. Chou, S. M. Nerbutorskih, J. P. Keiser, 1995, Choracterization Plan for the Proposed TWRS Treatmen Complex. WHC-SD-WM-PLN-109, Rev. 0, Westingtsouse Hanforr Companty, Richland, Washington.

Rucberford, W. A. I997, Letter to H. J. Hatte, Finor Danitd Harford, Inc., Contracr

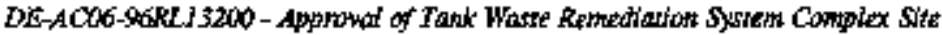
Evaluation Report, 97.SID.285, Dated July 10, 1997, U. S. Depariment of Energy. Richland, Washingeros. 
Shord, A. L., 1955, Tant Warte Remadiation Syatem Conplex Stie Evatuation Report, WHC-SD-WM-SE-D21, Rey. O, Weatinghouse Hanfort Company, Richlard, Washington.

Tenipleton, K. J., \$. J. Mitchell, P. M. Molion, 1994, "Low-Level Radiosctive Warte Dispasal Tecbnslogies User Outside the United States," Proceedings of the hrernational Toptcal Meeting on Naclear and Hazardass Haste Nanagement, Spertrum '94, Allanta, Georgia.

WHC. 1995, Other Options Data Package for the Tath Wase Rentediation System Enviranmental howpt Statement, WHC.SD-WM-EV-1)]6, Rey. 0, WestinghouseHautord Cocupany, Richland, Washington.

WHC, 1996, TWRS Fundolors and Kequirements, WHC-SD-WM-FRD-020, Revv. 1, Westimgtousse Hanford Company, Richiand, Waghington.

Whlson, C. E., 1996, Tatk Waste Remediatlost Sysem Flecal Year 1997 Mutt-Year Work Plam, WBS 1.1, WHC-SP-l lol, Rev, 2, Westinghouro Hanford Company, Richtand, Washiogion. 
HNF-SD-TWR-AGA-O04

Rev of

\section{APPENDIX A}

\section{COST ESTIMATING DATA}


HNF-SD-TWR-AGA-004

Rev 0

This patge tertentionally left blank. 
HNF-SD-TWR-AGA-OOA

Rev 0

\section{CONTENTS}

A1.0 BASELINE PLANNING CASE $\ldots \ldots \ldots \ldots \ldots \ldots \ldots \ldots \ldots \ldots \ldots$

A2.0 ALTERNATIVE PLANNING CASES $\ldots \ldots \ldots \ldots \ldots \ldots \ldots \ldots \ldots$ A-15 A2,1 DESIG'N BASIS FOR, ESTIMATE--JMMOBILIZED LOW-ACTTVITY

WASTE DISPOSAL COST ESTIMATES (OPTIONS 2A, 2B, 2C,

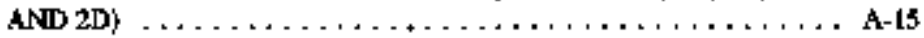

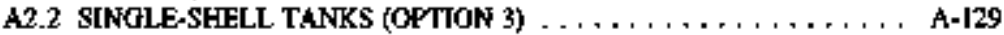
A2.3 CANYON BUILDLNGS (OFTIONS 4 A AND 4B) , . . . . . . . . A A.I\&4 A2.4 EXPANDED INTERIM STORAGE (OPTION 5) . . . . . . . A A-197 A2.\$ \$HIELDED OVERPACKS (OPTTONS 6A AND 6B) . . . . . . . . A-20\% 
HNF-\$D-TKR-AGA-OG4

Rey. 0

\section{LIST OF FIGURES}

A-1. Diamond wire Conting Technology $\ldots \ldots \ldots \ldots \ldots \ldots \ldots \ldots \ldots \ldots$ A-1T9

A-2. Overview of 48-Foot Diameter Hole in Single-Shell Taik $\ldots \ldots \ldots \ldots \ldots$ A-180

A-3. Overview of Saw Cat Paltera for 48-Foot Diameter Hoje in \$ingle-\$4ell Tank , A-181

A-4. Overyiew of 12-Fopt Diameter Hole in \$ingle-Shell Tank With Emelosire . . . A-182

A.5. Onerview of 48-Foot Diamerer Hote in Single-Shell Tank With Enclogure . . . A A-183

\section{LST OF TABLES}

A-1. Summary of Operating Staff Requirements for Baseline Immobslized Lop-Activity Wasto Container Disposal Baged on 1997 Multi-Year Program Plan (Option 1A) . A-9

A-2. Summary of \$taff Requirements and Costs for Batelime Immobllked Low-Acyivly Wagte Conbainer Disposal Based on 1997 Mului-Yeer Progran Plan (Option 1A) A-10

A-3. Summary of Estimated Capital Cost Details For Design, Construction and Closure of Baseline Imanobilined Low Activity Waste Disposal Based on Figcal Year 1997 Mulit-Year Work PJank (Option (A) $\ldots \ldots \ldots \ldots \ldots \ldots \ldots \ldots, \ldots, 14$

A-4. Estimated Infrastrucure Cose for Disposel of Remole or Contact-Handled Immobilized Low-Activity Wathe (Options $2 \mathrm{~A}, \mathbf{2 B}, 2 \mathrm{C}$, and 2D) , . . . . . A-29

A-5. Estimated Construction Cossts fotr Disposal of Renrote and Conact-Hanofled Immobjilized Low-Activity Waste (Options 2A, 2B, 2C and 2D) , . . . . . A A 49

A-6. Estimated Closure Costs for Disposal of Remote and Contact-Handled Immobilized low-Activity Wagce (Options $2 \mathrm{~A}, 2 \mathrm{~B}, 2 \mathrm{C}$, and 2D) . . . . . A-103

A.7. Summary of Estimated \$taff Requirements for Remote-Handled Immobilized Low-Activity Waste Contaiger Disposal Phase I and Phase II (Options 2A and 2B)

A.B. Summary of Estimated Staff Requinements and Costs for Remote-Handled Immobilized Low-Activity Wasta Container Disposal Phase I and Phase D (Options 2A and 2B) $\ldots \ldots \ldots \ldots \ldots \ldots \ldots \ldots \ldots \ldots \ldots \ldots \ldots \ldots$ 


\section{LIST OF TABLES (CONTINUED)}

A-9. Summary of Estirnted Staff Requiremente for Cottact-Handled Immobilized Lox-Activity Waste Siorage and Diopasal Phase I and Phate II (Options $2 C$

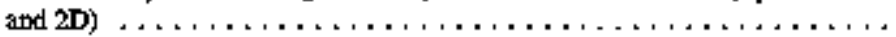

A-10. Surnmary of Estimated Staff Requiremenls and Costs for Conbact-Handled Immobilized Low-Activity Wapte Coutainer \$topagye and Disposal Pbase I and Phase I (Options 2C and 2D)

A-11. Summary of Estimated Consuruclion Cosis for Addition of Ore 12-Fook Diameter Openine to the Center of a Single-Sbell Tank Based on Dlamoud Wire Cuting

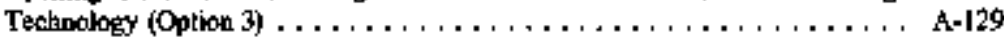

A-12. Sumarmary of Extimated Construction Coats for Addition of One 48-Foot Diameter Opering to the Center of a Single-Shell Tank Based on Diamoud Wire Culting Technology) (Option 3).

A-13. Surtuary of Eatimanted Coses for 22l-U Canyon Butding Dleposition Based on Inkernal and External Waate Disposa] Alterzatives of Phase I Feasibility Stady (FS Options 3 and 4)

A-14. Surmary of Adjasued Applicable Estimaled Congruction and closure Costs for Dispostition of Four Canpon Boildings Based on Internal and External Waste Disposal Altertatives of $221 . U$ (AGA Options $4 A$ and $4 B$ ). $\ldots \ldots \ldots \ldots$ A-185

A-15. Summary of Estimated \$taff Requiremenus for Remote-Handled Itrmobilized Low-Activity Whasio Contadner Disposal Phase I and Phase II (Optlon 4A) . . A-J86

A-16. Suramary of Estimated Staff Requbemenus and Coss for Remote-Hatudled Imntobilized Low-Activity Waste Container Disposal Pbase I and Ptase II (Option 4A) $\ldots \ldots \ldots \ldots \ldots \ldots \ldots \ldots \ldots \ldots \ldots \ldots \ldots \ldots \ldots, \ldots \ldots$

A-17. Surmary of Estirated Staff Recuinements for Remote-Handled Immobilized Low-Activily Waste Comtativer Disposal Phase I and Phase II (Opton 4B)

A-18. Summary of Estirnated Staff Requirements and Costs for Remote-Hatriled Imroobilized Low-Aclivity Waste Comeainer Disposal Phase 1 and Phase II (Option 4B)

A-19. Summary of Tolal Estimated Optraling Costs for Fotur Canyon Bullding Disposition Hased on Imternal and External Waste Dispossal Allernatives of 221-U atd Operating Crew for lramobilized Low-Activity Waste Disposal (AGA Oplions $4 \mathrm{~A}$ and 48)." $\ldots \ldots \ldots \ldots \ldots \ldots \ldots \ldots \ldots \ldots \ldots \ldots$ 
Rev. 0

\section{LST OF TABLES (CONTINUED)}

A-20. Cost Estimate for Project W-112 Enhanced Radioactive and Mixed Waste Swage $\ldots \ldots \ldots \ldots \ldots \ldots \ldots \ldots \ldots \ldots \ldots \ldots$ A-197

A-22. Summary of Infrastructure Cost Bstimate for Inwerien Storage Utite for ContwctHandled Mixed Immobilized Low-Activity Wate Conlaines Based on Projecl W-112

A-23. Summary of Cost Estimates for $\$ 040$ Coucrete and $\$ 040$ Carton Stoel \$hielded Overpacks for Digpogl of Kemole-Handled Immotl]izad Low-Activity Waste Containers (Options 6A and 6B)

A-24. Radialion Exposure Extimalo for Trasporting Remote-Handied Immotilized Low-Activity Wabio Containerg to Disposal Baged On Transportation Sheilding Requirements

A-25. Radiation Exposute Estirate for Transportint Contact-Handled Immobelized Low-Activicy Waste Containers to Dlsposal Eased on Transportation Shielding Requirements $\ldots \ldots \ldots \ldots \ldots \ldots \ldots \ldots \ldots \ldots \ldots \ldots \ldots, \ldots \ldots \ldots, 221$ 
HNF -SD-TWR-AOAA-904

Rer 0

APPEADIX A

\section{COST ESTMMATING DATA}

This appendix describes the basig for prapariog the life-cycke cust estimates for the baseline and al farnatives for dispasal of inmobilined low-activity wate (ILAW) containers.

\section{A1.i) BASELINE PLANINLG CASE}


HNF-SD-TWR-AGA-004

Rev. 마

This page intentiogally left blank. 
HONF-SD-TWR-AGA-004

Rev. 0

Table A-I. Sumcrary of Operating Staff Requirements for Baseline Inmabilized Low-Activity Waste Cantainer Diaposal Based on 1997 Militi-Year Program PJan (Option 1A).

\begin{tabular}{|c|c|c|c|c|c|c|c|}
\hline \multirow{2}{*}{$\begin{array}{l}\text { Posstion } \\
\text { All Wasle Remole Handied }\end{array}$} & \multirow[t]{2}{*}{ Type } & \multirow{2}{*}{\multicolumn{2}{|c|}{ Day shift }} & \multicolumn{2}{|c|}{ Throe Shiths } & \multirow{2}{*}{\multicolumn{2}{|c|}{ 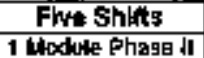 }} \\
\hline & & & & 1 Modultit & 里Ph860 I & & \\
\hline & $\mathbf{M}$ & 1 & & 1 & & - 1] & \\
\hline Cferk & $G$ & 0.5 & & 0.5 & & 0,5 & \\
\hline Ptent Englines & $\bar{E}$ & 1 & 25 & 1 & 25 & 1 & 2.5 \\
\hline 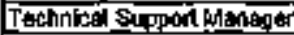 & hat & 1 & & 1 & & 1 & \\
\hline Clenk & E & 05 & & 0.5 & & 0.5 & \\
\hline Plentietrschoduler & $\mathbf{P}$ & 0.25 & & 0.25 & & 0.25 & \\
\hline Eudged Ana|y:t & $\bar{P}$ & 0.25 & & 0.25 & & 0.25 & \\
\hline 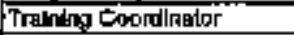 & 5 & 0.5 & & 1 & & 1 & \\
\hline Tomines & $\bar{P}$ & 0.5 & & 1 & & 1 & \\
\hline Fractdure Whitier & $\overline{\mathbf{P}}$ & 0.5 & & $\overline{0.5}$ & & 0.5 & \\
\hline Fiedrits Ensinger & $\bar{E}$ & 05 & & 直 & & 1 & \\
\hline Env. Compl. Englines & $\bar{E}$ & 05 & & 0.5 & & 0.5 & \\
\hline 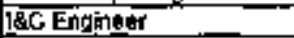 & EE & 0.5 & & 0.5 & & 0.5 & \\
\hline Mechanictol Englised & $\bar{E}$ & 0.5 & & 1 & & 1 & \\
\hline OKAnginiter & $\bar{E}$ & 0.5 & & 0.5 & & 0.5 & \\
\hline Oc inspoction & $T$ & 0.6 & 6.5 & 1.5 & 0.5 & 2.5 & 10.5 \\
\hline Production hanear & $M$ & 1 & & 2 & & 3 & \\
\hline Documbent clark & $\overline{8}$ & $\overline{1}$ & & 1 & & it & \\
\hline 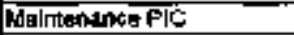 & $P$ & 1 & & 1 & & 1 & \\
\hline OsM Ptaner & $P$ & 0.5 & & 1 & & 1 & \\
\hline Inslummothl Tach & $\bar{T}$ & 1 & & 2 & & 2 & \\
\hline Erectriclares & c & 2 & & 2 & & 2 & \\
\hline Alechinnloal crafts & $c$ & 2 & & 2 & & 2 & \\
\hline Proctest Opetralors & $\underline{R}$ & 1 & & 3 & & $\mathbf{5}$ & \\
\hline HPTE & $\bar{T}$ & $\overline{0.5}$ & & 3 & & ज़ & \\
\hline Pontri operators & $\mathbf{R}$ & 1 & & 3 & & 5 & \\
\hline Erane Operstors & ह & 2 & & 3 & & 5 & \\
\hline Truck Dituers, Ho & C & 2 & 15 & 3 & $\overline{28}$ & 5 & $\sqrt{37}$ \\
\hline & & 24 & 24 & 38 & $3 B$ & 50 & 50 \\
\hline & & & & & & & \\
\hline & $\mathbf{M}$ & 3 & & 4 & & 5 & \\
\hline & E & 3.5 & & $\overline{4.5}$ & & 4.5 & \\
\hline & $\$$ & 0 & & 0 & & a & \\
\hline & $\mathbf{P}$ & 3 & & 4 & & 4 & \\
\hline & $T$ & 2 & & 8.51 & & 2.5 & \\
\hline & $\mathrm{c}$ & है & & 第 & & 象 & \\
\hline & $\mathbf{R}$ & 4 & & 9 & & 15 & \\
\hline & $\underline{L}$ & 0 & & 可 & & 可 & \\
\hline & 무 & 2.5 & & 3 & & 3 & \\
\hline & & 24 & & 39 & & so & \\
\hline & $w$ & 8.5 & & 12.5 & & 13.6. & \\
\hline & wo & 10 & & 18 & & 24 & \\
\hline & wo & 4.5 & & 8.5 & & 12.5 & \\
\hline & & 24 & & 38 & & 60 & \\
\hline
\end{tabular}




\begin{tabular}{|c|c|c|c|c|c|c|c|c|c|c|c|c|c|c|}
\hline \multicolumn{4}{|c|}{ 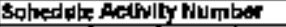 } & 874h5000 & \multicolumn{6}{|c|}{ 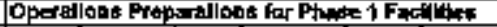 } & 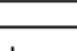 & \multirow{2}{*}{ 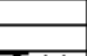 } & & \\
\hline J臣 & oc & $\overline{5}$ & $\overline{F T}$ & Doged & FE: & Momthe & Modn & th & $8 \%$ & To & 8,0 & & & \\
\hline$M(X) D$ & 73000 & 010 & 1 & mangont & $\frac{1}{2}$ & 24 & -1 & 7.40 & $\frac{6 \pi}{25}$ & $\frac{4}{2}$ & हासी & 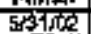 & & \\
\hline 5000 & T300 & काa & 1 & Englinas & 225 & 2 & 1 & 9,525 & 56 . & 2 & 9100 & 5मीयर & & \\
\hline 8000 & 73000 & 010 & 1 & siventiots & 0 & 24 & 7 & 0 & $\mathrm{D}$ & 2 & EN1/, & 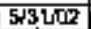 & & \\
\hline P000 & 73000 & 010 & 1 & 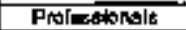 & 2 & 24 & $\bar{T}$ & 7,400 & 505 & 2 & $\mathrm{E} N 1 \mathrm{\pi}, \mathrm{t}$ & $6 \sqrt{31102}$ & & \\
\hline$T 000$ & 73000 & $0 Q 0$ & 1 & 7octunitatenta & 3.25 & 24 & 1 & 12029 & 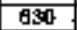 & 2 & $E M D$ & 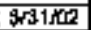 & & \\
\hline 0000 & 3000 & $0 \% 0$ & 1 & Crefifte & 3.5 & 24 & 1 & 12960 & 678 & 2 & Eन(10) & $6 \sqrt{3}$ & & \\
\hline ROOOO & 73000 & 060 & 1 & Operalats & 4.5 & 24 & 1 & 16,050 & 872 & 2 & $81+\infty$ & 5131002 & & \\
\hline LDOW & 73000 & 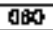 & 1 & Laborersts & 0 & 24 & 1 & 0 & D & 2 & $E$ Er & 5331,02 & & \\
\hline 2000 & $73 \times 0 \times 1$ & 000 & 1 & Clerltar & 1.5 & 24 & 7 & 5,550 & 178 & 2 & QN1000 & 553202 & & \\
\hline & 7300 & 140 & 1 & 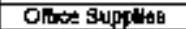 & 19 & 24 & 2 & & 16 & 2 & 6100. & $5 \sqrt{31002}$ & & \\
\hline & 73000 & 190 & 1 & Cothpule Hus & 19 & 24 & 2 & & 49 & 2 & Eला几र & EV3W02 & & \\
\hline & 73000 & 210 & 1 & Ontila fralning & 19 & 24 & 2 & & 24 & 2 & $8+\pi 06$ & Ex/2002 & & \\
\hline & 73000 & $4 C 0$ & 1 & $M P R$ & & 24 & 4 & & 4 & 2 & EMIDO & 6.33tot 2 & & \\
\hline & & & & & & & & 30,700 & +020 & & & & & \\
\hline Solhodivi & Acthin: & $\sqrt{1 / 4}$ & & S1Wh50015 & Hot $\mathrm{OP}_{\mathrm{P}}$ & Erationst & ar Fh & te $\mathrm{t} P \mathrm{x}$ & ties ths & inft ope & & & & \\
\hline JG & DE & 도 & FT & Deacripitith & PTEA & Monlhs: & Folsto & TAH & SK & Gurve & Stant & Frith & DNL & \\
\hline 14000 & 7300 & 010 & 1 & Maltigoers & 4 & $10 B$ & 1 & 66,600 & 4,541 & 0 & समत्रे & $5+31 / 17$ & & \\
\hline 5000 & 73000 & 010 & 1 & Enginofry & 4.5 & $10 B$ & 1 & 74.825 & 5,100 & $\bar{D}$ & लिखा? & 5r1/11 & & \\
\hline $5 \times 1+b$ & 730000 & D10 & i & Stlotitlats & 0 & 100 & 1 & $D$ & 0 & 0 & 6ride & 5 अवा1 & & \\
\hline$P(x)$ & F3000 & D1D & 1 & Proberalontals & 4 & $\sqrt{2}$ & 1 & 68,600 & 4,541 & 0 & 6iva & जुำ11 & & \\
\hline TO00 & 730000 & 080 & $\mathbf{1}$ & Tashniktane & B.5 & $10 \mathrm{O}$ & $T$ & $100,2 \times$ & 5,667 & a & WWO2 & $5 \times 31 / 11$ & & \\
\hline CDND & 73 :060 & $\operatorname{CAD}$ & 1 & Csafter & 7 & 100 & 1 & 116,550 & 6,100 & a & Aroz & 5xमा1 & & \\
\hline RDAO & 730000 & 000 & 7 & Cperogks & $g$ & I要 & 1 & 149,050 & 7846 & to & बivat & 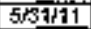 & & \\
\hline $\operatorname{Los} 0$ & 73000 & DaO & 5 & LAbof북 & 0 & 10 & 1 & 0 & b & to & E1N02 & 5ुग & & \\
\hline $\cos 0 x$ & $s=000$ & 000 & 1 & Chergal & 3 & 108 & 1 & 69,950 & 1602 & (5) & 6102 & 5अ1511 & & \\
\hline & $T 3000$ & 140 & 1 & 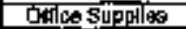 & 可 & 徆 & 2 & & 的 & 8 & ख1002 & जसी & & \\
\hline & 35000 & 780 & $T$ & Compistor HWwsw & $\overline{3 B}$ & Tर्म & $\frac{2}{2}$ & & 430 & t & FWOR & $5 \times 371$ & & \\
\hline & 73000 & 210 & 1 & Onsfet Treinilng & 38 & 108 & 2 & & 215 & to & A $151+02$ & \$NA1M1 & & \\
\hline & 73000 & 220 & 1 & Eltwtrichy & & 108 & 3 & & 67\% & $\theta$ & 6 61102 & $531 \pi 1$ & & \\
\hline & $730 \times 0$ & dW & 1 & Linndy Semito & 225 & tag & 3 & & 500 & 0 & EMाए人 & E/3Eस1 & & \\
\hline & 300 & 490 & 1 & Vething Itatink & & 108 & 3 & & 585 & 0 & BMIIM & Grtit & & \\
\hline & 1300 & 900 & 1 & 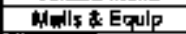 & & 106 & 3 & & $5 \%$ & 0 & MAOE & इड़ाल & & \\
\hline & 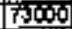 & 400 & 1 & TPF & & 108 & 4 & & 76 & 0 & ब्रास्त & $\$ 317 \%$ & & \\
\hline & & & & & & & & 2700 & 29,635 & & & & & \\
\hline
\end{tabular}




\begin{tabular}{|c|c|c|c|c|c|c|c|c|c|c|c|c|c|c|}
\hline Foher & Anthyle & Then & & 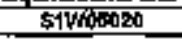 & Oenuth & tho of & 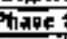 & Fom & & & & & & \\
\hline$\sqrt{c}$ & $\bar{\delta}$ & $\mathrm{CE}$ & $F$ & Depectiption & FTE: & Woralhe & Noto & स्मि & $\$ K$ & Curve & Stuf & Flnt:an & DNL & \\
\hline BWOF & $\sqrt{300}$ & 010 & 1 & Manseres & 2 & 12 & 1 & 3,700 & 252 & 3 & स्यारा & $5 \times 1 / 2$ & & \\
\hline ED00 & 73000 & 010 & 1 & Englönerte & 2.25 & 12 & 1 & 4,163 & 284 & $\sqrt[3]{ }$ & fस1s11 & 5Wत12 & & \\
\hline$\$ 000$ & 73000 & 010 & 1 & Spciqnitisty & 0 & 12 & 1 & D & 0 & 3 & कए1 & 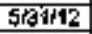 & & \\
\hline$P O 00^{\circ}$ & P3GOO) & 010 & 1 & Profesfibnalt & 2 & 12 & 1 & 3,700 & 252 & 9 & \$1ग11 & BOA1/2 & & \\
\hline 7000 & 73000 & 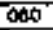 & $\overline{1}$ & Tetotsing & 3.25 & $\sqrt{2}$ & T & 0,073 & उतें & 3 & इसाप & काओतन & & \\
\hline colón & F 3000 & 000 & $i$ & Gints & 3.5 & 12 & 1 & 6,455 & 339 & 3 & स्रा1d11 & इक्जि & & \\
\hline RoD 0 & 7300 & 000 & 1 & Condtos & 4.5 & 12 & $T$ & 935 & 4 & 3 & झूता & अंदीय 2 & & \\
\hline 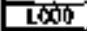 & $73 \times \bar{B}$ & 050 & 1 & Lebrax & 0 & 12 & 1 & 0 & 0 & 3 & orjat1 & 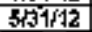 & & \\
\hline प्wस & 33000 & ofet & 7 & फ्ञpFiçd & 1.5 & 12 & 5 & $23+5$ & 要 & इ & E़ाt & इझझस & & \\
\hline & 73000 & 30 & 1 & 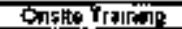 & 15 & 12 & $\frac{2}{2}$ & & 12 & 3 & बलामा & $5 \sqrt{31 / 12}$ & & \\
\hline & 73000 & $2 \sqrt{2 x}$ & 1 & Eextifly & & 12 & 3 & & हु & $\overline{3}$ & हला & $531 / 2$ & & \\
\hline & 73000 & $A L A$ & 1 & Laundry striction & 11.25 & 12 & $\sqrt[3]{ }$ & & 2 28 & 3 & बतार & $5 \sqrt{31} \sqrt{2}$ & & \\
\hline & $730[x$ & 400 & 1 & A APR & & 12 & 4 & & 0 & $\underline{9}$ & समf11 & ENWT2 & & \\
\hline & & & & & & & & 35,950 & 2,039 & & & & & \\
\hline Btretul & Aetivil & 개픈 & & $91 \% 0603$ & porgt & tens PDP & arrition & tor $74 x$ & 8F & 而国 & & & & \\
\hline & & & & & & & & & & & & & & \\
\hline s & bC & Cदू & FT & Dopipiption & FTEF & Tyomtho & Fipio & 내 & ak & Curva & Gilart & Findich & D些 & \\
\hline ANAth & bकow & D10 & 1 & 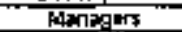 & 2.5 & 24 & 1 & 96 & a & 2 & 5100 & 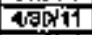 & & \\
\hline$E[x] 0$ & I & जo & $\overline{1}$ & Engtomints & 2.26 & 24 & & 0,325 & 58 & 2 & 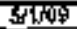 & $4 \sqrt{3}$ & & \\
\hline$s 000$ & 5300 & 010 & 1 & bentists & 0 & 24 & 1 & 0 & 0 & 2 & जा, & $280 \times 11$ & & \\
\hline POOD & $\sqrt{3000}$ & 010 & $T$ & Profopgionple & 2 & 34 & $T$ & 7806 & 800 & $\frac{\pi}{2}$ & Find & 4ख़ी & & \\
\hline TX6t & 1300 & $\infty$ & $t$ & Techuiclens & 4,76 & 24 & & $17,5+6$ & जूर & $\frac{2}{2}$ & इ10F & 4त्wन1 & & \\
\hline $\cos$ & 3idon & एकी & 5 & दत्री & 4,5 & 24 & $T$ & 18 650 & drk & 2 & इसर & 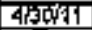 & & \\
\hline ROOD & $F$ & 060 & 9 & Dpointest & 7.6 & 24 & 1 & 27.700 & 1.463 & 2 & इतरकी & Aम & & \\
\hline $10 \times 0$ & 73,000 & 060 & 1 & L咩piots & 0 & 24 & 1 & 0 & 0 & 2 & Fine & $\overline{438011}$ & & \\
\hline CDOT & 73000 & $\infty 0$ & 1 & ciurical & 1,5 & $2 \phi$ & 1 & 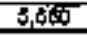 & 179 & 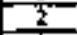 & 5ning & 413013 & & \\
\hline & P3000 & कीD & $\mathbf{1}$ & 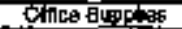 & 35 & 24 & $\overline{2}$ & & 19 & $\frac{a}{d}$ & जात्र & 43411 & & \\
\hline & 730010 & 160 & 1 & 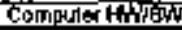 & 26 & 24 & 2 & & 这3 & $\frac{1}{2}$ & जीता & 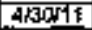 & & \\
\hline & 73000 & $2 \sqrt{20}$ & 1 & Conote Trolrseng & 25 & 24 & $\sqrt{2}$ & & 91 & 2 & $5 \sqrt{20}$ & alsont11 & & \\
\hline & 73000 & ब㛹o & 1 & 神k & & 24 & 4 & & 5 & 2 & $5 / 709$ & \$13011 & & \\
\hline & & & & & & & & 2500 & 5,238 & & & & & \\
\hline
\end{tabular}




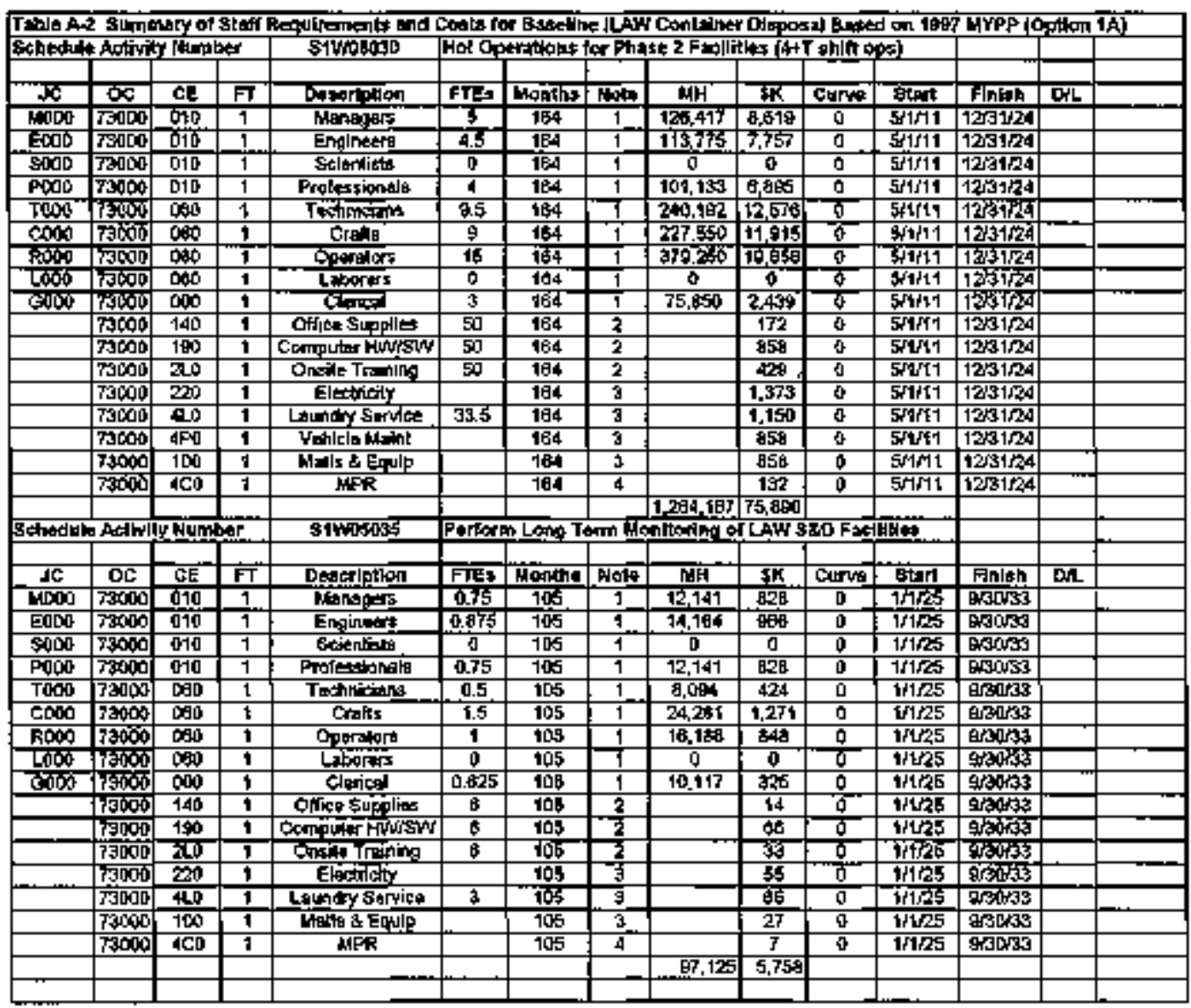




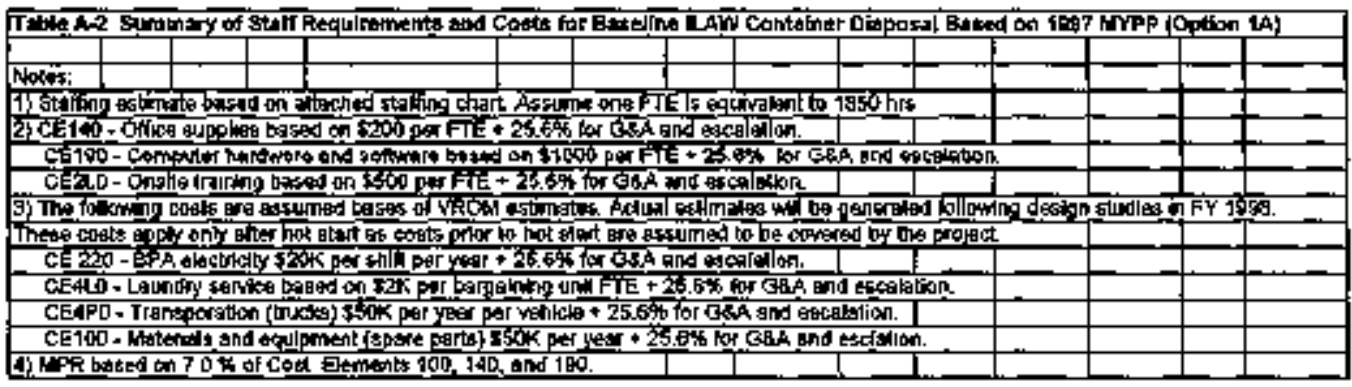


Rer. 0

Tabje A-3. Summary of Eatimatad Capital Cost Details For Design, Construction and Clospre of Boselline Iromobdilized Low Activly Waste Dioposeal Based on Fiscak Year 1997 Mult-Year Work Plan (Optoon $1 \mathrm{~A})$.

\begin{tabular}{|c|c|c|c|}
\hline Activity & $\begin{array}{l}\text { Moduk I } \\
\text { \$ milliog }\end{array}$ & $\begin{array}{l}\text { Moxtule } 2 \\
\$ \text { million } \\
\end{array}$ & $\begin{array}{l}\text { Module } 3 \\
5 \text { million } \\
\end{array}$ \\
\hline $\begin{array}{l}\text { Prepare Proliminary } \\
\text { Title I Design }\end{array}$ & & $1 . T$ & \\
\hline $\begin{array}{l}\text { Preparte Delailed } \\
\text { Title (I desirn }\end{array}$ & & $9.4^{4}$ & \\
\hline $\begin{array}{l}\text { Site PTep/Mobilize } \\
\text { Construction Foross }\end{array}$ & $20.6^{*}$ & 0.3 & - \\
\hline Construction & $130^{b}$ & 96 & 96. \\
\hline Closure & I3 & 13 & 13 \\
\hline
\end{tabular}

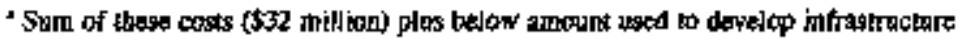
cost of Table 5-1.

- Differeuce between module I and other modules $\$$ \$130 million - $\$ 96$ million $=$ $\$ 34$ millon) added to above sum (\$32 million) to develop $\$ 60$ million total infrastructure cost of Table $5-1$. 
HNF-SD-TWR-AGA-004

Rev 0

\section{A2.0 ALTERNATTVE PLANNING CASES}

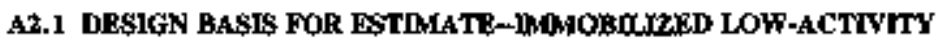
WASTE DISPOSAL COST ESTEMATES (OTTIONS 2A, 2B, 2C, AND 2D) 
HWNF-SD-TWR-AGA-00A

Rev 0

This page irlentionally left blank. 


\title{
A2.1 DESIGN BASIS FOR ESTZMATE-IHAOBILIEEN LOW-ACTIVTT WASTE DSFOSAL COST ESTTMATES (OPTIONS 2A, 2B, 2C, AND 2D)
}

\author{
DESIGN BASIS FOA ESTIMLTE \\ |LAN ofisposal cost Estinates
}

\section{0 socpt of Hork}

Foㅂ shall prepare throe (3) cost estinates for the ILAH Disposal project.

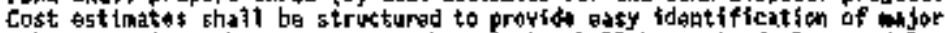
sabsystems in sich astiatse. Each estinate shall be a stand-alore acodule thak can be combined utlth other modales to establish the total capital cost for a varlety of possible disposal façility configurations.

The oftinates that will bo propariod ore as follow:

A) Disposal Project Infrastructura

B) Noduiar oisposal Units

- Renote Handlod Hixod Hasto

- Renote fondled hon-rosxod Woste

- Contact Handled Mlxed Waste

- Contser Handled Hon-MSxad Waste

c) Firs] Closure and Cap

- Prototyot Hanford Batrier

- Riternative I of lodified Hanforo Barrier

- A7tarnative 2 of Bodified Hanford Barsier

\subsection{Description of Merk.}

This gaction dascribes the minimun reguleed elenents of the total project and which estiaste they wirl fajd lnto. adofitianal elements that nay be derened

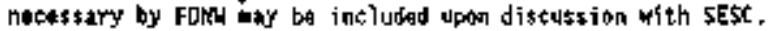

\subsection{Infrastruçture}

This estinate will includs all elenents of the project infrastructure that wold be comon to all configuratios. Speciffe elements to be included in this estimate are:

- Site ciear and grub

- Cut and f111 to rough prade

- Peringtar fencing and signs

- site dralnoge/stara water control

- Site 11ght ing

- Roadnays

- Utilities

- Eloctrterl

- Potabl * water

- FIra Nater

- Sanilary sewer

- Yolecommun itations

- Dperations butiding

- toatrol room

- Changoflocker roora

- Records storage room 
HLNP+SD-THR-AGTA-10014

Rey 0

- Traiping roon

- Adainistrative/offica space

- Majatentance berilditig

- Truck/forkl ift malutenance

- Crane neintendince

- \$acurity/access control

- Transport veficles

Desigu basig for estimeting Infrastructure ig as follows:

Sita arta is 90 atres. Location is soulhest of PERFX between 4 th strest and

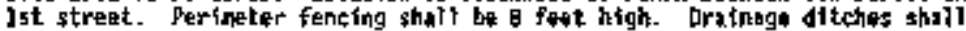
control rum-on and runaff of raimwater to and fron the dispesal site. Stte 1\{ghting sha]l be prordded for raadkays, parking lots, and fencé 1 ine.

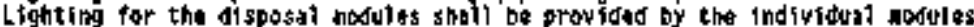
(see section 2.2\}. A pared central rosdway [10 meters wide) and perineter roads (8 maters widal shall prowide zccess to buth ends of atach ditpost enit.

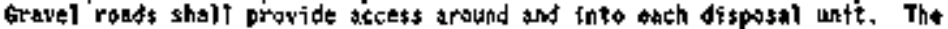
inforstructurg will proylde uggrades to Etrst street from the disposal site ontrance to the east fenteline of the 200 East area.

Utijites shall be proylded to the site and within the site. The project

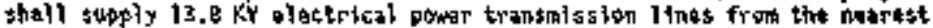
arallabie sultch gear to the disgosel site substation. The lafrastructure shall include $480 \mathrm{y}$ power distriłution to infrastructure-prordded belildings. Electricel distribution to the dispostil nodthes shall be ghrodiged by the individual nodules (see section 2.2).

Potable mater and fire water supply piping shall be provided to the dssposal s1to distribution cujfold. Valer distribut lom plping to infrastrveture.

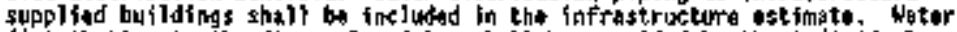
distribution to the disposal modelies shail be provided by the individasi rodula. Sanjtary semage disposal shall be provfded by the disposat site infrastructurk.

Telecomuntcations services to the disposs 7 site and within the oparations and mintendice buildings shall be provjoed by teifrastructure. Ielecomanications shall ficludo tetophone, local arta conputer natmork (HLAH), and inargtaky response carrunications systams.

Tha disposal site infrastracturi shall proidd a cantral operations Buitding consigtiog of control room, chengt/locker roow, rectords storagk rook, trafning row, and odnintstrattue office space. Oparations schedile will be 3 shifts

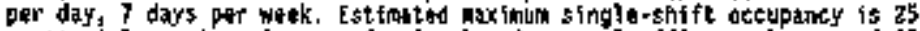
anagerial. engijeering, profassional and general offics workers, and 10 techloitas, traftsoen and operators. Iotal occupancy is estingted at 40

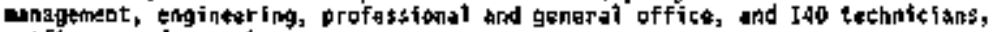
croftsman and operistors.

A alintedance ballding shall be provided by the infrastructure for seryicing wojor equifinette such is forklifts, trucks and crapes.

Sęcurity and access control shall bo proulded by guard houses at each active antranco/exit gate to the disgosal site. 
The disposal infratiructure shall provide 15 flatbed troctor-traller truckg for transportation of raste packages from the trastrent contractor site to tho disposal site. This adfitional transportatian capabtilty will bo rapuirod in 2010.

\subsection{Nodutar Disposal Units}

The estieste for the podmlar disposal units shall provide capital costs for construction of a single disposal unit for each of the faur dispasal unit concepts.

Destin features comon to all concepls:

The disposal units will be constructed in the cleared and graded area provided by the trfrastructure estimate. Utilitios spacific to the disposal unit such as is 1octumenicetions, *lectricsl and water distribution shatt be supplind by the disposal modules. pistribution run lengths tos "utility servicess shall be based on distances reflective of the avtrage [modian\} location of a disposa] unit.

Treated waste w171 be recelred fron the troatnont contractor iл a package. The packago size 15 J.B moters wide by 1.2 metters high by 1.2 naters ofep and includes alf appurtenances required for hankling by ouqthead crane or forklift. Maximm package woight is j0,000 kg. Rewole handled waste has a

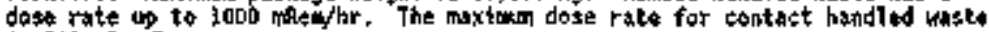
is 200 RRerlhr.

\section{Concopt 1: Reopte Handled Mixed Haste}

2

The disposal vait shall conxist of a shietded concrete raults divided into 5 cellg located adjacent to one another, with comon wolls seperating the yault calls. Each toll holds 840 packagts in an arrsy 10 wide by 6 high by 14 daep for \& total disposal unit capactty of 50t0 packages. The packigos shall bo separated by not less than 0.1 neter in both horizonta? difactians. in accesss roudhay funs the length of the yault on ond sfde, Internal diansions of exch

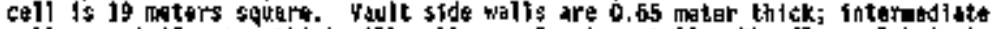

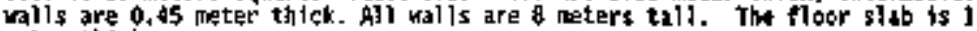
weter thick.

The disposal untt and access roadway are straddled by abile gantry/bridge crane building riding on rafls that ron the 3ength of the disposal init. The

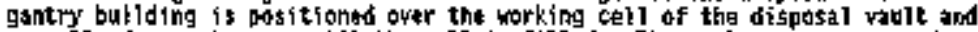

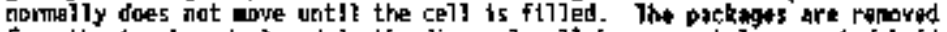
frox the track and placed in the disposal cell by a remotely operated bridge

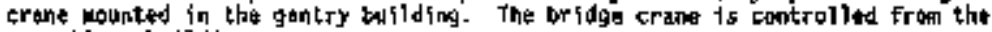
aperations bullding.

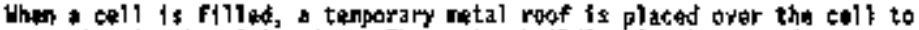
prevent rainwater Intrusion. The fantry bul]ding is then wored oreer to the next call. Whan all cells hare beton filted and a disposal decisjon has been cate, the cells will bo closed by renowing the tebporary metal roofs and back filling the disposal array with on inert filler waterial such as graded sand, and careved wth a 0.5 meter thick rainforced conereke roof slob. When all

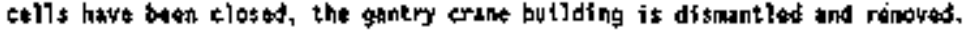


Beneath the disposth wht is a buble biner/loschate collectlon system. The distgrt bas is for the lingr and loschate coljection syaten shall be the save as Profect H-025, Lox Level Nixed Kaste disposal trenches. The liner systen consists of the fallaring layers (frow botion to top):

- Unxisturbed subgrade (botto-nost layer)

- Sexondary compacted adontx (bentonite alay/sotl\} 1 notur thick

- Secondary HDpE $15 n e r$

- Secondary geonet

- Ceotextile Typa B

- Secondary graqul orainage layer 0.3 mater thick

- Coptextile Type B

- Prinary conpactod amix (bentonitse thafsoit) 0.5 ogtor thick

- Primary HDPE l|jar

- Primary geonet

- Destaxtifle Typa B

- Prthaty drainaga graval Iayser 0.3 weter thick.

- Contextile Tyoe

- Qparations layes (graded sail/gravel) I meter thick (top layer)

Concept 2: Ropots Handlod Mon-mixed Maste

This concept is 10talicai to Concept 1 with the exception that the doublo Jiner and leachate collection system is not ragulyed.

\section{Concept 3: Contuct Handlod Nixad Wasta}

The dasposal unit is ar open trench with side slopes of 3H:1v. The disposal capecity of the moule shall bo notipatly 5040 packages, arranged in ath array Io wide by 6 high by gd rous deep. The packeges shal] be saparated by not 14:5 than 0.1 mator in both hortzootal directions. This provides a nominal dispagal array $25 z 8$ of 20 meters wids by 110 maters long by 7.2 meters tall.

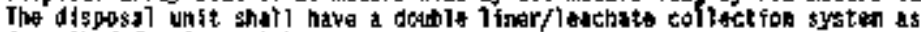
fescribed far concept ].

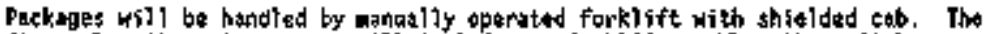
disposal unit cost estiate will include two forklifts. Shen the undt has beon ftiled and a dispostl dectsion has been made, the waste ptckage trray whll be back filled with an ingrt, parous filler matarial such as sand or grarsi.

Concept 4: Conţact Handlę mon-rolxed Waste

This concept is the sans as caticept 3 with the exteption that the double liker and leachate collection systen is not ropsirod. 


\subsection{Final closure and cap}

At closure intrk filler material (sand or gravet) will be added to the spaces arousd haste contasnors, + rainfored conerate ropf slab added to the raults, 'surfact protect|v tarthen tarrire placed over the disposal units and surface narkers iostalled. The barrier will inhibit infiltration of water and intrusion by pisnts, aniatis and hanans into the waste. Cisturbed land will rocontorred to conform rith the sirrounding terratin and revegototed it th locelly derived native plant species consistent rith stteride biological aitigation plans. The surface markers whit identify Jocation of the disposal uatis.

The estimate shat1 provide capital costs for addition of sand filler, addition of $0.5 \mathrm{~m}$ thick rqipforced conerate roof slabs to the vaules, construction of the protective barrier over each sisposal anit or groups of dispatal units. and addition of markerg.

There are 3 barrier concepts at this tine. Sepcific eleasnts to be frciuded in this est fuate are:

- Excavete/procure materizls

- Transpart salorials

- Roware tenoorary netal roof of yoults and olsposs

- Ndd required annunts of sand $f$; pler

- [nstal7 tainforted concrebe roof Elab on valts

- Add berster materials ard slopt os needod

- Resontour disturbed land areas

- Revegtate with natife plant spexías

- Procure and fistall surface warkers

The baseline berrier is the prototyoe Hanford surface Bartitr. It is about 4.4 in deep, 2 n outrhang, and has a vertical wajl encircling each disposal unit. The siternatjy barriers use the sard 5vrface barrier and overtiang but dIfferent sub loyers. The sub layers art used for water diversion and water conditionlag.

The barriers art surmarizod belon. The bold lecters are commo featuras.

Baseline: Hanford Surface Barriar

$1.0=\$ 11 t$ Logn/adnix Grava? K\$x

1.0. Silt Loarp

0.15 a sand Filter

0.30 a Gravel filtow

1.B - Fractured gasait Riprop

0.30 * Drainage firars?

0.15 a Asphal lic Concrete Coated w Flirid-Applited Asphat

2.0 - Barrier threrpang Geyond stoks of Each o1sposat linit

O.15 a Vertical Ring Asphattic Concrete Couted w Flahd-Applięd Rspalt to Encirclt Esch unit 
Rev 0

Altermative 1 Hodifted Hanford Bartier

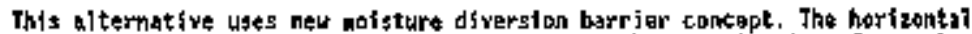
barrier is sand and slaping grarel. The vertical barriter ts rigg of gravel.

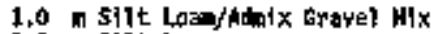

1.0 m silt Lot

0.15 in sond Filter

0,30 a grave] Fliter

1.5 F Fractured Basalt Riprap

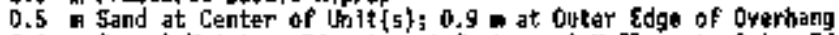

0.4 Gravet Moisture Divertar at tenkar - 3 y s7ope to Outer Edge of Ouertheng

2.0 - Barrler overbang Geyand \$des of Each Unit

1.0 V Vertical Ring of Graves to Exitcla Eter Unit

Alternative 2 Kodified Hanfard Barrier

This alternative uses a larger mosture diverter system than Al terastive I and a layer of crushed glass (frit) for water conditioning. Tha rertycal barrier is the sawe as Alternatiug 1 .

1.0 in Silt Low/Adalx Grayel H1X

1.o d silt Loan

0.15 a Sard Ffiter

0.30 on Gravol Filtes

1.5 而 Fractured Basalt Riprap

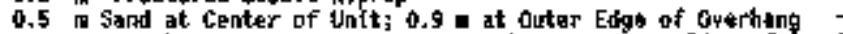

0.5 in Gravei hofstura Diverter $-3 \%$ Slopo to outer Edge of Duerhang

0.4 m Glass Frit Neter-Conditioning at Center $-3 \times$ Elope to Duter Edge of Oyewhang

2.0 m Barrite ovethang Boyond Sidus of Each Unit

1.0 m Vertical Ring of Gravel to Encircte Each Џa1t 
HNF-SD-TWR-AGA-004

Rev. 0

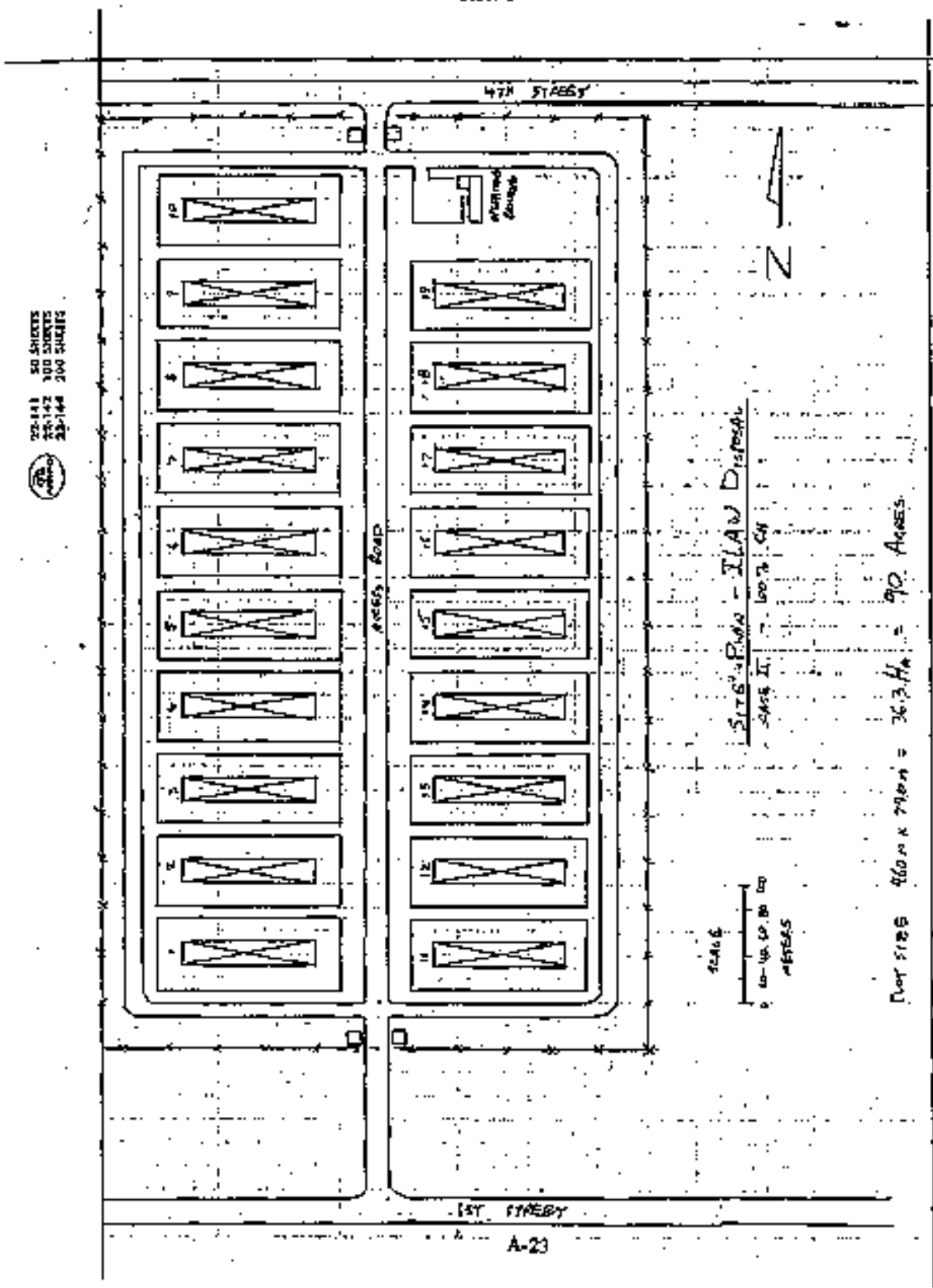




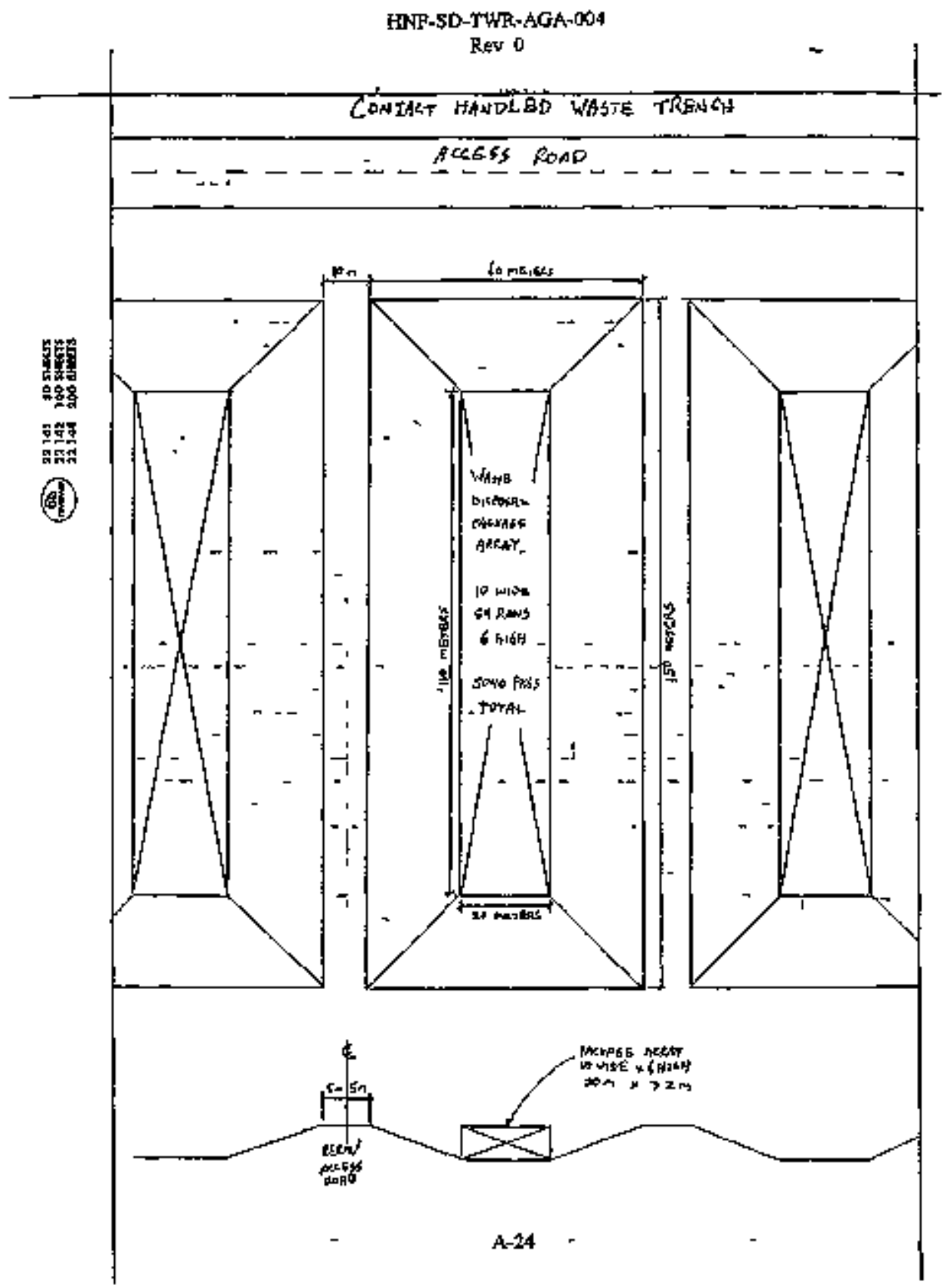




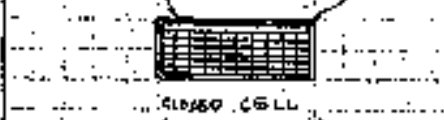

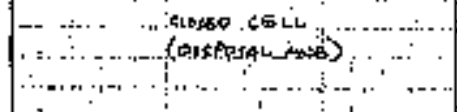

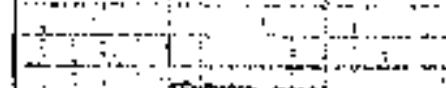

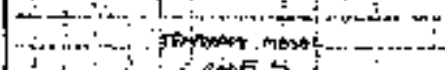

… $\therefore$ '

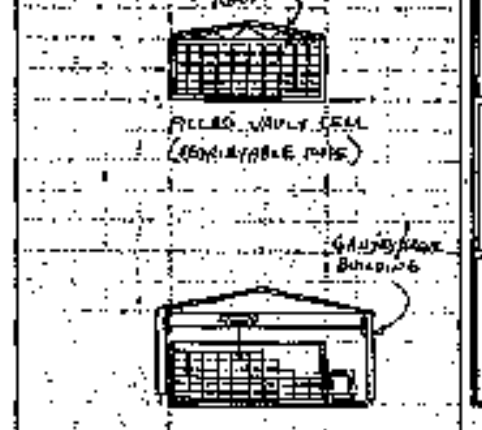

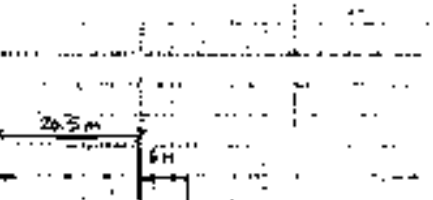




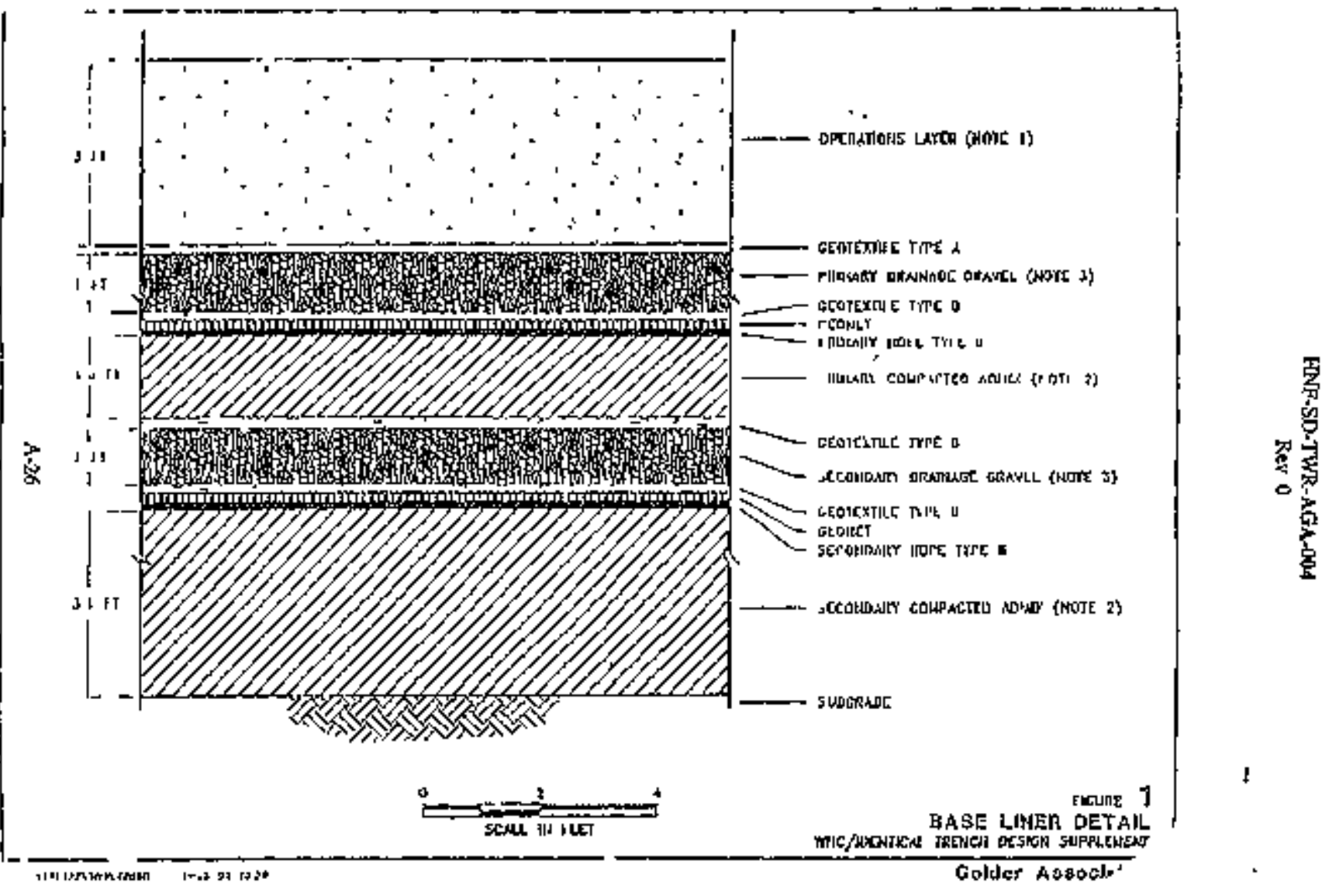


HDNF-\$D.TWR-AGA OOA

Rơv 0

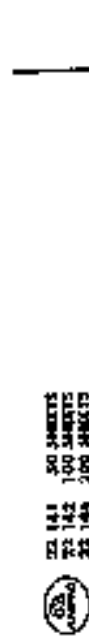

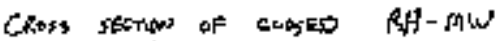

litar OIStGe DNIT

.

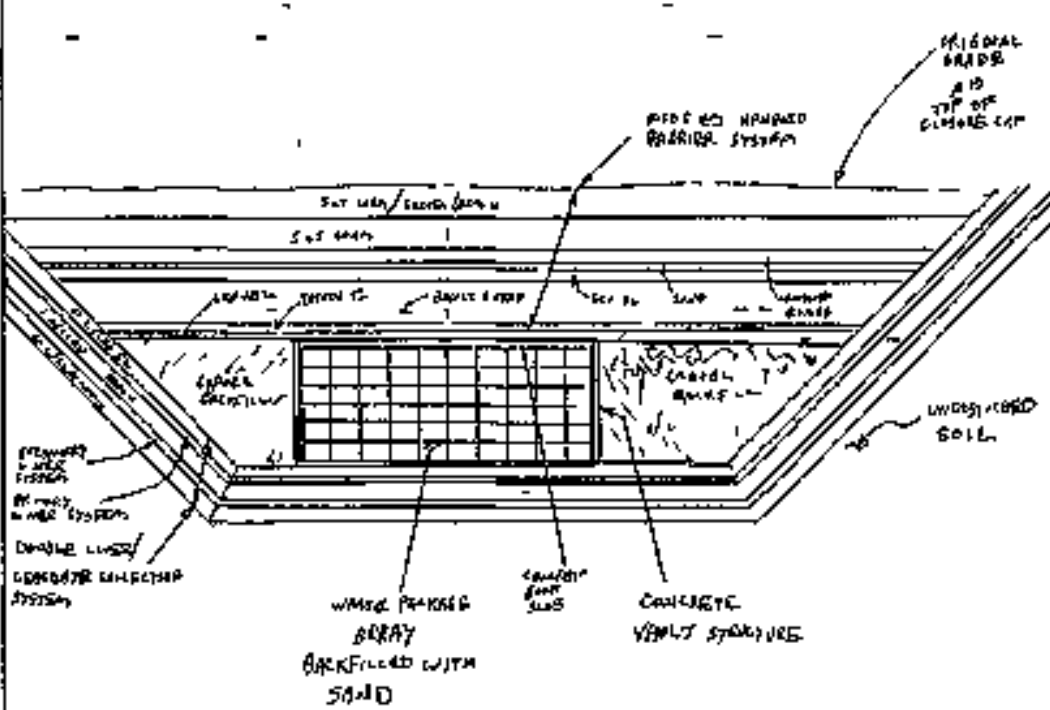




\section{NTERIM PERFORHANCE ASSESSTENT

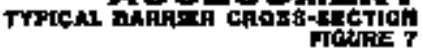

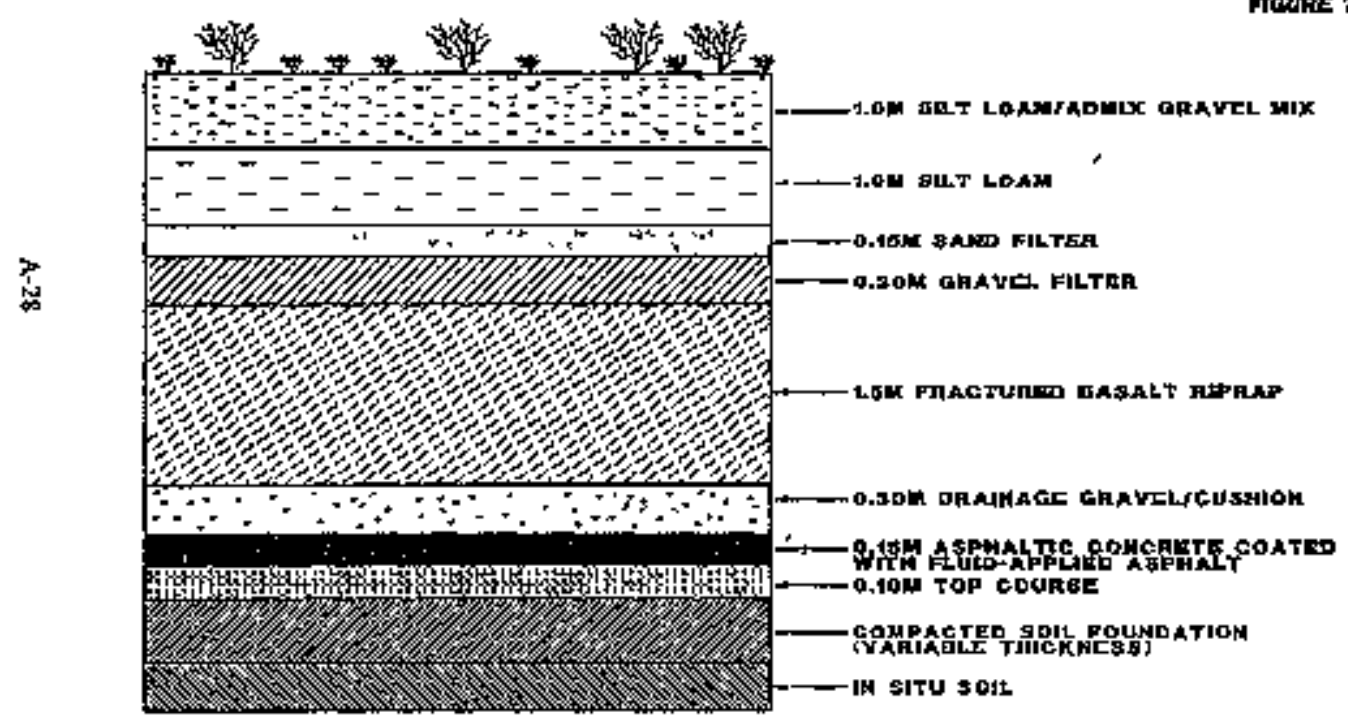


Table A-4. Estimated Iotrastructure Costs for Dippesal of Remote or Contact-Hatdled Immobulized Low-Activiry Waste (Optoun 2A, 2B, 2C, and 2D).

in

- Nate: The above estimale was developed based on June

i. 1997 dollars. It was escalated to September 30, 2062

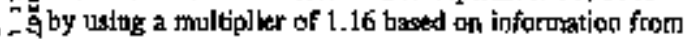

: $=$ FDHC on composite labor and materials.
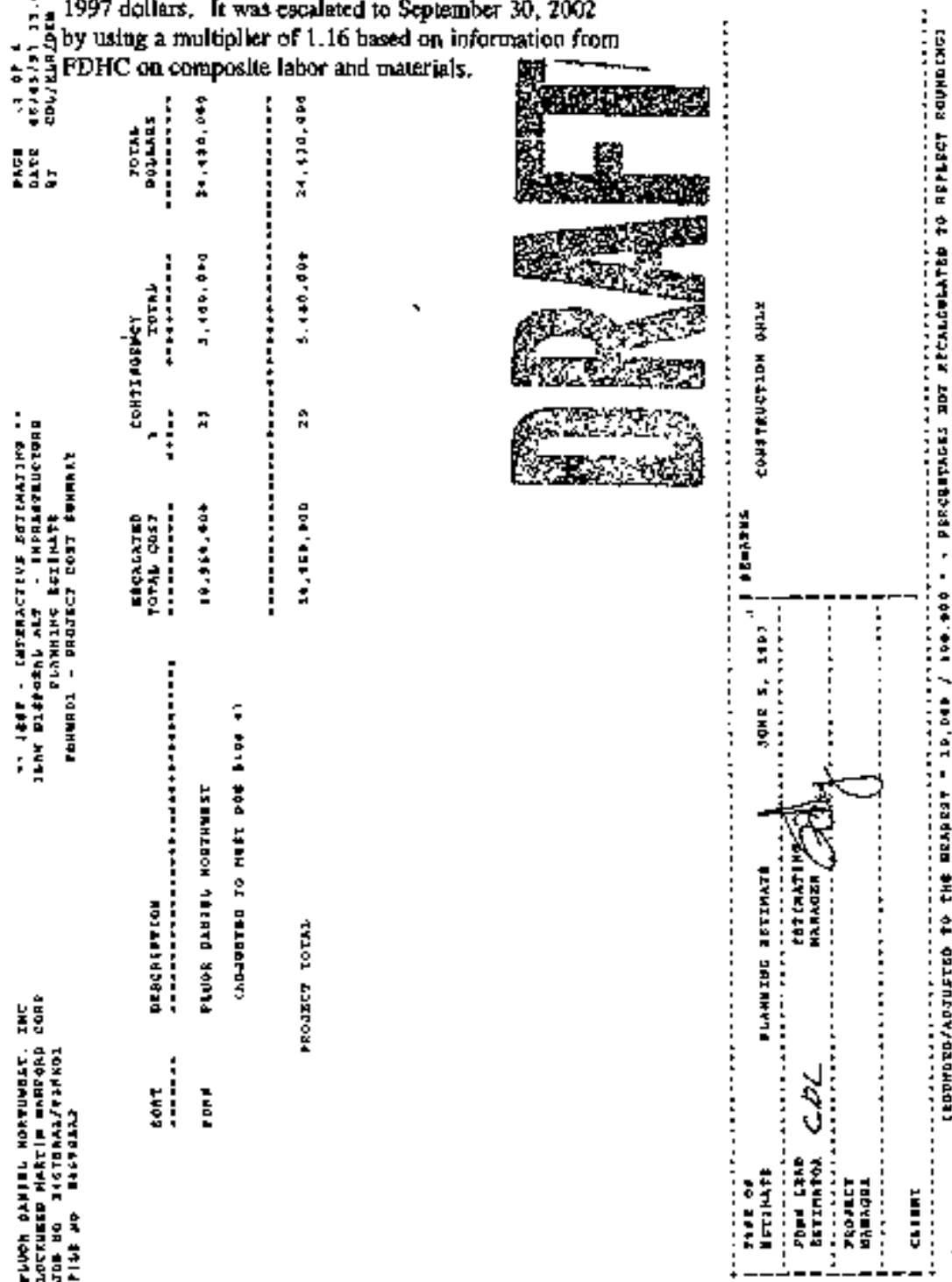


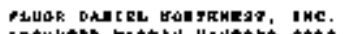

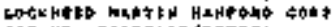

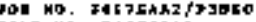

PrLE Ma. 146TARS

upo Defengition

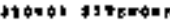

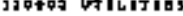

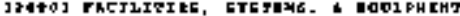

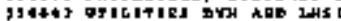

moject torat

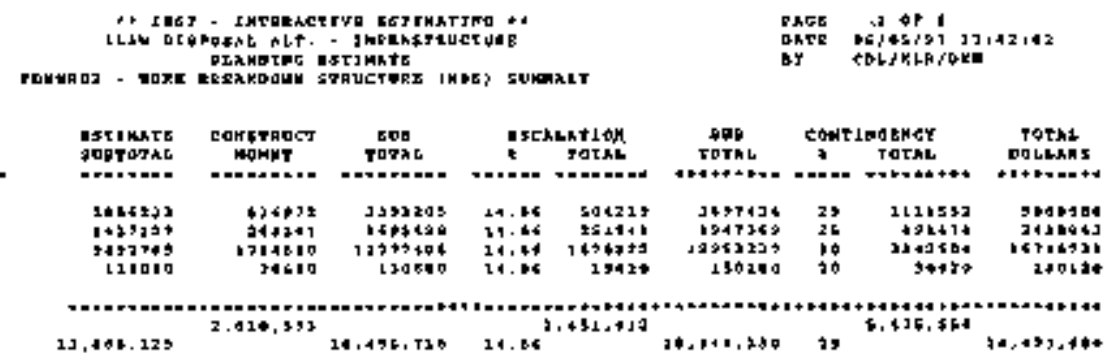




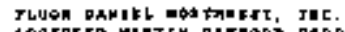
WOCEKED MUET CM RATEOAB ESRP

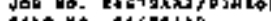

ILt No, \$167\$RLS

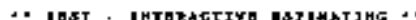

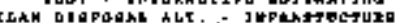

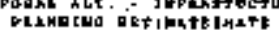

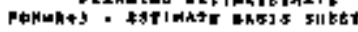

THOE 2 oT 0

$3.47,34$

ar

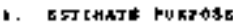

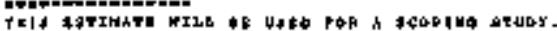

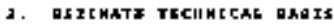

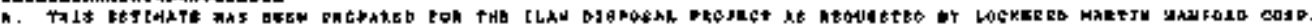

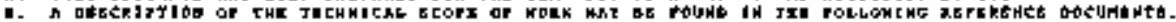

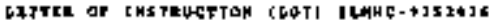

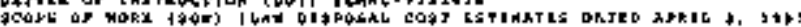

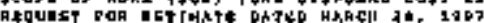

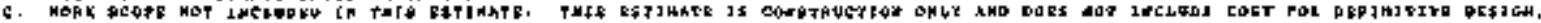

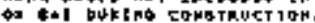

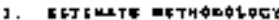

x.

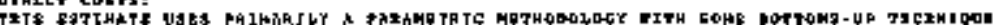

COHA

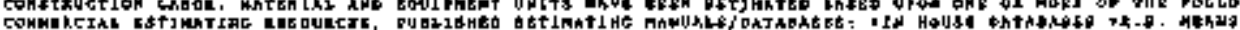

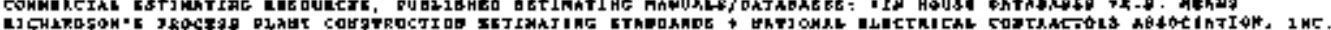

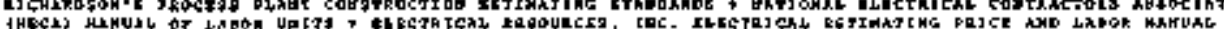

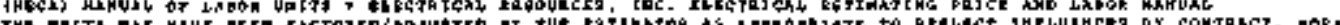

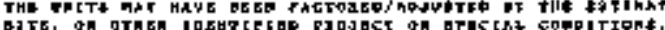

- BAREY COAT PXCTOP\$

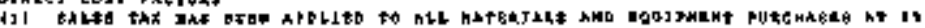

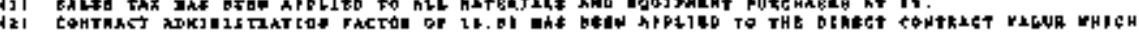

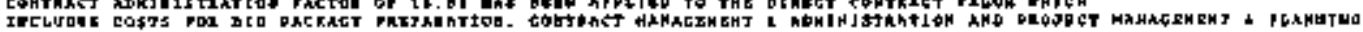
capoter

c. IDOAMBT tost:

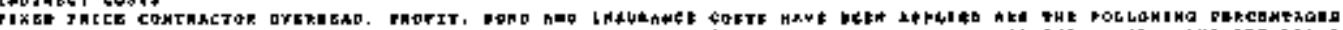

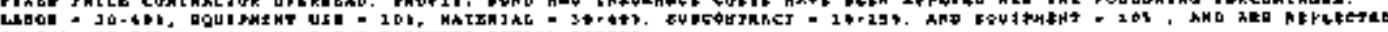

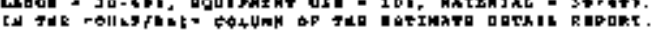

- मคTE.

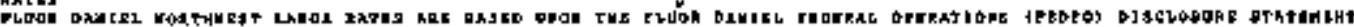

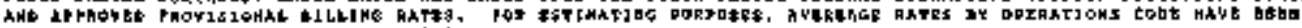

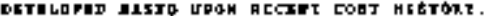




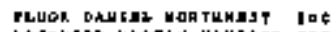

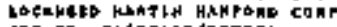

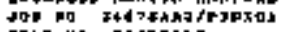

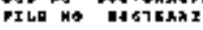

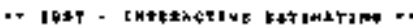
CLAX DIEPDEkE ALT, JoTESTECTURE VLAKEIPG IFTHKATE

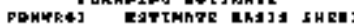

Dras 2 an

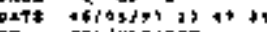

at Cob/KiRiaka

\section{FACH ४АTाQR}

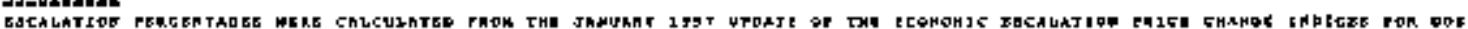

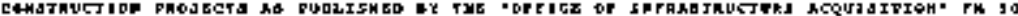

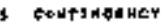

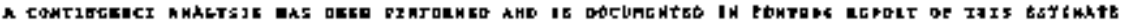

4 RLARI

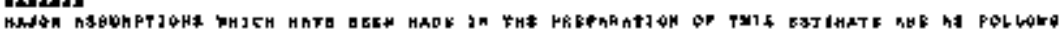

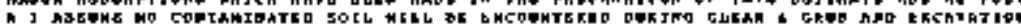

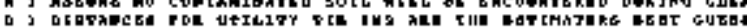

L J a TH 


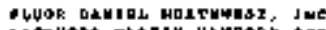

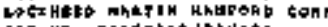

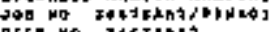

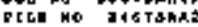

GotT

coberuat Dxsehirytor

460 ampLotemage TO LLH

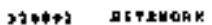

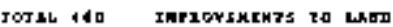

501 Pojtormas

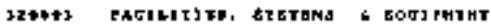
roth sB getcoteras

610 UชtLrtted

\section{7jo0:7 ajegron}

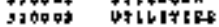

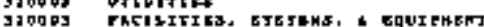

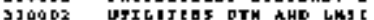

Total to. watiture

PLO HEXVY MQHILE FOUTPHEAT

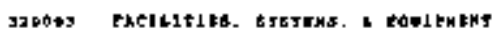

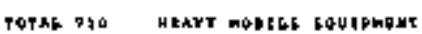

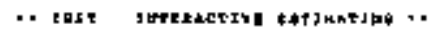

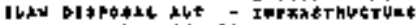

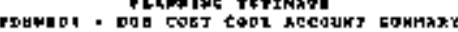

rhas a a a

45

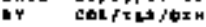

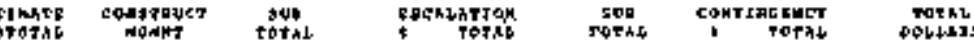

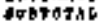

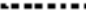

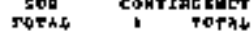

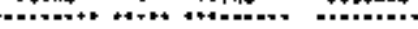

\begin{tabular}{|c|c|c|c|c|c|c|c|}
\hline $22,7 x+1$ & 457504 & 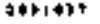 & 1916 & +21607 & 2320664 & סב & 126216 \\
\hline & 493504 & $2 \circ 51037$ & ro & $92+607$ & $3+20+1$ & 10 & 941151 \\
\hline
\end{tabular}

14 14 v+3 ta

14isist do

2)

$\cos +2+$

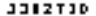

63939

4014143

14 .

\$ST174

081345910

$4010+13$

$+2570$

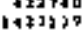

1240.

\section{0 \\ $\$ 0216 a$}

$26+342$

1010

ixpitis

$0+4+10$

$\begin{array}{ll}14 & 16 \\ 11 & \$ 6 \\ 19 & 06\end{array}$

74623

11010

421316

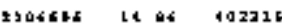

14 14

the

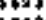

$19+74$

1047343
+34653

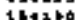

25

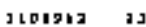

itim

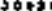

12310

$53+5$

Into

נथנ2 293

$52015+0$

5731310

2414+12

$4+4>2+2$

14 if $1022+13$

3902715

רוק

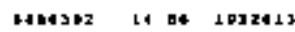

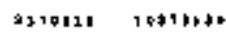

mostcy toghe

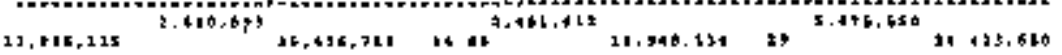




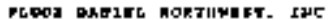

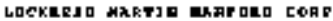

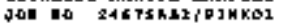

PI

CA I DERTITIOH

\section{Coretruction}
Qง गाт Еलक्र
11 equjertut
1] SPACIML Canstactian

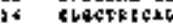

rogst CorEvRETJOH

phoster roth1

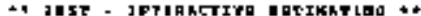

J1M DIS OOSpl

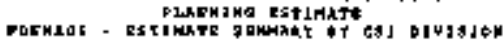

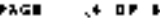

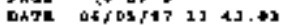

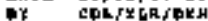

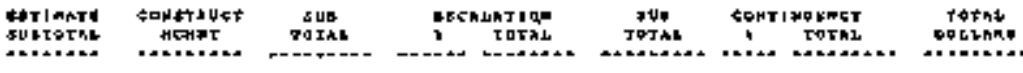

\begin{tabular}{|c|c|c|c|c|c|c|c|c|}
\hline $\begin{array}{l}2>03030 \\
6192564 \\
3303734 \\
1630463\end{array}$ & $\begin{array}{r}591015 \\
2101849 \\
435939 \\
201003\end{array}$ & $\begin{array}{l}2701054 \\
1464342 \\
1414643 \\
2166464\end{array}$ & $\begin{array}{ll}1+ & 16 \\
14 & 18 \\
2+ & 16 \\
21 & 16\end{array}$ & $\begin{array}{r}562561 \\
1022+13 \\
357175 \\
23+7 \$ 5\end{array}$ & $\begin{array}{l}1740635 \\
\text { t1 } 61715 \\
1619039 \\
2401824\end{array}$ & $\begin{array}{l}70 \\
90 \\
30 \\
70\end{array}$ & 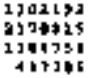 & $\begin{array}{l}51+2424 \\
20202824 \\
4010424 \\
2 \$ 6140\end{array}$ \\
\hline & $2,410.993$ & & & -451.412 & & & 479,381 & \\
\hline
\end{tabular}

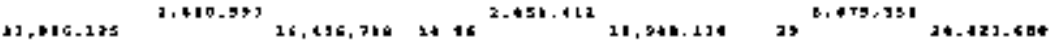

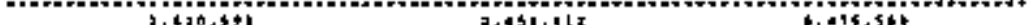

$13,019,124$

$16,196,71014,16$

is

24.123 .140 


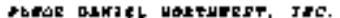

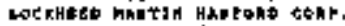

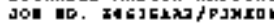

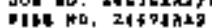

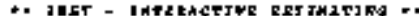

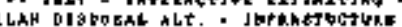
piryta
C10r a ga

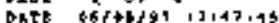

D. TOLSK ThK

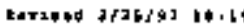

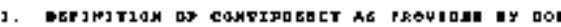

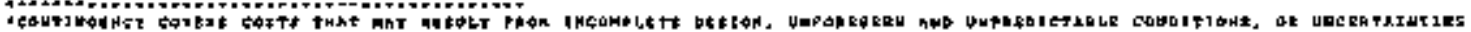

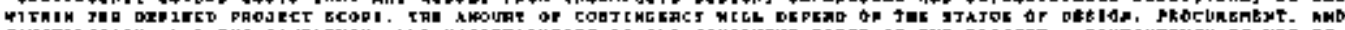

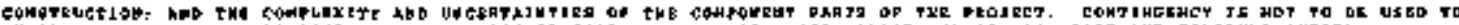

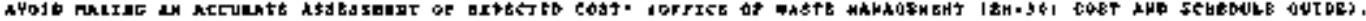

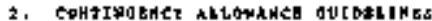

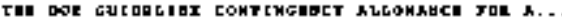

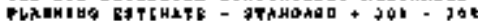

2.

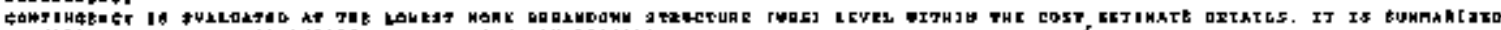

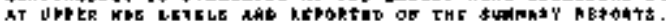

\section{3, LNRGTशI} An ASt EE

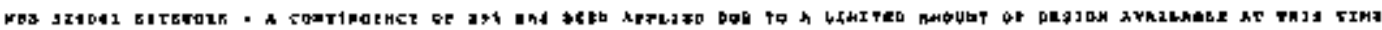

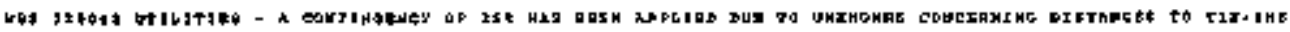

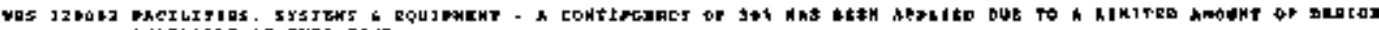

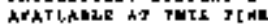

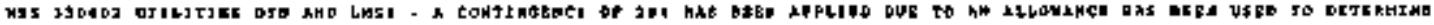
THE COOT 


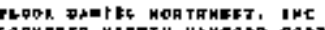

COCK WITE MAKTIH HAMFALD

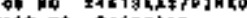

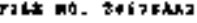

Pas sescitation

Con

321041 ETTEMER

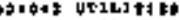

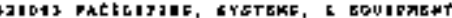

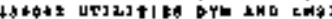

houltit tothl

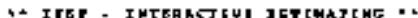

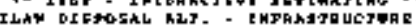
LREh s Ma gat lahto

DNeF का का

Dise oidotis? $13+212$

ar coctucotokn

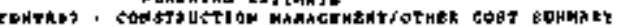

atinnt:

suldors

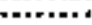

2156333

192124

247274

110400

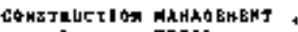

TDTS

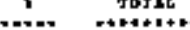

OTHEF

casta

$\rightarrow+r+\infty++4$

909

TOTAL

IB b* $53 \% 9 \mathrm{Ta}$

1. 04 agras

iN 10 2*\$1\$10

it at 206

\section{t tosast \\ 21130}

2101644

20014

19,00000000

$1,52+5,59$

$2,690,503$ 


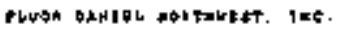

COCTHEED MARTJK HSETOAD CORE.

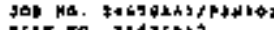

rEL no. aidTERs

necounz

MUNB ER

HESchjeftor

A ITEพ⿳亠口冋

970401

IITUVer

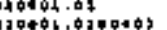

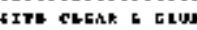

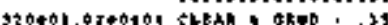

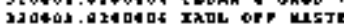

10070726 ATTEWep

Tofke $\cos \cos t i \cos$

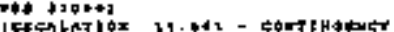

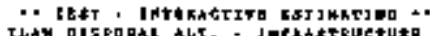

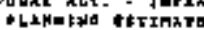

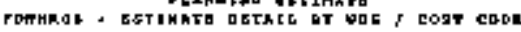

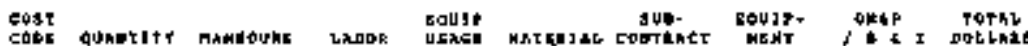

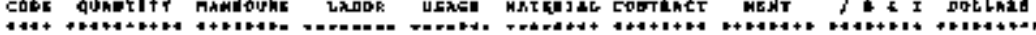

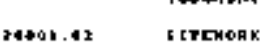

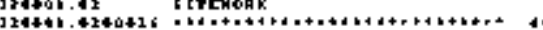

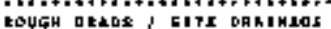

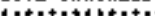

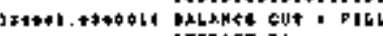

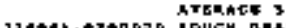

custars gizanakn

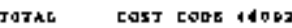

The $210 \bullet \bullet 1$

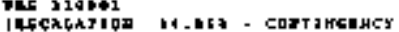

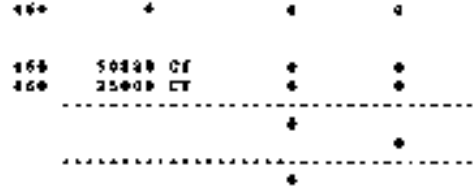

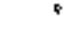

(n)

†IT\&MARK

$2 x+1+1,+7$

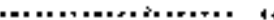

PEM JKETEX REMEIND FIDHF

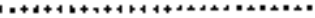

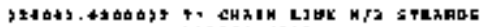
BAHBEO HIAE

JPOA1.02040JA 3B DAL GATE

J7004J. 0.040JE 926Ms

toutarnL tgttoroti

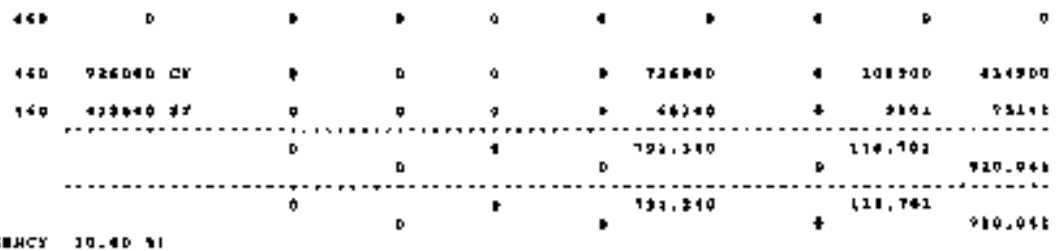

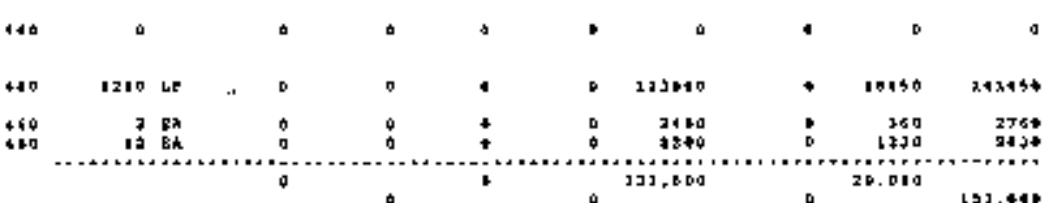


Rev. 0

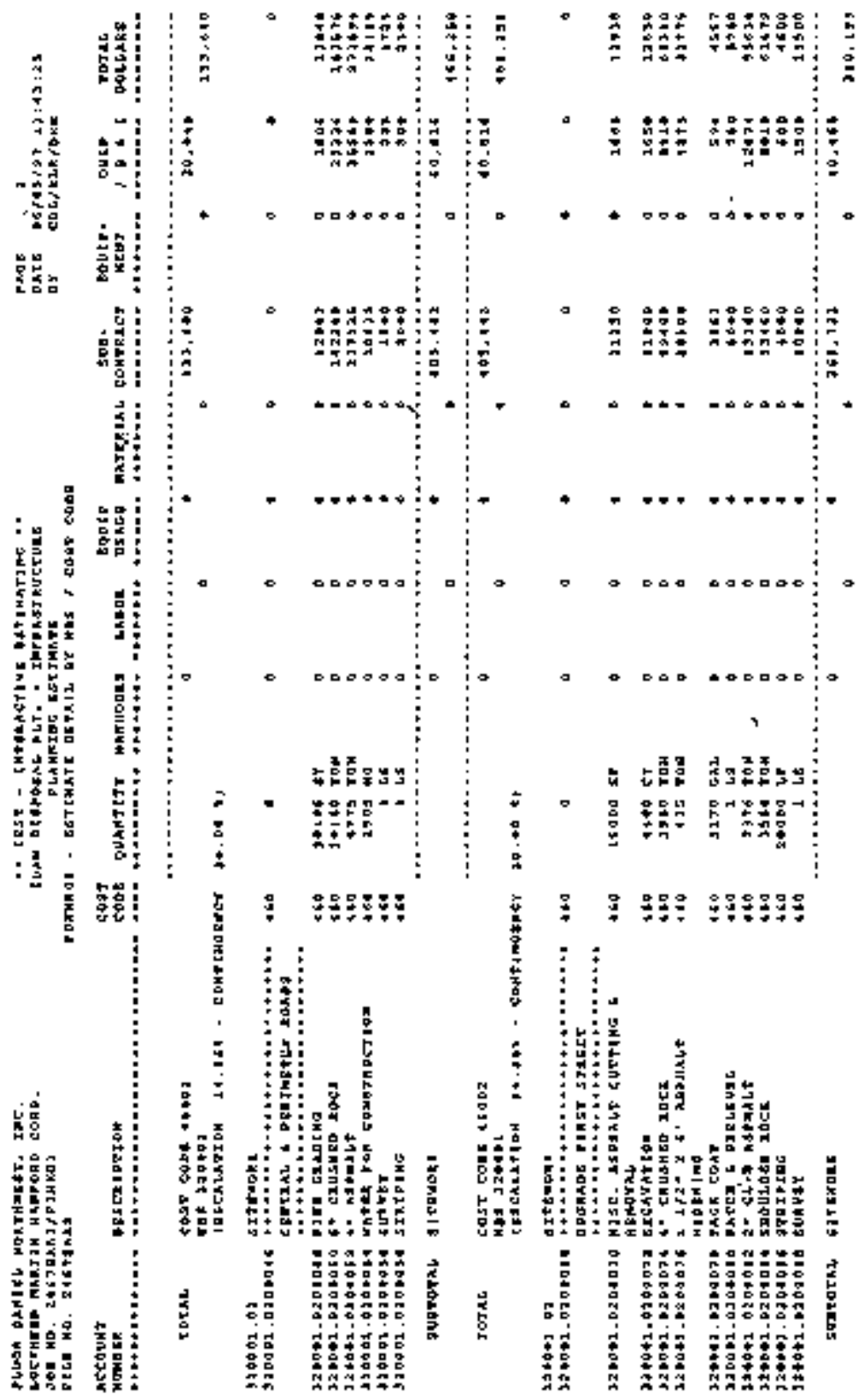




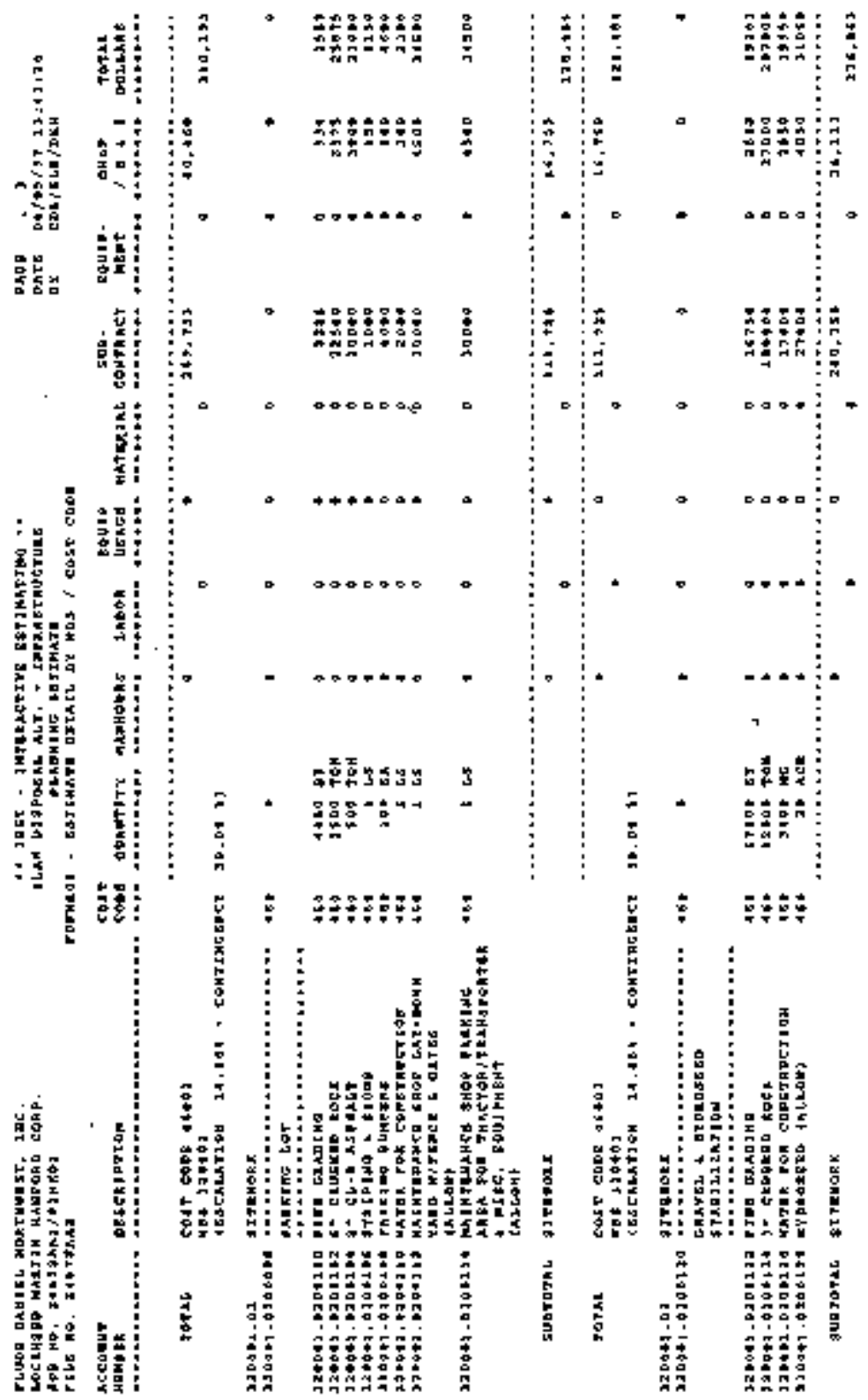




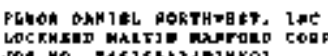

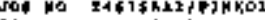

PIE MO Coriten?

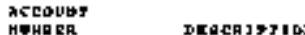
DEgerjoritas

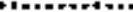

cost togt atos

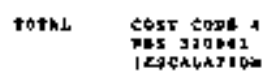

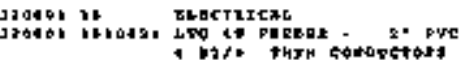

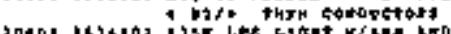

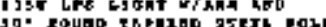

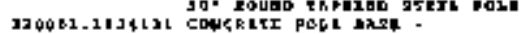

con

U $\mathrm{x}$

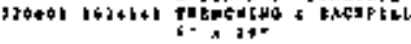

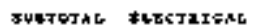

$$
\begin{aligned}
& \text { EALgg ThX I BA } \\
& \text { OHAP fOS HAETUPS OMLY }
\end{aligned}
$$

tothe

\section{cost coth 11311}

Mac 32004

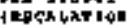

14 164 - colqunenç

Totkb det tabla ogtkwork

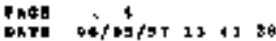

ey cagruciejayll tarkastrection

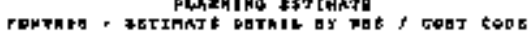

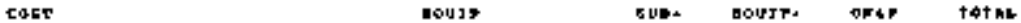

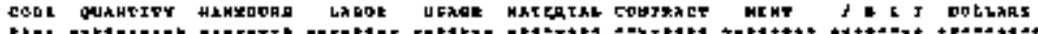

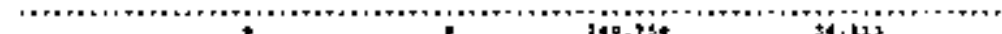

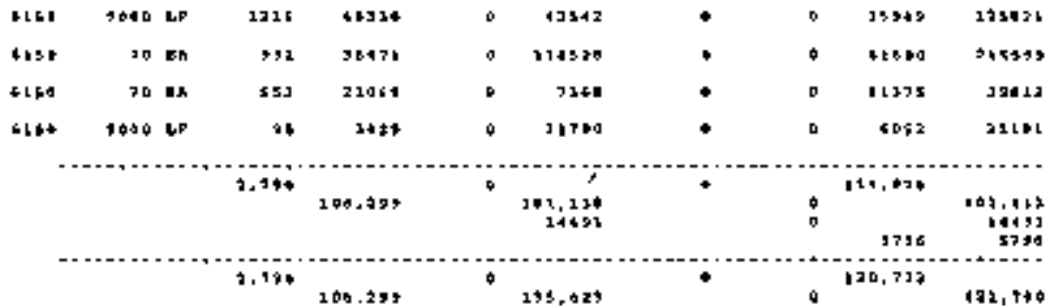

2410 ₹r

106.29,

1 נ, , $1: 4$

, Ist, s, s 


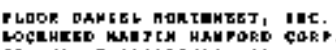

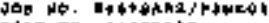

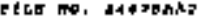

AceOFnT
MUMB Bh

Numb Bh

$27+469$ UTIGITRE

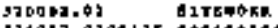

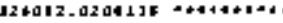
riak NAzkt

...........

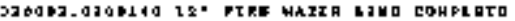

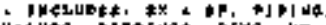

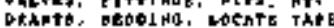

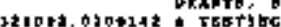

cuarosal suremak

FOHAL COST GOAE $6000 \mathrm{O}$ TDS 320402

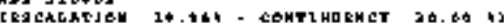

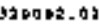
Artrethr.

IKN]

(1kp)

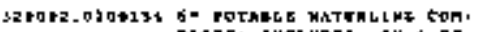

Hetr: inçuded. $2 k \leqslant$ a

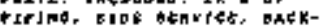

TLON PTEVINTCAH, HOCKTE TATO

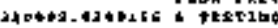

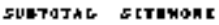

totk1

cast CObE $0+02$

aps 320042

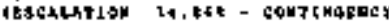

$100 \quad 2306 \mathrm{Lr}$

$6 \mathrm{ab}$

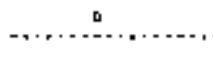

(

$20-0+1$ $s$ tranonk

920402.42

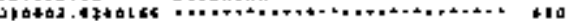

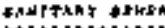

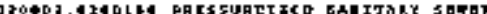

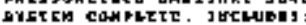

2són 19

10

12.5

0

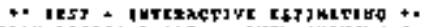

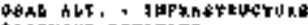

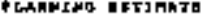

Phos +650,

CY CELSKLITEKU

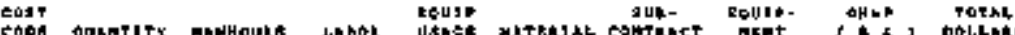

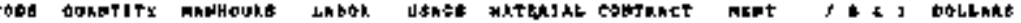
1014a-

1

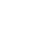

o

a

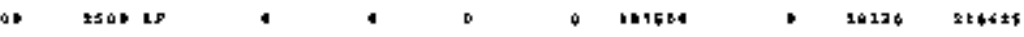

......

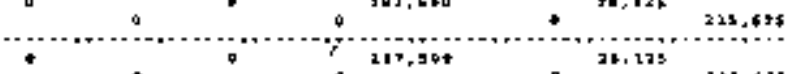

217,904 0 abizs

ats, tas

资

162.309

24, 3י

169,106

6

$118,0+5$

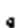

g

- งด 0400

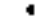




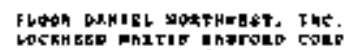

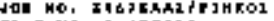
P1LE W0. $206752 \mathrm{Ka}$

\section{stcoup}

rowere

Daserietican

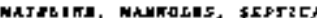

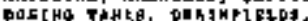

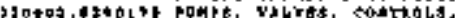

ELECTIEAL * TESTINA

sutgorte sRTHONK

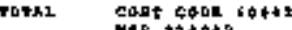

uns sidita

xiretrtcke

320402.

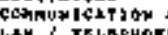

LAM TELBPHOE

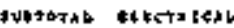

TOTKL

cost coos 1616

wot thota?

30003.1

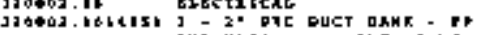

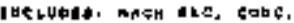

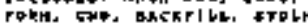

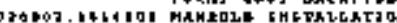

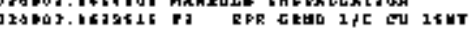

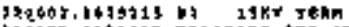
IAP TPACS POE 1].1 EV

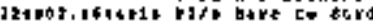

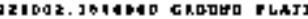

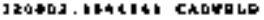

39002,301634 KFER $23 k \pi$ withmt

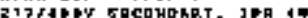

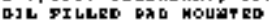

$$
\text { T5O KYA }
$$

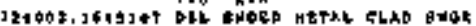

a. $12+0$ Hat

$4 \$$

$30.00+1$ EZESTILEAC

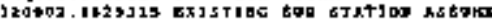

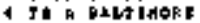

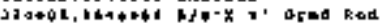

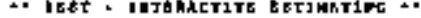

ILEM DaE POESL ALT. - IATRAETROCTUIE

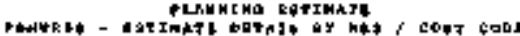

cott cot gouj

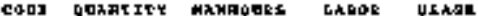

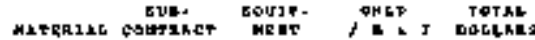

-....................

....................

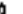

H.

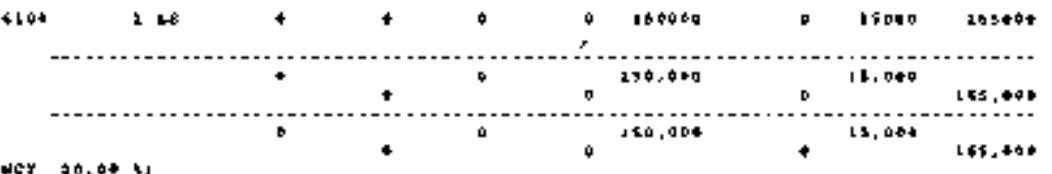

\begin{tabular}{|c|c|c|c|c|c|c|c|c|c|c|}
\hline bst & 2000 & $L F$ & 1 dos & $1+121$ & a & בוצנב & $\bullet$ & a & רפנפוג & 17210 \\
\hline $\begin{array}{l}6150 \\
6150 \\
6250 \\
1130\end{array}$ & $\begin{array}{r}3200 \\
19 \\
2\end{array}$ & $\begin{array}{l}300 \\
20 \\
\text { in } \\
0 .\end{array}$ & $\begin{array}{r}10 \\
210 \\
44\end{array}$ & $\begin{array}{r}3+40 \\
t>20 \\
014 \\
1+24\end{array}$ & $\begin{array}{l}0 \\
0 \\
0 \\
0\end{array}$ & $\begin{array}{r}13000 \\
1+344 \\
+14 \\
493+6\end{array}$ & 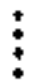 & $\begin{array}{l}0 \\
0 \\
0 \\
0\end{array}$ & $\begin{array}{r}1739 \\
9431 \\
532 \\
2+41\}\end{array}$ & 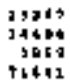 \\
\hline
\end{tabular}

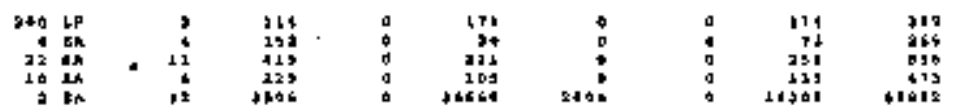

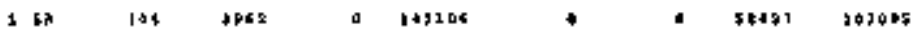




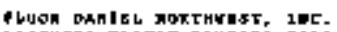

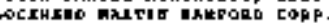

jol Na. 14614k2/01nkts

rot ma. Ititidis

neconнt

HUMa re

Dascrietrot

I-130 hap tIs

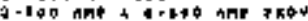

SOEJAJKL ELECTIGEAC

THLXE TAM 1.0.,

rotse cast c904 45316

IAECaLation

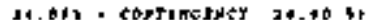

ORE TOP MKATUPS ONZY

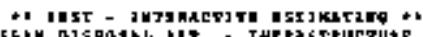

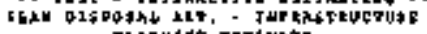

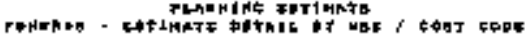

MRLE 5

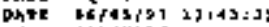

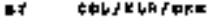

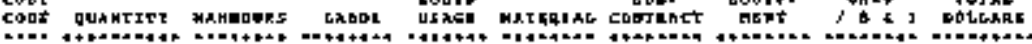

EOUI\$. $9 \mathrm{HLT}$ Tor

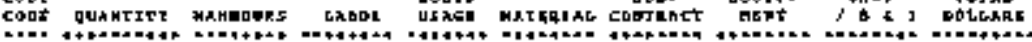

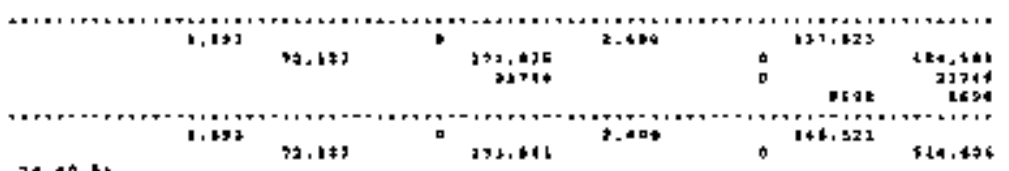

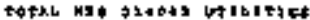

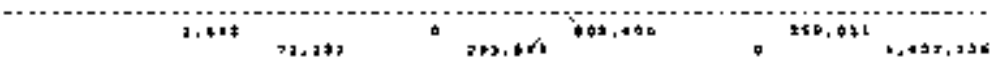


Rev. :

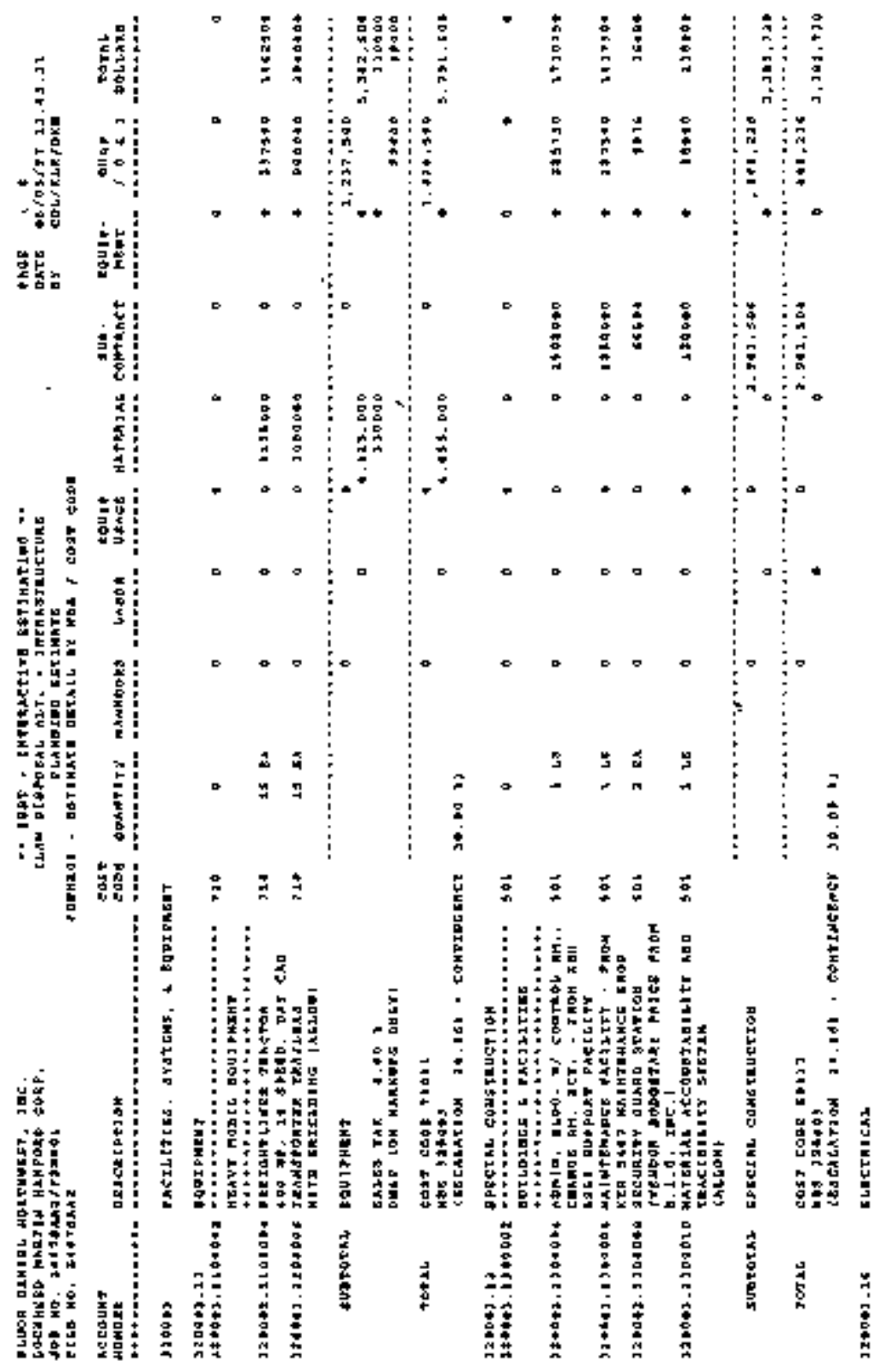


TLUDL DHFLEL HONTHMEET, IMC

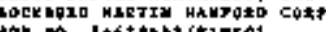

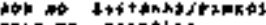

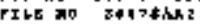

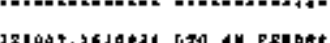

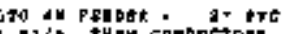

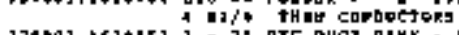

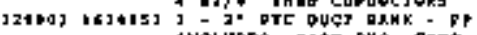
1Ncluose nata Exe, Gore.

TOAH. CNO, OKCKFILL. TTULH

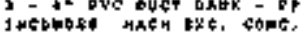

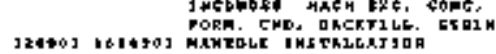

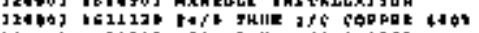

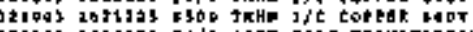

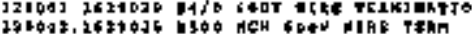

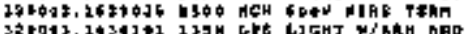

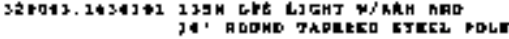

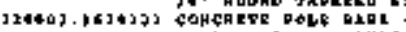

IDPin $x+$ somptuit

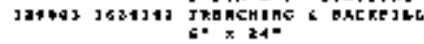

\section{tubtothl bletethich}

g1LES ThX E QI, OHLD TOM MRMKUPS OHLT)

TOTAL

cost codo 6 Hsie

mot d 7000 d

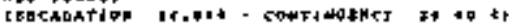

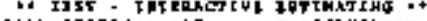

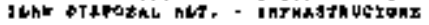

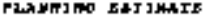

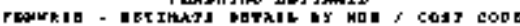

PX⿻上丨 1

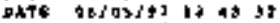

DY COL/CIMJOEH

movit

Sud-

Equta -

Оนธ D

tosx

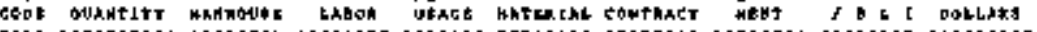

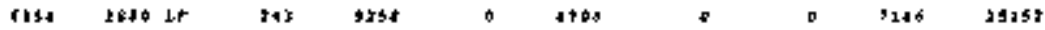

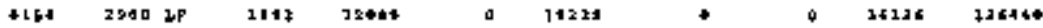

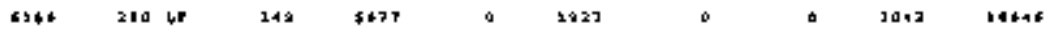

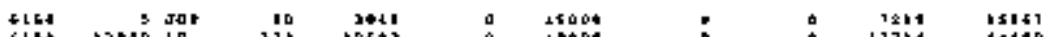

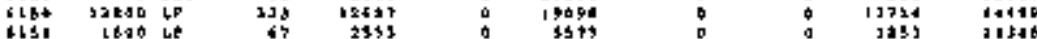

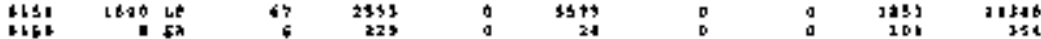

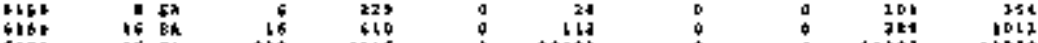

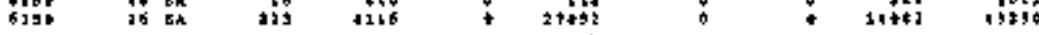

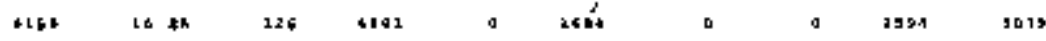

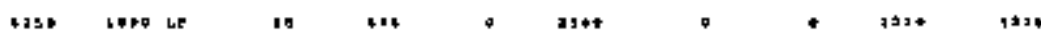

$\operatorname{ling}$

119. T49

(1)

Jigנ

3,147 219,748

$a, b=$

119,79
$9,940.584$

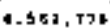

$1,41,7,16$ 


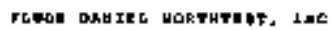

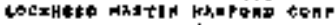

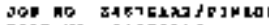

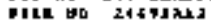

ictous

MaNFEF onsestrias

נ 0402 OTILJTFF EYT LNO LEG

J34\$\$2 IV XLETIICXL

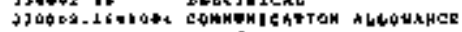
ThP $f$ TELFF

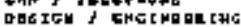

oustoahl dectnjent

TOTKL SQT $600 \mathrm{~B} 610,6$ Nos jutat

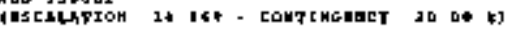

honta 11

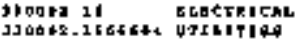

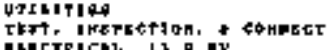
anctecenz I 3 g av

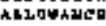

THQTOTAL FLECEKISHC

6110

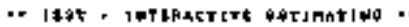

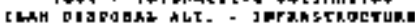

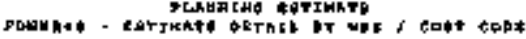

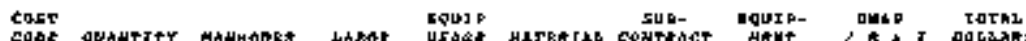

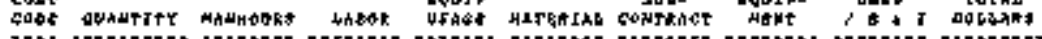

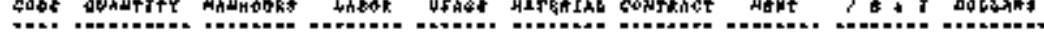

$6+\mathbb{1}$

1

$0 \quad 1+0+0$

$+\quad 6+64$

55000

(1)

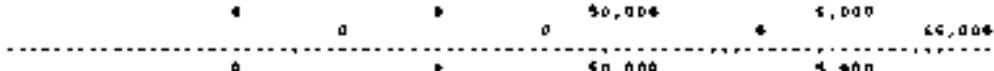

60,000

15, a04

11 t

I 1 s

$\varphi$

10000

$\$$ ó a

$\$ 404$

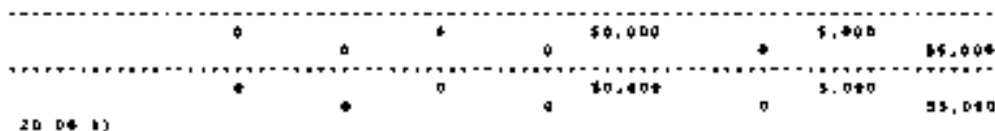

Cost CORE 81320

200411

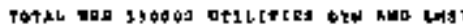

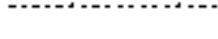

100,404
10, ata

[1F, 


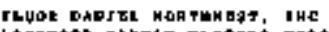

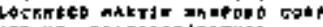

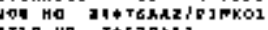

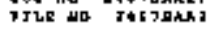

Mecaunt

rurepe

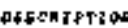

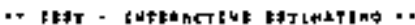

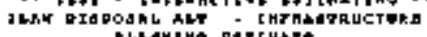
bhenito gischate

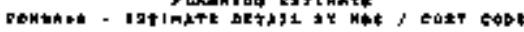

squт

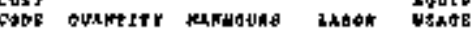

LUE-

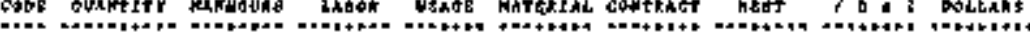

Hor 11

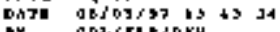

AY eQZJKLk/OKu

xq0CD- OHEY TOrML

hets $f$ a : Doltahs

\section{MERAJ TOTAL}

$t_{3,031,987^{1,164,069}}$

29127
$8,643,541$

$13,416,123$ 
HNE-SD-TWR-AGA-10)4

Rev. 0

This page intenionaliy Ieft byank. 
Rer 0

Table A-5. Estimated Construction Costs for Disposal of Remute and Corlact-Handleat Inmabilized low-Astrivity Wagto (Options 2A, 2B, 2C and 2D).

Nole: The above estimate was developed based on Juns 1997 dollars, It was eralated to September 30, 2002, by using a dnaltiplier of 1 . 16 based on infism thation from FDHC on composite labor and suaterals. 
HWF-SD-TWR-AGA-004

Rev. 0

This page intentionally left blank. 


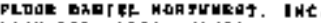

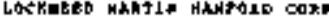

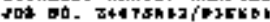

TIL Mo. TALISII

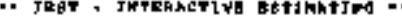

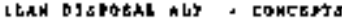

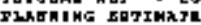

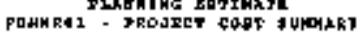

Puse $\triangle$ or 10

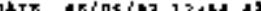

-T calfKLK/ bE. H

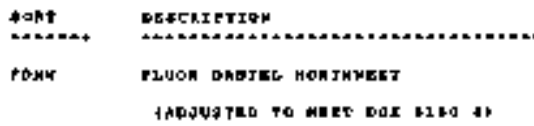

FHoJect tatak

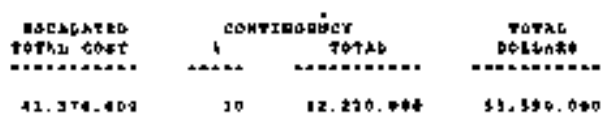

11.374.408

20

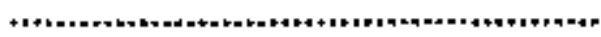

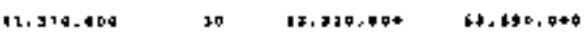

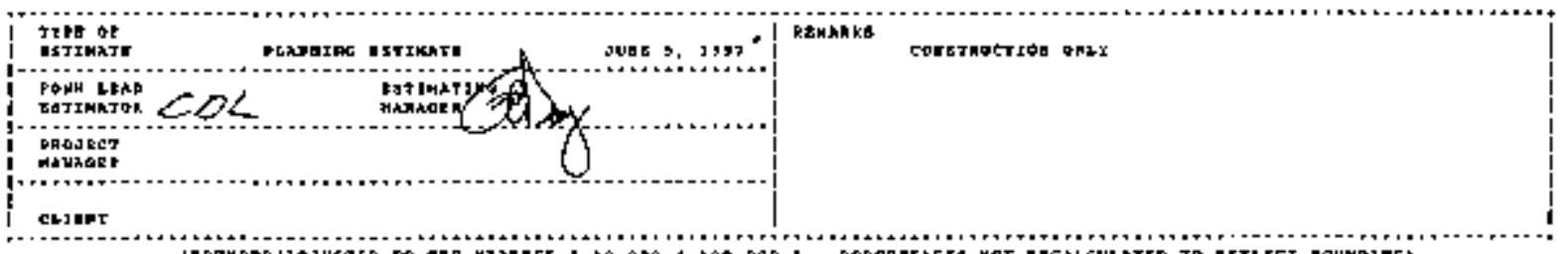

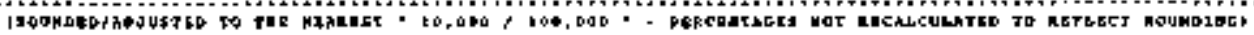




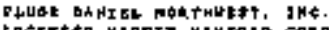

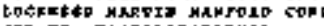
Sol on.

(n)

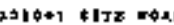

3วI

326043 CEOSYTrTate c t kTE

Jab4t Recess GRTVE Tas.

271046 GEHCWLTE LYPTEM

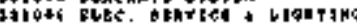

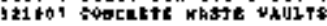

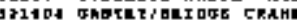

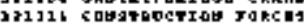

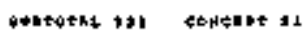

J22rag grturate

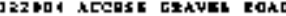

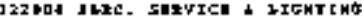

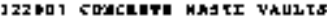

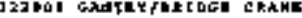

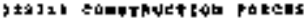

Evgrothl aza conchot to

sabst1 litt moke

$3230+2$ तोKIX LJE[3

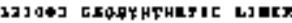

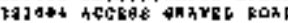

IJ3044 BEATHATE DTEJEM

3230 to ELC. EERVICE L GIOHTIMD

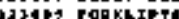

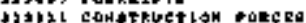

gortatsi aza coperpt a

\section{ABI SנTE MORX}

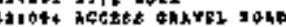

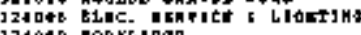

77904 TORKGIFT?

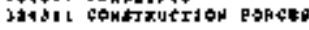

sureral bas corcary

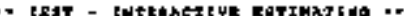

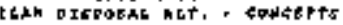

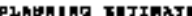

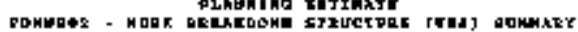

pade is on ho

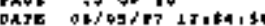

or culichion

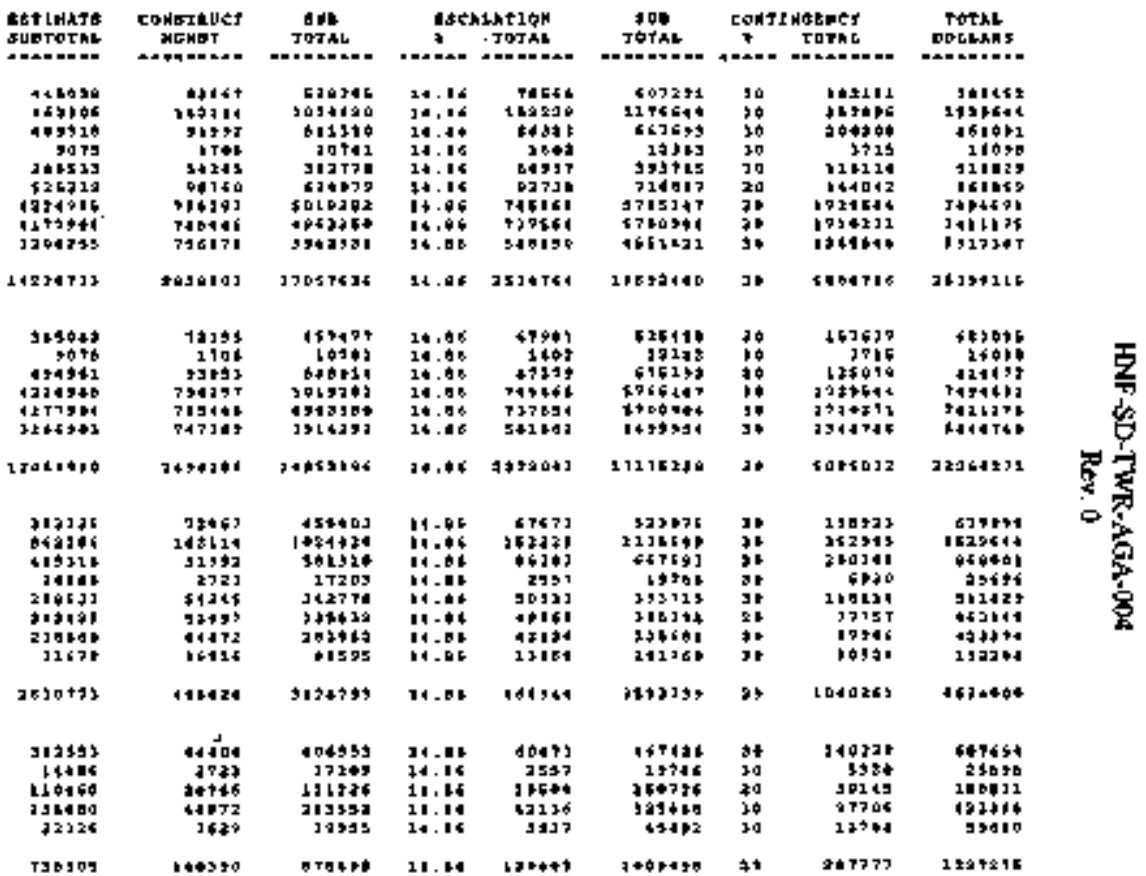




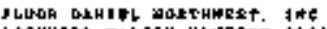
tockHt: matitin HANPonD tohr.

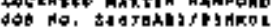

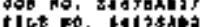

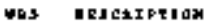

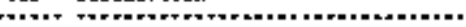

\section{gurgotal az

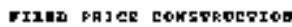

custotat

comatnvos ida

enostte TothL

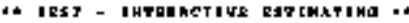

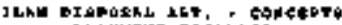

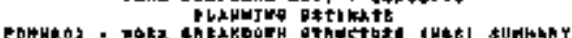

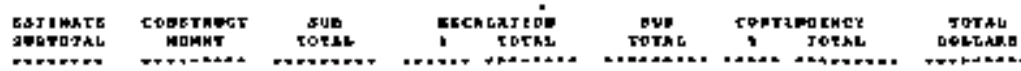

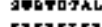

Tothe

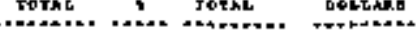

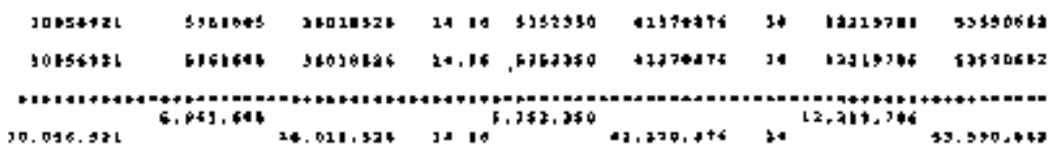




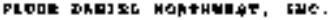

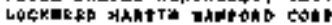

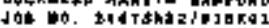

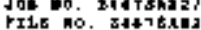

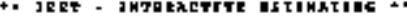

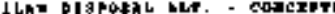

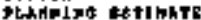

r गूरम
Fad 4 ar Do

DATE 06(45:5t+1):16,12

or

Cotrkinitox

1. Eatimite reppoden

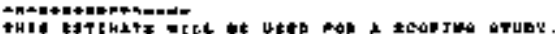

a. Tstrmhtr tecknachl ehsis

1. FHF

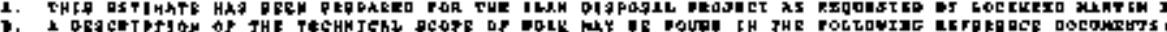

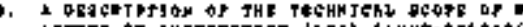

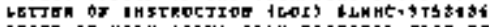

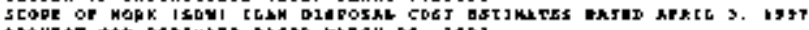

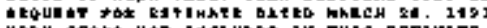

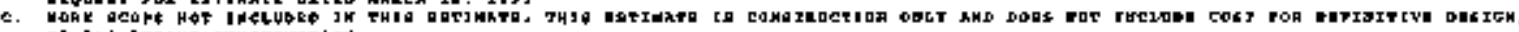

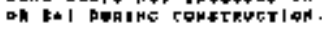

7. SQTTHKT E METEDOOLOOH

h. Q1hEct corva:

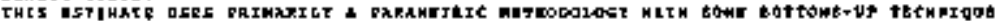

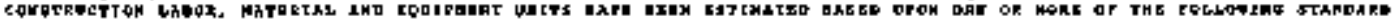

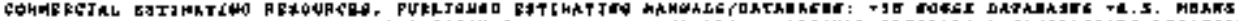

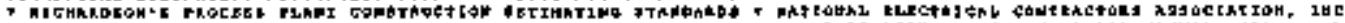

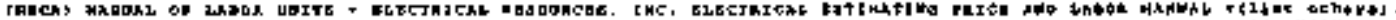

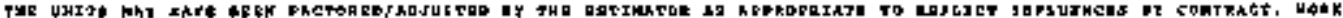

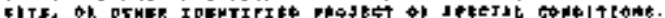

a. DiRxey cose phereng

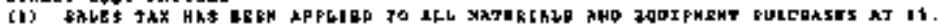

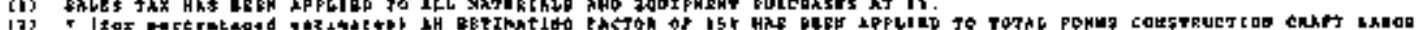

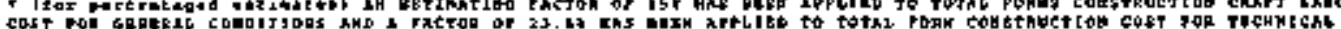

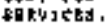

13) CDHS

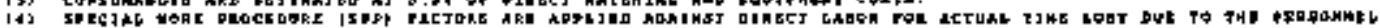

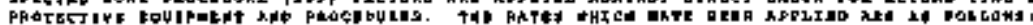

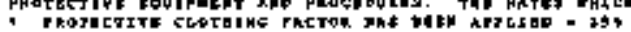

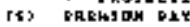

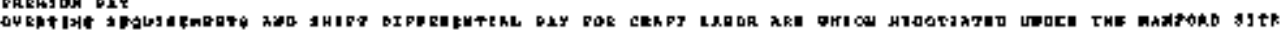

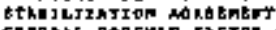

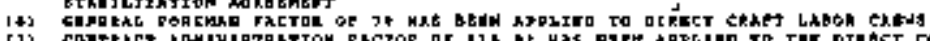

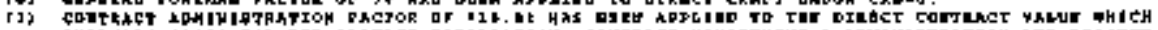

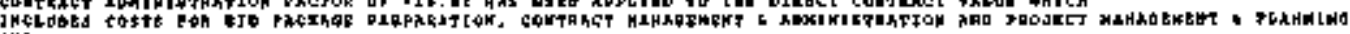
burpate

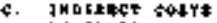

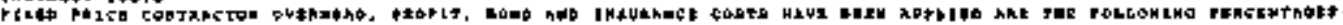

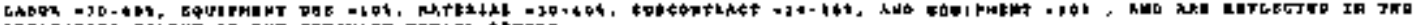

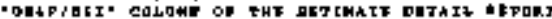




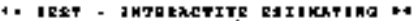

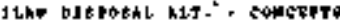

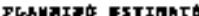

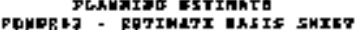

Chan th to

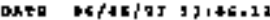

Is

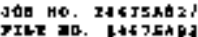

- Ehert

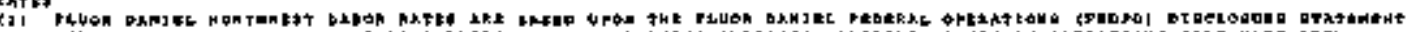
AN

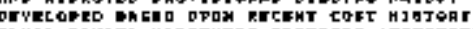

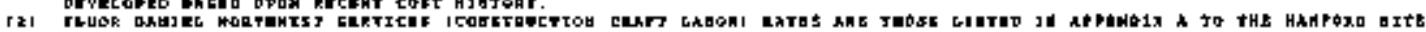

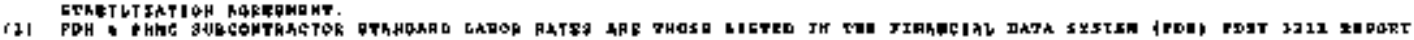

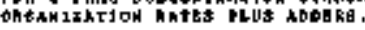

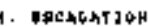

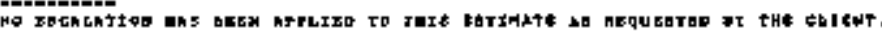

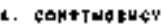

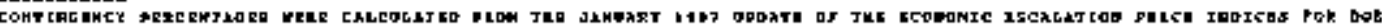

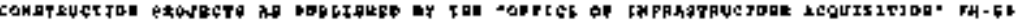

\section{b. Hehdiks}

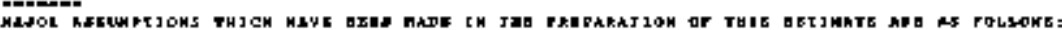

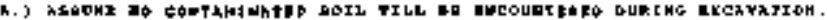

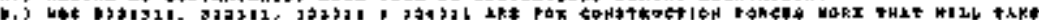

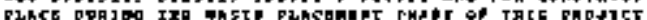

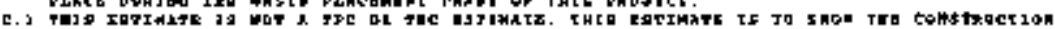

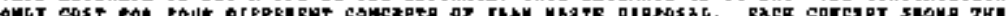

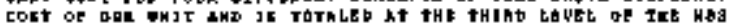

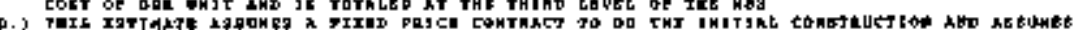

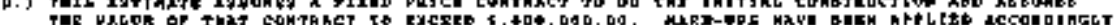

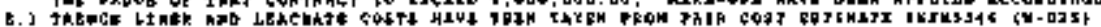

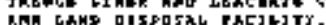




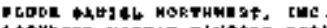

$\operatorname{son} r$

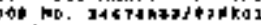

cat I

coderuph DERETPEJOH

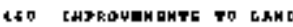

MLLa sto monk

Talob Nagix Linth

כ21BJ GQDOSTTKCTIC GTMER

J8LBA ACCAPA QRAVEL ROAD

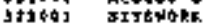

gapod acesat uravas nono

$32340 L$ CITI MORX

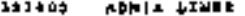

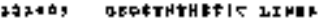

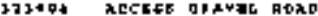

$23410 L$ GITE MORK

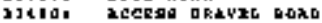

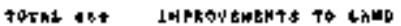

511 nutbajugs

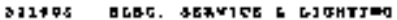

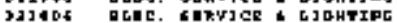

torses sol bullospos

\section{OTHEF ATEUCTUREY}

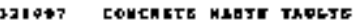

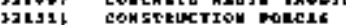

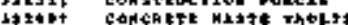

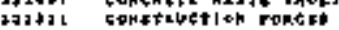

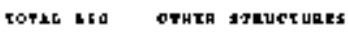

19* DTILIJIEE

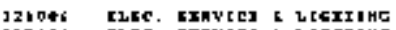

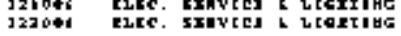

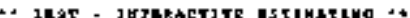

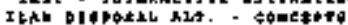

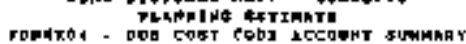

pxar 26 ar $2+$

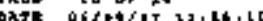

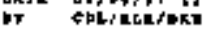

\section{cstrmara} satrarkl

cons reuct m[ats Toral

con

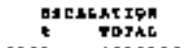
rol contrinteract JoTht $+.+1+1+*$

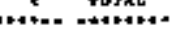
rogat

$+*++\cdots$

\begin{tabular}{|c|c|c|c|c|c|c|c|c|}
\hline+450$] 0$ & $1266]$ & 620705 & 16.84 & J0565 & 6172ב1 & I. & (19219) & $71+15 \%$ \\
\hline 945218 & leàbt & lotstto & 10,01 & 212130 & 1126649 & 70 & $93 a+96$ & 1620811 \\
\hline $\begin{array}{r}0+310 \\
0015 \\
3 \neq 5013\end{array}$ & $\begin{array}{r}+1962 \\
1106 \\
19116\end{array}$ & $\begin{array}{r}841314 \\
50394 \\
557471\end{array}$ & $\begin{array}{l}24.05 \\
24.04 \\
24.016\end{array}$ & $\begin{array}{r}10303 \\
1693 \\
4543\end{array}$ & $\begin{array}{r}012693 \\
92317 \\
635419\end{array}$ & $\begin{array}{l}10 \\
76 \\
70\end{array}$ & $\begin{array}{r}204340 \\
2765 \\
131637\end{array}$ & $\begin{array}{r}06+101 \\
16001 \\
617043\end{array}$ \\
\hline 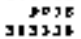 & $\begin{array}{r}j \times 09 \\
221007\end{array}$ & $\begin{array}{r}10712 \\
153403\end{array}$ & ge ed & 1907 & $\begin{array}{l}22343 \\
923076\end{array}$ & ק: & $\begin{array}{r}9715 \\
130323\end{array}$ & \\
\hline 462$\}<4$ & $26251+$ & $7+4+4=20$ & 14.44 & 252259 & 1236640 & 30 & thates & $152+4+4$ \\
\hline $41+114$ & -12182 & 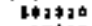 & $14-1$ & A6) & 44107 & $\$ 1$ & 2003010 & $060+01$ \\
\hline 29118 & 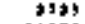 & 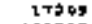 & 14.11 & 3469 & $19+44$ & *⿻ & 7090 & 24491 \\
\hline $\begin{array}{r}34553 \\
24116\end{array}$ & 1410t & $\begin{array}{r}106783 \\
17201\end{array}$ & $14-106$ & $\begin{array}{r}0473 \\
2553\end{array}$ & 467138 & 11 & 140230 & 607154 \\
\hline & & & & & & & & \\
\hline 1706219 & 044521 & $3 a b b+4$ & 16.61 & 760975 & 5096217 & 70 & 2769265 & $36310+1$ \\
\hline
\end{tabular}

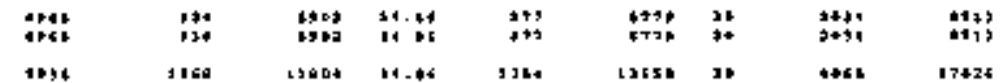


ThUSW DAHSEL DORTHнFs, $1 \pi \mathrm{C}$.

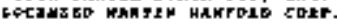

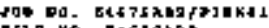

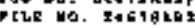

tost

$\cos 1 r u \neq 0$ Deser ibriah

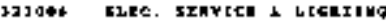

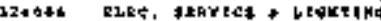

Jorke 1at OTIErita

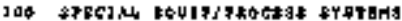

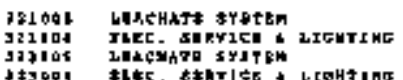

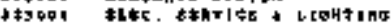

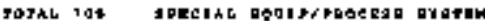

gu Iaxye hoblla soujputat

\section{GAETEY/ORIOGE CRAME

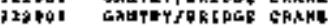

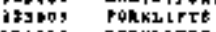

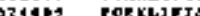

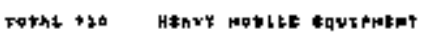

\section{Ho enmalition}

\begin{tabular}{|c|c|}
\hline 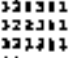 & 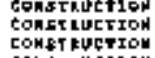 \\
\hline & \\
\hline
\end{tabular}

visit controustion rokck

JOJAL WD DaHOLIJIOA

PAONFGT TaThl

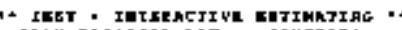

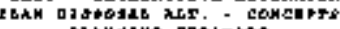

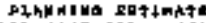

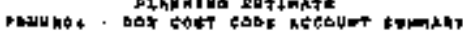

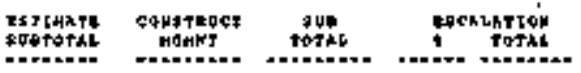

$2420<7$
$210+10$

26164

$14.16 \quad 14341$

Iofthe

ThE THELACY

erap 3 or it

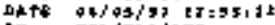

at Golixhkitoxa

135259

254263

LISTII

14.462345

i4jof:

14454020

3014

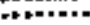

200325

25110

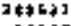

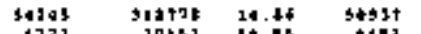

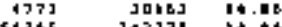

5425

247371

$2+1,10$

topa

$600+1$

intis

atiris

x+rin

1.:

210

+1619

24.16

$22003 t$

$12+10$

ง*

5 망

11114

4929

75*64*

Iinto

aisid

1301406

411704

13+1441

ân:1:

\section{restes \\ $+15354$}

+36455
$4475 ?$

16.05 T27154

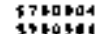

37506:

?4:

103421

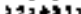

$\rightarrow 718$

14.08 Halsi

12160

19

14: เมे

T41675

12931"

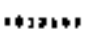

$31+*+2 *$

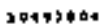

14.01 3aght

1001210

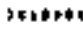

13⿻1917\%

\begin{tabular}{|c|c|c|c|c|c|c|c|c|}
\hline $\begin{array}{r}510172 \\
110111 \\
39671 \\
22126\end{array}$ & $\begin{array}{r}125129 \\
125013 \\
96016 \\
7671\end{array}$ & $\begin{array}{r}453231 \\
604652 \\
11505 \\
11965\end{array}$ & $\begin{array}{l}11.54 \\
14.24 \\
11.06 \\
11.06\end{array}$ & 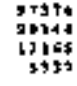 & $\begin{array}{r}757617 \\
61+404 \\
141714 \\
43 \$ 98\end{array}$ & $\begin{array}{l}30 \\
30 \\
20 \\
30\end{array}$ & $\begin{array}{r}23540 t \\
249046 \\
70601 \\
10964\end{array}$ & $\begin{array}{r}975417 \\
945044 \\
129714 \\
59644\end{array}$ \\
\hline $1,24+93$ & 2454916 & 2290432 & 14.16 & 204626 & 1597114 & Jo & 473136 & 2071215 \\
\hline
\end{tabular}

2454,16

2320433

14.16205626

159716930

173136

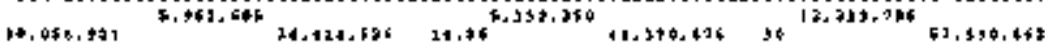




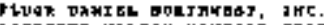

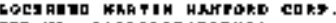

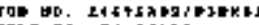

5xi axerjested

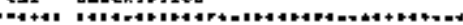

copsrkocsich

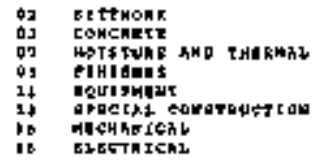

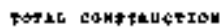

troutctitat
W IEET - IFTERSCTIVE EHTIMATIJO +o

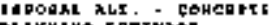

rent op 10

QY COF

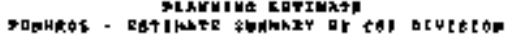

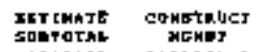

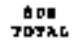

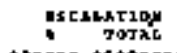

$\cos 4$

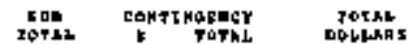
ata+o+*

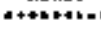
a.not+*

$++-\infty+4+\infty+\infty)$

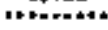

-on Tóphl
DQhesh 5

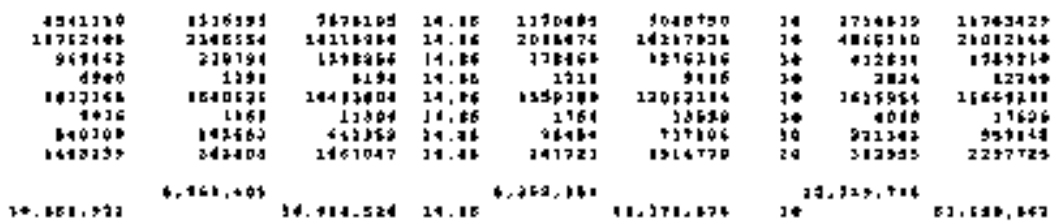

H, LEI, *23

$5.912,605$

34, 484, 131

$36,01+526$ J4.1

35,354

$11,3] 4,172$

10

12, $219, \mathrm{~T} 4$ 


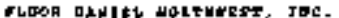

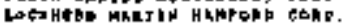

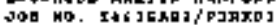

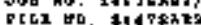

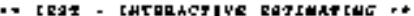

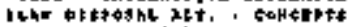

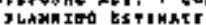

$0100+24$

$13,01,24$

(r) colikekioxh

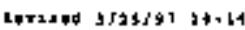

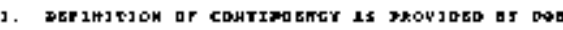

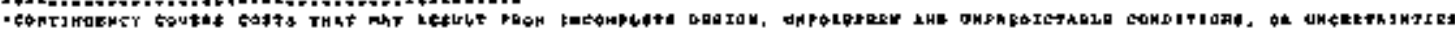

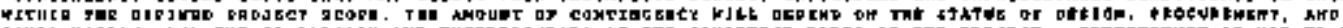

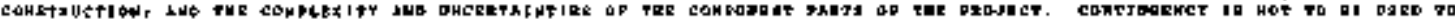

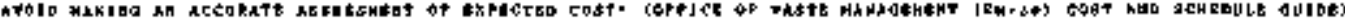

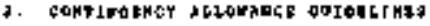

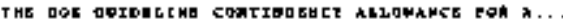

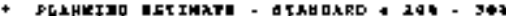

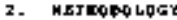

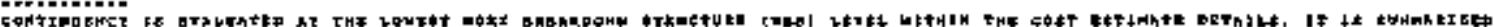

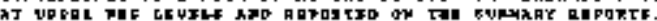

a. Merratis

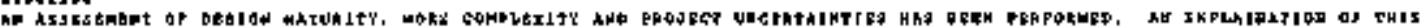

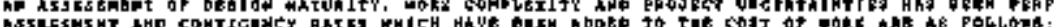

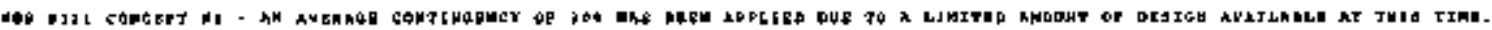

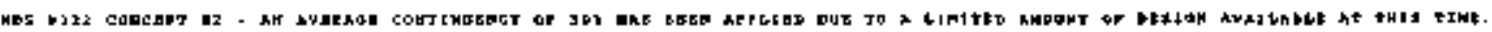

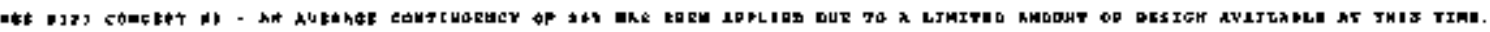

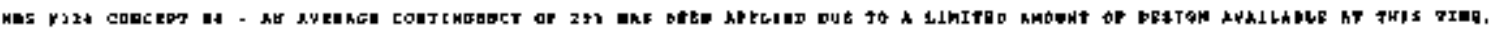




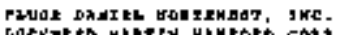

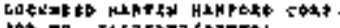

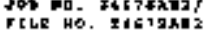

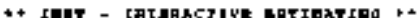

IGLU Q t*

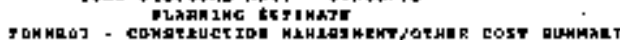

BADE $1+$ or 10

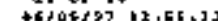

or coutkLkSBrH

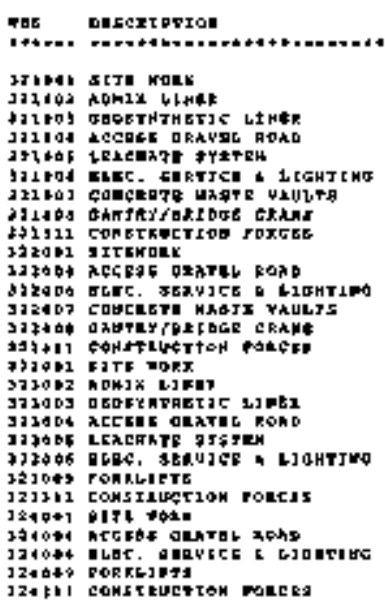

rmoject Tothl

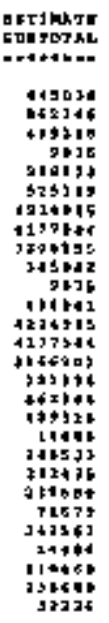

\subsection{4}

74.10

14, 9

14.00

11.10

14.10

11.10

13.00

14.60

19. 19

13.00

14.00

14.00

$2+-60$

14.60

10.0

10.09

10.00

10. 0

90.90

$1 \%, \$ 1$

23.6t

$17-4$

14.75

1).

$140 T$
26114

ज्ञात

1245

7.764

711201

thesed6

tosad

7 asy

jos

7706

93067

T94य)

restida

tido

10

103424

aty

54243

एव

14012

143 a

460

2+17

$x+1$,

IE
日8 Trod

bIt

162t14

120

$5+141$

96160

714127

716044

+4 $8+4+4$

ग219

7219

1706
$13+54$

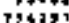

715.05

T)

गयS:

पมs

12104

tost

5)

914

14.

1413

64640

2727

बकाष

$+62$ 


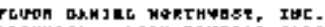

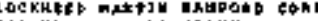

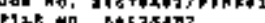

PIE 40. BQ6 75AS2

kecaura

Mบผx! PHPCFITT LOF

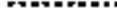

$221 * 0 \mathrm{n}$ \$Fra molrk aITamokr

9.201.42

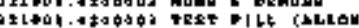

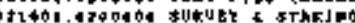

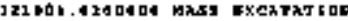

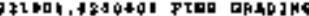

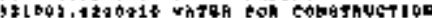

\begin{abstract}
gontajac EJTERER
\end{abstract}
Totat

50\%t 5005 1400

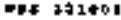

AUSCRLATIOM

14.164, EOHJIMGERT
- inst intaknstive eajchatina -

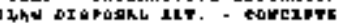

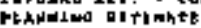

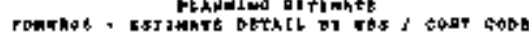

$\cos x$ bouts

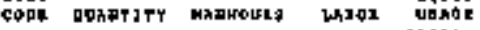

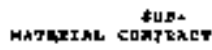

$\ln 4 \mathrm{P}>\mathrm{s}$

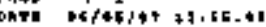

COL RKCHCOKT

Eoust. ake jotal

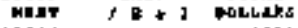

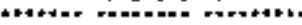

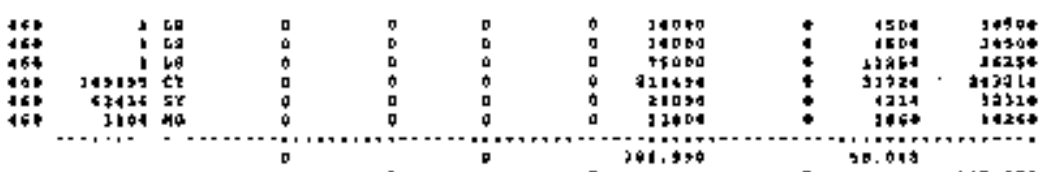

$+49+3 a$

40,43

904,930

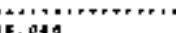




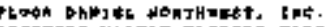

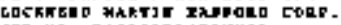

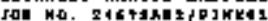

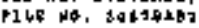

iccous unkere DHESRETLOH

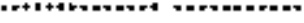

נa $1+0$ AOMEX MIER

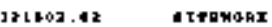

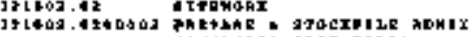

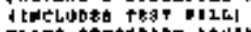

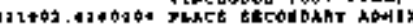

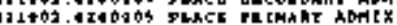

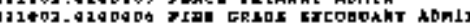

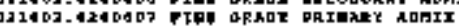

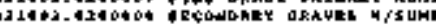

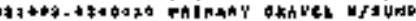

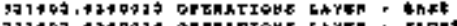

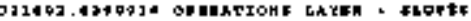
c geTJARL TIIL

SUEIOTRL CTTENOAX

rotst

$\cos \cos 14002$

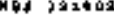
torchintiall

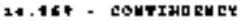

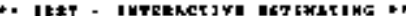

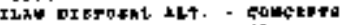

PLxmerec ectumite

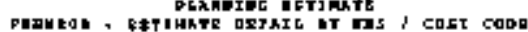

erag 2

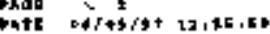

Cocikcatoke

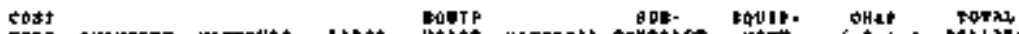

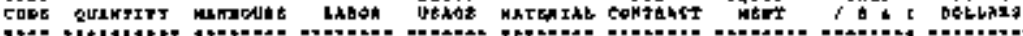

\begin{tabular}{|c|c|c|c|c|c|c|c|c|c|c|}
\hline 444 & סינ219 & cy & 0 & - & 0 & 0 & 939415 & 0 & D*to & $B \in 5+20$ \\
\hline+64 & $\begin{array}{r}29,96 \\
9438\end{array}$ & $\begin{array}{l}\text { cy } \\
\text { sy }\end{array}$ & D & : & a & : & $\begin{array}{r}17316 \\
72100\end{array}$ & a & $\begin{array}{r}13690 \\
1019\end{array}$ & $\begin{array}{r}19462 \\
01772\end{array}$ \\
\hline 140 & 163014 & $s$ & $\dot{\phi}$ & - & 9 & 0 & $3+376$ & 0 & 311 & $24+10$ \\
\hline 484 & סנדננו & se & 0 & - & 1 & 0 & 6463 & 0 & 249 & 5024 \\
\hline 444 & 2114 & $\mathrm{cy}$ & 0 & 4 & 0 & 0 & 24946 & 0 & 2012 & 21784 \\
\hline+44 & $2+42$ & CY & a & - & 0 & a & 23316 & a & 2312 & agrad \\
\hline 414 & $1 \neq 0$ & $\% \gamma$ & 0 & b & 0 & a & $3 \leq 0<0$ & $a$ & 2010 & 1704 \\
\hline 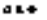 & 9710 & tr & $\phi$ & a & a & 6 & I $15 * 0$ & a & 1976 & 55795 \\
\hline
\end{tabular}




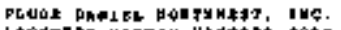

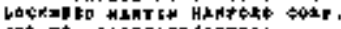

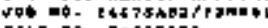

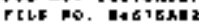

scconnt

GuMnR

CFachid dion

$2210 \div 3$

DEOETHTETLE LIMIN

$3260+3-40$ 112 axane

J

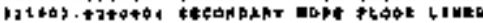

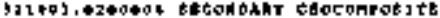

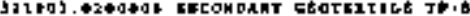

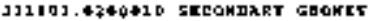

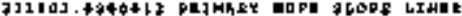

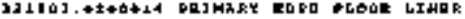

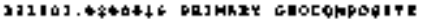

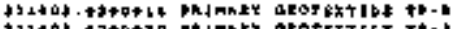

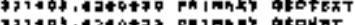

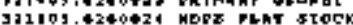

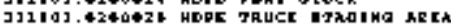

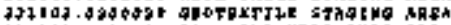

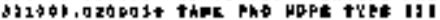

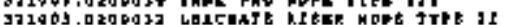

sug TOTAG SITEDORK

tathl cas cose thas

ubs gaboj

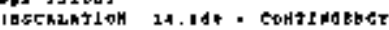

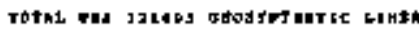

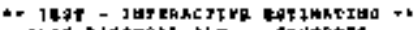

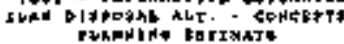

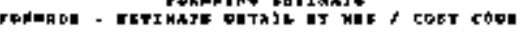

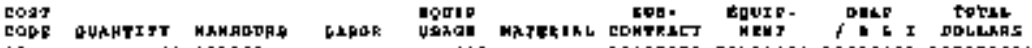

\begin{tabular}{|c|c|c|c|c|c|c|c|c|c|c|}
\hline 160 & $1260+2$ & ap & 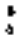 & $a$ & : & 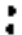 & $\begin{array}{l}62131 \\
12741\end{array}$ & : & $\begin{array}{l}1348 \\
1158\end{array}$ & $\begin{array}{l}21200 \\
1+1900\end{array}$ \\
\hline 160 & 123134 & t & $a$ & 6 & 0 & 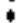 & 10:314 & 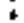 & $15)+1$ & $13+349$ \\
\hline 150 & 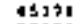 & A $P$ & 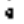 & 0 & 6 & : & t556! & . & ניy & 22161 \\
\hline Iat & 25630 & $t r$ & 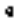 & 0 & 0 & a & 1601 & I & ונד 12 & 9 \\
\hline 161 & 120502 & 53 & a & D & 0, & a & ב4+404 & - & 0157 & נםידו \\
\hline 164 & d1Jo6 & व & $a$ & 0 & $a^{\prime}$ & 4 & ISOTA & . & $2 a 61$ & 17329 \\
\hline 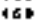 & 210101 & s & 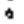 & D & $a$ & 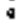 & כנסטסט & : & 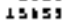 & 116170 \\
\hline 160 & tolst & al & $a$ & o & a & $a$ & 12174 & . & 1126 & $2+100$ \\
\hline if? & 30100 & $\bullet$ & $a$ & a & a & $a$ & gart & - & 2163 & 1263 \\
\hline 161 & צופול & tr & $\theta$ & $\phi$ & a & a & 1)15t & - & $76+1$ & 37472 \\
\hline 100 & & $t r$ & $\theta$ & 0 & 0 & 4 & 344 & - & 4 & 44 \\
\hline 100 & L & Is & 4 & n & a & 4 & 1931 & . & 190 & 6021 \\
\hline 46 & 10+5j & s & 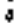 & 0 & a & a & גנםנ & 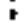 & 451 & 3461 \\
\hline 168 & $\$ 10$ & gip & 6 & . & 0 & 0 & 1306 & s & 702 & isits \\
\hline 144 & 10 & to & 0 & + & $\dot{\phi}$ & $\phi$ & $8+0$ & 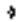 & 26 & $3+6$ \\
\hline
\end{tabular}

$125,4+2$

43,02

At: 121

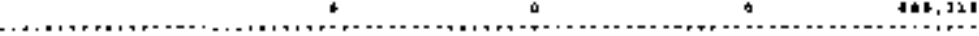

$431,4+2$

43.73

441, a14

94. $19+1$ 
PLUEh DHNTEL WOAJHLENY, JKe.

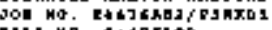

FILS Ua. EtTrkes

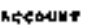

Herit $k$ h

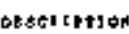

$1214 b 4$

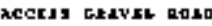

$021004,0 \mathrm{z}$ Butatokx

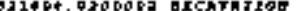

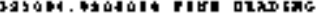

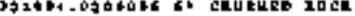

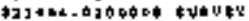

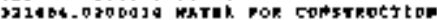

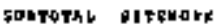

rosar

çopt coph 16012

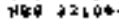

Nit

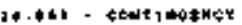

$30.40 \div 1$

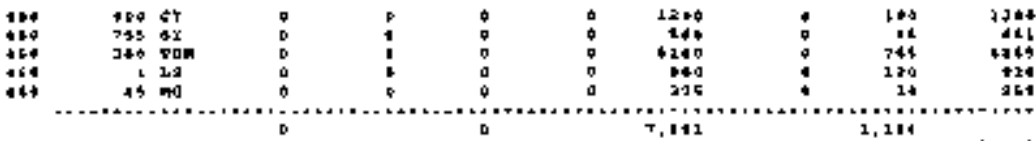

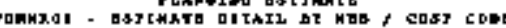

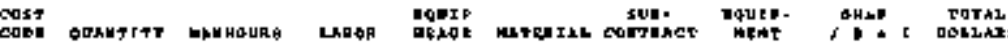

urat $>$

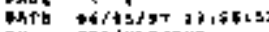

ar colikinition - -

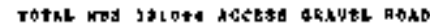

(1)

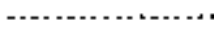

:

1,291

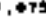

1,194

1,144

1, A TS 
PLWOF DAXIEL MOLTENEAT, IHC,

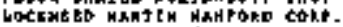

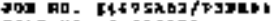
PILE MO. Z1615kE?

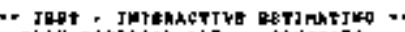

I LAN ditrotat nLI. TONCERTs

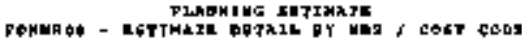

proz is 3

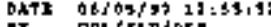

COt?

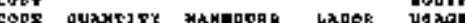

t): CaLf rLr, oKn

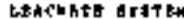

3200409 cortetion

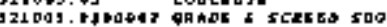

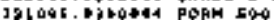

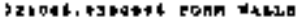

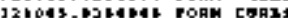

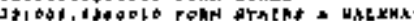

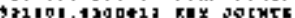

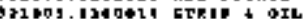

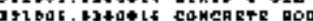

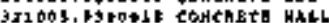

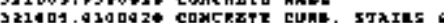
WhIMPY

121005,131002 t tuhine

givosing

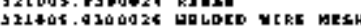

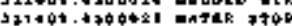

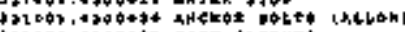

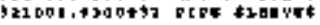

joldos.ajadast Trakri pIaIsk

\section{sutrofdi ctakktt}

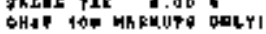

ratse

$\cos \cos 70403$

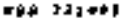

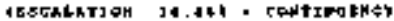

F|at\&Et*

$\$ 21464.0 \%$

coscros ctontima top

ARLCK

32300.9300002

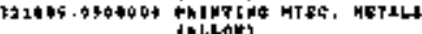

irt

\begin{tabular}{|c|c|c|c|}
\hline $\begin{array}{l}\text { JaD } \\
\text { JaD }\end{array}$ & $\begin{array}{l}\text { AE, } \\
\text { IT }\end{array}$ & $\begin{array}{l}\mathbf{E Y} \\
\mathrm{EY}\end{array}$ & 1' \\
\hline+90 & ו1י & \& & 1 \\
\hline $\mathrm{PaO}$ & 4 & GT & I \\
\hline 900 & 4 & 98 & 25 \\
\hline (0) & 21 & Gr & J \\
\hline 700 & 1201 & EF & 24 \\
\hline סם נ & נ & cT & 14 \\
\hline 900 & I & CT & 9 \\
\hline 704 & 4 & cr & $\bullet$ \\
\hline
\end{tabular}

SEgTathe DuIshe

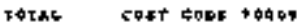

cot
$1 \cdot$

169

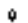
$\downarrow$

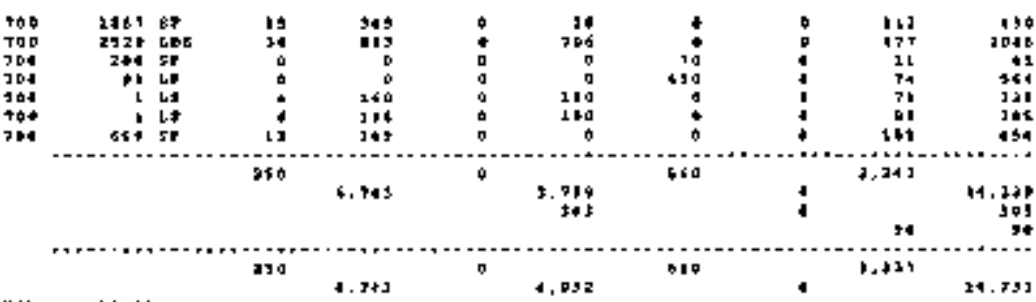

D o tsoto

1690

, n....

4225

.450

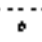

เ

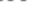

1,00

is




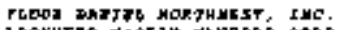

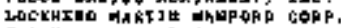

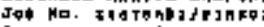

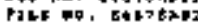

Accoum 1

ก๊น⿰ घค Detckiptom

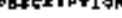

KESHRMICAL

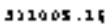
MECHXMTChL

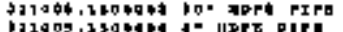

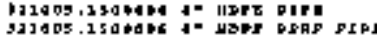

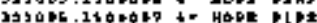

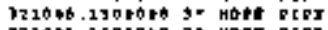

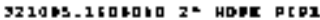

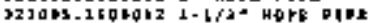

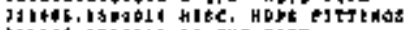
jives 1500015 .

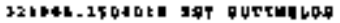

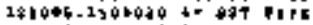

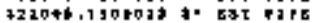

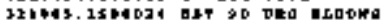

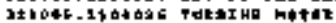

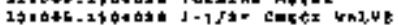

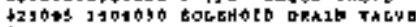

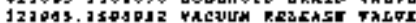

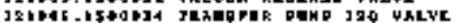

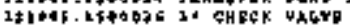

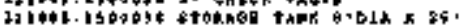

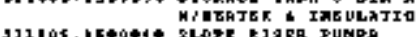
g 7108 B

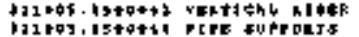

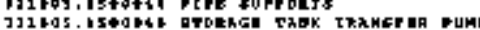
JjLG I J

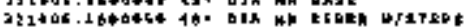
Ij1 115 ,

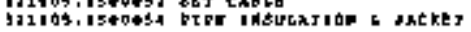

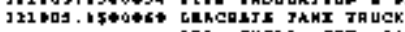

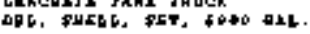

JUETDTAL MUTHABCAC

Tarke

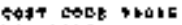

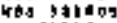

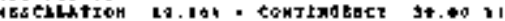

10.001

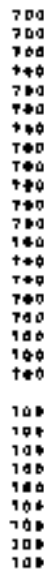

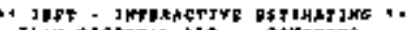

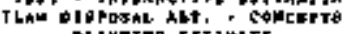

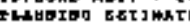

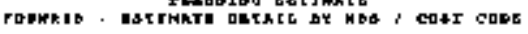

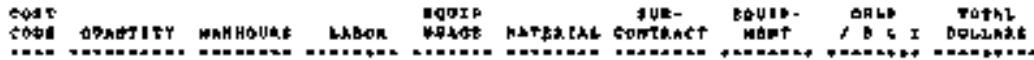

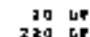

220
$1>10$

$20+6$ ir

$1102 \mathrm{r}$

120 L

120 Le

10 to

130 th

$130 \mathrm{Lr}$

90 1 1\%

101

口 2r

as

2 to

1 tक

1
3

i 18

2 Ek

3 r1

! 12

14

18

20

10. Lo

10 6t

:

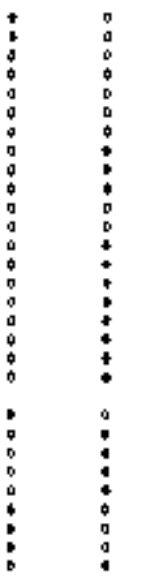

\begin{tabular}{|c|c|}
\hline & 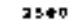 \\
\hline & 3340 \\
\hline & 20,60 \\
\hline & 8000 \\
\hline & 1230 \\
\hline & 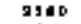 \\
\hline & $1+40$ \\
\hline & 135\% \\
\hline & 2100 \\
\hline & 1306 \\
\hline & 1359 \\
\hline & 140 \\
\hline & 10 \\
\hline & 216. \\
\hline & 100 \\
\hline & 273. \\
\hline & 45 \\
\hline & 2154 \\
\hline & 604 \\
\hline & 20404 \\
\hline & $4 \$ 04$ \\
\hline & 501 \\
\hline & 3601 \\
\hline & 204 \\
\hline & 1564 \\
\hline & 304 \\
\hline & $2+10404$ \\
\hline
\end{tabular}

:

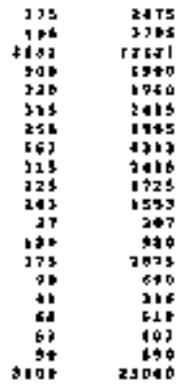

要

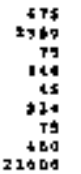

4171

$1+640$

570

$3+1$

194

$x+1$

JAS
$161 \mathrm{BaD}$

235,045

93,243

2rt. 35

J13, ot क

aro. 950

索 


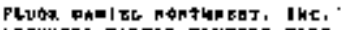

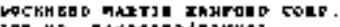

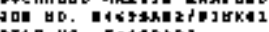

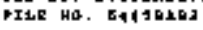

atconot

unean

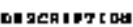

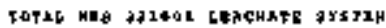

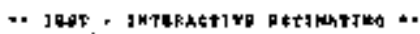

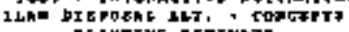

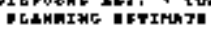

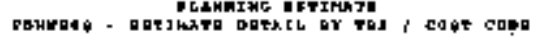

PNAT 1

Dht bithigt 1 a: is:at

ar colruchion

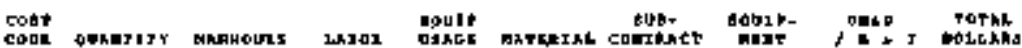

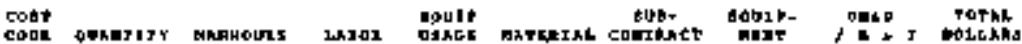

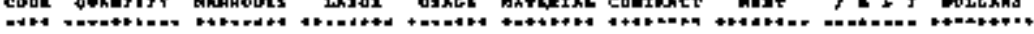

250

$1,+47$

1,023

as, HE

J1, 41.

נג, 21, 


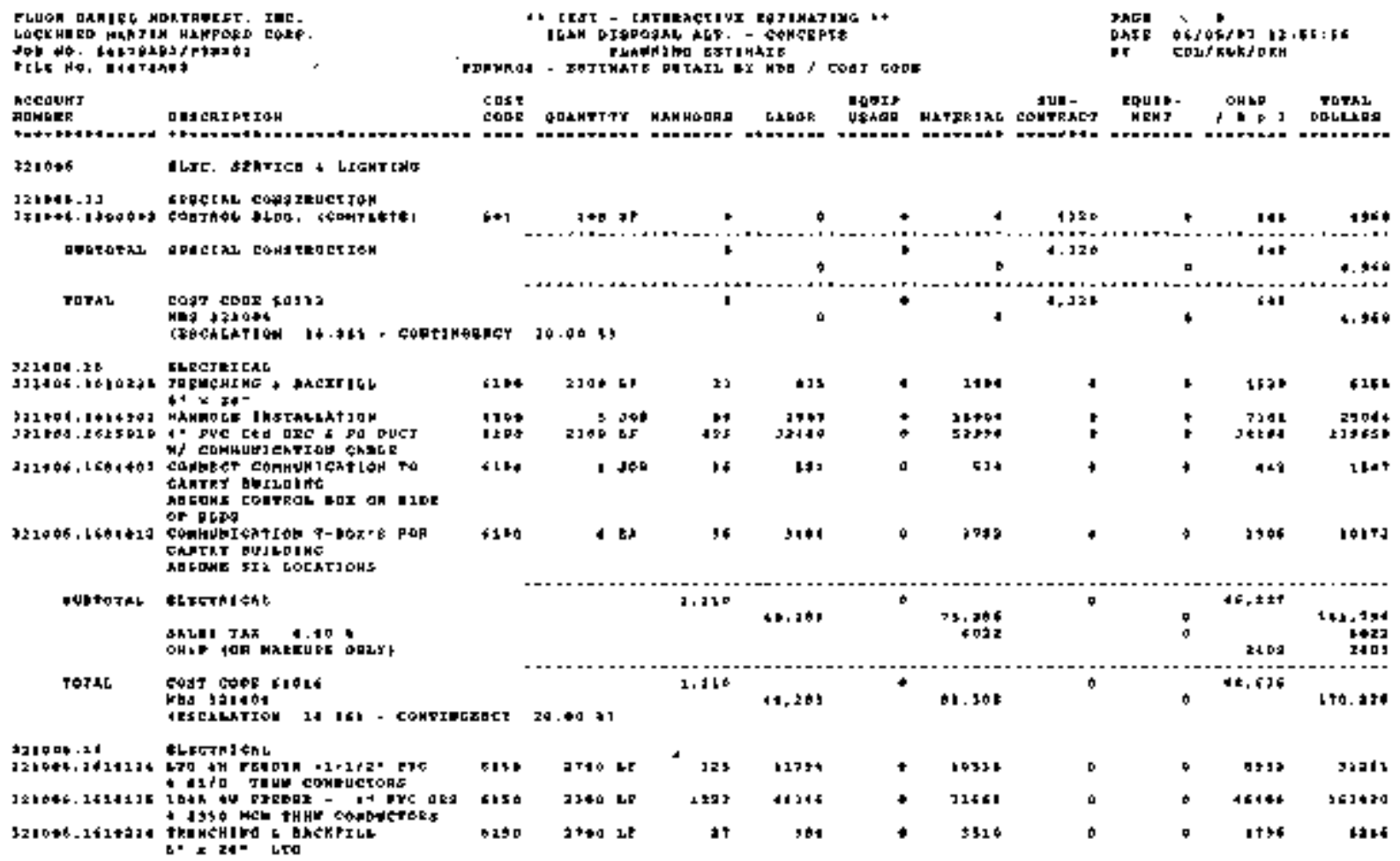




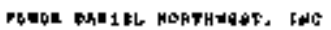
HockH:ll maxtiN Hagrohb cokr

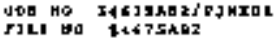

hockerter

kctole

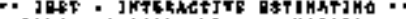

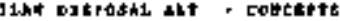

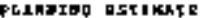

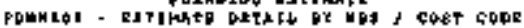

Pagen

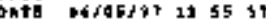

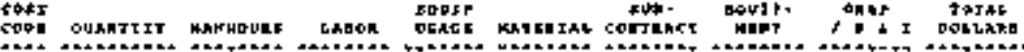

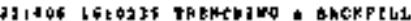

4. $x$ il:

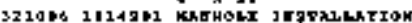

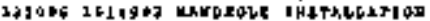

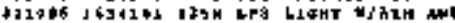

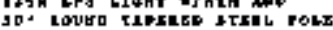

gareste

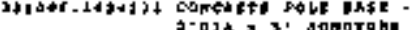

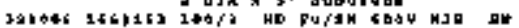

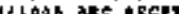

$\operatorname{ting}$ tot

Ist

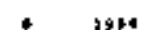

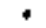

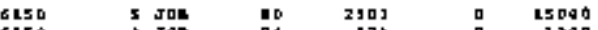

6150 10 590 in

4180

20420

18 $\leqslant$ th

[t5 [ ]

6 Lh

1742

$1+3 x$

650

conact ajor powlo

sisb

$1 \mathrm{stot}$

254

5020

146

ASEOHE COHTHOL BOx OH BIOE at abon

coutazhl

\section{mectrtchs}

Davet Thy in of

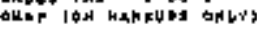

IDTa

\section{COET CODE 61320}

an $3 \geq 1 \% 0$

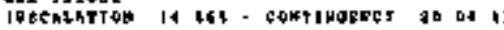

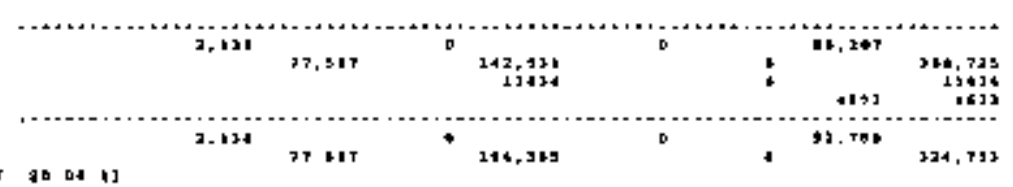

221104 10

ELECTEICA

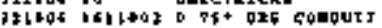

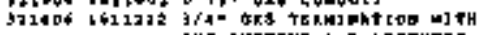

IMS BUERTME I LOCXMOSE

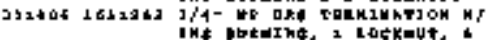

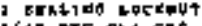

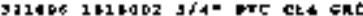

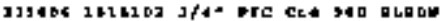

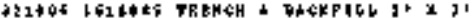

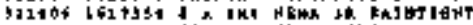

stRe coven e netros une

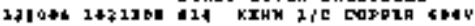

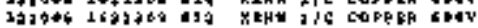

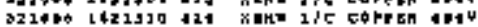

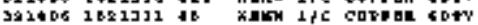

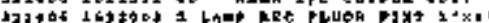

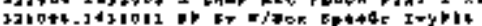

\begin{tabular}{|c|c|c|c|c|}
\hline $\begin{array}{l}2056 \\
2060\end{array}$ & $\begin{array}{l}90 \\
13\end{array}$ & $\begin{array}{l}6 T \\
\text { E. }\end{array}$ & 1 & $\begin{array}{l}145 \\
210\end{array}$ \\
\hline & 16 & 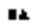 & 4 & $1+3$ \\
\hline$\lambda * 0$ & 640 & $2 \%$ & 24 & נו \\
\hline 7060 & 12 & $s \lambda$ & 10 & 363 \\
\hline 7060 & is & $6 \%$ & 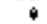 & 200 \\
\hline 1060 & 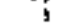 & E* & i & I \\
\hline & & 1. & 2 & \\
\hline 7060 & 1240 & 60 & 4 & ו1 \\
\hline 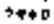 & 10 & LF & $\phi$ & + \\
\hline 7040 & $1 * 0$ & $2 F$ & 1 & ו \\
\hline 9060 & 1 & $\mathbf{k \lambda}$ & 1 & 26 \\
\hline$+* 60$ & 1 & Th & 9 & 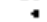 \\
\hline
\end{tabular}

31
16
24
$6+4$
119
19
19
10
64

$\bullet$
0
0
0
0
0
0




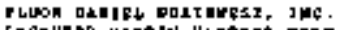

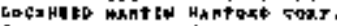

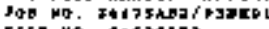

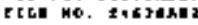

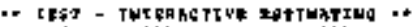

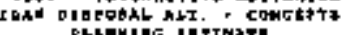

SLRENIMG IUTINATI

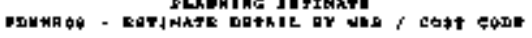

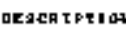

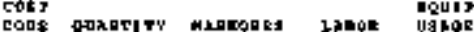

manic:

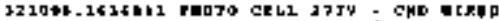

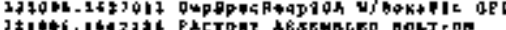

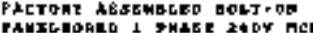
140 - 10

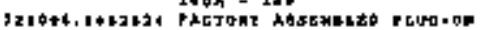

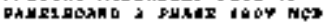
1915 - job

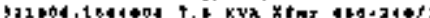

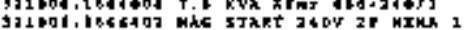

$$
\text { ctic } 0 \text { ahp }
$$

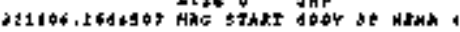
sTin

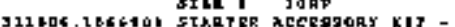

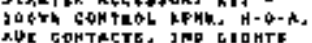

MUE GOHTACTE. JND CIOHTE

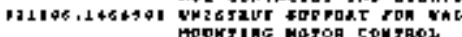

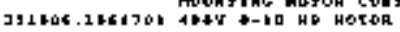

combatiand

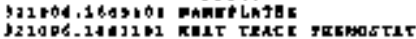

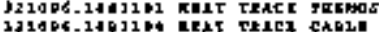

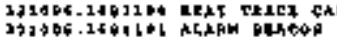

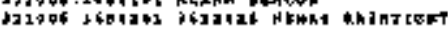
Enclosure

381004-14h212 1\%, CUT K.4.

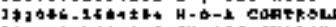

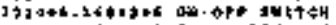

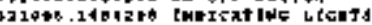

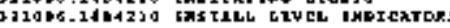

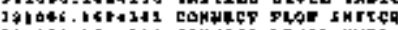

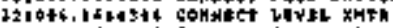

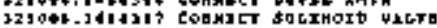

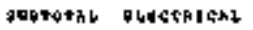

Bactat Thx b. Dt

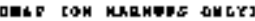

TOR 1

$\cos 6 \cos 2+616$

Iht 921 to

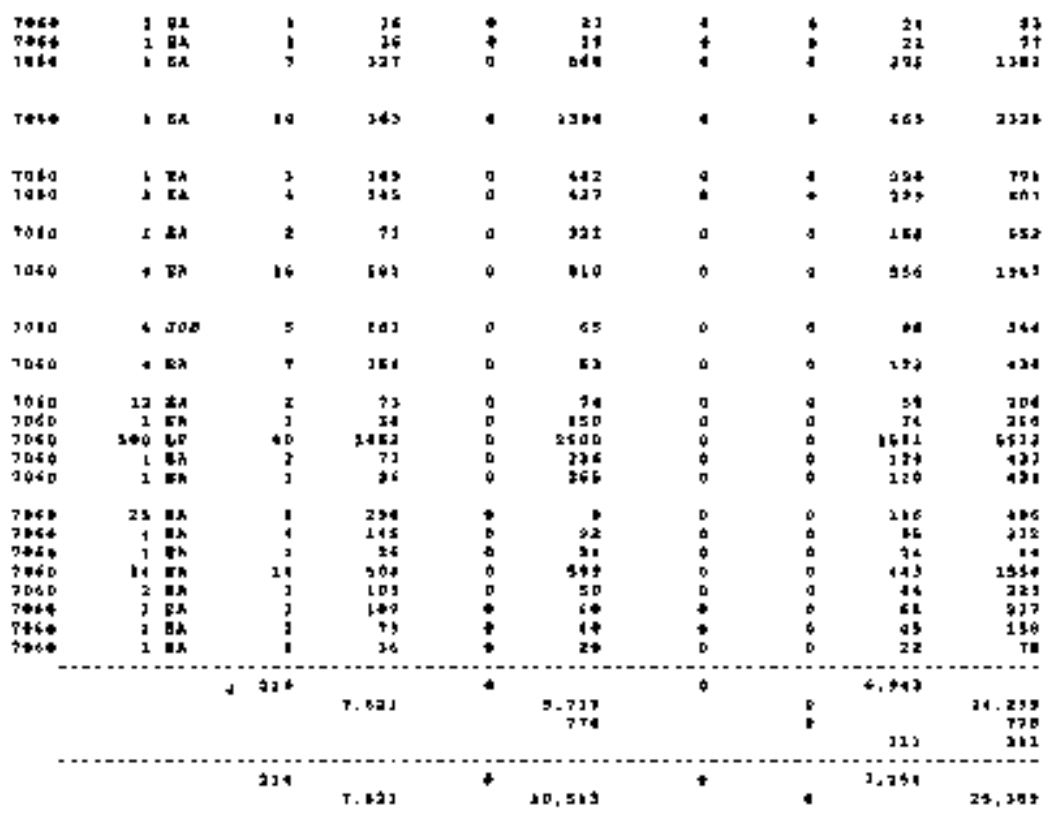

phas $>1$ to

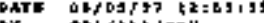
COU/RLK/DX. 
PLOOH ANHEL MOATHHFET. IHE.

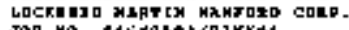

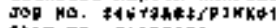

PLE GO. EQTFAOS

\section{nęcoumt}

Whatr.

bttenjuriton

ieschlagtar

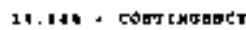

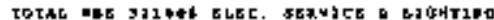

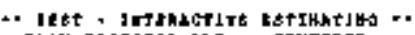

TLAY a 1SDOELC NuT. - caxcapss

MLNMFNG at ThATE

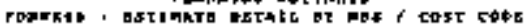

toulo

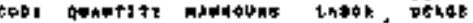

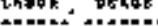

motektur sodax-

makint southitet

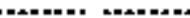

20.0411

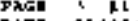

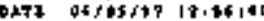

or tor frentoris

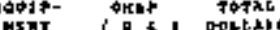

wswo

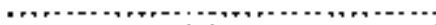

$1 .+30$

เม9,14

364,304
$+, 1 \geq 0$

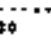

(1)

o
19,313

149 12

321,924 


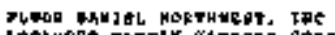

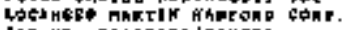

SOS HO IALJERA/FIMKO

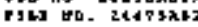

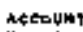

Howstin

betserarit on

ว่า1ด

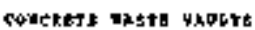

921007.03

Capcket $x$

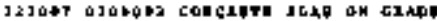

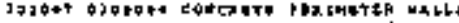

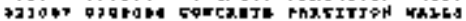

godrotal cagexira

terkl solf cobt buth

Hat 3 alot?

16 AB) - cowtracxucr

$3 \bullet . \bullet 0,1$

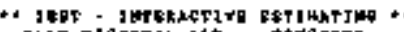

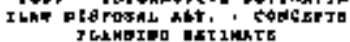

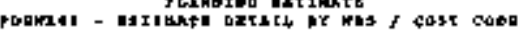

Daca $>12$

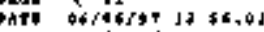

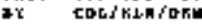

mong .

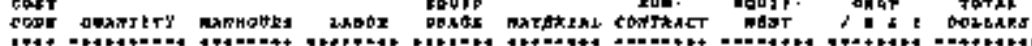

ond 4 t

Tat Thes

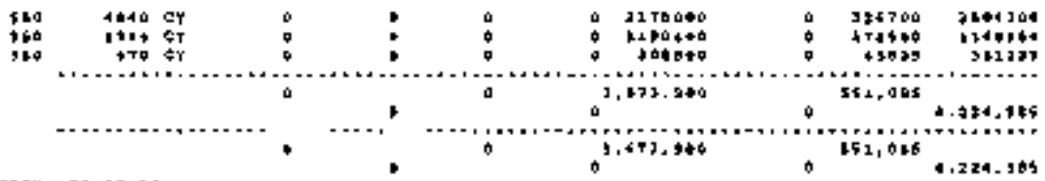

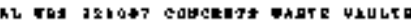

(2....................

-

เ

a

.671 .040

EE 2, tht

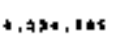




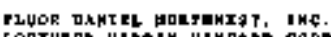

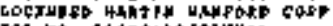

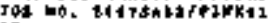

PTLE HO. ZALIERB?

Кесавнт

HบMㅁำ

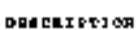

aver.

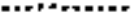

ARMTFY

191046

toptranant

$1210+6.11$

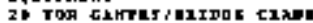

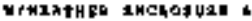

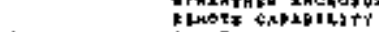

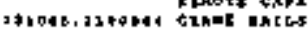

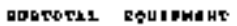

$\tan 26$

copt eq0\% 7143

Ho 11 th

195hla 140

LA, 46t - GonT [MOEHGS

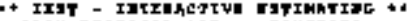

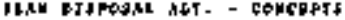

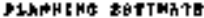

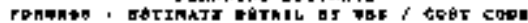

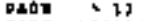

DAFE 66/61/27 t?:36,02

DY cblicteingm

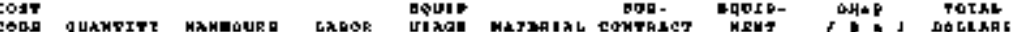
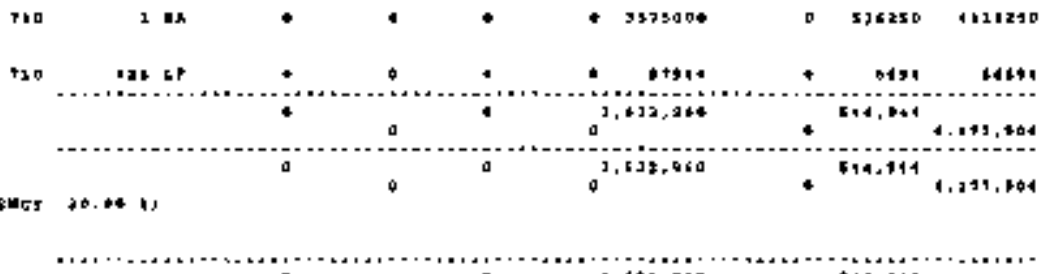

$3,1+3,10$

$\$ 44.14$ 


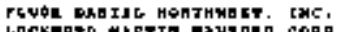

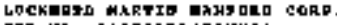

QRidirke1

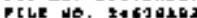

recondt

HWABth

betchrotint

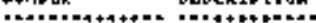

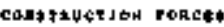

$3 a+1$

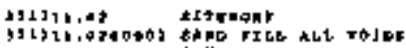
1 rautis

SUITOTKL SIJEMORK

collotrante 7,204

30129104

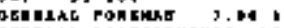

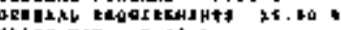

HhLEF TAX 0,06 ,

ONE Mar makeupy ater

tothl

$405 t$ cobk bi+os Hot 92131 L

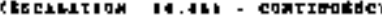

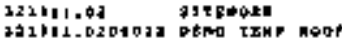

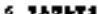

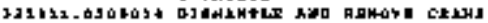

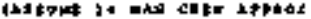

3.

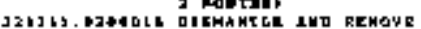

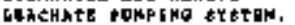

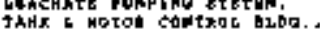

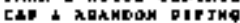

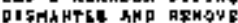

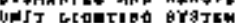

gobtothl artugh

COMS QMNDER 3.24 t

gho $\$ 1.046$

OtWERSL TORENAN T.00

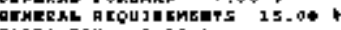

DAFEs ThX P. D4

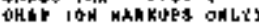

70Tत4

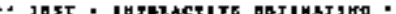

ILAD OIDPOEAL HT. - coucrppo PCKM 100 U⿴囗十 IMATE

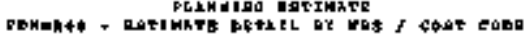

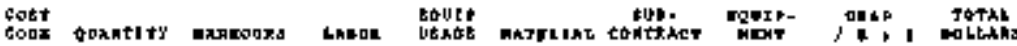
OH S

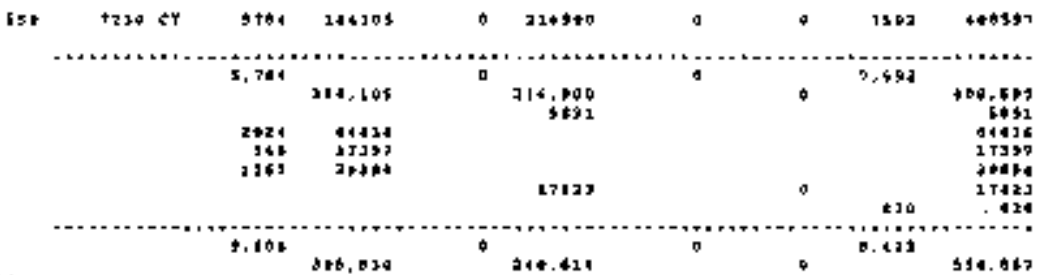

$20.40 \%$

bxal $04,1+4,9,17,66,12$

cr trictiolon

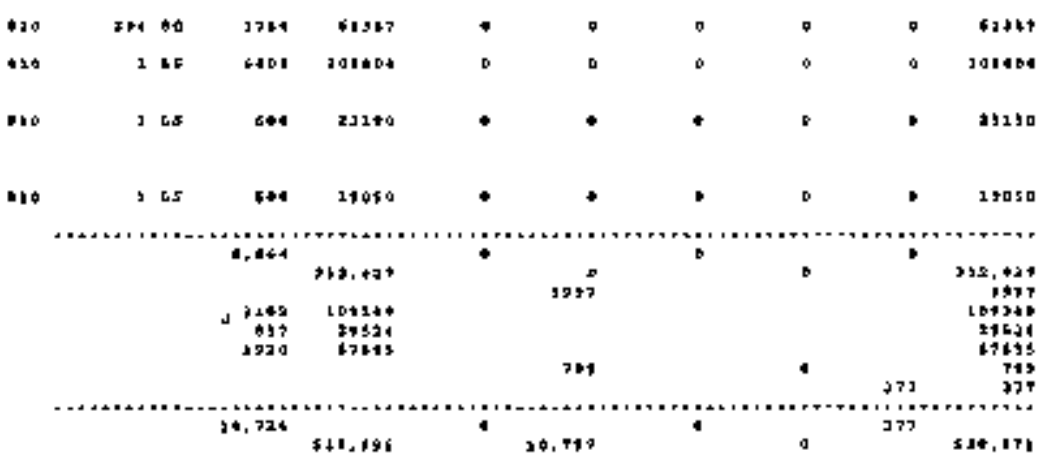


DLUQR DANIEL AOEFMERFF, LWE,

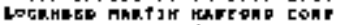

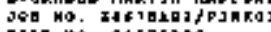

rien Ho. $24675 \pi$ as

Acepont

HU⿴囗十 $\mathrm{EH}$

absen BPtTon

atscaktion

CETAZhTLOH

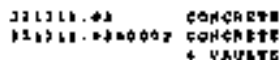

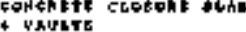

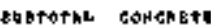

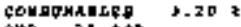

ito 33.04

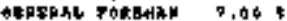

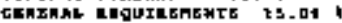

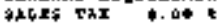

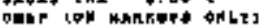

Tatal corp capt 16 th

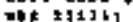

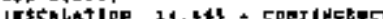

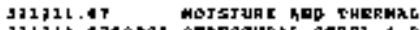

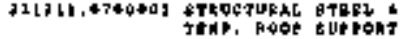

Ithi,

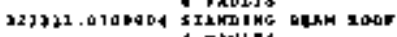

1 TसVथ

augrage wagatork hus thakml

comorumblez $3.3 * 1$

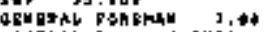

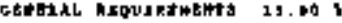

\$भ⿱中⿰㇀丶㇀

CH+中 tol nktaUPG opar।

70946

caty cose 60005

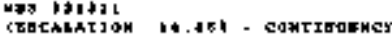

540

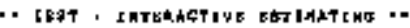

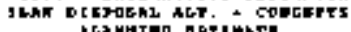

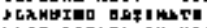

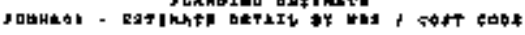

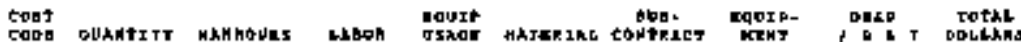

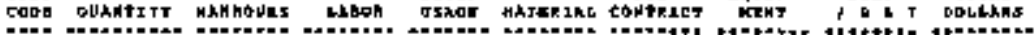

34.001

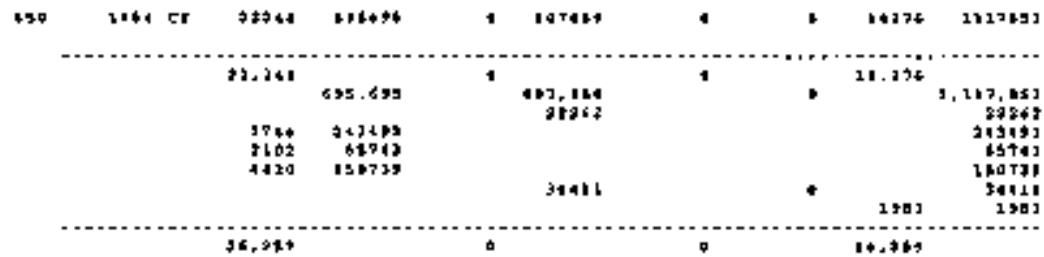

90.091

$1,345,4+3$

144,101

$1+\Delta+10$

\begin{tabular}{|c|c|c|c|c|c|c|c|}
\hline & & & a & 115240 & $\phi$ & 0 & 1072 \\
\hline
\end{tabular}

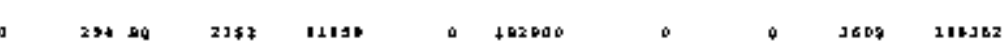

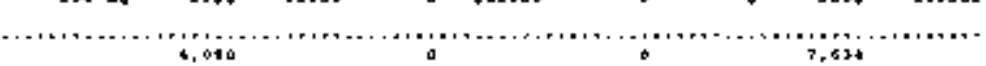

4,010

24, , 1

4,190

4145

4906

1491
305
179134

-1

713

10itis

I0161

गः

21, נas

$a 3 y,+\infty 0$

44,454

$+$

$41+1,2$

$30.04 *$
61. HE1

2, 216.945
$+16,4$

1 


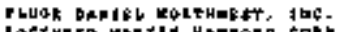

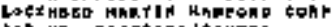

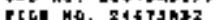

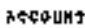

MoHa En TESCRIRTIOE

$x \geqslant 20+1$

$3220+2-09$

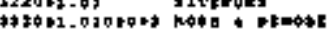

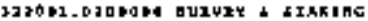

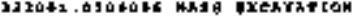

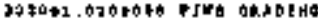

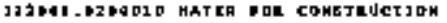

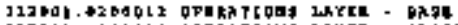

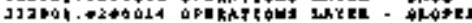

- aEMBMin PGCL

sarothl EITngat

roshe coss cops ifotr $\cos 20030$ (E)ChLATIOH

L4. E4t Toupamaney

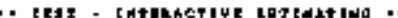

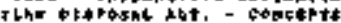

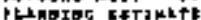

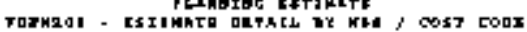

sate

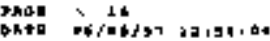

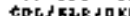
II qobukte

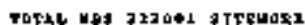

\begin{tabular}{|c|c|c|c|c|c|c|c|c|c|c|}
\hline 40 & 7 & La & $\phi$ & - & a & à & 20046 & a & $14+6$ & 74564 \\
\hline 460 & 1 & LF & 9 & . & 0 & $\phi$ & $760 \div 0$ & 0 & 11220 & 14234 \\
\hline 660 & $10+646$ & cr & 0 & . & व & च & 123040 & a & $11 * 11$ & 144671 \\
\hline+16 & $67+26$ & sy & 0 & 1 & $\theta$ & o & 21016 & a & $9 \geq 14$ & 12aze \\
\hline 440 & b & not & . & a & 0 & 0 & 12100 & 0 & $110 B$ & 14240 \\
\hline 164 & $+1 \leqslant 0$ & CY & • & a & • & D & $150 \$ 0$ & o & 2250 & $1 \pi 340$ \\
\hline 946 & 1740 & ar & - & 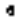 & b & D & 41046 & a & 256 & 56724 \\
\hline
\end{tabular}

a

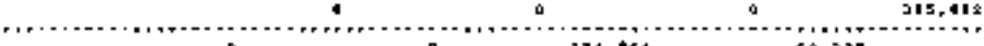

o

b

50,220

115. a12

2a.at 1
34,154

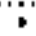

,

50,120

316.014 


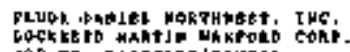

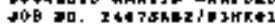

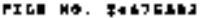

\section{Resothe}

لlumbin

phornjorat

dzat

$327044 \cdot 02$

LECELE SAMYIL EORO

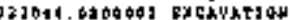

332014.020004 rinx chKoirn

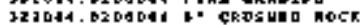

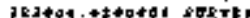

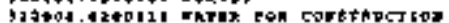

\section{Fogk SITUVer}

Tark1

\section{cost cove 16402}

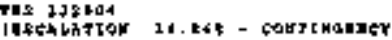

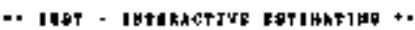

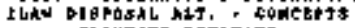

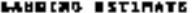

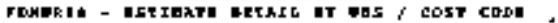

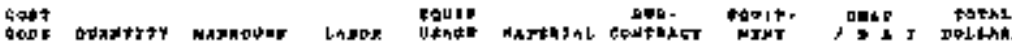

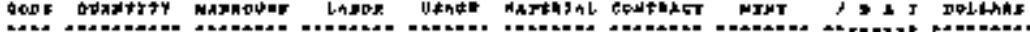

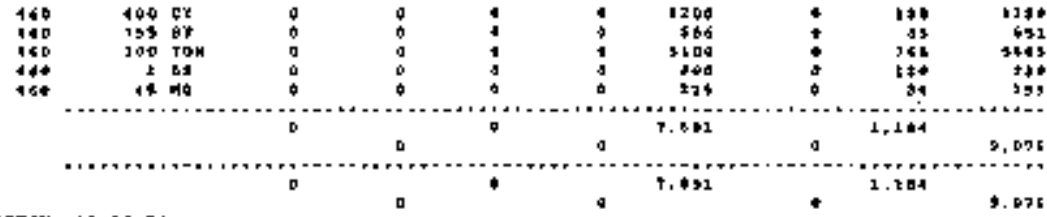

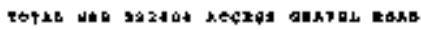

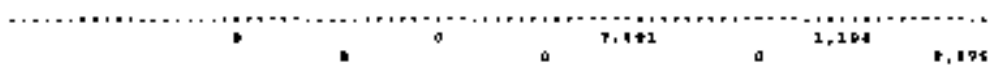




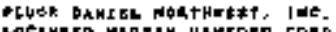
CONP.

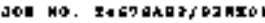

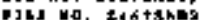

Atcount

กบหม E:

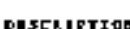

$+2 \pm+6$

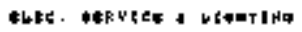

32004.16 GLCTRJenc

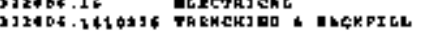

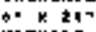

MLSTOM

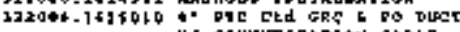

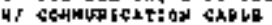

325044.1614012 cotsitct tednuarcat to to ahtriny BUILFI nsakn corJiob hax an stor QP Dtbo

$323044-164012$ CONetrmichtron T-nOT's rot axuray duICDita

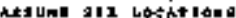

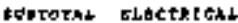

taleb the b. Di

axip I

rosta cost cado toL

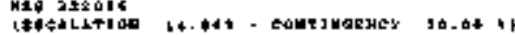

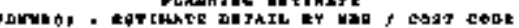

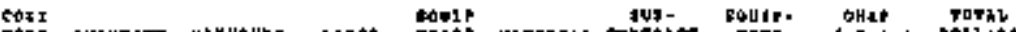

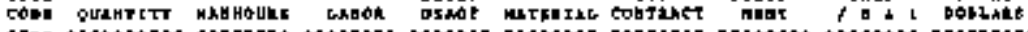

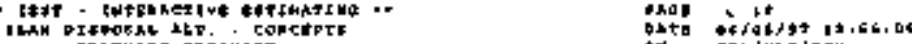

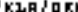

\begin{tabular}{|c|c|c|c|c|c|c|c|c|c|c|}
\hline 6140 & $a x+0$ & น" & a & 136 & 0 & $20+0$ & 0 & 4 & 1530 & 3055 \\
\hline $\begin{array}{l}6104 \\
6100\end{array}$ & 200 & $\begin{array}{l}\text { sob } \\
\text { ler }\end{array}$ & 19: & $\begin{array}{r}2964 \\
32164\end{array}$ & $t$ & $\begin{array}{l}15404 \\
52+94\end{array}$ & 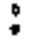 & $\begin{array}{l}0 \\
0\end{array}$ & $\begin{array}{r}1141 \\
34146\end{array}$ & $\begin{array}{r}85060 \\
91389\end{array}$ \\
\hline 6100 & 1 & $30 \mathrm{~B}$ & 11 & $\Delta t b$ & $\bullet$ & $3 \pm 1$ & 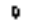 & 0 & 142 & 264 \\
\hline 100 & s & ah & 9 & 2404 & $\bullet$ & 3דף & $\phi$ & 0 & 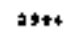 & a \\
\hline
\end{tabular}

B Bn

340

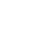

-

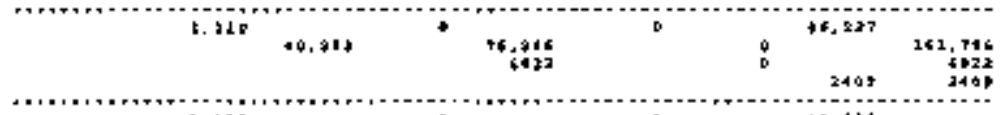

metrictict

323001.36

ty

1

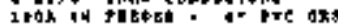

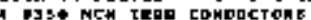

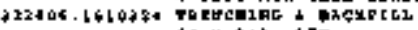

$\therefore m_{1}+2 z^{2}$

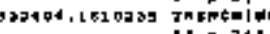

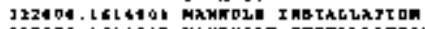

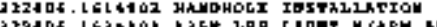

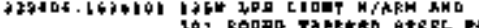

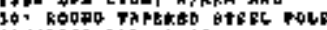

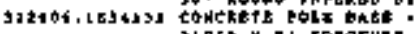

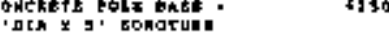

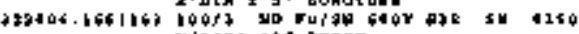

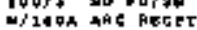

\begin{tabular}{|c|c|c|c|c|c|c|c|c|c|c|c|}
\hline 1154 & & 2306 & Gr & 925 & 11796 & $\bullet$ & 10939 & $\bullet$ & 0 & $0+92$ & $25 z+1$ \\
\hline 664 & & 2106 & $L P$ & 9272 & 94716 & $\bullet$ & 79665 & $\bullet$ & 8 & 16441 & 18240 \\
\hline 1150 & & 2704 & LF & 27 & 340 & 0 & סגבנ & 9 & 1 & $2=96$ & 6244 \\
\hline 6210 & & $2+\infty$ & $6 *$ & $\lambda+$ & $\omega 4$ & a & Át & a & 4 & 1531 & 5335 \\
\hline $\begin{array}{l}4150 \\
4150 \\
6169\end{array}$ & • & $\begin{array}{r}1 \\
21\end{array}$ & 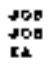 & $\begin{array}{r}10 \\
214 \\
210\end{array}$ & $\begin{array}{r}29+3 \\
071 \\
1673\end{array}$ & $\begin{array}{l}0 \\
0 \\
0\end{array}$ & $\begin{array}{r}14010 \\
1210 \\
24619\end{array}$ & $\begin{array}{l}0 \\
0 \\
0\end{array}$ & 1 & $\begin{array}{r}7161 \\
121 \\
13466\end{array}$ & $\begin{array}{l}23+46 \\
2+4 ; \\
14+19\end{array}$ \\
\hline 6930 & & $1 \%$ & ER & bat & 5257 & $\nabla$ & 1015 & a & 4 & 2419 & 81เ \\
\hline 4150 & & 6 & $\mathbf{r \alpha}$ & 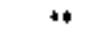 & 1742 & 0 & SOd & 0 & 4 & $2+04$ & $94 T 4$ \\
\hline
\end{tabular}


rLFon Datrac HONTHJEET. INE,

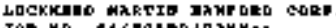

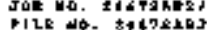

necolnt

oumbat. Detehroriag

C.-.".

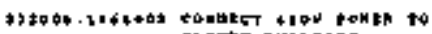

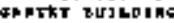

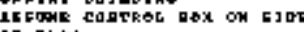
or 10 L

E UaTOJAC

ELtCTEICRL

SALES THY T, LO

CH०F tor wAh RUPG ONLY

totsl

cast Gode 61516

- 05 1 $37 x+66$

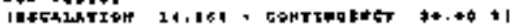

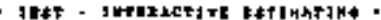

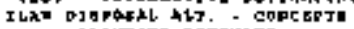

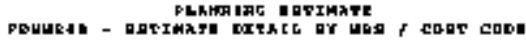

copt gedp

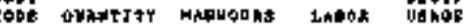

6150

1 sas

-

a.

Udinger

2,jit $+1,164$

4

142,07

11430

t, נ3:

จ , 5 เ

0

134,365

sum.

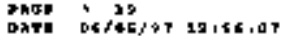

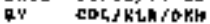

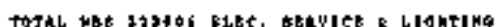

D

236,57

a
3,240

I, 12 .

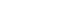




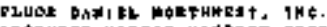
SOTEMAD MARTIR HANEORD CORE.

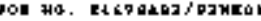

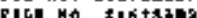

netcalus

urwara DEECET PTT ON

322047

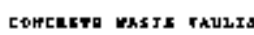

$2 \geqslant \$ \$ 4.03$ SOH+CH:TE

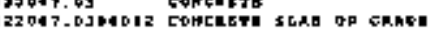

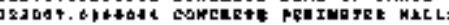

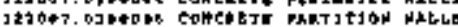

apotothb cauçere

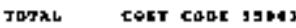

*16 320017

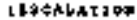

$14.014-$ -

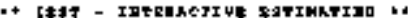

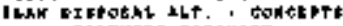

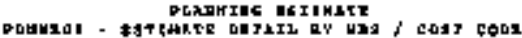

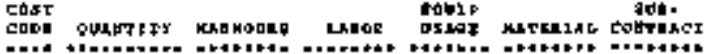

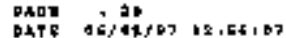
at CoISKIRFXH

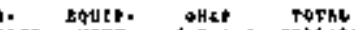
KETr $t$ C d abthis

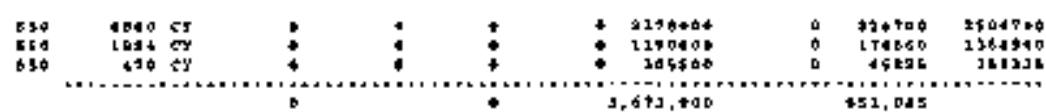
(. $1,226,216$ asi, ths

$+$
4

$6 r, 964$

$3,672,10$ 4 


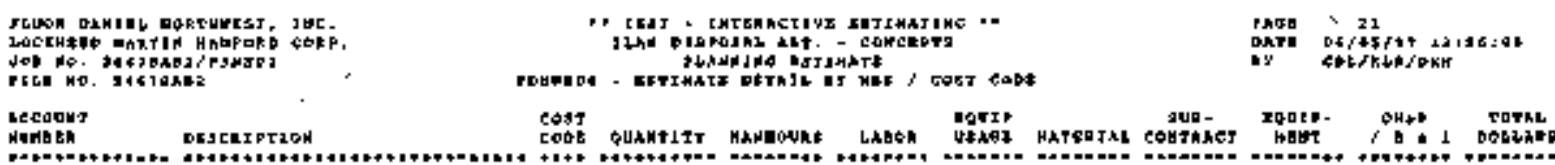

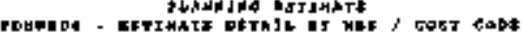

cesagur

Whith th

DESTEIPT2ON

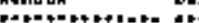

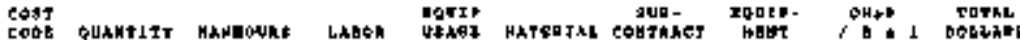

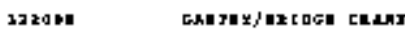

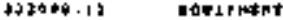

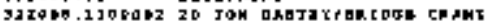

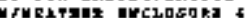

Alnotl EhPhILITY CRAMa $a+1$ th

gULTOThL GOOJPRKT

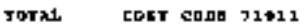

पर 32200.

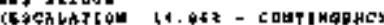

729

J $*$ n

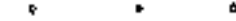

$0 \quad 26+40 * 6$

$\$ \$ 4 \$ 16 \quad 11+2816$

30

tat LE

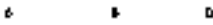

$0 \quad 57060$

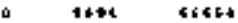

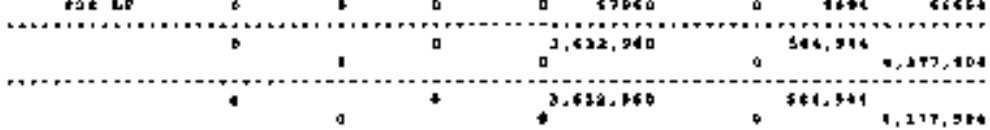

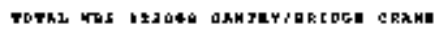

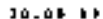

1

.

$3-43,500$

34,94

1,1 $17 . \$ 10$ 


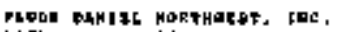

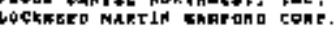

JaZ MO. S16TSMESTHUKaI

PLL No, EAt SARt

accogers

Dumbex

baceiditan

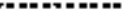

נมנมฺ

comajeuctron tances

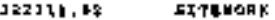

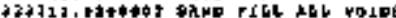

1 vauds

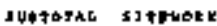

CaDsomhlors J.Ja?

GNP 33, DQt

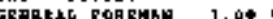

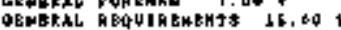

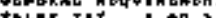

ShLES IAT S.t0

TOTAL COEJ COEQ 33907

ros jazila

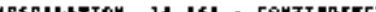

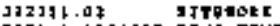

6 Yhets

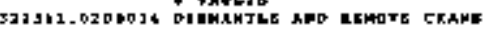

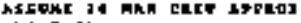

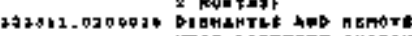

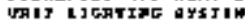

Evbtotal ajttobhx

tonsmiales 1.20 , cyp $35.0 * 1$

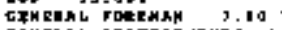

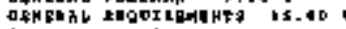

OALES TAR -D.

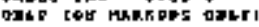

totrb

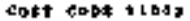

พम5 32231

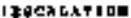

th.es - contimarmay

114

- IOST I IMTERACFIYE DATIHATIME -

LLA= DJEREAL ALt. - Codcerts

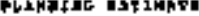

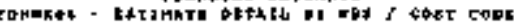

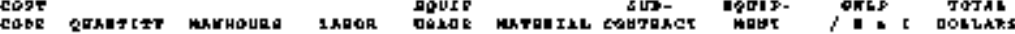

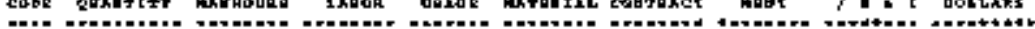

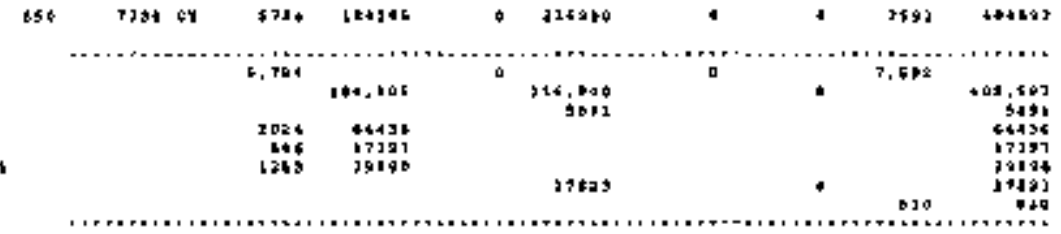

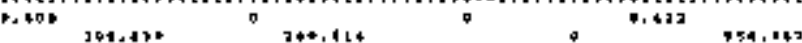

34.49 is

. 20

เ

1764 611

a

4

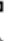

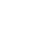

a 4137

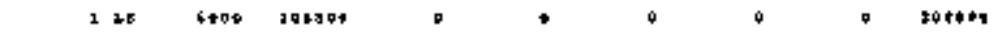

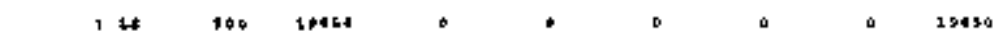

(...........

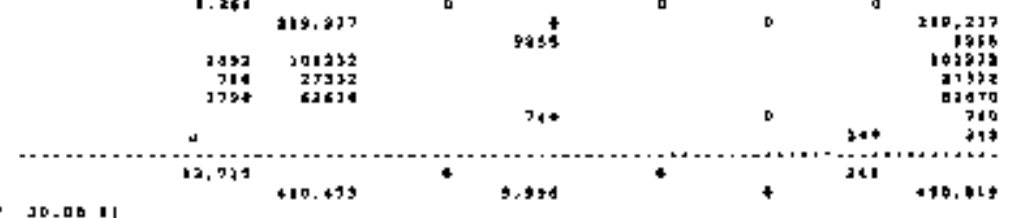

322313.01

Gonchet: 


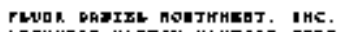

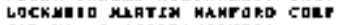

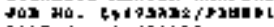

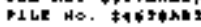

ACCOONT

AUHAX

anderstion

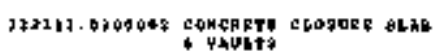

gorathl cokchuta

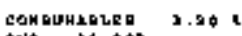

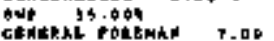

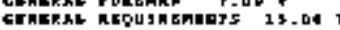

3ACEE TR T

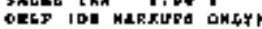

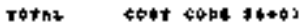

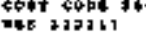

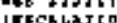

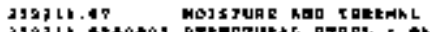

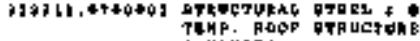
Truite

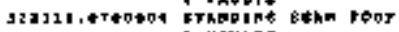
i ravita

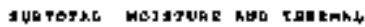
CORJUMh BLEE, $2 *$,

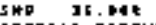

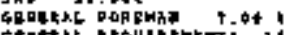

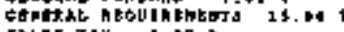

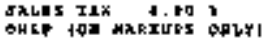

rothl

\section{$\operatorname{cost} \cos 9501$}

Fic jazjas

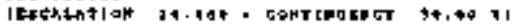

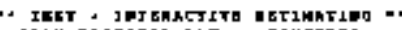

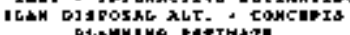

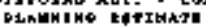

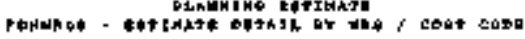

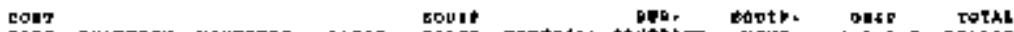

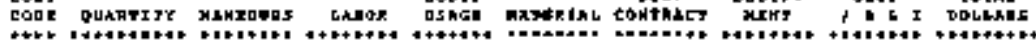

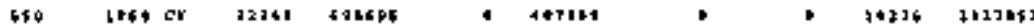

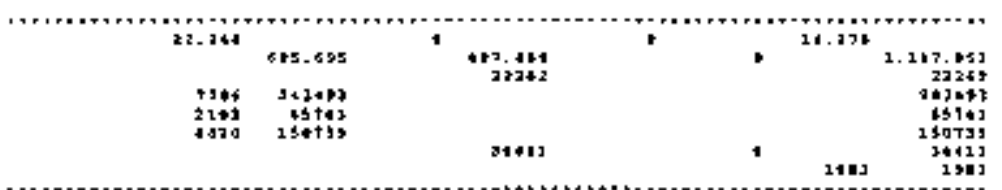

$+4,+5 *$

1, aڤ1, 13

a 164,45

$a+2+,+6 \%$

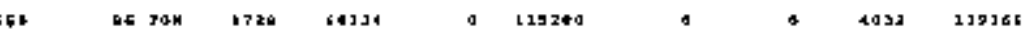

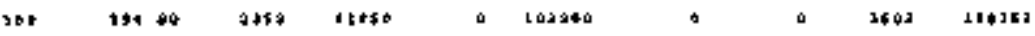

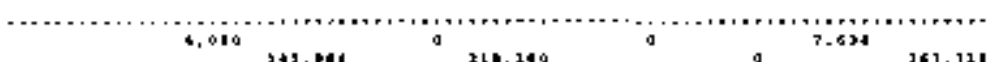

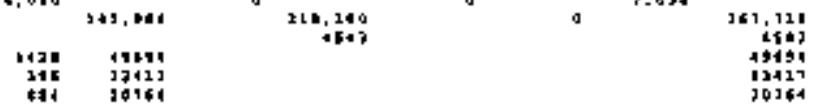

49d 20161 20164

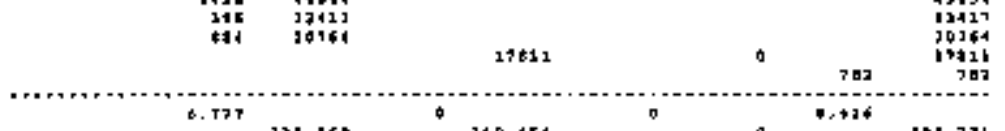

$$
64,013
$$

a. 1+1, 193
4 $\phi$

$x^{2}$

13. 147 
PLUAE PhWT CL MOHTMMLET, INS.

LOTKNE EO WRMTI MAMPQD COIF.

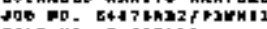

PLL HO. ITEJERE?

kc5ount

elublek

DTECRITto

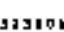

รI I. Makk

Sa3IOL.12 SITHMORX

33I0L.12 SITHMORX

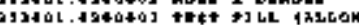

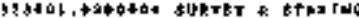

$13910 \mathrm{~L}, 42+6+01$ WaEE ExChvation

$3 a 310 L, 1240404$ RIMT GRAOIBSC

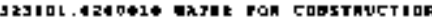

suatotse

TTMORK

Tothas

$\cos \cos +4+0$,

पad $j 23+01$

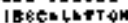

14. L6t - compinagery

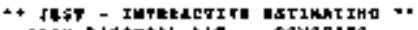

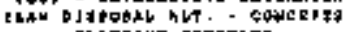

PLABMIMC EAJTART

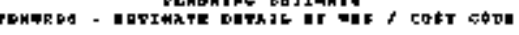

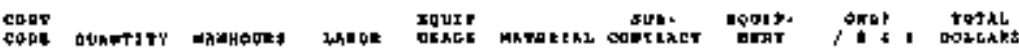

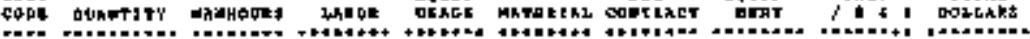

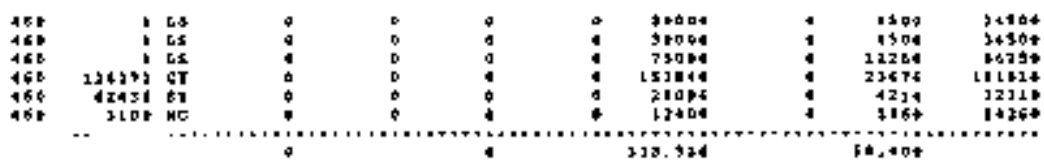

F⿻十 +2

243,336

$+$

[a, tos

243.234 


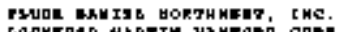

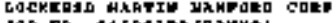

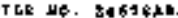

\section{ntrofowt}

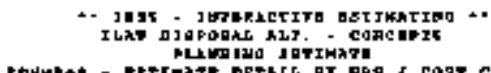

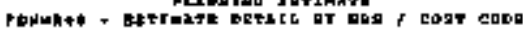

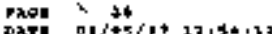

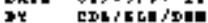

tott

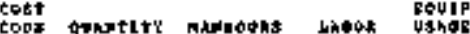

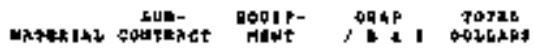

\section{$323 \times 2$ \\ ADNIX LuUn:}

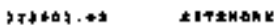

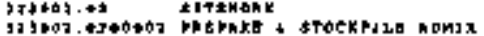

I I RCLUDE THST TIL,

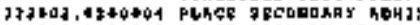

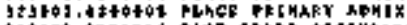

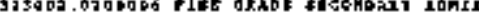

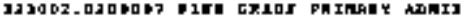

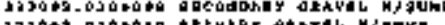

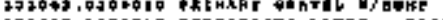

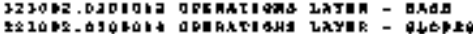

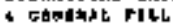

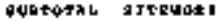

reset cost cant 4003

पas

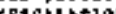

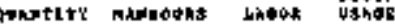

aqkat

Hawt

11 ouknas

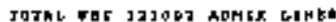

\begin{tabular}{|c|c|c|c|c|c|}
\hline idv & 20,14 & er & 4 & a & 4 \\
\hline $16 \%$ & $\begin{array}{l}40424 \\
2+28\end{array}$ & $\begin{array}{l}\text { ct } \\
\text { cY }\end{array}$ & a & D & 4 \\
\hline 60 & 1,1504 & st & 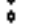 & $\bullet$ & 0 \\
\hline+10 & 33730 & $s \mathbf{r}$ & 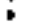 & 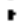 & 0 \\
\hline 160 & 11140 & cr & - & $\bullet$ & 0 \\
\hline+46 & $14+45$ & $5 Y$ & 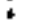 & 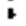 & a \\
\hline tha & $13+0=$ & $\mathrm{Er}$ & - & 1 & 0 \\
\hline+60 & 1740 & $\mathrm{cr}$ & : & i & 0 \\
\hline
\end{tabular}

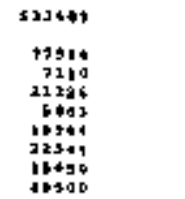

1740 ar

10300

11691

1291

21:

759
$2+42$

itio

a>30

7214

1910

חנים

7410

142

בנרא

atis

$1+100$

53715

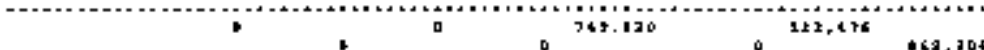

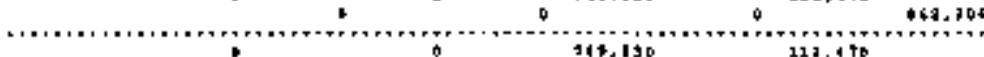

112.1 to

a. $1,3 * 4$ 


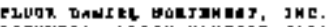

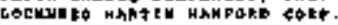

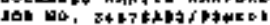
116. 10 .

nCcauar

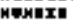
Frgerotion

\section{GEOTMTKETIC LIMZE}

323905

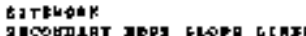

$3 \rightarrow 04 \$ .92$

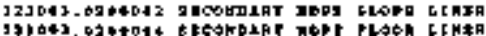

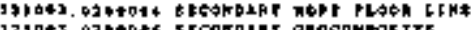

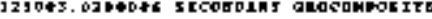

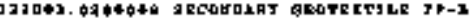

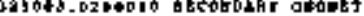

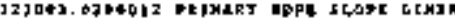

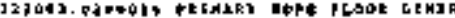

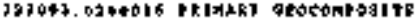

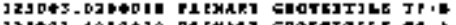

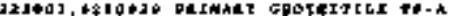

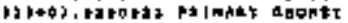

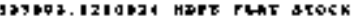

12903.

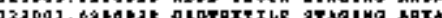

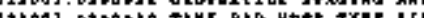
ThO

\section{gDVTath शנזTMank}

roenL

\section{$\cos 2 \cos 4640$}

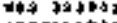

ietencottur 19.04 ,

CQHTIHEERS

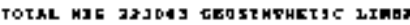

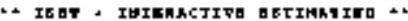

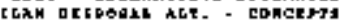

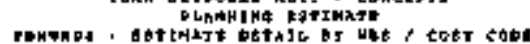

PXOF $>11$

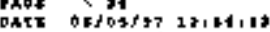

वT

cals resian aut

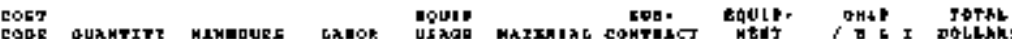

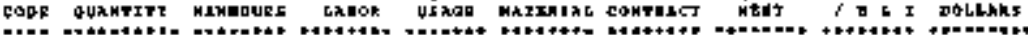

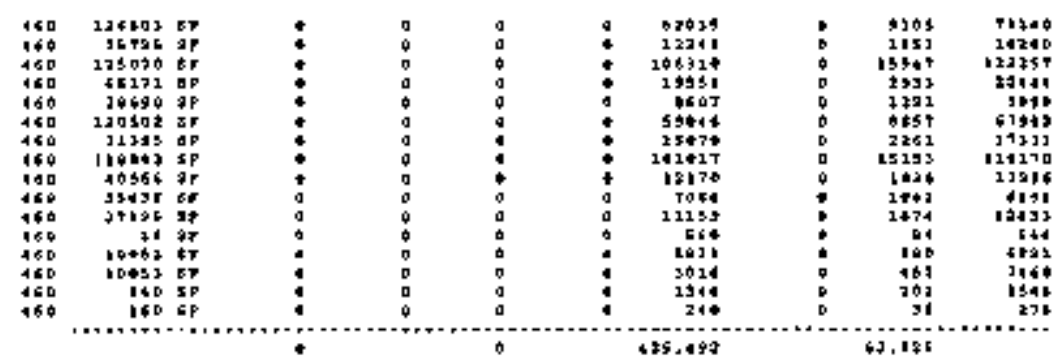

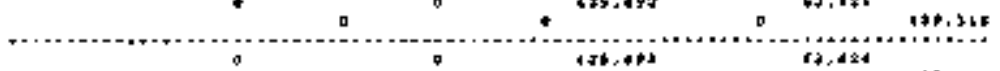

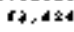

14, a 3

$10 .+413$ 


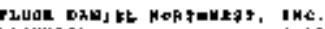

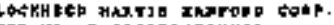

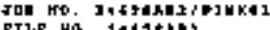

PILE HO. IA11\#ABS

necount

vukank gasenimitas

92900 hCcFes anTEL nohd

1t+\$1, t I I*HकhK

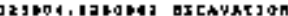

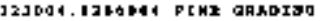

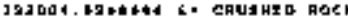

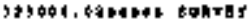

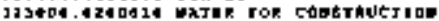

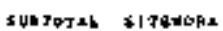

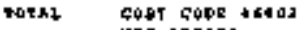
$408 \quad 273+0,1$ inschicator

14. W6 . COHJ

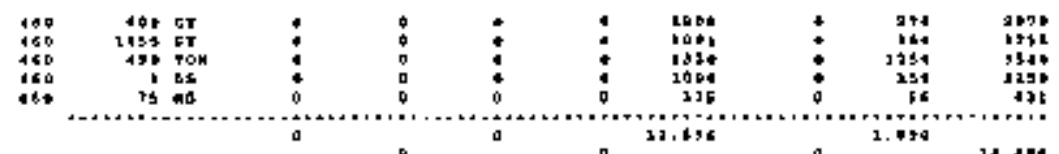

a

14,40

a

a

$12, \cdots$

.98

$14,4+6$

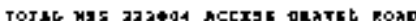

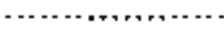

$\phi$
That

1645:19T $17196-1]$

CLLJKLFRKE 
TLOON DAHIR MORTUVAST, thC.

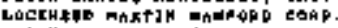

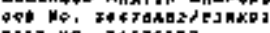

rib] MO. aAt TSREz

तecsunt

M४Hए Eम

betchIt+19

223405

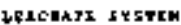

$3230,0,02$

comenesz

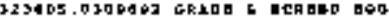

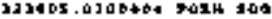

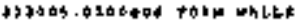

333105.0300401 ro14 cules

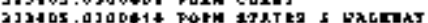

ggy+05.0206012 R. J JO1mtt

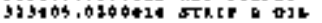

123109.0300416 cajckerx s06

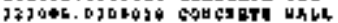

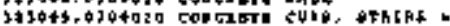

\section{congsis}

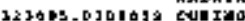

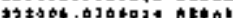

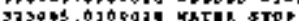

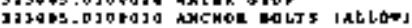

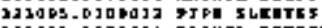

政

SUDTOTAL concolte

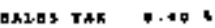

Tarel $505+\cos$ rodos

rod yajed

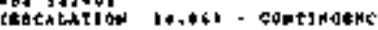

323043.0

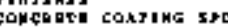

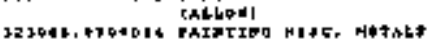
CAL LaWI

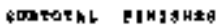

yotal

COEJ COaE Tathe

พละ 329045

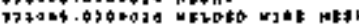

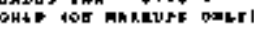

- jast - jertohtojte estimating -

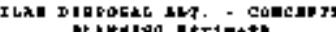

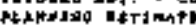

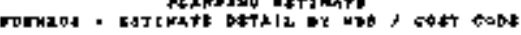

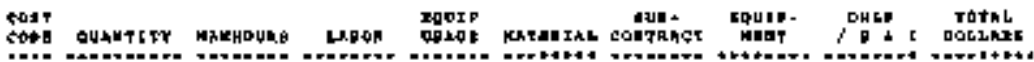

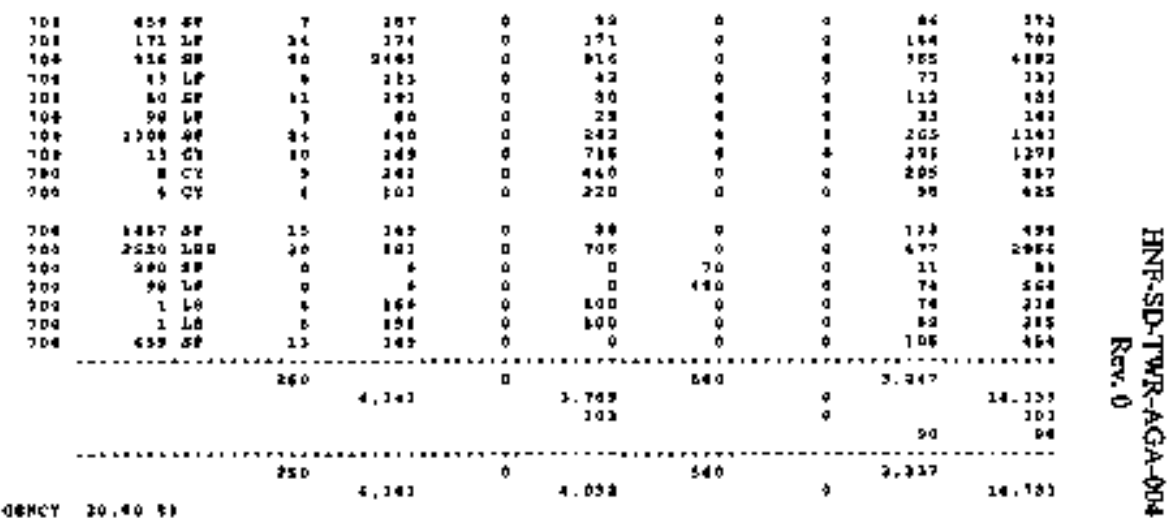

㤎

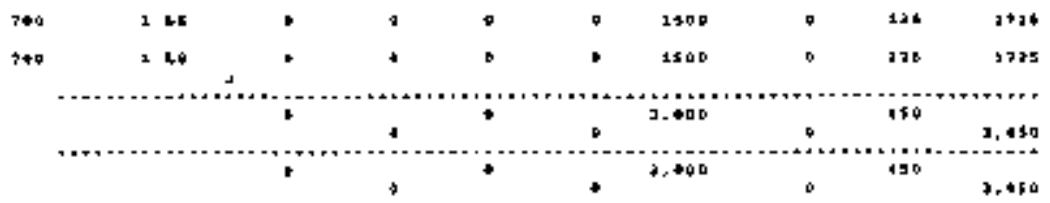




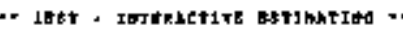

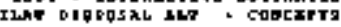

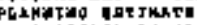

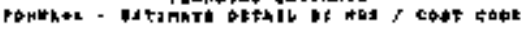

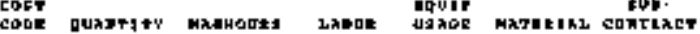

phat 24

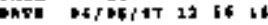

I 7 GELIKLITEKa

MQIS- OEL TETKL

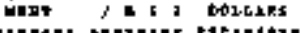

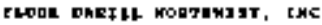

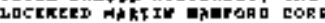

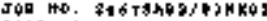

pink do asterea

secoors

Dunknk

bething?

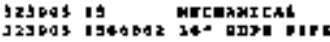

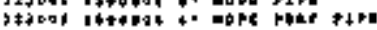

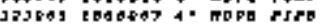

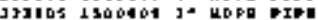

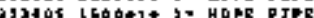

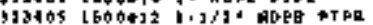

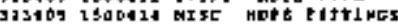

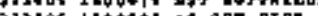

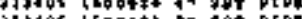

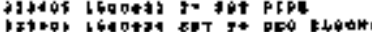

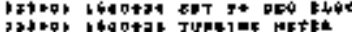

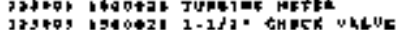

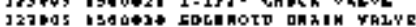

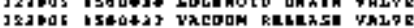

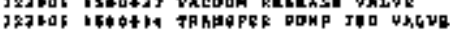

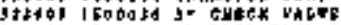

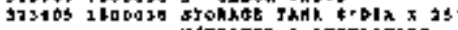

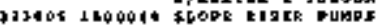

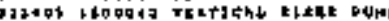

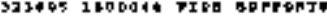

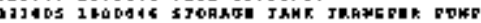

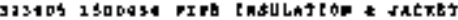

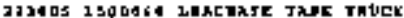

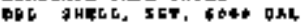

aUgajac machsojeh

TETAE COAT CODE thos

mal jzjab

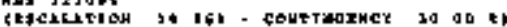

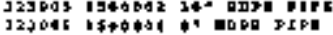

jaldos 1200416 We PIF

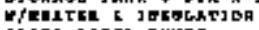

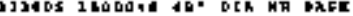

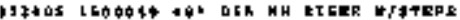

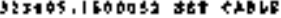

HeY 20 Q 1

\begin{tabular}{|c|c|c|c|c|c|c|c|c|}
\hline $\begin{array}{l}7 \notin 0 \\
7 * 0\end{array}$ & $2 \geq$ & $\begin{array}{l}6 r \\
E\end{array}$ & 1 & ? & $t$ & $\begin{array}{l}2601 \\
2100\end{array}$ & $\begin{array}{l}285 \\
193\end{array}$ & ל, \\
\hline$++b$ & 134 & LF & 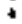 & 0 & $\bullet$ & 14344 & 1902 & 13121 \\
\hline$+\infty$ & $200 \mathrm{r}$ & 6 & 4 & 0 & * & Cobe & 100 & Got \\
\hline ז & b) & LF & 0 & - & 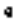 & 1620 & 330 & $1+0$. \\
\hline $10 \mathrm{t}$ & 124 & LE & a & 0 & a & 2100 & & $2+21$ \\
\hline Tat & 311 & WF & a & D & a & i7na & 284 & lose \\
\hline 101 & I1 & Ex & $\phi$ & b & $\phi$ & 2916 & 567 & 1212 \\
\hline זםר & 121 & LE & 0 & 9 & 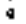 & 2600 & 311 & dis \\
\hline • & 21 & $E \lambda$ & 0 & 4 & 4 & asod & 221 & Sבד \\
\hline ja. & 31 & $6 \%$ & 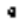 & 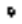 & 4 & 1334 & נa & 1353 \\
\hline rob & 3 & $6 *$ & $\bullet$ & o & 4 & 114 & 23 & 207 \\
\hline tos & ' & 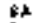 & $\bullet$ & 0 & + & $A B$ & 121 & Wat \\
\hline tob & a & 12 & $\bullet$ & b & + & 2504 & 275 & It) \\
\hline 700 & a & $t \boldsymbol{t}$ & $a$ & a & + & God & 01 & 640 \\
\hline 108 & 2 & 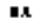 & 4 & 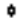 & 4 & $2+3$ & A & 364 \\
\hline TOO & J & 11 & 4 & 9 & 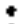 & 434 & 61 & 111 \\
\hline$T A B$ & j & F2 & 4 & 0 & 4 & 334 & 31 & 103 \\
\hline 904 & i & 8 & 0 & - & 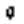 & $c * 0$ & 90 & 690 \\
\hline 104 & $i$ & th & 0 & B & 0 & 24040 & JaBd & $7300 *$ \\
\hline 704 & 2 & $\mathbf{x \lambda}$ & 0 & 8 & a & $10+0$ & T & SLI \\
\hline got & $\neq$ & $\neq \lambda$ & 0 & • & a & 17010 & 2330 & $1+354$ \\
\hline 104 & i & t\% & 6 & t & 0 & いか & 15 & 575 \\
\hline 7 & ב & $\nabla \lambda$ & $\phi$ & $b$ & $\phi$ & $5 \in+6$ & 419 & $64+$ \\
\hline 704 & l & $I \lambda$ & 0 & 8 & 0 & aro & 15 & $2+5$ \\
\hline ב 04 & 13 & rv & D & D & a & 1340 & का० & 199 \\
\hline J04 & b & ใ? & a & b & a & $s \geqslant 0$ & ts & $3+4$ \\
\hline $10+$ & +40 & if & 0 & B & 0 & 3060 & 450 & $343 *$ \\
\hline 101 & i & $\mathbf{x x}$ & 0 & 4 & 0 & $61+010$ & 21000 & $161 \bullet 0 *$ \\
\hline
\end{tabular}
$0+9,+4 t$ ass, + L in....................
$2+0,934$ 
TLUOM REHILL MORTHEET, IFC. LOCKHTEB MAETIM IIABTOED CONP.

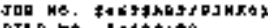

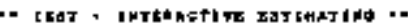

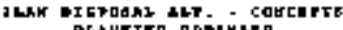

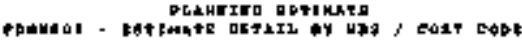

cost Eders

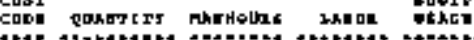
gachiog tom

AGColuet

Dunbal (3)

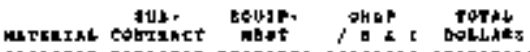

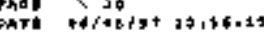
ELLKLARE

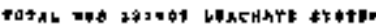

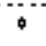

$\boldsymbol{1}, \boldsymbol{n t}$

$23 \pm, 6+1$
250

4,74 
TLUAX DKUIEL NORJMHE IT, FHC.

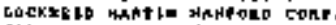

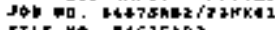

TILE HO. IAGIEAR?

secobut

NUmb tro

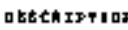

drathet

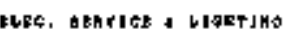

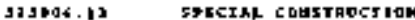

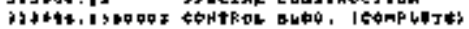

sararkl pracrkl cubaracriah

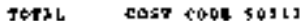

Tp 232106

istehintion

Leceratrab

732405,66

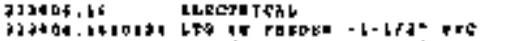

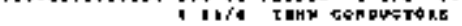

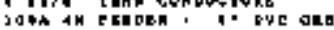

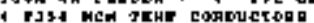

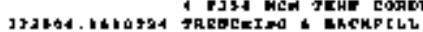

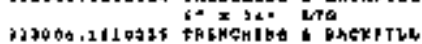

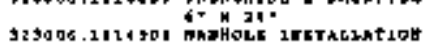

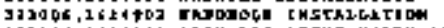

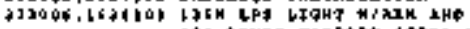

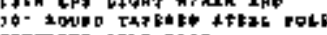

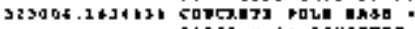

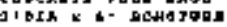

SURJOTAL TLFCTUIChL

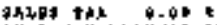

OH $\rightarrow P$ tol hankete orkt

T.712

cosi conx wsis

wOS $192+\$ 6$

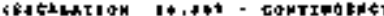

Sxan: 71

Pntt $66 r+4 f+19,16,16$

at Gestrintorie

sist.

Doulr.

OH OO TOT25

maxt, ind golitihs

D I ITtaiesi

2230 क6. 16

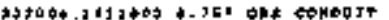

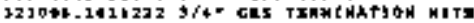

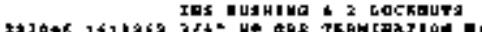

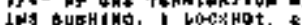

a) bet

1304 L7

$230 * 4$

150028

$\$ 50 \mathrm{~s}$

3 and

2. $2 x$

10 5त

$$
+, T
$$

נחים

נasa

at

19.

เ

54t

150
6190

1190

$24 \quad 2903$

24 17?

a)

ניא

aथ 2 เb

แb 0 1t+

$20 \mathrm{sus}$

$1++16$

उ3:

1350

1300
1200

30479

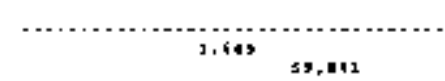

124

pos

$+\infty$

1. 20

12 th

16 ox.

1.649

51, 141

b

11. 105

$i+04$

$124,21\}$

(

232,016

$4,220 \quad 0 \quad 640$

t, 2 24

a

cad

1, 14 ,

\begin{tabular}{|c|c|c|c|c|}
\hline * & $\begin{array}{l}16 \\
16\end{array}$ & 1 & : & נג \\
\hline$\bullet$ & 26 & I & 1 & 61 \\
\hline
\end{tabular}




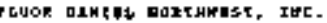
COCEMARO HANTEN HAHPOKQ COEF,

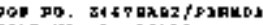
FILI tro. F+14 takn?

netodnt nombtin Decererejon 1 BEALJTEC LOGKHUT

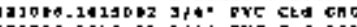

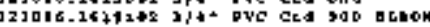

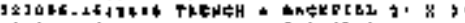

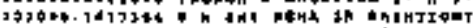
CER KU COVER MMCLOHORT

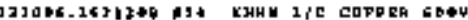

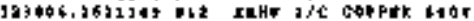

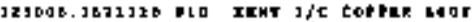

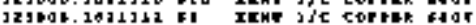

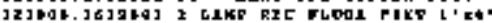

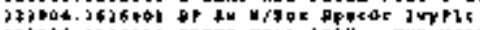

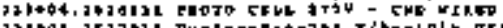

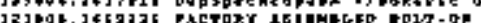

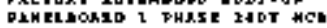

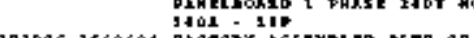

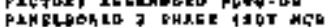

3agob, 61406 a

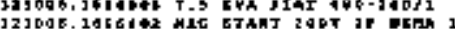

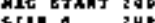

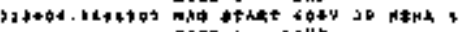
SIz: $\mathrm{IOH}$

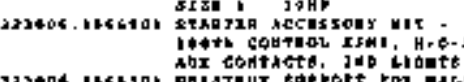

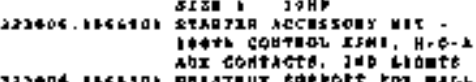

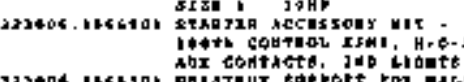

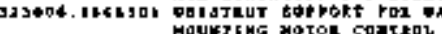

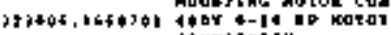
cowretion

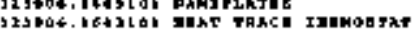

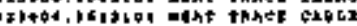

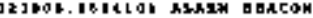

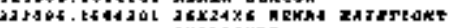

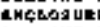

$32+046.16012021 \%$, tog k-Q.

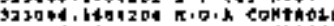

323004.5404206 OM=DTF ENITEH

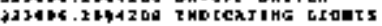

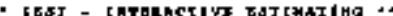
1*b\$all abF. - coucapre

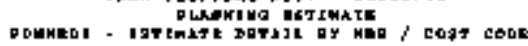

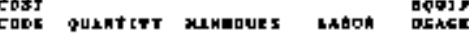

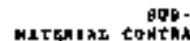
gQp-

rand 32

part offolith 12154:12

at colixutal

$\cos 20$

ager Tothe Ment, $y$ I botekint

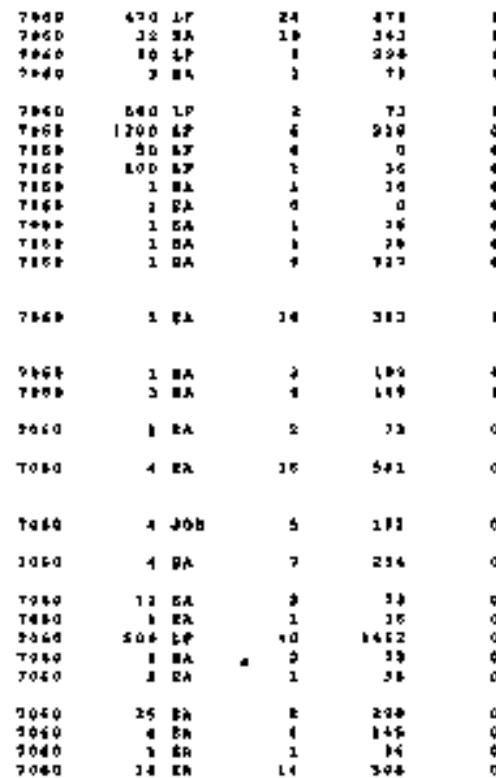

104
104
67

19:

10

2)

629

1304

$+43$

II

21

010

45

33

130

$r 20$

$\$ 16$
705

a7

14 rh 97
597 


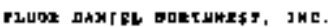

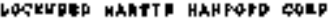

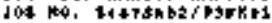

TLE MO. IALרRE?

recount

minktk

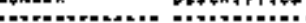

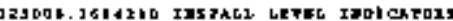

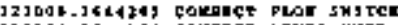

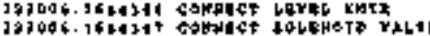

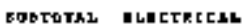

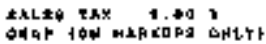

tothl cott cotoc 1011

Mar 323096

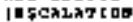

L. W6 - Cakringrecs $20.00+1$

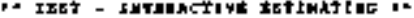

CLAM DTAPOPL ALT. - EDNCEPIS

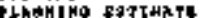

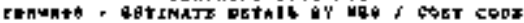

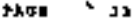

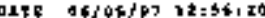

DT $602 / K L R / D x$ 
PLWOP DRHILL NONTHAEET, JWG.

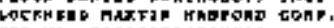

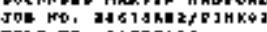

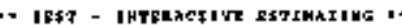

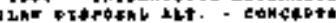

reanira astiknti

necoun?

MUнसЕ日 DtektRtJoH

323403

Pakcitere

Lbtab.L2 toul Pakpt

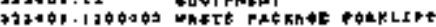

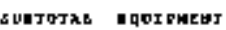

\$h20S TAK 1.40

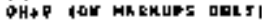

TOThC

cott sode raOal

Nas 3230, ,

inschestion

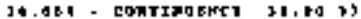

CRE $>9$

ר: $156: 2$

-Y CEIKLRIOKN

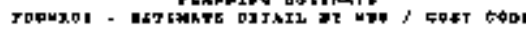

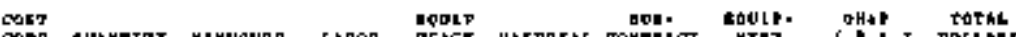

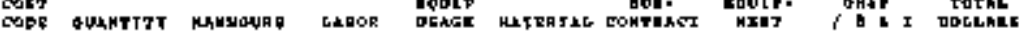

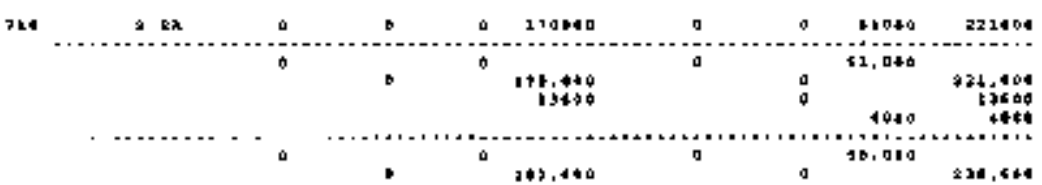

(1)

4

$202,4+0$
9

52,064

931, 64 


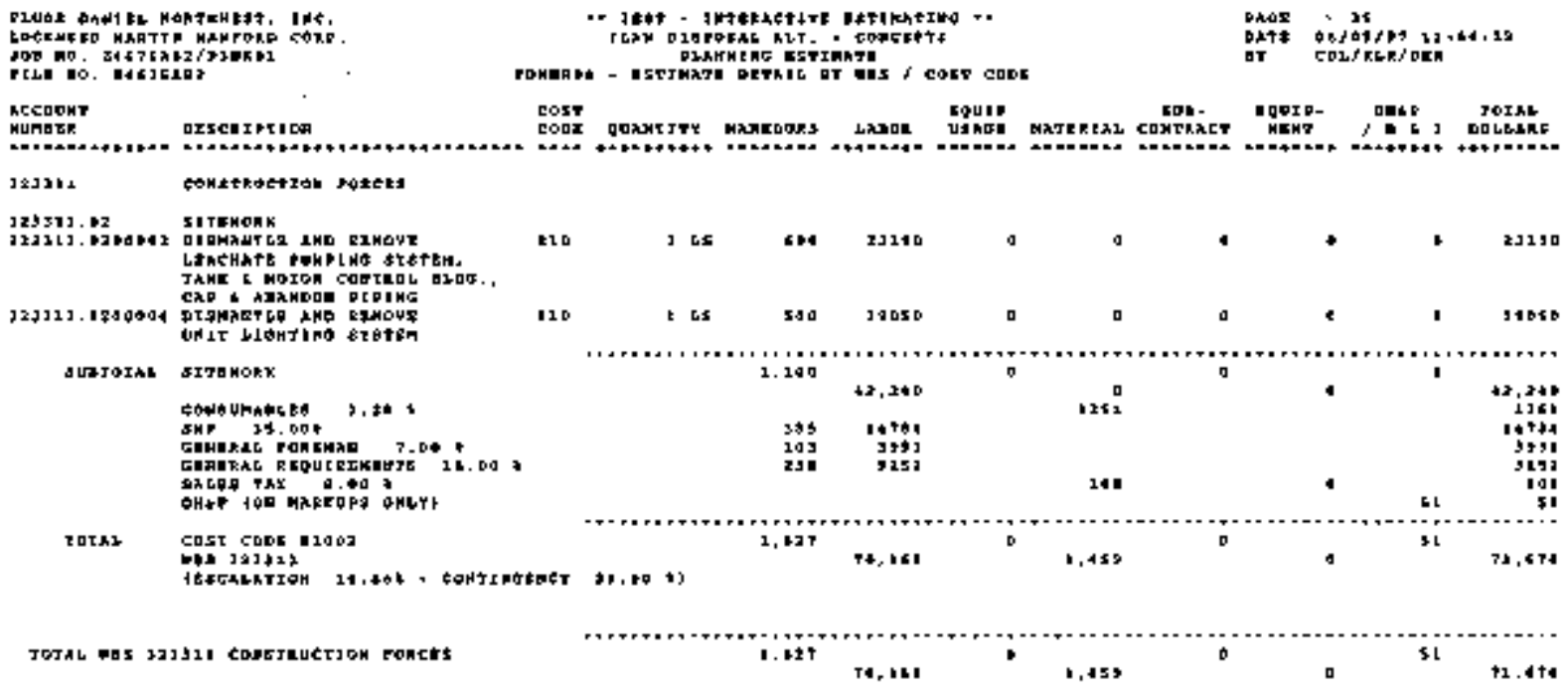


DLUSA BRHIEL JQRTHaEgT, rac.

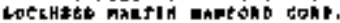

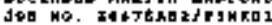

IJE G0. $246 \sin 2$

AC coun J

Mtoll EA

onckrotzan

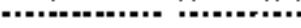

324001

sIrd MOKK

1*10+1.02

1tEmoted

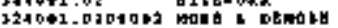

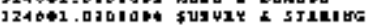

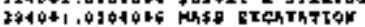

321041 0701010 THE 01405MG

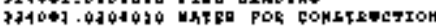

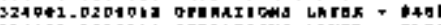

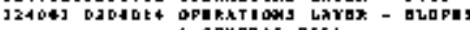

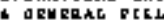

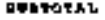

I TEMaR日

JOTAR

cout cote itora

Wald $9240+1$

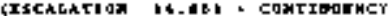

Toth2 MSA $2400+1$ a

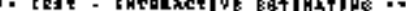

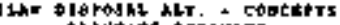

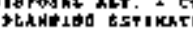

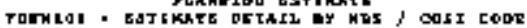

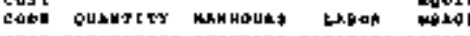

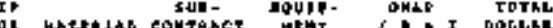

onta ec/os/21 $12,56.87$

QhT tor

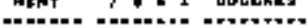

\begin{tabular}{|c|c|c|c|c|c|c|c|c|c|c|}
\hline $\begin{array}{l}640 \\
409\end{array}$ & $\begin{array}{r}1 \\
1 \\
7 \Perp 0<1\end{array}$ & $\begin{array}{l}1 \cdot \theta \\
16 \\
\text { ct }\end{array}$ & : & i & $\begin{array}{l}0 \\
0 \\
0\end{array}$ & D & $\begin{array}{l}j 0 \leqslant 0 \\
T \leq \geqslant 00 \\
10120\end{array}$ & : & $\begin{array}{l}4540 \\
11390 \\
17304\end{array}$ & $\begin{array}{r}16160 \\
012 \$ 0 \\
20190\end{array}$ \\
\hline 40 & $\begin{array}{l}62436 \\
93 * 0\end{array}$ & $\begin{array}{ll}1 \% \\
\mathrm{nc}\end{array}$ & 0 & 1 & 0 & : & $\begin{array}{l}2 \$ \$ 96 \\
12400\end{array}$ & $:$ & $\begin{array}{l}1130 \\
1160\end{array}$ & $\begin{array}{l}23210 \\
11210\end{array}$ \\
\hline+40 & $\begin{array}{l}17+0 \\
0 T+0\end{array}$ & $\begin{array}{l}\text { cy } \\
\text { cy }\end{array}$ & 8 & 1 & : & : & 15060 & : & $\begin{array}{l}\text { a } 244 \\
7273\end{array}$ & $\begin{array}{l}19+104 \\
53775\end{array}$ \\
\hline
\end{tabular}

(1)

242,553

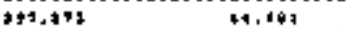

$+$

$20.00,1$

$2,2,12$ 


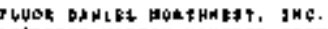

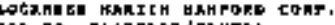

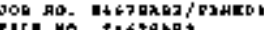

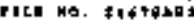

\section{Ассорнт}

MDMRE DESchetPFion

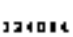

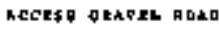

\subsection{2}

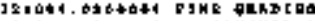

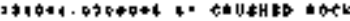

121044.0204001 50R सT:

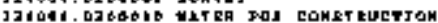

tubtoth1 sitekok

JOTHL CEET COOY 14012

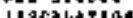

4.,6s- costingsmay

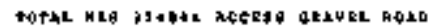

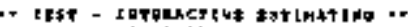
Difr brand Altr. - concarte

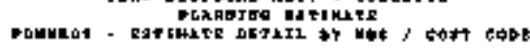

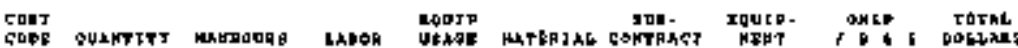
Gם

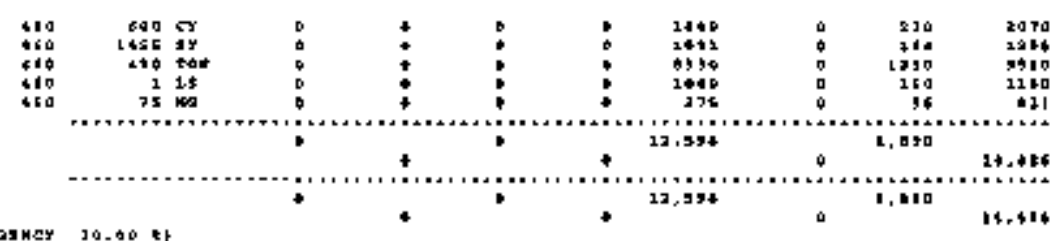

$10-00<1$

a) 3 \$

$1, \$ 0$

14.418 


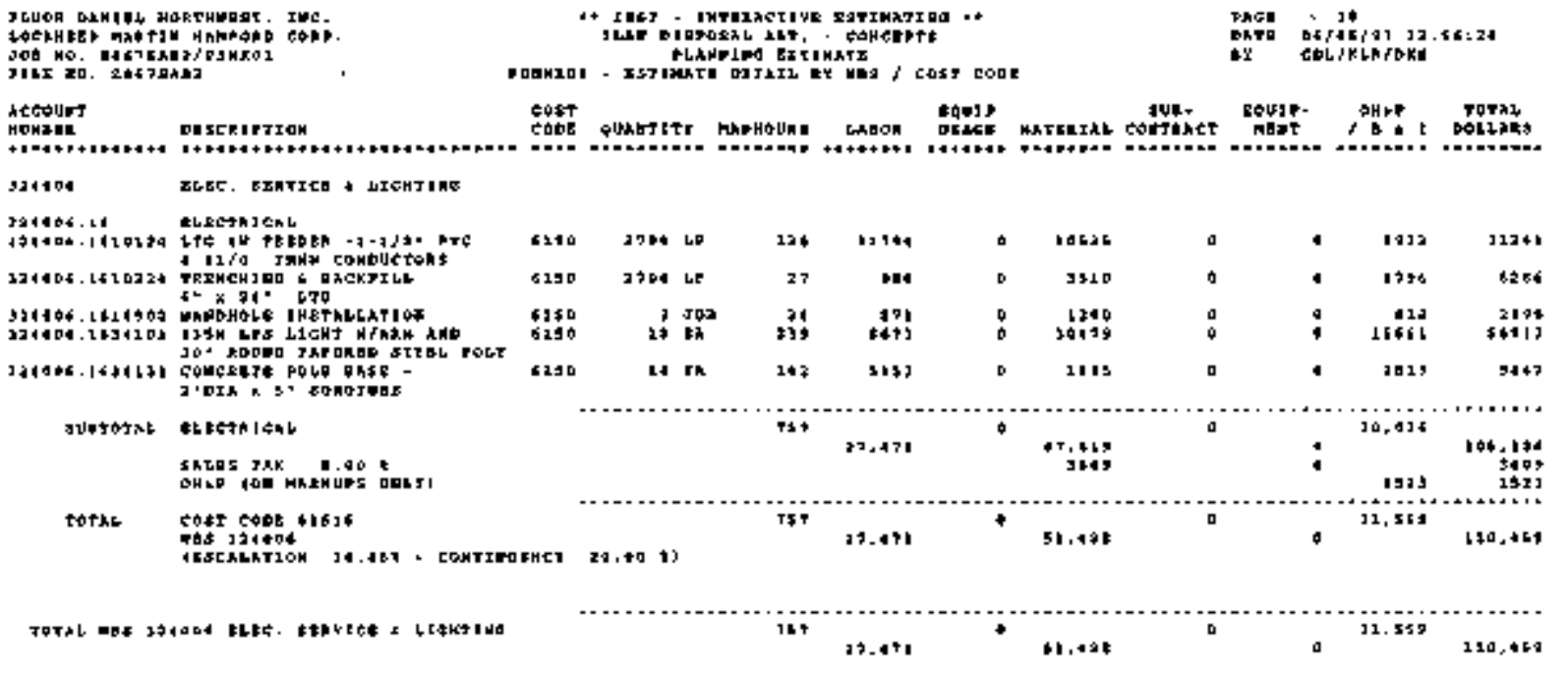




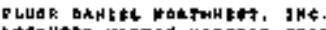

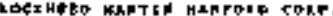

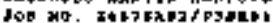

PICE wo. T4615ke?

Recouns

HัM⿻ Eด

.......

\section{PQHK+IPT*}

321042.11 gQUน MHг Кт

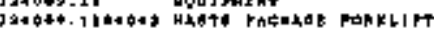

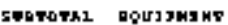

thete tax o. ob

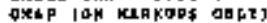

Totkb

Wet $12+093$

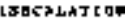

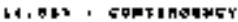

$\operatorname{cost} \cos 3 r+19$

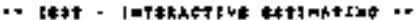

ILAU DISPOFA ALT, CONGHTT

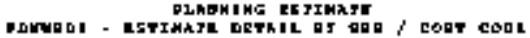

SOT T.

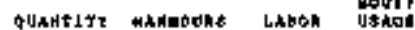

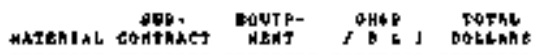

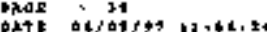
CoLs KIRS ox.

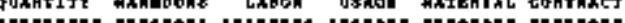

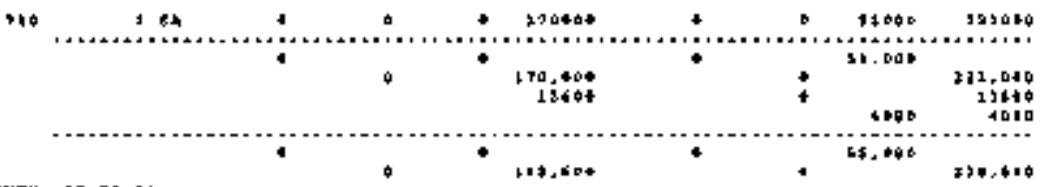

wothl uns iztoag roRkeIgts

$20,0+11$

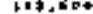

20.4.

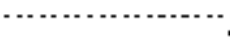

4

15, va

117,604

2, $2, \in 0$ 


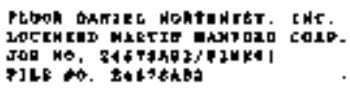

necourt I

COST

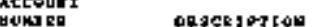

... - .

$3 a+311 \quad$ constauction rohere

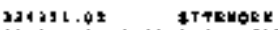

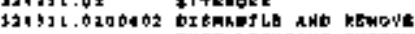

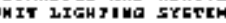

IUPTOTKS STTEKOKK

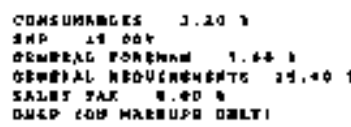

Togac

\section{$\cos x \cos$ isato}

was $3903 \mathrm{Lin}$

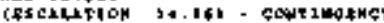

114
L L

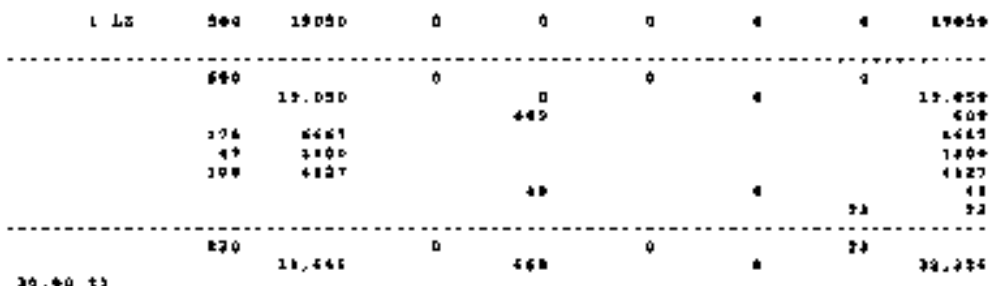

a

o

n

4

4 2\%⿺st

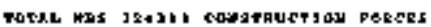

(......................

120.64

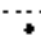

...

$16+$

a

92

3a. 2 20 
HNF-SD-TWR-AOA-004

Reri 0
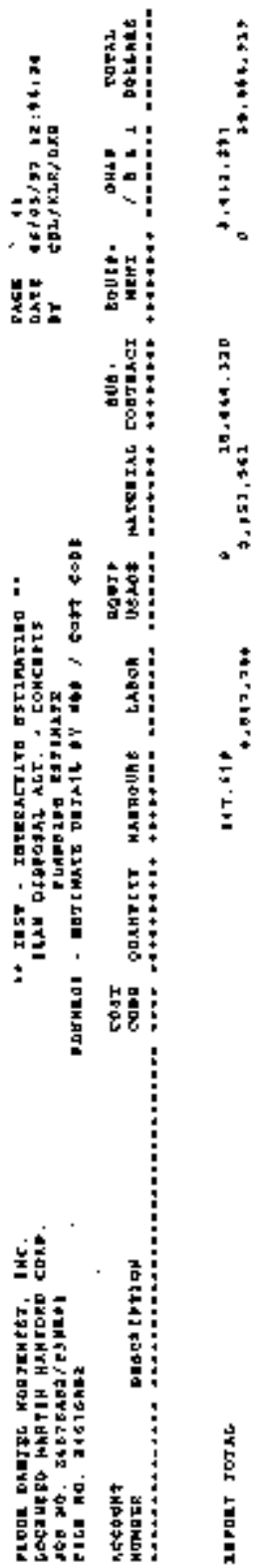


\section{HNF-SD-TWR-AGA-014}

\section{Rev of}

This page intentiondally lefi blank. 


\section{HNF-SD-TWR-AGA-DO4}

Rev 0

Table A-6. Estimated Cosure Costs for Disposal of Remote and Comtach-Handled Imuabilized Low-Actrvity Waste (Cptions 2A, 2B, 2C, and 2D).

Note: The above estunate was developed based on Jone 1997 dollars. It was escalated to September 30,2002 , by using a multiplier of 1.16 beased on jntowrmation from FDHC on compossite labor and materials. 


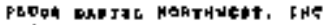
LOSKRESO MKRTIF UABJ OLO COM

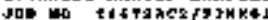

Prat Wo tatialch

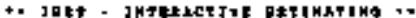

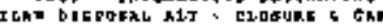

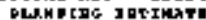

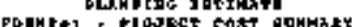

PATH 2 की

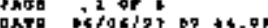

QY COL/KIAROKH

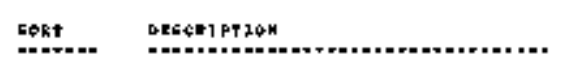

rous

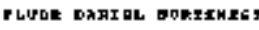

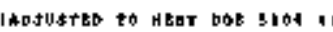

FAOTECI TERKL

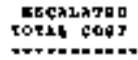

$5,600,004$

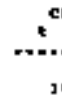

cam I manنंcy

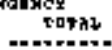

g

1. $54 a, 4 b$

DOLLAF:

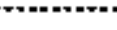

$7,204,010$

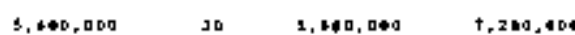

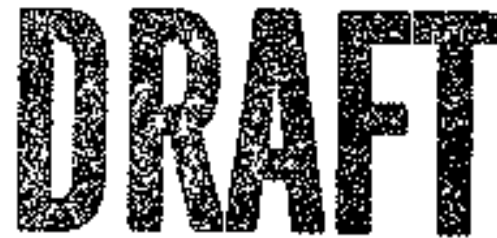

党

the

guNE \&. 392,

Mrakp

COH ETMECT LOH OMLT

porar endip

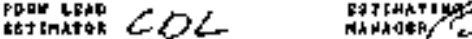

parasca

Mithene

$\operatorname{ctg} \operatorname{sis}$

AhNA-10

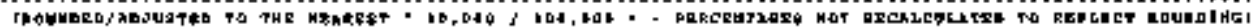

占 


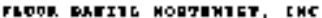

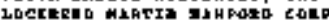

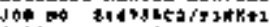

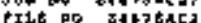

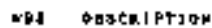

wos pastalprjon

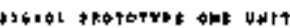

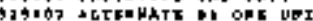

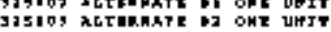

Dow ret rothe

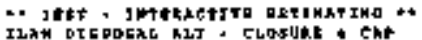

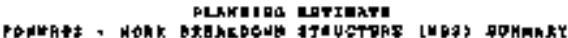

Pran 2 Q.P.

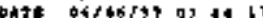

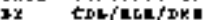

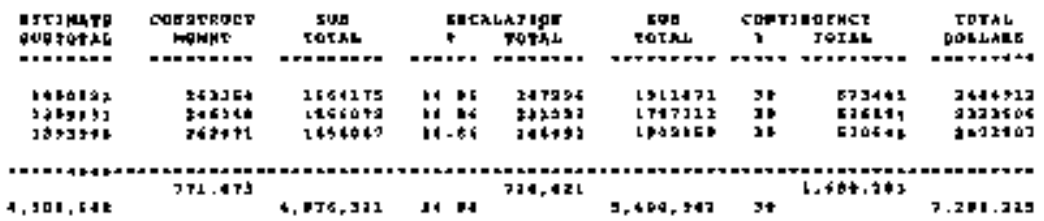


PLYQ́. OARIXL HONJHNEET, INC,

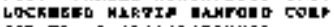
tav Do. 194t+kesiojuKA1

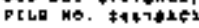

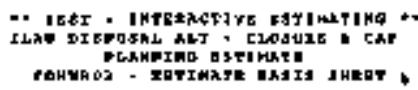

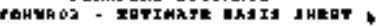

DRG 5 or

phta oistis, 12:41:36

EY CELjKLRIOKH

1. QS3

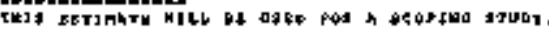

a. ESTIHATE TETHITAL BABI7

h. TH

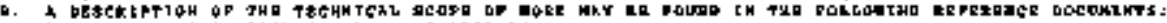

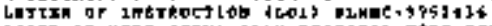

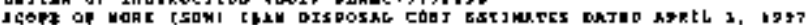

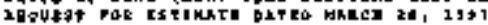

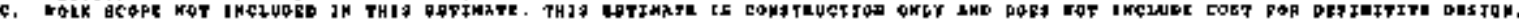

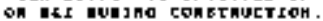

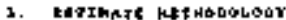

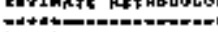

h, binet sofTs,

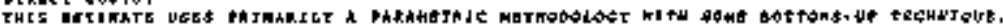

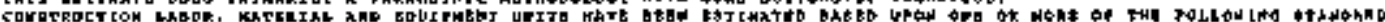

cown

ch (2)

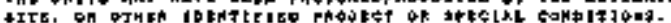

- eriger cost pacroze

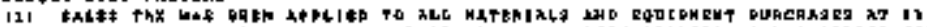

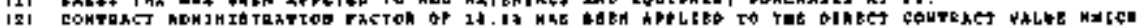

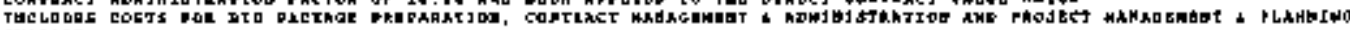
GWH PQRT

C. TRäREI COSTE

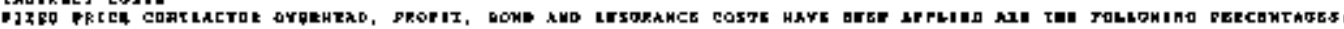

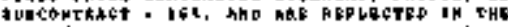

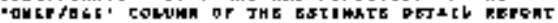

1. Matk

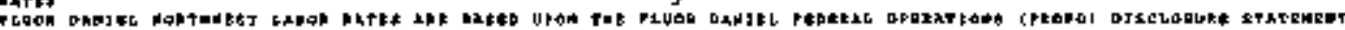
AHQ

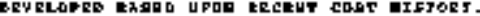


TLUOR OAHIEL MORTMIST, INC.

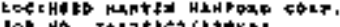
Tot

tCt: Ho, aAs Thic

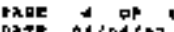

3, a. 38

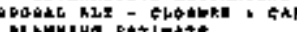

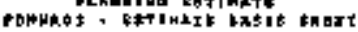

\section{Kacklithol}

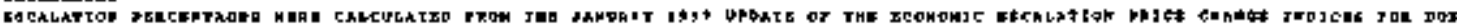

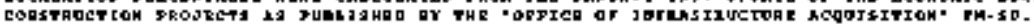

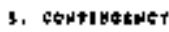

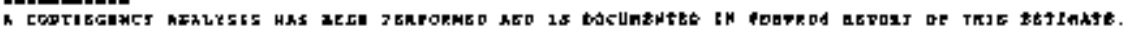

\section{Rmankus}

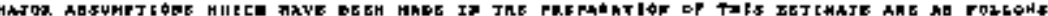

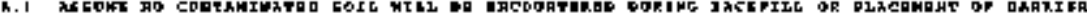
96 [2. POUHOKTI'

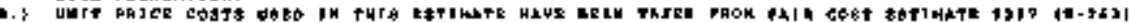

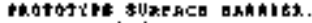

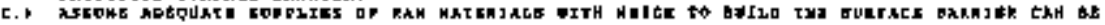

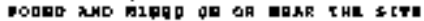

D.) TElo EAT

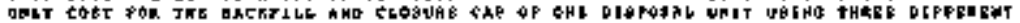
TYGX OT GAKETER:

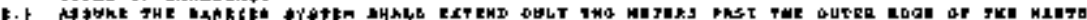

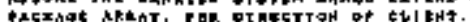




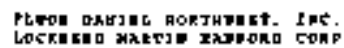

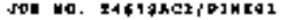

FLI मa. C+69RतC

安䉼

coditiog

Dischitian

4to inpronsmetrs to Lato

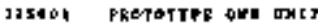
dastiog PLTEInATE EL ONE UIT

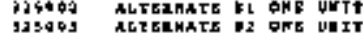
rotht 4

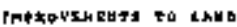

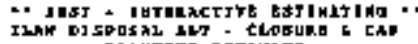

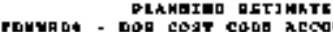

HAOE is OP 1

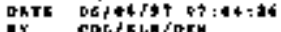

Ir

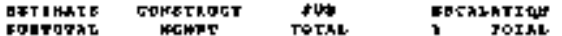

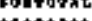

Terst.

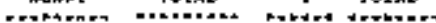

500 COMIIHETHEY

TOThl + TOTAL

TrTA1

Dochlect

\begin{tabular}{|c|c|c|c|c|c|c|c|c|}
\hline $\begin{array}{l}1+401+1 \\
15+4051 \\
13+3+94\end{array}$ & $\begin{array}{l}763754 \\
241214 \\
262011\end{array}$ & $\begin{array}{l}1664115 \\
2656017 \\
1454047\end{array}$ & $\begin{array}{l}19.96 \\
19.84 \\
14.04\end{array}$ & $\begin{array}{l}711296 \\
351230 \\
244030\end{array}$ & 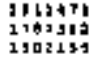 & $\begin{array}{l}34 \\
38 \\
30\end{array}$ & $\begin{array}{l}677415 \\
196194 \\
370414\end{array}$ & $\begin{array}{l}9404+17 \\
23 a 35+4 \\
2472<+1\end{array}$ \\
\hline 1201410 & $1+2,31$ & $1+46+71$ & 14,44 & $23142 x$ & $1+0+517$ & 10 & 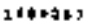 & 7701073 \\
\hline
\end{tabular}

PRALCt tothl 
PBDOR OKRIAL HQHTHEEPT, IDS.

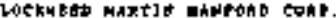
Job HO. IASTSHCZFH 3 WKOI

FILE $00.347 s+C ?$

TE D DESCETIDOM

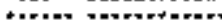

conszederou

12 \$1tดwok

TORhL SOWPTA USTIOH

PROJECT TOThL

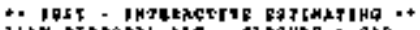

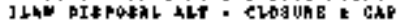
PloHgl got Bstimhte

Whe 4 ar a

DSTE 46/06/D7 $47,41,15$

IT COLJKEJOCE

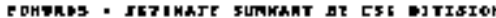

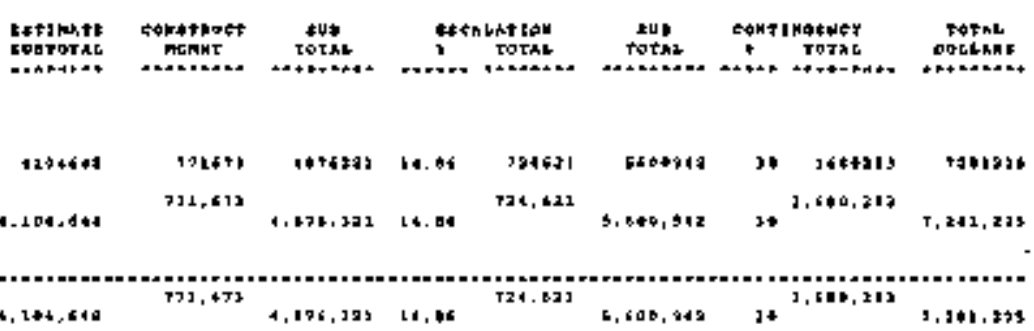


PLUER DKNI EL MoETEMEAT, JME.

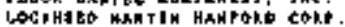
JOQ

rila

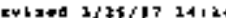

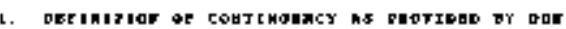

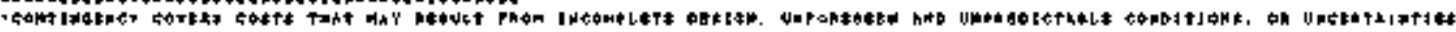

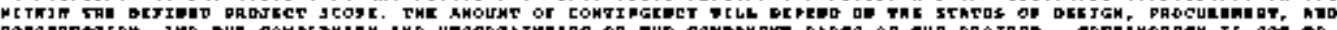

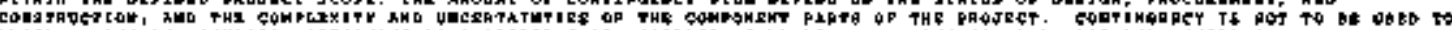

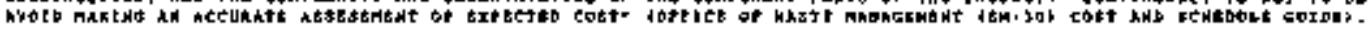

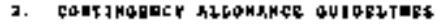

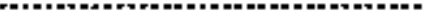

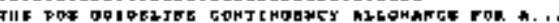

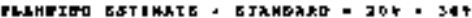

2. mktHobtob

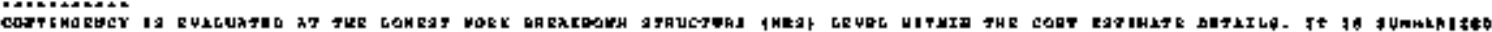

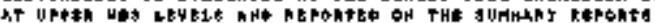

3. SMLCET:

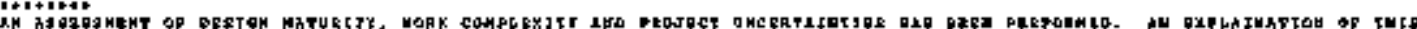

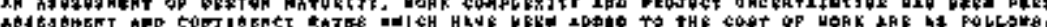

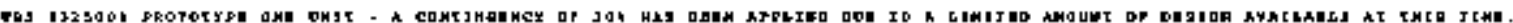

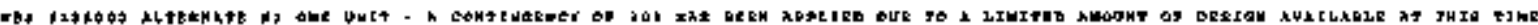

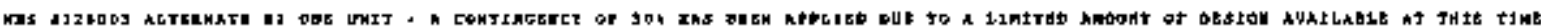




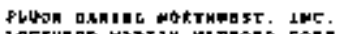

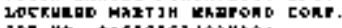

JOB MO. \$1619RCaYATHROA

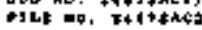

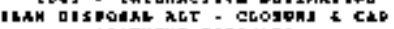

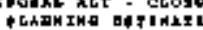

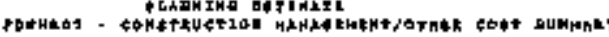

Pack 1 or

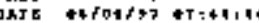

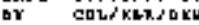

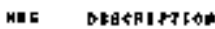

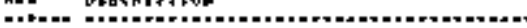

yabad peosotire otr urI

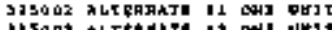

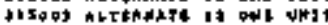

DeOdEC toval

\section{actumke}

9070942

-

3044 23

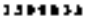

$7+3 \times 194$
Cogatgoct tah mathagmase,
Tothl
ner.
11.14 243354
11.14 54.161
$11.1+$

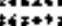

\section{Drkik}

costs

.

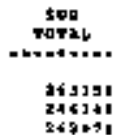

$2463+1$

$262+1$

$0.20 .304,540$

$1+1+4$

$1+1,171$ 


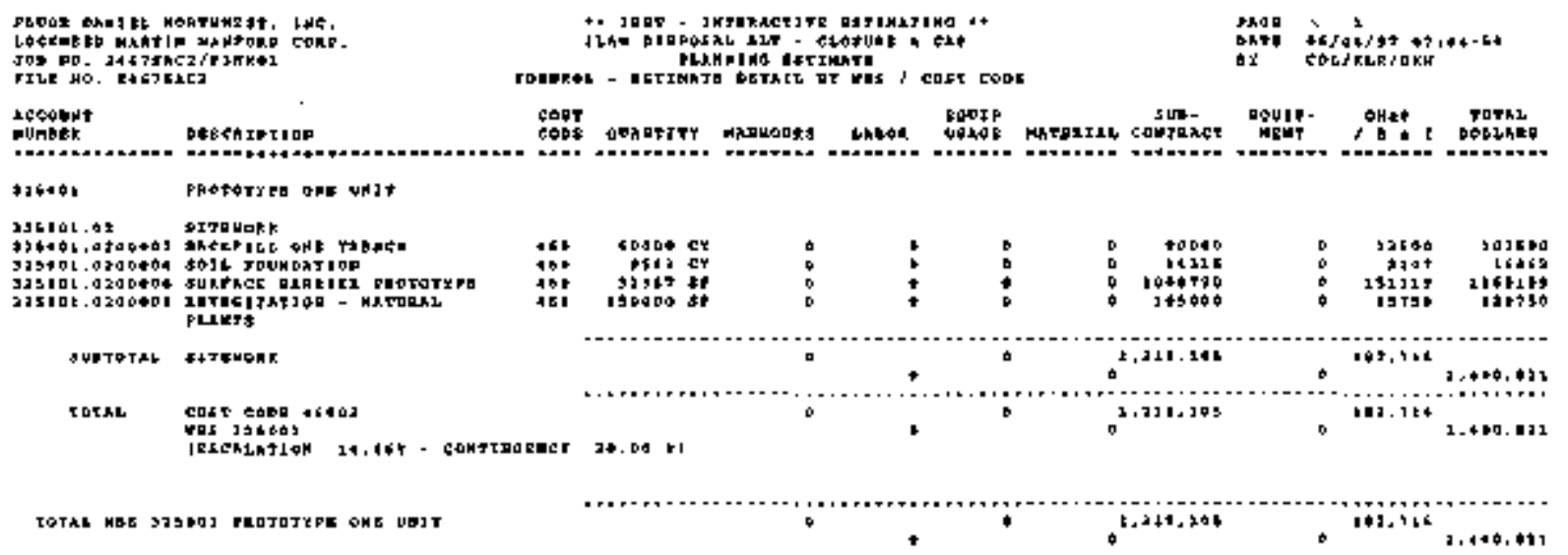




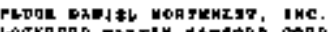

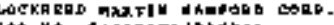

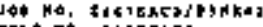

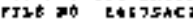

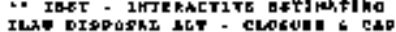
PLNWTWG ditikite

that:

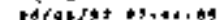

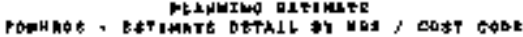

COST Equr

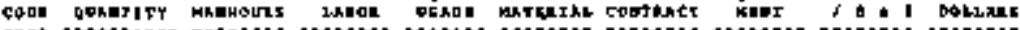

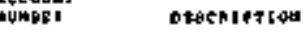

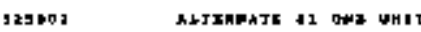

ITSDA. IS ETTEMOAK

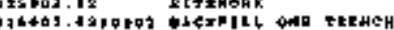

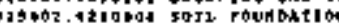

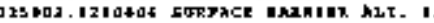

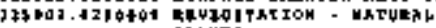

a

$\begin{array}{ll}0 & 0 \\ 0 & 0 \\ 0 & 0 \\ 0 & 0\end{array}$

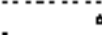
alnint

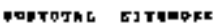

rotse

6 6at 000216002

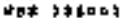

45 TCRLPTION

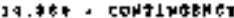

cotod cr

QSil et

3at? $\mathrm{cr}$

נab4

(-2)-

10.0.

,

$\therefore$

(1.2.2......

1.134 .94

$\begin{array}{ll}0 & 24010 \\ 0 & 14315 \\ 0 & 171659 \\ 0 & 109010\end{array}$

i...-

170.047

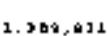

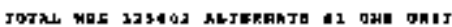

0
(1) b. bld, tal
130,40 
TLUOK DASTEL HORTH MEST, EMC.

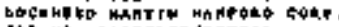

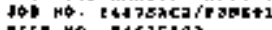

rell ho. J46iescr

Recoum?

NUMO EP

DESGRTPT JOH

325003

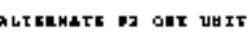

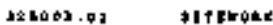

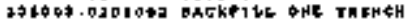

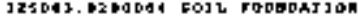

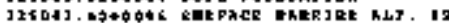

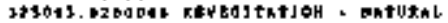

$$
\text { DLRFis }
$$

PHaTOTAL EIJKHAPh

Tothl cost coon tobar

$1+1 \neq 0+1$

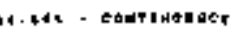

st. p.

+d JUET - JOTERETIY ISTIMLIIHO -

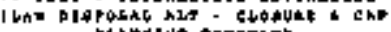

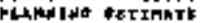

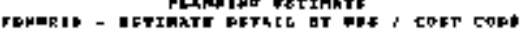

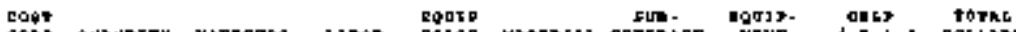

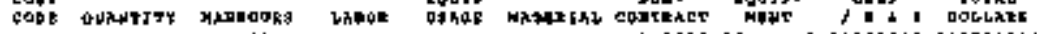
n.e. - .

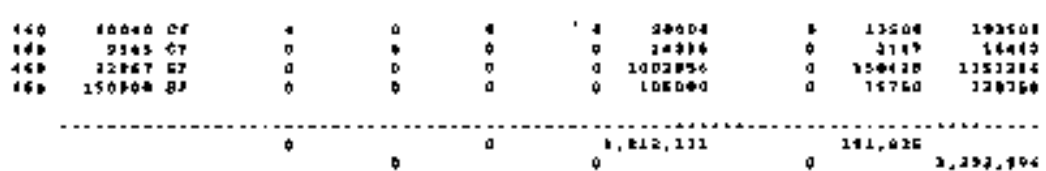

a

0 a $1.26 a 10 b$

$1+1.029$

$3,23,104$

1. 33,936

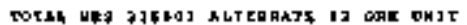

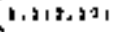

o
औ $1, \notin j 6$

1, 253. 938 


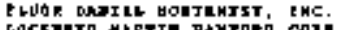

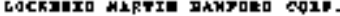

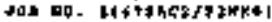

PTLE Ho. tortides

account

ank:

DAtकkg T T

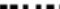

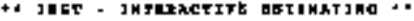

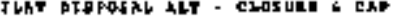

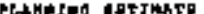

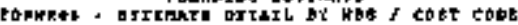

cose

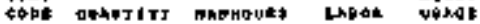

1 पाँ -

1un-

migit

rath

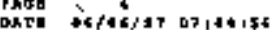

DF CQL $/$ KLAROKH hespht tork1

口

3.963 .260 
Table A-7. Summary of Estimaut Staif Requiresrents for Renote-Fandled Immobilized

Low-Actipity Waste Containter Disposal Phase I and Phase If (Options 2A ald 2B).

\begin{tabular}{|c|c|c|c|c|c|c|c|}
\hline Profiltion & Typt & plase & Three shifis & & Pfunst & \multicolumn{2}{|c|}{ erra bffitís } \\
\hline Rerade Hand ad Whatlo & & 1 & 2 D*aposal thitag & & II & \multicolumn{2}{|c|}{4 Dtapxeal thints } \\
\hline 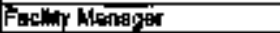 & H & & -1 & & & 1 & \\
\hline Eleak & 6 & & 6.5. & & t & 7 & \\
\hline \multirow{2}{*}{ Fand Enginger } & $\mathbf{E}$ & & 1 & 2.5 & & I) & 3 \\
\hline & & . & & & & & \\
\hline 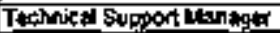 & 4 & & नां & & & 5 & \\
\hline Cleork & G & & 0.65 & & & 1 & \\
\hline Pbennerrecheduler & $\boldsymbol{P}$ & & (4.25 & & & 1 & \\
\hline 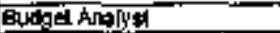 & $P$ & & 0.2 守 & & & i & \\
\hline \multicolumn{8}{|l|}{ Trapining cotoldintor } \\
\hline Tralner & $\bar{P}$ & & 业 & & & 1. & \\
\hline Próesdura Whiter & $\mathrm{P}$ & & 05: & & & 1. & \\
\hline Elextrket Entinetr & $\bar{E}$ & & 1 & & & 1: & \\
\hline Em. Compl En:ahnar & $\mathrm{E}$ & & प5] & & & 1] & \\
\hline HEC Enginger & $\bar{E}$ & & D.s. & & & 1) & $=$ \\
\hline Alachanical Enginger & E & & 1 & & & 4, & \\
\hline 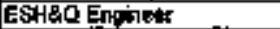 & $\mathbf{E}$ & & 1 & & & 1: & \\
\hline \multirow[t]{2}{*}{ OĆ Inspector } & $\mathbf{T}$ & & 3 & 11.6 & & ह1 & 20 \\
\hline & & & & & & & \\
\hline Frodurdion Mandgat" & 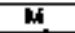 & & 1 & & & 1 & \\
\hline 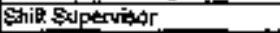 & $\mathbf{k}$ & & 3 & & & 10 & \\
\hline Dociment Cles $x$ & 直 & & 1 & & & 2 & \\
\hline 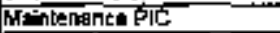 & $\mathbf{p}$ & & 1 & & & 5 & \\
\hline D\&s Panner & $F$ & & 1: & & & 3) & \\
\hline 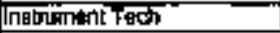 & $T$ & & 2⿺⿻一𠃋十斤 & & & 2 & \\
\hline Evotriciants & ç & & 2 & & & 4 & \\
\hline 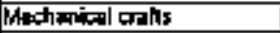 & ç & & b] & & & 10 & \\
\hline Thwolghts & $c$ & & 3 & & & 20 & \\
\hline Proposs Dparalces & $\mathbf{R}$ & & 6! & & & 201 & \\
\hline HPTs & $T$ & & $3 !$ & & & 10 & \\
\hline Ponete detritors & $\boldsymbol{R}$ & & 3 & & & s & \\
\hline Crand Detoptors & $\mathbf{R}$ & & Ef & & & 25 & \\
\hline Truck Ditrecth, HE & $\mathrm{C}$ & & को & & & 25 & \\
\hline \multirow[t]{3}{*}{ Ferklit Driners } & C & & Di & 44 & & of & 144 \\
\hline & & & SE. & 58 & & 16: & $15 ?$ \\
\hline & & & 1 & & & & \\
\hline Mlartaptets & $\mathbf{M}$ & & $8^{-}$ & & & 13 & \\
\hline Erginç̣tara & $E$ & & 8 & & I & 91 & \\
\hline Prefeesterns's & $\vec{P}$ & & $4^{r}$ & & $\perp$ & 14 & \\
\hline Techinkenns & $T$ & & $\bar{B}$. & & . & 177 & \\
\hline Coretita & C & & It] & & & का & \\
\hline Oparakst & $\mathbf{R}$ & & $15 i$ & & & 501 & \\
\hline \multirow[t]{2}{*}{ Gik; } & $G$ & & 3 & & & 䙷 & \\
\hline & & & 59: & & I & 167 & \\
\hline Exphy & Wh & & $15^{\circ}$ & & & 39: & \\
\hline 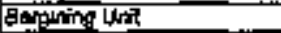 & फs & & 32 & & & 1D舟) & \\
\hline \multirow[t]{2}{*}{ Pun exeringlinon bagg } & WB & & $11 \mathrm{j}$ & & & 221 & \\
\hline & & & 36. & & i & 16त्र & \\
\hline
\end{tabular}




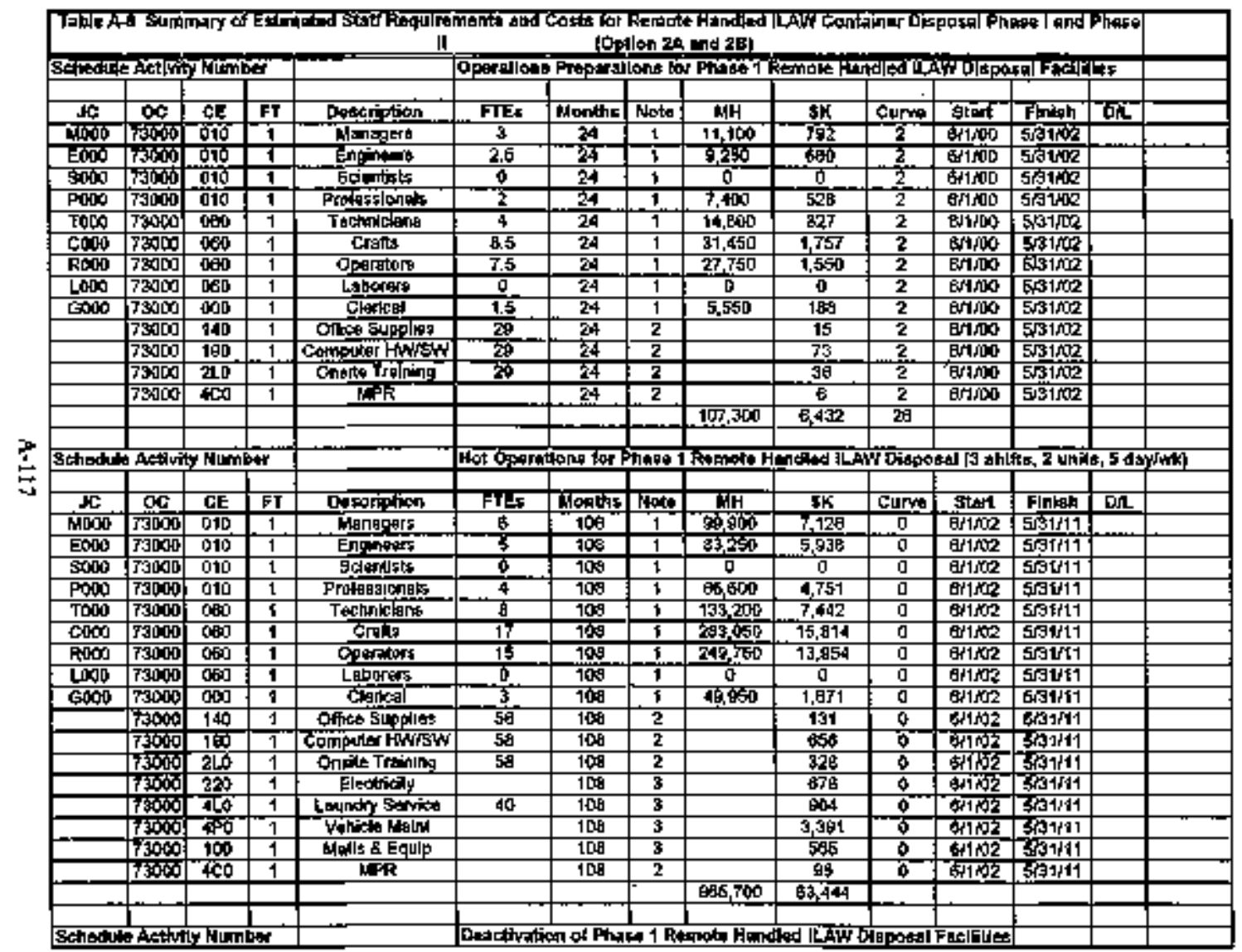




\begin{tabular}{|c|c|c|c|c|c|c|c|c|c|c|c|c|c|c|}
\hline \multicolumn{14}{|c|}{ 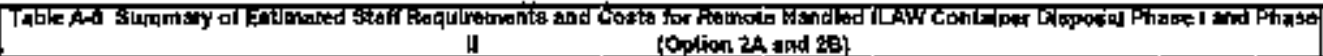 } & \\
\hline 鸟 & क्ट & E्क & FT & 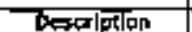 & PTEs & Montha & NDole & $\mathbf{T H}$ & $\bar{F}$ & cure: & start & Fाn & Dद & \\
\hline DWFoto & Yर्णिए & की0 & 1 & * & 3 & $\sqrt{2}$ & 1 & 750 & 806 & 3 & E्राt1 & इस3नाय & & \\
\hline EOT) & 73000 & 010 & 1 & Enolnoers & 2.5 & $\sqrt{2}$ & 1 & 4025 & 330 & $\frac{5}{3}$ & बसमा & $5 \sqrt{3142}$ & & \\
\hline $\sin 00$ & 73000 & 010 & $\frac{1}{1}$ & Bxithesta & 0 & 12 & 1 & 0 & D & $\frac{2}{3}$ & 6N1+11 & 5/42 & & \\
\hline$P 000^{\circ}$ & 73000 & 010 & 1 & Prolestingals & 2 & 12 & 1 & 3.700 & 28 & 7 & BMtII & $5 \times 31 / 2$ & & \\
\hline Tित्र० & $73 \times 100$ & DOO & 1 & Jestnictaly & 4 & 12 & 1 & 1.400 & A13 & 3 & aris1 & $531 / 12$ & & \\
\hline 0000 & 73000 & 000 & 1 & Crgits & 85 & $\sqrt{2}$ & 1 & 73.75 & 878 & 3 & QMN1 1 & $5 \sqrt{31} \times 12$ & & \\
\hline RaD? & 73000 & $0 \geqslant 0$ & 1 & oparators & 7.5 & $\sqrt{2}$ & $\overline{1}$ & 13.575 & 75 & 3 & हलता & 53142 & & \\
\hline LONO & 73000 & 10 & $\frac{1}{1}$ & Letorers & 6 & $\sqrt{12}$ & 1 & 0 & 0 & 3 & aicliti & $5 \sqrt{3172}$ & & \\
\hline \multirow[t]{7}{*}{ G0खा } & $73 \times 100$ & DID & $i$ & Clarica & 1.5 & 12 & 1 & 2,775 & 93 & 73 & बrill & $5 \sqrt{31 / 12}$ & & \\
\hline & 73000 & $\mathbf{2 0}$ & 1 & Conste Tralning & 29 & 12 & 2 & & 98 & 3 & $\theta+1 \mathrm{H} 1$ & $5 \sqrt{31 / 12}$ & & \\
\hline & 73000 & 200 & 1 & Eotricity & & $\sqrt{2}$ & 3 & & 25 & 3 & 6M/A1 & $5+36 / 12$ & & \\
\hline & 73000 & ماه & 1 & Loundy Servicet & 20 & 12 & 3 & & 50 & 3 & AHMT & SRIt12 & & \\
\hline & 73000 & $4 C 0$ & $\mathbf{1}$ & KFPR & & 12 & 2 & & 0 & 3 & APIMT & 5rat/t? & & \\
\hline & & & & & & & & 53,650 & 3,243 & & & & & \\
\hline & & & & & & & & & & & & & & \\
\hline \multicolumn{4}{|c|}{ 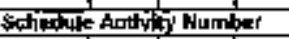 } & & \multicolumn{9}{|c|}{ 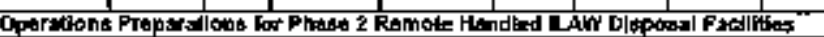 } & \\
\hline & & & & & & & & & & & & & & \\
\hline $\mathbf{I C}$ & OC & CE & FT & Deteription & TTE* & Monthe & Wote & T्रr & $\mathbf{\$ K}$ & Corve & Stant & Finith & Oالㄴ. & \\
\hline $1000^{\circ}$ & 73000 & 010 & 1 & Milangers & 6.5 & 24 & 1 & 24, प्SE & 1,715 & 2 & छा & ABQD1 & & \\
\hline$=0$ & 80000 & 和 & 1 & En: & 4.5 & 24 & 1 & 18,650 & $1,180^{4}$ & $\frac{7}{2}$ & 5970 & 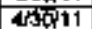 & & \\
\hline हैँ & 73000 & 010 & 1 & Sccintlists & 0 & 24 & I & 0 & 0 & 2 & 5409 & ABQ11 & & \\
\hline P地, & 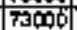 & 010 & 7 & Professiants & 7 & 24 & 1 & 25,800 & 1,947 & 2 & DNEROA & $4 \times 3011$ & & \\
\hline अणुण & 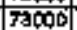 & 080 & 1 & Tochmitiant & 8.5 & 29 & 1 & 31,450 & 1,757 & 2 & $51 \times 9$ & Aracr1 & & \\
\hline दरOOO & $7 a x+0$ & De्ष0 & 1 & Crals & 29.5 & 24 & $\mathbf{1}$ & 102,150 & 800 & 2 & 51108 & dPar11 & & \\
\hline 8000 & 73000 & $\theta$ & 1 & Opartatsts & 25 & 24 & 1 & 82,500 & 5,168 & $\frac{7}{2}$ & 5 रारण & $4 \sqrt{30}[11$ & & \\
\hline LOO' & 73000 & 000 & 1 & Labortars & 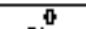 & 24 & 1 & D & 0 & 2 & 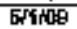 & $4 \sqrt{30}-1$ & & \\
\hline काष & 73000 & 000 & 1 & Cleriogal & 2.5 & 24 & $\frac{1}{4}$ & 8250 & 310 & 2 & इस1, & Aअव 1 & & \\
\hline & 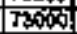 & 140 & 7 & Ofikes Sufpitat & 83.5 & 24 & 2 & & 42 & 2 & 5 प100 & Aसम2011 & & \\
\hline & 730000 & 790 & 1 & Comporer HWWEN & 83.5 & 24 & 2 & & 210 & 2 & Eनत्प & 4 काष्या1 & & \\
\hline & 73000 & $\mathbf{2 1 0}$ & 1 & Onsilı Training & 83.5 & 24 & 2 & & 105 & 2 & Eनसम & $4 \sqrt{30}+1$ & & \\
\hline & Tअण00 & 400 & 1 & NPR & & 24 & 2 & & to & 2 & 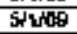 & $4 \sqrt{3} / 1$ & & \\
\hline & & & & & & & & उOB,960 & 18,450 & & & & & \\
\hline
\end{tabular}




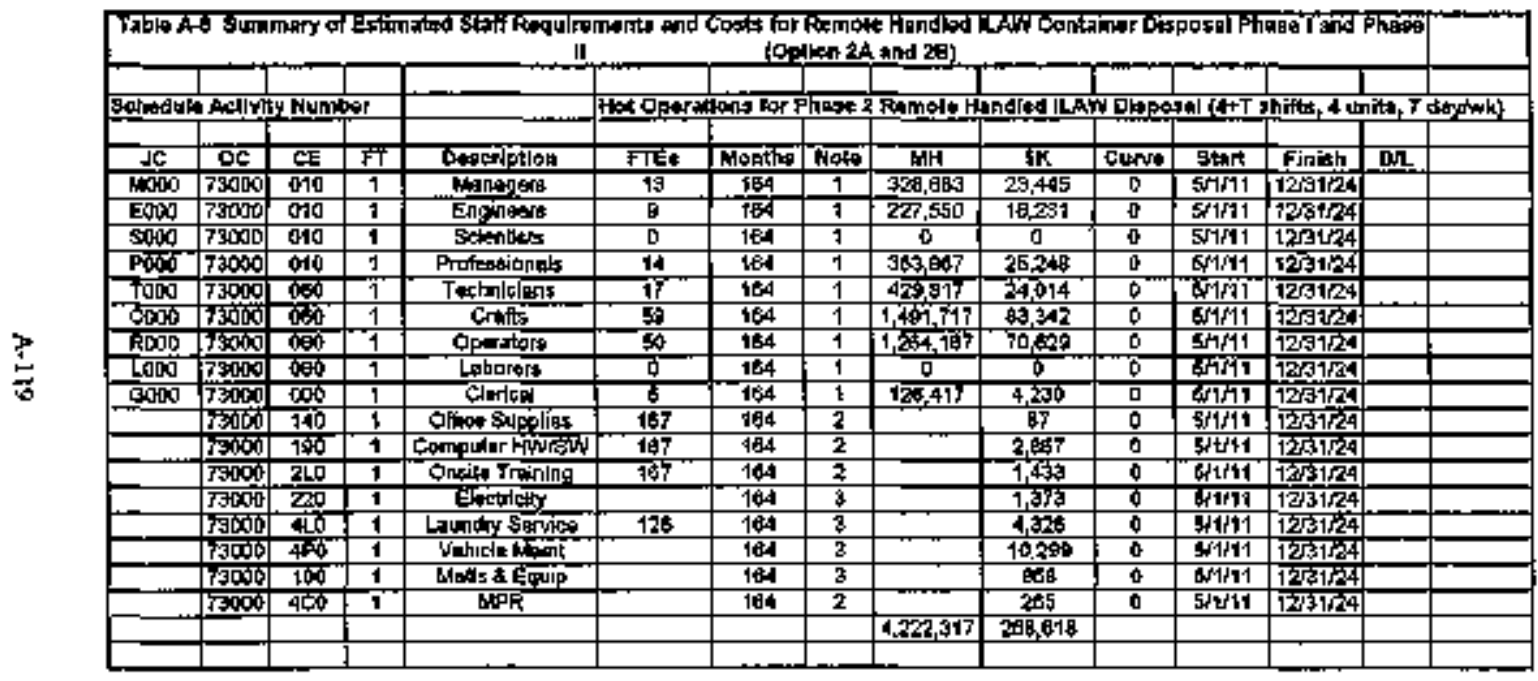




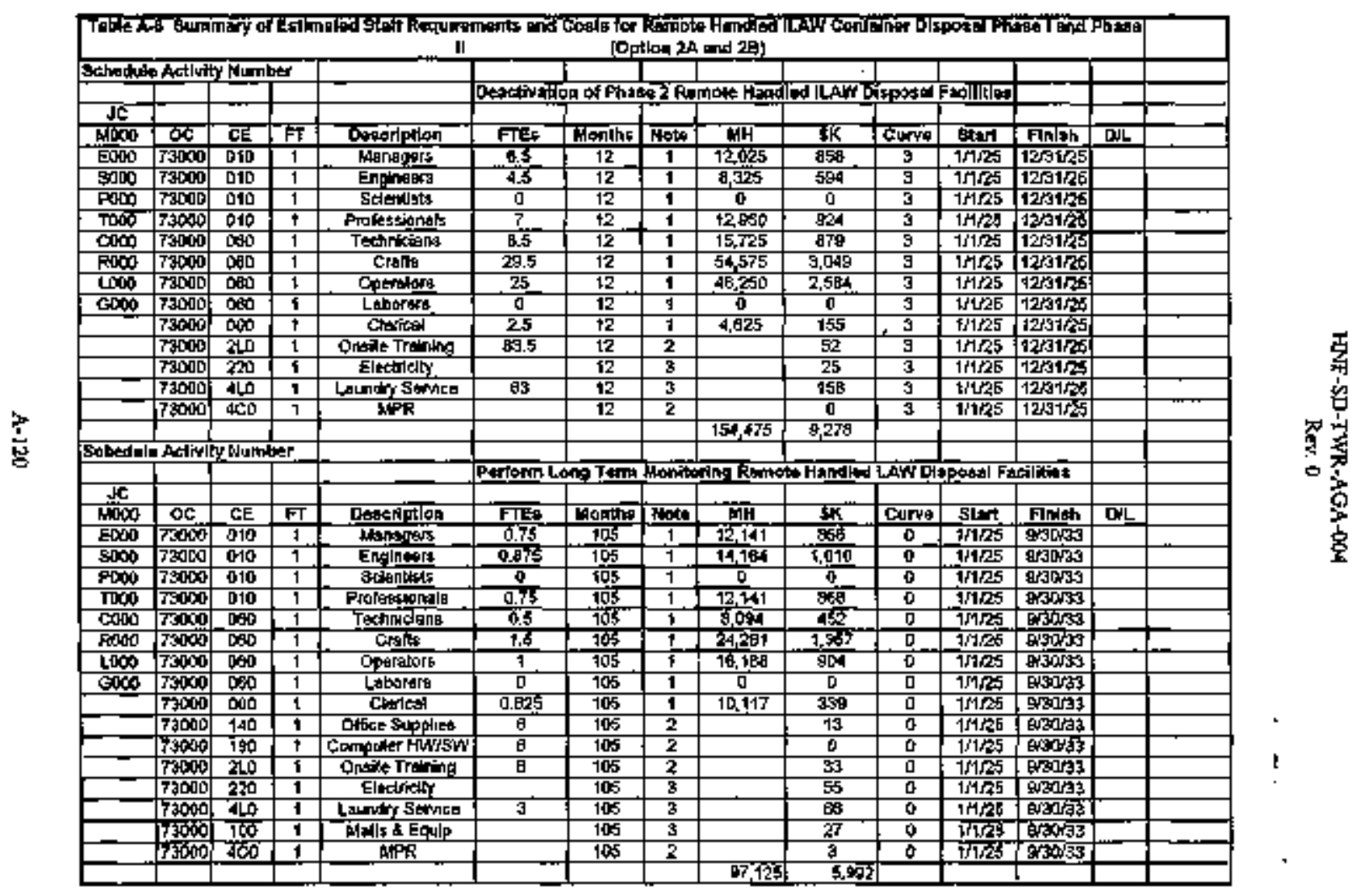




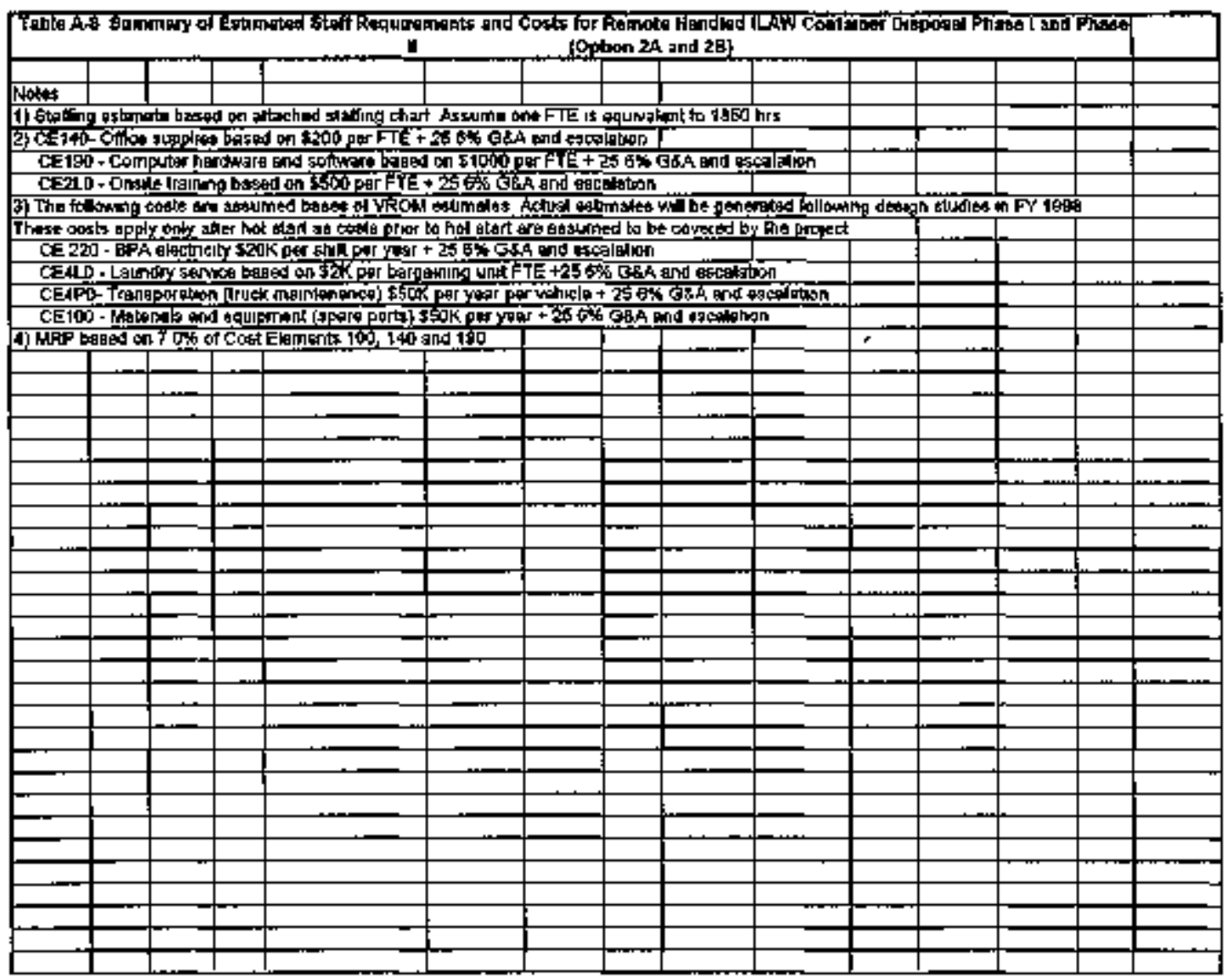


Table A-9. Surnmary of Estimated Staff Requirements for Conkact-Handled Immobilized Low-Activity Waste Storage and Disposal Phase I and Phase I (Options 2C and 2D).

\begin{tabular}{|c|c|c|c|c|c|c|c|}
\hline Posittipn & Type & Fhage & Thres Shifts & & Pheset & Three Sh & hE姆 \\
\hline Conlact Handleof Wabte & & 1 & 1 Storgag Unit & & ॥ & \$ Disposel U & Undite \\
\hline Faxdlity Whanager & $M$ & & 1 & & & 1 & \\
\hline Clęrk & 它 & & 0,5 & & & 0.5 & \\
\hline Plart Ënglneef & E & & 11 & 2.5 | & & 1 & $\overline{25}$ \\
\hline & & & & & & & \\
\hline Fechnical Bupport Meneger & B4 & & 1 & & & 1 & \\
\hline ctork & $\mathrm{G}$ & & $\overline{0} .5$ & & & 0.5 & \\
\hline Planner'schaduler & $\mathbf{P}$ & & 0.25 & & & 0.25 & \\
\hline Euxdget $\overline{A n}$ Blyst & $\mathbf{P}$ & & $0 . \overline{25}$ & & & 0.25 & \\
\hline Training Coometintioc & $\underline{G}$ & & 1 & & & 1 & \\
\hline Tringer & $\bar{P}$ & & 1 & & & 1 & \\
\hline Procedur Writer & P & & 0.5 & & & 0.5 & \\
\hline हlectrical Englneer & $\bar{E}$ & & 1 & & & 1 & \\
\hline Env, Compl. Engingar & $\mathbf{E}$ & & 0.5 & & & 0,5 & \\
\hline ISC Englnaer & $\bar{E}$ & & 0.5, & & & 0.5 & \\
\hline Moctianical Ergingetr & E & & t & & & 1 & \\
\hline 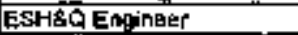 & E & & $\mathbf{1}$ & & & I & \\
\hline QC Inspector & $T$ & & 3 & 12 & & 3 & 11.5 \\
\hline & & & & & & & \\
\hline PToduclan Manger & in & & 1 & & & 1 & \\
\hline Shift Supenisot of & $m$ & & 3 & & & 可 & \\
\hline 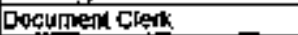 & $G$ & & 1 & & & 1 & \\
\hline Makndanance PIC & $\vec{p}$ & & 1 & & & 2 & \\
\hline OSMPlanner_- & $\mathrm{P}$ & & 1 & & & 3 & \\
\hline Instrument Tech & $T$ & & 2 & & & 2 & \\
\hline Elediric|ang & $\mathrm{C}^{-}$ & & 2 & & & 2 & \\
\hline Hechan & $\bar{c}$ & & 3 & & & B & \\
\hline Prpocasa opersaloss & $\mathbf{R}$ & & 3 & & & 8 & \\
\hline HPTs & $\bar{T}$ & & 3 & & & $\ddot{\mathbf{B}}$ & \\
\hline Poppar Cporalops & $\mathbf{R}$ & & 3 & & & 3 & \\
\hline Сгапе Gperalors & $\overline{\mathbf{R}}$ & & 0 & & & $\overline{0}$ & \\
\hline Trupk Drivets, HO & $\underline{c}$ & & 4 & & & 12 & \\
\hline Forkilfin Drtwers & $\mathrm{c}$ & & 4 & 31 & & 12 & 59 \\
\hline & & & 45 & 46 & & 73 & 3 \\
\hline & & & & & & & \\
\hline Manaosis & $M$ & & 6 & & & 是 & \\
\hline Enploesta & $\bar{E}$ & & 5 & & & 5 & \\
\hline Propepsisonal & $\mathrm{P}$ & & 4 & & & 7 & \\
\hline TEchnicans & $F$ & & 8 & & & 11 & \\
\hline Crofis & C & & 13 & & & \$ي & \\
\hline Opertitors & $\mathbf{R}$ & & 6 & & & 9 & \\
\hline C9erke & G & & 3 & & & 3 & \\
\hline & & & 45 & & & 79 & \\
\hline & & & & & & & \\
\hline Exकmpt & W7 & & F5 & & & 18 & \\
\hline 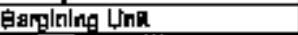 & Wh & & 1司 & & & $\$ 1$ & \\
\hline 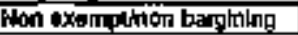 & Wh & & 11 & & & 14 & \\
\hline & & & 45 & & & 73 & \\
\hline
\end{tabular}



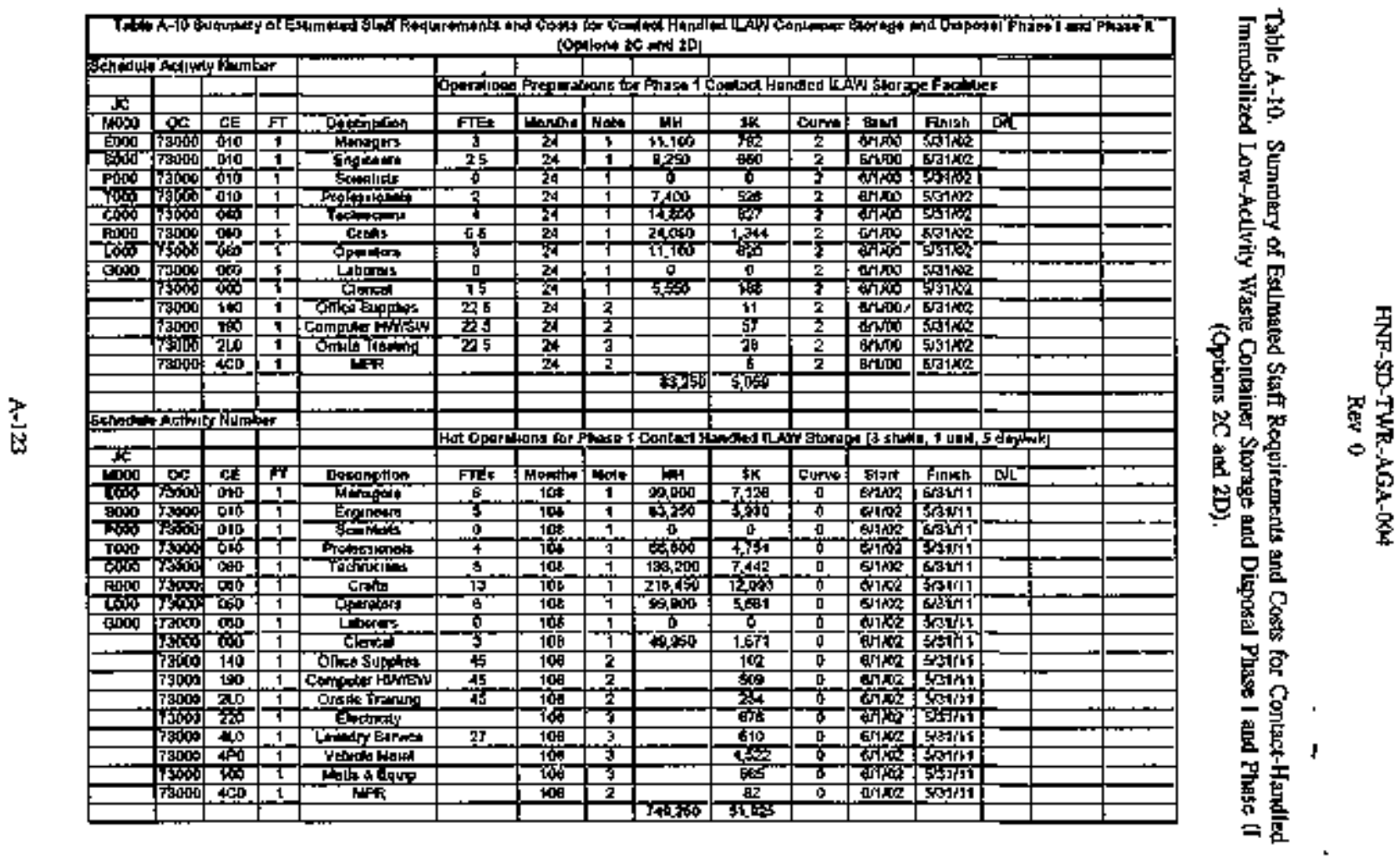


\begin{tabular}{|c|c|c|c|c|c|c|c|c|c|c|c|c|c|c|c|}
\hline \multicolumn{4}{|c|}{ 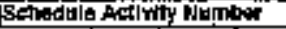 } & & & & & & & & & & & & \\
\hline & & & & & \multicolumn{7}{|c|}{ 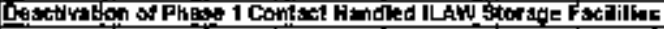 } & & & & \\
\hline 点 & & & & & & & & & & & & & & & \\
\hline जिए & $\mathbf{O C}$ & CE & ET & Dexariptian & MTs & Monthe & Wote & MH & $\$$ & tack: & \$क्षारे & 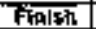 & DL & & \\
\hline E 000 & 300 & 610 & 7 & 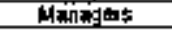 & 3 & 12 & 7 & $5,6600^{-}$ & 290 & $3^{-}$ & इसा11 & झुजार & & & \\
\hline sim & 73000 & ถิ10 & $\mathbf{1}$ & Entinter & 2.6 & 12 & 1 & 4,625 & 130 & 3 & 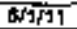 & उता/2 & & & \\
\hline PO000 & 73000 & 010 & 1 & Sconilutets & 0 & 12 & 1 & 0 & 0 & $\overline{3}$ & $8 / 9611$ & $8 / 31 / 2$ & & & \\
\hline$T 0, \infty$ & 75000 & 90 & 1 & Prufessionsle & 2 & 92 & 1 & 3,300 & 284 & 3 & E/1T & 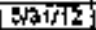 & & & \\
\hline$C(x) 0^{\circ}$ & $\gamma \$ 000$ & 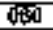 & 1 & Tectun|cistents & 4 & $\sqrt{2}$ & 1 & 3,400 & 413 & 3 & BलMF & इअगयर & & & \\
\hline ROOD & 73000 & 0 & 1 & Crastos & 8.5 & 12 & 1 & 12,025 & 872 & 3 & EMM1 & $5 \sqrt{31} 142$ & & & \\
\hline 100 & 73000 & $0 \Leftrightarrow 0$ & 1 & Cophitomt & 3 & 12 & 1 & 5,550 & 310 & $\frac{3}{3}$ & एखया & \$\$3 & & & \\
\hline 5000 & Isonos & $\infty$ & 1 & Labandis & 0 & 12 & 1 & 0 & 0 & 3 & सा & बराप1 & & & \\
\hline & 5000 & 000 & 1 & clopical & 1.5 & 12 & 1 & $2 \pi 5$ & 实 & 3 & सारा & क्षारा & & & \\
\hline & 73000 & $2 \mathrm{LO}$ & 1 & Ongte Train̈ritg & 22.5 & $\sqrt{2}$ & 2 & & 14 & 3 & Erlf17 & Eखा1 & & & \\
\hline & Pand & 220 & 7 & Eloctinthy & & 72 & 3 & & $2 \mathrm{~B}$ & 3 & EMST & 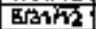 & & & \\
\hline & 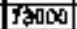 & ALE & 1 & Laundry Banices & 13.5 & 12 & 3 & & 34 & 3 & ब्ञागी & 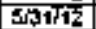 & & & \\
\hline & 3300 & 40 & 1 & MPR & & 12 & 2 & & 0 & 3 & बाग & एक्षार & & & \\
\hline & & & & & & & & 41,日面 & 2561 & & & & & & \\
\hline
\end{tabular}




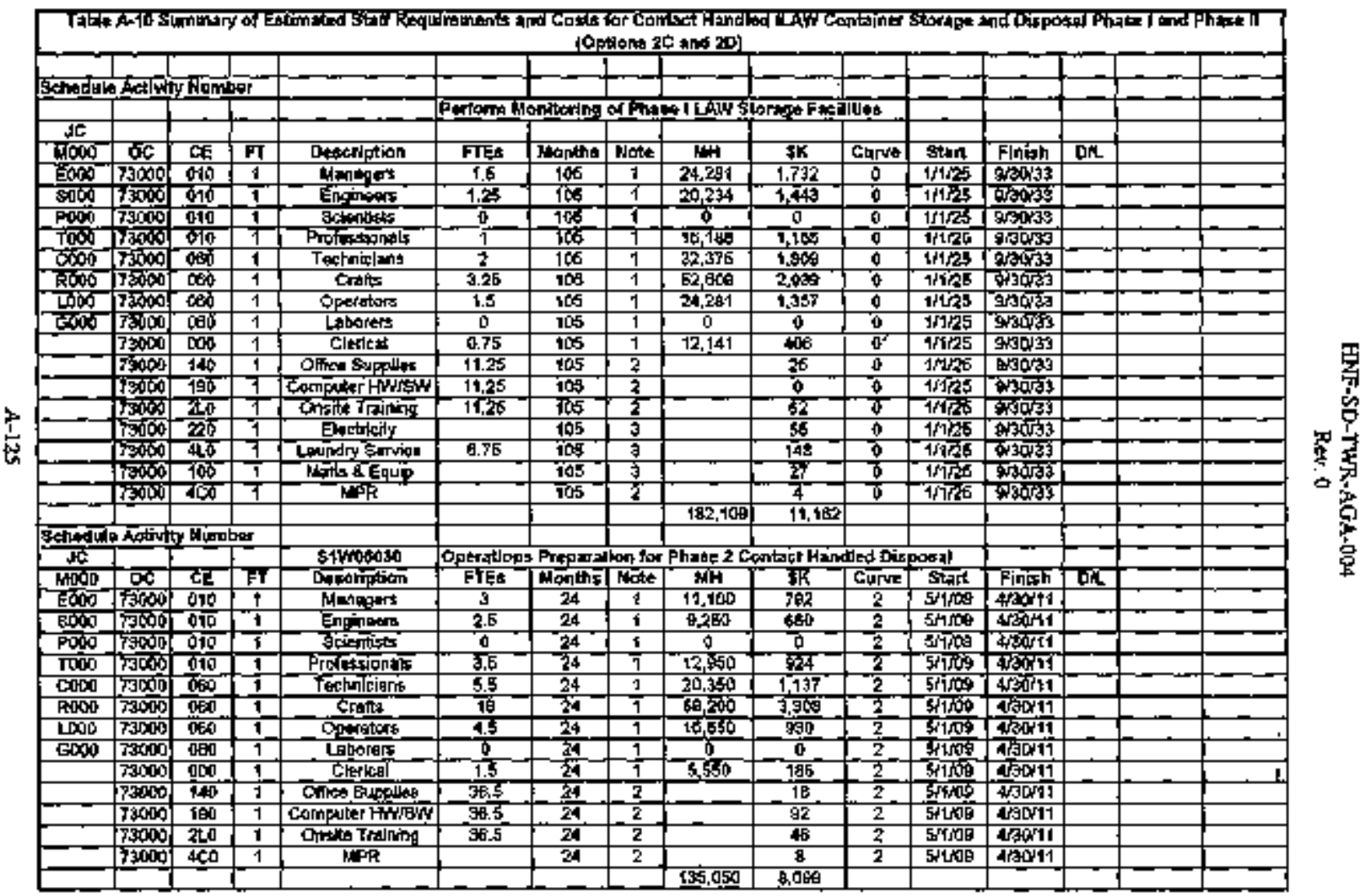




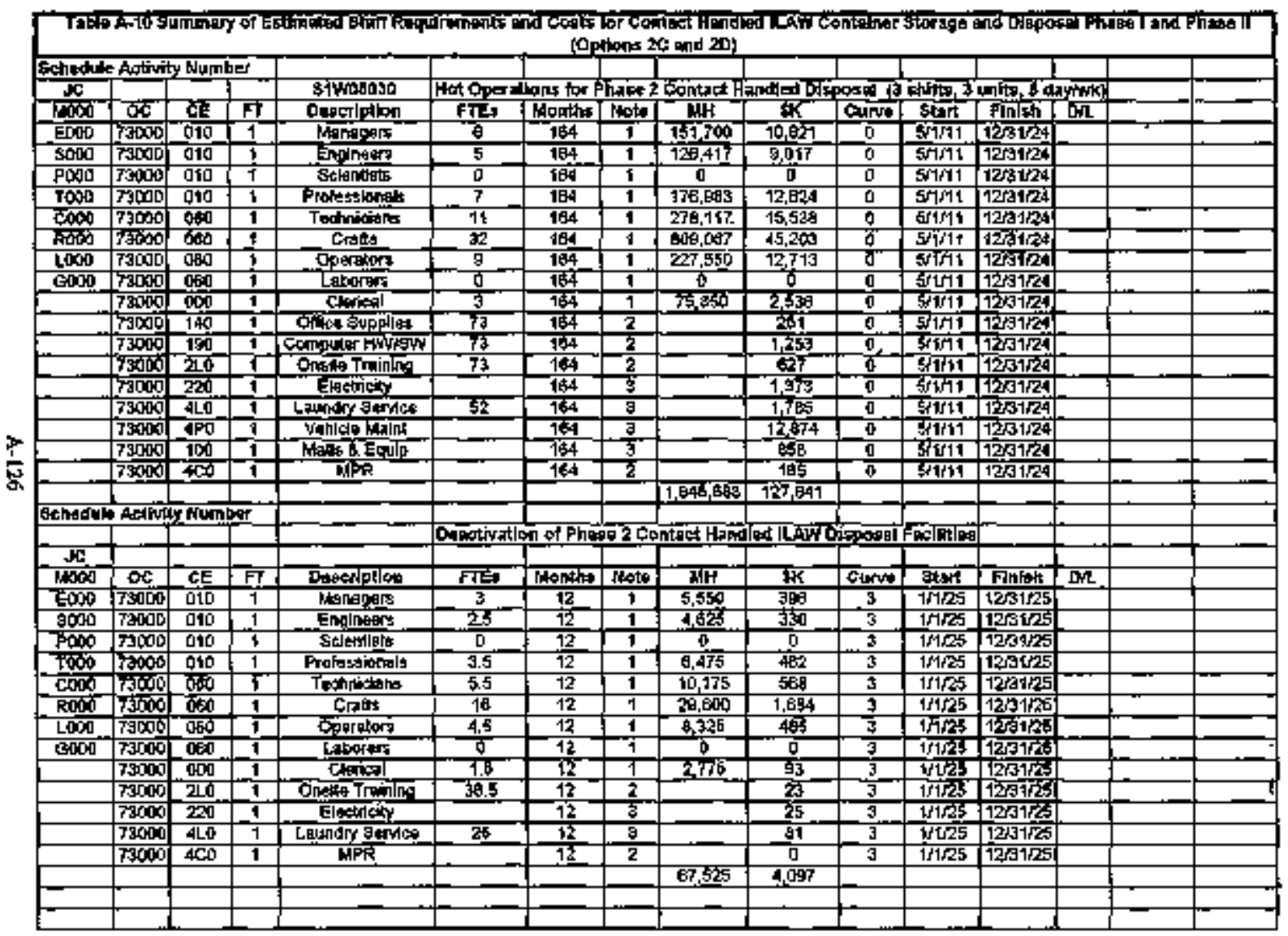




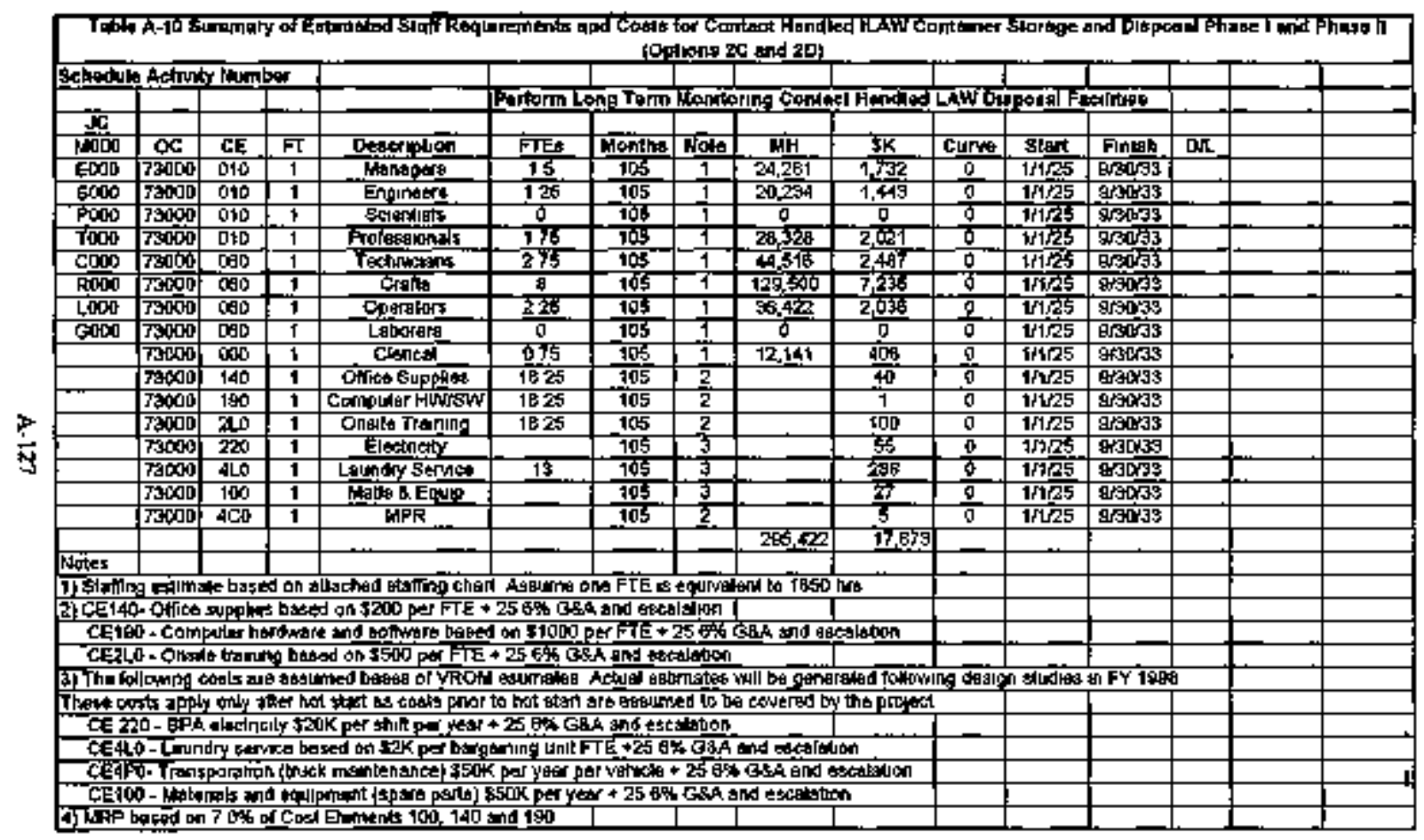


HNF-SD-TWR-AGA-004

Bey. 0

This parps inkendonally keft blank. 
Rev 0

\section{A2.2 SNGLESHELL TANKS (ONTION 3)}

Table A-11. Summary of Estimaled Corsifuction Costs for Addition of One 12-Font Diarieter Opening w the Cender of a Single-Shell Tank Based on Diamond Wine Cutting Technolozy (Option 3).

Note: The aborve astimate was derckped hased on Auguat 1990 dollars. For Tahle 5-8, demolition cost was escalptod to Septernber 30, 2002, by using multipliers of 1.26 (bared on

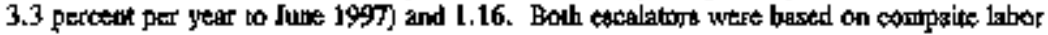
and materials information from FDHC. Sec Figures A-I though A-4 tor tectiniqulte. 
THISE EN TIHE ENS MAHFOAP

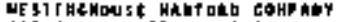

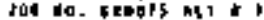

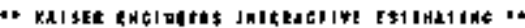

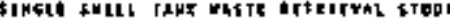

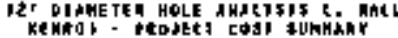

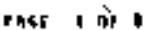

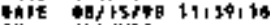

4Y cbitult

\begin{tabular}{|c|c|c|c|c|c|}
\hline $\begin{array}{l}\cos 1 \\
\cos 1 \\
\cos 1\end{array}$ & 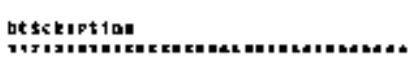 & 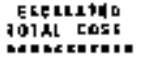 & $x_{x=10}^{504}$ & 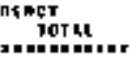 & 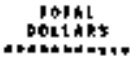 \\
\hline $00 \bullet$ & EPIMEEErW & 1.360 .040 & SD & 770,000 & $5,316,400$ \\
\hline 10 & betroLillon & $\Delta, 5, \theta+, 0+\theta$ & so & $2,300,000$ & $r, 1 \omega_{1}, 00$ \\
\hline
\end{tabular}

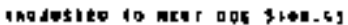

Phodecl Itint

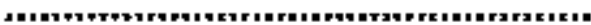

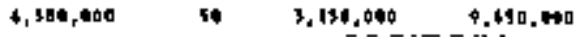

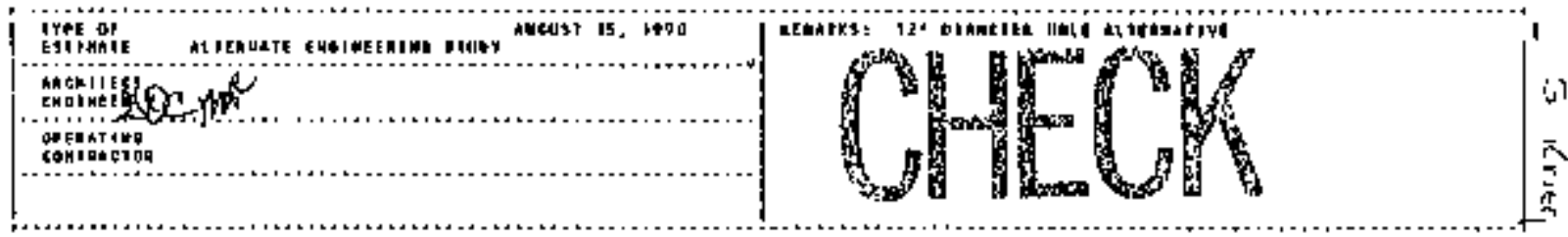

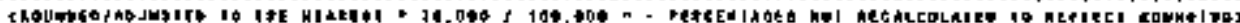

5. 


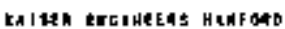

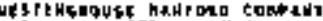

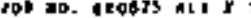

\section{Jus DECCRIPTIOA}

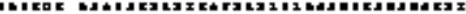

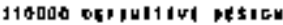

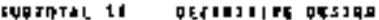

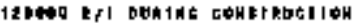

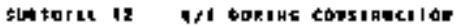
sdbiolat todmenime

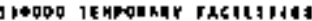
sispol CArIMHOAK

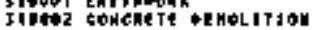

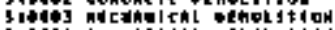

gioded terciejthi otholgtion

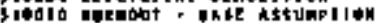

liokzo nat bistosil iels

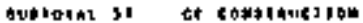

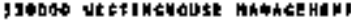

suniaikl 1 couslklott1eh

egontct taghe

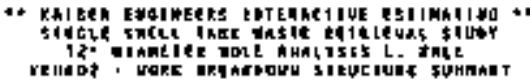

\begin{tabular}{|c|c|c|c|c|c|c|c|c|}
\hline 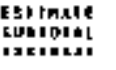 & 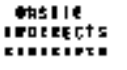 & 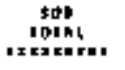 & $\begin{array}{c}\operatorname{lsch} \\
t \\
0=10\end{array}$ & & $\begin{array}{c}\operatorname{swn} \\
\operatorname{xog} 12 \\
0+10,1 \mathrm{ra}\end{array}$ & 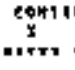 & $\begin{array}{l}\text { wetwey } \\
\text { intint } \\
\text { omatian }\end{array}$ & 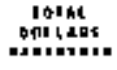 \\
\hline 158000 & 0 & $0 \$ 2900$ & $\bullet .60$ & $\bullet$ & $43<940$ & 50 & 476550 & 1628750 \\
\hline 043900 & 0 & p\$2000 & $\bullet .40$ & $\bullet$ & \$32700́ & 34 & 676650 & $142+350$ \\
\hline 476450 & 9 & 476470 & 0.0 & 9 & 674654 & 10 & 230327 & $39+475$ \\
\hline 674630 & $\bullet$ & f3+4so & 0.06 & 0 & 176450 & 50 & 230225 & 714475 \\
\hline 1429354 & - & 16 ז्9354 & 1.P4 & $\bullet$ & $1+20150$ & 30 & 7144 is & 214607 \\
\hline 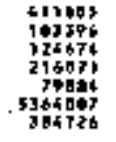 & $\begin{array}{l}0 \\
0 \\
0 \\
0 \\
0 \\
0 \\
0\end{array}$ & 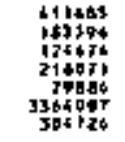 & $\begin{array}{l}0.00 \\
0.00 \\
0.00 \\
0.00 \\
0.0 \% \\
0.06\end{array}$ & $\begin{array}{l}0 \\
0 \\
0 \\
0 \\
0 \\
0\end{array}$ & 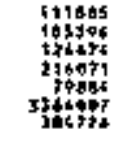 & $\begin{array}{l}50 \\
50 \\
50 \\
50 \\
90 \\
50 \\
50\end{array}$ & 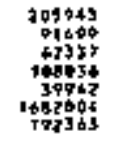 & 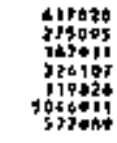 \\
\hline 6765467 & $\bullet$ & 6746463 & 0.00 & $\bullet$ & $4764 d 43$ & 30 & 2542324 & 71444 \\
\hline 114240 & $\bullet$ & pitite & 0,00 & $\bullet$ & $152+60$ & 30 & $31+20$ & 1313 \\
\hline 4ars8B3 & $\bullet$ & 6824643 & 0,00 & $\bullet$ & c87atat? & St & 2451644 & T316327 \\
\hline
\end{tabular}

Her ? H

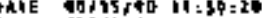

U soluts

4 epsos

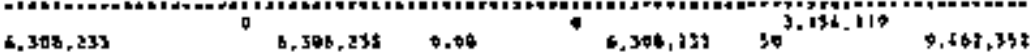


Phtat I nit

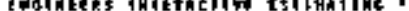

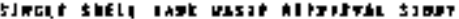

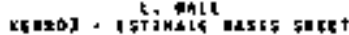

padr ungerto $96: 2011 \mathrm{~A}$

ni ahksuls
1. Hocynters RHQ H MNIHCS

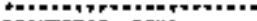

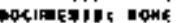

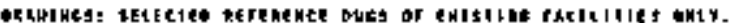

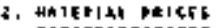

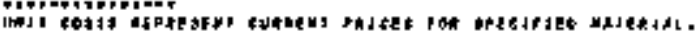

3. Habk telte:

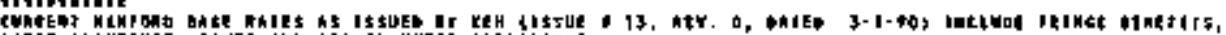

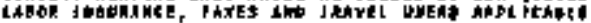

4. Se RenAL We

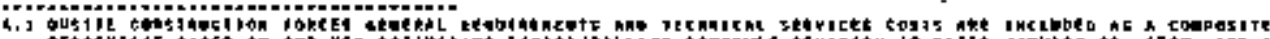

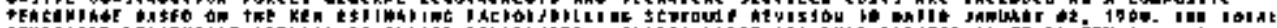

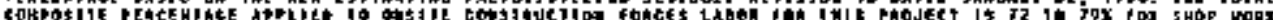

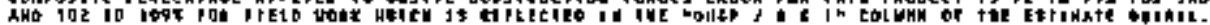

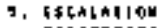

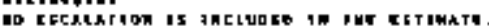

b. Doubrias. CIMC Itens

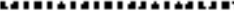

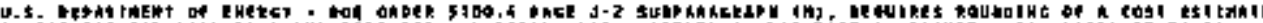

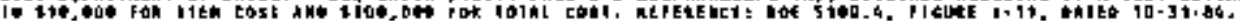


7. Etadkk:

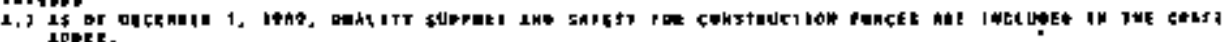

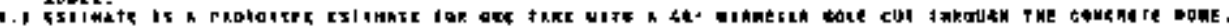

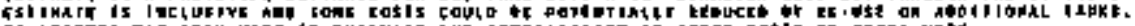

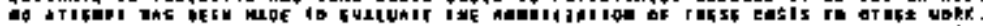

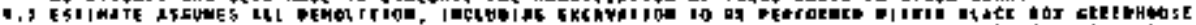

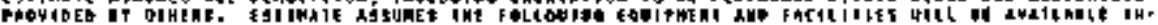

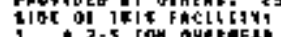

1. 2-5 COH OHE

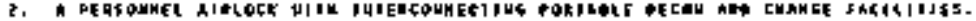

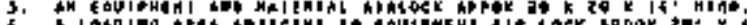

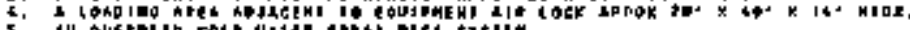

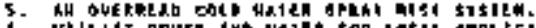

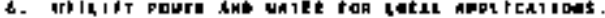

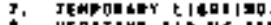

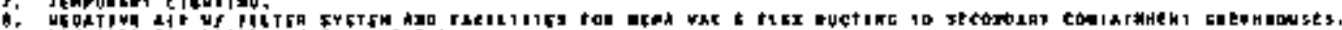

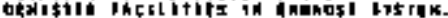

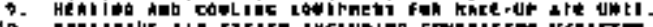

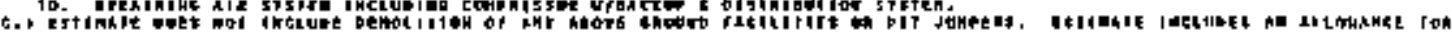

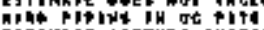

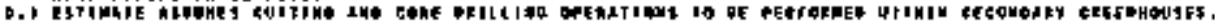

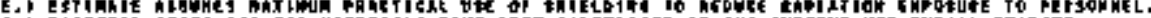

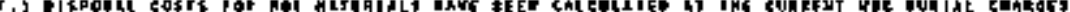

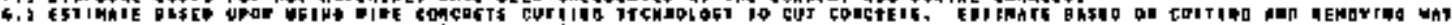

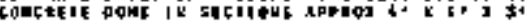

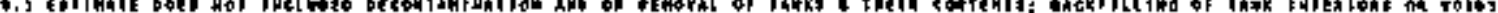

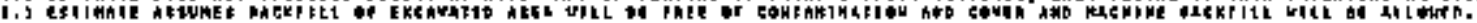

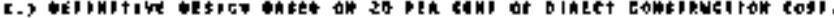

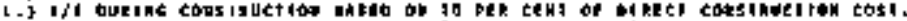

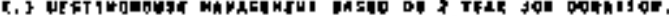

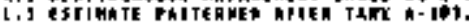

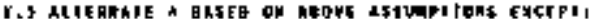
IAUF.

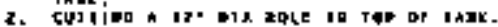

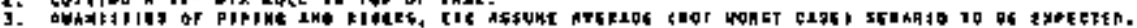




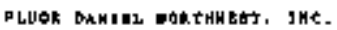

COC1HELO WARTIM MAHTDND CakF.

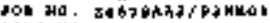

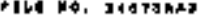

\section{cant}

cobirnbs

ottechstrot

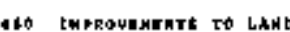

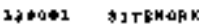

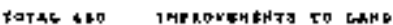

34 Ge[LRHES

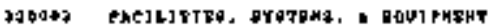

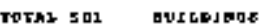

\section{UTthitge}

IMOAL SITEMORK

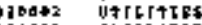

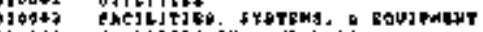

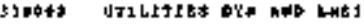

TOTSC CDO UTILITES

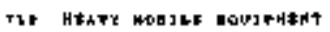

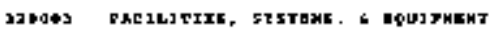

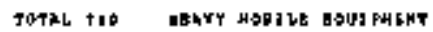

PROAL TOThl

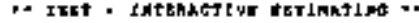

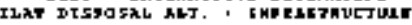

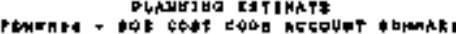

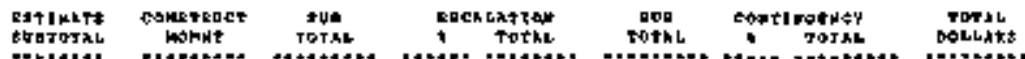

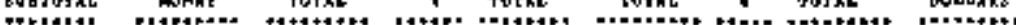

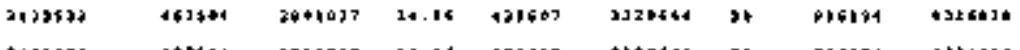

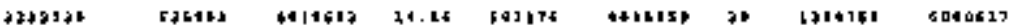

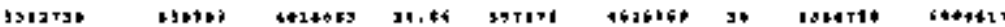

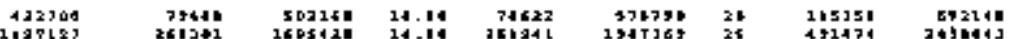

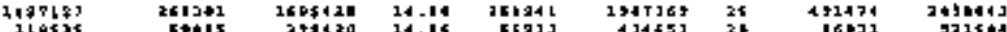

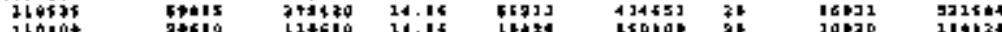

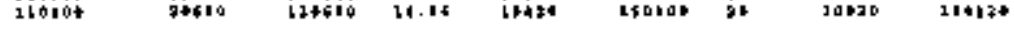

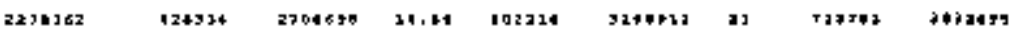

\begin{tabular}{|c|c|c|c|c|c|c|c|c|}
\hline 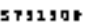 & 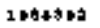 & $\cos +4 b a$ & 19,56 & |ratal\} & $70+216$ & Jt & 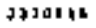 & 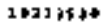 \\
\hline $191 \div 00$ & 740dedz & 6010342 & 14.14 & בום622 & $7342 \geqslant 19$ & 34 & 2270315 & 0נ102139 \\
\hline
\end{tabular}

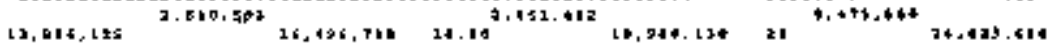




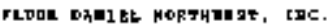

LOSKHERB WNTIIB HAMPOAD SOAP.

Jos HO, JIGJSAAZ/FSHKI

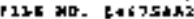

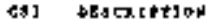

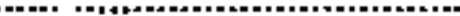

couptewct a ox

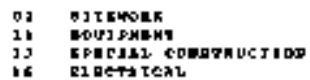

is

TOTसG GOHstaUcT2OH

poduct tathu

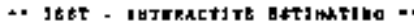

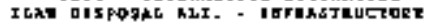

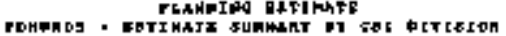

PAeE 6 tot

Dhta OA

aY CPLSKLPABKE

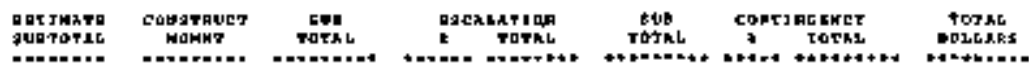

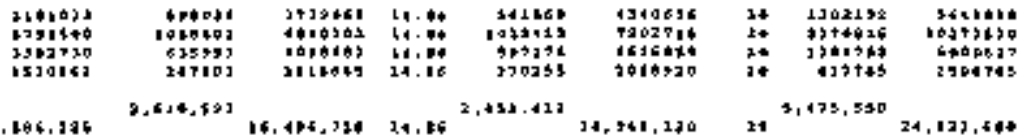

1. 1A6, gat

$1,433,910$

$94,473,810$ 


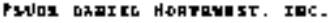

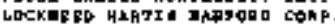

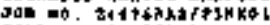

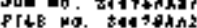

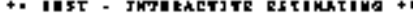

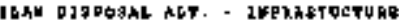

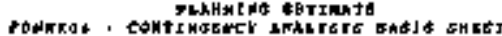

Aat $\rightarrow$ of

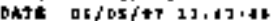

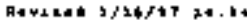

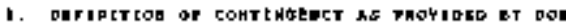
in+1+1+4.

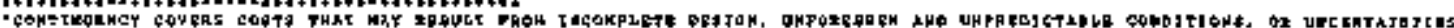

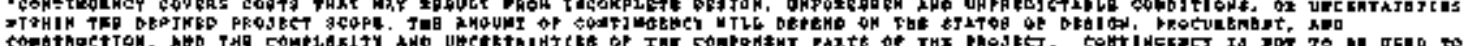

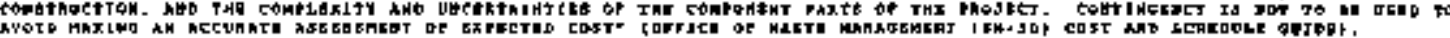

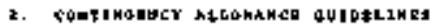

Th

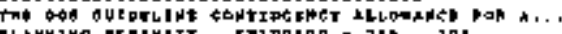

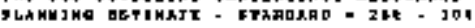

7. mEthQDQLOQO

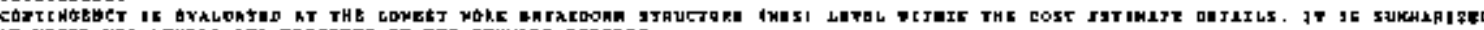

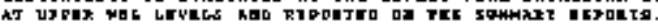

1. ANabt+1+

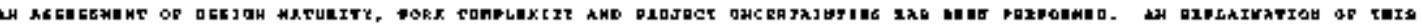

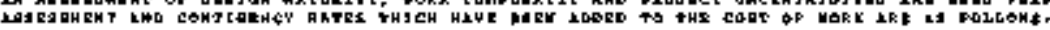

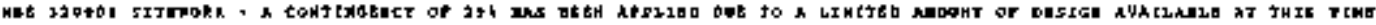

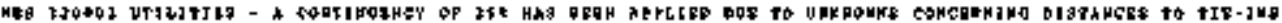

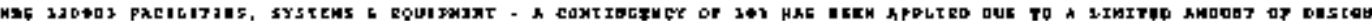

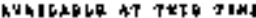

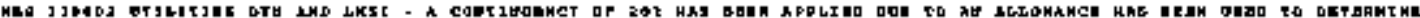
TIIt \$0\&5. 
Rev. 0

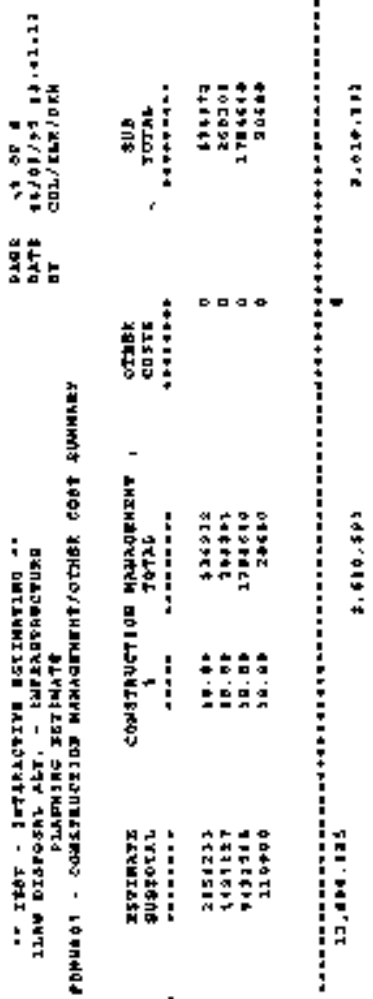

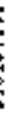




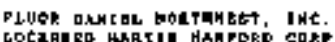

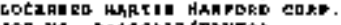

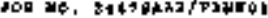

PIVI G0. Intatsing

hecrut?

VDMER

Dagentotion

icterate

$32+041$ Gu Temokn

$320041-09$

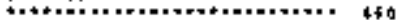

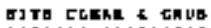

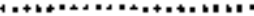

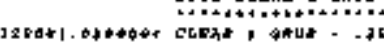

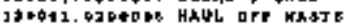

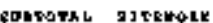

TOTAK COAT CODE 16007

HBS j20401

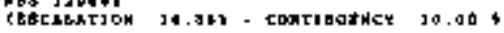

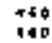

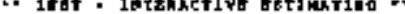

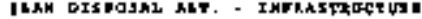
PthaNING BOTIMATE

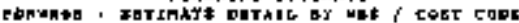

Pati: $>1$

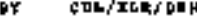

gaj $00703 / 3713: 43: 23$

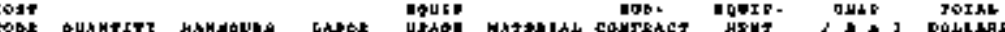

150

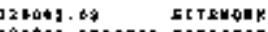

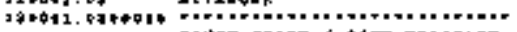

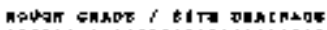

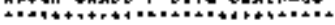

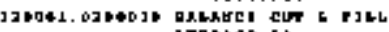

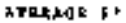

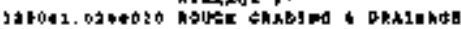

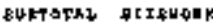

707264 $\cos \cos 46012$

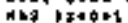

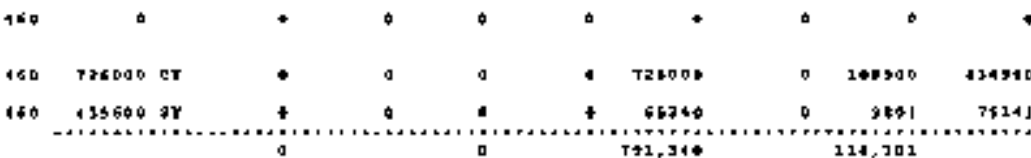

$T+1,34 *$

மR, od

$+$

$T+1,34 *$

11. Tा 1

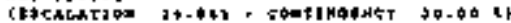
a jequars

$33 \times 041.04$

1तkt?

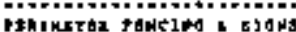

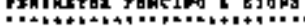

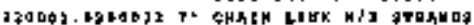

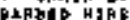

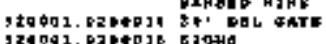

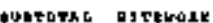

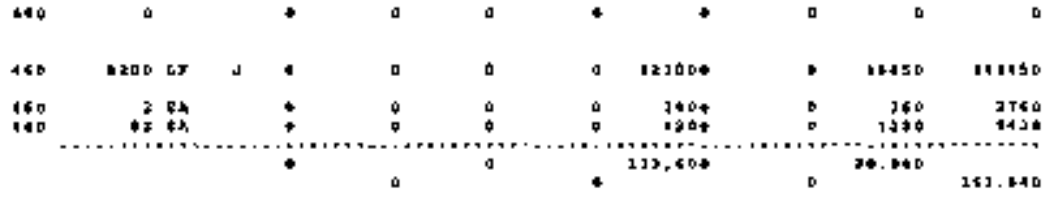




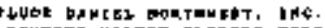

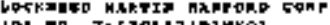

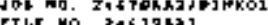
ILE MO. 2 th

Mecount RUDP $1 \mathrm{~F}$ DESCETPF10R

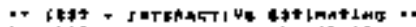

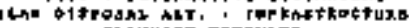

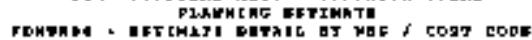
cosp gqujs phat

$0,10932713: 43,27$

ot colikeroin

TOTA

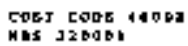

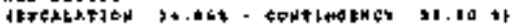

Hבด・. a S J Trapken

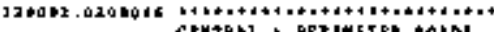

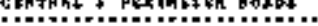

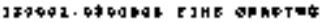

J21001.0204090 E. EMUзHER MOEK

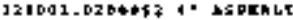

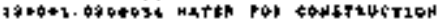

37001.0304036 G0KRAY

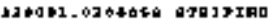

todtoThL EJTEmbXK

TOTAL COAT SODE 1600 ?

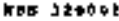

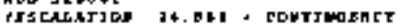

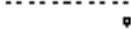

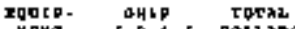
REHT $T$ T DOLRAT

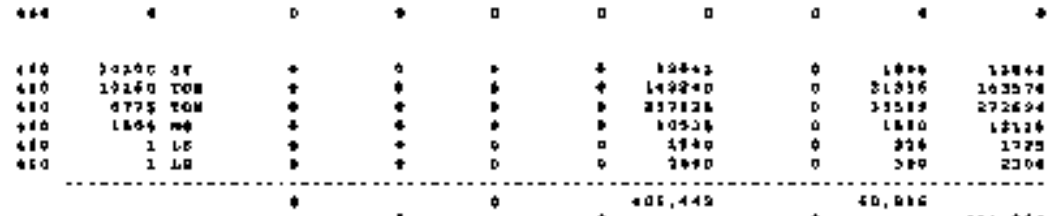

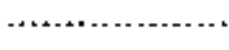

thatast

0 193,412 a 10,age 165,251

$x \neq a$ ob 2 . ba H IY knak

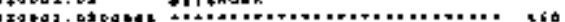

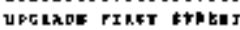

and

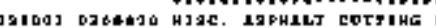

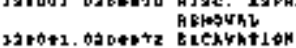

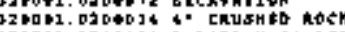

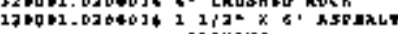

nognjog

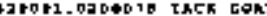

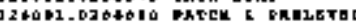

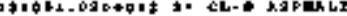

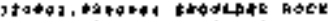

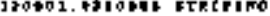

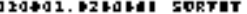

quprothl gitimodk

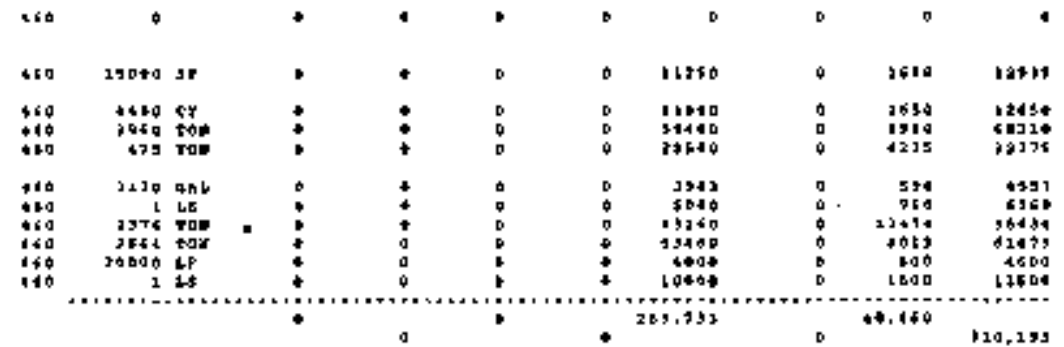




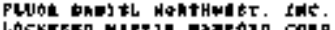

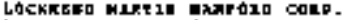

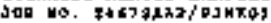

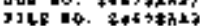

sçourt

mukroa

Degcmistar

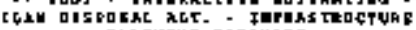
PLARKJUA DATIMSTE

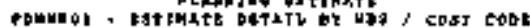

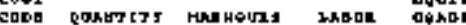

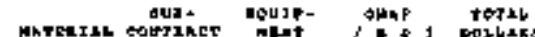

rathl cast cape $18+0 \mathrm{~d}$

(1..................

a

a

a $60,73 \mathbf{3}$

DRED 2

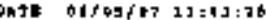

IT

coulKLAfoKx

9r. to to

$2,9010 \mathrm{~L} .09$

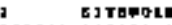

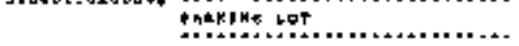

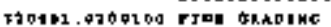

330401.070010252 TROAKED RDCE

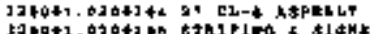

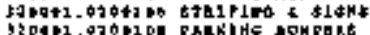

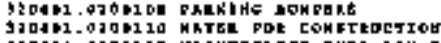

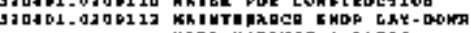

YREO WJFENGE \& ARTES

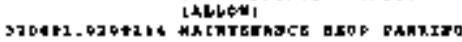

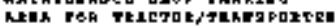

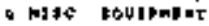

[ALLOWI

\section{tuttors tetrouk}

$707 \times 4$

\section{co9t c601 16042}

$2 x+6+1$

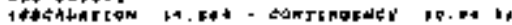

J2400. 12 E[J]MORK

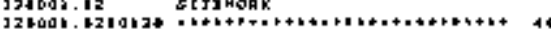

Ga גYel a HrazoneEs

staillizerion

7Forol.

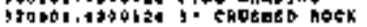

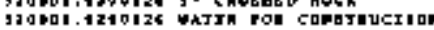

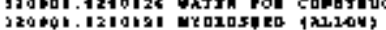

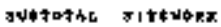

\begin{tabular}{|c|c|c|c|c|}
\hline 460 & a & & 0 & 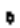 \\
\hline $\begin{array}{r}160 \\
+60 \\
460 \\
640 \\
110 \\
110 \\
460\end{array}$ & $\begin{array}{r}1400 \\
1040 \\
500 \\
1 \\
100 \\
1 \\
1\end{array}$ & $\begin{array}{l}1 y \\
\operatorname{Tan} \\
\text { Ton } \\
15 \\
18 \\
10 \\
10\end{array}$ & $\begin{array}{l}0 \\
: \\
: \\
0 \\
0 \\
0\end{array}$ & $\begin{array}{l}0 \\
0 \\
1 \\
1 \\
1\end{array}$ \\
\hline
\end{tabular}

460

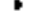

$\varphi$

$\varphi$

02235

ariotio

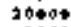

1400

$+40$

$2+40$
$i \oplus 40$

,

$\bullet$

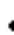

111.715

14,799

txit

$29+3$

154

2300

160

1600

I, 190

$13+4$

(

74510

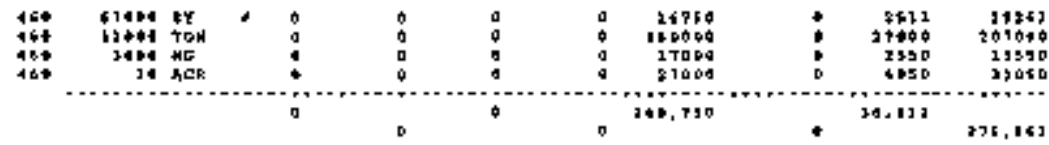




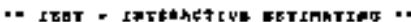

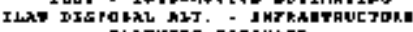

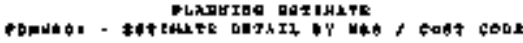

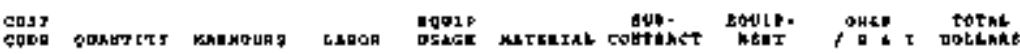

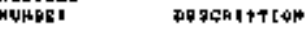

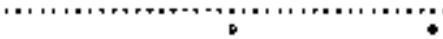

404,750

a

$1+113$

$\rightarrow 0+320+41$

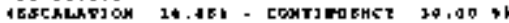

S3

$32040 \mathrm{~L} .1+20074$ LJO ik Freoch - z- erc

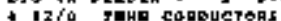

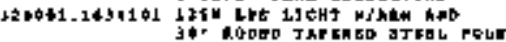

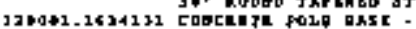

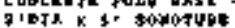

3204BL.1431111 TKENTHIRE G GACKFIL

$4074^{\circ}$

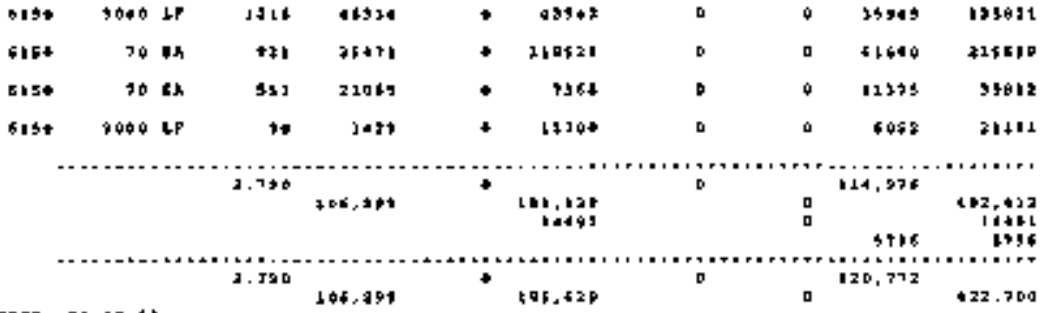

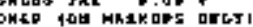

co5T 5006 4263\%

Iros jabat

106,99

505,62

Terhe wad 3204ob grtamak

194,210

\section{D}

$105 .+29^{2,124,366}$

Lo6, a01

2.050.231 


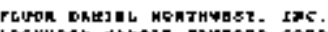

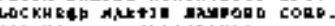

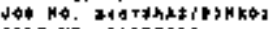

PJLE NO. Bt TFAR?

nccount

HubBt

boentogtom

j2000

320042.62 UจT LTTEA

6s 5 tsimonk

c

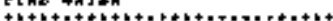

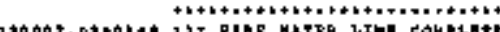

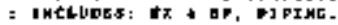

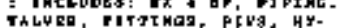

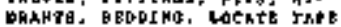

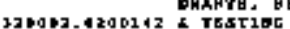

\section{sutfothl sltgmokx}

toth1

$\cos \cos 60002$ $10 \pm 126402$

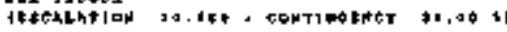

320402.42

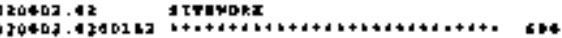
THETRARY MATER

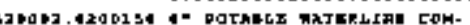
PLFTE, INCTLUDS QK. DP TIPIMD, EIDE BEMTECE. BACK. FLON TREVINJIDE. LOCRTA IRPE

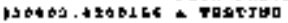

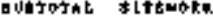

Totras

COST CODE CDOOZ

404200407

TEStaLATIOH

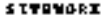

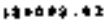

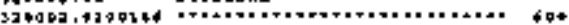

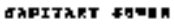

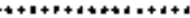

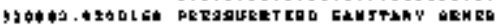

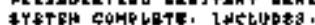

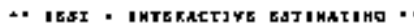

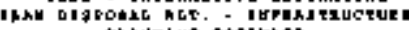

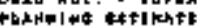

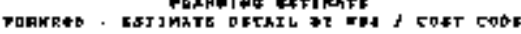

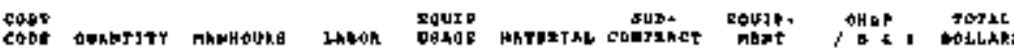

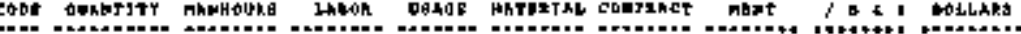

100

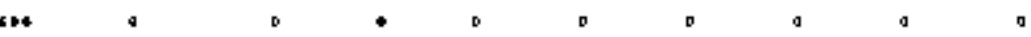

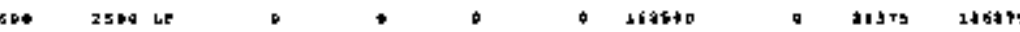

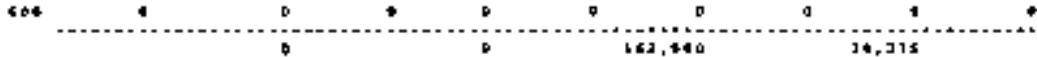

6

206,195

b

atio th 4 D

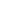

1 La

$+$

D

- 3า4

$9 \quad 4390$

345404 


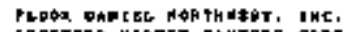

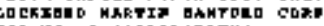

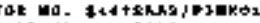
PI LB No, Itotbiha

\section{1есопнт}

TUM? DEActition

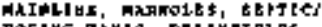

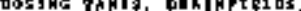

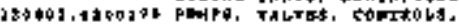

EHETTXICAL * TESTEMG

AUATETEL DETRONK

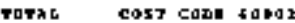

\section{$\cos c \cos 2$}

190

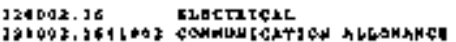
LAN ' TELE FHOE

guotorkL gLacktch

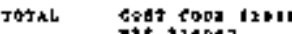

mit $3 a 4042$

i rtextidita

325002.16 CLeTkR\&L

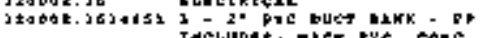
racluost, mote kpt, edac,

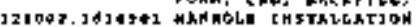

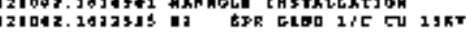

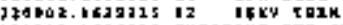

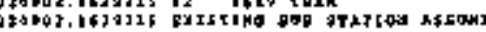
ano opact motit is

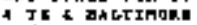

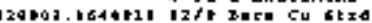

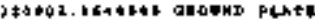

$330+62,161+2+1<\alpha B=140$

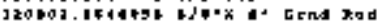

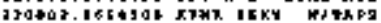

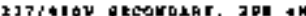
QIL PILLE HAt modNTAD +16 kys

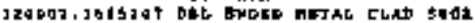

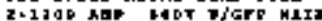

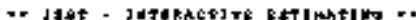

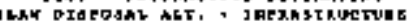

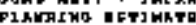

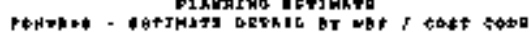

race

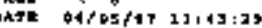

or

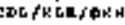

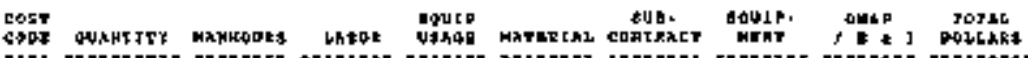

\begin{tabular}{|c|}
\hline 60 \\
\hline
\end{tabular}

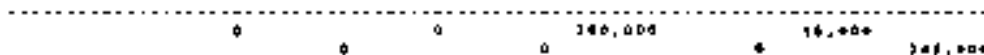

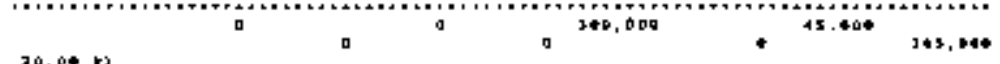

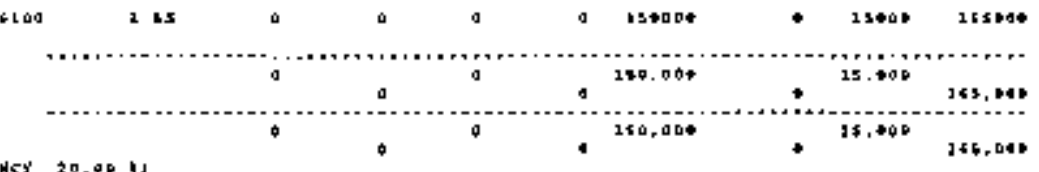

154

\begin{tabular}{|c|c|c|c|c|}
\hline $\begin{array}{l}61 b+ \\
6 \text { b } 34\end{array}$ & 17006 & $\begin{array}{l}\text { Jal } \\
\text { LF }\end{array}$ & 19. & $\begin{array}{l}300 \\
1300\end{array}$ \\
\hline 4130 & 12 & EA & 24 & 314 \\
\hline
\end{tabular}

$+$

- 2431T 47310

卓

2125

24014

$+114$

20412

1,200

tog

1130

$+130$

4150

$20+17$
1104
102
102

119
112
119
315

2 as

נSוs

0
0
0
0

$1+2$
30
221
240
3406

$\underset{20}{4}$

$+$

124
+5
254
135
1490

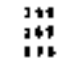

173

1130

1 es.

184

1962

Q 14itos

$4 \quad 54471$

$7+7+15$ 


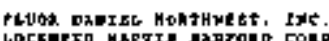

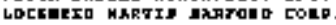

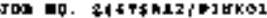

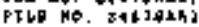

\section{מсCоUNт}

Tuman 8

$1-120+\sin$ TנE

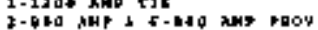

terterse bitetteter

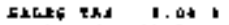

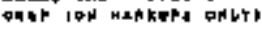

$\operatorname{ros} 21$

Nin 320042

ryexidtan

14. н6t - coutimanнсу cont cand $616 \mathrm{~L}$

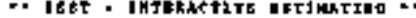

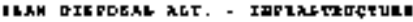
CLNHAIML IDTTMATE

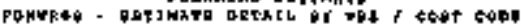

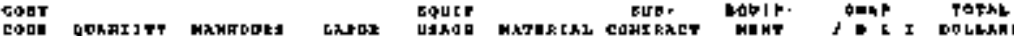

n.

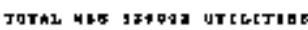

bis

12,123

$\cos 40$

359,021

1.427 .124 


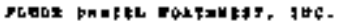

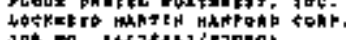

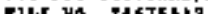

ncesowt totthrerion

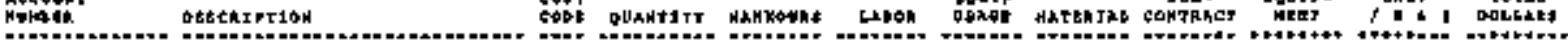

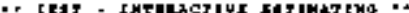

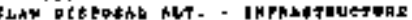

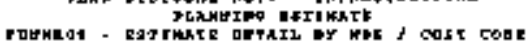

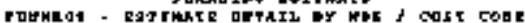

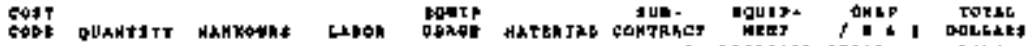

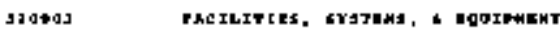

$+2009,12$

jagrd. Geurrhers

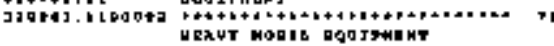

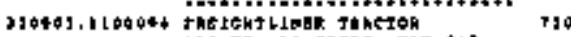

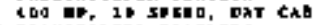

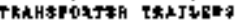

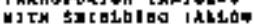

suerotsl EQUTENRI

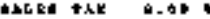

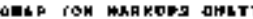

TロTA2

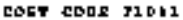
MED $2240 \% 3$

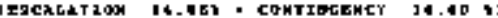

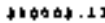

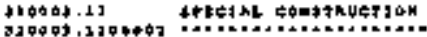

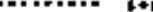

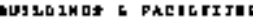

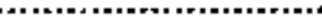

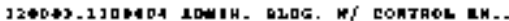

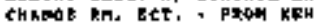
Sal suppat Ficility

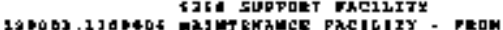

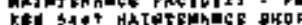

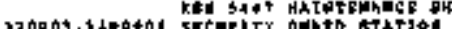

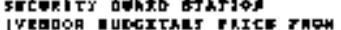
IVRagoh durcer

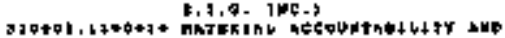
Threjbicist eteTEM |xLLors

sumbrat sorcice cassinucion

TQTㅅ․ tott cobs 50112 tas $320 \mathrm{Oat} 3$

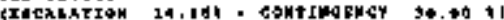

ILetratch
Fint $v$

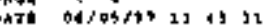

Thr Dichiory
$2+442-28$
720

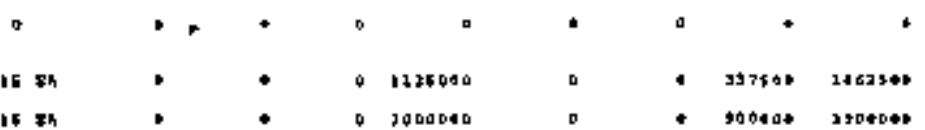

..

............

( ,$\ldots$

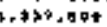

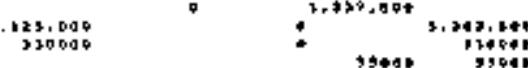

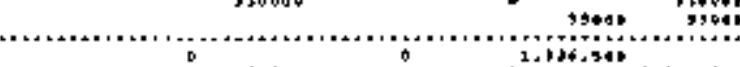

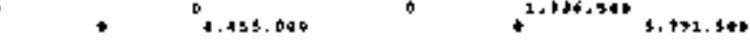

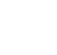

$+1$

9

1 t5

I 4

21

$2 \mathbf{1}$

$+2$

4

n

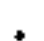

$+$

- $1345+00$

34

(4)

64

a

a

o

a

I 12adrap

o

b Itteon 14jegoo

- 65504

a

$7016 \quad 36+10$

1 katov

D $14000 \quad 13000$

n....


FLUOR RLMERL DOETHMAST, IJC

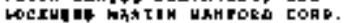

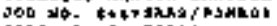

ile mo titisikt

\section{ACCOdHT \\ MPAB EW \\ DESERPTrot}

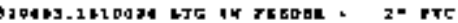

+ 12/a trany conacrors

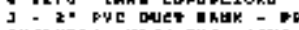

I Welubes wAeh EXc, tave,

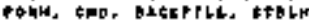

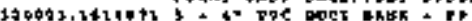

I reluess, hich Eje, eare,

Fakh, cod. Gectribl, stmik

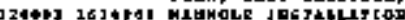

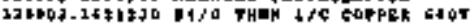

hatoblet

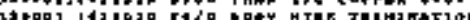

(30 נ)

1044

3945.

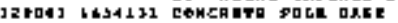

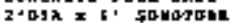

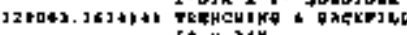

$5+240$

E*TETAL GLETEERL

1तlese tha 4,0 ,

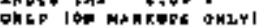

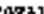

cost cand th 16

$2+1201015$

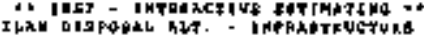

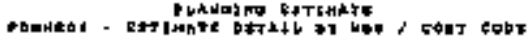

bhet 2 ,

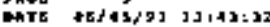

BT COL/KahIDKH

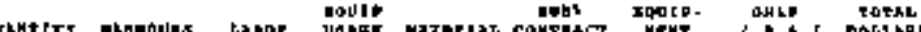

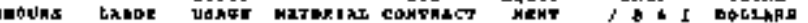

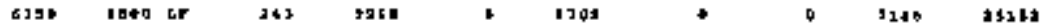

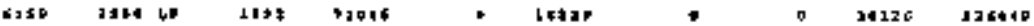

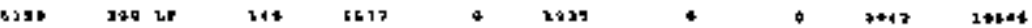

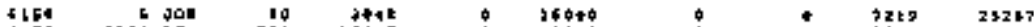

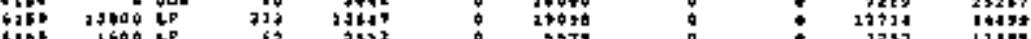

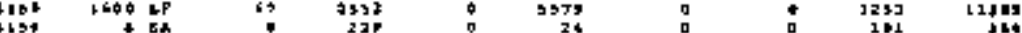

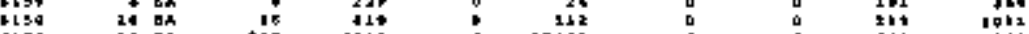

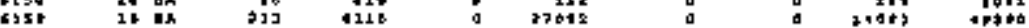

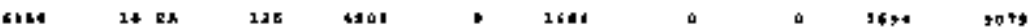

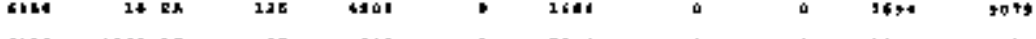

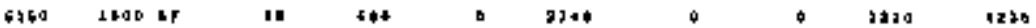

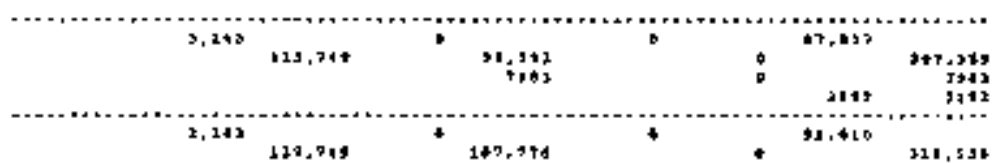

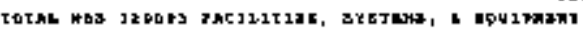

$3,2+3$

$2,3+1,304$

$1,1+4,73$, 


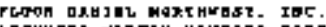

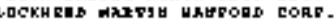

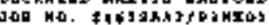

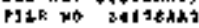

iccount

บukne 1 Dencriotar

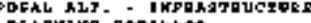

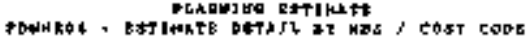

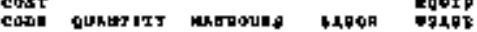

\section{rera}

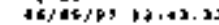

or coljkinjokk

\section{g300a} DTJITEE DYE NED LTEI

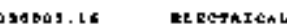

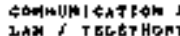

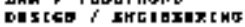

SUATOTAL EGETRTER

Tarkl Cast cabl G1414

rot $2+0+0$,

AEschlotioh

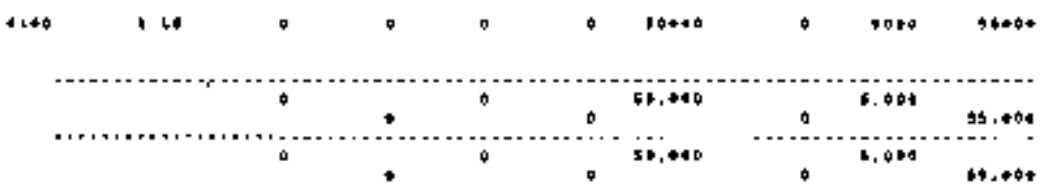

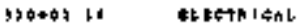

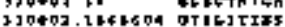

4150

aira 1

THOTD- QHLP TOTत2. MEWT r D I Doliand

Test, respecton, c conant ERc7pTCA2 17.4KU

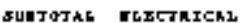

TrThL

COET COFE $+2+11$

Mac 320402

2 L.t

30040

14.16k - contrinagect

$0, \ldots+\ldots$
0

0

ș, 4 a4

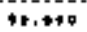

a

(.,

24.

9. $\div 4$ 


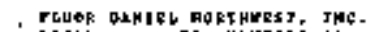

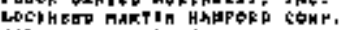

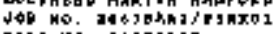

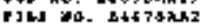

hccódut

MTwh th

DEECH $(\mathrm{P}+\mathrm{T} O H$

Ravony gotal

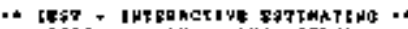

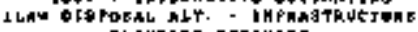

PLAMEIAd getimkte

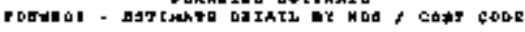

एकor

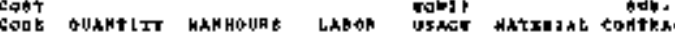

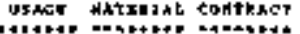

PAOE 11

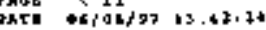

QY COLIKLR/OKH

Eavir- OHA R TatkL

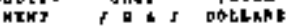

3,191

2), irt

$\bullet+, 0,1,+1,300,410$

$0,575,041,00,125$ 


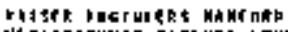

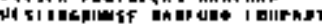

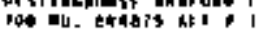

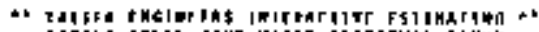

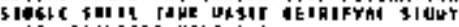

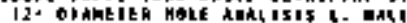

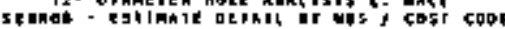

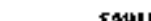

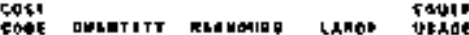

hesceipitan ton

seas.

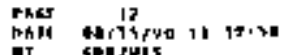

accour

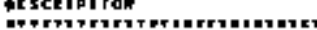

ECECIFICAL OEMOLITLA

J repes

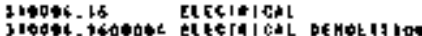

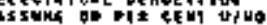

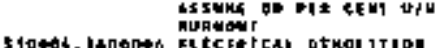

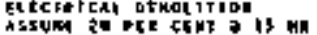

SUPIDIAL ERECIEICIL

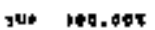

Lf tfrti iofing

cotsuHinit $5.00^{3}$

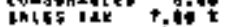

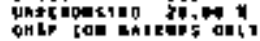

reani cot? cote $110+4$

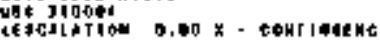

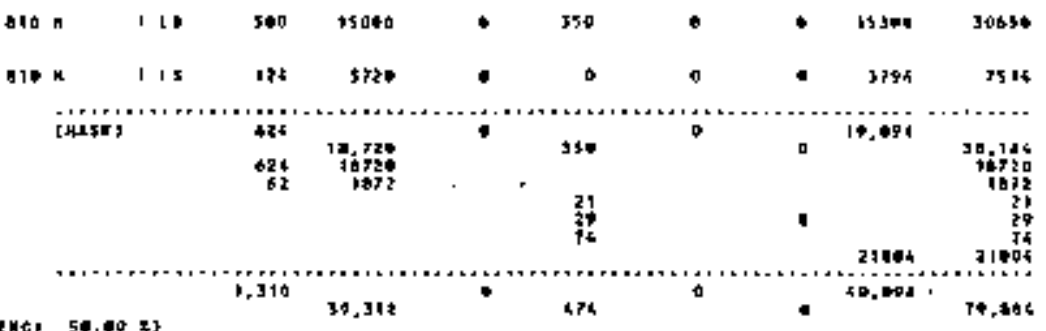

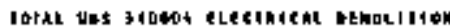

\section{o}

434 


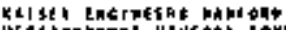

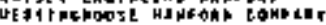

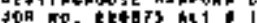

ArCeytet

WUHAEA OECKIFIIQLU

OEFEIFIIOU

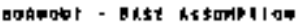

310810

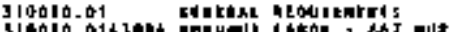

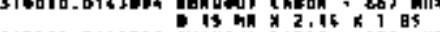

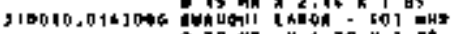

a 70 w. $k+.20 \times 1.04$

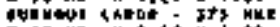

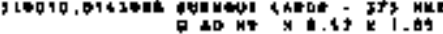

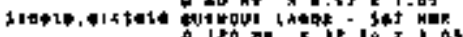

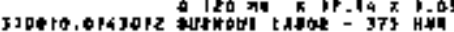

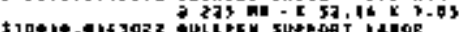

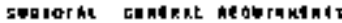

Tolsh

\section{cos t tond atebl}

wis gido

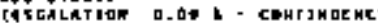

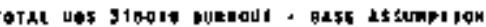

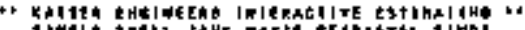

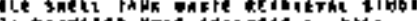

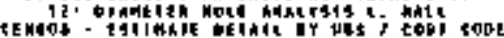

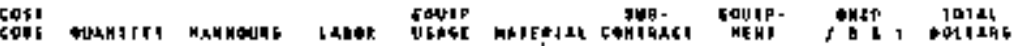

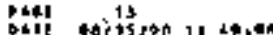

CY SUR TUTS

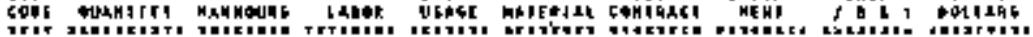

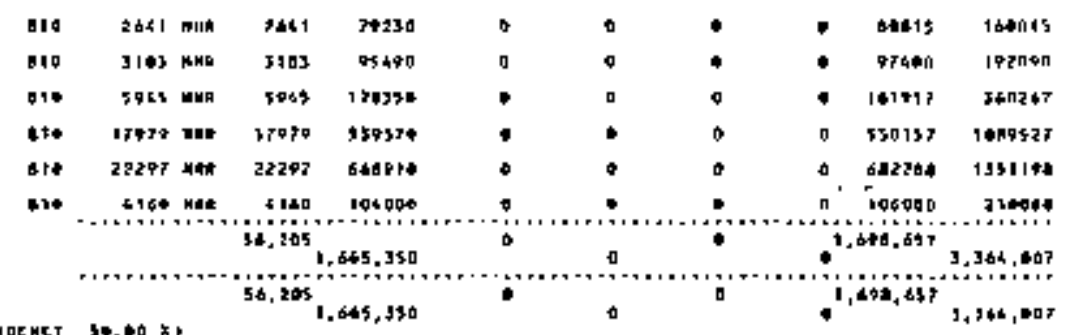




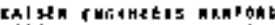

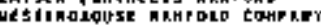
ral vo. Inogts $A$ L $T$,

\section{ictonet}

auntid

Brfchirilag

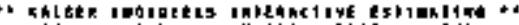

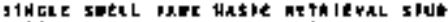

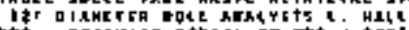

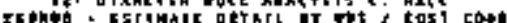

C45 rast

की

- 7 cos puls

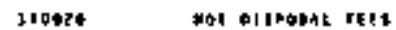

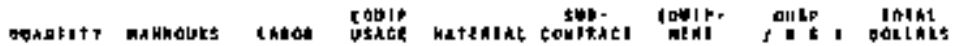

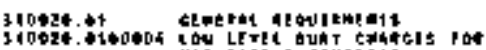

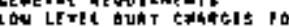

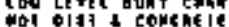
at

320030,019004 Wr Moj DUT C colca EIE

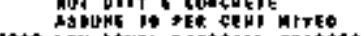
ou kerel oistajak cashotes 10

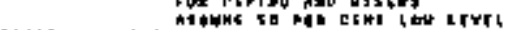

110420,0100010 HIX्रE

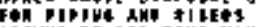



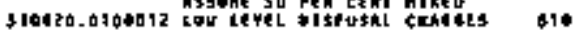

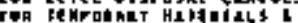

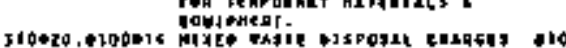

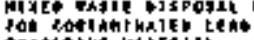

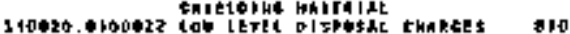
D4 DOHE toHenE $1 \mathrm{z}$

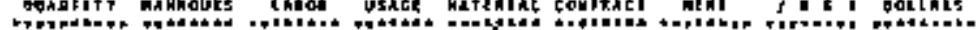

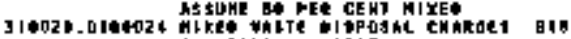
ion obut conchel $E$ sunt rece ctwi aixet

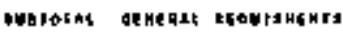

101 int

\section{$\cos 100 \mathrm{E}, 010 * 1$}

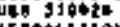

cercininan

HQ 2 - Conctagenct

550 to

240 4

271 te

4363 if

tor ch,

150 c ㄱ

15065

35 CF

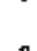

0

p

o

-

$\bullet$

จ

口

$\bullet$

o

a

0

.

\begin{tabular}{|c|c|c|c|c|}
\hline & 704116 & $\bullet$ & 0 & $2+0916$ \\
\hline$\phi$ & 70214 & 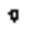 & 0 & $5+275$ \\
\hline a & GTIA & 0 & a & SI: \\
\hline$\bullet$ & 14. & $\bullet$ & 0 & 186 20 \\
\hline D & 24443 & $\bullet$ & 0 & $20+45$ \\
\hline & 20136 & $\bullet$ & $\bullet$ & $2+174$ \\
\hline$\theta$ & 30307 & 0 & $\bullet$ & $5 * 5 * 7$ \\
\hline & 3012 & $\bullet$ & 0 & 5812 \\
\hline
\end{tabular}
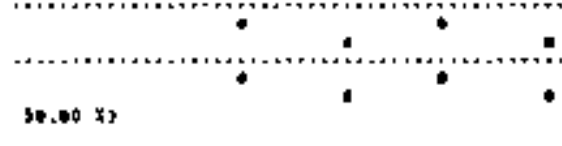

$3 \pm 4.370$

3at, 20

jes, $7 \%$

.

386,726 


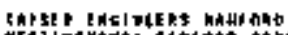

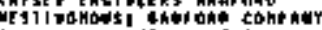
dag wa, rdouts aci i

\section{htcourer}

ตена ти

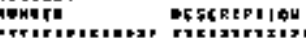

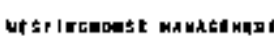

30ub*

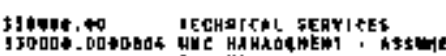
G जQHIHT

a๑b

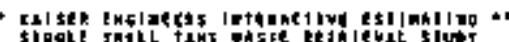

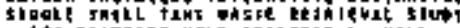

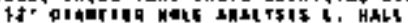

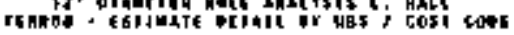

cost cost

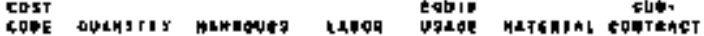

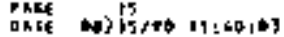

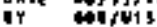

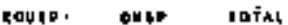

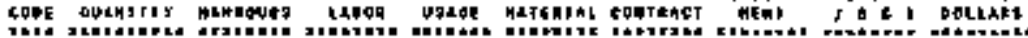

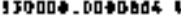

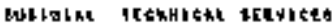

901AL

tot I towe orads

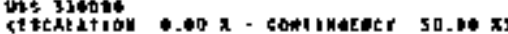

14

136411426

$+6$

a

6

- I1t+4t4

1, 346

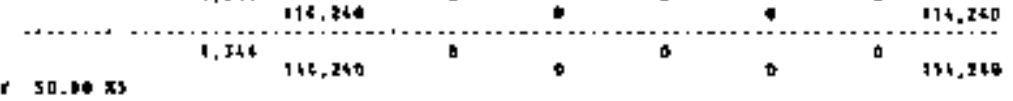

$---$

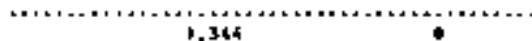

19,240
(1)

0

$\bullet$ 


\section{Rev 0}

Table A-12. Summary of Estimated Construction Costs for Addition of One 48-Foot Diameter Opening wo the Center of a Single-\$hell Tank Based on Diarrond Wire Cuttrng Technology (Options 3).

Not: The above estimale para developed based on August 1990 dollars. For Table 5-B, demolition coss was escalated to September 30, 2002, by using multipliess of 1.26 (based on 3.3 percent per gear to hune $(99 \mathrm{~T})$ and 1.16 . Both escalators were based on compostie labor

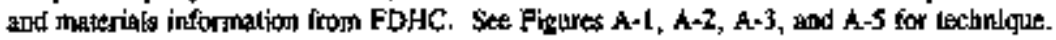


HNF-SD-TWR-AGA-004

Rey o

This page intentionally left byank. 


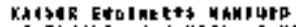

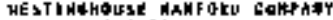
100. 10. toars

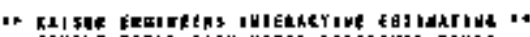

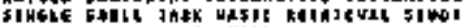

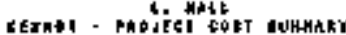

+han in of

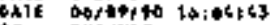

ir BKA TuI
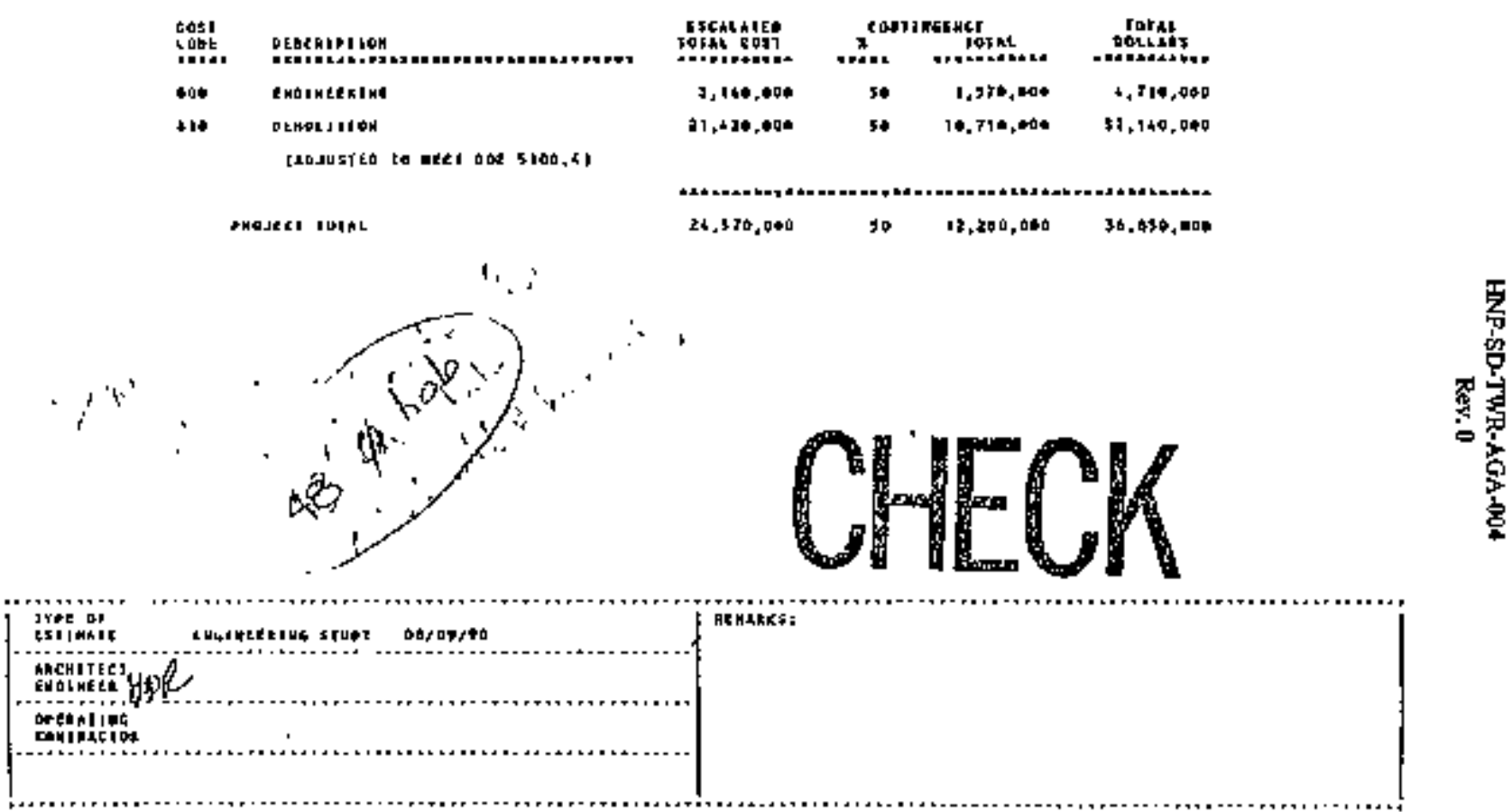

1J DHELE

$\frac{1}{n}$ 


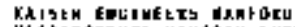

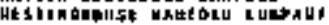
Ja) M. CHOL's

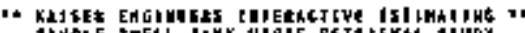

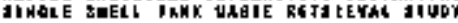

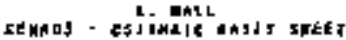

PhEt 3 ar

DAIF OB,

$t$

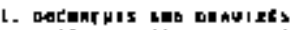

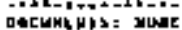

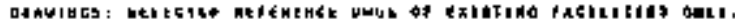

A, ankbink ralab

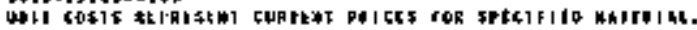

d.

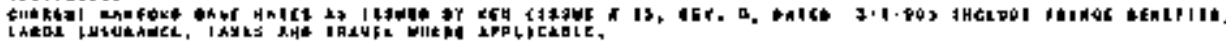

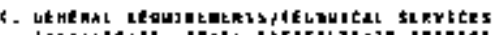

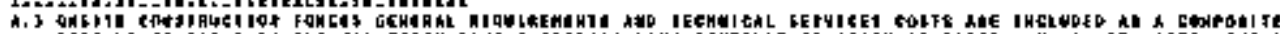

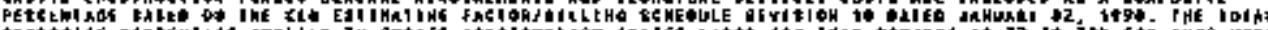

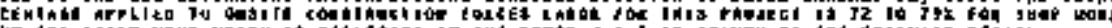

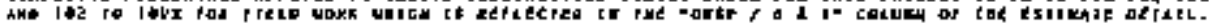

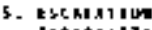

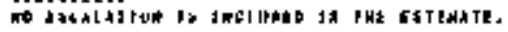

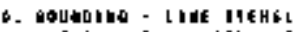

Sations

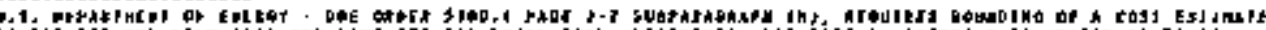

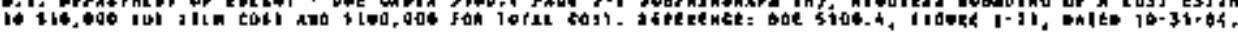


KMIEFT EUSI UEERL WAHFAOD

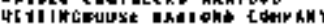
don No, teqos

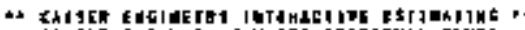

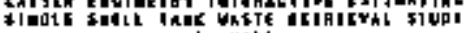
L. IIst rits of

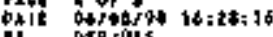
oth rû́:

f. ICLNHYS

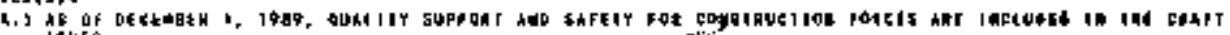

HUE

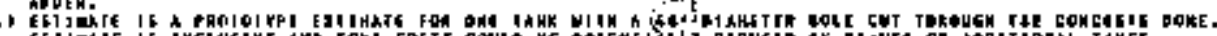

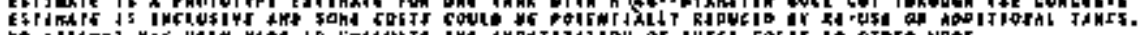

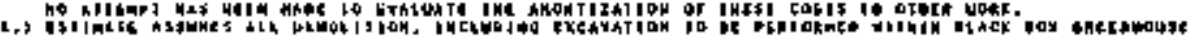

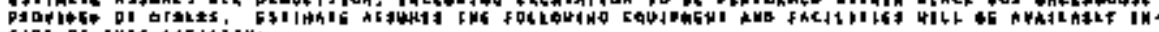

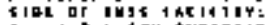

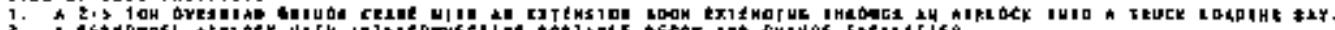

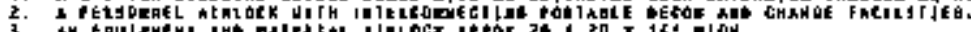

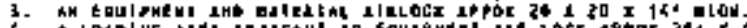

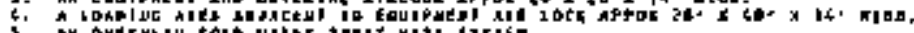

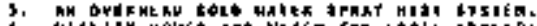

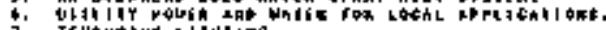

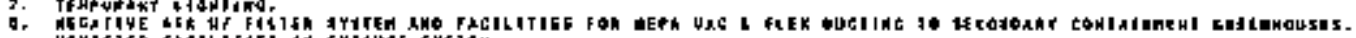

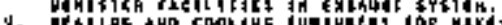

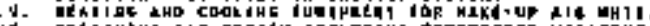

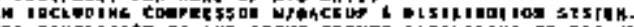

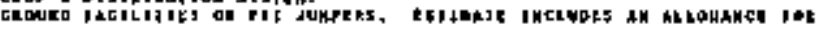

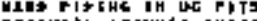

(a)

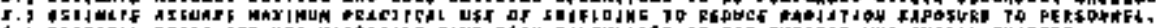

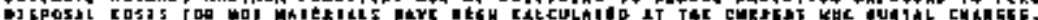

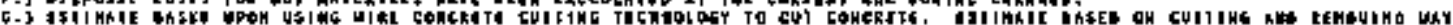

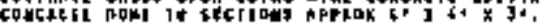

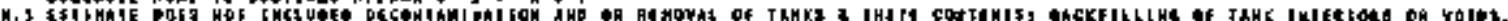

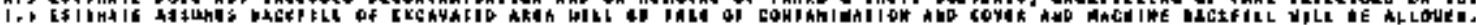

R.?

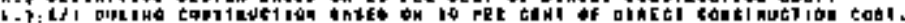

K- 


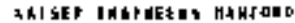
WE Thing

HLS DESERTII\%H

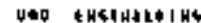

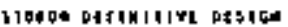

1700 6o

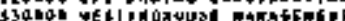

IOJAL TQ6 EMEITIABIHE

\section{Ho athot ition}

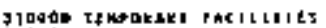

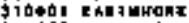

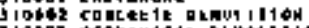

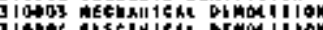

1.

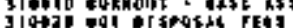

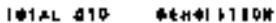

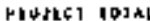

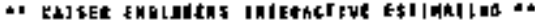

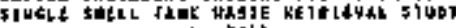

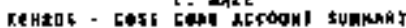

ETTIMATE

Sutiota

ดH口115

1.PIDETT

Tolist.

TSCMnitote

$x$ TOThL

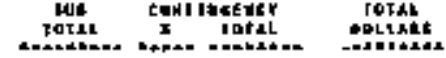

Fage 5 of

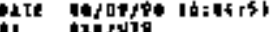

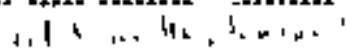

1849437

the

1499916

0.00

$+$

$317 \times+90$

9.00

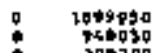

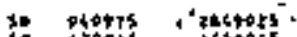
299200

297200

30 istors

54000

3439000.00

3130180

50 156959 4forfo
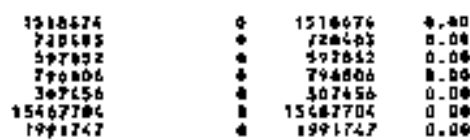

+0
0.06
0.06
0.06
0.00
0.0
15144t5
597652
TPGHet
30746.
g6ertod

5. 239337

50 29403

50 370t?

5o 7ityest

10,2725

liosit

tolit

15467306

o 21620722 4.40

aquata

10

21\{2A 22 t770x+1]

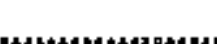

$24,567,402$<smiles>[Li]</smiles>

24.347 .902

D.04

$24,367,702$

10

a.203, pos!?

1 3s,4\$1,454

罢
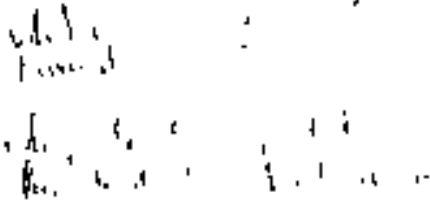
Wur be BChIPI 10y

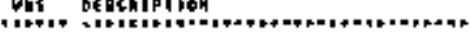

\section{TAC INE H I H4}

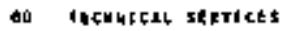

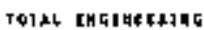

\section{carsengerian}

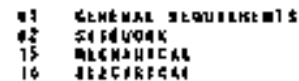

It 2 CIRESA

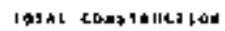

PIOATC tolAL

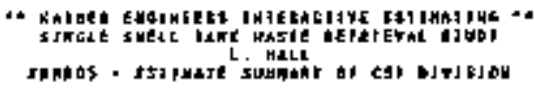

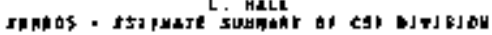

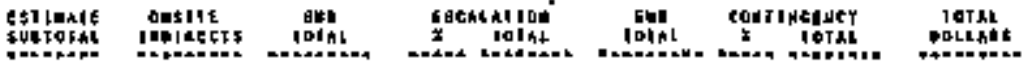

\begin{tabular}{|c|c|c|c|c|c|c|c|c|}
\hline $313710 \mathrm{~A}$ & 4 & $31391 \leqslant 0$ & $6 . \bullet 0$ & 0 & J1391:0 & 5B & 134056巾 & 4740734 \\
\hline 3134160 & 0 & $31+41$ at & •.๑ú & 0 & 3134180 & so & 1349790 & d70t8 \\
\hline
\end{tabular}

\begin{tabular}{|c|c|c|c|c|c|c|c|c|}
\hline $\begin{array}{r}10996125 \\
17+2335 \\
796468 \\
207535\end{array}$ & 1 & $\begin{array}{r}13990125 \\
1340335 \\
740504 \\
347694\end{array}$ & $\begin{array}{l}0,00 \\
0,00 \\
0,00 \\
0,00\end{array}$ & 4 & 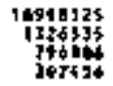 & $\begin{array}{l}50 \\
50 \\
50 \\
50\end{array}$ & 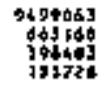 & 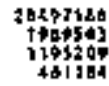 \\
\hline 29620727 & 9 & z15roraz & $0, \oplus 0$ & D & E16ror3z & & 10716362 & \\
\hline
\end{tabular}

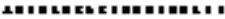

$24,347,902$
Q $26,307,902$ ค. pisterrz

34 10716307

37143040 


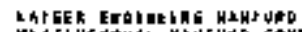

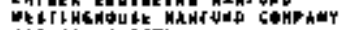
jod to. EReur?

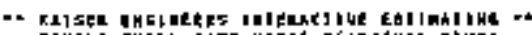

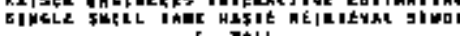

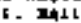

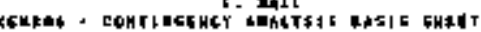

Phet 7 oे ta

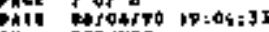

ir orhotis?
karkkH4T:

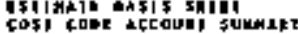
Fite
3 of 1
rob

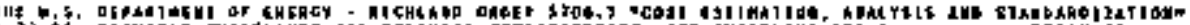

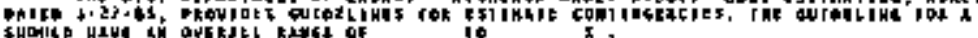

CSI binate

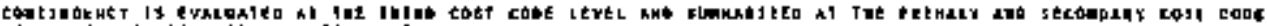

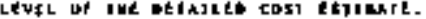

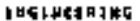

CONIINGCIAH 


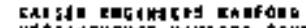

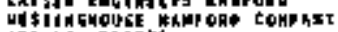

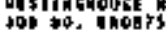

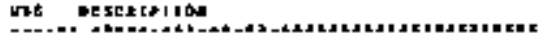

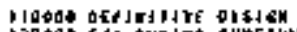

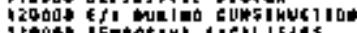

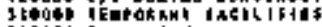

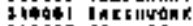

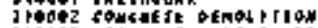

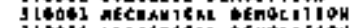

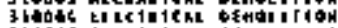

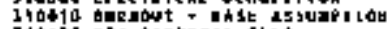

310bit dor pistesil frks

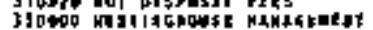

fLAFt 1G1L

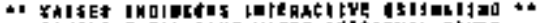

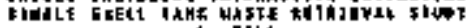

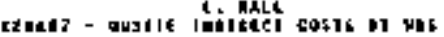

\begin{tabular}{|c|c|c|c|c|c|}
\hline 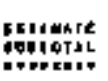 & corrantet & 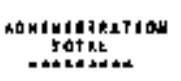 & 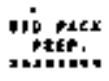 & 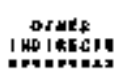 & 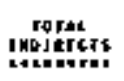 \\
\hline 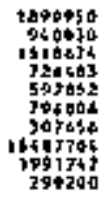 & $\begin{array}{l}0.00 \\
0.00 \\
0.00 \\
0.00 \\
0.00 \\
0.00 \\
0.00 \\
0.00 \\
0.00 \\
0.00\end{array}$ & $\begin{array}{l}0 \\
0 \\
0 \\
0 \\
0 \\
0 \\
0 \\
0 \\
0\end{array}$ & $\begin{array}{l}: \\
0 \\
0 \\
0 \\
0 \\
0 \\
0 \\
0\end{array}$ & $\begin{array}{l}0 \\
0 \\
0 \\
0 \\
0 \\
0 \\
0 \\
0\end{array}$ & $\begin{array}{l}0 \\
0 \\
0 \\
0 \\
0 \\
0 \\
0 \\
0 \\
0 \\
0\end{array}$ \\
\hline
\end{tabular}

that of

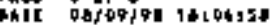

ar bentus 


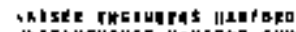

JH + INGHOUKE HOMFOTD CUMYAUY

Jat MQ. ETHA is

15

CF SEKRIIQH

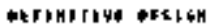

turlolind Etengichb tenvite

Iolke cast tank butato

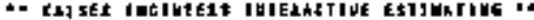

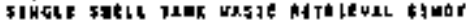

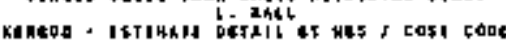

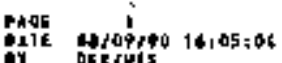

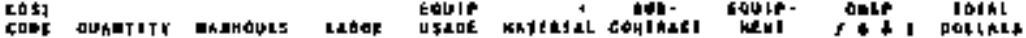

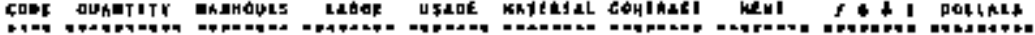

PECHMICAT TGKYICES

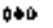

Is 20730 1204030

4

-

0

प

(1497850

27,230

1.496.910

1

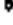

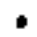

1, 198. 450

IEFG II

29,230

$1, \$ 19,050$

$\therefore$

50.00 कर

1

I, IPP, p10

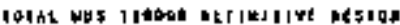

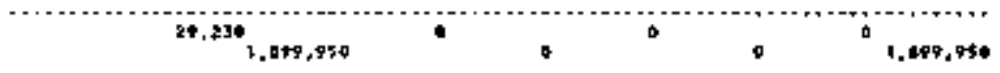


HAISEA GHEIEEE $\rightarrow$ HAH DAP

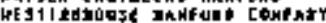
Jat Mo. [tods

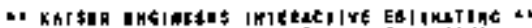

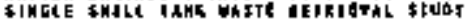

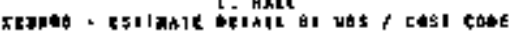

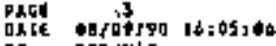

er ortinls

arcout L

tumbrt

LESkIr610h

that I covIP

SUl,

SU1,

ceuiv. DItr tolihe

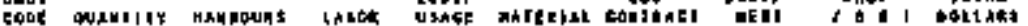

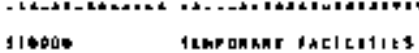

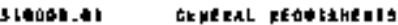

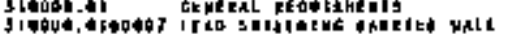
1rum

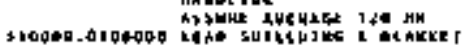
rot sars

rok Lahf

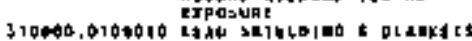

aj whic is

disunt hYlance go all

trotatile

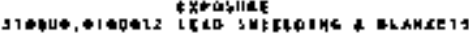

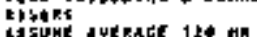

cicume a

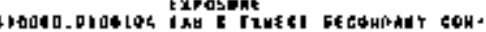
IAI HसLM

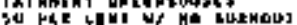

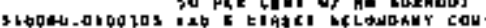
1 SL UKE 1 Cate HLOWE:

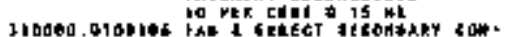

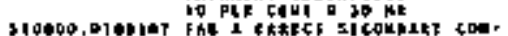

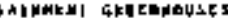

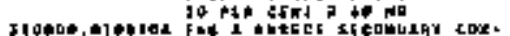

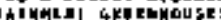

10 PLII CLWT $2120 \mathrm{KE}$

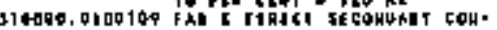

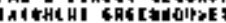

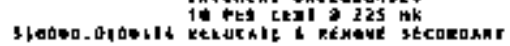

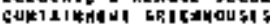

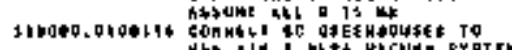

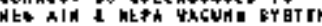
AS YUNE $\rightarrow$ LL $A$ is $\mathrm{at}$

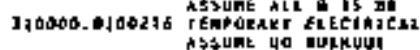

41. $n$ Is 240

115

200

604

aubo

tato

125

104

2500

ato

I,

140

esto.

110 n sure

Q10 2005 51

410 ก 1000 18

Q16 M 1402

-10 4

H* 4 10te at

Q10

$010 \mathrm{n}$

$1 \mathrm{LS}$

$1 \mathrm{kB}$

690

1.5

1200

30000

\begin{tabular}{|c|c|c|c|c|c|c|}
\hline 4 & $\bullet$ & a & $\bullet$ & 6920 & & 12120 \\
\hline$\Delta$ & 15400 & a & ó & 10200 & 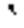 & $352 \mathrm{at}$ \\
\hline - & 5046 & $\bullet$ & 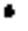 & 2550 & d & I tobst \\
\hline a & soted & a & 4 & 2354 & 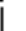 & 16 asto \\
\hline 0 & 1000 & 9 & $\nabla$ & 3025 & 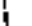 & 9473 \\
\hline$\bullet$ & 306 & - & a & Jas & 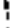 & 1dos \\
\hline$\bullet$ & 30 & $\bullet$ & 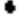 & 765 & h & 1845 \\
\hline 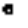 & 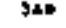 & $\bullet$ & $\mathbf{8}$ & 183 & 1 & 1073 \\
\hline a & 904 & 0 & $\bullet$ & 767 & i & 1495 \\
\hline 8 & 300 & D & 口 & 763 & i & 1695 \\
\hline - & labt & 0 & $\nabla$ & $1 \oplus 2 * 0$ & , & 21200 \\
\hline 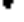 & 2540 & b & a & $7+16$ & & 17065 \\
\hline - & 1000 & $\theta$ & 0 & Istr?0 & & 77720 \\
\hline
\end{tabular}


LAISTE LARINELAS IIAUA IIED

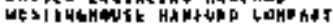

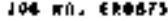

\section{ACLOUWI}

中unuf $\mathrm{k}$ 中téripiagh

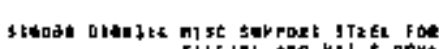

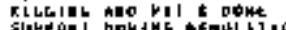

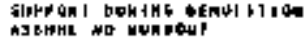

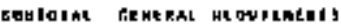

\section{4\% 10úcós}

G5 HARE TONIELA $5,10 \mathrm{C}$

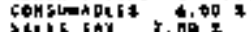

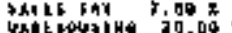

oast 10H Hakzits unt?

chat shol cont bide

Mus blutón.

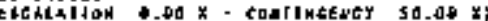

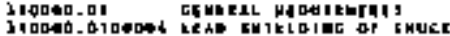

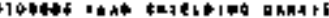

ilopo, 010464

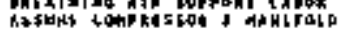

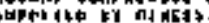

Jlaueu u

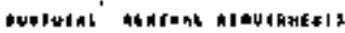

twi 15,001

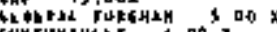

Cunsumauts

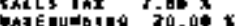
OHEY tod hatkers aniz

10ike cosi cune biat

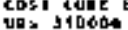

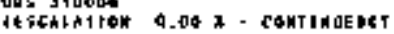

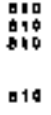

010

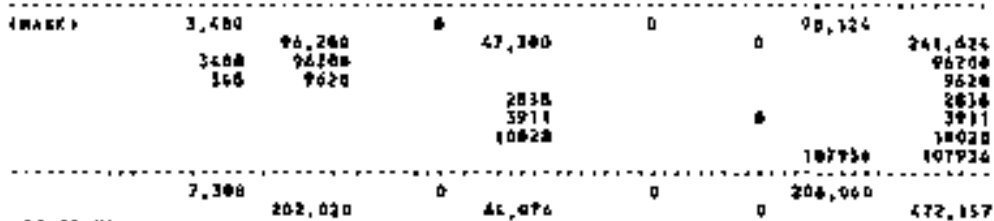

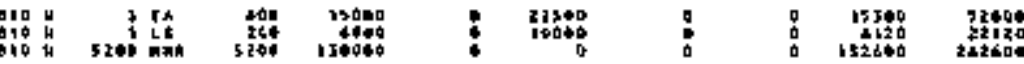

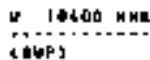
inipi

14,640 rets.

260400 49:406

10.

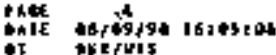

$\bullet$

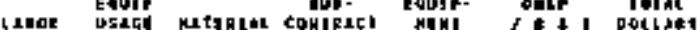




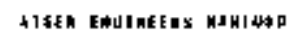

CITESHOUSE DAUFUA EOHFAKT

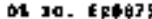

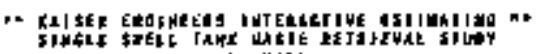

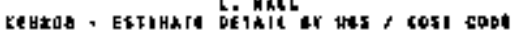

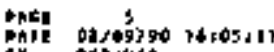

iy uretits

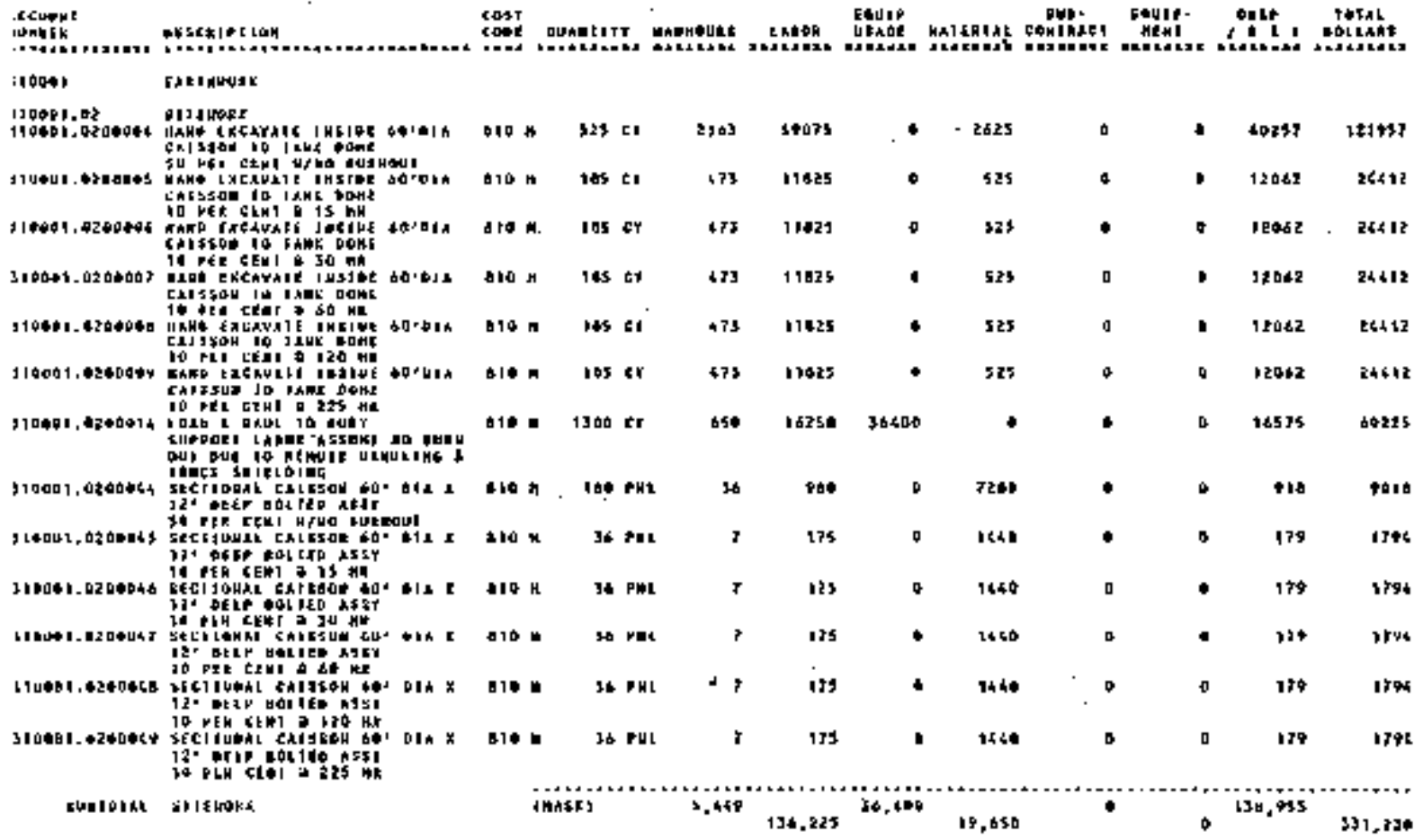




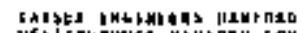

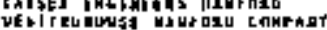
Jo4 MO. Ck4⿴囗十

\section{0u I I}

DIM日过 DESTMIPIITH

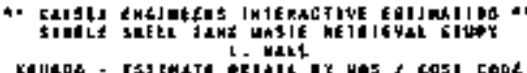

Coll

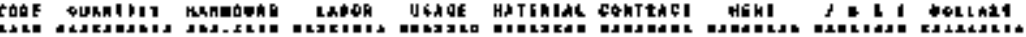

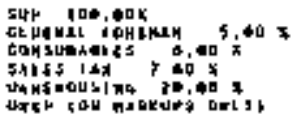

lorkh

cot coug otod 2

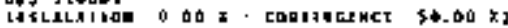

S1EUDE

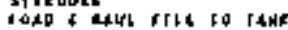

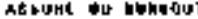

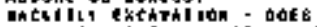

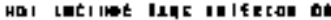

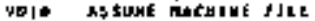

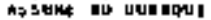

\$HUItolnt

Etuteri gatrand 5.0.

ando foM MLabups aury

1 1中:15:

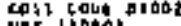

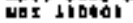

ALSCARIIAU

\begin{tabular}{|c|c|c|c|c|c|c|c|c|}
\hline 1100 & I yod $\leq x$ & \{\$3 & 11379 & 92400 & $\bullet$ & $\bullet$ & $\bullet$ & 11607 \\
\hline 10 & $1304 t$ & 453 & 11575 & $1 \% 500$ & 0 & $\bullet$ & d & 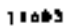 \\
\hline
\end{tabular}

IOIAE Hes J1000l EAklilvôth

,

.

o

1,000

27.571

38,906

5.,

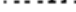

12,542

113.543

6. .540

2d, פנ,

-

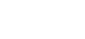

i $310,020 \quad 320,603$ 
46 couing

turatin

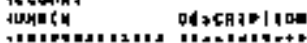

, a 0

34002,48

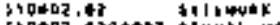

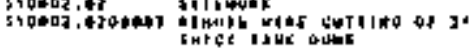

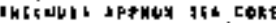

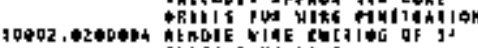

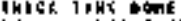

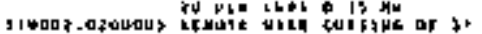

IFISK TREE BOHL

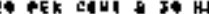

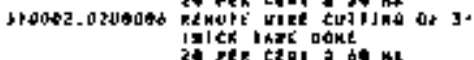

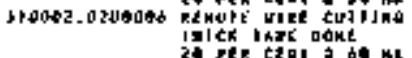

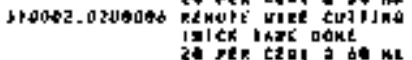

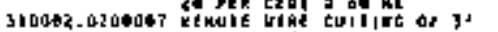

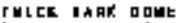

U l'tn CEHJ I20 HL

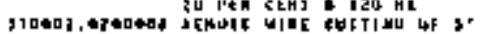

I MI CX INUK DOHF

?O PG CIMI

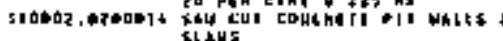

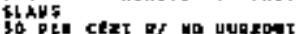

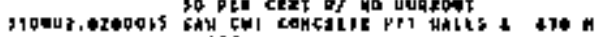
HAas

T 25

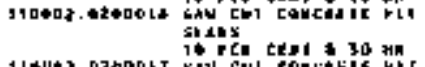

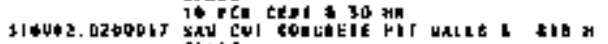

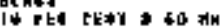

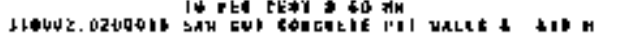

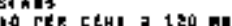

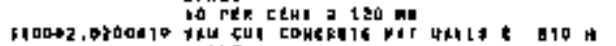
उ人,

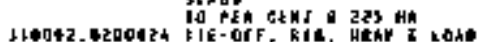
cnictitgé stcilíą

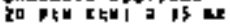

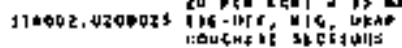

Q10 $\mathbf{n}$

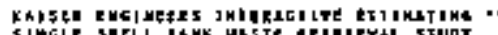

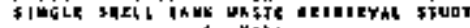

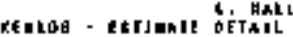

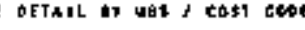

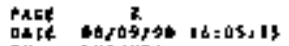

ar BKETH

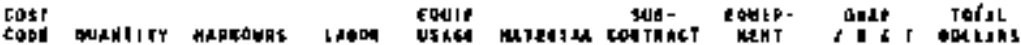

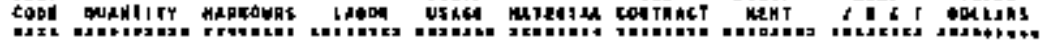

1910

16 it 162

sedo

1200

8) *1 747

40

12006

fol tedat

a10 M $1625 t$

fob 12000

49 M 36851

$400 \quad 12 * 00$

$361 \mathrm{br}$

Irs 14

ด1 2006

27 15

d1 Is

11

tr?

1127

$25 \mathrm{EH}$

11275

$23 \mathrm{kP}$

1123

25 if

$$
+11
$$

275

125

192

5376

14

$1 \uparrow 2$

5376 


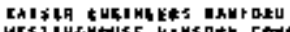

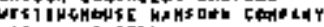

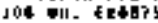

\section{$\operatorname{setadinj~}$ \\ แuพg I}

DESCRIPII:H

1h00tion

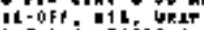
concmile setiloni

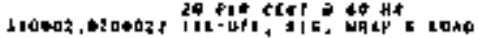

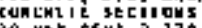

T) conentit setilans conckit setions at

b10402.020002t ilt

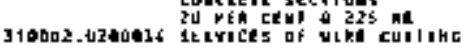

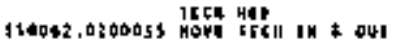

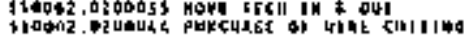

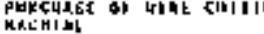

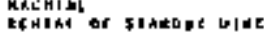

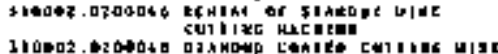

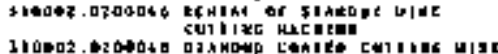

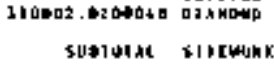

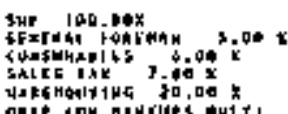

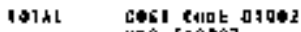

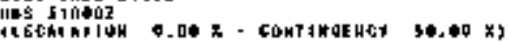

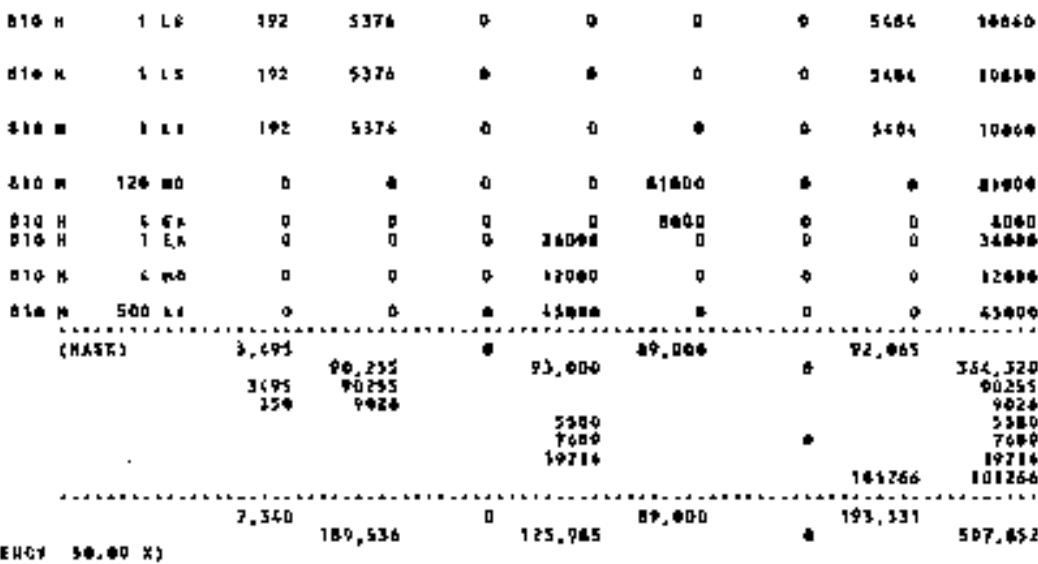

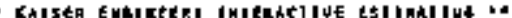

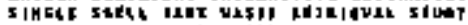

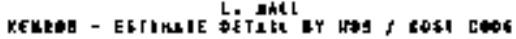

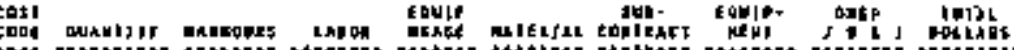

H. DE 


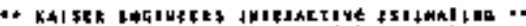

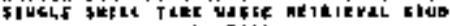

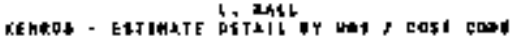

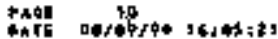
t1 brinfirid

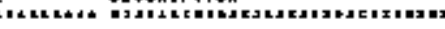

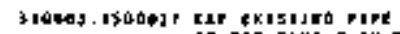

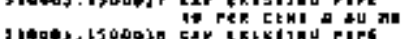

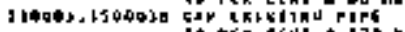

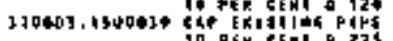

10 PEN CENI 223 HA

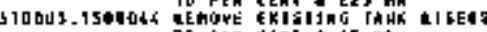

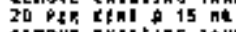

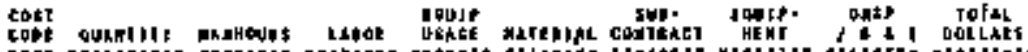

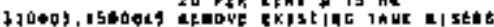

zo

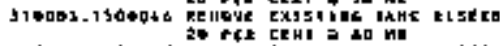

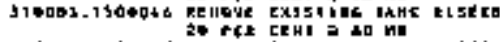

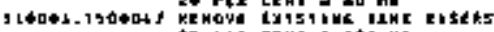

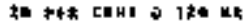

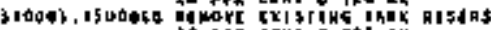

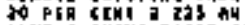

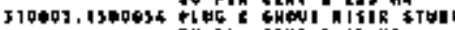

Z⿱ PEL CINI a is HA

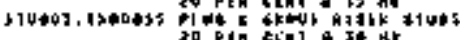

70 Din tind st $\mathrm{Hr}$

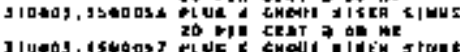

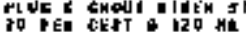

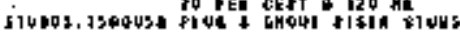

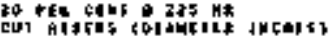

aró $x$

2697

$410 \Rightarrow+100 \%$

वION $N$ DO

a.*

61.

SEk

$74 \boldsymbol{1}$

int3

$1+23$

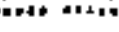

古以 5 I I I

110 $\mathrm{S}$ S Ih

Q1* $M$ S E h

Q14 $M S A$

a 6 S $5<\mathrm{k}$

H. $\pi$ Ste

$\rightarrow \mathbf{t a}$

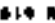

$10+1$

gat 01

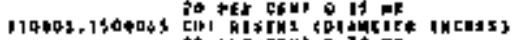

10.

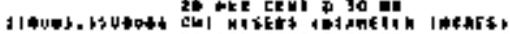

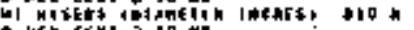

$105 \div 1$

IS5 B.

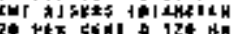

DIO $M$

186 . 1

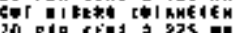

res ine is

$410 \mathrm{~m}$

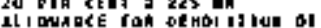

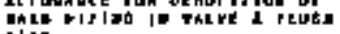

$1 \mathrm{k}$

195une so yanerus!

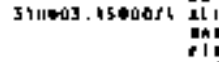

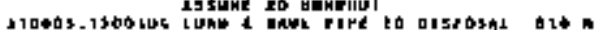

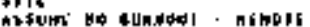

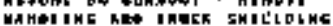




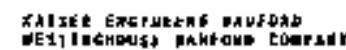

tot op. tk4tis

\section{Ntedanil \\ जчमקAk

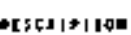

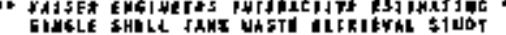

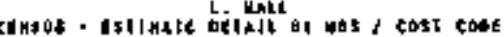

PAgr 11

ir DKaruls

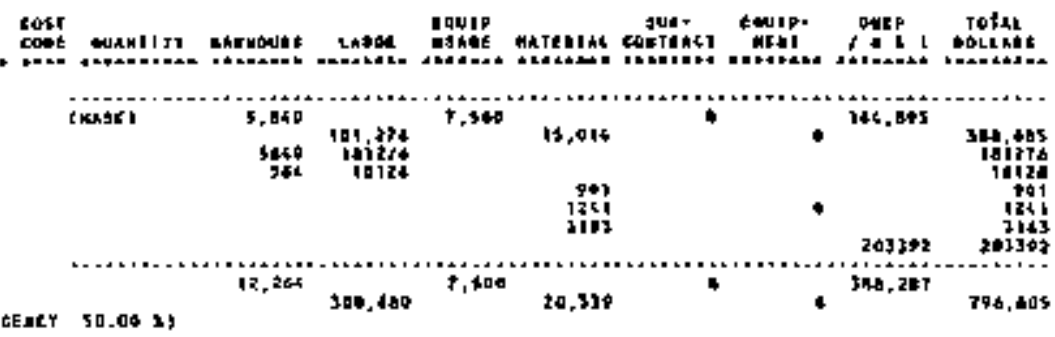

smater1L

MkCAMICAL

sur 100,40.

dEKETAI IOEE HAL 5,

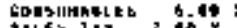

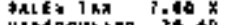

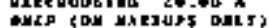

onine

cora saph oras

Mat 3 1.

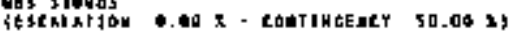

$12,10+$

180,464

7,500

20.339

d

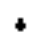

3at, ?67

7td, ubs 


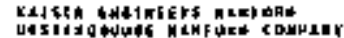
100 Me. En Dits

\section{necennI}

necwint

atsceiralos

blobot

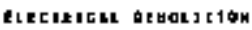

31040 , 18

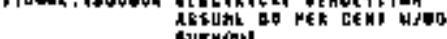

3̧18005 - 1

is sumt zó $v$ ip cteis is an

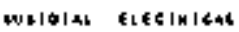

tup lab.obx

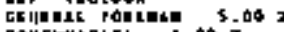

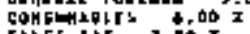

SHLET IAT $7,8+7$

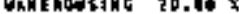

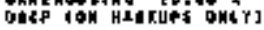

I tolne

\section{tall cote acoto}

uas bioug

cetthkilion

*.M z - coni Intener

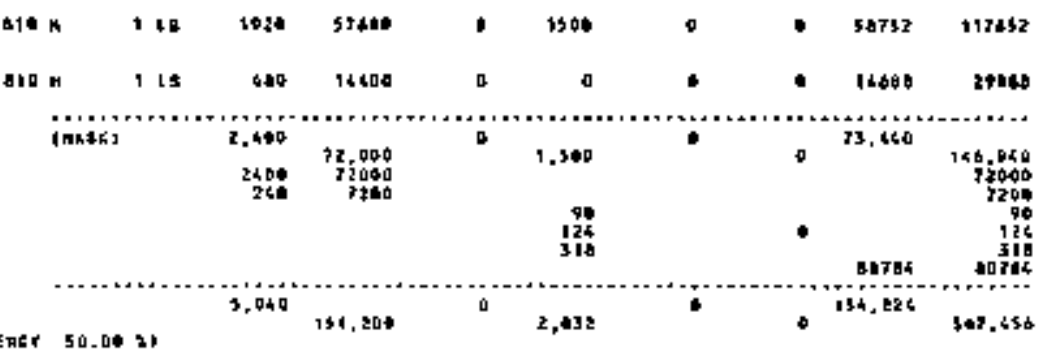

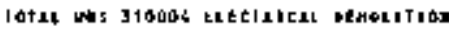

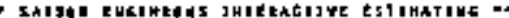

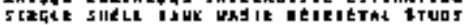

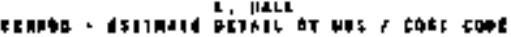

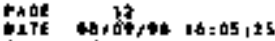

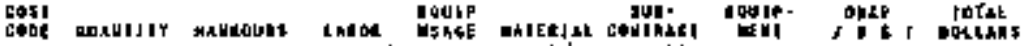

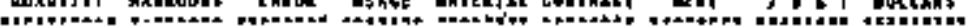

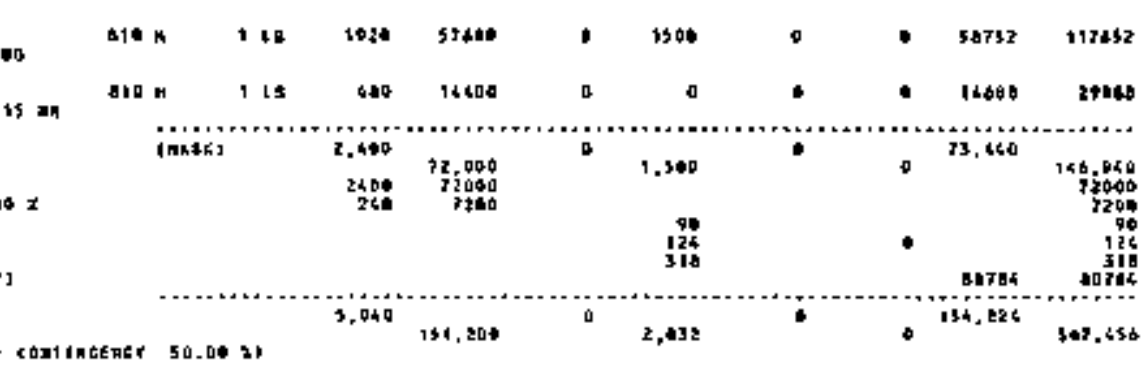


clitg fmolnten* MAnfanm

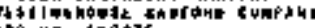
190 MO. L20 a

\section{A5CDUtT}

WUHLE:

QE IEM IPI I

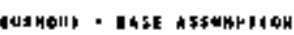

210014

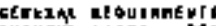

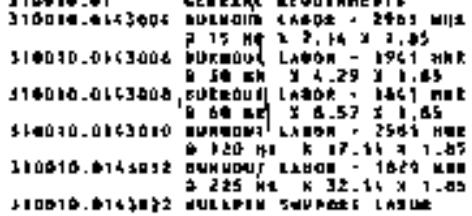

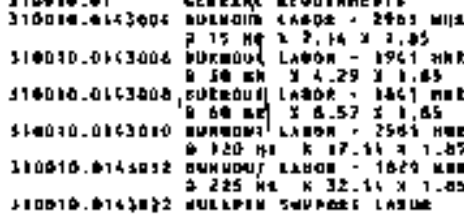

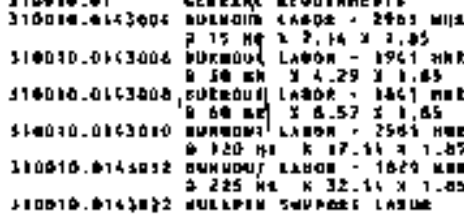

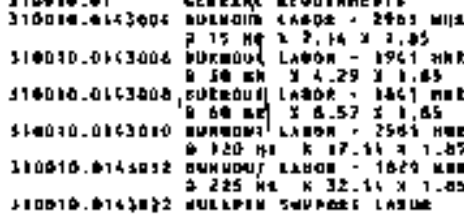

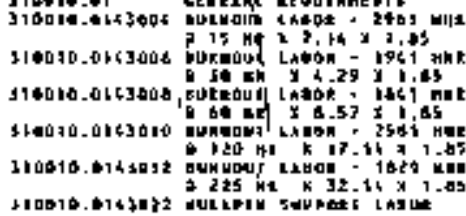

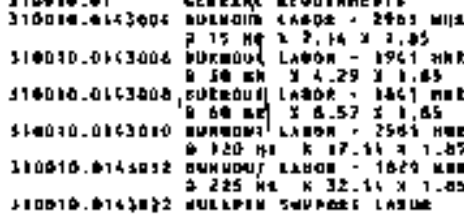

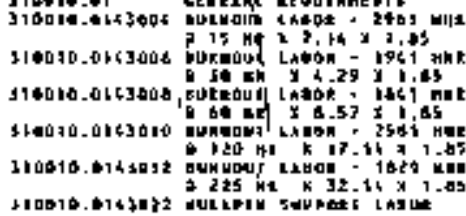

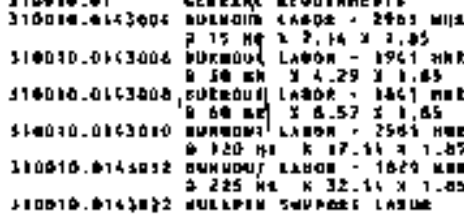

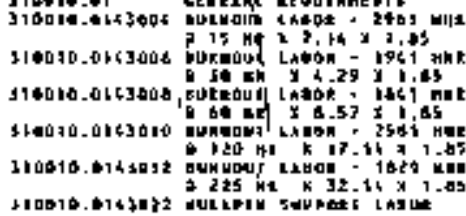

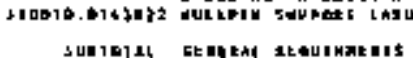

lol ks c091 top D1001 นะ 5 ind 10 TEECARE IOH

Je $z$ - cont inetert

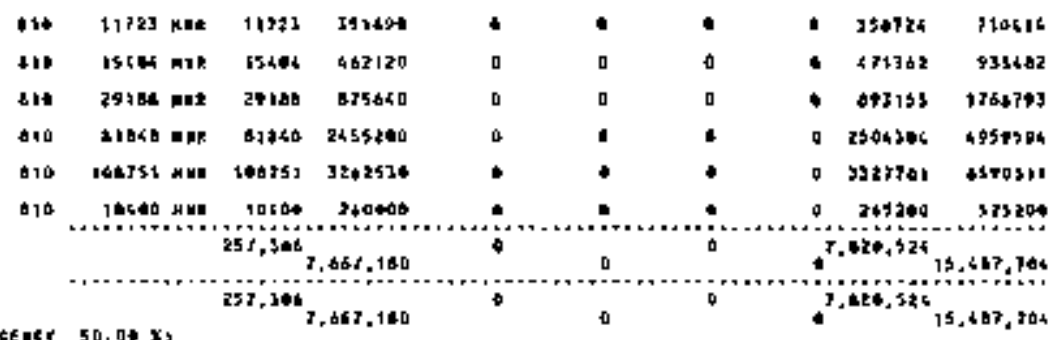

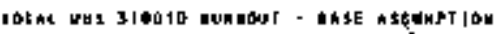

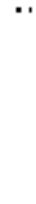

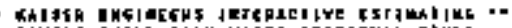

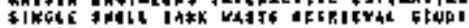

That 13

$04096 \% 1460926$

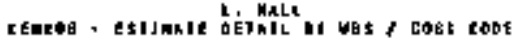
oxitrat

\section{tentr}

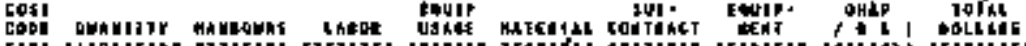

มี1 :

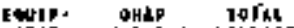




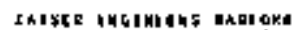
VI 1 [ हMG JUA HA, CIOAT?

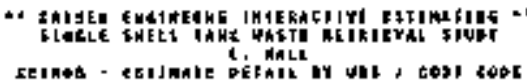

P10

par

IT

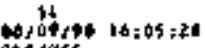
aterast

EAUIP- DHAP loline

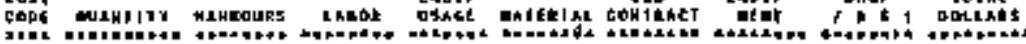
iftictisan MUI DStPQER TEEs UE

310040

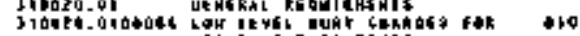

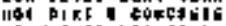
MIXEY U,

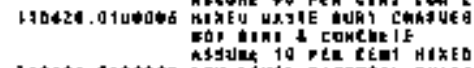

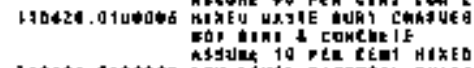

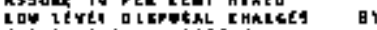

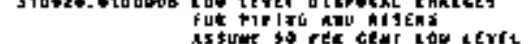

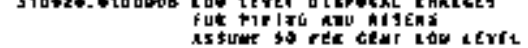

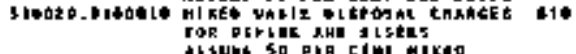

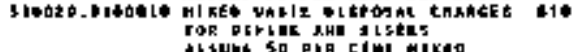
作

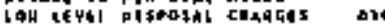

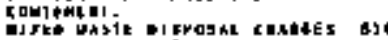

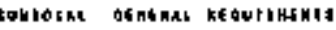

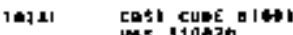

uns joazt

itsenth|

a. on 1 - conarimgency

1

1086 \&f

$2 \$ 313$ if

2023 $\mathrm{CF}$

5) C

5046

2506

1000 s6

5344 5

. . . . . . . . . . . .

0

(1)

o

(n)

$50,00 \%$

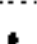

o

$0 \quad 002647$

a 31769

- 15rzz

- 5\$067

a Patrs

- 112130

- 4areos

- 12uts $\bullet$

-

o

$\bullet$

-

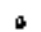

$\bullet$ $\bullet$

- 121773
- $4026+8$

a Mitas

1 15725

- 56065

- Tot 75

- 192330

- 4arots

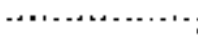

1.981 .747

$1,4+1,7+7$ 
HNF-SD-TWR-AGA-004

Rov 0

Figure A-1. Diamogd W'ire Curulng Techrology.
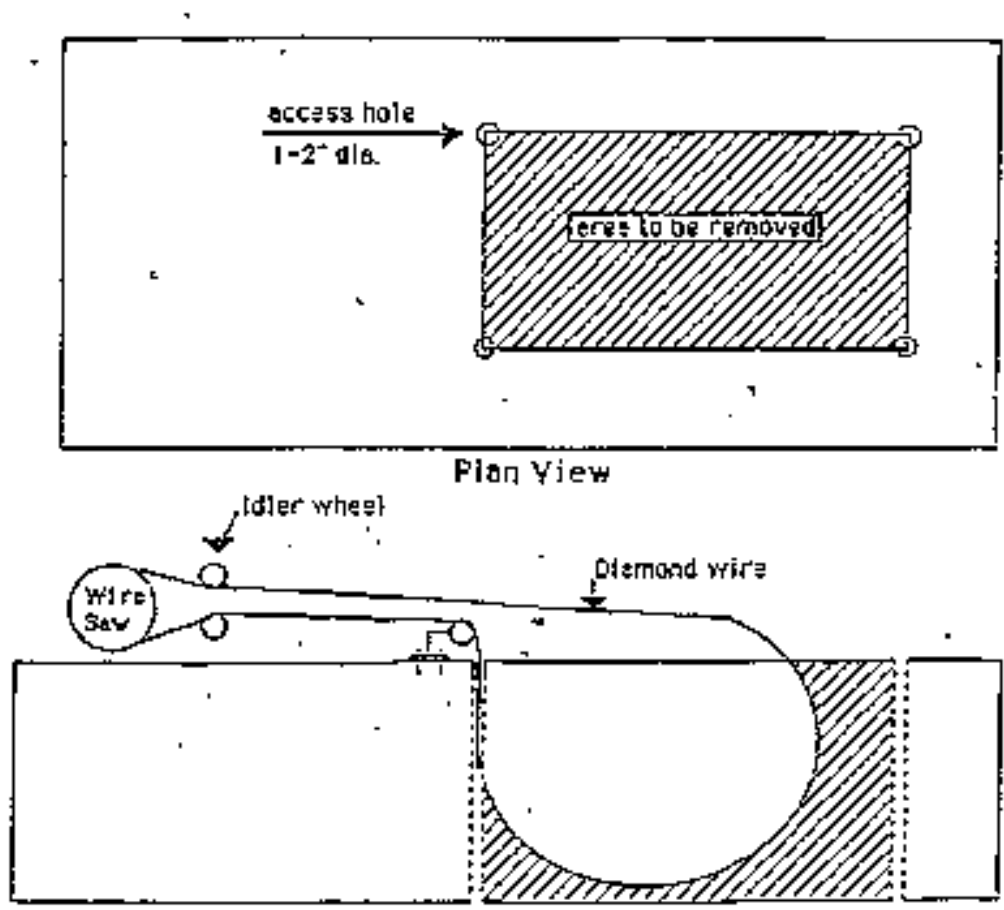

Seckion Yies 
HNF-SD-TWR-AGA-DO4

Rev. o

Figure A-2. Overview of 48-Font Diameter Hole in Single-Shell Tank,
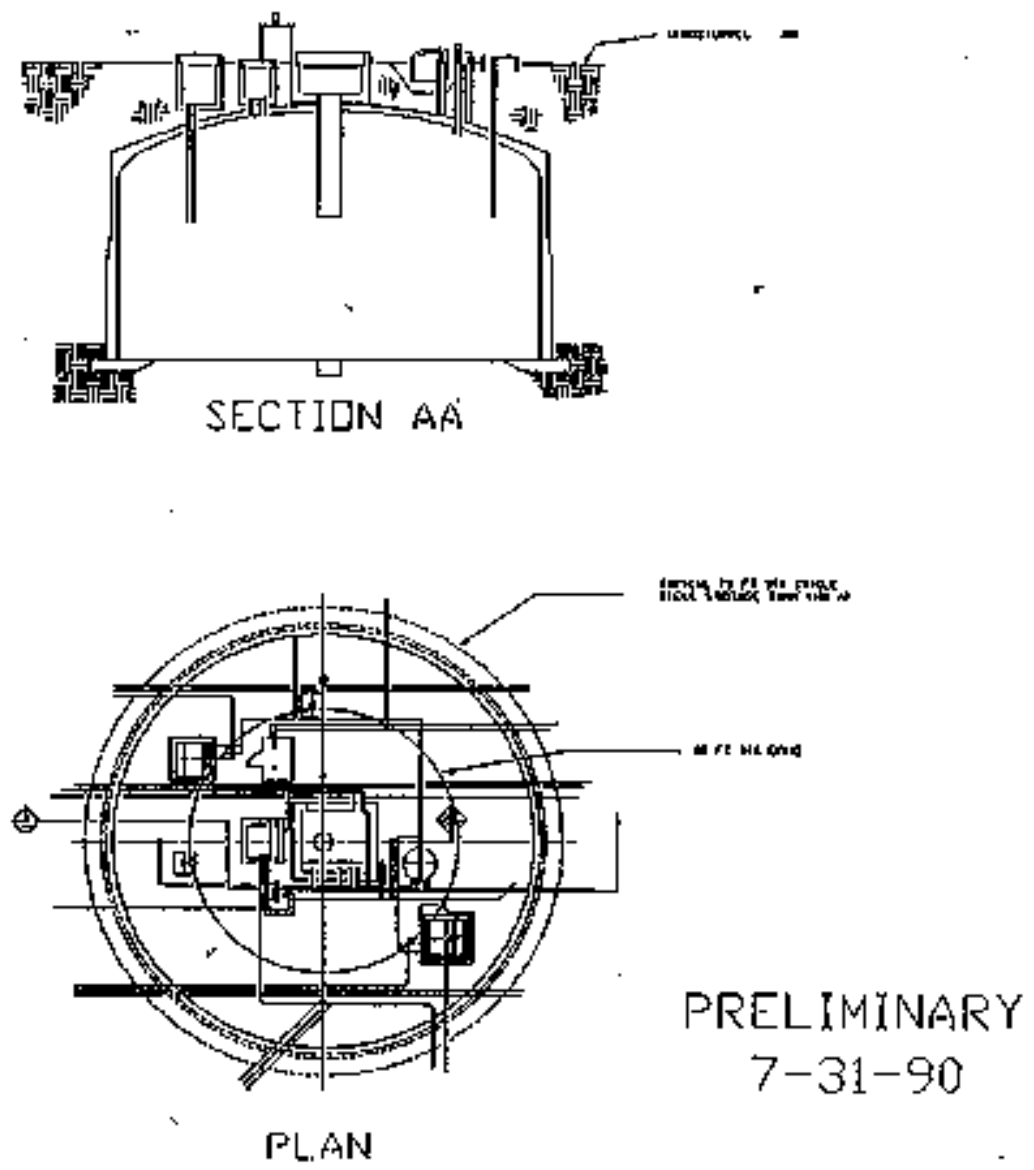


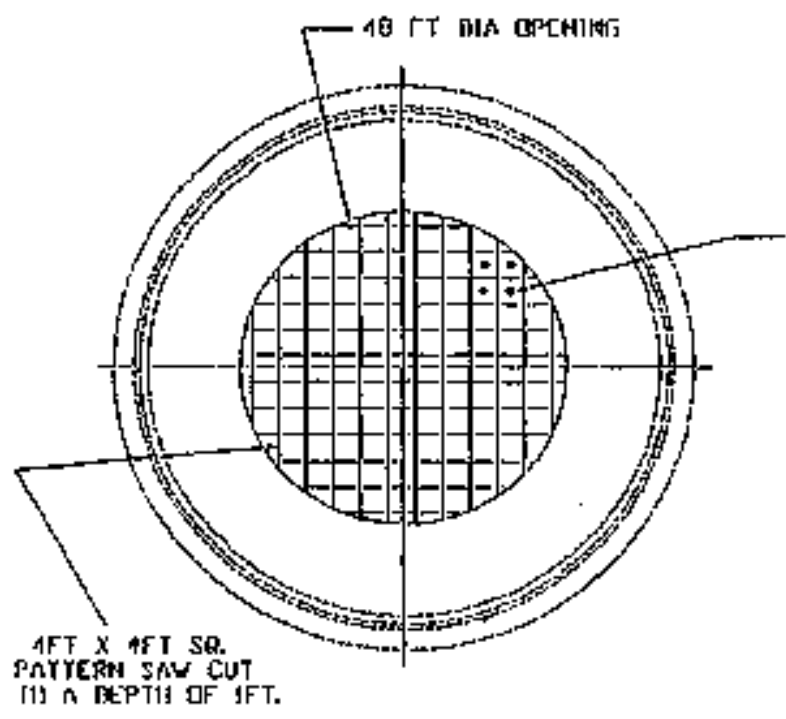

ATTACA A LIF TIMG LUT

YO ENCI INOAE SECTITW

TD DC RCH]VF.P \&TYP>,

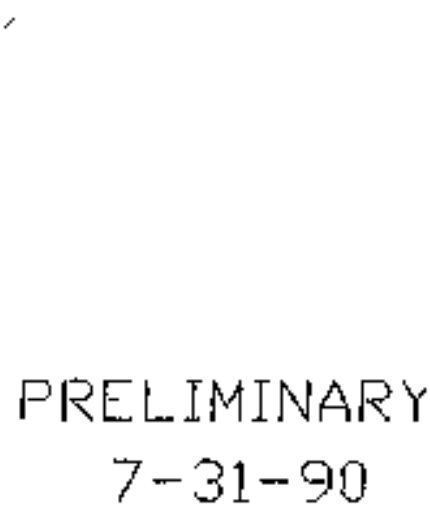

DE WNDE GY REMCJTE CUYTING DEVICE LOCAYED

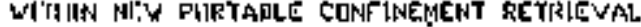

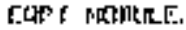




\section{Rev 0}

Figue A-4. Overvitw of 12-Foot Diameter Hole in Single-Sheld Tank Wilh Enclosute.

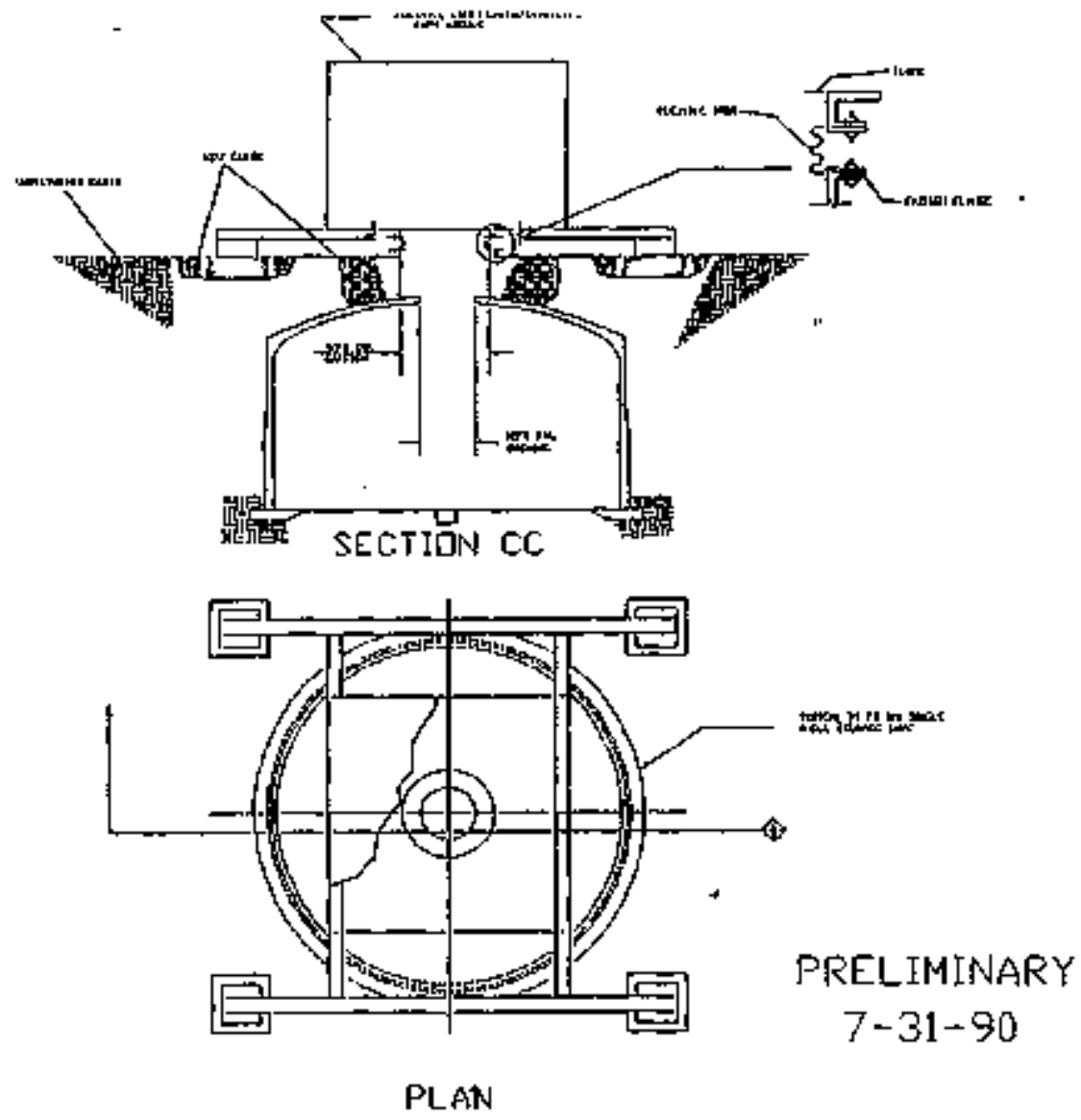




\section{ReV 0}

Figure A-5. Overyiew of 48-Foot Diamever Hole in Single-Shell Tark, With Epciosure.
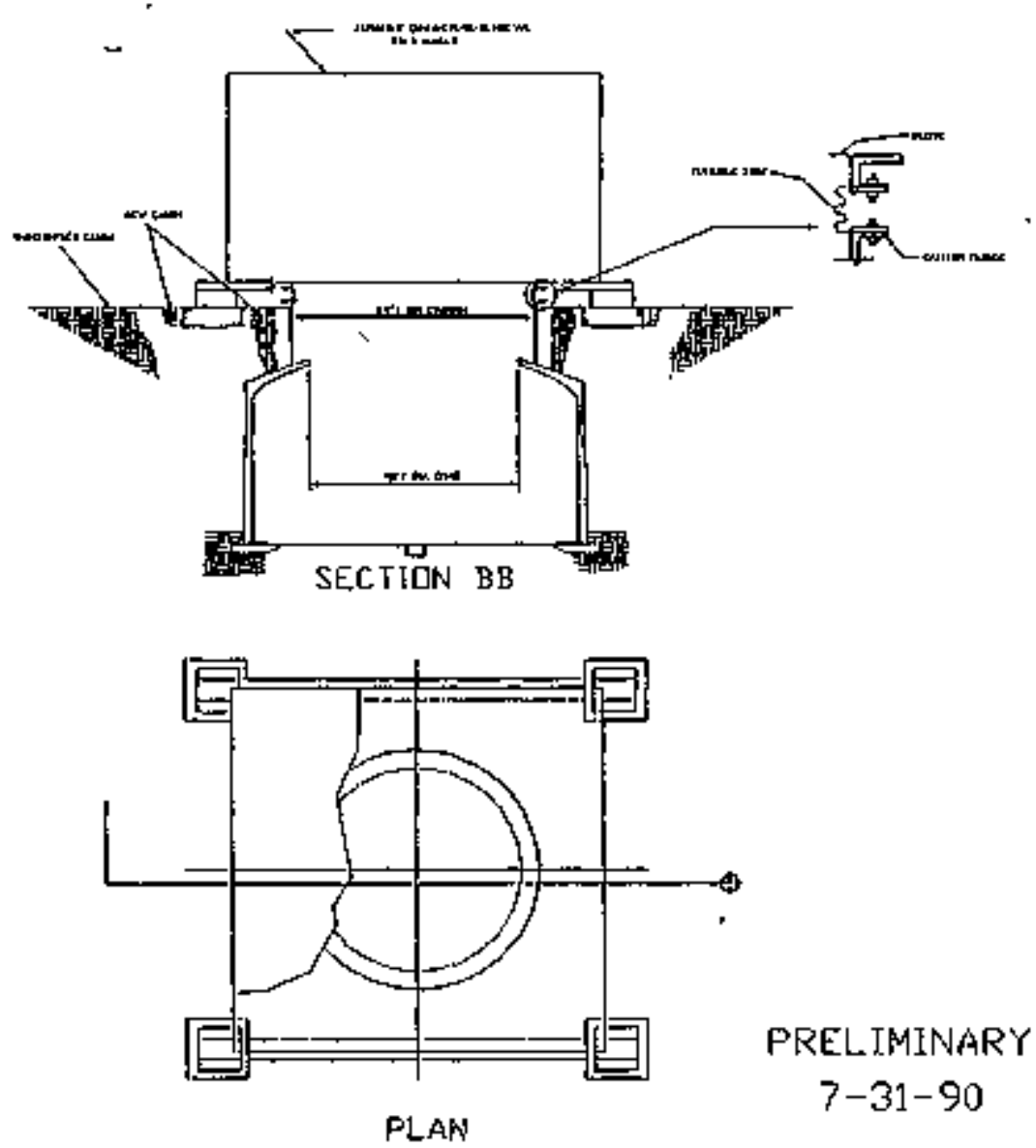

PRELTMINARY $7-31-90$ 
HNF-SD-TWR-AGA-DA4

Rev. 0

\section{A2.3 CANYON BUILDINGS (OPTTONS 4A AND 4B)}

Table A 13. Summary of Estinatted Coosts for 221-tu Canyon Bnilding Disposition Based on

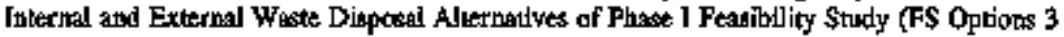
and 4)." (2 Sheets)

\begin{tabular}{|c|c|c|c|c|}
\hline \multirow{2}{*}{ Alletagtire } & \multicolumn{2}{|c|}{ Internal pragte disposal } & \multicolumn{2}{|c|}{$\begin{array}{l}\text { Internal texternal waste } \\
\text { disposal }\end{array}$} \\
\hline & $\$$ million & Apqlicable & S million & Applicablet \\
\hline \multicolumn{5}{|l|}{ 1.0 Prepare Existing Complex } \\
\hline Establish Hazarda Protection & 0.87 & 0 & 1.35 & No \\
\hline Eglablish Infragtrocture & 2.30 & 0 & 2.30 & No \\
\hline Modify Facility & IS.I & 9.1 & I5.1 & 9.1 \\
\hline Mopdify External Area & 17.6 & $\mathrm{ONO}$ & 15.5 & 6.0 \\
\hline Manage Hexsordous Materials & 0.58 & 0.58 & 0.58 & $Y_{e s}$ \\
\hline 1.0 Applicable Subtatal & 36.5 & 9.7 & 34.8 & 15.7 \\
\hline \multicolumn{5}{|l|}{ 2.0 Operate the Conplex } \\
\hline $\begin{array}{l}\text { Decontaminate Surfaces, } \\
\text { Equipment asd Stroctores }\end{array}$ & 0 & 0 & 0 & 0 \\
\hline $\begin{array}{l}\text { Remove Equlpment and } \\
\text { Structures }\end{array}$ & 0 & 0 & 0 & 0 \\
\hline Jdentlfy Waste Sources & 0.1 & 0.1 & 0.1 & 0.1 \\
\hline Process Wraste & 0.76 & 0 & 0.76 & 0 \\
\hline Dispose of Waste Makrials & 15.5 & 15.5 & 41.4 & 20.7 \\
\hline $\begin{array}{l}\text { Maintain Walt Operations } \\
\text { Capability }\end{array}$ & 0.76 & 0.76 & 0.76 & 0.76 \\
\hline Demolish and Excarate Building & 0 & 0 & 0 & 0 \\
\hline Collapse Mait Strukture & 0 & 0 & 0 & 0 \\
\hline 2.0 Applicable Soblotal & 17.1 & 16.4 & 430 & 21.6 \\
\hline \multicolumn{5}{|l|}{ 3.0 Close the Complex } \\
\hline Equablish Closuce \$ystejns & 1.51 & 0 & 1.51 & 0.8 \\
\hline Install Final Barriers & 24.8 & 0 & 24.8 & 12,4 \\
\hline Sugtain Post Closure & 2.40 & 0 & 2.40 & 1.2 \\
\hline 3.0 Appllable Subtotal & 28.7 & 0 & 28.7 & 14,4 \\
\hline
\end{tabular}


Rey, 0

Table A-13. Sumbary of Estimalted Costs fit 221-U Canyon Bujilding Ditposition Based on Internal and External Waste Dioposal Alternatives of Phase [ Peagibility Study (FS Options 3 and 4)." (2 Sheets)

\begin{tabular}{|c|c|c|c|c|}
\hline Alternativive & \multicolumn{2}{|c|}{ Internal wrasto disposal } & \multicolumn{2}{|c|}{$\begin{array}{c}\text { Internal/exterfal watut } \\
\text { disposal }\end{array}$} \\
\hline Subtotal All Applicable & B2.3 & 28.4 & 106.5 & 49.7 \\
\hline Contitisency at $35 \%$ & 28.8 & 9.9 & 37.3 & 17.9 \\
\hline Applicable Total Capisal Cost & 111.1 & 38,3 & 143.8 & 67,1 \\
\hline
\end{tabular}

'Source of information is DOE/RL-97-1L (FS Options 3 and 4).

- Activity evalnated as applicable to capital or operating cost for subgequent complorison.

Table A-14. Summary of Adjusted Applicable Estimated Consunction and Choure Costs for Disposition of Foor Canjon Buildings Based on Intertal and Extental Waste Disposal Alamatives of 2Rl-U (AGA Options 4A and 4B).'

\begin{tabular}{|c|c|c|c|c|}
\hline Option & $\begin{array}{c}\text { Constrixction } \\
\$ \mathrm{M}\end{array}$ & $\begin{array}{c}\text { lofragerstare } \\
\$ M\end{array}$ & Clooure \$ M & Totol \$ M \\
\hline $\begin{array}{l}\text { 4A - Internal Waste } \\
\text { Disposal }\end{array}$ & $65.1^{b}$ & $o^{d}$ & $\theta^{\circ}$ & 6.1 \\
\hline $\begin{array}{l}\text { 4B - Internal and } \\
\text { Bxternol Waate } \\
\text { Disposal }\end{array}$ & $105.4^{4}$ & $a^{d}$ & 83. $2^{\mathrm{a}}$ & 188.6 \\
\hline
\end{tabular}

- Scurce of information is Table A-13 and below calcalations.

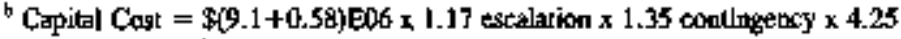
Equivalent Buildings $=\$ 65.1$ million

"Capital Cost = \$(IS.T)E06 x I.17 escalation X 1.35 contingency $\times 4.25$ equivalent buildings $=\$ 105.4$ milion

4 Infrastructure $C$ ost $=\$(0) E 06 \times 1.17$ eacalation $\times 1.35$ comlingency $\times 4.25$ equirakent builitings $=\$ 0 \mathrm{~m}$ لlion

Closure Cost $=\$(12.4) E 06 \times 1.17$ escalation $\times 1.35$ comtinizency $\times 4.25$ equivaleal buildingg $=\$ 63.2$ millon, 


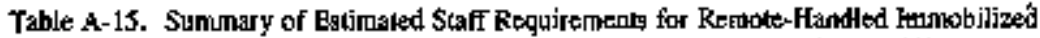
Lor-Activity Whaste Contajiner Disposal Phase I and Pbase $D$ (Option 4A).

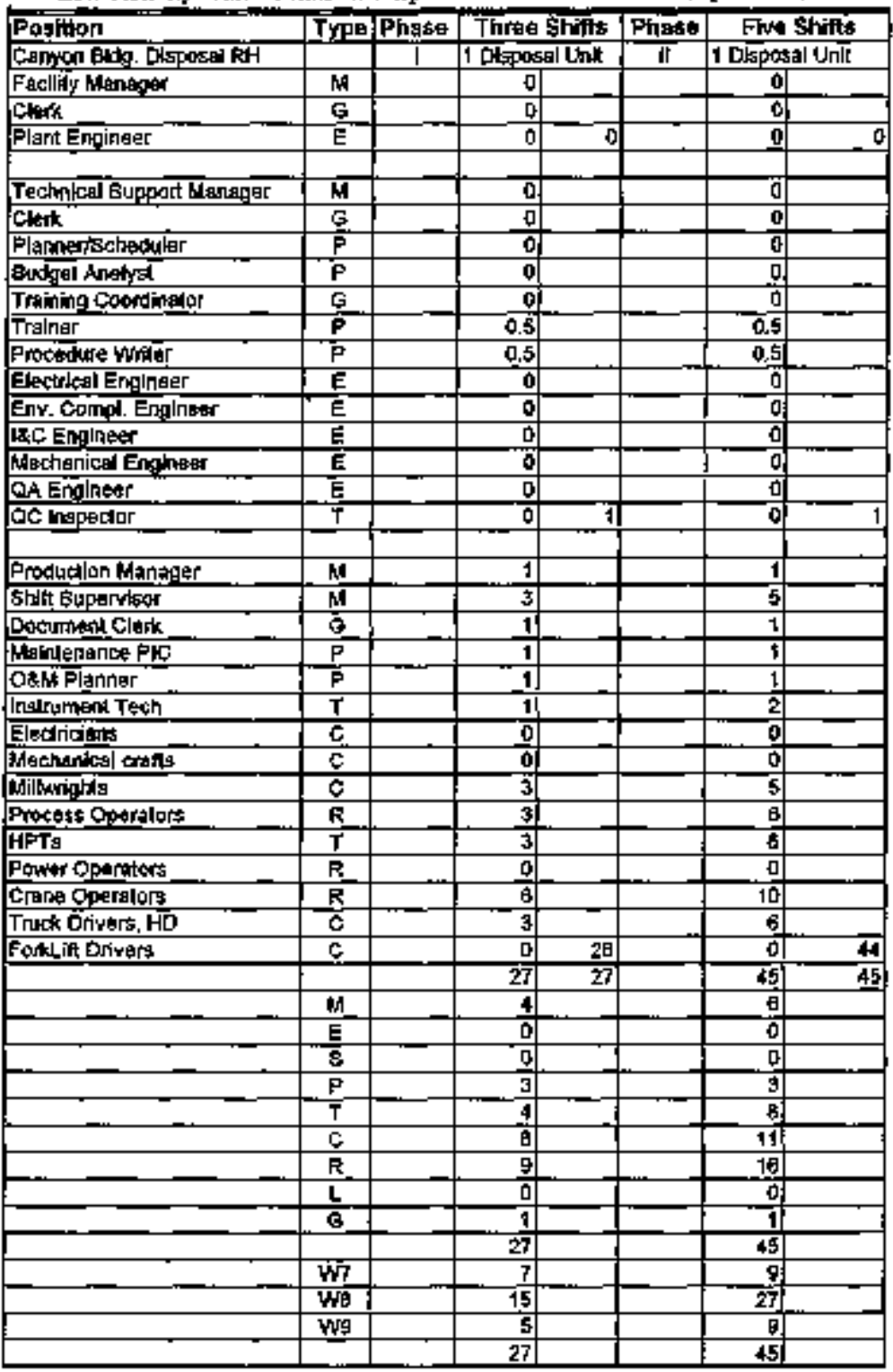


Twis A-15 \$ \$

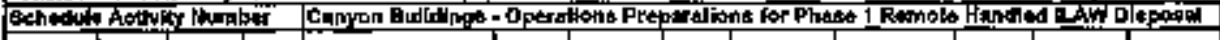

\begin{tabular}{|c|c|c|c|c|c|c|c|}
\hline & & & & & & & \\
\hline FTL & Flonths & Noth & MK & $7 \%$ & Curve & Stant & Floled \\
\hline 2 & 74 & 1 & 7,400 & 520 & 2 & FUOO & 613602 \\
\hline $\bar{D}$ & 24 & 1 & 8 & 0 & 2 & 5400 & Bavor \\
\hline 1.5 & 27 & 1 & 5,550 & 35 & 2 & Bron & 5rotoz \\
\hline 2 & 24 & 1 & 7.4006 & 413 & 2 & EXito & $5 \times 31+06$ \\
\hline 3 & 24 & 1 & 11.100 & $\mathrm{E} 20$ & 2 & हर्म०ण & जतमू \\
\hline 4.5 & 21 & 1 & 16.50 & Fुo & 2 & Eराम्र & इअवर्म \\
\hline 0 & 24 & 1 & 0 & 0 & 2 & W1P0 & 539102 \\
\hline 0.5 & 24 & 1 & 1,50 & 52 & 2 & हलग00 & \$सरकी \\
\hline 13.5 & 24 & 2 & & 3 & 2 & Er 1000 & \$31, \\
\hline 15.5 & 24 & 2 & & 34 & 2 & ENलSO & $631 \pi_{2}^{2}$ \\
\hline 15.5 & 24 & 2 & & i? & 2 & हलग0 & $5 \$ 102$ \\
\hline & 24 & 4 & & 3 & 2 & sitnot & 503102 \\
\hline & & & 49800 & 90 & & & \\
\hline & & & & & & & \\
\hline
\end{tabular}

\begin{tabular}{|l|l|l|}
\hline & & \\
\hline & & \\
\hline & & \\
\hline & & \\
\hline & & \\
\hline & & \\
\hline & & \\
\hline & & \\
\hline & & \\
\hline & & \\
\hline
\end{tabular}

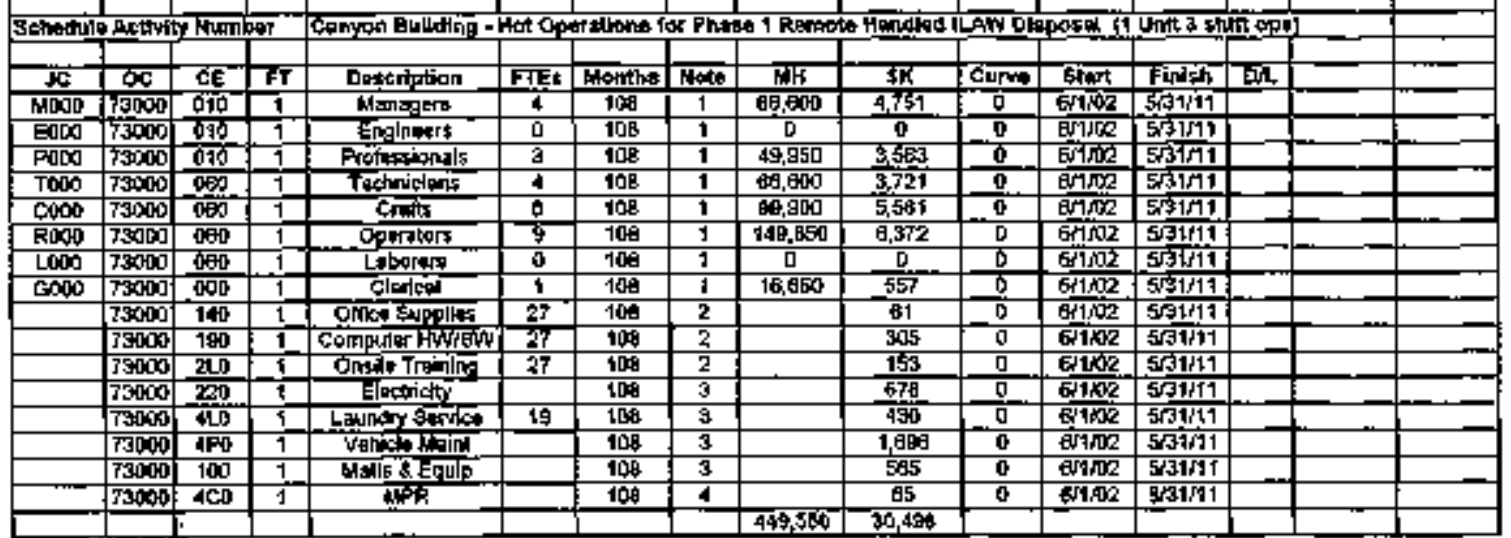




\begin{tabular}{|c|c|c|c|c|c|c|c|c|c|c|c|c|c|c|c|}
\hline \multicolumn{4}{|c|}{ 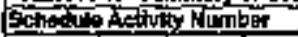 } & \multicolumn{3}{|c|}{ 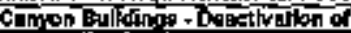 } & \multicolumn{6}{|c|}{ 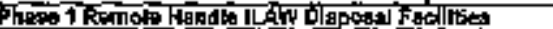 } & \multicolumn{2}{|c|}{\begin{tabular}{|l|l|} 
& \\
\end{tabular}} & 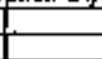 \\
\hline$\sqrt{5}$ & OC & $\mathrm{CE}$ & Fi & Oabelporion & FE: & Wonth. & Thole & कास & 5 & Eurve & Start & Pinlsh & DIL & & \\
\hline MoO0 & 33000 & 010 & $\overline{1}$ & Mantegtry & 2 & 12 & 1 & $\widehat{3,700}$ & $2 E 4$ & 3 & Exit1 & $501 / 2$ & & & \\
\hline$E 000$ & 73000 & 010 & E & Englonts & 0 & 12 & 1 & 0 & 0 & 3 & स्ता & ज小112 & & & \\
\hline$P \operatorname{son} 0$ & $7+300$ & Q10 & $T$ & Proplessionatit & 1.5 & 12 & 1 & $2, \mathrm{JPB}$ & TDQ & $\mathrm{a}$ & 61151 & ज्ञातर & & & \\
\hline$\sqrt{100}$ & उड़OCO & 整 & T & Tochricions & 2 & 12 & 1 & 3,700 & 207 & 3 & कासह1 & प्रता12 & & & \\
\hline con & 73000 & Don & 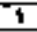 & Crahs & 3 & 13 & 1 & ह5,55 & 310 & 3 & छका & 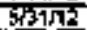 & & & \\
\hline Pुbo & 73000 & $0 \$ 0$ & 1 & Opersifont & 4,5 & 12 & 1 & 0,256 & 485 & 3 & 6119 & $\sqrt[3]{512}$ & & & \\
\hline 100 & 73000 & OE & 1 & Laborers & 0 & 12 & 1 & 0 & 0 & 3 & घ1111 & इन/ & & & \\
\hline उत् & F⿻大 & 0,0 & $T$ & Clatual & 0.5 & 12 & 1 & 825 & उद & $3^{7}$ & घातन & इनाकान & & & \\
\hline & 7300 & $2[0$ & T & Buter Tring & 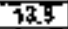 & 12 & 2 & & 1 & 3 & प्रा11 & इतुगय & & & \\
\hline & 73500 & 220 & 1 & Elociricity & & 12 & 3 & & 25 & 3 & Eखा1 & $5 \sqrt[3142]{2}$ & & & \\
\hline & 5 & 4 & 1 & Lalonx & 25 & 15 & $\bar{a}$ & & 24 & $\overline{3}$ & 6111 & इसुजाल & & & \\
\hline & $73 \times 00$ & $4 \mathrm{CD}$ & $T$ & AMPR & & $\frac{1}{12}$ & 1 & & 0 & $\frac{7}{3}$ & E्रात1 & 531/12 & & & \\
\hline & & & & & & & & 2495 & TES & & & & & & \\
\hline \multirow{2}{*}{\multicolumn{4}{|c|}{ 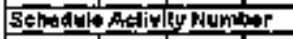 }} & & & & & & & & & & & & \\
\hline & & & & \multicolumn{3}{|l|}{ 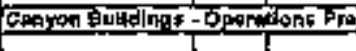 } & & $\operatorname{Aog} P$ in & 2 kin & 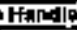 & ताएकास & 10pates & & & \\
\hline & & & & & & & & & & & & & & & \\
\hline$\pi$ & $\boldsymbol{\alpha}$ & EE & FI & DE:Fcisitom & Files & Fonfin: & Wyote & 쌕 & 彦 & Clave & stan & Finatin & DEL & & \\
\hline 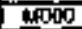 & 7 काष्ठ & $0 \overline{0}$ & 1 & 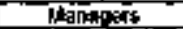 & $-\frac{3}{7}$ & 24 & 1 & 11.100 & 782 & -2 & 51208 & 45211 & & & \\
\hline EOD & $7 \sqrt{30000}$ & भक & $\frac{1}{1}$ & Englinas: & 6 & 34 & 1 & 0 & to & 2 & $5 \sqrt{1,08}$ & $\sqrt{3} 211$ & & & \\
\hline Poot & $\sqrt{2}$ & $8 \sqrt{10}$ & 1 & Frolesenonals & 1.5 & 24 & 1 & 5,300 & जिए & $\frac{2}{2}$ & हुवान्म & $4 \sqrt{617}$ & & & \\
\hline $\min x$ & 73000 & 000 & 1 & 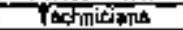 & 4 & 24 & T & 14, Bकाप & 87 & 2 & 5ग0 & युजस & & & \\
\hline CDo 0 & 7 హో & 080 & 1 & Conts & 6.6 & 26 & 1 & 20,350 & 1.137 & $\frac{2}{2}$ & SMM & $4 x+1$ & & & \\
\hline R600 & 7306 & 0,0 & $i$ & Opentisar & 8 & 24 & 7 & 29,000 & 1,654 & 2 & इएवक्ष & क्षoरf & & & \\
\hline 1000 & ऊळ & 000 & 7 & Lotomes? & 0 & 24 & 1 & 0 & 6 & 2 & बल西 & 40011 & & & \\
\hline Eण00 & 8,3000 & 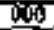 & & CाFint & 0.6 & 24 & 1 & 1,850 & 可 & 2 & आबक्ष & 40111 & & & \\
\hline & 7000 & ब柿 & 1 & of & 2.5 & 24 & 2 & & $\pi$ & 2 & $\sqrt{3 \sqrt{n}}$ & dis & & & \\
\hline & 7300, & 196 & 1 & Eomenter HWh & 25 & 24 & 2 & & 57 & 2 & $\sqrt{4}, 02$ & \$क्श & & & \\
\hline & $\sqrt{3000}$ & 20 & 1 & 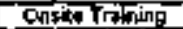 & 25 & 24 & 2 & & खु & 2 & $5 \mathrm{sin} 2$ & सअज्ञा1 & & & \\
\hline & $\sqrt{3000}$ & बद & 5 & I I & & 24 & 4 & & 3 & 2 & 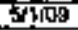 & बक्या11 & & & \\
\hline & & & & & & & & $8,2,260$ & 46 & & & & & & \\
\hline
\end{tabular}




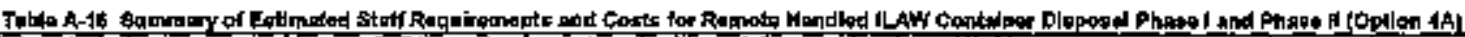

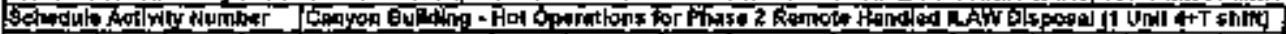

\begin{tabular}{|c|c|c|c|c|c|c|c|c|c|c|c|c|c|c|c|}
\hline & & & & & & & & & & & & & & & \\
\hline $\mathbf{K}$ & EC & ck & Fu & Doteriotion & Fit: & Thonts: & Wots & T्रF & झू & Ĕarve & ; & Finlsh & D, & & \\
\hline 2000 & 73000 & प्राण & 1 & Mantagdra & 6 & 184 & 1 & 157.76 & FD, Eून & 0 & EMAdit & $12 \sin ^{2}$ & & & \\
\hline $\operatorname{EONO}$ & $730 \times 6$ & 010 & 1 & Engineers & 0 & 164 & $t$ & 0 & 0 & 0 & ENA1 & $12 \sqrt{3} 1 / 24$ & & & \\
\hline 5000 & 75000 & 010 & 1 & Scoinifins & 0 & 198 & 1 & 0 & 0 & 0 & $5 M A 14$ & 1203124 & & & \\
\hline 1000 & 73000 & पio & 1 & Profossignnets & $\mathbf{a}$ & 184 & $\mathbf{1}$ & J5, 050 & $5410^{\circ}$ & $0^{\circ}$ & S़স & $12 \sqrt{112}$ & & & \\
\hline TW0 & 73000 & 5 政 & $\frac{1}{1}$ & Fochonklans & $\sigma^{-1}$ & 184 & 1 & 202,87 & प1. ख़्य & $\frac{7}{0}$ & समता & $12 \pi 3124$ & & & \\
\hline हDo & $73 \times 0$ & 000 & i & 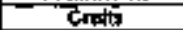 & 11 & $18 \mathrm{~A}$ & $\mathbf{1}$ & 278,17 & $16,5 \times$ & d & हमाना & 12तiाय & & & \\
\hline 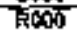 & 73000 & 060 & 1 & Ophaturat & 16 & $\sqrt{134}$ & 7 & 404,535 & 22,61 & 0 & जा/1 & 12/3124 & & & \\
\hline 2000 & 73000 & $\overline{0} 60$ & 1 & Lefborass & 0 & 184 & 1 & D & 0 & $0^{\circ}$ & इ्सम & 12म174 & & & \\
\hline 0000 & $7 \times 0$ & 00 & 1 & Cinericil & 1 & $1 \leqslant 0$ & 1 & 26,363 & प़ेष्ब & 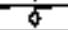 & उत्रण 1 & $12+31 / 2$ & & & \\
\hline & 5300 & 140 & 1 & 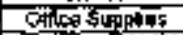 & $4 d$ & 164 & 2 & & $15 \overline{4}$ & D & अपना 1 & 12जa & & & \\
\hline & 100 & igh & 1 & conouker fHMSHY & 45 & 164 & 2 & & 7 & 8 & जूला & 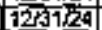 & & & \\
\hline & 730 & 20 & 1 & comete traing & 45 & 便 & 2 & & 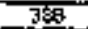 & 0 & इसमा? & 12खा & & & \\
\hline & 73000 & 20 & 1 & EHomety & & 184 & 3 & & 1,373 & 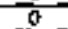 & 51/11 & 120120 & & & \\
\hline & 73000 & HD & 1 & 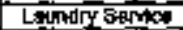 & $\sqrt{5}$ & 164 & 3 & & 1,202 & 0 & जराम? & $12+3124$ & & & \\
\hline & $5 \% 0$ & $\triangle P 0$ & 1 & Verth & & 154 & $\overline{3}$ & & 724 & 6 & इस्जा & $12 \sqrt{312}$ & & & \\
\hline & sow & 100 & $\frac{7}{1}$ & 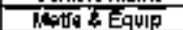 & & 164 & $\$$ & & 粗 & $\frac{2}{6}$ & इमला & 1235124 & & & \\
\hline & 73000 & $4 \mathrm{CD}$ & 1 & 陑家 & & 154 & 4 & & 113 & $\frac{6}{6}$ & 51011 & $13 \sqrt{3124}$ & & & \\
\hline & & & & & & & & & 78,5 & & & & & & \\
\hline thedur & Inethy & $\mathbf{N a}$ & & 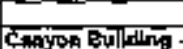 & 아에 & paron as & & Rpectie & intod & Tapo & $\bar{F} F=$ & & & & \\
\hline & & & & & & & & & & & & & & & \\
\hline 曾 & $\overline{O C}$ & $\overline{G E}$ & FT & Dosentiption & PT[5] & 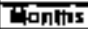 & ropto & न्रम & कार & 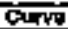 & 5क्रा & Fामक़्र & & & \\
\hline Mाएक & TFO & उन & 1 & Gв & 3 & 72 & 1 & 6,550 & 304 & 3 & ipti25 & 1030125 & & & \\
\hline 5000 & 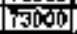 & 870 & 1 & Eng now & D & 17 & 1 & 0 & i & 3 & 1) 185 & 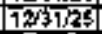 & & & \\
\hline Fखल & $5 \times 00$ & मू० & $\overline{1}$ & 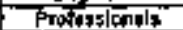 & 1,4 & 18 & 1 & 3776 & T然 & द & सा & 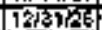 & & & \\
\hline$\pi \times 0$ & Tिरण & क्षण & $\bar{T}$ & Techruatas & 7.6 & $1 \frac{12}{2}$ & $\overline{1}$ & $2,7+5$ & 155 & $\overline{3}$ & $1 / 125$ & $-12 \sqrt{31 \Omega 5}$ & & & \\
\hline 0000 & 73000 & abo & 1 & Cा & 4 & $\overline{12}$ & $\overline{1}$ & 7.90 & d15 & 3 & $1 / 125$ & $12 \sqrt{3125}$ & & & \\
\hline Fidex & Т\$ONG & $\cos 2$ & 1 & Opporstose & 5.5 & 12 & 1 & 10,175 & 5 & 3 & 91125 & $12 \sqrt{3125}$ & & & \\
\hline 1600 & 73000 & D我 & 7 & Lebor & $\mathrm{B}$ & 12 & i & $14,8 \times 00$ & 0 & 3 & T/125 & $\mid 2 / 125$ & & & \\
\hline जा & 73000 & 00 & 1 & 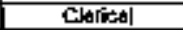 & 0 & 12 & 1 & 0 & 0 & 3 & ग1R & $\mid 12 / 31 / 25$ & & & \\
\hline & $7 \% 00$ & 20 & 1 & ons't Titinhm & 23.5 & 12 & 2 & & 75 & 3 & $1 / 25$ & $12 \sqrt{31,25}$ & & & \\
\hline 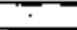 & 73000 & 236 & 1 & Eloctioily & & 12 & 3 & & 25 & 3 & IS1及5 & $12 / 31 / 2 B$ & & & \\
\hline & rison & 46 & 1 & LFindy' Sowvica & 19 & 12 & 3 & & 48 & 3 & ग4र & 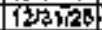 & & & \\
\hline & अ०00 & 束 & 1 & - W & & 12 & 4 & & $\pi$ & 8 & Forb & 12 मुद्य & & & \\
\hline & & & & & & & & 43475 & 1314 & & & & & & \\
\hline
\end{tabular}




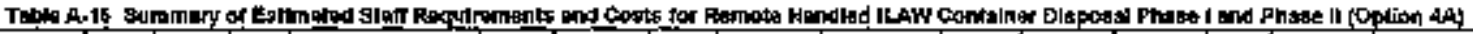

\begin{tabular}{|c|c|c|c|c|c|c|c|c|c|c|c|c|c|c|c|}
\hline \multirow{2}{*}{\multicolumn{4}{|c|}{ 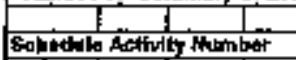 }} & - & \multirow{2}{*}{\multicolumn{4}{|c|}{ 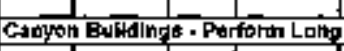 }} & 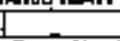 & \multicolumn{2}{|c|}{1} & 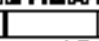 & & \\
\hline & & & & \multirow[t]{2}{*}{ 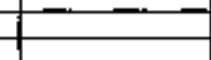 } & & & & & Terith Pland & $\cos \theta$ & ILAW D & poost $F_{0}$ & |litiex & & \\
\hline & & & & & & & & & & & & & & & \\
\hline $\mathrm{SC}$ & क्ष & CE & FT & 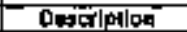 & FrES & nontho & Dote" & R. & $\$ K$ & G्पिWO & Stgif & सतnsh & WL & & \\
\hline $\tan$ & 33000 & 010 & 1 & Hnars & 15 & 106 & 1 & 24,289 & 6.732 & 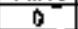 & 仵视 & 93033 & & & \\
\hline ED狂 & 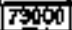 & 510 & 1 & Engingrs? & 0 & 10 & 5 & 0 & 0 & $\overline{0}$ & 1/125 & Exhors & & & \\
\hline$P Q D D$ & Fon: & D10 & 7 & Frolosstomale & 275 & -105 & $\bar{T}$ & 12,141 & 8 & 0 & $1 / M$ & A30093 & & & \\
\hline$\tau 000$ & 730000 & $0 \% 0$ & 1 & Tetrinleath & 2 & 105 & $\mathbf{T}$ & 32,375 & 1,809 & $D$ & $151 \sqrt{25}$ & क्रणपखर & & & \\
\hline $\cos 0$ & 73000 & 080 & 1 & Conlts & 275 & 105 & 1 & 44,518 & 2.497 & 0 & $1 / 1 / 25$ & extars & & & \\
\hline ROCOO & 73000 & 100 & 1 & Opertiont & 4 & 70 & 9 & 6,750 & 3518 & 0 & $1 / 1 / 5$ & 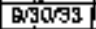 & & & \\
\hline EOOOt & 330000 & $1 \%$ & 1 & Labertiry & 0 & बह & 1 & 0 & $\bar{b}$ & 0 & t1fer5 & 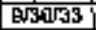 & & & \\
\hline $\operatorname{soc} x$ & JSODOD & 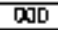 & 1 & Clenger & 025 & 16 & 1 & $4,0 \mathrm{DT}$ & 135 & 0 & $H 1 / 25$ & EN30/33 & & & \\
\hline & 70000 & 1की & 1 & 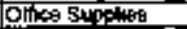 & 113 & 706 & $\frac{1}{2}$ & & 25 & 0 & 1/135 & Ex & & & \\
\hline & 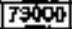 & 190 & $\bar{E}$ & Condorter HWF & 1125 & 106 & 2 & & 124 & 0 & 2125 & Dis $30 \sqrt{33}$ & & & \\
\hline & T3000 & चा & $T$ & Oneite Traqnung & 1125 & 10 & $\overline{2}$ & & 82 & 0 & $\$ 1 / 25$ & Ersoris3 & & & \\
\hline & $7300 \times 0$ & 20 & 1 & Eoctority & & 105 & 3 & & 55 & 0 & v1/25 & Eusolas & & & \\
\hline & 73000 & D & 5 & Laundry service & 87 & 105 & 3 & & 102 & 0 & W125 & ex:30333 & & & \\
\hline & 7\%000 & 100 & 1 & 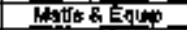 & & 108 & $\overline{5}$ & & 27 & 0 & N1/25. & 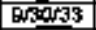 & & & \\
\hline & $730 \times 0$ & $A C D$ & 1 & MPR & & 104 & 4 & & 12 & $\underline{9}$ & 11025 & $9 \sqrt{3 \times 3}$ & & & \\
\hline & & & & & & & & 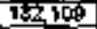 & 15,144 & & & & & & \\
\hline & & & & & & & & & & & & & & & \\
\hline Notel= & & & & & & & & & & & & & & & \\
\hline 1) Etam & costentint & 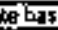 & 可的 & 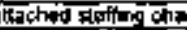 & $\sqrt{45} 50, \pi$ & 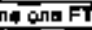 & $\mathbf{E}$ & Yationto & $960 \mathrm{hr}$ & & & & & & \\
\hline 2) & 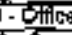 & gever & 18 292 & flon & $12 \overline{60}$ & Or & 70 & glwan & & & & & & & \\
\hline CED & 5. Eलm & Juter $h$ & 10 & $869 d$ antiwn & 01510 & 00 par Ft & $E+1$ & 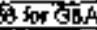 & Bne pecale & & & & & & \\
\hline$\vec{c}=21$ & a-Ond & 0 trang & aba & 0 on 3500 p p fie & +1236 & for 5 the & ind 998 & 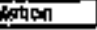 & & & & & & & \\
\hline Mvinet & letang & 0005 & 6.59 & {$[\mathrm{NA}$ d bo: } & क्सारण & $4 \%$ Aक्काय & 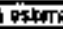 & 605 will & Panelotodo & wompong & 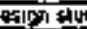 & 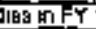 & 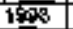 & & \\
\hline Thesect & ste 6 epol & काly & $\pi \in T_{k}$ & t gtort os costs pnos & tothot象 & Fitar & क्याओ & 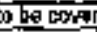 & adtother & 사t & & & & & \\
\hline CE2 & 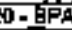 & enexin & Ny & 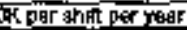 & +1256 & BrOAB & जid & Genon & & & & & & & \\
\hline c्EA & D-2Emith & 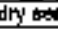 & $n \rightarrow b$ & 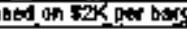 & gaming & nittifie- & 125 & CGBA all & Itatalabon & & & & & & \\
\hline 64 & $0+$ Tran & gpórtat & $\ln [0$ & 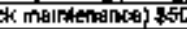 & K per ye & Er per yea & $\operatorname{tote}+1$ & 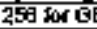 & BA and & Abon & & & & & \\
\hline CEII & $a-\mathrm{M}_{3}+\mathrm{H}$ & told an & 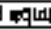 & inoth tepole partsts & Eock pes & $y=a x+1$ & 5 for & 3 sen and $\mathrm{e}$ & fesalabtora & & & & & & \\
\hline d) 400 & MPR b & $x \in d$ or & 708 & of Cod Udents 10 & 0,1400 & a 796 & & & & & & & & & \\
\hline & & & & & & & & & & & & & & & \\
\hline & & & & & & & & & & & & & & & \\
\hline & & & & & & & & & & & & & & & \\
\hline & & & & & & & & & & & & & & & \\
\hline & & & & & & & & & & & & & & & \\
\hline & & & & & & & & & & & & & & & \\
\hline & & & & & & & & & & & & & & & \\
\hline & & & & & & & & & & & & & & & \\
\hline
\end{tabular}


Table A-17. Sumbingy of Estimated Staft Requirements for Remote-Handled lamobilized Lour-Activity WHaste Container Disposal Ptase I and Phase II (Option 4B).

\begin{tabular}{|c|c|c|c|c|c|c|c|}
\hline Position & Typo & Phasa & Thres & Sherits & Phis: & Threse & eshifs \\
\hline 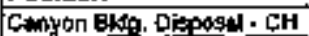 & & 1 & $50 \operatorname{sos} x$ & sal Un & II & 3 Doppose & 3al Unlls \\
\hline Facllpy Hanegar & $\mathbf{M}$ & & 0 & & & 이 & \\
\hline Clek & G & & 0 & & & 0 & \\
\hline Flant_Englnger & $E$ & & o & to & & 0 & 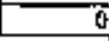 \\
\hline 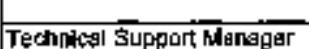 & $\sqrt{14}$ & & 0 & & & & \\
\hline Clark & $G$ & & 0 & & & 0 & \\
\hline 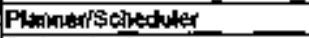 & $\bar{p}$ & & $\underline{0}$ & & & to & \\
\hline Bactgal Analyzs & $\bar{P}$ & & $\overline{0}$ & & & 0 & \\
\hline Fralning Coordinalar & $G$ & & 모 & & & a & \\
\hline Tralner & $\bar{F}$ & & 0,5 & & & 0.5 & \\
\hline Frosedure hînor & $\mathbf{P}$ & & 0,5 & & & 0.5 & \\
\hline Eectropli Englnger & $\bar{E}$ & & $\hat{0}$ & & & 0 & \\
\hline Ënv.Coracl. Enqinas & $\mathbf{E}$ & & 0 & & & 여 & \\
\hline IEC Engineer & $\mathbf{E}$ & & g & & & 0 & \\
\hline hochanlced Enginetir & $\mathrm{E}$ & & 0 & & & (2) & \\
\hline OA Ëpinster & $\mathbf{E}$ & & 0 & & & 0 & \\
\hline oc insoectar & $\mathbf{T}$ & & ㅁ & 1 & & D) & 1 \\
\hline & & & & & & & \\
\hline Prodikftion Mananger & hat & & 1 & & & 1 & \\
\hline 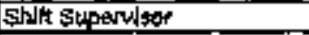 & $m$ & & 3 & & & \$1 & \\
\hline Odcument clark & $\bar{G}$ & & 1 & & & 1 & \\
\hline Matndenanos PlC & $\mathbf{P}$ & & 1 & & & 1 & \\
\hline QEM PlenneГ & $\overline{\mathbf{P}}$ & & 1 & & & 可 & \\
\hline 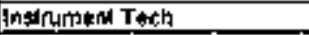 & $\mathbf{T}$ & & $\mathfrak{t}$ & & & 2 & \\
\hline Elecirlcians & $\overline{\mathbf{C}}$ & & 0 & & & 0 & \\
\hline Mochanical gans & $c$ & & $\theta$ & & & 可 & \\
\hline 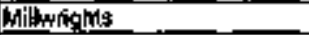 & $\mathbf{c}$ & & 0 & & & 可 & \\
\hline Process Oparalors & $\overline{\mathbf{F}}$ & & 3: & & & 9 & \\
\hline HPTs & $\mathbf{T}$ & & 3 & & & 텨 & \\
\hline Power Op日rators & $\mathbf{R}$ & & t: & & & 0 & \\
\hline Crant orotalors & $\mathbf{R}$ & & t) & & & a & \\
\hline Tratk Drlyers, HQ & ¿ & & 4 & & & 12 & \\
\hline Forkfin Oper donsts & $\mathrm{c}$ & & 4 & 22 & & 12 & 50 \\
\hline & & & 20 & 25 & & & 鸟 \\
\hline & $M$ & & 4 & & & 4 & \\
\hline & $\mathbf{E}$ & & 0 & & & D & \\
\hline & $\underline{s}$ & & 0 & & & (3) & \\
\hline & $\bar{P}$ & & 3 & & & st & \\
\hline & $\mathbf{T}$ & & (4) & & & B) & \\
\hline & c & & 可 & & & 24 & \\
\hline & $\vec{R}$ & & 3 & & & 包 & \\
\hline & $\mathrm{L}$ & & t) & & & 可 & \\
\hline & $G$ & & 1 & & & -1) & \\
\hline & & & 28 & & & 51 & \\
\hline & $m$ & & 7 & & & 9 & \\
\hline & Wh & & 11) & & & 33 & \\
\hline & wo & & 5 & & & 0 & \\
\hline & & & 23 & & & $5 !$ & \\
\hline
\end{tabular}




\begin{tabular}{|c|c|c|c|c|c|c|c|c|c|c|c|c|c|c|c|}
\hline \multicolumn{4}{|c|}{ 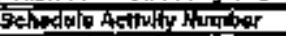 } & \multicolumn{9}{|c|}{ 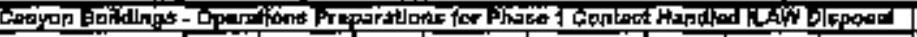 } & \multicolumn{2}{|c|}{\begin{tabular}{l|l} 
& \\
\end{tabular}} & 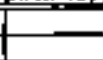 \\
\hline JC & $\mathrm{DC}$ & CE & FT & bosecriotion & F辰 & Womth: & Tiote & 网 & द्रद & C्पrvo & start & FIngh & D. & & \\
\hline$x+000$ & ग3000 & 070 & 1 & Motson & 2 & $2 \overline{4}$ & 1 & 7,400 & 528 & 2 & 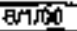 & $5 \times 1, \sqrt{202}$ & & & \\
\hline $\mathrm{E} 000$ & $7 \%$ & Dib & 1 & Enjibert & 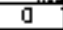 & 24 & $T$ & D & $\mathrm{D}$ & 2 & बMño & $5 \times 31 \pi 02$ & & & \\
\hline FODS & ग3000 & 014 & 1 & PToketelonals & 1 & 24 & $\mathbf{1}$ & 3,700 & 204 & 2 & enino & $5 \times 3102$ & & & \\
\hline $\operatorname{Top}$ & 73000 & 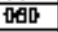 & 1 & Tochniclayte & 2 & 24 & 1 & 7,400 & 413 & 2 & BMת & ER1, & & & \\
\hline COND & 73000 & Dodo & 1 & Cratis & 4 & 24 & 1 & 14,800 & 827 & 2 & BMnth & $5 \times 31002$ & & & \\
\hline ROAT & 73000 & D度 & 1 & Optraters & 1.5 & 24 & 1 & 5,550 & 310 & 2 & MN0 & $5 \sqrt{31 / 02}$ & & & \\
\hline 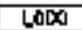 & $7 \times 500$ & as & 1 & Laborero & 0 & 24 & $\overline{1}$ & 0 & 0 & 2 & बतino & $531 \times 02$ & & & \\
\hline ç000 & 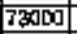 & DOD & 1 & Cierxet & D.5 & 24 & 1 & 1.80 & 62 & 2 & 6नात0 & $5 \times 31 \wedge 2$ & & & \\
\hline & 73000 & 190 & 1 & 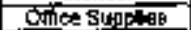 & 11 & 24 & द & & 8 & 2 & 日n西家 & $5 \sqrt{3} 1 \times 12$ & & & \\
\hline & T3000 & 190 & 1 & 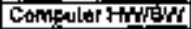 & 11 & 24 & 2 & & 28 & 2 & बiriso & $5 \sqrt{3102}$ & & & \\
\hline & 73000 & 20 & 1 & Cratte Erainuge & 11 & 24 & $\bar{z}$ & & 14 & 2 & $\theta+1 \times 0$ & 5\%WA2 & & & \\
\hline & $\mathrm{T}$ आOCW & $\triangle \mathrm{ACD}$ & 1 & MPR & & 24 & 4 & & 2 & 2 & 6100 & $5 / 2102$ & & & \\
\hline & & & & & & & & 40.700 & $2, \mathbf{4 5 9}$ & & & & & & \\
\hline & & & & & & & & & & & & & & & \\
\hline Scthodu & Aetivit & Namm & 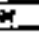 & Canpton bullining. & ot be & 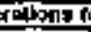 & Phas & 1 (50pta & Hind & AW Dा: & 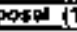 & Unll 39h & it 오요 & & \\
\hline $\mathrm{JE}$ & CE & 屯芒 & Fi & Denstiotion & FTfo & Panths & Rote & 새 & th & CurP. & Stri & कानक & & & \\
\hline 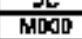 & $\frac{13000}{20}$ & $\frac{15}{010}$ & $\frac{m}{1}$ & $\frac{1}{\operatorname{mon}}$ & $\frac{15+9}{4}$ & $\frac{10 \mathrm{~s}}{40}$ & 1 & 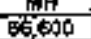 & $\frac{34}{4.751}$ & $\operatorname{con} 6$ & $\frac{5150}{61102}$ & Findih & 몐 & & \\
\hline 5000 & 73000 & 40 & 1 & Englomers & $\overline{0}$ & $\sqrt{1 \times 8}$ & $T$ & 0 & 0 & 0 & QN1F2 & ज्ञाती & & & \\
\hline$F(x)$ & 73000 & Ma & 7 & 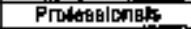 & 2 & I08 & 5 & 39 , & 2375 & 0 & E्ञाति2 & $531 / 11$ & & & $\cdot$ \\
\hline T000 & 73000 & DEO & 1 & Tectholelang & 4 & 行的 & i & 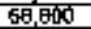 & 37 & 0 & Exp2 & 531M1 & & & \\
\hline $\cos 0$ & 73000 & $\sqrt{n}$ & i & Cratho & 5 & 108 & $\bar{t}$ & 139.200 & $\overline{7,442}$ & 8 & EMth & 5391710 & & & \\
\hline$R 000$ & 73000 & Dera & 1 & Goeratory & 3 & 108 & $T$ & व9, 950 & $2 \sqrt{31}$ & 0 & ESIT:R2 & इउसान & & & \\
\hline 1000 & 72000 & DOAD & 1 & LAbGETE & $\overline{0}$ & 108 & 1 & 0 & 0 & $\bar{D}$ & हा1य02 & 5Bगय11 & & & \\
\hline 5000 & $7 \not 0 \%$ & 000 & 1 & Clefict & 1 & 108 & $T$ & 1日, 650 & 55 & $\bar{D}$ & $817 \pi 02$ & $5 \times 31511$ & & & \\
\hline & 73000 & 140 & 1 & Offos Suppliat & 22 & 198 & $\overline{2}$ & & 50 & 0 & AS150Z & EN3IJ11 & & & \\
\hline & 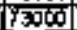 & 190 & i & Campardm HWFFW & 22 & 108 & 2 & & 240 & $\overline{0}$ & (17) 02 & E\$1/11 & & & \\
\hline & 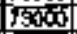 & 70 & 1 & 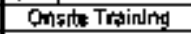 & 2 & 708 & 2 & & 124 & 0 & बित्री & Q51/19 & & & \\
\hline & 39000 & 20 & 1 & Electincty & & 108 & 3 & & 678 & 0 & ब110? & 531141 & & & \\
\hline & Fैअ00 & 400 & 1 & 1 endidy \$ermoe & 15 & 100 & $\overline{3}$ & & $\$ 39$ & $\theta$ & स40 & 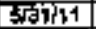 & & & \\
\hline & $73+00$ & $4 \times 0$ & $\frac{1}{i}$ & VonkलF Minl & & 100 & 3 & & 1,49 & 6 & $61 x=2$ & 5्रता & & & \\
\hline & 15000 & 100 & 1 & Kons \& Eq & & 100 & 3 & & 589 & 0 & 04102 & इड़ा1 & & & \\
\hline & Tअ00 & 460 & 1 & FF & & 106 & 4 & & 80 & రे & $5+102$ & $5 \sqrt{3+11}$ & & & \\
\hline & & & & & & & & 365,306 & ह5.356 & & & & & & \\
\hline
\end{tabular}




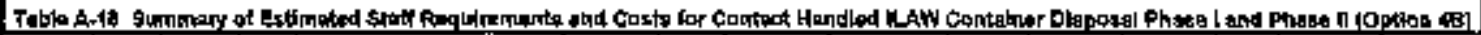

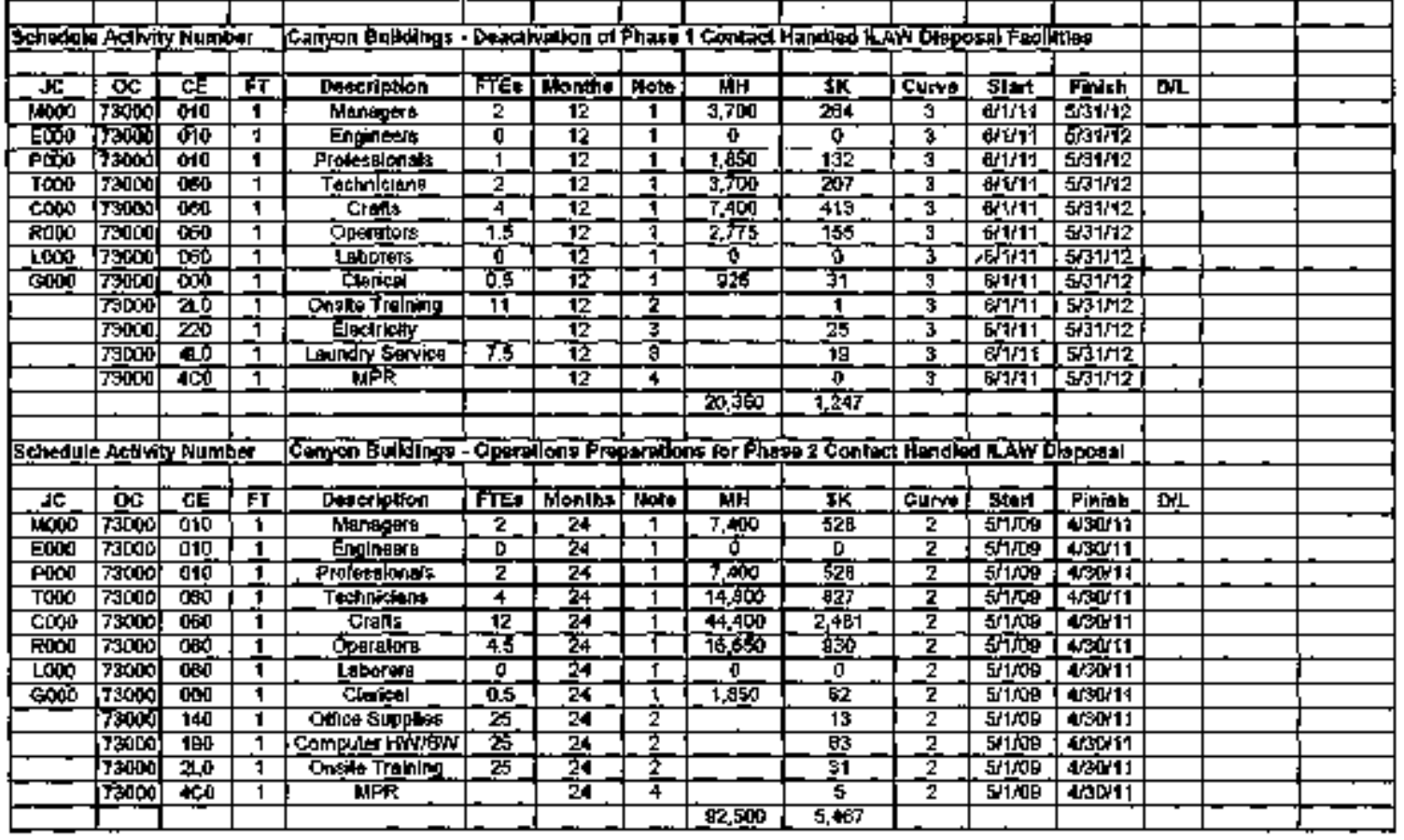




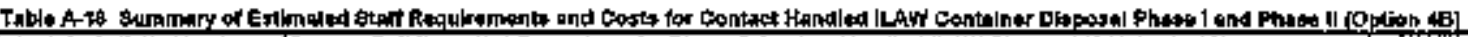

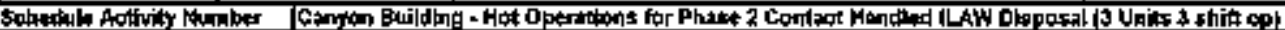

\begin{tabular}{|c|c|c|c|c|c|c|c|c|c|c|c|c|c|c|c|}
\hline \multicolumn{4}{|c|}{ 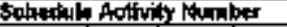 } & \multicolumn{11}{|c|}{ 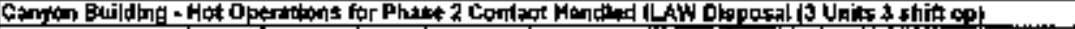 } & \\
\hline 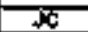 & 更 & CE & F & Opacilplan & FTEF & Mapthe & Wete & $\mathbf{M A H}$ & $\mathbf{E}$ & Euryin & Bttant & Flakg & ORL & & \\
\hline 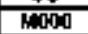 & 7 Fo, & 010 & 1 & 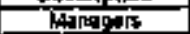 & 4 & $1 \hat{q}^{2}$ & -1 & 101,13" & $5 \sqrt{214}$ & 0 & इसा1 & $12 \sqrt{24}$ & & & \\
\hline EXOD & 73000 & 010 & $\frac{1}{1}$ & Examerar & í & TE्र & $\mathbf{T}$ & D & D & 0 & 511511 & 120124 & & & \\
\hline$S \times 00$ & 73000 & 010 & 1 & Ectentigth & to & 原 & 1 & 0 & b & 0 & SH1Mt & 128126 & & & \\
\hline$P(x)$ & 73000 & Q⿻一丿𠃌 & 1 & Profetetionalo & $\overline{4}$ & वह्त & $\overline{1}$ & $101,1 \%$ & 7,214 & 0 & ज्ञf11 & $7232 / 24$ & & & \\
\hline T0\%0 & 73000 & 080 & 1 & Tegrinistant & 8 & 104 & 1 & $20,2,267$ & 11,\%o1 & 0 & $5 N 111$ & $12 \times-124$ & & & \\
\hline $\operatorname{con} 0$ & 73000 & 0,50 & 5 & Cot6 & $\overline{24}$ & T武 & $\overline{1}$ & $605 \sin 2$ & 3902 & 0 & ज्ञाया & 123120 & & & \\
\hline$k 000$ & 8,9000 & एक & 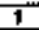 & 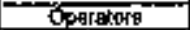 & 8 & 184 & 1 & 227350 & 12,713 & 0 & $\$ 1111$ & 123124 & & & \\
\hline 1000 & F⿻에 & ठळू & 1 & Latpornt & 6 & 1BA & 4 & 0 & की & 0 & इसा11 & $1231 \pi$ & & & \\
\hline 5000 & Foo & $\overline{0}$ & 1 & Cle्काद्या & i & $10 \overline{4}$ & 1 & 25263 & 846 & 0 & झासा1 & $12 x+\sqrt{4}$ & & & \\
\hline & sisons & 140 & 1 & 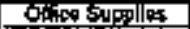 & 60 & 784 & 2 & & 72 & $\overline{0}$ & एगा & $12{ }_{12}$ & & & \\
\hline & $\operatorname{tin} 0$ & 160 & i & Comprintw & 50 & 164 & 2 & & 63 & 0 & \$11111 & $1231 \sqrt{24}$ & & & \\
\hline & 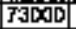 & $2 \mathrm{LO}$ & 1 & Optistrealng & 30 & TBA & $\overline{2}$ & & 429 & (0 & $51 / 11$ & $12 \times 3-124$ & & & \\
\hline & Tsa00 & 220 & $\mathbf{1}$ & Elactroty & & 184 & $\overline{9}$ & & 1,379 & to & $51 d^{\prime \prime 11}$ & $12 / 31 \sqrt{2} \mid$ & & & \\
\hline & 73000 & ALO & 1 & Latinty Berulce & 91. & 154 & 可 & & 1,400 & 0 & $5+1 / 11$ & $12 \sqrt{3} 1 / 24$ & & & \\
\hline & 73000 & APD & 1 & Wohlele xhint & & is4 & 3 & & 7.724 & to & 5Uस & $1281 / 24$ & & & \\
\hline & 73000 & 100 & 1 & Mote B. Explp & & 184 & 3 & & 693 & to & FWा1 & $1231 \sqrt{24}$ & & & \\
\hline & 73000 & 40 & 1 & MPR & & 184 & 9 & & 120 & 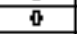 & 5 SW13 & $1231 \sqrt{24}$ & & & \\
\hline & & & & & & & & $1,2804,67$ & 85,857 & & & & & & \\
\hline & & & & & & & & & & & & & & & \\
\hline \multicolumn{4}{|c|}{ 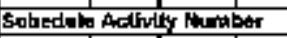 } & \multicolumn{12}{|c|}{ 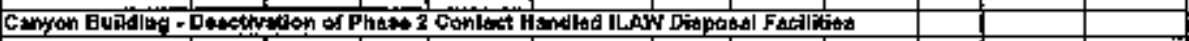 } \\
\hline & & & & & & & & & & & & & & & \\
\hline$\sqrt{6} 6$ & $\infty$ & CE & $\mathbf{F T}$ & Dotarystan & FTEB & Montins & Fote & $\mathrm{MH}$ & 5 & Corve & Start & Finlett & DIL & & \\
\hline 10000 & 73000 & D10 & 1 & Mansopars. & 2 & 12 & 1 & $3, \sqrt{300}$ & 206 & 3 & $7 \sqrt{1 / 25}$ & $52 \sqrt{31 / 25}$ & $\cdot$ & & \\
\hline$E \infty O B$ & 73000 & 010 & 1 & Engindert & 0 & 12 & 1 & b & a & $\mathbf{3}$ & 1HR & |2/31/25 & & & \\
\hline "150 & $7 \$ 000$ & 010 & 1 & Prolecionslo & 2 & 12 & 1 & 3,300 & 254 & 3 & 1FIAC & \begin{tabular}{|l|}
$12 / 3125$ \\
\end{tabular} & & & \\
\hline 7000 & $7=000$ & $\infty$ & 1 & Tofingints & 2 & 12 & 1 & 3,700 & 207 & 3 & $1 M \sqrt{6}$ & 12बव125 & & & \\
\hline $\operatorname{col} \cos ^{\circ}$ & 7000 & $0 \$ 0$ & 1 & Capt & 4 & 12 & 7 & 7,400 & 413 & $\mathbf{a}$ & $1 M \sqrt{45}$ & $12 \mathrm{an} / \mathrm{s}$ & & & \\
\hline Rxto & f $\sqrt{3000}$ & D\$ & i & Drarabors & 12 & 12 & 1 & $z, 200$ & 1,240 & $\boldsymbol{z}$ & $1 \mathrm{MrF}$ & 12 12. & & & \\
\hline LOOO & 33000 & DE & $T$ & Linoorts & 4.5 & 12 & 1 & 5,426 & $\sqrt{6}$ & $\overline{3}$ & This & 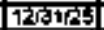 & & & \\
\hline GaCo & 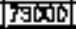 & 60 & 1 & 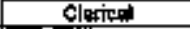 & $\theta$ & 12 & 1 & 0 & 0 & 3 & $1 M \sqrt{25}$ & 121312s & & & \\
\hline & 73000 & $2[0$ & $T$ & Conste Treining & 25 & 12 & 2 & & 17 & 3 & 1/质 & 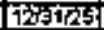 & & & \\
\hline & 73000 & 220 & 1 & ENatricty & & 12 & $\frac{3}{3}$ & & 26 & 3 & $1+1 \sqrt{2}$ & 12 3125 & & & \\
\hline & 73000 & $A L O$ & 1 & Laundery seryteq & 2.6 & $\sqrt{2}$ & $\overline{3}$ & & 67 & $\overline{3}$ & 1f1 & 1飞3125 & & & \\
\hline & 73000 & $4 C 0$ & 1 & BPR & & 12 & 4 & & 0 & 3 & $1+125$ & 123125 & & & \\
\hline & & & & & & & & Q8, त25 & 2,087 & & & & & & \\
\hline
\end{tabular}




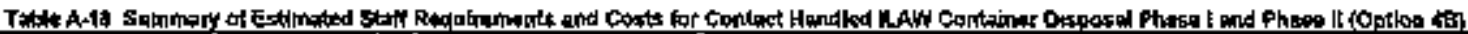

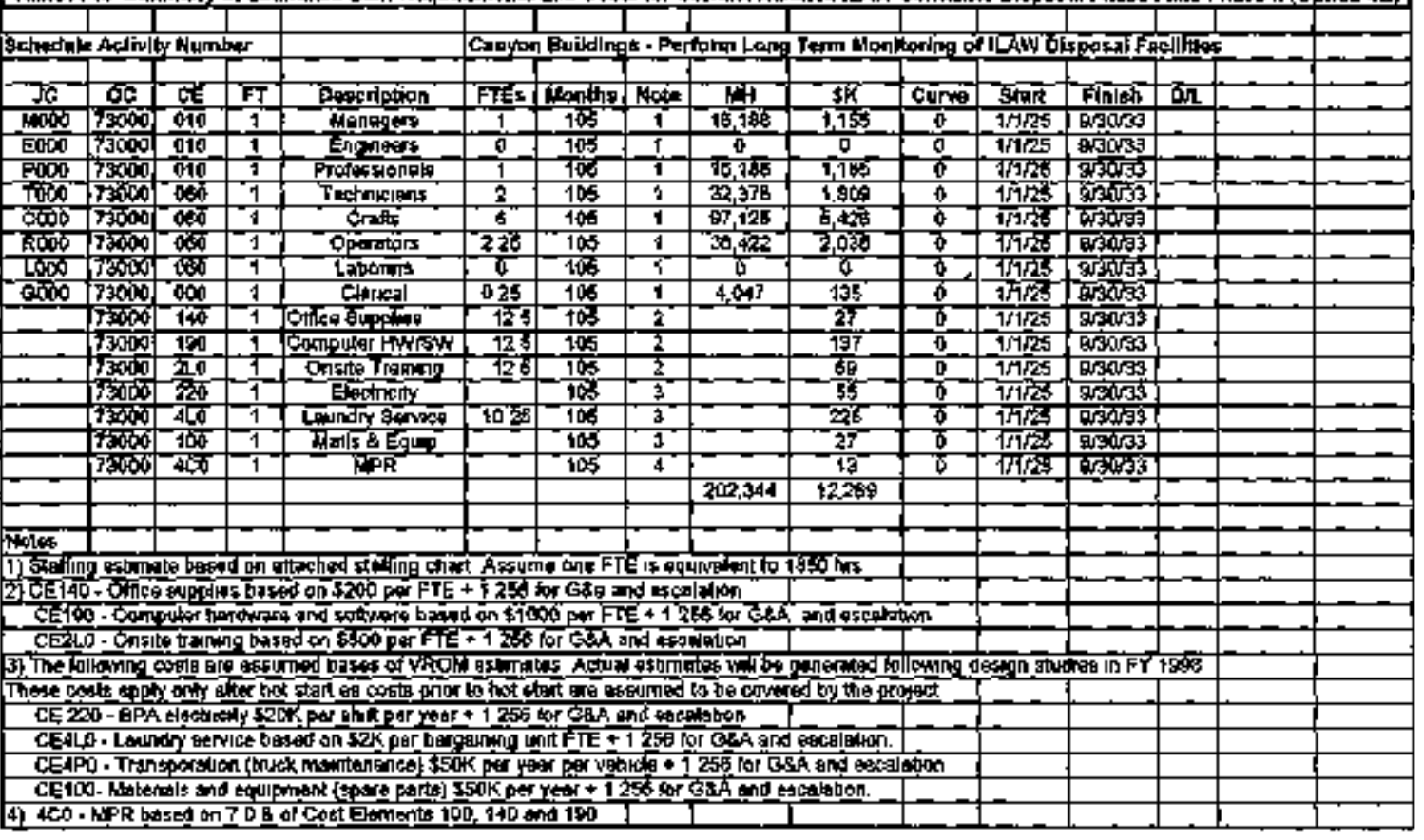


Tahte A-19. Stramary of Total Estionsted Operating Costs for Foer Canyon Building Disposition Based on Internal and Exterial Waste Diapseal Alternatives of 221-U and Operaring Crew for Immobilized Low-Acưvity Wagte Dipposal (AGA Options 4A and 4B).

\begin{tabular}{|c|c|c|c|c|c|}
\hline Option & $\begin{array}{c}\text { Operationtal } \\
\text { preparalion } \\
\$ M \\
\$ M\end{array}$ & $\begin{array}{c}\text { Hol operation } \\
\text { \$ M }\end{array}$ & $\begin{array}{l}\text { Deactivation } \\
\text { \$M }\end{array}$ & $\begin{array}{c}\text { Subtotal } \\
\text { \$ M }\end{array}$ & $\begin{array}{c}\text { Grand tocal } \\
\$ M\end{array}$ \\
\hline $4 A_{1}=$ & \multicolumn{2}{|c|}{$117 . g^{b}$} & $\sigma^{d}$ & 117.8 & \multirow[t]{2}{*}{146.8} \\
\hline $\begin{array}{l}\text { (23,330 } \\
\text { ILAW } \\
\text { Containurs) }\end{array}$ & 1.9 & $26.3^{t}$ & 0.8 & 29.0 & \\
\hline \multirow{2}{*}{$\begin{array}{l}\text { 4B - } \\
\text { lnternal and } \\
\text { External } \\
\text { Waste } \\
\text { DHsposal } \\
(98,200 \\
\text { ILAW } \\
\text { Contanners) }\end{array}$} & \multicolumn{2}{|c|}{155.7} & $14.4^{d}$ & 170.1 & \multirow[t]{2}{*}{292.5} \\
\hline & $7.8^{2}$ & $111.0^{+}$ & $3 . \theta$ & 122,4 & \\
\hline
\end{tabular}

- Soutce of information are Table A-13, Table A-16, Table A-18 and belows caliculations.

Operating Cost $=\$(0.1+15.5+0.8) \mathrm{EO} 6 \mathrm{6} \times 1.256$ escalation $\times 1.35$ contingency $\mathrm{x}$ 4.24 equiqalent buildings $=\$ 117.8$ million (Table A. 13 )

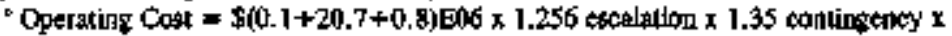
4.25 equivalent buildungs $=\$ 155,3$ million (Table A-13)

Deactivation Cost $=\$ 2.0 \mathrm{E} 06 \times 1.256$ escalation $\times 1.35$ coutingeocy $\times 4.25$ equivaknt buildings $=\$ 14,4$ million (Table A-13)

'Operation prep cost $=\$(\$ .0+\$ .0) \mathrm{EOG}$ × $0.2 \$=\$ 1.9$ million (Table A-16)

' Hot operauton cost $=\$(30.5+78.9)$ E06 $\times 0.24=\$ 26.3$ million (Table A-16)

- Deactivalion cost $=\$(1.5+J .8)$ E06 $\times 0.24=\$ 0.8$ million (Table A-J6)

- Operation prep cost $=\$(2.4+5.4) \mathrm{E} 06 \times 0.76+\$ 1.9606=\$ 7.8$ million (Tabie 18 and nole " above)

' Hot operation cost $=\$(25.4+\$ 6.0) \mathrm{E} 26 \times 0.76+526.3 \mathrm{E} 06=\$ 11 \mathrm{~L} .0$ million (Table A-18 and note ${ }^{\dagger}$ above)

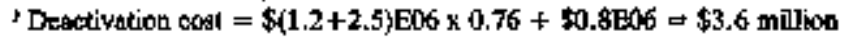
(Table A-18 and bote ${ }^{\star}$ aboye). 


\section{A2.4 EXPANDED RVTERIM STORAGE (OPTTON 5)}

Table A-20. Cost Estinate for Project W-112 Erbanced Radioscrise and Mixed Waste Sturage.

DON'Y SAY IT -..- Nrite It!

TD: Matke J. Kiem H5-27
DATE: Alugust 6, 1997

FROM: Erte 6. Erpenbeck

TeTephong: 376-8032

SUBGECT: OLD ICF XH ESTIHAIE FOR LONG TERH DRLA STORAGE BUILDIRH 2404 SEFIES

Per you request, attached are copjes of an estimate used on Project N-112 for the $2404 \mathrm{~N}$ sertes building constructed at the Hanford Site Central Waste complex. This sstieate is for a pre-engineered metal cutler bulding with a concrete chated fluar for the storage of hadloactive Mixed Waste. Thas factlity is a double profile sloped roof with 20 ' eave hejght, a dry pipe sprinklèr and alaro system, INo 16,700 CFH roof ventilators, dverhead coll doors on both ends of the buflding and a flued head air sampler with cantrol cablut to verify the absence of radloactive contanination.

I hope this Information will be tsaful in materer applications you are considering.

Hery Truly Yours,

Eric 6. Erpanbecík

Project Engineer 
EXISAR EAOLHE ERG MAUP DAP

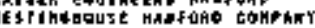
tol $r$. W.112, $178 k+3$

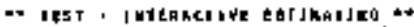

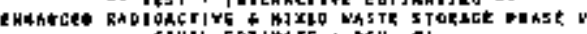

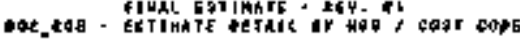

CR5C

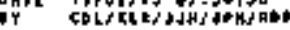

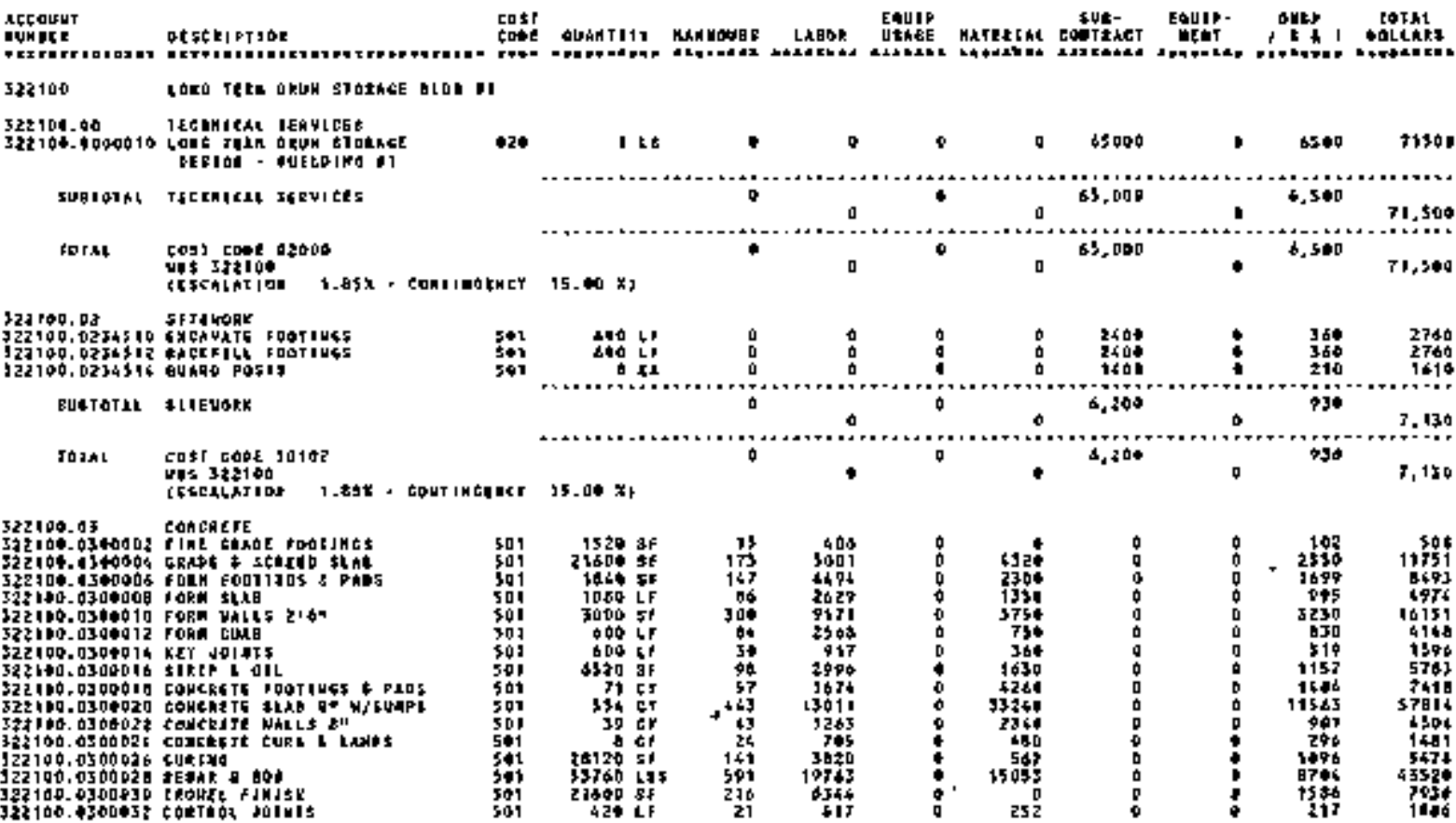


FAISAR ETCLIUERF HATSQRO

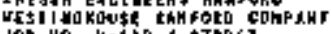

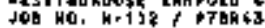

ACc⿻ U*:

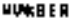
PetragPIOK

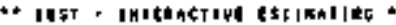
EUHARCE RADIOACIITE E MIXED WASTE GTQRALE PHAEE

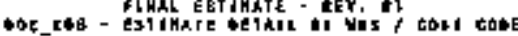

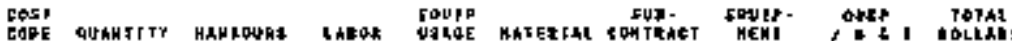

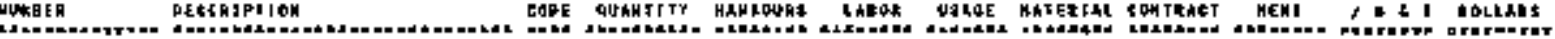

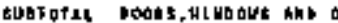

GALEF TAK T.

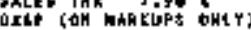

TOIML LOET COPE 30100 It: 322100

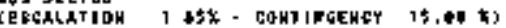

\section{PInjsuts}

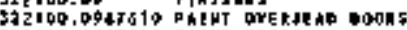
322104092712 PAIKT D00R

Coxrche Ploans 327400.0907515 ing

5EDIOIAL PIMASHES

TOTAL CF\&1 SPOE $5 * 140$ Het $32210 \%$ itschingion

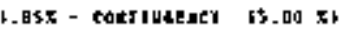

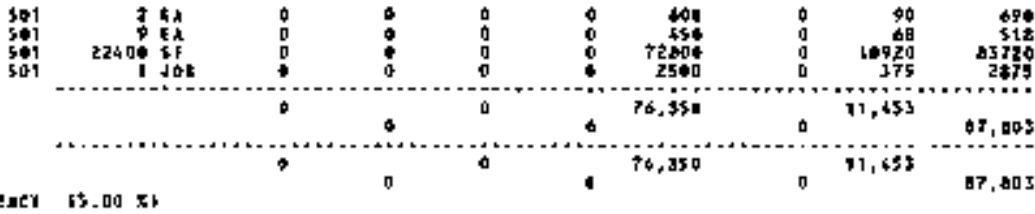

322140.10 FPESIALTIBF

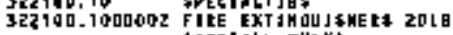
(BOSIALL OHLY)

suttarac thitiadiet

Jorht toET cabs sol MBC 32 L LOD

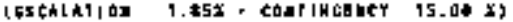

9,136

3,286
+

4

$4 \leqslant 0$

1120

398

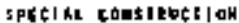

322104,13 SPKC1K2

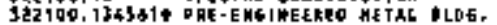

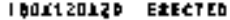

uflike ilser t esec. Panel

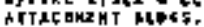

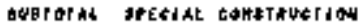

4 ES

4

243

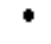

0

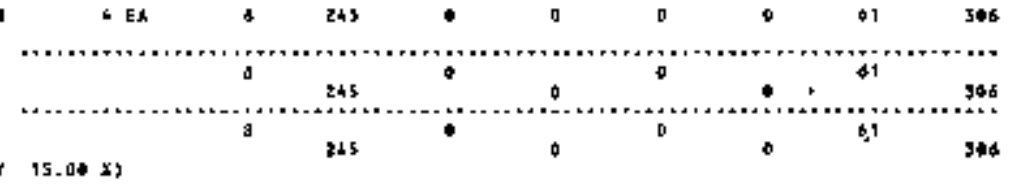

30

200000

4 1212* 323020

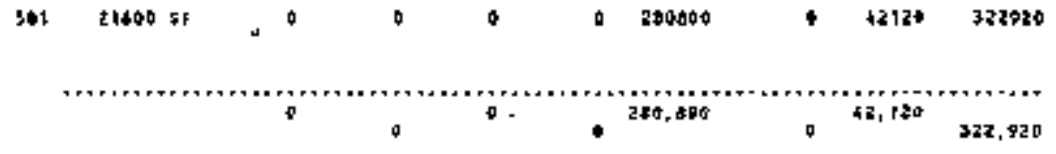


KAI SED EUAIMTEMS HAUP and UETIMGUQUSE HAHSOR TDMPAMY

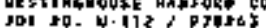

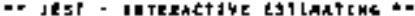

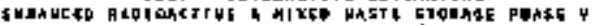
DOE_ROB - EETIKAIE OETSIL OY HEA SOST COOE
ThG 43

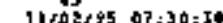

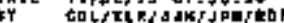

Acequalf
Aumete

$1017650515005 \$ 0113$

uts 322100

SEFG1ATIDH $1.63 X$, COHIEEGEKCY

Mnçusalche

322100.13

fl RE bedtection CPAITKCE G

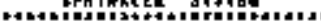

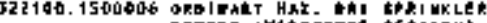
STSTOH (WhiEroOs? SEtripu) I HCLUOSF SThI IRLGIHG EXTHA

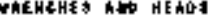

122100,150000 ORE

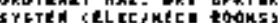

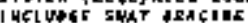

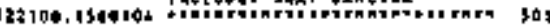
Fike progection

\section{कि}

3210 15 the

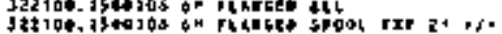

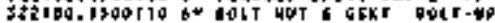

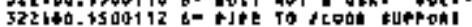

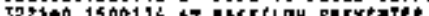
Th J2 2 200 J2. 7

32 32ClAd. 15Q0120 TaE hLALH HOR

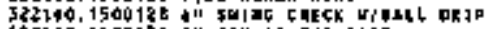
32f

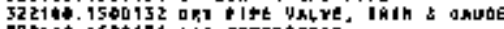
322140,1500134 Ald CQDEPLE $\$$ \$OR

321109,150013, IJAHESE COULE 1J OH

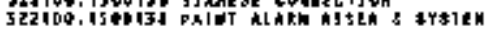
$0 * A$ In

SUBJDIAL MECWHULAL

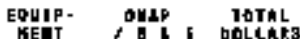

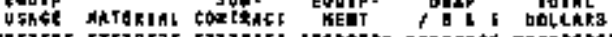

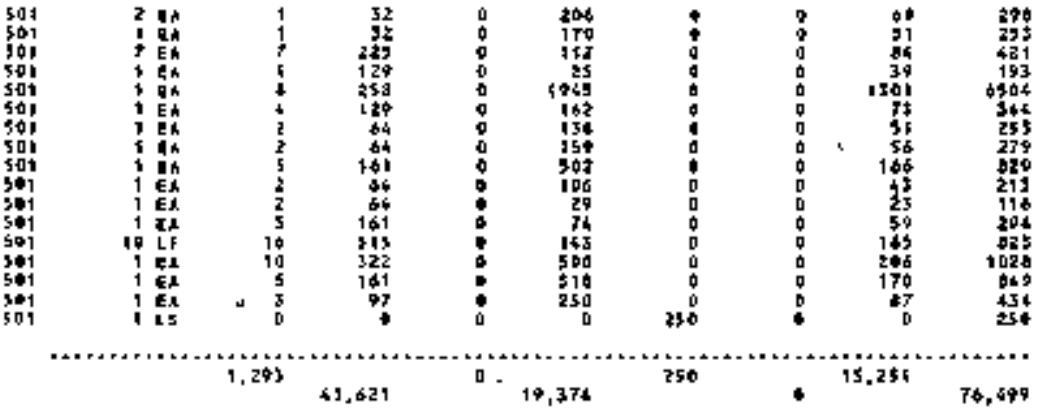




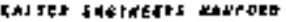

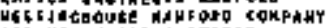
hat

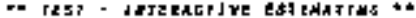

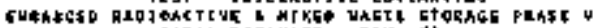
E13 TL EsTIHATE. RIY

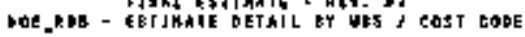

Phat fit

fos $07: 30: 31$

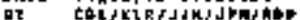

\begin{tabular}{|c|c|c|c|c|c|c|c|c|c|c|c|}
\hline 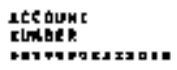 & 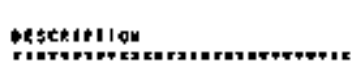 & $\begin{array}{l}\text { to\$? } \\
\text { topi } \\
\operatorname{lin} r\end{array}$ & 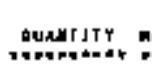 & manguls & IXBen & 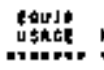 & 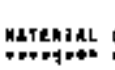 & 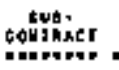 & 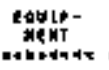 & 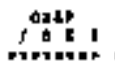 & 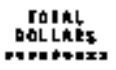 \\
\hline & 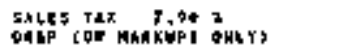 & & & & & & 1530 & & $b$ & 302 & $\operatorname{lig}_{\substack{3 \\
02}}$ \\
\hline $10 \mathrm{Ch}$ & 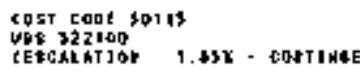 & E*ET & $15.00 x$ & 1,203 & 61,021 & 6 & 20,706 & 236 & 8 & 15,638 & 70,492 \\
\hline $\begin{array}{l}322304.13 \\
322100,1550109\end{array}$ & 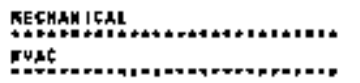 & sol & $\bullet$ & 0 & $\bullet$ & D & $\bullet$ & $\bullet$ & $\mathbf{0}$ & 9 & $\mathbf{t}$ \\
\hline $522304,130020 \%$ & 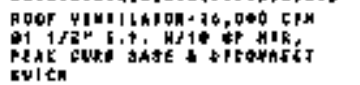 & $\$ 01$ & $\leqslant$ en & 92 & 1bey & $\bullet$ & 6454 & $\bullet$ & 0 & $\xi 1 \times 1$ & $9 z 7+$ \\
\hline $122160.190<202$ & 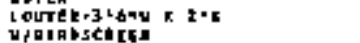 & 501 & $13 \mathrm{EK}$ & 26 & Bb2 & 4 & 2418 & 0 & 0 & 940 & 6290 \\
\hline $\begin{array}{l}32 z 100.1304206 \\
32100.1500200\end{array}$ & 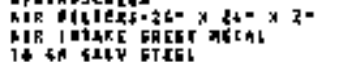 & $\begin{array}{l}501 \\
\text { sol }\end{array}$ & 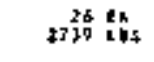 & 266 & 9281 & 4 & 1370 & 0 & a & $\begin{array}{r}296 \\
3196\end{array}$ & $130 \%$ \\
\hline $\begin{array}{l}322100.1500208 \\
322 \ln 40.1500210\end{array}$ & 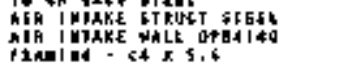 & $\begin{array}{l}501 \\
501\end{array}$ & $9+0,104$ & $\$ 7$ & 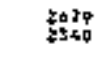 & $\bullet$ & $\begin{array}{l}350 \\
912\end{array}$ & $\stackrel{0}{0}$ & $a$ & 911 & $\begin{array}{ll}3246 \\
3506\end{array}$ \\
\hline 922600.1900212 & 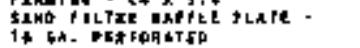 & 501 & 12011 & 12 & 407 & $\uparrow$ & 520 & 8 & 0 & 24 & 1216 \\
\hline 322100.1500214 & 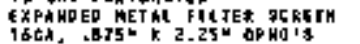 & $s+1$ & $1075 \mathrm{~F}$ & 11 & 373 & 0 & 161 & ô & $\mathbf{B}$ & 160 & $6+4$ \\
\hline 322100,1500218 & 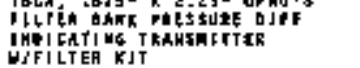 & $\mathbf{5 \$ 1}$ & is Ex & 13 & $5+1$ & 0 & 2646 & 0 & $\nabla$ & $\$ 68$ & 3751 \\
\hline 328100,1500216 & 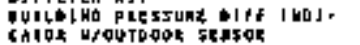 & 541 & $1 \mathrm{E \lambda}$ & $\mathbf{I}$ & 36 & 0 & 76 & 0 & 0 & $y z$ & 140 \\
\hline 322100.1500220 & 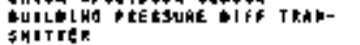 & 341 & $1 \mathrm{E \lambda}$ & 1 & 96 & $\nabla$ & $1 \div \xi$ & 0 & q & 10 & 212 \\
\hline $322100.15 \$ 0222$ & 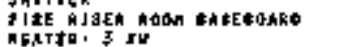 & 591 & $1 \mathrm{EA}$ & $\mathbf{1}$ & 94 & $\nabla$ & 4 & $\nabla$ & $b$ & 26 & 103 \\
\hline 322100.1500224 & 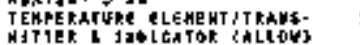 & 501 & $56 h$ & 3 & 102 & 0 & 100 & $\bullet$ & $\phi$ & 21 & פI \\
\hline 3221100.1304220 & 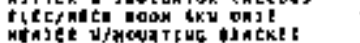 & sôl & I th & 3 & 102 & 0 & 330 & $\bullet$ & 0 & $13 *$ & 562 \\
\hline $3421+0.1904220$ & 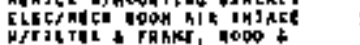 & 501 & 1 in & 10 & 337 & o. & 35,6 & 4 & $\phi$ & 807 & 196. \\
\hline
\end{tabular}


CHISIR EACI HE TRS HAMSOHO HEST JMEHOUSE IAMPOA* COMFHH

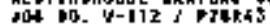

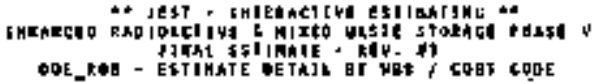

DATE $\$ 5$

QHTE $11 \% 02,9507: 30: 31$

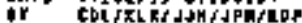

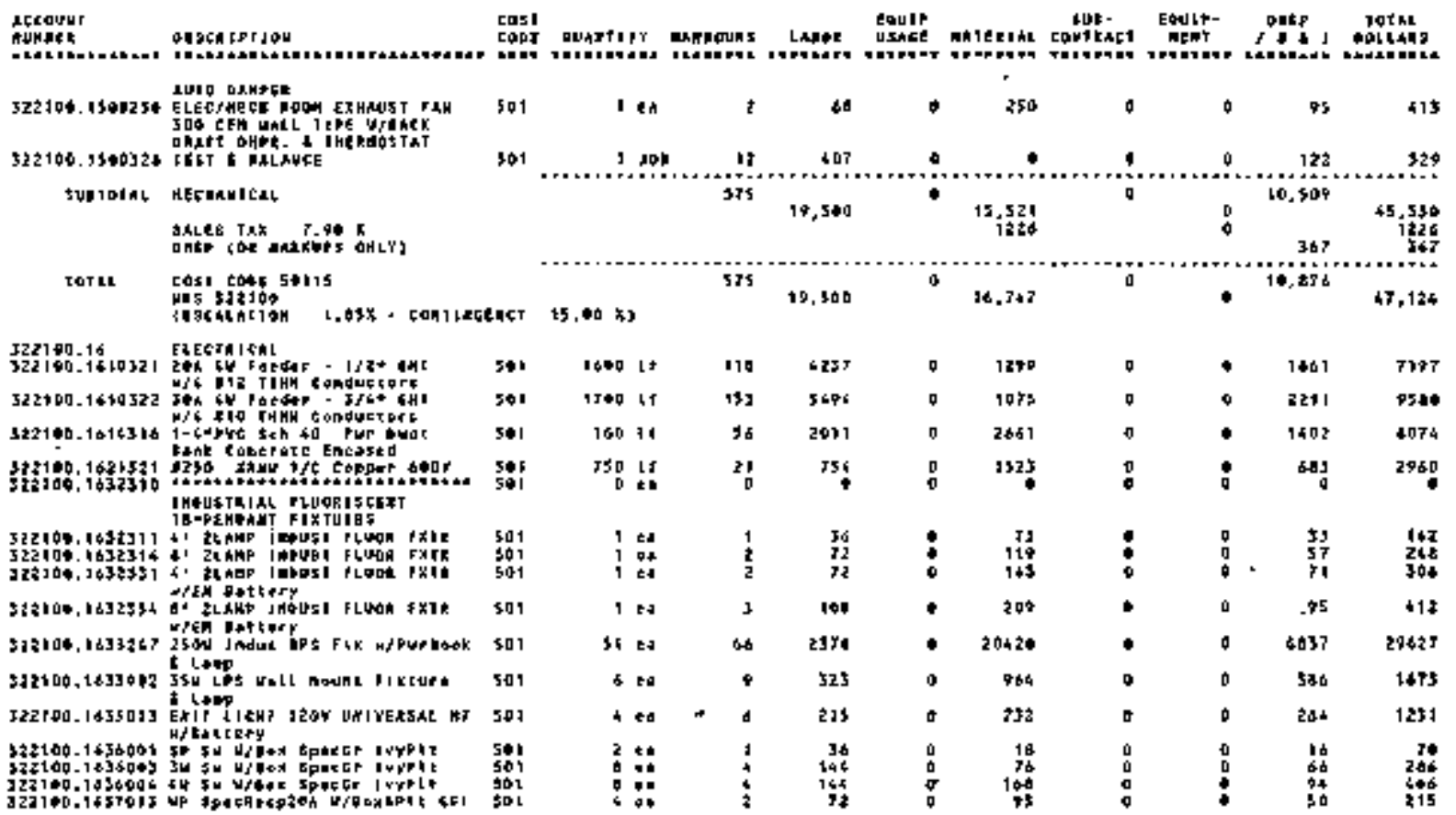

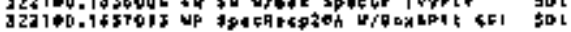

点 


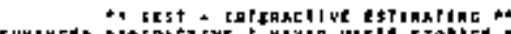

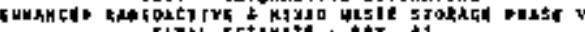

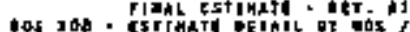

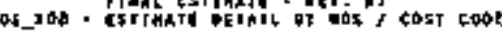

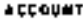

DECKIPTiah

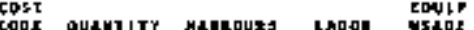

Мस대

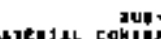

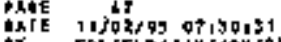

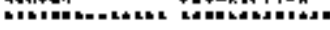

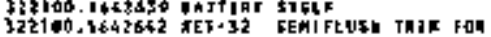
Th=37 Ance Osoke

3z21\%0.1\$42\$16 Fid. AIP क TeEt Allowant?

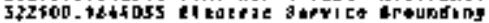

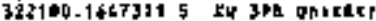

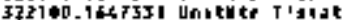
$32210.14+532$ 3x Uninge Bthe

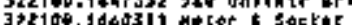

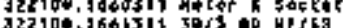

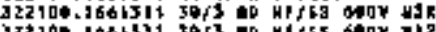
grरlop 1006s?

aटz

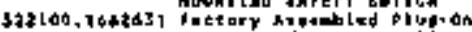

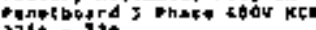
$224 \%-320$

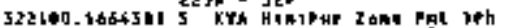

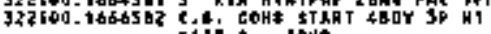
site 1 Ionto

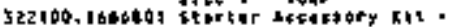

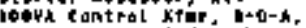

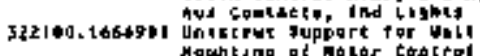

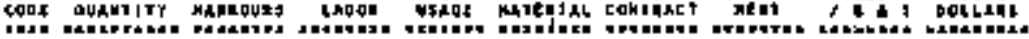

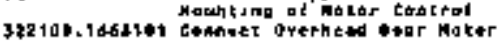
oparatar

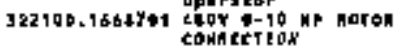

str TaT1K ELECTEIChL

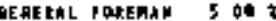

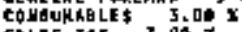

ShLE TAE TH,

OHIP TOM KALEUPS OHCT

TOTAS COST, çoot $301 \mathrm{tb}$

[Etiexto

505
501
501
501
501
501
501
301
501
501
509
501
501
501
501
501
501

5)t

501

301

$5 \% 1$

301

301

501

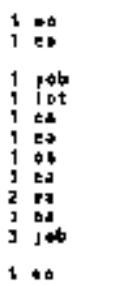

$\begin{array}{rrr}2 & & \\ 2 & & \\ 16 & & 5 \\ 24 & & \\ 2 & & \\ 0 & & \\ 2 & & \\ 5 & & 1 \\ 2 & & \\ 1 & & \\ 3 & & \\ 15 & & 50\end{array}$

501

5eI

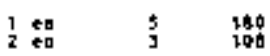

2.* 283

$\begin{array}{ll}0 & \\ 0 & \\ 0 & 2 \\ 0 & 2 \\ 0 & \\ 0 & 1 \\ 0 & 1 \\ 0 & 1 \\ 0 & \end{array}$

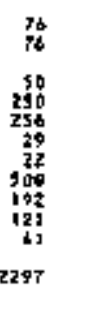

\begin{tabular}{|c|c|c|c|}
\hline $\begin{array}{l}0 \\
0\end{array}$ & $\begin{array}{l}0 \\
0\end{array}$ & $\frac{d}{46}$ & $\begin{array}{l}162 \\
102\end{array}$ \\
\hline $\begin{array}{l}0 \\
0 \\
0 \\
0 \\
0 \\
0 \\
0 \\
0\end{array}$ & $\begin{array}{l}0 \\
0 \\
0 \\
0 \\
0 \\
0 \\
0 \\
0\end{array}$ & $\begin{array}{r}160 \\
314 \\
90 \\
9 \\
24 \\
182 \\
74 \\
47 \\
65\end{array}$ & $\begin{array}{r}113 \\
1446 \\
626 \\
30 \\
132 \\
790 \\
343 \\
204 \\
106\end{array}$ \\
\hline 8 & 口 & 80 & 2640 \\
\hline
\end{tabular}

\begin{tabular}{|c|c|c|c|c|c|c|c|c|c|c|}
\hline 986 & $z$ & pob & 2 & $7 t$ & 0 & 20 & $\emptyset$ & 0 & 30 & 190 \\
\hline 301 & z & 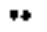 & $\theta$ & 207 & 0 & 510 & 0 & $\bullet$ & 236 & 1023 \\
\hline 501 & $t$ & 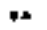 & 3 & $10 t$ & a & 23 & 0 & $\bullet$ & 39 & 170 \\
\hline
\end{tabular}




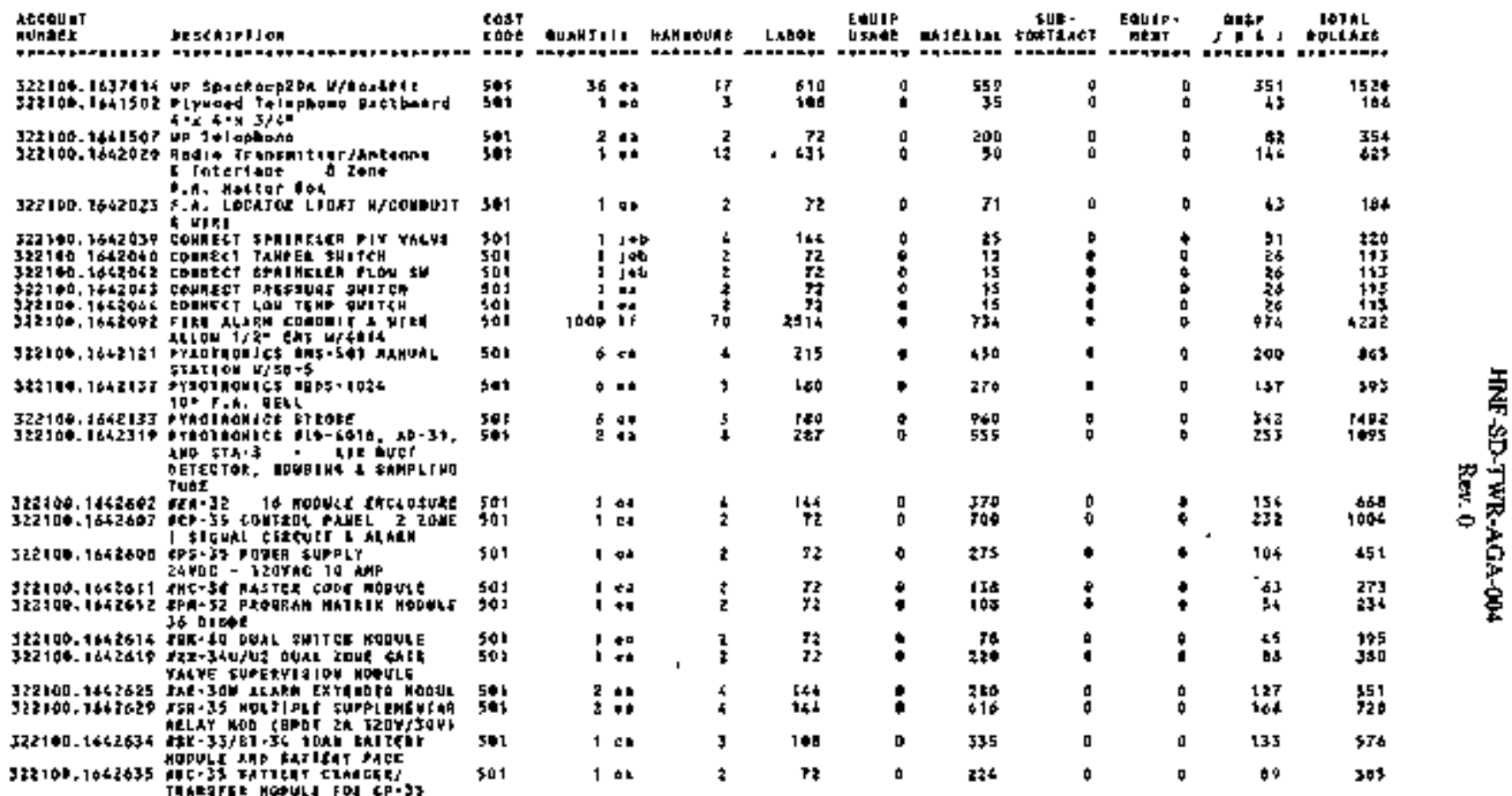

总 


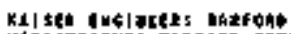

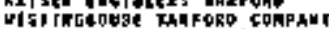

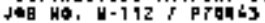

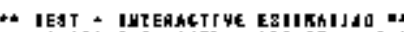

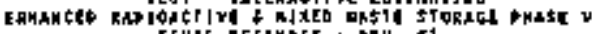

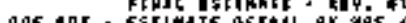

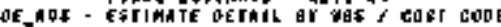

DAGE 4.

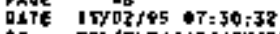

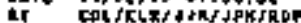

\section{Atconu $\mathrm{c}$}

aUMPG 5

Q5TCEIPTIOH

CaAT touly

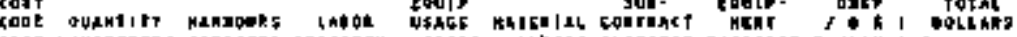

$322100.168+104$ hosर Trate Tubing

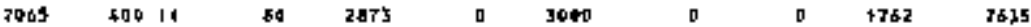

322100.162410 t $4000 \mathrm{n}$ ह

ILthe

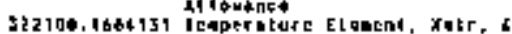

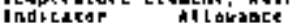

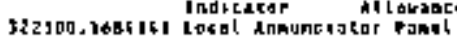

522300.1685161 locol dnume

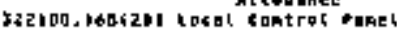

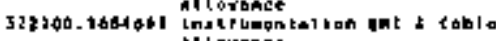

H l lownes.

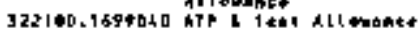

furioliti recthichl

TE MERAE FOREKMH $5.00 \mathrm{y}$

COWS UNA:LES J. $00^{3} \bar{x}$

SALEE 1AK T.

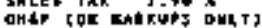

IOIAL ERT CODE $7 \$ 616$

dat 322300

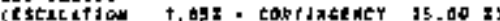

joas 1 I E

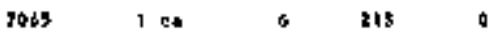

$7045 \quad 1530070$

7085 $1 \mathrm{et}$ in sts

$7065 \quad 75 \% 1155 \quad 1975$

ro6s

$1 \mathrm{dab}$

340

150

750

754

seto

$\ldots . . .2$ dab 31

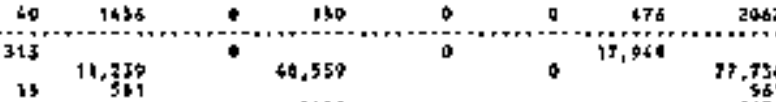

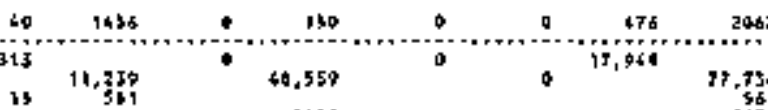

77.756

3931000951

36

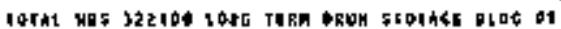


Table A-21. Surnmary of Cost Estimate for Constroction of Interim Storage Units for Contaxt-Handled Mixed ILAW Conbinets Bared on Project W-112 (Cptipn S)

\begin{tabular}{|c|c|c|c|c|}
\hline \multirow[t]{2}{*}{ Description } & \multicolumn{2}{|c|}{ W.112 Project Estimate } & \multicolumn{2}{|c|}{ CH-MW ILAW Storage Estimate } \\
\hline & \$ million & Vahe & $\$$ million & Value \\
\hline Unit I. & 1.03 & $\$ \$ 15 / m^{2}$ & 2.06 & $5515 / \mathrm{m}^{2}$ \\
\hline \multicolumn{5}{|l|}{$\begin{array}{l}\text { Adjusiments to } \\
\text { Plocr \$lab }\end{array}$} \\
\hline Conctele & None" & $\$ 116 \mathrm{~m}^{3}$ & $0.31^{b}$ & $\$ 116 / \mathrm{m}^{3}$ \\
\hline Rebarar & Nopo* & $\$ 27 / \mathrm{m}^{3}$ & $0.23^{\mathrm{b}}$ & $\$ 87 / m^{3}$ \\
\hline Subtotal & 1.03 & $\therefore$ & 2.60 & $\$ 650 \mathrm{~m}^{2}$ \\
\hline Escalation to $2002^{\mathrm{C}}$ & . & . & 0.57 & . \\
\hline Total 1 Unit & 1.03 & $\mathbf{S S L S / \mathrm { m } ^ { 2 }}$ & 3.17 & $5793 / \mathrm{m}^{2}$ \\
\hline Totgl 2 Units & 2.1 & $\mathbf{s} 15 / \mathbf{m}^{2}$ & 6.3 & $\$ 793 / \mathrm{m}^{2}$ \\
\hline
\end{tabular}

- Baseline tloor slab is $2000 \mathrm{~m}^{2}$ area and $0.25 \mathrm{~m}$ thick.

bNew floos slab requirement is estiulated at $4000 \mathrm{~m}^{2}$ and $0.5 \mathrm{~m}$ thick.

- Baged on 5.2\% from Niovember 1995 to Jume 1997 and 16\% to September 30, 2002.

'Based on information in Table A-20. 
HNF-SD-TWR-AOA-A04

Rev 0

Table A.22. Sommery of Infrastructure Coss Estionate for Interim Swrage Units for Contace-Handled Mired Immobiliwed Low-Axtivity Wagte Containers Baged on Project W-1L2.

\begin{tabular}{|c|c|}
\hline Description. & Toual Cost sK \\
\hline Infrastructare Support & 39 \\
\hline $\begin{array}{l}\text { Procuremen of Radio Fire Alarm Report } \\
\text { Boxes }\end{array}$ & 38 \\
\hline Electrical Tie-Ins and Furniture & 34 \\
\hline Ganeral Conditions & 104 \\
\hline Water Line & 375 \\
\hline Site Grading & 204. \\
\hline Pagred Roads & 355 \\
\hline Paved Parking and Drives. & 114 \\
\hline Sidewalks and Fencing & 62 \\
\hline Soir Stabilization & 158 \\
\hline Saniary Sewer & 311 \\
\hline Sagatbrush Mikigarion & 500 \\
\hline $\begin{array}{l}\text { Hanford Local Area Network (HLAN) atd } \\
\text { Telephone Services }\end{array}$ & 314 \\
\hline Sybtotal. & 2,648 \\
\hline Escalation' & 582 \\
\hline Total & 3.230 \\
\hline
\end{tabular}

"Based on $5.2 \%$ from November 1995 to Jume 1997 and $16 \%$ to Septernber 30, 2002. 
HINF-SD-TWR-AGA-004

Ror o

\section{A2.5 SHIELDED OVERPACKS (OPTIONS 6A AND 6B)}

Table A-23. Sumunary of Cost Estimates for 5040 Concrete and 5040 Carbon Steel Shielded Overpacks for Dispogal of Remote-Handlod Imamobilized Low-hetivity Waste Comtainero (Opirons 6A and 6B). 
HNF-SD-TWR-AGA-004

Rev. o

This page intentionally left blamk. 
TLUOR BANTEL HEOTEMrsJ, IMC LOCAHIEO MANIIN HANPOKQ GOEO

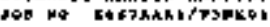

TICI To EATARA1

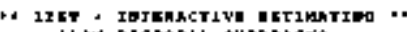

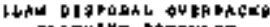

ERMMINA EHJXATE

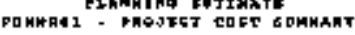

rear $>2$ or?

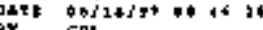
CaL
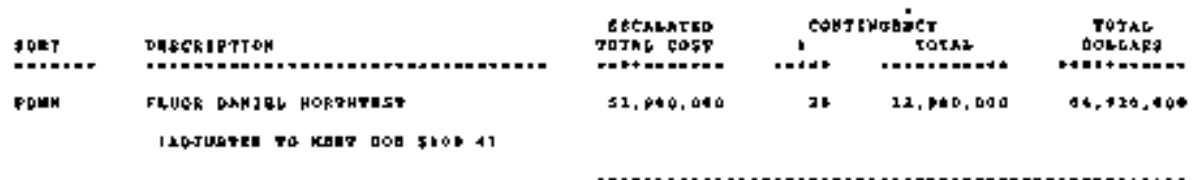

honser torac

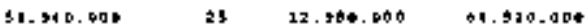
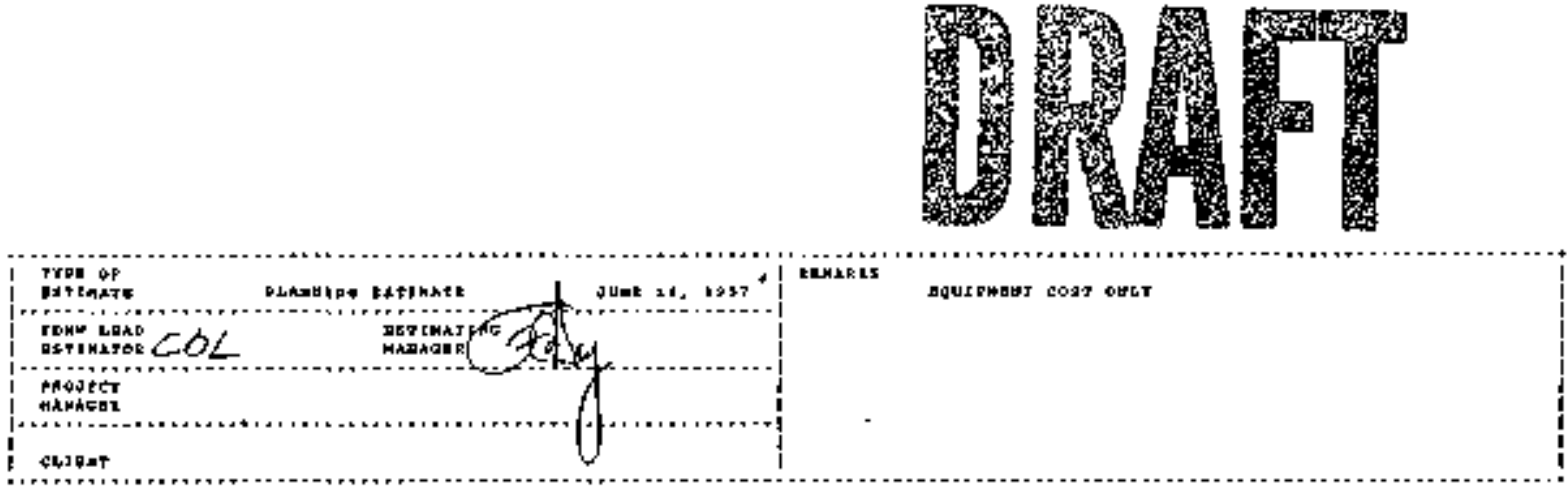

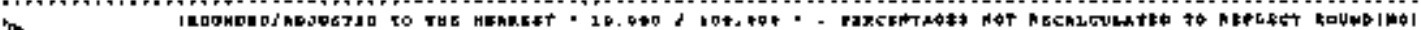

N 


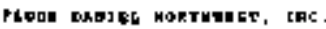
LOSKHEEP MALTIM HMBEOHD SOFE J08 HQ, IAGJNGAIJTHEI

FILE Da. 2tethest

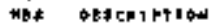

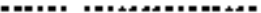

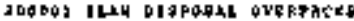

nougcs rotk

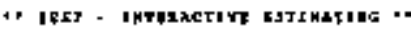

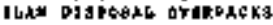

DLNGTEG EJTIMLTE

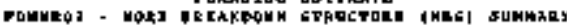

buat itopt

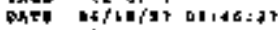

EI

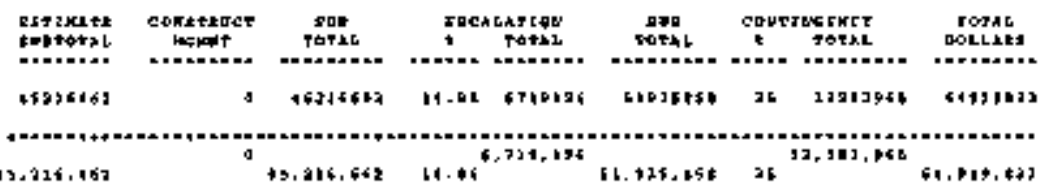


Rey. 0
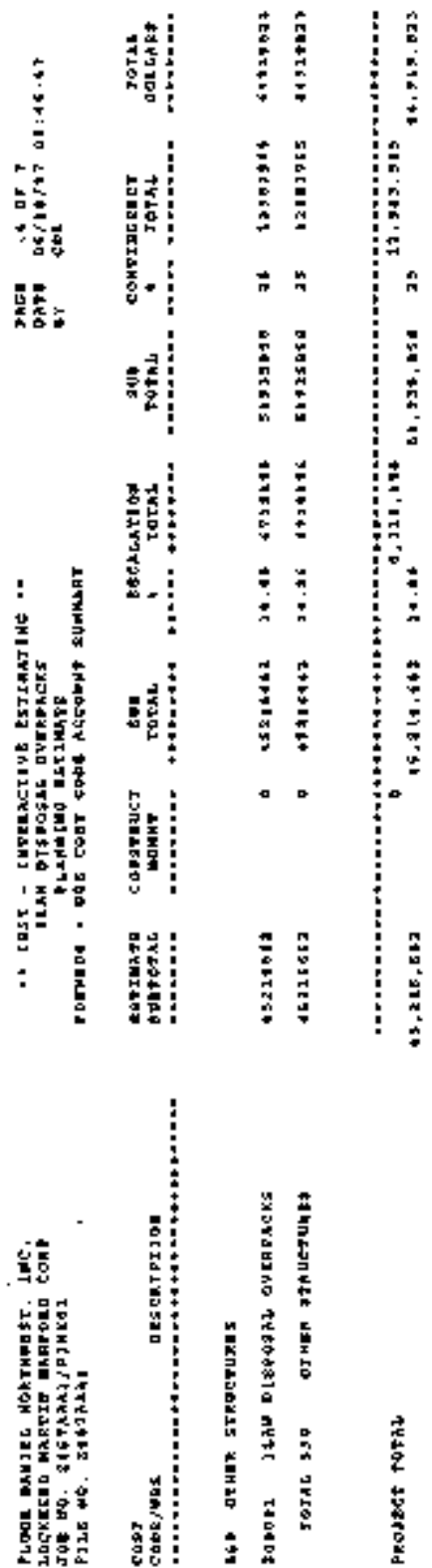


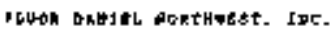

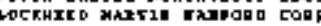

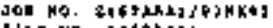

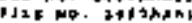

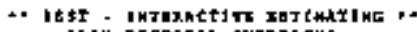 \\ a lah pIgporil orgepheKs

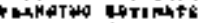

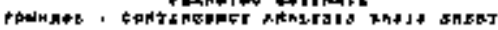

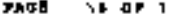

MATI OG/10/9t 01:11:21

$\rightarrow$

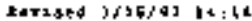

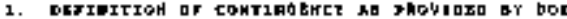

: coupjugp

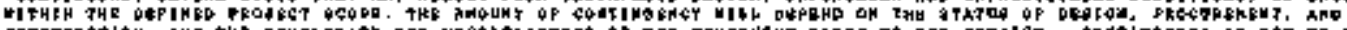

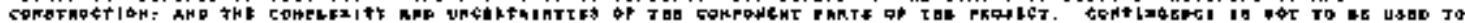

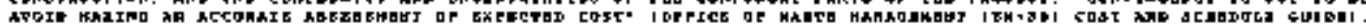

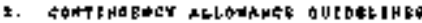

A.M.

T3:

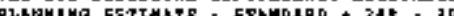

1. Mrtmagobov

40

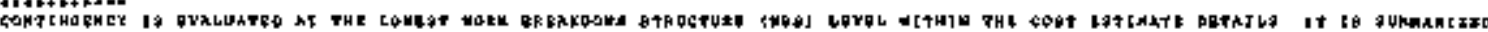

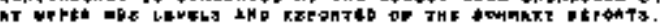

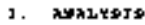

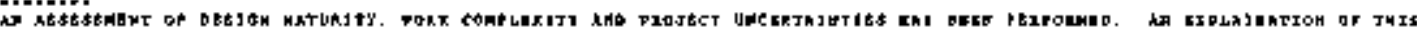

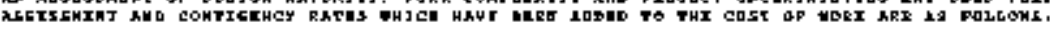

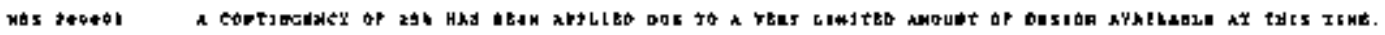


Rev. 0
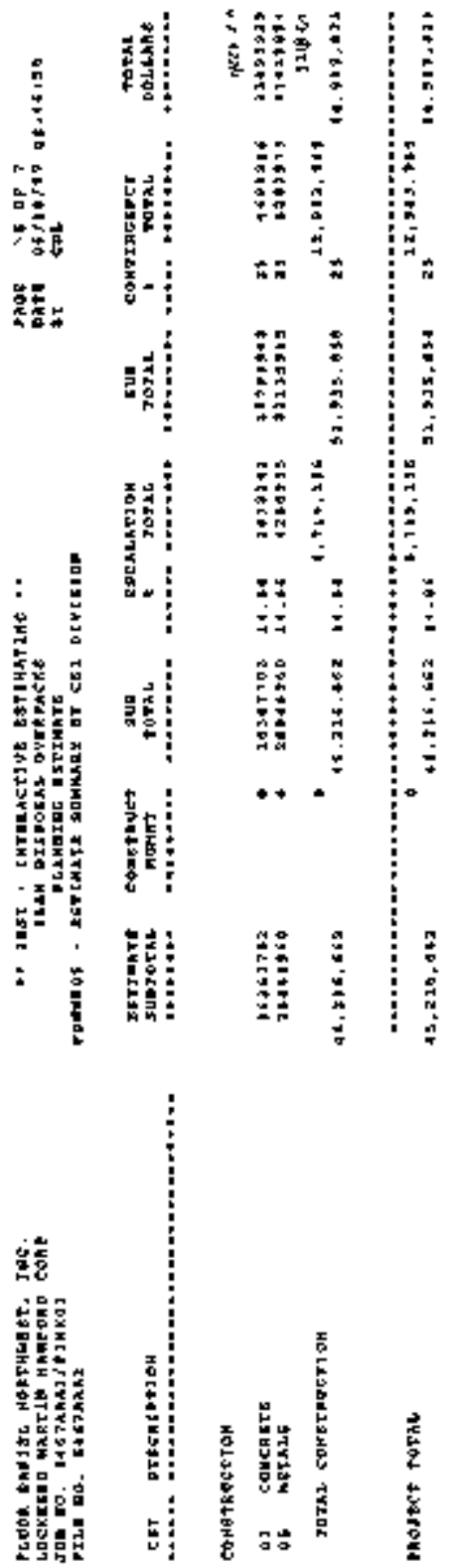

PCWOA DSMIDE NOATHERPT, LPC.

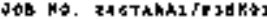

TILE AD - ItB?hAR:

\section{-* IEAT - IHTDLXCTIVE RATIHATIHB -

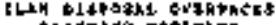

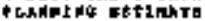

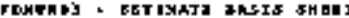

Plate is De +

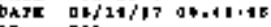

$\rightarrow$ e $\mathrm{LL}$

l. EotEmata DUAREE

THEQ BotiMht

a. tot LTATE ttemBical gatit

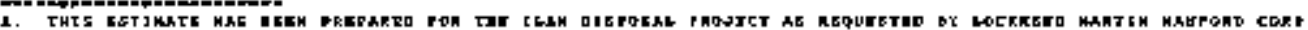

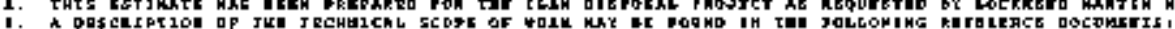

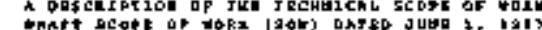

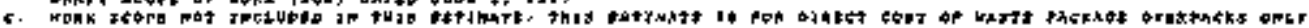

3. astrkhe METratoloar

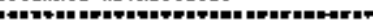

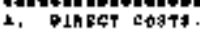

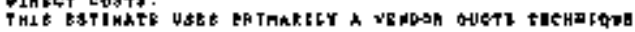

d. DIRTCt COLJ ractaks

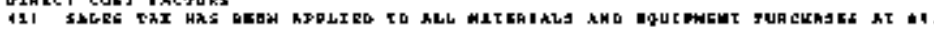

e. INGSRECY COATS

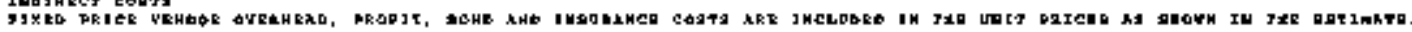

1. t\$ChGAITH

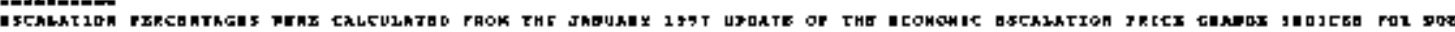

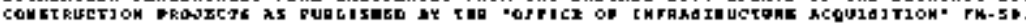

L, 5 a

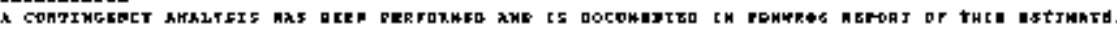

6. HBHBR KE

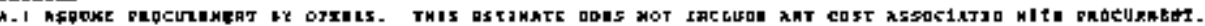

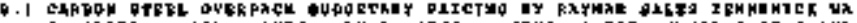

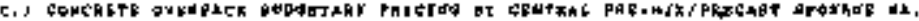

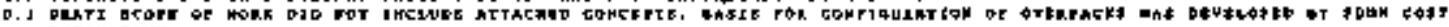

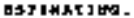

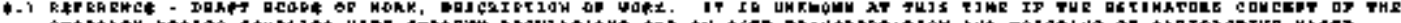

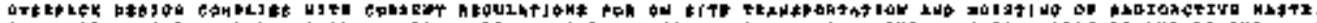

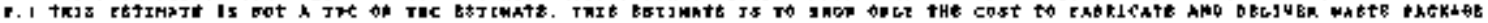
DYREREKE. 
Rev. 0
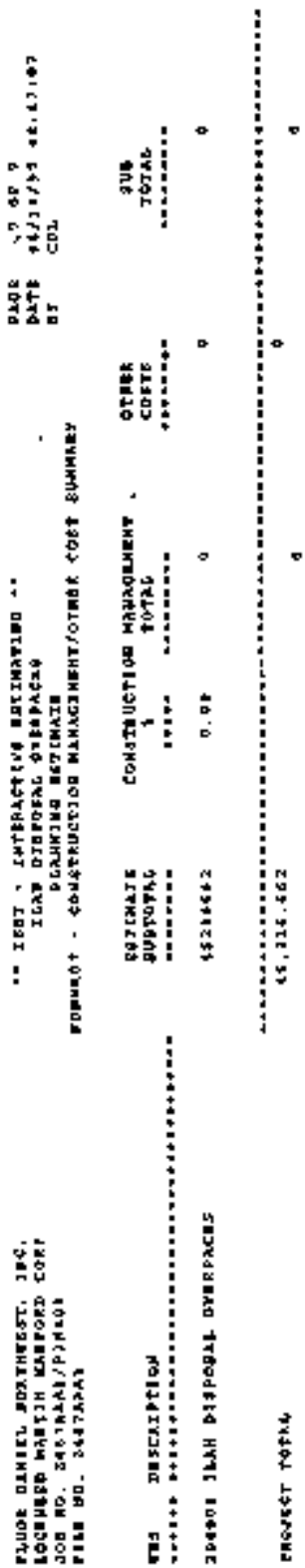


\section{HNF-SD-TWR-AGA-DO4}

Rev. 0

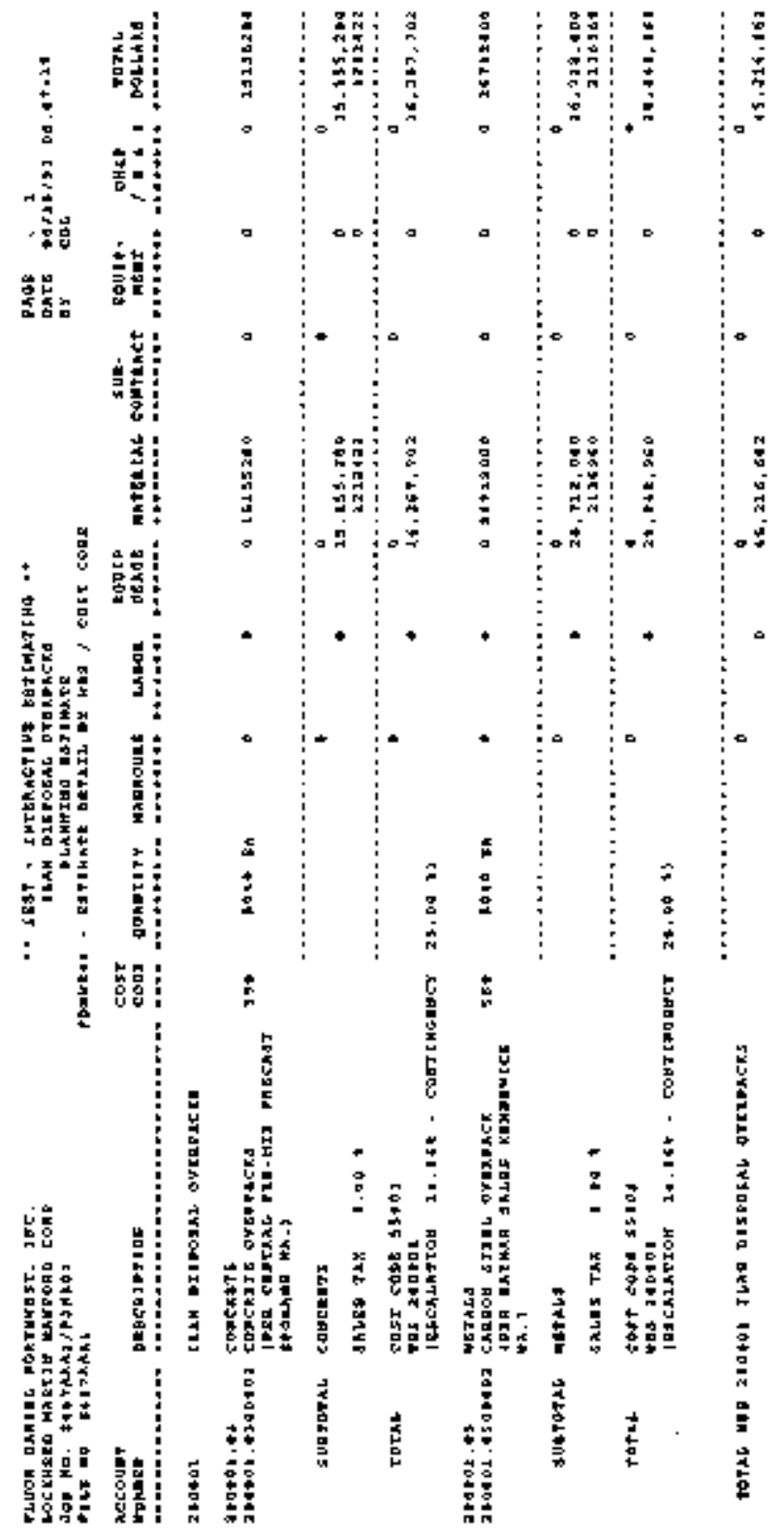


TLUOR ganitz monthHBBt, IHe, COEIHE EE WHATSM HAMTDEB CORE

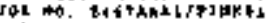

PLLE NO, IIBThShI

ACLODNT

Menbth

DEEÇEI

vanas.

MEPOIT TOTRE

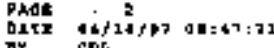

TlNo DJMOSAL QVERTACRE

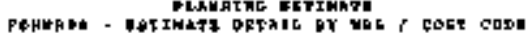

Cots

$\cos 0$

EQVI:

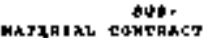

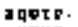
45

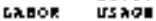

and Toth

1.

1.. -...

$+46,925,669$

0

$41,214,462$

管 


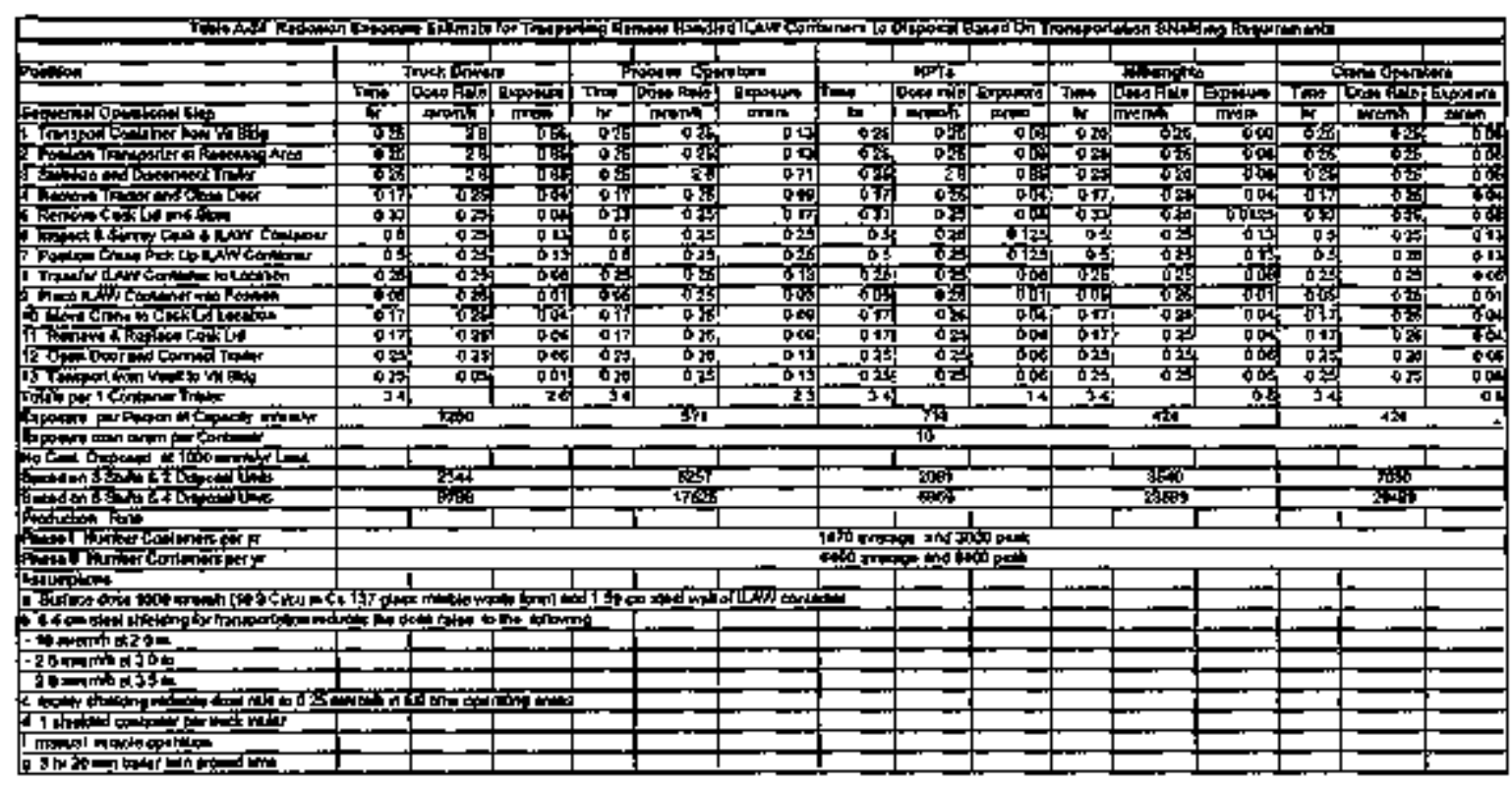




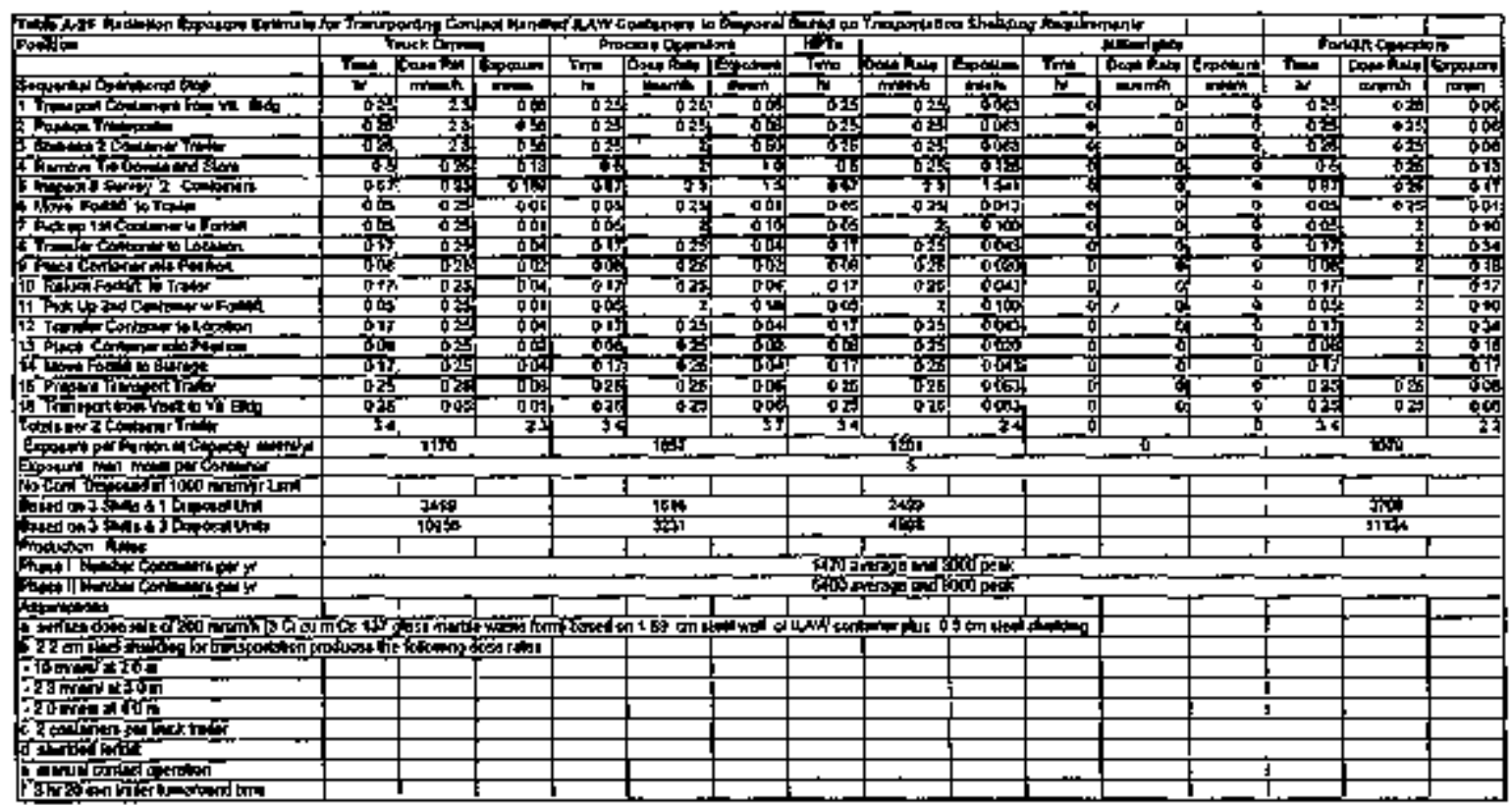


HNF-SD-TWR-AGA.00A

Rer 0

This parse intealiogally left todank. 


\section{HNF-SD-TWR-AOA-004}

Rev. 0

\section{APPENDIX B}

\section{CONSTRAINTS ON DESIGN OF THE IMMOBILIZED LOW-ACTIVITY WASTE DISPOSAL FACILITY}


HNF-SD-TWR-AOA-00\#

Rew, 0

This page invedionally left blank.

E-2 


\section{APPENDIX B}

\section{CONSTRAINTS ON DESIGN OF THE MMOOBLIZED LOW-ACTVITY WASTE DLSPOSAL FACHATY}

Ilatic sype = requirement was added to RDD 100 bastline before Fob review of Boriemarisurbank/Klem.

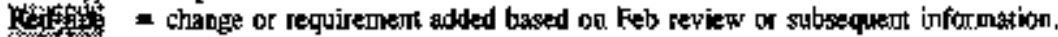

Code of Federal Regulations

\begin{tabular}{|c|c|c|c|}
\hline Document Identifier & Tilk & Applicable & Justification \\
\hline \multicolumn{4}{|c|}{$\begin{array}{l}\text { TTILE 10 ENERGY } \\
\text { PART 61 LCENSING REQUIREMENIS FOR LAND DISPOSAL } \\
\text { OF RADIOACTIVE WASTE }\end{array}$} \\
\hline 10 CFR 61.4I & $\begin{array}{c}\text { Subpert C. Performance } \\
\text { Objoctives } \\
\text { Protecion of the general } \\
\text { population from releages of } \\
\text { redimalivity }\end{array}$ & $\mathrm{Y}$ & 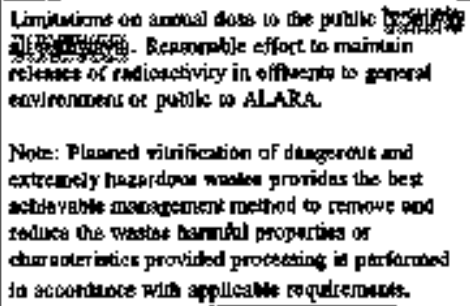 \\
\hline$I 0 \mathrm{CFR} 61.42$ & 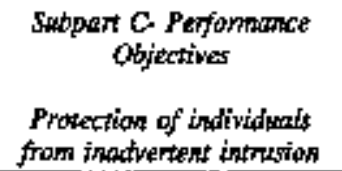 & $Y$ & 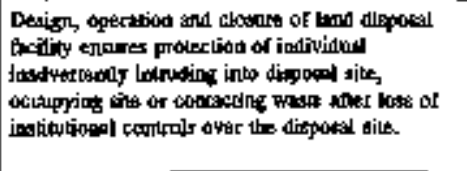 \\
\hline
\end{tabular}


Code of Federal Regulationes

\begin{tabular}{|c|c|c|c|}
\hline Docuument kitentifier & Tille & Applicable & Juslification \\
\hline$I 0 \mathrm{CFR} 61,43$ & $\begin{array}{l}\text { Subpart C-Performance } \\
\text { Objectiver } \\
\text { Protection of indilyiduals } \\
\text { during operation }\end{array}$ & $\mathbf{Y}$ & 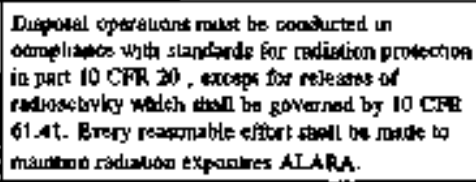 \\
\hline 10 CFR 61.44 & $\begin{array}{l}\text { Subpart C - Performaber } \\
\text { Objectives } \\
\text { Stability of the disposal } \\
\text { site after closute }\end{array}$ & $\mathbf{Y}$ & 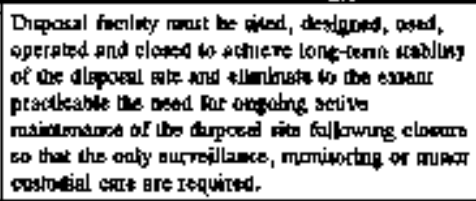 \\
\hline Ans & 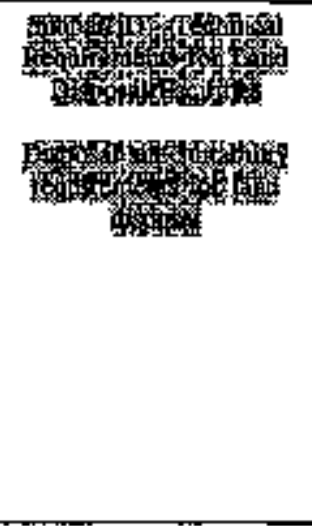 & 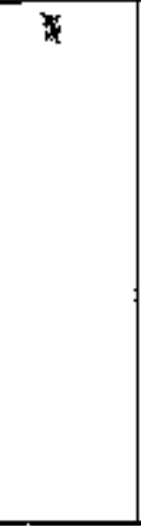 & 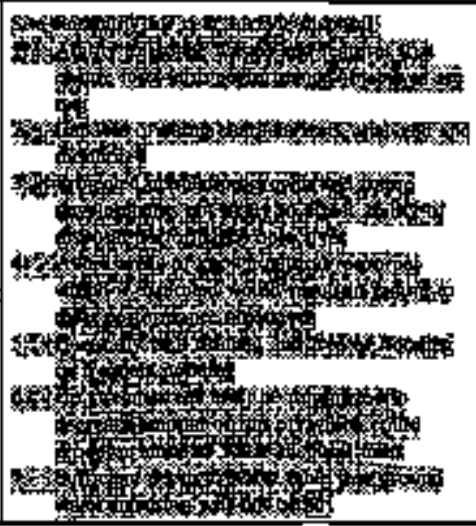 \\
\hline
\end{tabular}


Code of Federal Regulations

\begin{tabular}{|c|c|c|c|}
\hline Documetet Identifier & THue & Applicable & JustiEveation \\
\hline $10 \mathrm{CFR} 61.51$ & 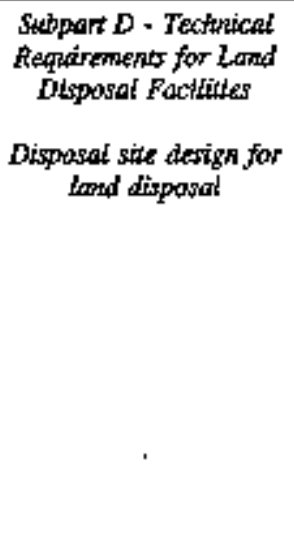 & $Y$ & 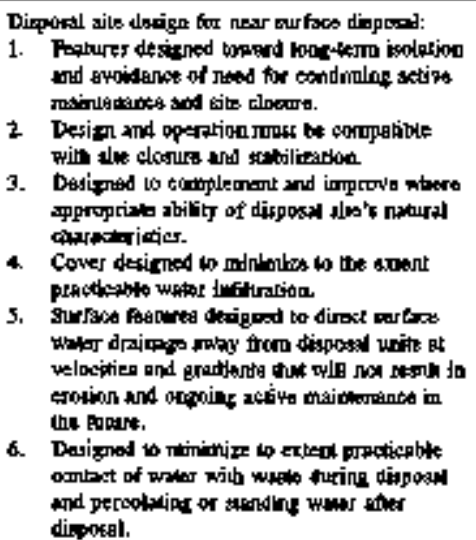 \\
\hline
\end{tabular}


Code of Pederal Regulations

\begin{tabular}{|c|c|c|c|}
\hline Docurspent Identifïr & Tide & Applicable & Justifkealion \\
\hline 10 CFR 61.52 & $\begin{array}{l}\text { Subpart D - Tochnical } \\
\text { Requirements for Land } \\
\text { Disposal Factillits } \\
\text { Land dosposal faciltty } \\
\text { operation and disposal site } \\
\text { closure }\end{array}$ & $Y$ & 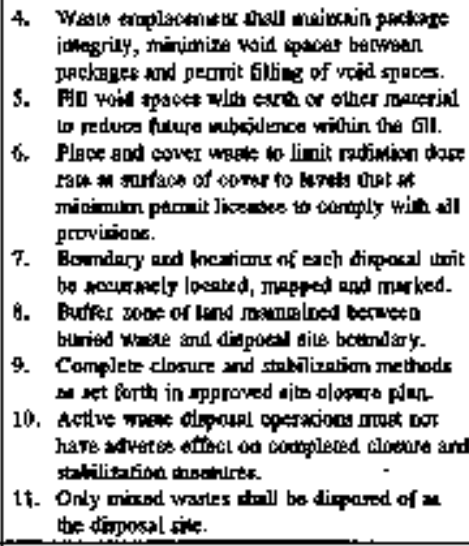 \\
\hline $10 \mathrm{CPR} 61.53$ & 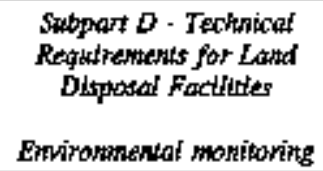 & $Y$ & 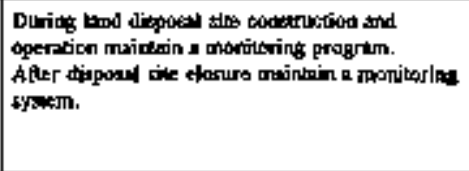 \\
\hline 10 CFR 61,55 & $\begin{array}{l}\text { Subpart D - Technical } \\
\text { Requirements for Land } \\
\text { Disposal Facilities } \\
\text { Waste classification }\end{array}$ & $\mathbf{N}$ & 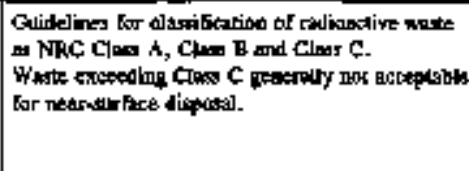 \\
\hline
\end{tabular}


Code of Federal Regulations

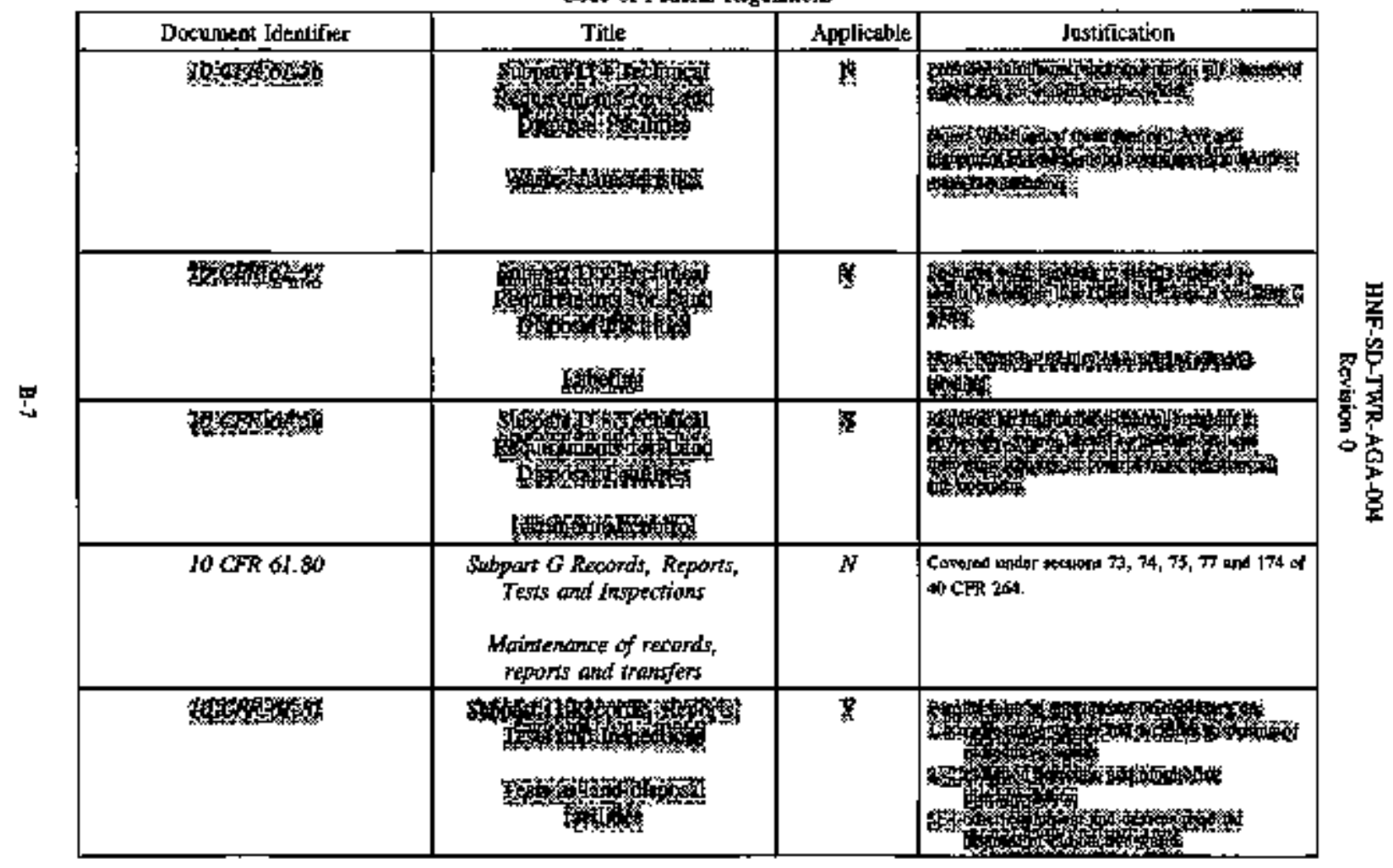


Cods of Federal Regolations

\begin{tabular}{|c|c|c|c|}
\hline Documenu Identifier & Titte & Applieable & Iustiticantion. \\
\hline 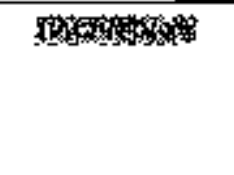 & 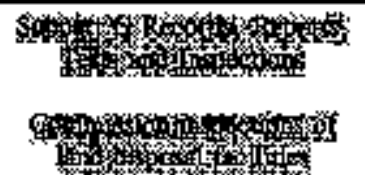 & 要 & 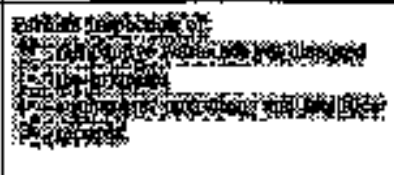 \\
\hline
\end{tabular}

詈 
Code of Federal Regrigations

\begin{tabular}{|c|c|c|c|}
\hline Docament Jdentificer & Titile & Applicable & Justification \\
\hline \multicolumn{4}{|c|}{$\begin{array}{l}\text { TTILE } 10 \text { ENERGY } \\
\text { SUSBPART \$\$O NUCLEAR SAFETY MANAGEMIENT }\end{array}$} \\
\hline 10 CFR $830.120 \mathrm{a}-\mathrm{C}$ & $\begin{array}{l}\text { Subpart A - General } \\
\text { Provisions } \\
\text { Quality Abarrance } \\
\text { Requirements }\end{array}$ & $\mathbf{Y}$ & 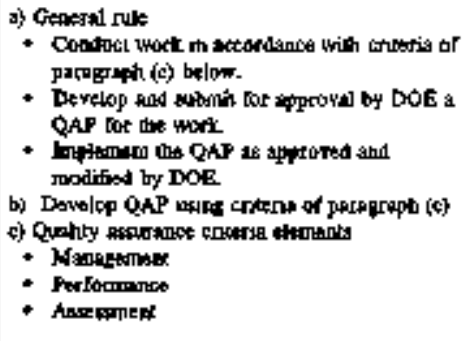 \\
\hline W & 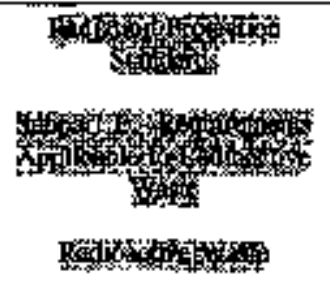 & 程 & 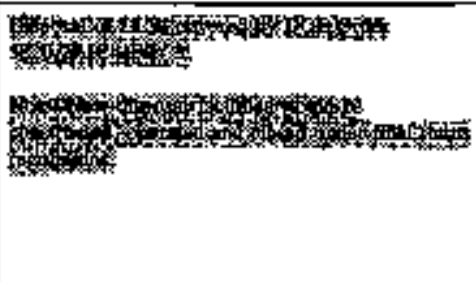 \\
\hline
\end{tabular}


Code of Federal Reqularions

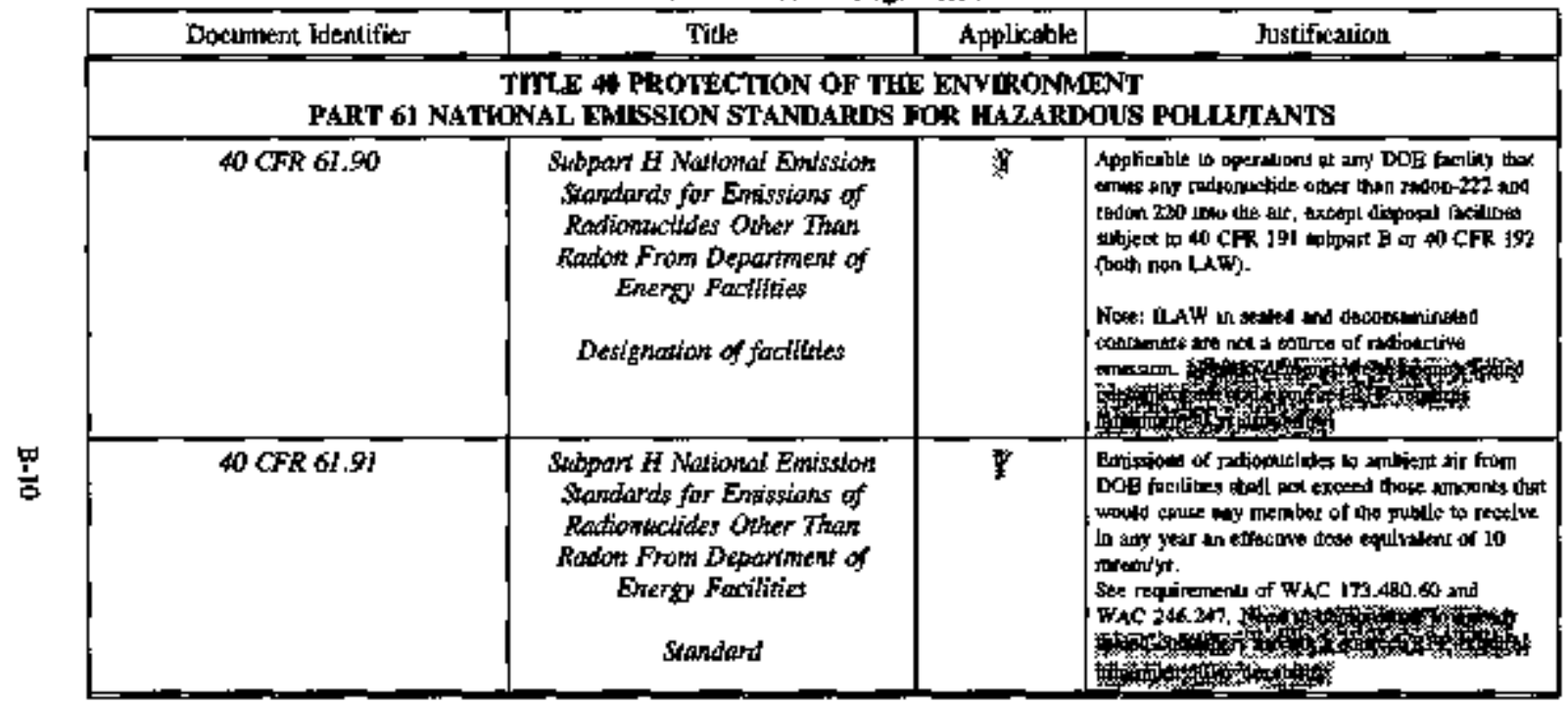




\begin{tabular}{|c|c|c|c|}
\hline \multicolumn{4}{|c|}{ Code of Federal Regulations } \\
\hline Document Identifier & Tilke & Applicable & Justification \\
\hline \multicolumn{4}{|c|}{$\begin{array}{l}\text { TTILE 40 PROTECTYON OF THE ENVIRONMENT } \\
\text { PART 200 HAZARDOUS WASTE MANAGEMENT SYSTEM: GENERAL }\end{array}$} \\
\hline 40 CFR $260.22 a-d, f, h-l$ & 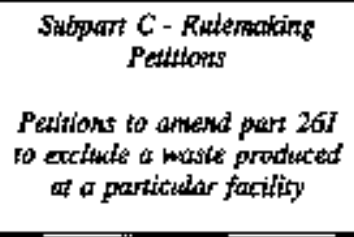 & $\mathbf{Y}$ & 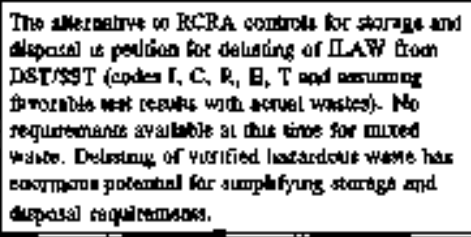 \\
\hline \multicolumn{4}{|c|}{$\begin{array}{l}\text { TITLE 40 PROTECTYON OF TKE ENVTRONMIENT } \\
\text { PART 264 STANDARDS FOR OWNERS AND OPERATORS OF BAZARDOUS WASTE TREATMENT, } \\
\text { STORAGE ANI DLPOSAL FACIIITIRS }\end{array}$} \\
\hline 40 CFR $264.13 a$ & $\begin{array}{l}\text { Subpart B - General } \\
\text { Facilly Stondards } \\
\text { Genemal utaste anabysis }\end{array}$ & Y & 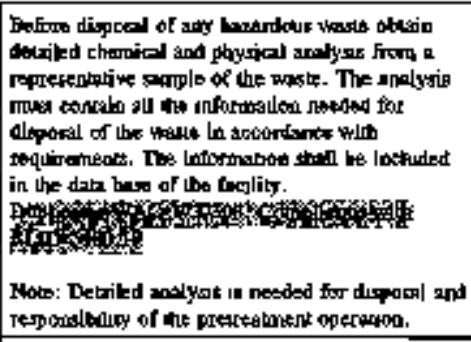 \\
\hline 10 CFR 264.140 & 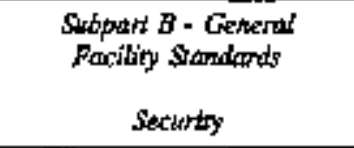 & $y$ & 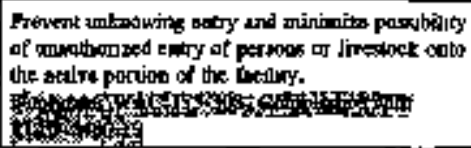 \\
\hline
\end{tabular}


Code of Federal Regulations

\begin{tabular}{|c|c|c|c|}
\hline Documentik İdemtifiter & Tidk & Applicabite & Justifictation \\
\hline 40 CPR 264.15 a-d & 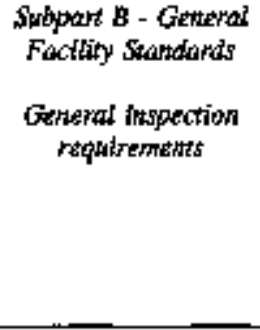 & $\boldsymbol{Y}$ & 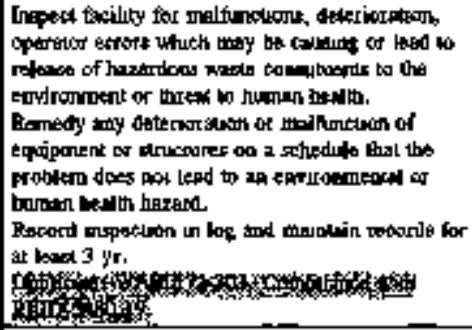 \\
\hline 40 CFR 264.16 a.e & $\begin{array}{l}\text { Sabpant B - Genenal } \\
\text { Factiliy Shandards } \\
\text { Persontel iradring }\end{array}$ & $y$ & 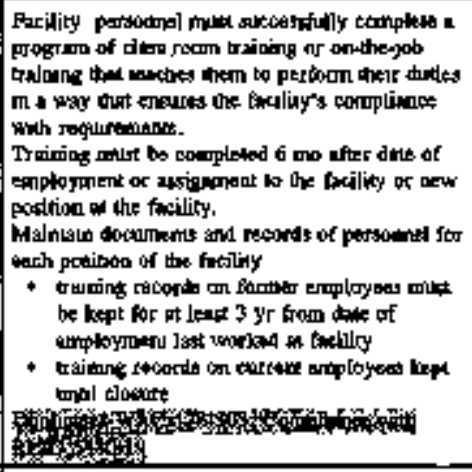 \\
\hline 40 CFR $264.18 \mathrm{db}$ & $\begin{array}{l}\text { Subpart B - Getherds } \\
\text { Facility Shanderds } \\
\text { Lachation Standards }\end{array}$ & $y$ & 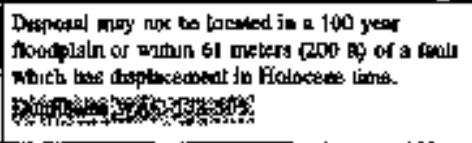 \\
\hline
\end{tabular}


Code of Federel Regulations

\begin{tabular}{|c|c|c|c|}
\hline Doxument Identifier & Tilk & Applicable & Instification \\
\hline 40 GPR $264.3 I$ & $\begin{array}{l}\text { Sebpart C. Preparediness } \\
\text { and Prevention } \\
\text { Designt and optration } \\
\text { of focitity }\end{array}$ & $Y$ & 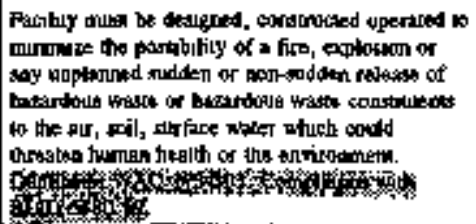 \\
\hline 40 CFR 264.32 add & 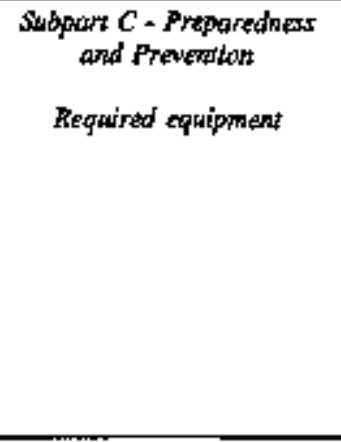 & $F$ & 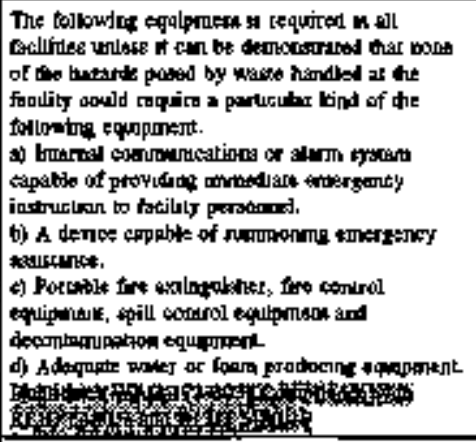 \\
\hline 40 CFR 264.33 & $\begin{array}{l}\text { Subpart C. Preparedress } \\
\text { and Prevertitort } \\
\text { Testing and matintesapoce of } \\
\text { equipment }\end{array}$ & $Y$ & 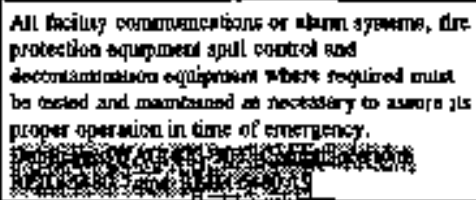 \\
\hline
\end{tabular}


Code of Federal Regulations

\begin{tabular}{|c|c|c|c|}
\hline Document Idestifier & Tile & Applicable & Justification \\
\hline 40 CFR 204.34 ab & $\begin{array}{l}\text { Subpart C - Preparedness } \\
\text { ond Prevestion } \\
\text { Access to commurstcations } \\
\text { or alam system }\end{array}$ & $\mathbf{y}$ & 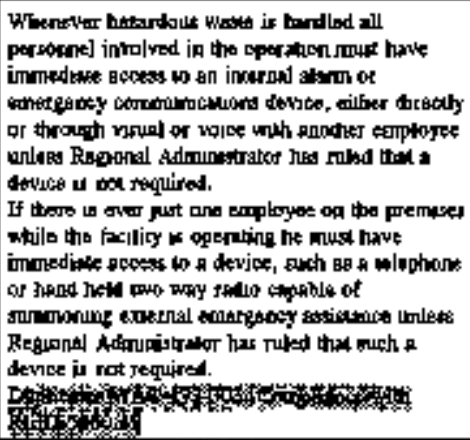 \\
\hline 40 CFR 264.35 & $\begin{array}{c}\text { Subpart } C \text { - Preparedivess } \\
\text { and Prevention } \\
\text { Reguired aiste spoct }\end{array}$ & $y$ & 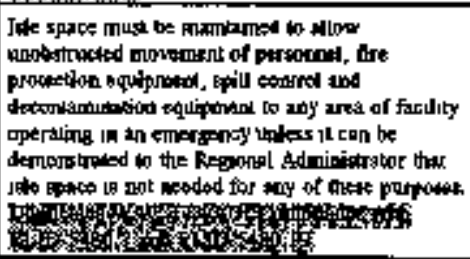 \\
\hline $40 C F R 26477 a$ & 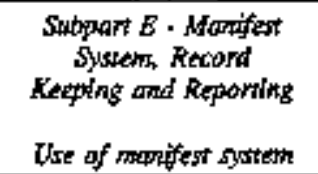 & $y$ & 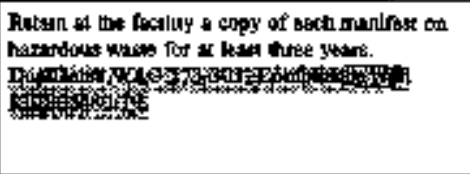 \\
\hline
\end{tabular}


Code of Pederai Regalations

\begin{tabular}{|c|c|c|c|}
\hline Document Identifier & Titale & Applicable & Juktifikeation \\
\hline 40 CFR $264.73 a b$ & 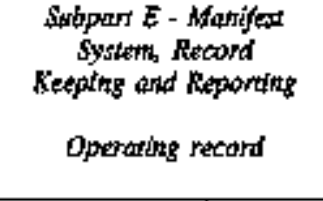 & $r$ & 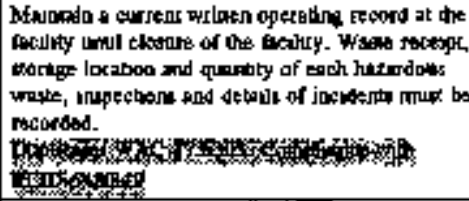 \\
\hline 40 CFR $264.74 a-c$ & 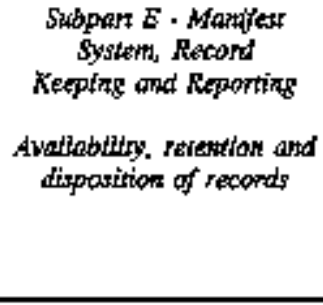 & $Y$ & 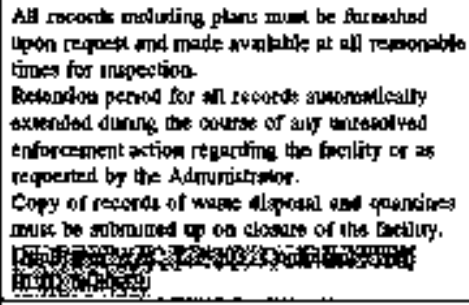 \\
\hline 40 CFR 264.75 & $\begin{array}{l}\text { Subpart E- Manifest } \\
\text { System, Recond } \\
\text { Ketpling attd Reportitg } \\
\text { Bierpial report }\end{array}$ & $x$ & 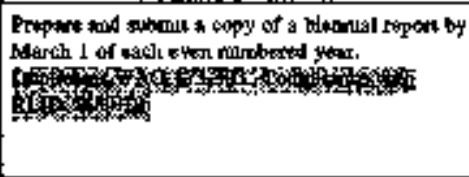 \\
\hline $40 \mathrm{CFR} 264.77 a-\mathrm{C}$ & 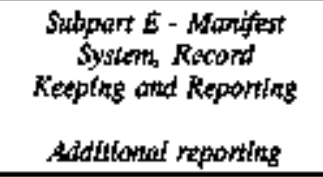 & $r$ & 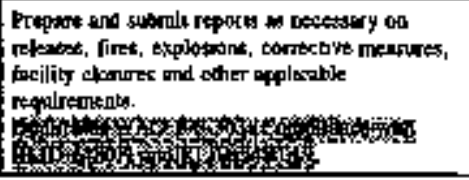 \\
\hline
\end{tabular}


Code of Federal Regulations

\begin{tabular}{|c|c|c|c|}
\hline Doxument Idealifier & Tiule & Applicable & Justification \\
\hline$\$ 0$ CNK 264.90 ab & 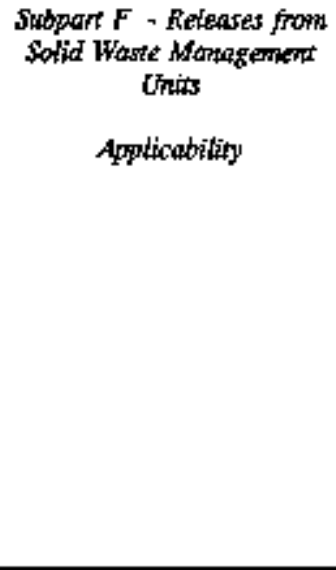 & $y$ & 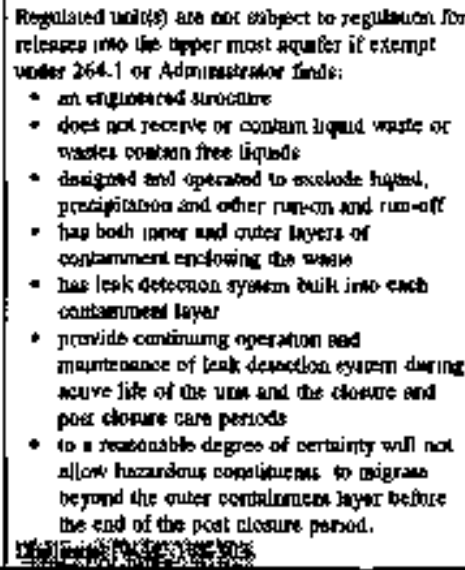 \\
\hline $40 \mathrm{CFR} 264.10 \mathrm{db}$ & 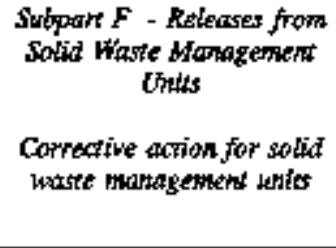 & $y$ & 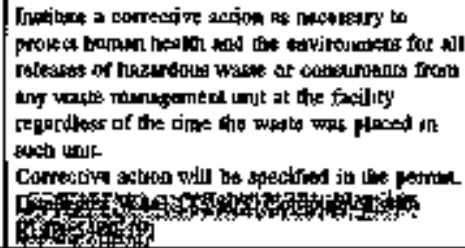 \\
\hline
\end{tabular}




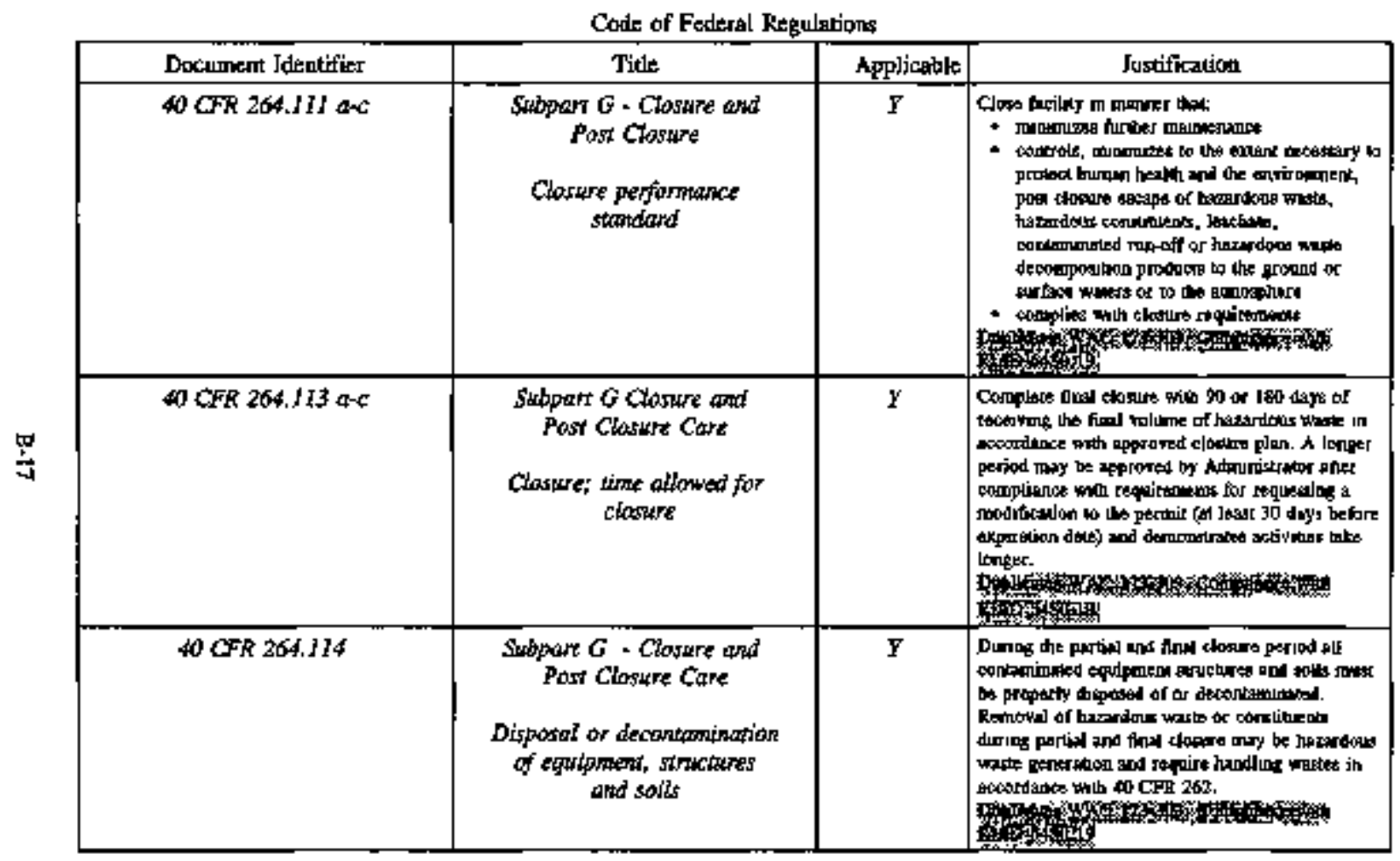


Code of Federal Regulations

\begin{tabular}{|c|c|c|c|}
\hline Docunent ldentifier & Tille & Applicable & Jusuification \\
\hline 40 CFR 264.117 & $\begin{array}{l}\text { Skbpart G - Closure and } \\
\text { Pose Closure Care } \\
\text { Post-closure care and we } \\
\text { of property }\end{array}$ & $\gamma$ & 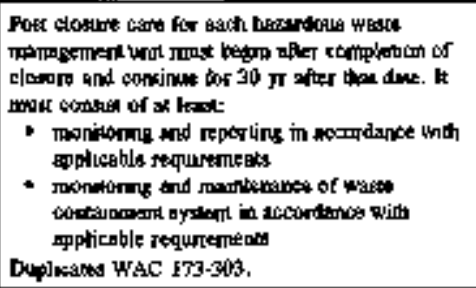 \\
\hline 40 CFR 264.171 & $\begin{array}{l}\text { Subpart I - Use and } \\
\text { Management of Containers } \\
\text { Condition of containers }\end{array}$ & $\mathbf{N}$ & 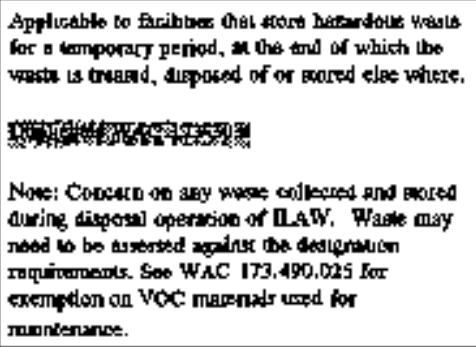 \\
\hline 40 CPR 264.172 & $\begin{array}{l}\text { Subpart I - Use and } \\
\text { Managtemitent of Contadrexs } \\
\text { Comparibilly of waste } \\
\text { with conkainers }\end{array}$ & $\mathbf{N}$ & 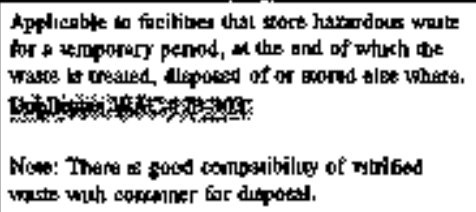 \\
\hline
\end{tabular}


Code of Federal Regulations

\begin{tabular}{|c|c|c|c|}
\hline Docuarequt Identificer & Tite & Applicablei & Justificeation \\
\hline 40 CFR 264.173 ab & $\begin{array}{l}\text { Subpart I - Use and } \\
\text { Mamagement of Contajines } \\
\text { Management of containers }\end{array}$ & $\mathbf{N}$ & 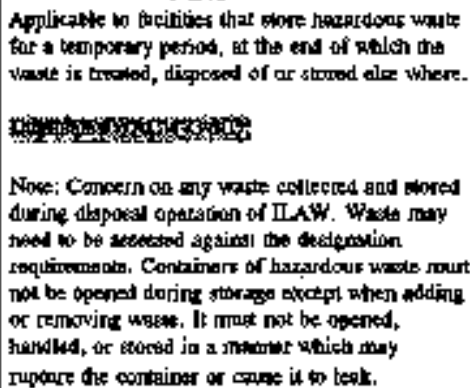 \\
\hline $40 C P R 264.174$ & $\begin{array}{c}\text { Sabpart i - Use and } \\
\text { Management of Comtadres } \\
\text { Inspections }\end{array}$ & $N$ & 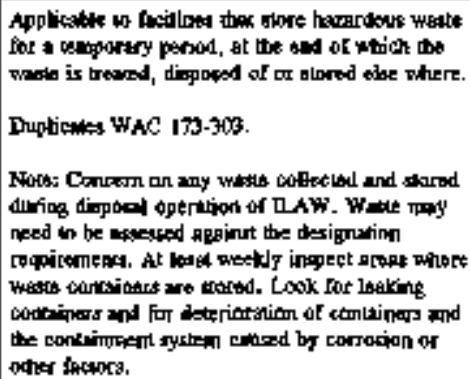 \\
\hline
\end{tabular}


Code of Federal Regulations

\begin{tabular}{|c|c|c|c|}
\hline Document fdentitier & Title & Applicable & Justification \\
\hline 40 CFR $264.175 \mathrm{c}$ & $\begin{array}{c}\text { Subpart I - Use and } \\
\text { Maakgement of Containers } \\
\text { Containnent }\end{array}$ & $\mathbf{N}$ & 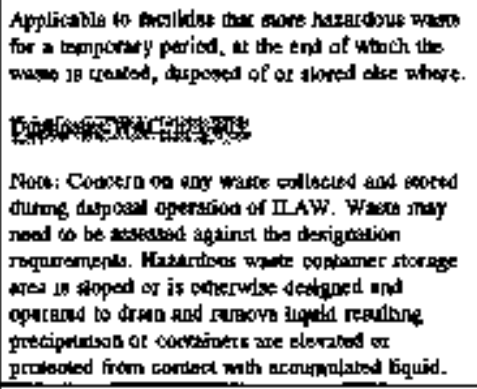 \\
\hline $40 \operatorname{CFR} 264.176$ & $\begin{array}{l}\text { Subpant } 7 \text { - Use and } \\
\text { Managentent of Containers } \\
\text { Speciad requiranents for } \\
\text { ignitable or reoctive waste }\end{array}$ & $\boldsymbol{N}$ & 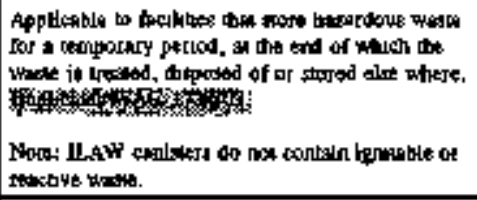 \\
\hline 40 CFR 264.177 a,c & $\begin{array}{l}\text { Subpart } 1 \text { - Use and } \\
\text { Management of Combainers } \\
\text { Spechal requirements fior } \\
\text { incompalibie waste }\end{array}$ & $\mathbf{N}$ & 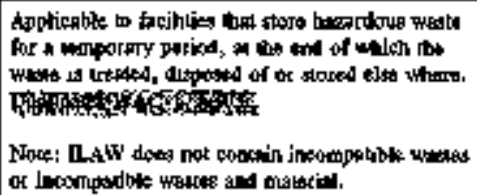 \\
\hline
\end{tabular}


Code of Federal Regulations

\begin{tabular}{|c|c|c|c|}
\hline Dxpuprent kentifier & Title & Applicable & Justifikntion \\
\hline $40 \mathrm{CFR} 264.178$ & $\begin{array}{c}\text { Subpart I - Use and } \\
\text { Mantexement of Containers } \\
\text { Closure }\end{array}$ & $\mathbf{Y}$ & 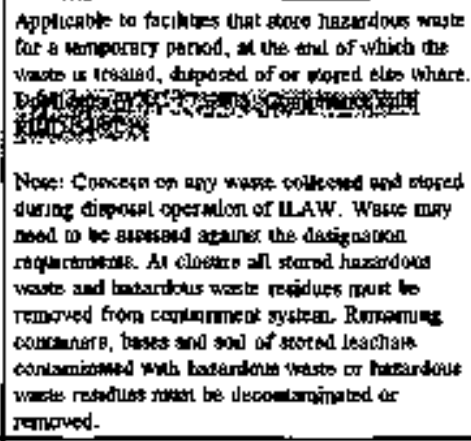 \\
\hline $40 \mathrm{CHR} 264,603$ & $\begin{array}{c}\text { Sobpart X - Misceldanecous } \\
\text { Uniss } \\
\text { Post closurc care }\end{array}$ & $\mathbf{N}$ & 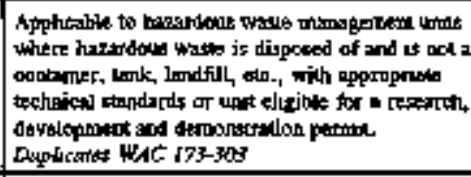 \\
\hline 40 CFR $264.1032 \mathrm{a}$ & $\begin{array}{c}\text { Subpart AA - Air Emistion } \\
\text { \$tapdardg } \\
\text { Process vents }\end{array}$ & $\mathbf{N}$ & 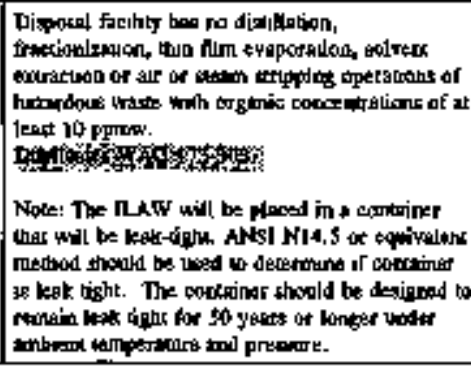 \\
\hline
\end{tabular}


Code of Foderal Regrulations

\begin{tabular}{|c|c|c|c|}
\hline Document ldentifier & Title & Applicable & Iustificalion \\
\hline 40 CFR 264. 1054a-c & $\begin{array}{c}\text { Subpart BB - Air Emlssion } \\
\text { Standards } \\
\text { Pressure telief } \\
\text { devices in gasivapor } \\
\text { service }\end{array}$ & $\mathbf{N}$ & 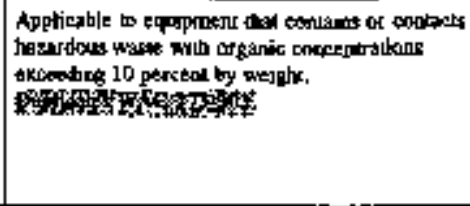 \\
\hline 40 CFR 264.1102 ab & $\begin{array}{l}\text { Subpart DD - Contabmeme } \\
\text { Buildingt } \\
\text { Closure and post closwrt } \\
\text { cart }\end{array}$ & $\mathbf{N}$ & 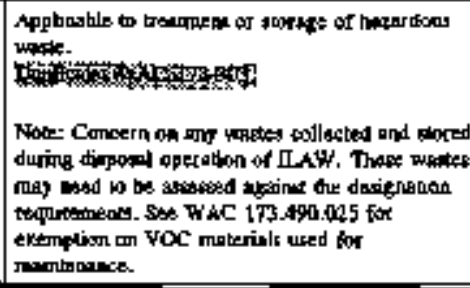 \\
\hline \multicolumn{4}{|c|}{ 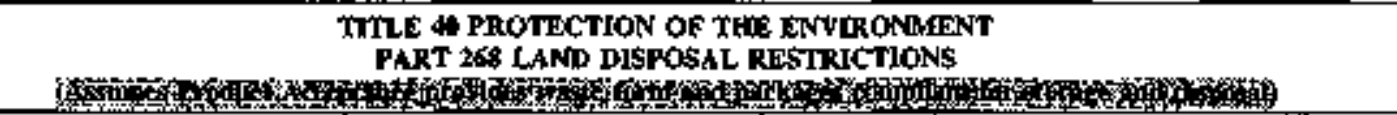 } \\
\hline 40 CPR 268.1 a,c & $\begin{array}{l}\text { Subpart A - General } \\
\text { Purpose, scope and } \\
\text { applicability }\end{array}$ & $\mathbf{N}$ & 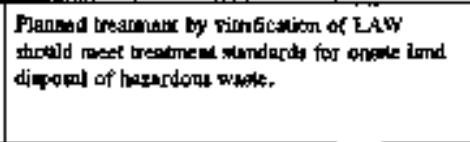 \\
\hline 40 CFR 268.50 a 2 & $\begin{array}{l}\text { Switpart E - Prohibitions on } \\
\text { storage } \\
\text { Prohibitions on storage of } \\
\text { restricted watate }\end{array}$ & $\mathbf{N}$ & 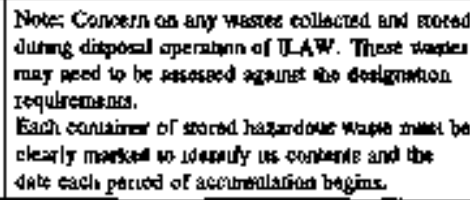 \\
\hline
\end{tabular}


Code of Federal Regnalations

\begin{tabular}{|c|c|c|c|}
\hline Doxument Idenlifier & Titk & Applicable & Justification \\
\hline 40 CFR 268.35. j & $\begin{array}{l}\text { Subpart C - Prohibirions on } \\
\text { Laud Disposgal } \\
\text { Waste specitic prohibitions } \\
\text { - Third waste }\end{array}$ & $\mathbf{N}$ & 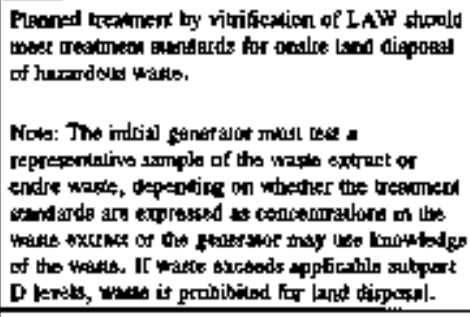 \\
\hline 40 CFR 2618.37 & 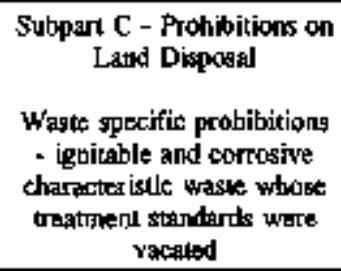 & $\mathbf{N}$ & 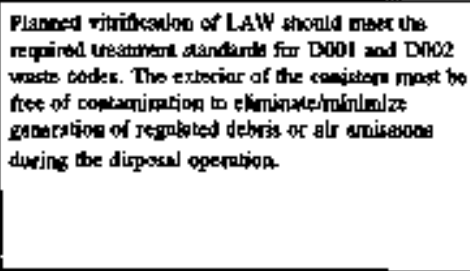 \\
\hline 40 CFR 268.40 & $\begin{array}{l}\text { Subpart D - Treatnent } \\
\text { Stamdards } \\
\text { Applicability of trealment } \\
\text { standards }\end{array}$ & $\mathbf{N}$ & 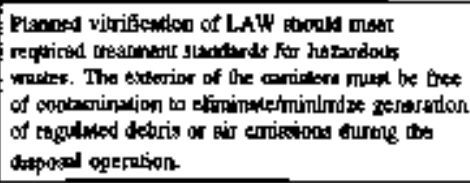 \\
\hline 40 CFR $268.41 \mathrm{atc}$ & $\begin{array}{l}\text { Subpart D - Treatment } \\
\text { Standards } \\
\text { Treatureat standards } \\
\text { expressed as concendration } \\
\text { in poaste extract }\end{array}$ & $N$ & 5 and 4 CFR 268.40 \\
\hline
\end{tabular}


Code of Federsil Regulalions

\begin{tabular}{|c|c|c|c|}
\hline Documenl Identifiter & Title & Apjlicab & Justification \\
\hline 40 CFR $263.42 \mathrm{a}$ & 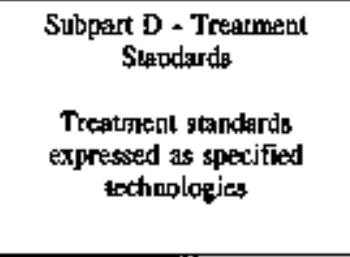 & $\mathbf{N}$ & 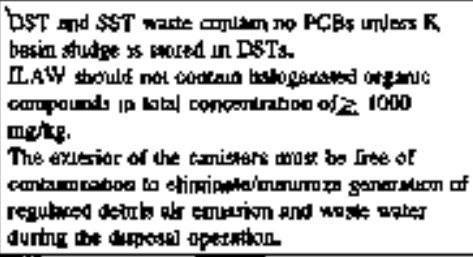 \\
\hline \multicolumn{4}{|c|}{ 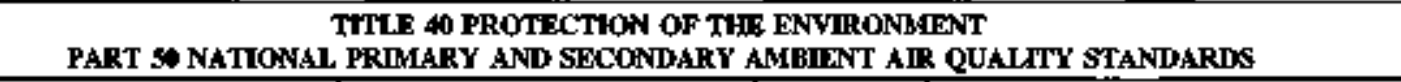 } \\
\hline 40 CFR 50.4 & $\begin{array}{l}\text { Sobpent C - Air Froprams } \\
\text { Marional primary ambient } \\
\text { air quality standards for } \\
\text { sulfur oxides } \\
\text { (sulfur dioxide) }\end{array}$ & 密 & 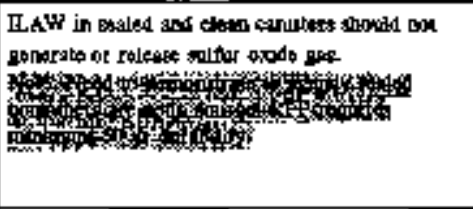 \\
\hline 40 CFR 50.5 & $\begin{array}{l}\text { Sobpart C - Air Proprams } \\
\text { National stecondary ambient } \\
\text { air quality staxdards for } \\
\text { sulfur oxides } \\
\text { (sulfur dioxide) }\end{array}$ & 空 & 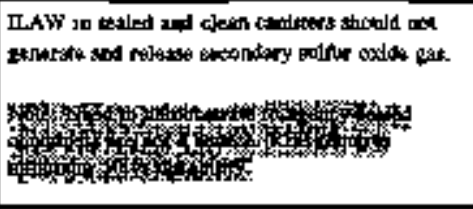 \\
\hline 40 CFR $\$ 0.6$ & $\begin{array}{l}\text { Subpart C - Air Protidams } \\
\text { National primary and second } \\
\text { ambient air quality } \\
\text { stamdards for particulate } \\
\text { matter }\end{array}$ & PX & 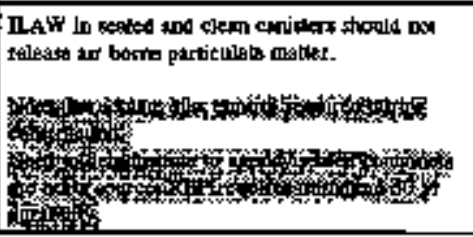 \\
\hline
\end{tabular}


Code of Federal Regulations

\begin{tabular}{|c|c|c|c|}
\hline Document Identifler & Title & Applisable & Justificertion \\
\hline 40 CPR 50.8 .9 & $\begin{array}{l}\text { Subpart C - Air Programs } \\
\text { National primary ambiedr } \\
\text { ait quality } \\
\text { otandards for } \\
\text { carbon moroxide }\end{array}$ & 蒾 & 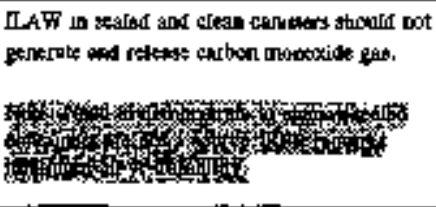 \\
\hline 40 CFR 50.9 & $\begin{array}{l}\text { Subpart C - Air Prograins } \\
\text { National prinary atd secoud } \\
\text { ambjeal gir quality } \\
\text { andandards for ozore }\end{array}$ & $\$$ & 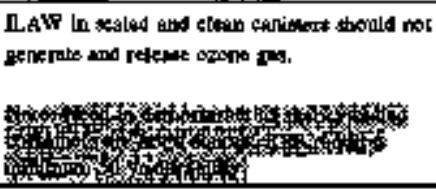 \\
\hline 40 CFR 50.11.ab & $\begin{array}{l}\text { Subpart C - Air Programs } \\
\text { National primary and } \\
\text { secondary ambient air } \\
\text { quality standard for } \\
\text { nitrogen dioxide }\end{array}$ & $\bar{z}$ & 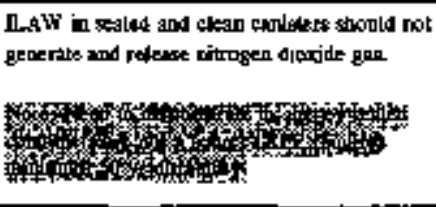 \\
\hline 40 CPR $\$ 0.12$ & $\begin{array}{l}\text { Subpart C - Air Programts } \\
\text { National primery and } \\
\text { gecondary ambient air } \\
\text { quality for lead }\end{array}$ & 愁 & 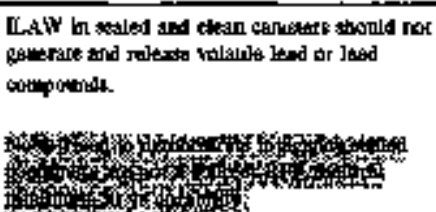 \\
\hline
\end{tabular}


Corstraints on Design of the ILAW Disposal Facility

Washinaton Adrainistrative Code

\begin{tabular}{|c|c|c|c|}
\hline Doçument Identifięr & Title & Applicable & Justificcation \\
\hline \multicolumn{4}{|c|}{$\begin{array}{l}\text { TYTLE } 173 \text { DEPARTMENT OF ECOLOGY } \\
\text { CHAPTER } 303 \text { DANGEROUS WASTE RECULATIONS }\end{array}$} \\
\hline WAC $173.303 .140,4,5$ & $\begin{array}{l}\text { Latd disposal restrictionts } \\
\text { Lathi disposal restrictions } \\
\text { ard prohibitions } \\
\text { Treatment in land disposd } \\
\text { facilities }\end{array}$ & $\begin{array}{l}\text { s } \\
y \\
y\end{array}$ & 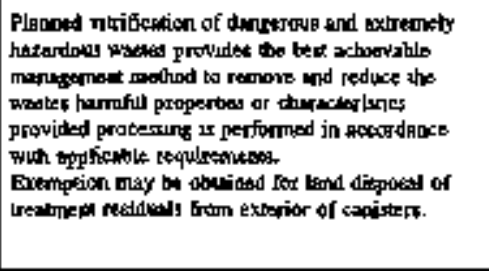 \\
\hline 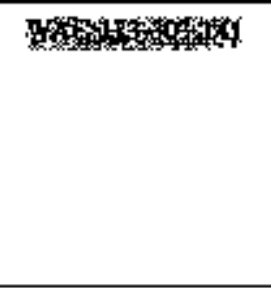 & 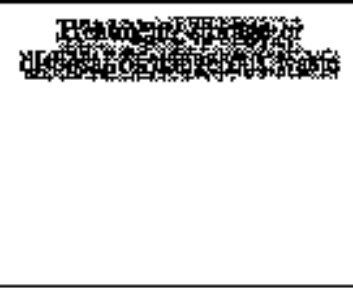 & 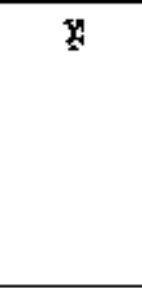 & 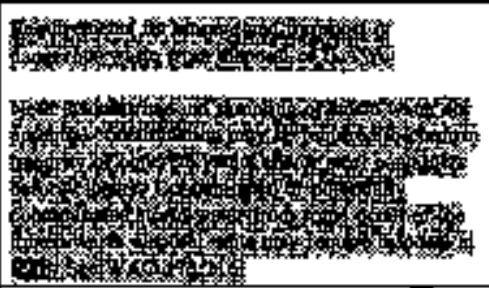 \\
\hline WAC 173.303 .145 .3 & $\begin{array}{l}\text { Spitts and discharges to } \\
\text { ite emowdrontent } \\
\text { Witigation and control }\end{array}$ & $\mathbf{Y}$ & 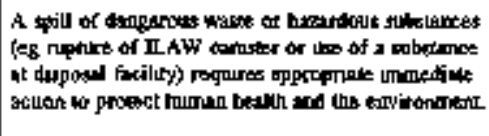 \\
\hline
\end{tabular}


HNF-SD-TWR-AGA-004

Revislon 0

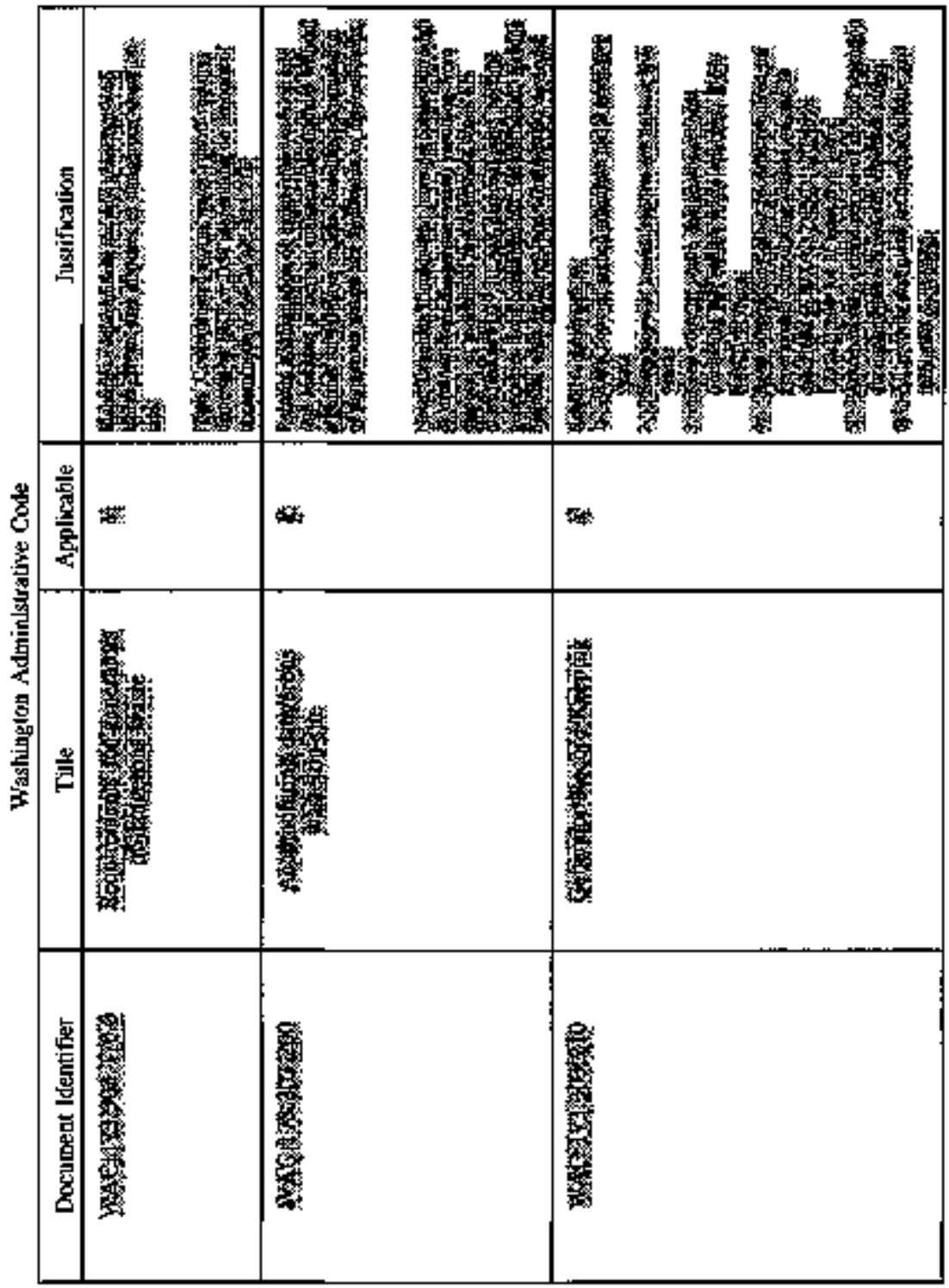


Washington Administrative Code

\begin{tabular}{|c|c|c|c|}
\hline Document ldentifier & Tikle & Applicable & Justificcation \\
\hline Bffer & 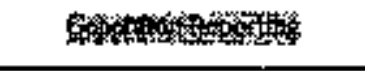 & 要 & 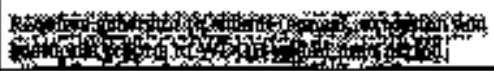 \\
\hline 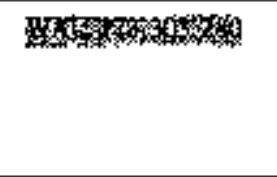 & 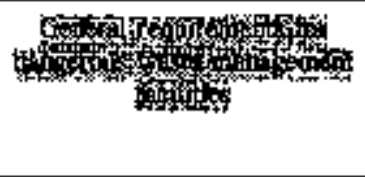 & 8 & 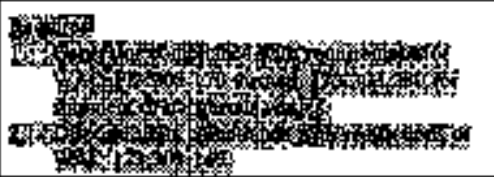 \\
\hline 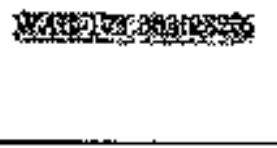 & 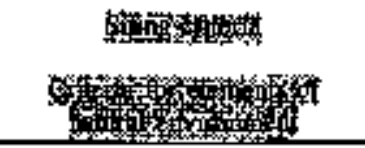 & t & 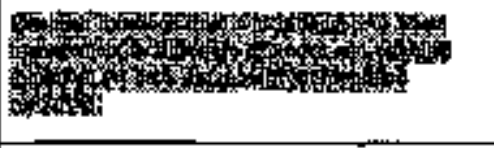 \\
\hline 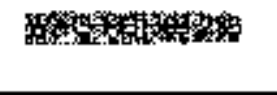 & 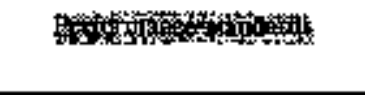 & $\forall$ & Sols \\
\hline WAC 173.309 .300 & General waste analysis & $\boldsymbol{y}$ & 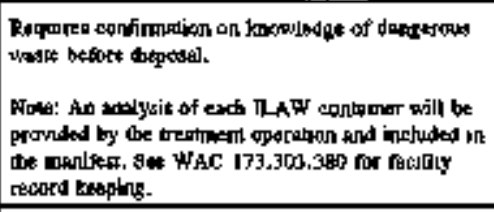 \\
\hline WAC $173.303 .310 .1,2$ & 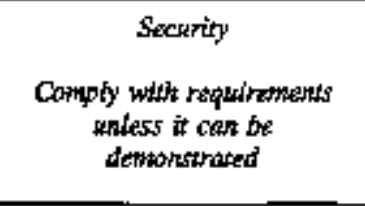 & $y$ & 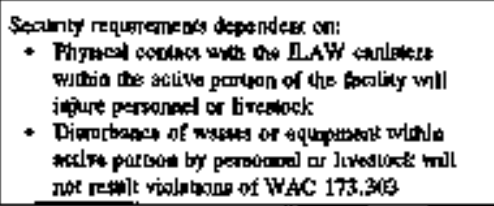 \\
\hline
\end{tabular}


Wasthingon Adminlapratre Code

\begin{tabular}{|c|c|c|c|}
\hline Docuntent Identifier & Titke & Applicable & Justification \\
\hline WAC 173.303 .310 .2 & $\begin{array}{c}\text { Securiny } \\
\text { Facility requirements }\end{array}$ & $Y$ & 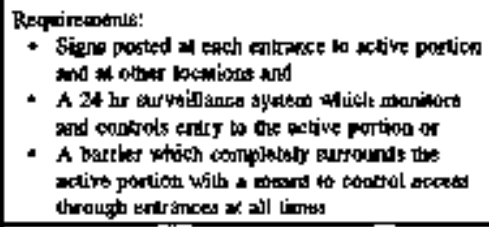 \\
\hline WAC 173.303 .320 & General trepectiont & $\boldsymbol{Y}$ & 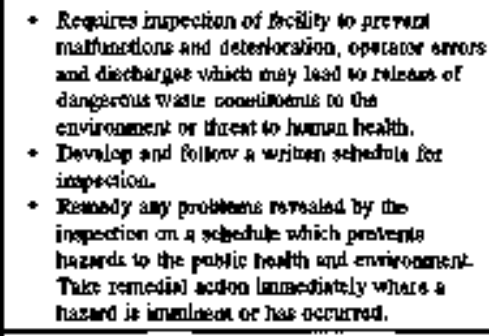 \\
\hline WAC 173.303 .330 & Personitel troinding & $\mathbf{Y}$ & 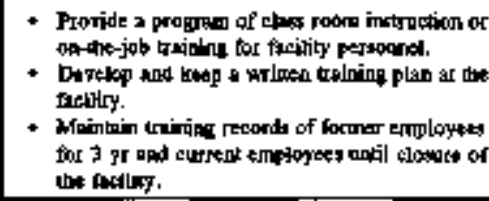 \\
\hline HAC 173.303 .340 & Preparedies and prevertion & $\mathbf{r}$ & 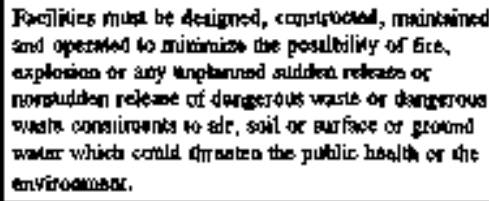 \\
\hline
\end{tabular}


Wasthingion Adminigrative Code

\begin{tabular}{|c|c|c|c|}
\hline Document Identlfies & Title & Applicable & Justification \\
\hline WAC 173.303 .340 .1 & $\begin{array}{l}\text { Preparedness and prewentiont } \\
\text { Required equiphtarl }\end{array}$ & $Y$ & 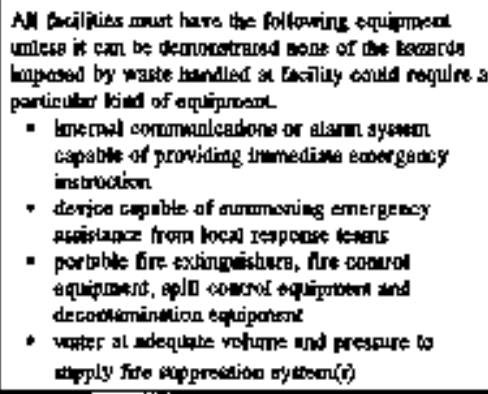 \\
\hline WAC 173.303 .340 .2 & $\begin{array}{c}\text { Preparethess and preverdion } \\
\text { Access to comintenications } \\
\text { or atams }\end{array}$ & $\boldsymbol{Y}$ & 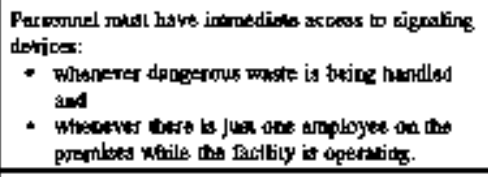 \\
\hline WAC 173.305 .340 .3 & $\begin{array}{c}\text { Preporedness and provention } \\
\text { Alsle space }\end{array}$ & $Y$ & 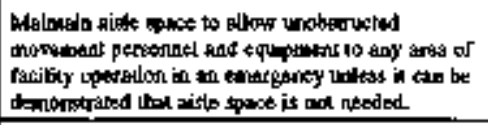 \\
\hline WAC 173.303 .380 & Facitiby record keeping & $\boldsymbol{Y}$ & 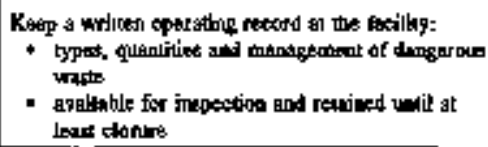 \\
\hline
\end{tabular}


Washingwn Administrative Code

\begin{tabular}{|c|c|c|c|}
\hline Document Identiter & Title & Applicable & Justification \\
\hline WAC 173.303 .390 & Facility reportisg & $\boldsymbol{Y}$ & 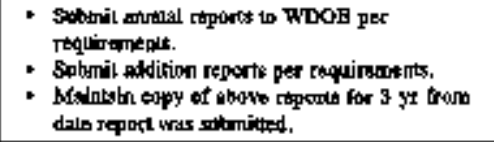 \\
\hline WAC 173.303 .395 & Other general reguirements & $y$ & 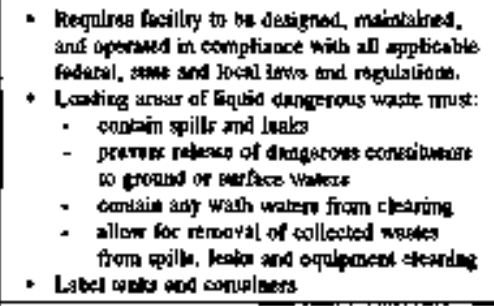 \\
\hline WAC 173.303 .610 .2 & $\begin{array}{c}\text { Closure and pest closure } \\
\text { Applicability }\end{array}$ & $y$ & 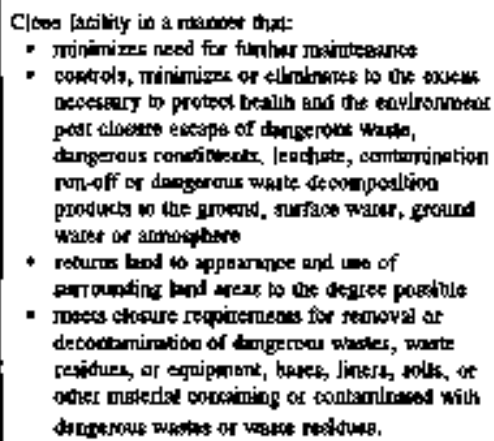 \\
\hline
\end{tabular}


Washingeon Adnunistrarive Code

\begin{tabular}{|c|c|c|c|}
\hline Doctumeat Identifier & Titre & Applicable & Josutitcation \\
\hline WAC $173,303.610 .4$ & $\begin{array}{l}\text { Closere and post closkre } \\
\text { Clasure: tine alloned for } \\
\text { closbre }\end{array}$ & $Y$ & 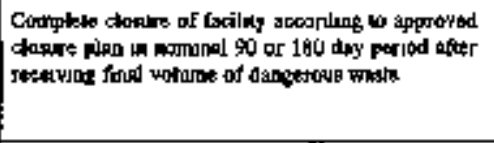 \\
\hline WAC I73.303.610.5 & 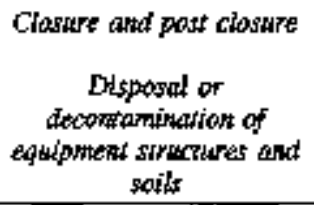 & $Y$ & 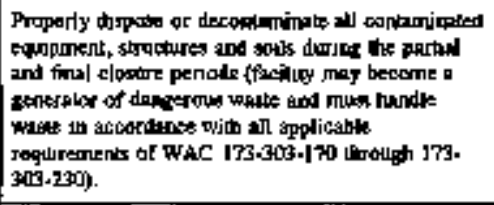 \\
\hline WAC 173.303 .610 .7 & $\begin{array}{l}\text { Closure and past closure } \\
\text { Post closure and use of } \\
\text { propetry }\end{array}$ & $Y$ & 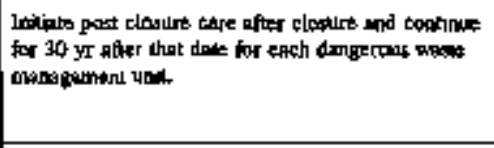 \\
\hline WAC 173.303.630 & $\begin{array}{c}\text { Uge and management } \\
\text { of conlaibers }\end{array}$ & $\mathbf{q}$ & 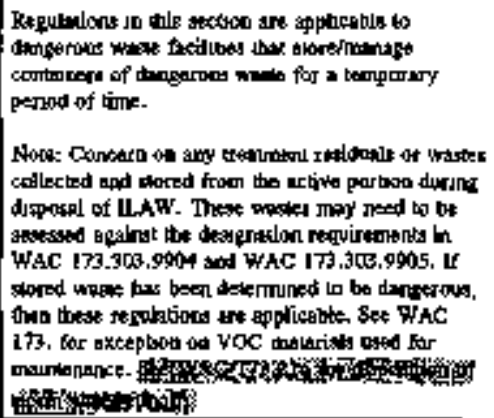 \\
\hline
\end{tabular}


Washington Administralive Code

\begin{tabular}{|c|c|c|c|}
\hline Documenl Identifier & Tide & Applicable & Jostification \\
\hline WAC I73.303.640 & Tank systens & 裙 & 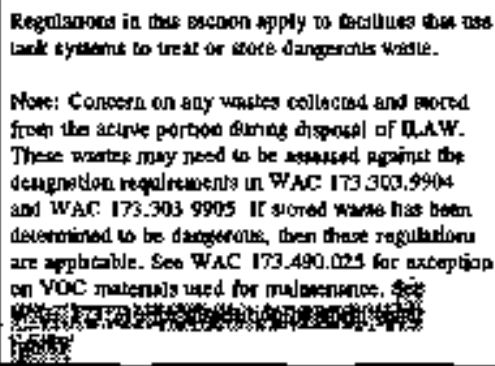 \\
\hline WAC 173.303 .645 & $\begin{array}{l}\text { Releases from regulaued } \\
\text { units }\end{array}$ & $Y$ & 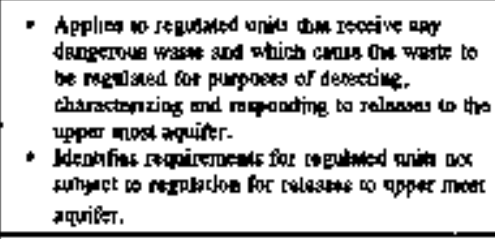 \\
\hline WAC $\{73.303 .646\}$. & $\begin{array}{l}\text { Corrective axtion } \\
\text { Purpose and applicublity }\end{array}$ & $Y$ & 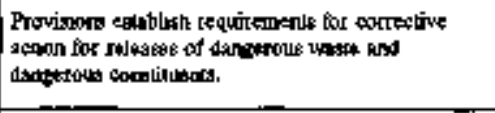 \\
\hline$W A C I 73.303 .645 .2$ & $\begin{array}{l}\text { Corrective ation } \\
\text { Requirenents }\end{array}$ & $\boldsymbol{Y}$ & 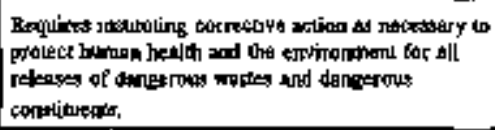 \\
\hline WAC 173.303 .665 .1 & $\begin{array}{l}\text { Landfills } \\
\text { Applicebility }\end{array}$ & $\mathbf{Y}$ & 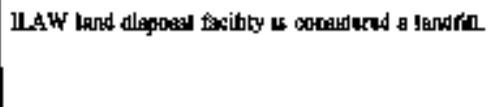 \\
\hline
\end{tabular}


Washington Administrative Code

\begin{tabular}{|c|c|c|c|}
\hline Documenu Identifier & Tiule & Applicable & Instification \\
\hline WAC $173.303 .665 .2 . \mathrm{a}, \mathrm{J}$ & $\begin{array}{l}\text { Landfills } \\
\text { Design and opereattro } \\
\text { condilions }\end{array}$ & $\mathbf{Y}$ & 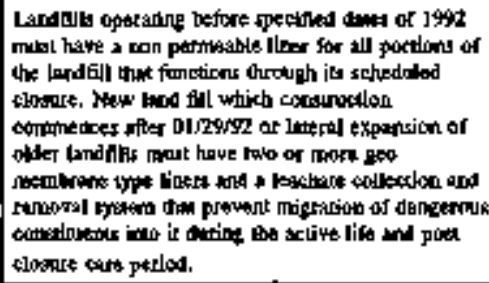 \\
\hline WAC 173.303.665.2.c & $\begin{array}{l}\text { Lardtills } \\
\text { Design and operatiog } \\
\text { conditions }\end{array}$ & $\mathbf{Y}$ & 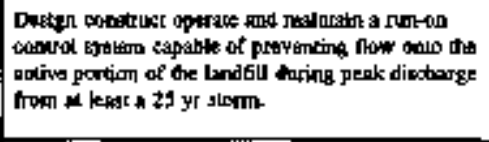 \\
\hline WAC 173.303.665.2.d & $\begin{array}{l}\text { Landfills } \\
\text { Design and operatiog } \\
\text { condlitions }\end{array}$ & $\mathbf{Y}$ & 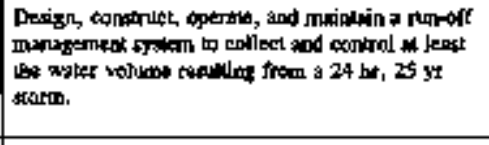 \\
\hline WAC $173.303 .665,4, a, b$ & $\begin{array}{l}\text { Landitls } \\
\text { Monthoring and inspection }\end{array}$ & $Y$ & 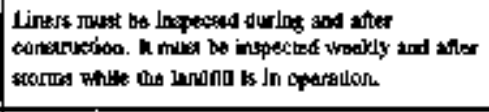 \\
\hline WAC 173.303 .665 .6 & $\begin{array}{l}\text { Landfills } \\
\text { Chosure and post closure }\end{array}$ & $\mathbf{Y}$ & 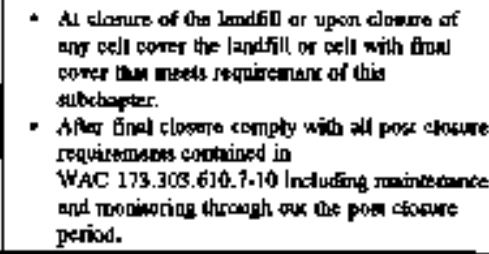 \\
\hline
\end{tabular}


Washington Administative Code

\begin{tabular}{|c|c|c|c|}
\hline Document Identifler & Tithe & Applicable & Justification \\
\hline WAC 173.305 .691 .1 & 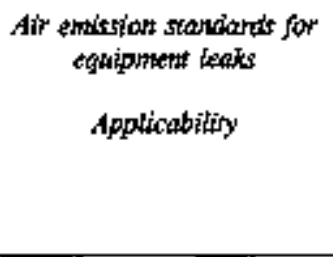 & $\boldsymbol{N}$ & 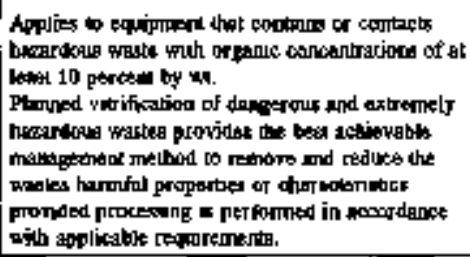 \\
\hline \multicolumn{4}{|c|}{ 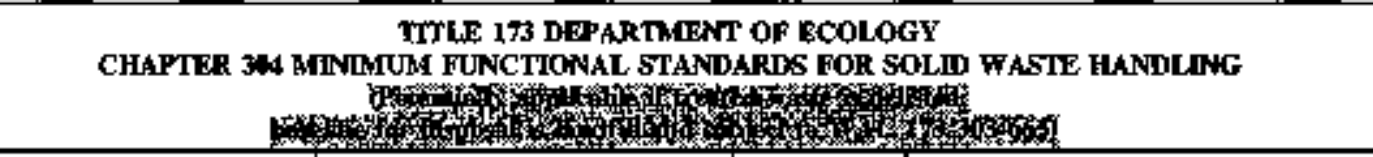 } \\
\hline WAC 173.304 .130 .1 & $\begin{array}{l}\text { Locational standards for } \\
\text { disposal stites } \\
\text { Applicability }\end{array}$ & 新 & 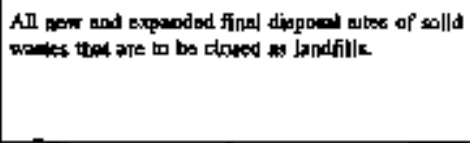 \\
\hline WAC $] 73,304,130.2$ & $\begin{array}{l}\text { Locational standaris for } \\
\text { ditpasal sites } \\
\text { Lacationat standards }\end{array}$ & 离 & 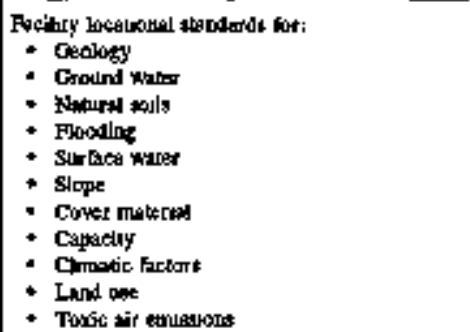 \\
\hline
\end{tabular}


Washington Adrainistrative Code

\begin{tabular}{|c|c|c|c|}
\hline Document Identifier & Title & Applicable & Justifination \\
\hline$W A C[73.304 .460]$. & $\begin{array}{l}\text { Laufielting standarats } \\
\text { Applicablity }\end{array}$ & 要 & 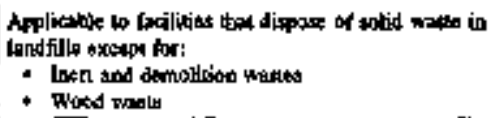 \\
\hline WAC 173.304 .460 .2 & $\begin{array}{l}\text { Landifiting standards } \\
\text { Miniman functionat } \\
\text { standands for perfomonte }\end{array}$ & 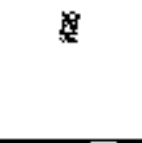 & 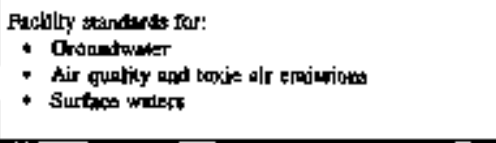 \\
\hline WAC I75.304.460.3 & $\begin{array}{l}\text { Lawditling stardards } \\
\text { Minimum fortctional } \\
\text { standards for design }\end{array}$ & $\underline{Y}$ & 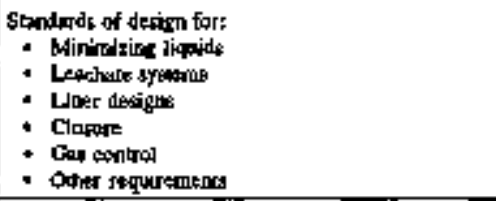 \\
\hline WAC $[73.304 .460 .4$ & 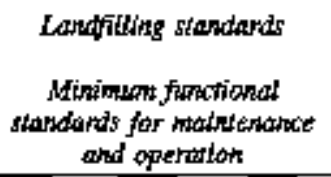 & W & 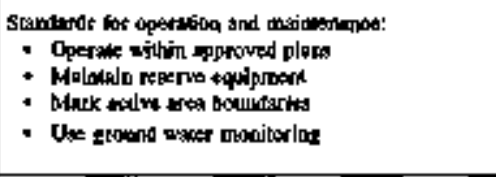 \\
\hline WAC 173.304 .490 .1 & $\begin{array}{l}\text { Groupd water motiforitg } \\
\text { requirements } \\
\text { Applicablety }\end{array}$ & $\mathrm{f}^{2}$ & 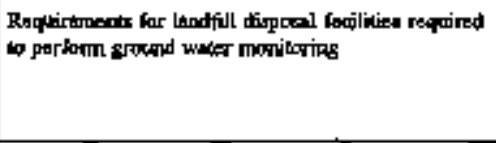 \\
\hline WAC 173.304 .490 .2 & $\begin{array}{l}\text { Growod water monitoritg } \\
\text { requirements } \\
\text { Growhd water montitoritg } \\
\text { reguirements }\end{array}$ & $\frac{4}{4}$ & 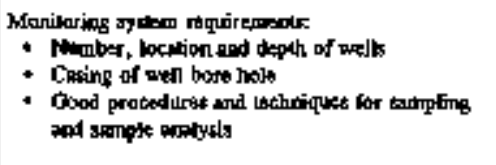 \\
\hline
\end{tabular}


Washington Administrastive Code

\begin{tabular}{|c|c|c|c|}
\hline Dopument Identifier & Tritk & Applicable & Justification \\
\hline \multicolumn{4}{|c|}{$\begin{array}{l}\text { TITLE } 173 \text { DEPARTMENT OF ECOLOGY } \\
\text { CHAPTER 46, CONTROLS FOR NEW SOURCES OP TOXIC AIR POLLITTANTS }\end{array}$} \\
\hline WAC 173.460 .030 .1 & $\begin{array}{l}\text { Requiremenus, } \\
\text { applianbility and } \\
\text { exemptions } \\
\text { Applicability }\end{array}$ & $\mathbf{Y}$ & 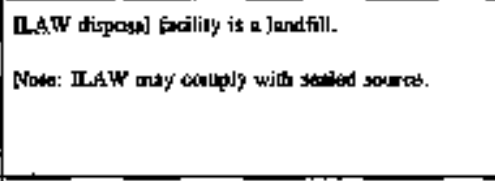 \\
\hline WAC 173.460 .030 .2 & $\begin{array}{l}\text { Requirements, } \\
\text { applicability and } \\
\text { exemptions } \\
\text { Exempl scarces }\end{array}$ & $\mathbf{Y}$ & 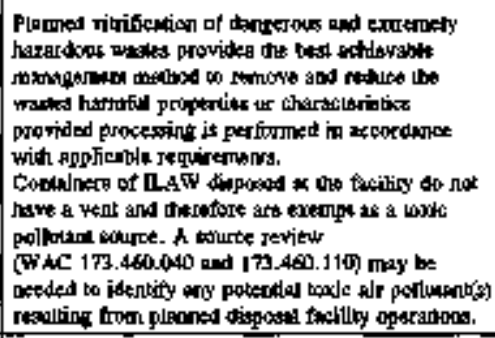 \\
\hline WAC I73.460.040.1 & $\begin{array}{l}\text { New scurce review } \\
\text { Applicability }\end{array}$ & $\mathbf{Y}$ & 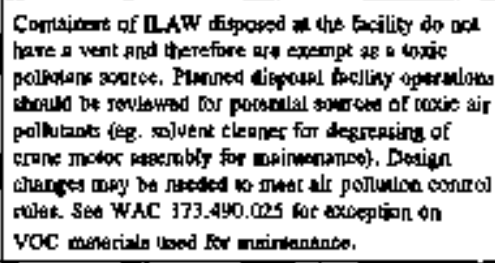 \\
\hline
\end{tabular}


Wastington Administrative Code

\begin{tabular}{|c|c|c|c|}
\hline Documend Identifier & Tites & Applicable & Justification \\
\hline WAC 173.460 .050 .2 & $\begin{array}{l}\text { Requirenents to quantify } \\
\text { conissions } \\
\text { Small quantily sonurtes }\end{array}$ & 政 & 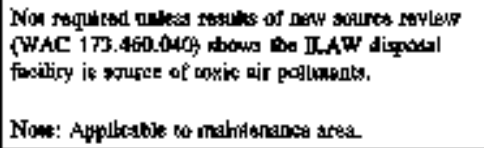 \\
\hline WAC 173.460 .060 .5 & $\begin{array}{l}\text { Control bchnology } \\
\text { requirements } \\
\text { Stchunt melal cleaners }\end{array}$ & 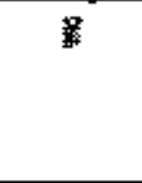 & 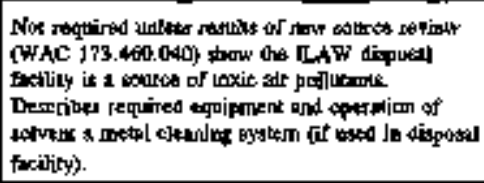 \\
\hline WAC 173.460 .070 & Anbien impact requirtanets & x & 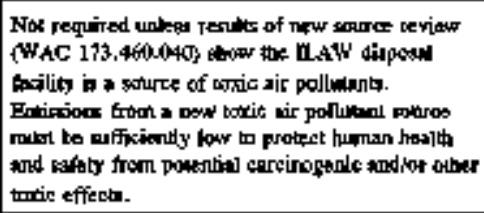 \\
\hline WAC 173.460.080.3 & $\begin{array}{l}\text { Demonsirating ainblent } \\
\text { inipact compliapce } \\
\text { Criteria for compliance }\end{array}$ & $\mathbf{N}$ & 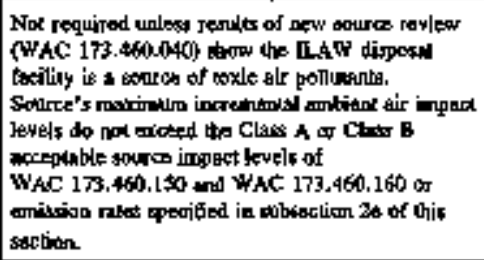 \\
\hline WAC 173.460 .090 & Second tier analysis & $\mathbf{N}$ & 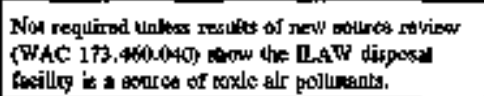 \\
\hline
\end{tabular}


Washington Administratiwe Code

\begin{tabular}{|c|c|c|c|}
\hline Dxxomens Jdentjfikr & Title & Applicable & Jnstification \\
\hline WAC 173.460.100 & $\begin{array}{l}\text { Request for rdst } \\
\text { management decision }\end{array}$ & $\mathbf{N}$ & 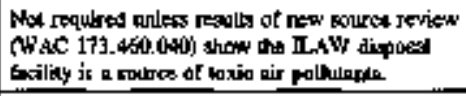 \\
\hline WAC 173.460.110 & $\begin{array}{c}\text { Acoppisble source impacts } \\
\text { levels }\end{array}$ & $\mathbf{N}$ & 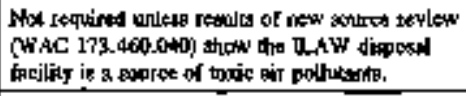 \\
\hline WAC $173,460.120$ & $\begin{array}{l}\text { Scientific revitw and } \\
\text { ameodment of acoeptable } \\
\text { sontece impact kevels and } \\
\text { Jists }\end{array}$ & $\mathbf{N}$ & 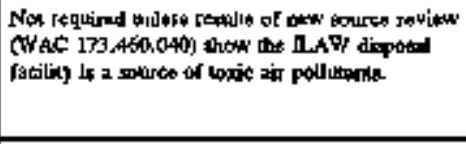 \\
\hline WAC 173.460.130 & Fees & $\mathbf{N}$ & 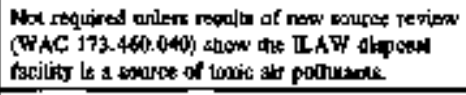 \\
\hline WAC 173.460 .140 & Reinedies & $\mathbf{N}$ & 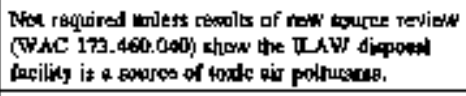 \\
\hline WAC 173.460 .150 .1 & 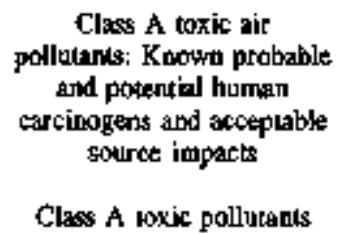 & $\mathbf{N}$ & 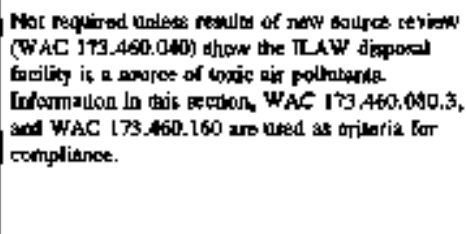 \\
\hline
\end{tabular}


Washington Admlnistralive Code

\begin{tabular}{|c|c|c|c|}
\hline Document Ideatifier & Title & Applisable & Justitieation \\
\hline WAC 173.460 .150 .2 & 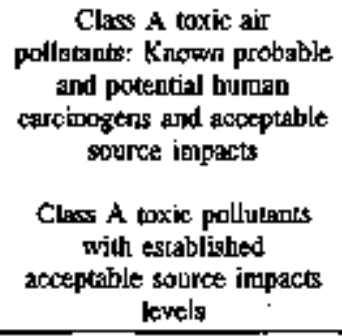 & $\mathrm{N}$ & 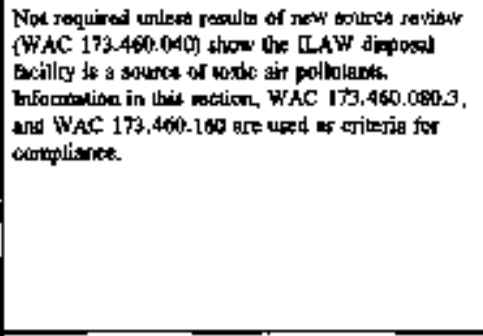 \\
\hline WAC 173.460 .150 .3 & $\begin{array}{c}\text { Class A toxic air } \\
\text { pollutants: Known probable } \\
\text { and potential haman } \\
\text { carcinogens and acceptable } \\
\text { source inipatts } \\
\text { Class A loxic polfutaats } \\
\text { with specíl } \\
\text { accoptable sowrce impacts } \\
\text { levels }\end{array}$ & $\mathbf{N}$ & 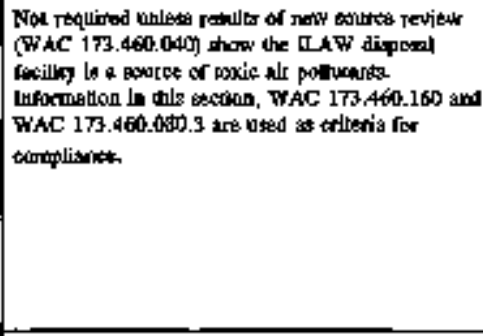 \\
\hline WAC 173.460 .160 & $\begin{array}{l}\text { Class B toxic air } \\
\text { pollutants and acceptable } \\
\text { impact levels }\end{array}$ & $\mathbf{N}$ & 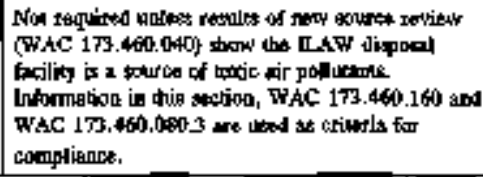 \\
\hline \multicolumn{4}{|c|}{$\begin{array}{l}\text { TITUE } 173 \text { DEPARTMENT OF ECOLOGY } \\
\text { QUALITY STANDARDS FOR CARBON MONOXIDE OZONE AND NITROGEN } \\
\text { DIOXWE }\end{array}$} \\
\hline
\end{tabular}


Waghingem Afmimistralive Code

\begin{tabular}{|c|c|c|c|}
\hline Document Identifler & Tille & Appiliesble & Justificalion \\
\hline $\mathrm{HAC} 173.475 .030,1,2,3$ & Atr qually standards & $\dot{\mathbf{x}}$ & 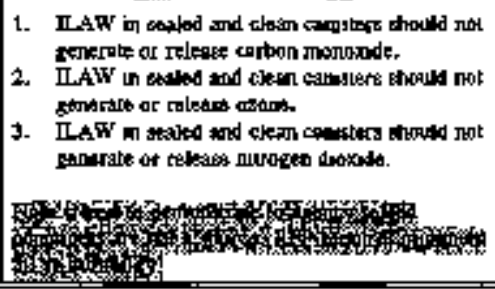 \\
\hline \multicolumn{4}{|c|}{$\begin{array}{l}\text { THTE } 173 \text { DEPARTMENT OF ECOLOOY } \\
\text { CHAPTER 4B0 AMEIENT AIR QUALIYY STANDARDS AND EMSSION LDITIS FOR RADHONUCLIDES }\end{array}$} \\
\hline WAC 173.480 .60 & $\begin{array}{l}\text { Emission randards for new } \\
\text { and modified emissiopt } \\
\text { units }\end{array}$ & $\mathrm{N}$ & 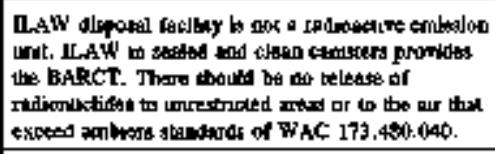 \\
\hline WAC IT3.490.70 & $\begin{array}{l}\text { Emission monitorind and } \\
\text { compllance procedures }\end{array}$ & $N$ & Erempered by WAC 246.300.020.2. \\
\hline
\end{tabular}


Washington Administratiye Code

\begin{tabular}{|c|c|c|c|}
\hline Drocumenı Identifier & Tritle & Applicable & Justification \\
\hline \multicolumn{4}{|c|}{$\begin{array}{l}\text { TITUE } 173 \text { DEPARTMENT OP ECOLOGY } \\
\text { CHAPIER } 490 \text { FMMSSON STANDARUS ANB CONIROLS FOR SOURCES EMITTING VOLATLE ORGANIC } \\
\text { COMPOUNDS (VOC) }\end{array}$} \\
\hline WAC I73.490.025.4 & $\begin{array}{l}\text { Getreral Applicabiaty } \\
\text { Exenpieal roc sourtes }\end{array}$ & $Y$ & 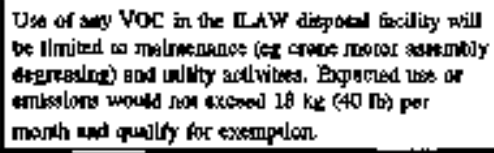 \\
\hline \multicolumn{4}{|c|}{$\begin{array}{l}\text { TTILE 2\$6 DPPARTMIENT OF BIEALTH } \\
\text { CEAPTER t20 RADLATION PROTECTION - GENERAL PROVISIONS }\end{array}$} \\
\hline WAC 246.220.007 & Staterixent of philosophy & $\mathbf{Y}$ & 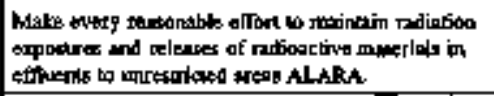 \\
\hline WAC 246.220.020. I & Records & $\mathbf{Y}$ & 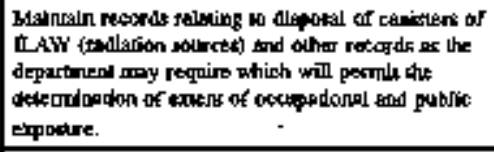 \\
\hline WAC 246.220 .030 .1 & Intpecition: & $Y$ & 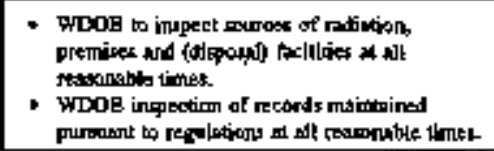 \\
\hline
\end{tabular}


Wastington Administranve Code

\begin{tabular}{|c|c|c|c|}
\hline Documem Idearifier & Trte & Applicable & Justification \\
\hline$W A C 246.220 .040 . I$ & Tets and Sureys & $\boldsymbol{Y}$ & 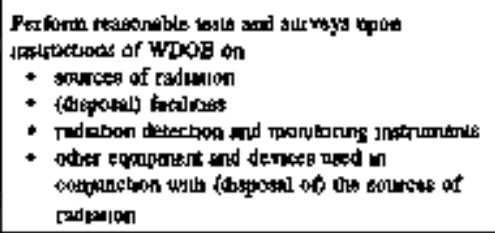 \\
\hline \multicolumn{4}{|c|}{$\begin{array}{l}\text { TITLE 246 DEPARTMENT OF HEALTH } \\
\text { CHAPTER 247 RADIATHON PROTECTION AIR EMTSSIONS }\end{array}$} \\
\hline WAC 246.247.001 & Porpose & $\mathbf{Y}$ & 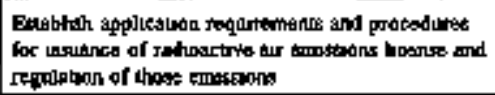 \\
\hline WAC 246.247 .002 & Authonty & $\mathbf{y}$ & 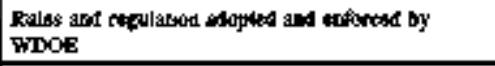 \\
\hline WAC $246.247 .010 .1,2$ & Applocabyluy & $\mathbf{Y}$ & 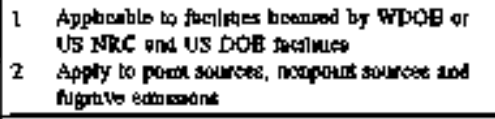 \\
\hline WAC $246,2 \mathrm{~A}\} .0201,1,2$ & Exemptons & $\mathbf{Y}$ & 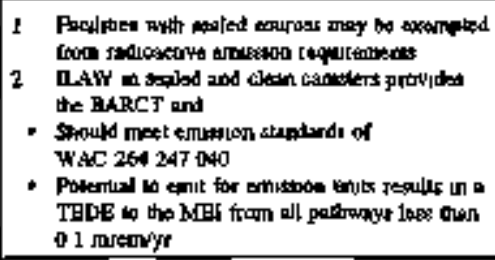 \\
\hline WAC 246,247.030 & Defiditrons & $\mathbf{Y}$ & Infoxmigton purpores onty \\
\hline
\end{tabular}


Washington Administrative Code

\begin{tabular}{|c|c|c|c|}
\hline Dhcumeent Identifięr & Title & Applicable & Jugtification \\
\hline WAC $246.247,040.1,3,6$ & Slendards & $\mathbf{Y}$ & 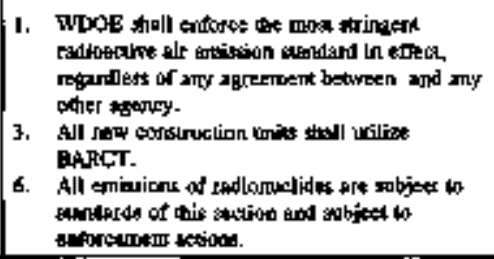 \\
\hline WAC 246.247 .60 .7 & $\begin{array}{l}\text { Applications, registration } \\
\text { and licensing }\end{array}$ & $\mathrm{Y}$ & 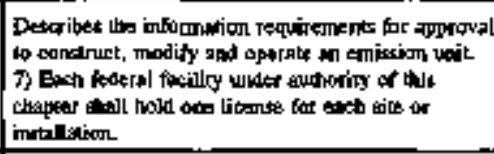 \\
\hline WAC 246.247.65.1 & Fens & $\mathrm{N}$ & 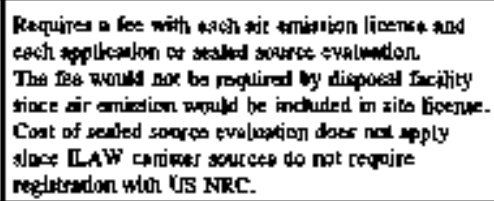 \\
\hline
\end{tabular}


Washingeon Adminidutrative Code

\begin{tabular}{|c|c|c|c|}
\hline Dopenment Identifies & Tille & Appliçable & Justificaliont \\
\hline WAC $246.247 .75 .3,4,6,7$ & $\begin{array}{l}\text { Monibring, testimg and } \\
\text { quality asemsanct }\end{array}$ & $\mathbf{Y}$ & 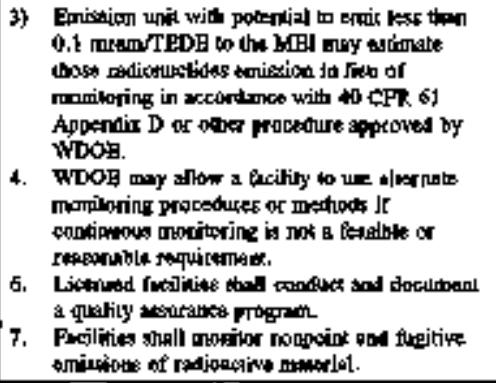 \\
\hline WAC 246.247. 80 & $\begin{array}{l}\text { Inspections, reporting and } \\
\text { recoitd keepint }\end{array}$ & $\mathrm{Y}$ & 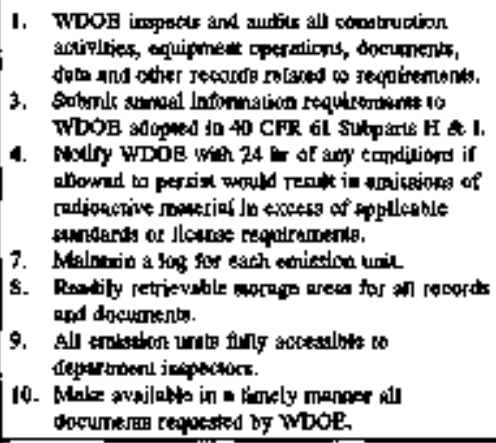 \\
\hline WAC 246.247.85 & $\begin{array}{l}\text { Compliance, determination } \\
\text { for existing emission } \\
\text { units and facilicies }\end{array}$ & 部 & 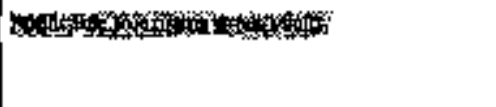 \\
\hline
\end{tabular}


Wastilugton Adminlstrative Code

\begin{tabular}{|c|c|c|c|}
\hline Docknmens Identifier & Title & Applicable & Justification \\
\hline WAC 246.247.100 & Enforcement acxions & $\mathbf{Y}$ & 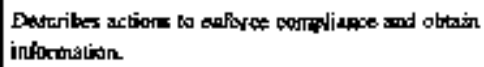 \\
\hline
\end{tabular}

点 
Waghington Administrative Code

\begin{tabular}{|c|c|c|c|}
\hline Dopcument Identifier & Tidte & Applicable & Justifization \\
\hline \multicolumn{4}{|c|}{$\begin{array}{l}\text { TTLE 24 DEPARTMENT OF HEALTH } \\
\text { CHAPTER 250 RADIQACTYVE WASTE - LICENSING LAND DISPOSAL }\end{array}$} \\
\hline WAC 246.250.00I & Puphose ard Scope & $N$ & 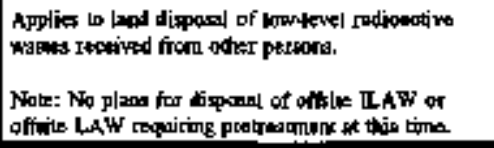 \\
\hline \multicolumn{4}{|c|}{$\begin{array}{l}\text { THLe } 296 \\
\text { DEPARTMENT OF LABOR AND INDUSTRIES }\end{array}$} \\
\hline WAC 296.24 & $\begin{array}{l}\text { General Siffey and Health } \\
\text { Steredards }\end{array}$ & $\boldsymbol{Y}$ & 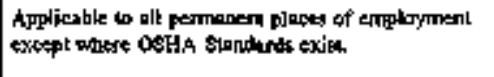 \\
\hline WAC 296.62 & 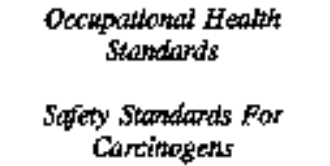 & $N$ & 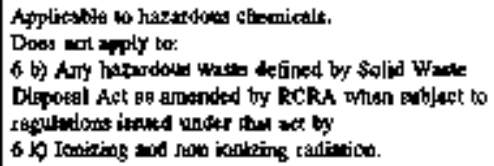 \\
\hline
\end{tabular}


Constraints on Detign of the ILAW Dispasal Facilly

U.S. Department of Entrigy

\begin{tabular}{|c|c|c|c|}
\hline Document Identifier & Title & Applicabje & Justification \\
\hline DOE-RL 93.08 & Hanford LSigsicon Plan & $\gamma^{y}$ & 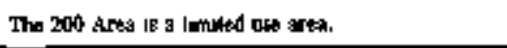 \\
\hline DOE 4330.4b & $\begin{array}{l}\text { Maintenand Management Plab. } \\
\text { Polcy }\end{array}$ & 圆 & 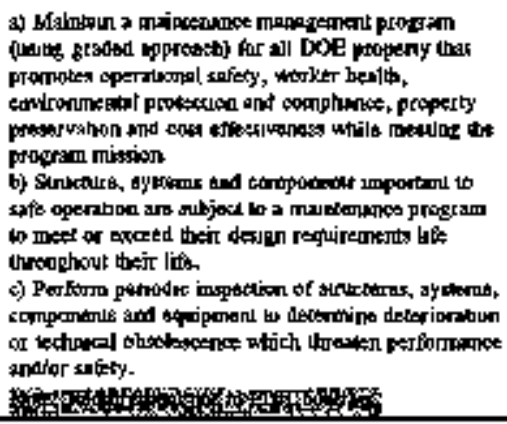 \\
\hline 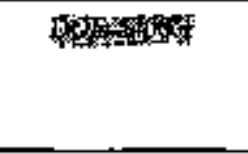 & 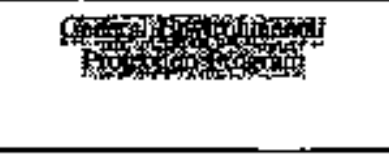 & S & 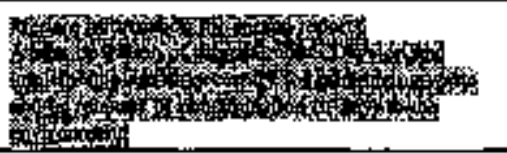 \\
\hline$D O E 500.5 .2 .4 . a$ & $\begin{array}{l}\text { Radiatian Prowection of the } \\
\text { Public and Envinomint } \\
\text { Public dose timit - all } \\
\text { exposture madrs }\end{array}$ & $\gamma$ & 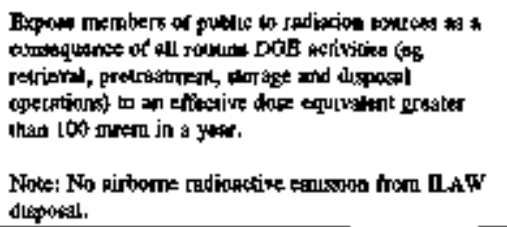 \\
\hline
\end{tabular}


U.S. Department of Energy

\begin{tabular}{|c|c|c|c|}
\hline Document Identifier & Tide & Appijtable & Justificalion \\
\hline DOE $\$ 400.5 .2 .1 .6$ & $\begin{array}{l}\text { Radiation Protectiont of the } \\
\text { Public and Envirowment } \\
\text { Aimbome entssions only . } \\
\text { all DOE sources }\end{array}$ & $\mathbf{x}$ & 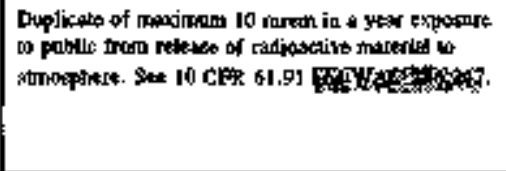 \\
\hline DOE $\$ 400.5 .2 .1 . d$ & $\begin{array}{l}\text { Radiation Protection of the } \\
\text { Pablic and Enyirounent } \\
\text { Drialcing Water Pathway - } \\
\text { all DOE sources }\end{array}$ & $\mathbf{Y}$ & 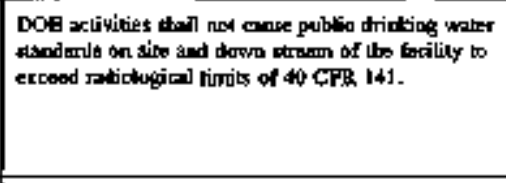 \\
\hline Dos $\$ 480.19$ & $\begin{array}{c}\text { Conduct of Operation for } \\
\text { DOE facilities }\end{array}$ & $\mathbf{Y}$ & 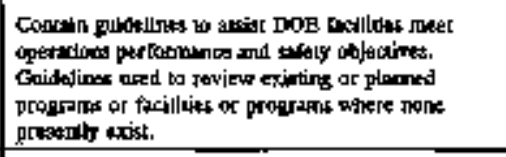 \\
\hline DOE 5480.20 .2 & $\begin{array}{l}\text { Personnel Selection, } \\
\text { Qualificaton and Traluing } \\
\text { for DOOE Nuclear Facilitiess }\end{array}$ & $\mathbf{Y}$ & 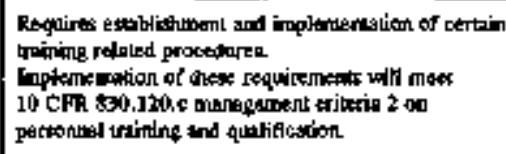 \\
\hline DOE 5480.21 & Unreviewad Safety Queation & $\mathbf{Y}$ & 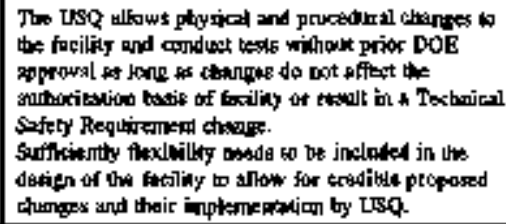 \\
\hline
\end{tabular}


U.S. Depariment of Energy

\begin{tabular}{|c|c|c|c|}
\hline Document lóentufier & Title & Applicable & Iustufication \\
\hline DOE 548022.9 .4 & $\begin{array}{c}\text { Technucal Safuy } \\
\text { Requrements }\end{array}$ & $\mathbf{Y}$ & 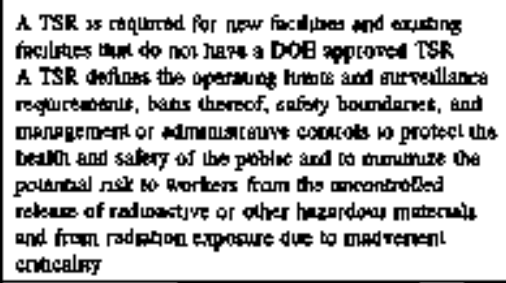 \\
\hline DOE 5480.23.8 & $\begin{array}{c}\text { Nuclear Safety Analysis } \\
\text { Repunt }\end{array}$ & $\mathbf{Y}$ & 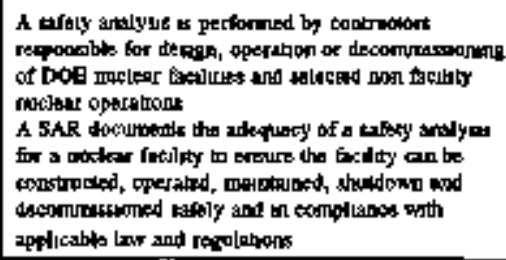 \\
\hline DOE 5480.24 .7 & Nuclear Crutkeality Safuly & $\mathbf{N}$ & 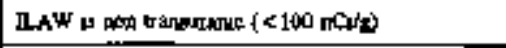 \\
\hline DOE 5480.28 & $\begin{array}{l}\text { Natural Phenoment } \\
\text { Hazards Milngalion }\end{array}$ & y & 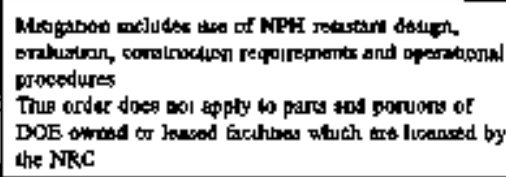 \\
\hline DOE 5480.4 & $\begin{array}{l}\text { Enviratimentig] Protection, } \\
\text { Safery and Health } \\
\text { Protection }\end{array}$ & $\mathbf{Y}$ & 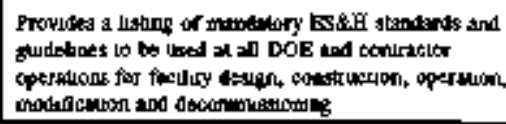 \\
\hline
\end{tabular}


U.S. Department of Energy

\begin{tabular}{|c|c|c|c|}
\hline Document Identifier & Titte & Applicable & Mustification \\
\hline DOE 5490.7A & Fire Protection & 粲 & 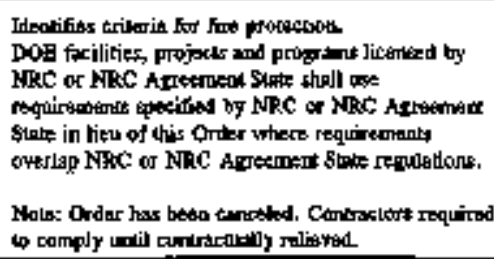 \\
\hline DOE 5483.1A & $\begin{array}{l}\text { Oecupational Safety and } \\
\text { Health Poogram for DOP } \\
\text { Contractor Enoplojess at } \\
\text { Government Ohwned Contractor } \\
\text { Operated Facilities }\end{array}$ & $\mathbf{X}$ & 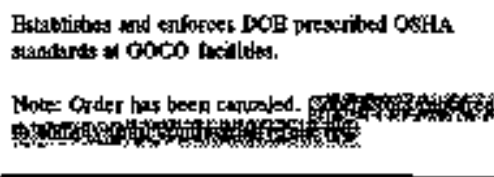 \\
\hline DOE $5700.6 \mathrm{C}$ & Quality Assurance & TBD & 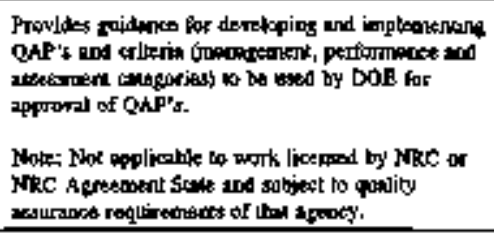 \\
\hline DOE $5820.2 A .3 .2 . \mathrm{C}$ & $\begin{array}{l}\text { Radoactwe Waste } \\
\text { Management } \\
\text { Management of Low Lovel } \\
\text { Waste } \\
\text { Policy }\end{array}$ & $\mathbf{Y}$ & 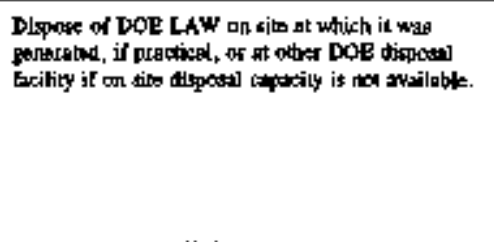 \\
\hline
\end{tabular}


U.S. Depariment of Energy

\begin{tabular}{|c|c|c|c|}
\hline Document Identifiter & Tille & Applicable & Instificalion \\
\hline DOE $5820.2 \mathrm{~A} .3 .3 . \mathrm{b} . \mathrm{t}$ & $\begin{array}{c}\text { Radioactive Waste } \\
\text { Mauagement } \\
\text { Management of Low Level } \\
\text { Waste } \\
\text { Requirements - Performance } \\
\text { agsessment }\end{array}$ & $\mathrm{Y}$ & 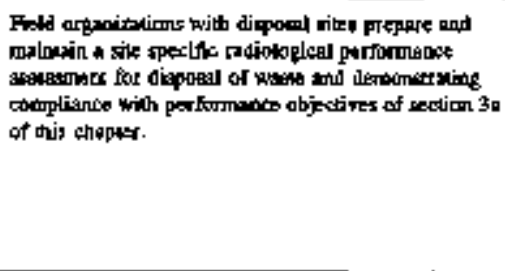 \\
\hline DOE $58201.2 A, 3.3 . b .2$ & $\begin{array}{c}\text { Radioaclive Waste } \\
\text { Management } \\
\text { Mannagement of Lon Level } \\
\text { Waste } \\
\text { Requirements - Perfocmance } \\
\text { assessment }\end{array}$ & $\mathbf{Y}$ & 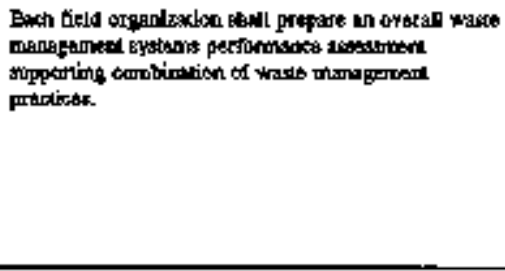 \\
\hline DOE 5820.2A.3.3.6.3 & $\begin{array}{l}\text { Radionctive Waste } \\
\text { Management } \\
\text { Management of Lonv Level } \\
\text { Waute } \\
\text { Requitemenus - Performanct } \\
\text { assessment }\end{array}$ & $\mathbf{Y}$ & 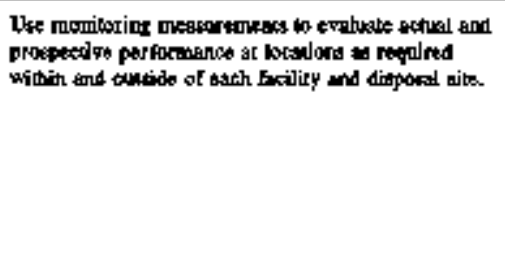 \\
\hline
\end{tabular}


Џ.S. Defarment of Energy

\begin{tabular}{|c|c|c|c|}
\hline Documeut Idendfiet & Tiule & Applicable & Justification \\
\hline DOE 5820.2A.3.3.4.4 & $\begin{array}{l}\text { Radioactke Wask } \\
\text { Mantagement } \\
\text { Managgement of Low Level } \\
\text { Wase } \\
\text { Requirements - Wogte } \\
\text { generation }\end{array}$ & 䇣 & 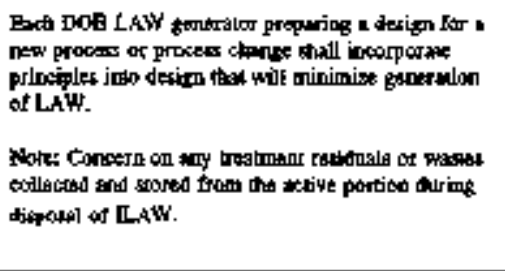 \\
\hline DOE $5820.2 A .3 .3 . \mathrm{E} 1$ & $\begin{array}{l}\text { Radiogative Waste } \\
\text { Management } \\
\text { Management of Low Level } \\
\text { Wask } \\
\text { Requdrements - Wase } \\
\text { acceptance criteria }\end{array}$ & $x$ & 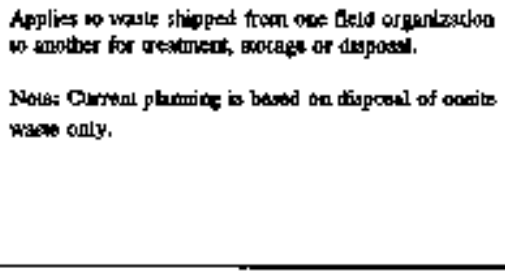 \\
\hline DOE 5820.24 .3 .3 .22 & $\begin{array}{l}\text { Redioactive Waste } \\
\text { Alanagement } \\
\text { Management of Low Level } \\
\text { Waste } \\
\text { Requintmemts - Wagte } \\
\text { acceptance crjerta }\end{array}$ & $Y$ & 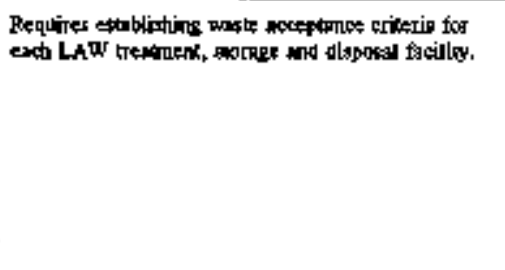 \\
\hline
\end{tabular}


U.\$. Bepartunent of Energy

\begin{tabular}{|c|c|c|c|}
\hline Decarment denuifier & Title & Applicable & Iustification \\
\hline DOE $5820.2 A, 3.3 . \mathrm{e} .3$ & $\begin{array}{l}\text { Radioactive Waste } \\
\text { Marasgemtent } \\
\text { Management of Low Lewel } \\
\text { Waste } \\
\text { Requlrements - Wagte } \\
\text { actepptance criteria }\end{array}$ & $\mathbf{Y}$ & 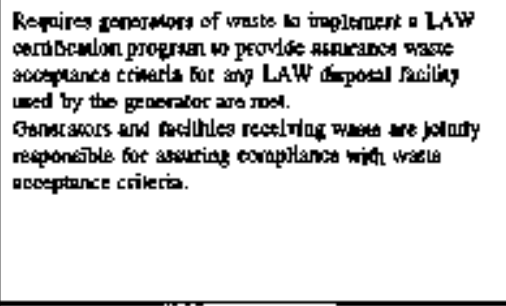 \\
\hline DOE S820.2A.3.3.e.5 & $\begin{array}{l}\text { Radionctive Wagte } \\
\text { Management } \\
\text { Management of Low Lasel } \\
\text { Waste } \\
\text { Reguirements - Wasce } \\
\text { acteptanct criteria }\end{array}$ & $\mathbf{Y}$ & 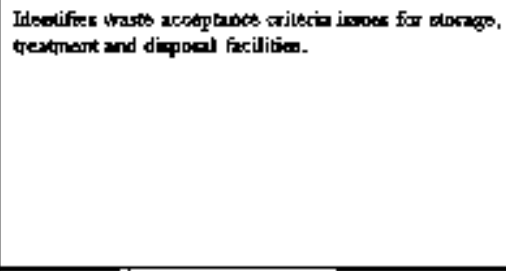 \\
\hline DOE 5820.2A, 3.3.h. J-4 & $\begin{array}{l}\text { Radiowctive Wask } \\
\text { Management } \\
\text { Managerient of Low Level } \\
\text { Waste } \\
\text { Requ[rements - Loug-term } \\
\text { stor was }\end{array}$ & $\mathbf{N}$ & 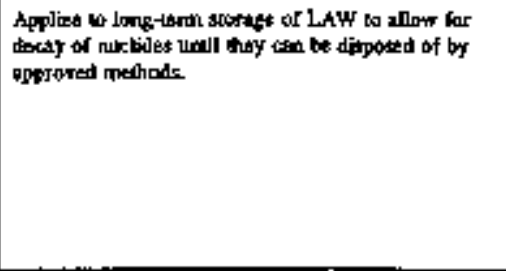 \\
\hline
\end{tabular}


U.S. Department of Energy

\begin{tabular}{|c|c|c|c|}
\hline Decordenl Identifier & Title & Applicsble & Justification \\
\hline DOE $\$ 820.2 A$.3.3.i.1 & $\begin{array}{c}\text { Radioscliwe Waste } \\
\text { Management } \\
\text { Management of Lor Level } \\
\text { Waste } \\
\text { Requirements - Disposal }\end{array}$ & $\mathbf{Y}$ & 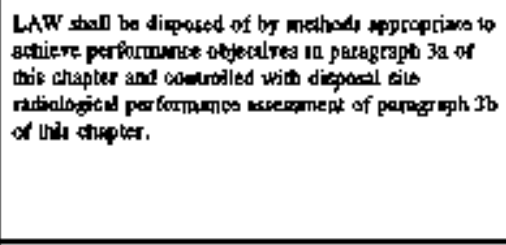 \\
\hline DOE $5920.2 \mathrm{~A} .3 .3 .12$ & $\begin{array}{c}\text { Radionacive Waste } \\
\text { Managememt } \\
\text { Management of Low Level } \\
\text { Waste } \\
\text { Requbements - Disposal }\end{array}$ & $\mathbf{Y}$ & 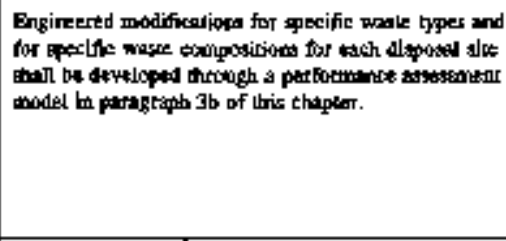 \\
\hline DOE 5820.2A.3.3.i.3 & $\begin{array}{l}\text { Radionctive Waste } \\
\text { Management } \\
\text { Management of Low Level } \\
\text { Waste } \\
\text { Requirementy - Disposal }\end{array}$ & $\mathrm{Y}$ & 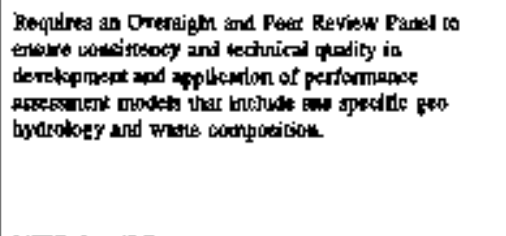 \\
\hline DOE $5820.2 A-3.3 . i .4$ & $\begin{array}{l}\text { Radifactive Waste } \\
\text { Manage } \\
\text { Managent } \\
\text { Waste } \\
\text { Requirements - Disposal }\end{array}$ & 莎 & 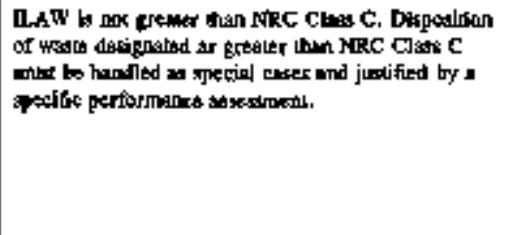 \\
\hline
\end{tabular}


U.S. Department of Energy

\begin{tabular}{|c|c|c|c|}
\hline Document Idethifiter & Title & Applicable & Justification \\
\hline DOE 5B20.2A.3.3.is & $\begin{array}{l}\text { Radionctive Waste } \\
\text { Mantagetuent } \\
\text { Managerment of Low Leval } \\
\text { Waste } \\
\text { Requirements - Disposal }\end{array}$ & $\mathrm{Y}$ & 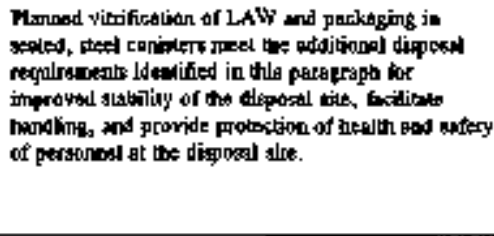 \\
\hline DOE SB20.2A.3.3.1.6 & $\begin{array}{l}\text { Radionctive Waste } \\
\text { Management } \\
\text { Managersent of Low Level } \\
\text { Waste } \\
\text { Requirements - Disposal }\end{array}$ & $\mathbf{N}$ & 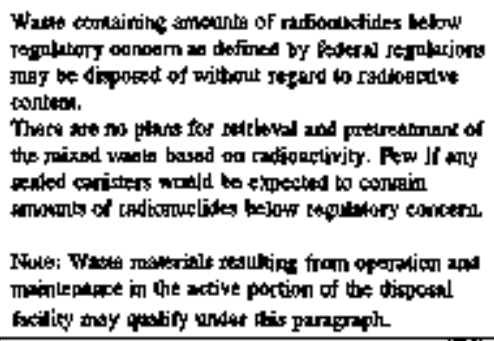 \\
\hline DOE 5B20.2A.3.3.j.? & $\begin{array}{l}\text { Radioactive Wasle } \\
\text { Mabtgemeul } \\
\text { Managernent of Low Level } \\
\text { Wabte } \\
\text { Requiremeats - Dispotsal }\end{array}$ & $\mathbf{Y}$ & 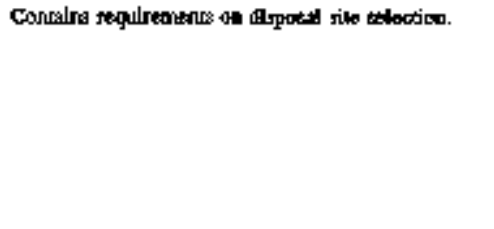 \\
\hline
\end{tabular}


U.S. Department of Energy

\begin{tabular}{|c|c|c|c|}
\hline Docoment Identifer & Titelc & applicable & Jastification \\
\hline DOE 3820.2 A.3.3.1.8 & $\begin{array}{l}\text { Radioxaclye Woste } \\
\text { Managernent } \\
\text { Management of Low Losel } \\
\text { Waste } \\
\text { Requirements - Dispogal }\end{array}$ & $\mathbf{Y}$ & 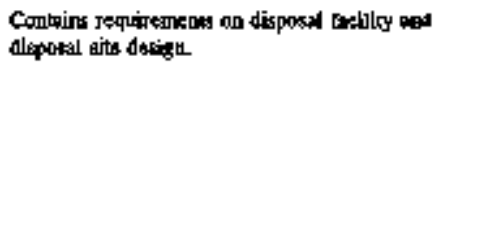 \\
\hline DOE $\$ 920.2 \mathrm{~A} .3 .3 .1 .9 . \mathrm{b}$ & 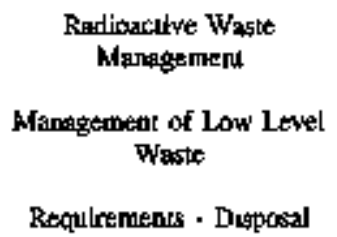 & $\mathbf{Y}$ & 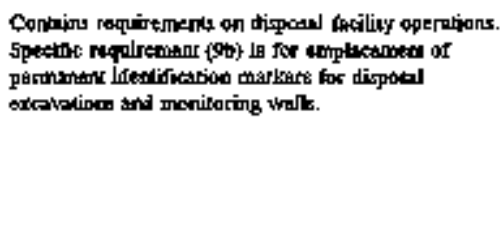 \\
\hline DOE $5820.2 A-3.3-j .1$ & 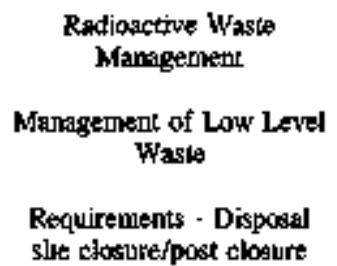 & $\mathbf{Y}$ & 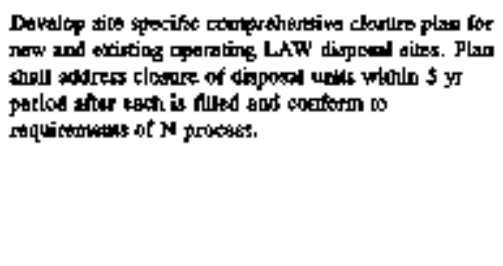 \\
\hline
\end{tabular}


U.S. Deparimint of Enercy

\begin{tabular}{|c|c|c|c|}
\hline Document Idestiflex & Tiule & Applicable & Jusuificattors \\
\hline DOE $5820.2 \wedge .3 .3 . j .2$ & $\begin{array}{c}\text { Radionctive Waste } \\
\text { Matagement } \\
\text { Managemedt of Low Level } \\
\text { Waste } \\
\text { Requitements - Disposal } \\
\text { sice cloeme/post closure }\end{array}$ & $\mathrm{Y}$ & 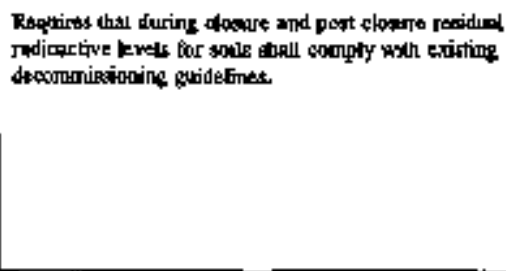 \\
\hline DOE $5820.2 \mathrm{~A} .3 .3 . \mathrm{J} \cdot 3$ & $\begin{array}{l}\text { Radioactue Waste } \\
\text { Maragement } \\
\text { Marapement of Low Level } \\
\text { Waste } \\
\text { Requiremeuts - Disposal } \\
\text { sire clogure/post closure }\end{array}$ & $Y$ & 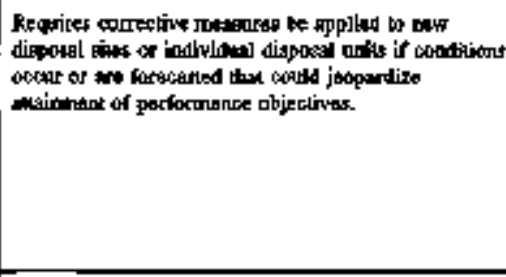 \\
\hline DOE 5820.2A.3.3.j.4 & 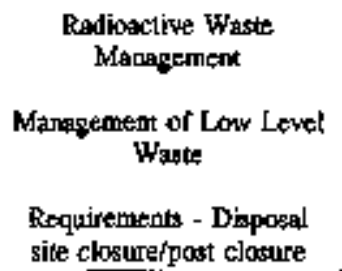 & 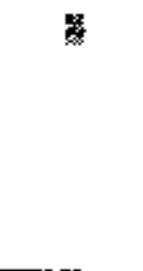 & 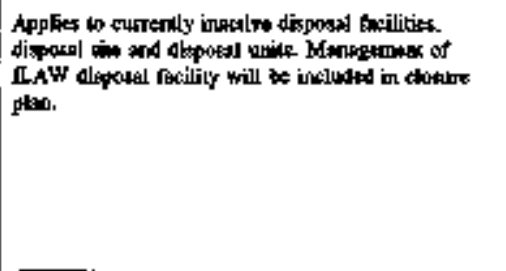 \\
\hline
\end{tabular}


V.S. Depariment of Energy

\begin{tabular}{|c|c|c|c|}
\hline Document Identifier & Title & Applicable & Jostification \\
\hline DOE $5820.2 A, 3.3, j .5$ & 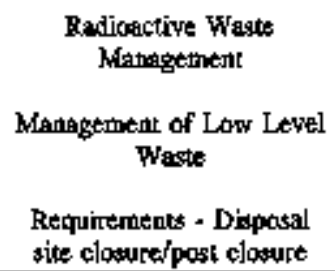 & $\mathbf{Y}$ & 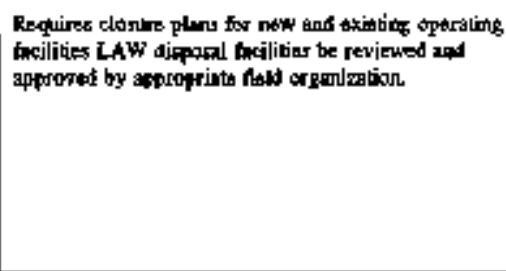 \\
\hline DOE $5820.24 .3 .3 . j .6$ & $\begin{array}{c}\text { Radbactive Waste } \\
\text { Management } \\
\text { Management of Low Level } \\
\text { Waste } \\
\text { Requirentents - Dlsposal } \\
\text { site closurefpost closure }\end{array}$ & $\mathrm{Y}$ & 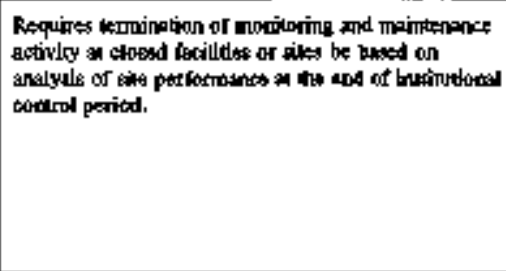 \\
\hline DOE 5820.2A.3.3.k I & $\begin{array}{c}\text { Radioactive Waste } \\
\text { Managemeal } \\
\text { Managemeul of Low Leyel } \\
\text { Waste } \\
\text { Requirtmenus - } \\
\text { Encikonmental monitoring }\end{array}$ & $\mathrm{Y}$ & 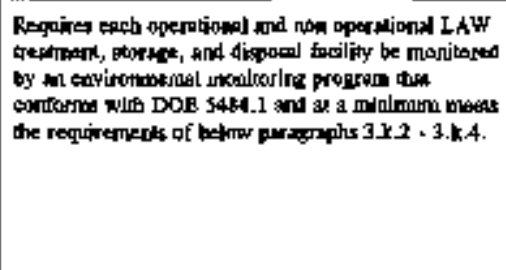 \\
\hline
\end{tabular}


U.S. Depariment of Energy

\begin{tabular}{|c|c|c|c|}
\hline Doctument Identifies & Title & Applicable & Justification \\
\hline DOE 5 $20.2 \mathrm{~A} \cdot 3.3 . \mathrm{k} 2$ & 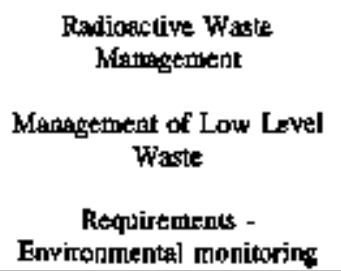 & $\mathrm{Y}$ & 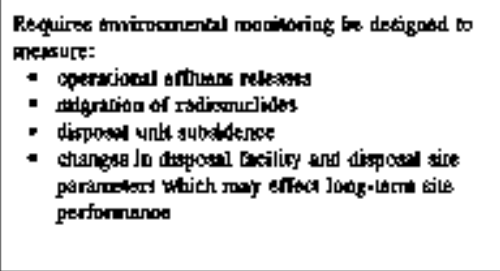 \\
\hline DOE $5820.24 .3 .3 . \mathrm{k} .3$ & $\begin{array}{c}\text { Radloaculve Woste } \\
\text { Maragement } \\
\text { Monagement of Low Level } \\
\text { Waste } \\
\text { Requirements - } \\
\text { Environmental monitoring }\end{array}$ & $\mathbf{Y}$ & 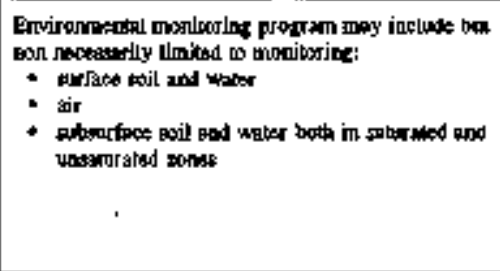 \\
\hline DOE $5820.2 A .3 .3 .5 .4$ & $\begin{array}{c}\text { Radioactive Waste } \\
\text { Marragement } \\
\text { Management of low Level } \\
\text { Waste } \\
\text { Requirtments - } \\
\text { Enfiroumental monitoring }\end{array}$ & $\mathbf{Y}$ & 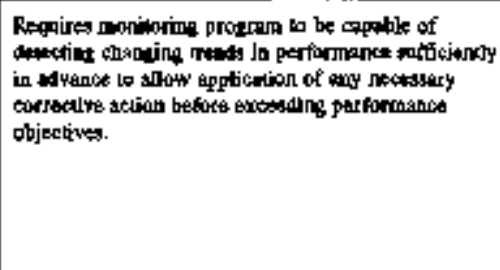 \\
\hline
\end{tabular}


U.S. Deparument of Enerey

\begin{tabular}{|c|c|c|c|}
\hline Drocurbent Identifier & Title & Applicable & Justification \\
\hline DOE \$820.2A.3.3.m.l & $\begin{array}{c}\text { Radiaticlive Waste } \\
\text { Managentent } \\
\text { Management of Low Leyel } \\
\text { Waste } \\
\text { Requirements - } \\
\text { Recouds and stports }\end{array}$ & $\gamma$ & 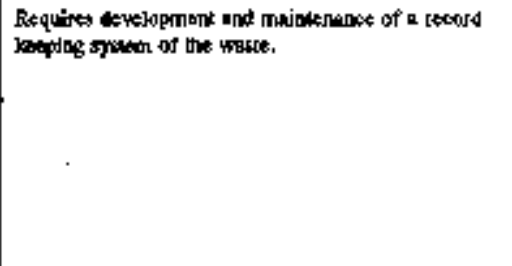 \\
\hline DOE 5820.2A.3.3.m.2 & $\begin{array}{l}\text { Radikasctive Wasto } \\
\text { Management } \\
\text { Management of Low Level } \\
\text { Waste } \\
\text { Requirements - } \\
\text { Reconds and reports }\end{array}$ & $\mathbf{Y}$ & 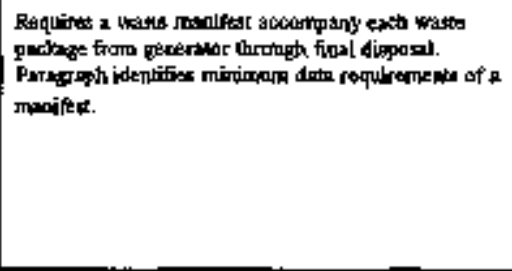 \\
\hline DOE 5820.24 .5 .3 .9 .2 & $\begin{array}{l}\text { Radionctive Waste } \\
\text { Managentent } \\
\text { Decommissioning of } \\
\text { Rediosctively Contaminated } \\
\text { Focslities } \\
\text { Requitements - Genteral }\end{array}$ & $Y$ & 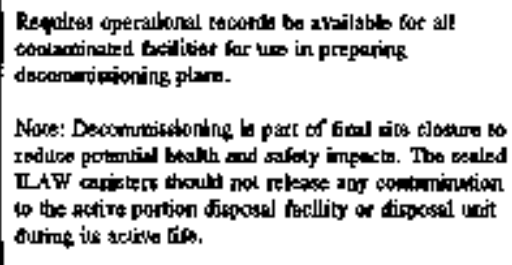 \\
\hline
\end{tabular}


U.S. Department of Exerey

\begin{tabular}{|c|c|c|c|}
\hline Document Identifier & Tite & Applicable & Justification \\
\hline DOE 5820.2A.5.3. a.5 & $\begin{array}{l}\text { Radiosctive Waste } \\
\text { Management } \\
\text { Decommissioning of } \\
\text { Radioactively Consamıated } \\
\text { Farilities } \\
\text { Requirements - General }\end{array}$ & 弯 & 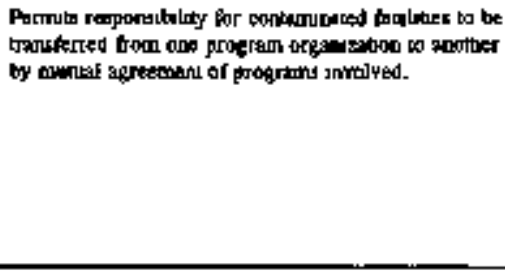 \\
\hline DOE $\$ 820,2 A .5 .3 .0$ & 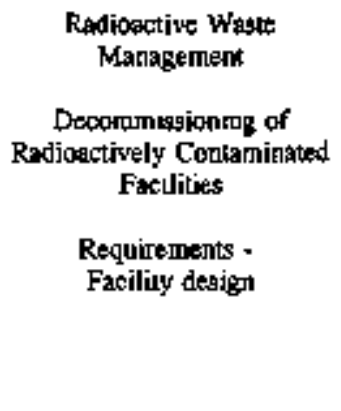 & $Y$ & 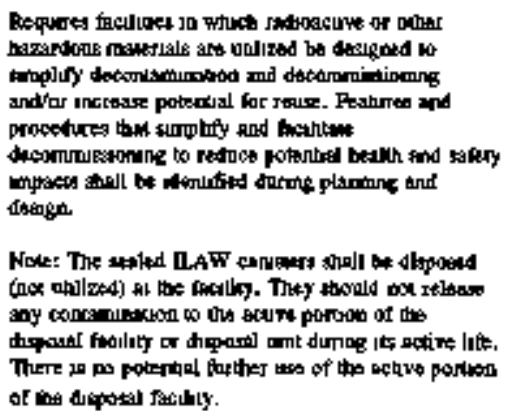 \\
\hline
\end{tabular}


U.S. Departuxeal of Energy

\begin{tabular}{|c|c|c|c|}
\hline Document Identifier & Title & Applictable & Iustiffeation \\
\hline DOE $5820.24 .5 .3 . c$ & $\begin{array}{c}\text { Radiosctive Waste } \\
\text { Mantagtmed } \\
\text { Decommitssioning of } \\
\text { Redioactively Contaminated } \\
\text { Facilltes } \\
\text { Requirements - } \\
\text { Post operational actirilies }\end{array}$ & $\mathbf{Y}$ & 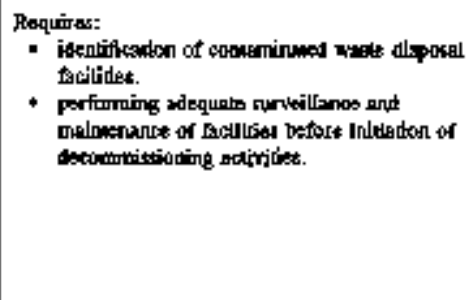 \\
\hline
\end{tabular}

总 
Congtraints on Desigy of the ILAW Disposal Pacility

\begin{tabular}{|c|c|c|c|}
\hline \multicolumn{4}{|c|}{ Miscellaneous } \\
\hline Document Identifier & Titke & Applicable & Justification \\
\hline 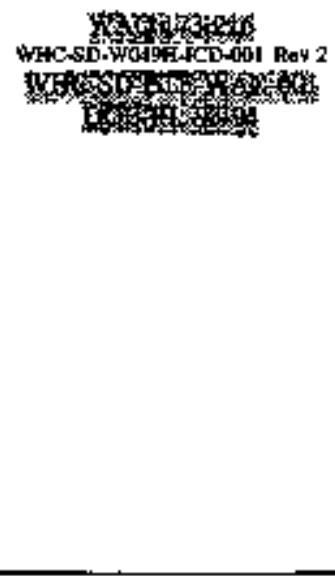 & 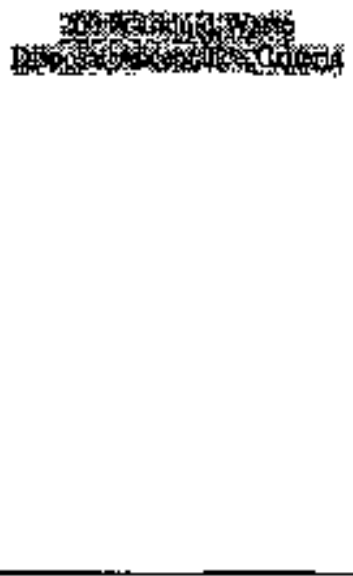 & $\mathbf{Y}$ & 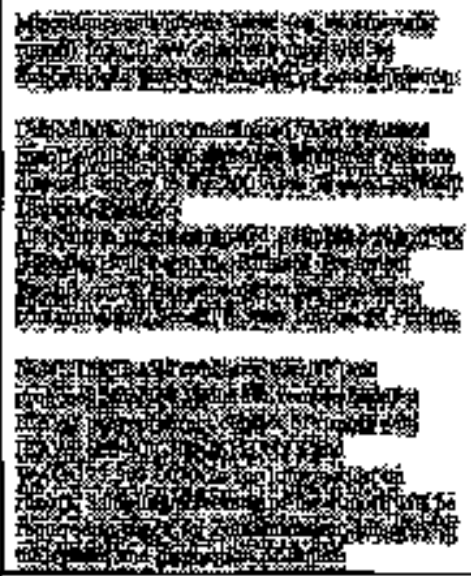 \\
\hline WAS JT3.400 & $\begin{array}{l}\text { General Regulations } \\
\text { for Air Poltution Sources }\end{array}$ & & 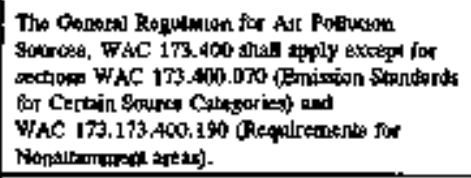 \\
\hline $\begin{array}{c}\text { DOE } \$ \$ 20.2 \mathrm{~A} \\
\text { DOE } 5280.2 \mathrm{~A} .3 .3 . \mathrm{e} 3\end{array}$ & Hanford Site Perrmilts & $Y$ & 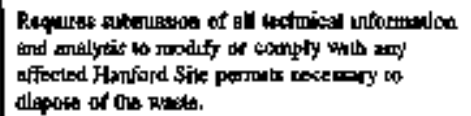 \\
\hline
\end{tabular}


Miscellaneous

\begin{tabular}{|c|c|c|c|}
\hline Document Identifier & Tithe & Applicable & hostifikation \\
\hline $\begin{array}{c}\text { "Bader k0 Trago" Letier } \\
\text { 95-PRIÁfT3 }\end{array}$ & $\begin{array}{c}\text { Estimated TWRS Project } \\
\text { Schedule }\end{array}$ & $Y$ & 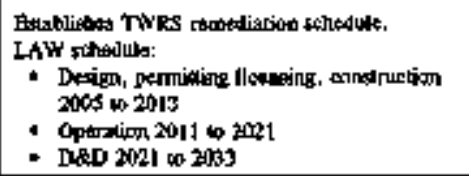 \\
\hline None identified & $\begin{array}{l}\text { SDWP Permit } d S 0 \mathrm{~h} \\
\text { and Fact Sheet }\end{array}$ & $\mathbf{N}$ & 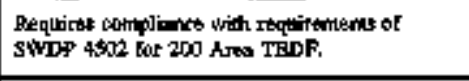 \\
\hline $\begin{array}{l}\text { WAC } 173.440 .141 \\
\text { WAC } 173.460 .060 \\
\text { WHC-CM-7.5 }\end{array}$ & $\begin{array}{l}\text { Toxic Air Emissiont } \\
\text { (Hazrardous Air Pollutants) }\end{array}$ & $\mathbf{N}$ & 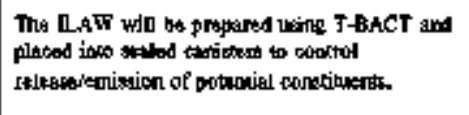 \\
\hline None identified & Rain Waver Infiltration & 慈 & 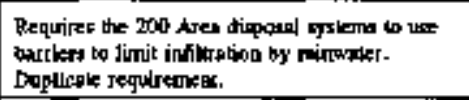 \\
\hline WHC-SD-ETF-WAC-001 & $\begin{array}{l}\text { Eftluent Treatment Facility } \\
\text { Waste Acceptance Criterta }\end{array}$ & 4 & 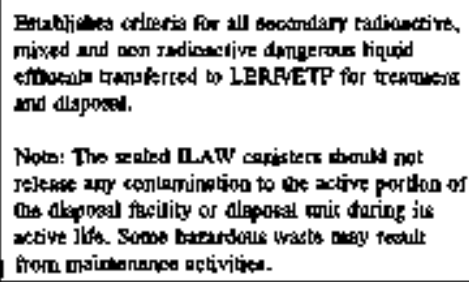 \\
\hline
\end{tabular}


Miscellaneous

\begin{tabular}{|c|c|c|c|}
\hline Docurtent Identifięr & Titule & Applicable & Justification \\
\hline $\begin{array}{l}10 \text { CFR } 20.1301 \\
\text { J0 CFR } 20.1601 \\
10 \text { CFR } 20.1602 \\
10 \text { CFR } 20.1603 \\
10 \text { CFR } 20.1701 \\
10 \text { CFR } 20.1702 \\
40 \text { CFR } 191.03 .2 \\
40 \text { CFR } 192 \\
40 \text { CFR. } 193\end{array}$ & NRC Requirerpents & 湿 & 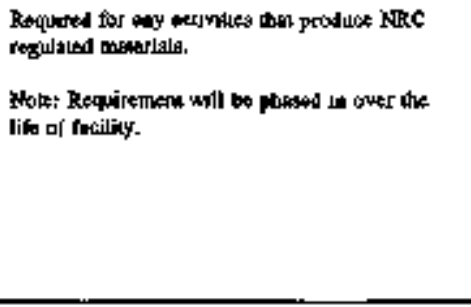 \\
\hline DOE 5400.5.2.1.d & Public Drknking Waker & 18 & 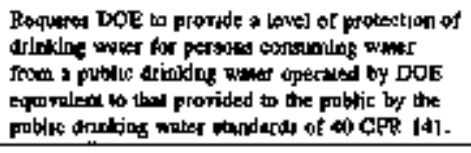 \\
\hline 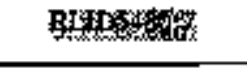 & 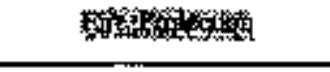 & 畒 & 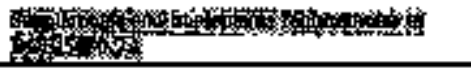 \\
\hline $\begin{array}{l}\text { DOEJEIS-0189 } \\
\text { SEN-35-91. }\end{array}$ & Radloxactive Air Emistions & $\mathbf{Y}$ & 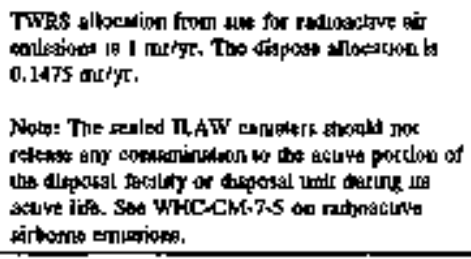 \\
\hline
\end{tabular}


Miscellaneout

\begin{tabular}{|c|c|c|c|}
\hline Dowmenl Identifier & Title & Applicable & Josuification \\
\hline $\begin{array}{c}\text { DOE 5820.2.3.3.g } \\
\text { WHC-EP-0063 }\end{array}$ & $\begin{array}{l}\text { Radioactive and Radinoctire } \\
\text { Dangerous Solid Waste } \\
\text { Forecasting }\end{array}$ & 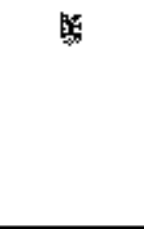 & 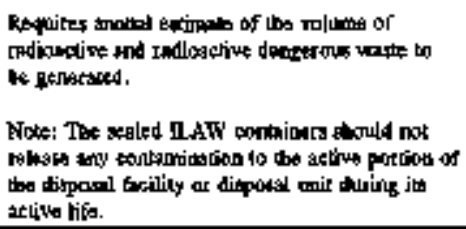 \\
\hline $\begin{array}{c}\text { Decition Condlal Paptet } \\
\text { \#33, Decision 3 } \\
\text { DOE 5820.2A.3.2.c }\end{array}$ & $\begin{array}{l}\text { Radloactlye and Radioactive } \\
\text { Mlixed Waste Disposil }\end{array}$ & $\mathbf{Y}$ & 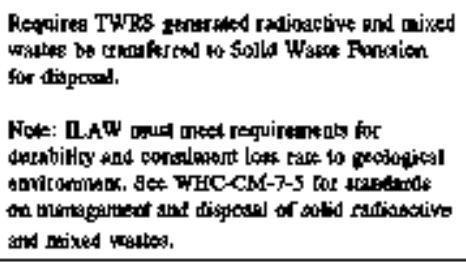 \\
\hline Nons Identified & $\begin{array}{l}\text { ILLW Product Repork } \\
\text { and Retrievability }\end{array}$ & $\mathbf{N}$ & 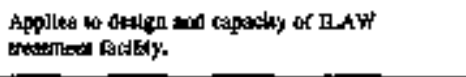 \\
\hline $\begin{array}{l}\text { Dec. Comtrol Paper } 33 \\
\text { Decision } 1,3,4,5,8,11-16\end{array}$ & $\begin{array}{l}\text { A railability of Utilities } \\
\text { and Services }\end{array}$ & $\mathbf{Y}$ & 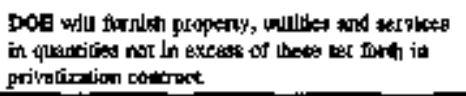 \\
\hline $\begin{array}{l}\text { Fubur for Hanford Џed } \\
\text { and Cleanup (Durmmond) } \\
\text { WHC-\$D-WH-\$E-021 Rev } 0\end{array}$ & Land Use & $\mathbf{N}$ & 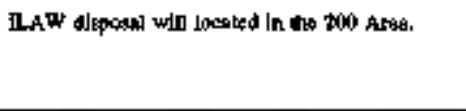 \\
\hline
\end{tabular}


Mjscellaneous

\begin{tabular}{|c|c|c|c|}
\hline Dopument Identifier & Title & Applicablt & Justjficalion \\
\hline $\begin{array}{l}\text { Bemero 1993.1 } \\
10 \text { CFR } 61.55\end{array}$ & Incidendal Waste & $\mathbf{Y}$ & 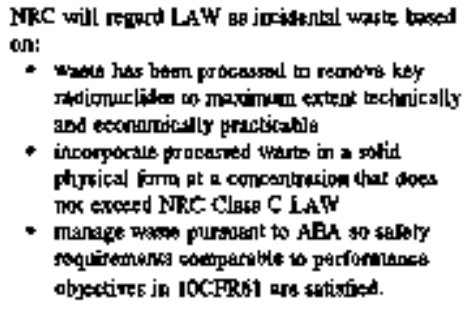 \\
\hline 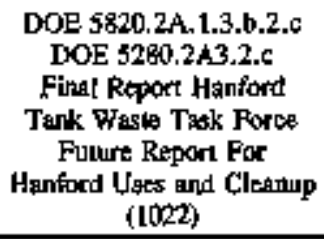 & Disposal Site Location & 歌 & 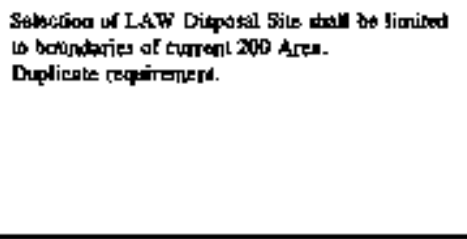 \\
\hline $\begin{array}{c}\text { DOe } 5820.2 A .3 .3 .6 \\
(1030)\end{array}$ & $\begin{array}{l}\text { ILLW Ptrytical } \\
\text { and Cherrical-Propertios }\end{array}$ & $\mathbf{x}$ & 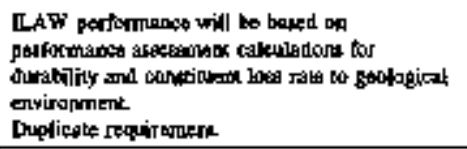 \\
\hline $\begin{array}{l}\text { DOE 5820.2A } \\
\text { WAC (73.303 } \\
\text { (1033) }\end{array}$ & $\begin{array}{c}\text { Dangerous Waste } \\
\text { Mandgemedi }\end{array}$ & 嵒 & 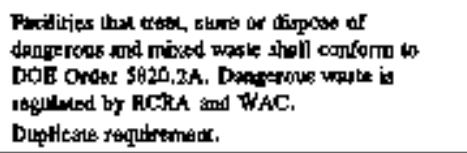 \\
\hline
\end{tabular}


Miscejlaneous

\begin{tabular}{|c|c|c|c|}
\hline Document Identifier & Title & Applicable & Justification \\
\hline $\begin{array}{l}\text { ETF State Discharge } \\
\text { Permil } \\
\text { WHC-CN-7-5 } \\
(1039)\end{array}$ & Ufntreaked Liquid Effluents & 委 & 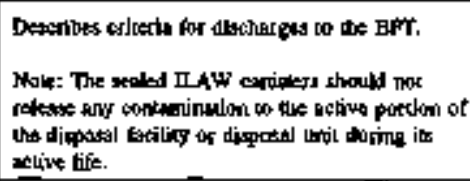 \\
\hline $\begin{array}{l}\text { DOE Direction for } \\
\text { Privatization } \\
(1040)\end{array}$ & $\begin{array}{c}\text { Nonradipnoclide Ainborne } \\
\text { Emissions }\end{array}$ & $\mathbf{N}$ & 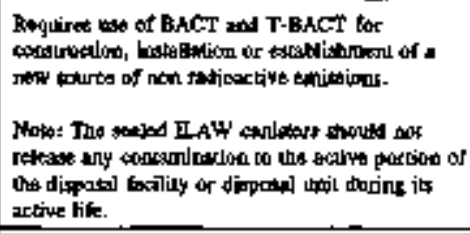 \\
\hline $\begin{array}{l}\text { WAC } 2 A 6.247 \\
\text { WHC-CM-7.5 } \\
(1041)\end{array}$ & $\begin{array}{l}\text { Notification of Startup, } \\
\text { Shutdown or Other Change } \\
\text { That Mty Affect Emisslons } \\
\text { Mesamement }\end{array}$ & 8 & 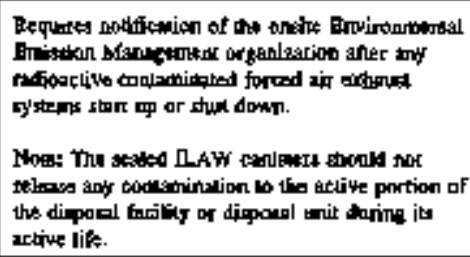 \\
\hline $\begin{array}{l}\text { DOE 6430.IA } \\
(1065)\end{array}$ & Safety Class & $\mathbf{Y}$ & 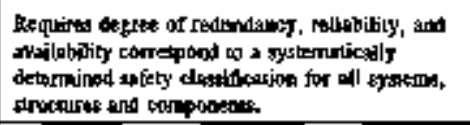 \\
\hline $\begin{array}{c}\text { DOE 6430.1A, 0200-99.0.4 } \\
\text { PNL 4622) } \\
\text { PNL 6415 Rev } 6 \\
(1503)\end{array}$ & Enviroumental Conditions & $\bar{Y}$ & 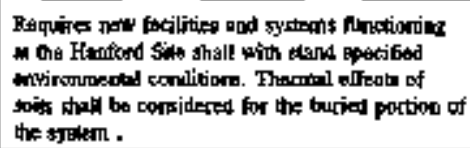 \\
\hline
\end{tabular}


MSisctilansous

\begin{tabular}{|c|c|c|c|}
\hline Doxwrixent Identifier & Title & Applicable & Institieation \\
\hline $\begin{array}{l}\text { DOE 6430.1A, 1300-12.1 } \\
\text { DOE 6430.1A, 1300-12.4.11 } \\
\text { DOE 6430.1A, 1300-12.4.2 } \\
\text { DOE 6430.1A, 1300-12.4.6 } \\
\text { (1512) }\end{array}$ & Humá bactots & Y & 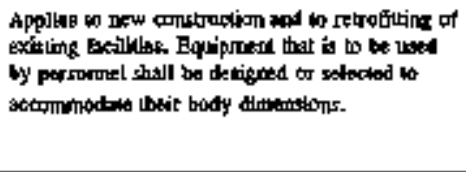 \\
\hline $\begin{array}{l}\text { DOE 6430, IA, 1300-[.3 } \\
\text { WHE-EP-DT50,1],9.10 } \\
\text { (1514) }\end{array}$ & ALARA Design & $\mathbf{Y}$ & 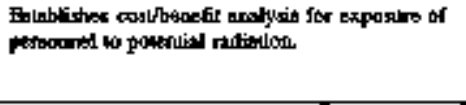 \\
\hline $\begin{array}{c}\text { DOE } 2 \text { EIS }-0189 \\
\text { (2010) }\end{array}$ & Site Bounkary & $\mathbf{N}$ & 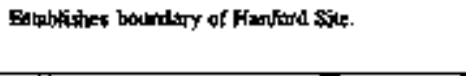 \\
\hline $\begin{array}{l}\text { DOE } 1540.1 \\
\text { DOE-95-5WT-166 } \\
\text { WHC-CM-2-14 } \\
\text { WHC.FP.0063 } \\
\text { (2019) }\end{array}$ & $\begin{array}{c}\text { Radioactive Solid Wagte } \\
\text { Trantsfer }\end{array}$ & $\mathbf{N}$ & 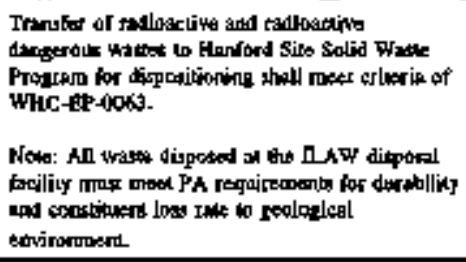 \\
\hline $\begin{array}{c}10 \text { CFR } 835 \\
\text { WHC-\$D-GN.DGS-30,011 } \\
(2023)\end{array}$ & Shiceldiling Criterta & 量 & 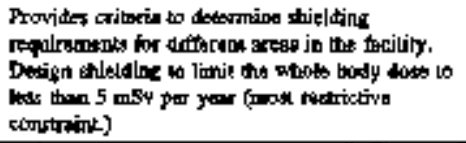 \\
\hline
\end{tabular}


Miscellaneous

\begin{tabular}{|c|c|c|c|}
\hline Dopeument ldentifies & Tidte & Applicable & Justifichation \\
\hline $\begin{array}{l}\text { ANSLANS-3.2-88 } \\
\text { ASWE-NQA-1-1-1989-1A } \\
\text { ASWE-NQA-1-1994-IA } \\
\text { DOE-STD-1073-93 } \\
\text { DOE 5500.7b } \\
(2031)\end{array}$ & Documentation & $\mathbf{Y}$ & 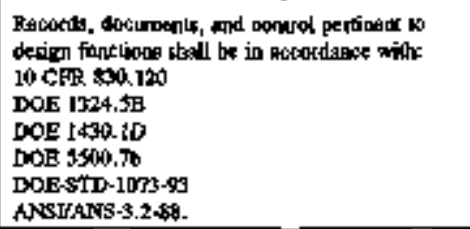 \\
\hline $\begin{array}{l}\text { Dop Dectslou Paper: } \\
\text { Specification } \\
\text { of Radionuclide } \\
\text { Concentrabions in } \\
\text { THRS Low-Activity Waste } \\
(2040)\end{array}$ & Glass/Malrix Waste Form & $\mathbf{N}$ & 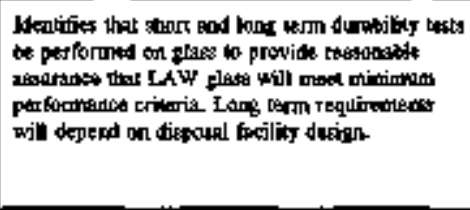 \\
\hline $\begin{array}{c}10 \text { CFR 6) } \\
10 \text { CFR 6I.55 } \\
10 \text { CFR } 193 \\
\text { DOE Order } \$ 820.2 \mathrm{~A} \\
(205 \mathrm{I})\end{array}$ & Dieposal Facility & $\mathbf{Y}$ & 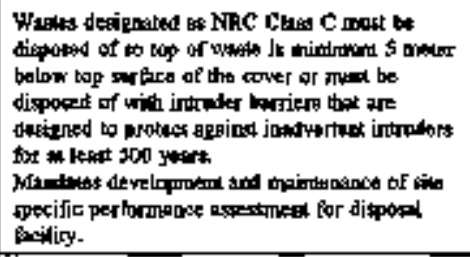 \\
\hline $\begin{array}{c}\text { DOE } 5280.2 A .3 .3 . j .9 \\
(2052)\end{array}$ & $\begin{array}{c}\text { Dlsposal Site } \\
\text { Excavation Alarkers }\end{array}$ & 菻 & 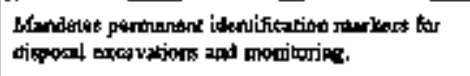 \\
\hline
\end{tabular}


Miscellaneous

\begin{tabular}{|c|c|c|c|}
\hline Document Identifier & Title & Applicable & Intification \\
\hline $\operatorname{Sen} .35 .91$ & Nuclear Safely Podicy & $\mathbf{Y}$ & 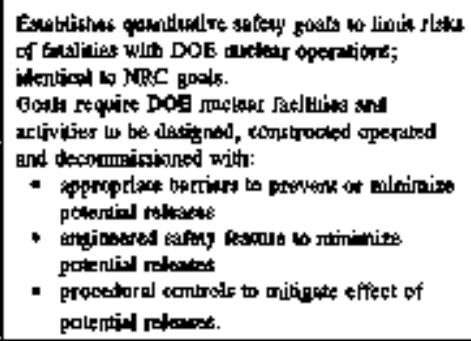 \\
\hline $\begin{array}{l}\text { Deciaion Conirol Paper } \\
\text { "33, Decistern I6 } \\
\text { (PDC,TR.83) }\end{array}$ & Transportadlon Container & $\mathbf{Y}$ & 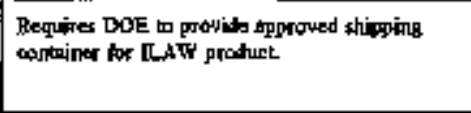 \\
\hline $\begin{array}{l}\text { Decision Control Paper } \\
\text { \#33, Decision 16 } \\
\text { (ROC.TR.6) }\end{array}$ & Transpontalion Vebicles & $\mathrm{Y}$ & 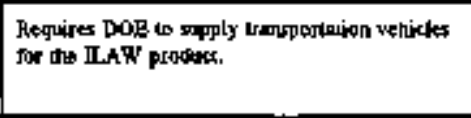 \\
\hline $\begin{array}{l}\text { Decision Control Paper } \\
\text { F3, Decision 16 } \\
\text { DOE 96-RTY-035 } \\
\text { (POC,TR.4) }\end{array}$ & $\begin{array}{l}\text { Itifrastructure Support } \\
\text { for Transportation }\end{array}$ & $\mathbf{Y}$ & 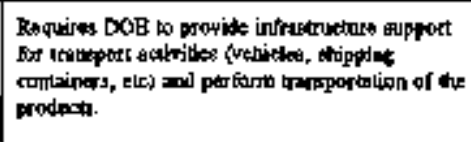 \\
\hline
\end{tabular}


Miscellanomus

\begin{tabular}{|c|c|c|c|}
\hline Bocument Identifier & Tiuk & Applicable & Justurlcatbon \\
\hline DOE 5480.11 & $\begin{array}{l}\text { Worket Rodidologicad Strety; } \\
\text { Hanford Sice Radiological } \\
\text { Contrd Mandal Chapter 1, } \\
\text { Parı } 1 \text { Section } 11 \text { I and I12 }\end{array}$ & $\mathbf{Y}$ & 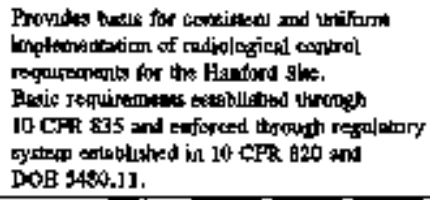 \\
\hline $\begin{array}{c}\text { Unkni⿱宀女n } \\
\text { (PDC.B.1\} }\end{array}$ & ILLW' Shipping frequtacy & $\mathbf{T B D}$ & Urowan \\
\hline
\end{tabular}

幽 
Constraints an Design of the I.

Weslingbouse Hanford Company

\begin{tabular}{|c|c|c|c|}
\hline Document ]dentifier & Tille & Applicable & Justification \\
\hline WHC-CM-2-14 & $\begin{array}{c}\text { Hazardous Malerial } \\
\text { Packaging ad Shippiug }\end{array}$ & $\mathbf{N}$ & 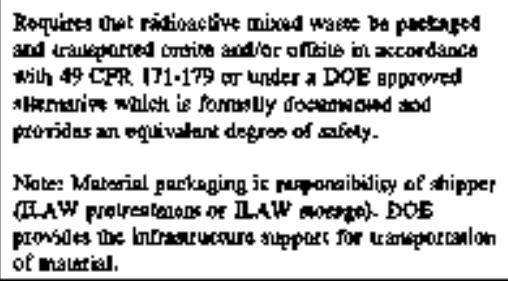 \\
\hline WHC-CM-7-5 & $\begin{array}{c}\text { Radicactive Air Emiations } \\
\text { Sile Wide }\end{array}$ & $\mathbf{Y}$ & 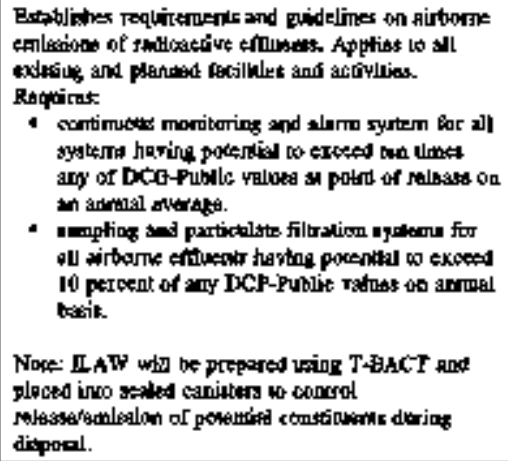 \\
\hline
\end{tabular}




\begin{tabular}{|c|c|c|c|c|c|}
\hline \multirow{2}{*}{$\begin{array}{l}\text { Fo } \\
\text { Distributtan }\end{array}$} & \multirow{2}{*}{\multicolumn{3}{|c|}{ From }} & \multicolumn{2}{|l|}{ Page 1 of 1} \\
\hline & & & & \multicolumn{2}{|c|}{ Dete $10 / 03 / 97$} \\
\hline \multicolumn{4}{|c|}{ Project Titlentwork Ordes } & \multicolumn{2}{|c|}{ EOT Ha. 622687 } \\
\hline \multicolumn{4}{|c|}{$\begin{array}{l}\text { Wnolysis of hiternatives for lomobllized Low-Activity Waste } \\
\text { Disposal, HAF-SD-ThR-AGA-004, Rev. 0 }\end{array}$} & \multicolumn{2}{|l|}{ ECN No. } \\
\hline Name & MSN & $\begin{array}{c}\text { Toxt } \\
\text { Wrth Al } \\
\text { Att:alo, }\end{array}$ & Toxt Onlr & $\begin{array}{l}\text { Attachit } \\
\text { Apporndix } \\
\text { Outly }\end{array}$ & $\begin{array}{l}\text { EDTrECN } \\
\text { Draly }\end{array}$ \\
\hline
\end{tabular}

Central F1tac

pol toonting toon

d. W. hl l bart

I. T. Arfibrat

ㅁ. J. dahley

s. K, herer

v. F. purila

t. R, coriech

u. L. Itoren 20

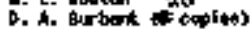

K. c. Detrot

L. H. Gldiran

R. D, talutut

1. c c lam

A. D, troht

k. क. CIx力口卄

4. Cox

P. A. Crala

4. DoLemet Int

6. G. Erlendoas

d. P. Ertradlado

1. L Fart

K. h. acapar

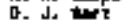

J. I. Indarzon

k. t. UIskk

J. D. Mankaten

h. D. hetyby

A. d. HIom

b. R. Lures

F, M. Howim

R. J. Wurtounki

k. d. Paralin

J. A. Parcier

C. A. Pateroth

d. E. Palper .

J. H. Ehode

Q. 3 in

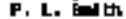

4, 1. Thoratpon

R. L. Treat

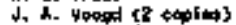

d. J. Hackent tolder

I. F. Yonctr

E. บ. Danke tooes

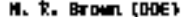

P. E. Lowang toof]

R. W. LTER (DOE)

R. H. Uifuralp Case?
$01-67$
91-65

ค1-65

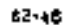

ㅁ. 41

T2.

月5- 47

ค3-1

月3.5

t5-21

H3- 27

H5-03

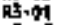

45-2?

แ. 27?

mi-5s

แ. 69

ha-ys

ตร-21

c)-61

202-36

12-36

15.61

$1+3-37$

중ำ

15.14

500

다-기

15-1.

+5.27

T4-012

1.)-31

武 10

16-49

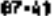

is-zT

\$5.2t

15- 7

ต-71

다마

하-21

К.0

16-63

$57 \cdot d 0$

[3- 07

57.53

K.

$\$ 7.53$

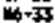

15.11

त

$$
\text { x }
$$$$
4
$$$$
\text { x }
$$

$$
\begin{aligned}
& k \\
& k \\
& k
\end{aligned}
$$

$$
\begin{aligned}
& k \\
& \text { K } \\
& \text { g }
\end{aligned}
$$

$$
\text { . }
$$$$
\begin{aligned}
& x \\
& x
\end{aligned}
$$$$
x
$$$$
x
$$$$
x
$$$$
\underset{x}{x}
$$$$
\mathbf{x}
$$$$
\begin{aligned}
& x \\
& x \\
& x \\
& x
\end{aligned}
$$$$
\begin{aligned}
& x \\
& x
\end{aligned}
$$$$
x
$$$$
\begin{aligned}
& \mathbf{x} \\
& \mathbf{x} \\
& \mathbf{x}
\end{aligned}
$$$$
x
$$$$
\begin{aligned}
& x \\
& x \\
& x \\
& x
\end{aligned}
$$$$
\begin{array}{r}
\mathbf{x} \\
\mathbf{x}
\end{array}
$$$$
\begin{aligned}
& \mathbf{x} \\
& \mathbf{x} \\
& \mathbf{k}
\end{aligned}
$$$$
\begin{aligned}
& \mathbf{x} \\
& k \\
& k
\end{aligned}
$$ 